\title{
Large Eddy Simulation of Turbulent Flow over a Backward-Facing Step
}

\author{
Edmond Shehadi
}

Institutionen för informationsteknologi 


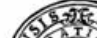 \\ (1) \\ UPPSALA UNIVERSITET}

Teknisk- naturvetenskaplig fakultet UTH-enheten

Besöksadress:

Ångströmlaboratoriet

Lägerhyddsvägen 1

Hus 4, Plan 0

Postadress:

Box 536

75121 Uppsala

Telefon:

$018-4713003$

Telefax:

$018-4713000$

Hemsida:

http://www.teknat.uu.se/student

\section{Abstract \\ Large Eddy Simulation of Turbulent Flow over a Backward-Facing Step}

Edmond Shehadi

This work studies the effect of grid resolution and subgrid scale modeling on the predictive accuracy of Large Eddy Simulation. In particular, the problems considered are: turbulent flow over a backward-facing step and fully-developed turbulent channel flow. A SGS-free model and four subgrid scale models are used: Smagorsinky, k-equation, dynamic k-equation and WALE. Different combinations of streamwise and spanwise grid resolutions are considered along with different strategies for the cell-size distribution in the wall-normal direction.

First, a detailed study pertaining to fully-developed turbulent channel flow is presented for target friction Reynolds numbers 180 and 300. A symmetric three-layered wall-normal meshing strategy in conjunction with a SGS-free model is shown to be the best compromise between computational efficiency and agreement with benchmark data. Overall, the accuracy of the results was observed to be most sensitive to the spanwise resolution of the grid. Cell sizes in wall units ranging in between 25-28 and 12-20 in the streamwise and spanwise directions, respectively, yielded excellent agreement with reference data.

Second, a detailed study of turbulent flow over a backward-facing step at a step-height Reynolds number of 5100 is conducted. This includes analysis of first- and second-order statistical moments of the velocity field for three different grid refinement levels and several SGS models. Additional quantities are computed on the finest grid, such as high-order statistics, one-dimensional spanwise power spectral density and spatial autocorrelation of the velocity, turbulent kinetic energy budget, as well as the first- and second-order statistics of the vorticity field. In line with the channel flow study, the WALE and SGS-free models produce the best results when compared with experimental and numerical benchmark data. All presented datasets are made available online for public use.
Handledare: Saleh Rezaeiravesh Ämnesgranskare: Timofey Mukha Examinator: Jarmo Rantakokko IT 18009

Tryckt av: Reprocentralen ITC 
"Out of clutter, find simplicity. From discord, find harmony. In the middle of difficulty lies opportunity."

- Albert Einstein 
THIS PAGE IS INTENTIONALLY LEFT BLANK 


\section{Acknowledgments}

I would like to start off by expressing my gratitude to Uppsala University for all the resources I had access to. Also, I would like to thank all the members involved in the evaluation of my report, such as my supervisors, reviewer and examiner. I highly appreciate the time investment they put forth into helping me improve my thesis.

More particularly, I would like to thank my two supervisors: Timofey Mukha and Saleh Rezaeiravesh. They both helped introduce me to turbulence modeling as well as bolstered my motivation for advanced CFD studies. Moreover, the exciting scientific discussions pertaining to CFD were fun and essential to this work's fruition and my career development. Thank you for offering me this amazing opportunity to work on something I really enjoy.

On a personal level, I would like to express my eternal gratitude to my wonderful parents: Antoine, George, Aida and Kenaan. They were/are always there for me, despite the distance. You perpetually advocate me to become a better person and consistently place very high emphasis on my education, while making sure I have everything I need to achieve it; you believed in my success, even when I, myself, did not. I cannot find words to express how indebted I am to your unceasing support. Additionally, I thank my dear uncle, Antoine, for all his support throughout the recent years of my academic life. Also, I cannot but mention my friends (especially Michel), both near and far, that helped me along my academic journey and made it a little more fun and much more bearable.

Last, but definitely not least, I want to thank God. In my humble opinion, while I did my part and worked diligently, yet none of the outstanding opportunities could have ever been possible for me if it wasn't - and still isn't - for him. Also, I thank God for all the delightful people I have met, and still meet, along my journey.

The computations were performed on resources provided by the Swedish National Infrastructure for Computing (SNIC) at PDC Centre for High Performance Computing (PDC-HPC). 
THIS PAGE IS INTENTIONALLY LEFT BLANK 


\section{Contents}

1 Introduction 1

2 Turbulence Modeling $\quad 7$

2.1 Navier-Stokes Equations . . . . . . . . . . . . . . . . . . 7

2.2 Direct Numerical Simulation . . . . . . . . . . . . . . . . . . 9

2.3 Reynolds Averaged Navier-Stokes . . . . . . . . . . . . . . . . . . . . 11

2.4 Large Eddy Simulation . . . . . . . . . . . . . . . . . . 13

2.4.1 Smagorinsky Model . . . . . . . . . . . . . . . . . 16

2.4.2 SGS Turbulent Kinetic Energy Equation Model . . . . . . . . 18

2.4.3 Dynamic SGS TKE Equation Model . . . . . . . . . . . . 19

2.4.4 Wall-Adapting Local Eddy-viscosity (WALE) Model . . . . . . 20

2.4.5 Subgrid-Scale (SGS)-Free Model . . . . . . . . . . . . . 23

3 Numerical Implementation $\quad 25$

3.1 The Finite Volume Method . . . . . . . . . . . . . . 25

3.1.1 Spatial Discretization . . . . . . . . . . . . 27

3.1.1.1 Convective Term . . . . . . . . . . . . 27

3.1.1.2 Diffusive Term . . . . . . . . . . . . . . 27

3.1.2 Temportal Discretization . . . . . . . . . . . . . 28

3.1.3 System of Algebraic Equation . . . . . . . . . . . . . . . . 29

3.2 Pressure-velocity Coupling . . . . . . . . . . . . . . . . . 31

3.2.1 SIMPLE Algorithm . . . . . . . . . . . . . . . . . . . . 31

3.2.2 PISO Algorithm . . . . . . . . . . . . . . . 32 
$3.2 .3 \quad$ PIMPLE . . . . . . . . . . . . . . . . . . . 32

4 Wall-bounded Turbulence 35

4.1 Wall-bounded Flows . . . . . . . . . . . . . . . 36

4.1.1 Turbulent Boundary Layer Flow . . . . . . . . . . . . 36

4.1.2 Turbulent Channel Flow . . . . . . . . . . . . . . . 38

4.2 Near-wall Flow Characteristics . . . . . . . . . . . . . . . . . 40

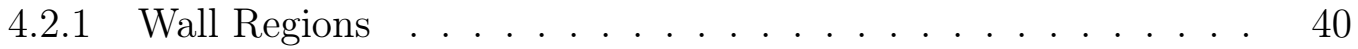

4.2 .2 Velocity Fluctuation Behavior . . . . . . . . . . . . . 42

5 Turbulent Channel Flow $\quad 45$

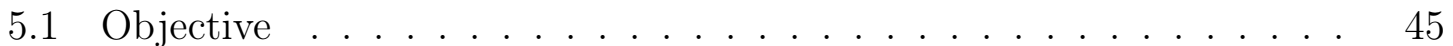

5.2 Literature Review . . . . . . . . . . . . . . . . . . . 46

5.3 Simulation Set-up . . . . . . . . . . . . . . . . . . . . . . 48

5.3 .1 Computational Domain . . . . . . . . . . . . . . . . . . 49

5.3.2 Initial and Boundary Conditions . . . . . . . . . . . . . . 51

5.3 .3 Physical and Flow Parameters . . . . . . . . . . . . . . . 52

5.4 Results and Discussion . . . . . . . . . . . . . . . . . . 52

5.4.1 Numerical Simulations for a Target $\operatorname{Re}_{\tau}$ of $180 \ldots \ldots$

5.4.1.1 Mean Velocity Profiles . . . . . . . . . . . . . . 54

5.4.1.2 Reynolds Stress Components . . . . . . . . . . . 56

5.4.1.3 Vorticity Fluctuations . . . . . . . . . . . . . 58

5.4.1.4 Turbulent Kinetic Energy Budget . . . . . . . . . . 60

5.4.1.5 High-order Central Statistical Moments . . . . . . . 62

5.4.2 SGS Study on the Same Wall-normal Grid Resolution . . . . . 65

5.4.2.1 Mean Velocity Profiles . . . . . . . . . . . . . 67

5.4.2.2 Reynolds Stress Components _. . . . . . . . . . 67

5.4.3 SGS Study on Different Wall-normal Grid Resolutions . . . . 73

5.4.3.1 Mean Velocity Profiles . . . . . . . . . . . . . . . 74

5.4.3.2 Reynolds Stress Components _. . . . . . . . . . 75

5.4.3.3 Subgrid Scale Viscosity _ . . . . . . . . . . 78 
5.4.4 Spectral Analysis of SGS Models and Grid Resolutions . . . . 78

5.4.4.1 One-dimensional Streamwise Power Spectral Density 81

5.4.4.2 One-dimensional Spanwise Power Spectral Density · 92

5.4.4.3 Convergence of the Friction Velocity . . . . . . . . 102

5.5 Concluding Remarks . . . . . . . . . . . . . . . . . . . 103

6 Turbulent Flow over a BFS 107

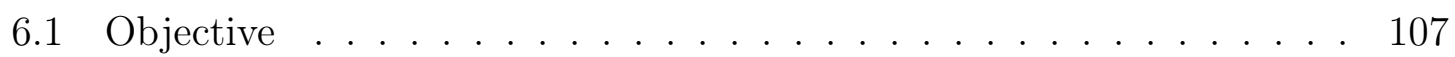

6.2 Literature Review . . . . . . . . . . . . . . . . . . . . 108

6.3 Simulation Set-up . . . . . . . . . . . . . . . . 110

6.3 .1 Computational Domain . . . . . . . . . . . . . . . . 112

6.3.2 Initial and Boundary Conditions . . . . . . . . . . . . 114

6.3.3 Physical and Flow Parameters . . . . . . . . . . . . 115

6.4 Results and Discussion . . . . . . . . . . . . . . . . 116

6.4.1 Numerical Simulation: Coarse Mesh . . . . . . . . . . . . . 117

6.4.1.1 Mean Streamwise Velocity . . . . . . . . . . . . 118

6.4.1.2 Reynolds Stress Components . . . . . . . . . . . 119

6.4.1.3 Pressure and Skin-friction Coefficients . . . . . . . 121

6.4.1.4 Friction Velocity and Friction Reynolds Number . . . 122

6.4.2 Numerical Simulation: Intermediate Mesh . . . . . . . . . 123

6.4.2.1 Mean Streamwise Velocity . . . . . . . . . . . . . 124

6.4.2.2 Reynolds Stress Components _. . . . . . . . . 126

6.4.2.3 Pressure and Skin-friction Coefficients . . . . . . 128

6.4.2.4 Friction Velocity and Friction Reynolds Number . . . 128

6.4.3 Numerical Simulation: Fine Mesh . . . . . . . . . . . . . . 129

6.4.3.1 Mean Streamwise Velocity . . . . . . . . . . . . 130

6.4.3.2 Reynolds Stress Components . . . . . . . . . . . 131

6.4.3.3 Pressure and Skin-friction Coefficients . . . . . . 132

6.4.3.4 Friction Velocity and Friction Reynolds Number . . . 133

6.4.4 Comprehensive Numerical Simulation on a Fine Mesh . . . . . 134 
6.4.4.1 Mean Pressure Profiles . . . . . . . . . . . . . . . 134

6.4.4.2 Mean Velocity Profiles . . . . . . . . . . . . . . 134

6.4.4.3 Reynolds Stress Components _. . . . . . . . . . 139

6.4.4.4 Vorticity . . . . . . . . . . . . . . . . . 142

6.4.4.5 High-order Statistical Moments . . . . . . . . . . 147

6.4.4.6 Turbulent Kinetic Energy Budget . . . . . . . . . . 160

6.4.4.7 One-dimensional Spanwise Spectra . . . . . . . . 167

6.4.4.8 Auto-correlation Function in the Spanwise Direction 172

6.4.4.9 Probability of Backflow and Reattachment . . . . . 178

6.4.5 Effects of Grid Refinement . . . . . . . . . . . . . . . . 179

6.4.5.1 Cell Sizes . . . . . . . . . . . . . . . . . 179

6.4.5.2 Subgrid Scale Viscosity _ . . . . . . . . . . . 181

6.4.5.3 Mean Streamwise Velocity and RMS of Wall-normal Velocity . . . . . . . . . . . . . . . . 181

6.4.5.4 Flow Reattachment Estimation . . . . . . . . . 183

6.5 Concluding Remarks . . . . . . . . . . . . . . . . . . . 183

$\begin{array}{lll}7 & \text { Conclusion and Future Work } & 187\end{array}$

$\begin{array}{ll}\text { A Energy and Power Spectral Density } & 199\end{array}$

A.1 Energy/Power Spectra in One Dimension . . . . . . . . . . . . . 199

A.1.1 Continuous Space . . . . . . . . . . . . . . . . . . . 199

A.1.2 Discrete Space. . . . . . . . . . . . . . . . . . . . . . . . . 201

A.1.3 Parseval's Relation to (homogeneous) Turbulence . . . . . . . 202

A.2 Power Spectra in One Dimension . . . . . . . . . . . . . . . 204

A.2.1 Relation between PSD and ACF . . . . . . . . . . 208

B Turbulent Kinetic Energy Budget $\quad 211$

B.1 Convection . . . . . . . . . . . . . . . . . . . . . . . . . 212

B.2 Production . . . . . . . . . . . . . . . . . . . 213

B.3 Viscous Dissipation . . . . . . . . . . . . . . . . . 213 
B.4 Transport by Viscous Diffusion _... . . . . . . . . . . 213

B.5 Transport by Velocity-Pressure Gradient . . . . . . . . . . 214

B.6 Transport by Turbulence . . . . . . . . . . . . . . . . . . . 214

C Implementation of Some SGS Models in OpenFOAM 215

C.1 Smagorinsky Model . . . . . . . . . . . . . . . . . . . . . 215

C.2 SGS Turbulent Kinetic Equation Equation Model . . . . . . . . . 218

D High-order Statistics $\quad 219$

D.1 Third Moment. . . . . . . . . . . . . . . . . . 220

D.2 Fourth Moment . . . . . . . . . . . . . . . . . 220

D.3 Fifth Moment . . . . . . . . . . . . . . . . . 220

E Additional Flow over a BFS Figures 221

E.1 Coarse Grid . . . . . . . . . . . . . . . . . . . . . . . . . 222

E.2 Intermediate Grid . . . . . . . . . . . . . . . 226

E.3 Fine Grid . . . . . . . . . . . . . . . . . . 230 
THIS PAGE IS INTENTIONALLY LEFT BLANK 


\section{List of Figures}

4-1 Sketch illustrating how the mean flow develops over a boundary layer (a) and a 2D slice taken from a LES depicting the instantaneous velocity magnitude $(\mathrm{m} / \mathrm{s})$ of a developed TBL $(\mathrm{b})$. . . . . . . . . .

4-2 Sketch illustrating how the mean flow develops over a channel flow (a) and a $2 \mathrm{D}$ slice taken from a LES depicting the instantaneous velocity magnitude $(\mathrm{m} / \mathrm{s})$ of a fully-developed turbulent channel flow (b). . . .

4-3 Mean streamwise velocity component versus the wall-normal coordinate taken from a fully-developed turbulent channel flow with a target $\operatorname{Re}_{\tau}$ of 300 using LES, expressed in outer scaling (a) and inner scaling

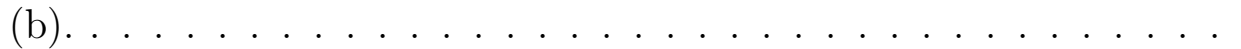

4-4 Scaling of Reynolds stress components as a function of the wall-normal coordinate near the wall. These are taken from a LES of fully-developed turbulent channel flow for a target $\operatorname{Re}_{\tau}=300 . \ldots \ldots$. . . . . . .

5-1 Channel computational domain with respect to the global Cartesian coordinate system (dark surfaces correspond to walls). . . . . . . . . 49

5-2 Transient phase for $u_{\tau}$ taken from a LES with target $\operatorname{Re}_{\tau}=300$. . . $\quad 52$

5-3 Inner-scaled grid resolution of the wall-normal direction up to $\delta$ for a target $\operatorname{Re}_{\tau}=180 \ldots \ldots \ldots \ldots \ldots \ldots$

5-4 Inner-scaled (a) and outer-scaled (b) mean streamwise velocity profile

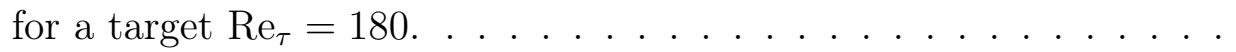

5-5 Inner-scaled (a) and outer-scaled (b) mean spanwise velocity profile for a target $\operatorname{Re}_{\tau}=180 \ldots \ldots \ldots \ldots \ldots \ldots$ 
5-6 Inner-scaled $u_{r m s}^{\prime}(\mathrm{a}),-\left\langle u^{\prime} v^{\prime}\right\rangle$ (b), $v_{r m s}^{\prime}$ (c) and $w_{r m s}^{\prime}$ (d) for a target

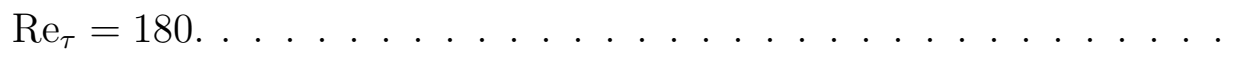

5-7 Outer-scaled $u_{r m s}^{\prime}(\mathrm{a}),-\left\langle u^{\prime} v^{\prime}\right\rangle(\mathrm{b}), v_{r m s}^{\prime}(\mathrm{c})$ and $w_{r m s}^{\prime}(\mathrm{d})$ for a target

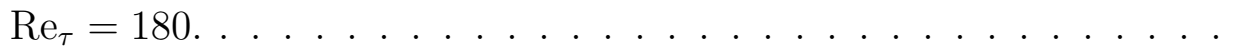

5-8 Non-dimensionalized RMS of the streamwise vorticity versus the innerscaled (a) and the outer-scaled (b) wall-normal coordinate for a target $\operatorname{Re}_{\tau}=180 \ldots \ldots \ldots \ldots \ldots \ldots \ldots \ldots$

5-9 Non-dimensionalized RMS of the wall-normal vorticity versus the innerscaled (a) and the outer-scaled (b) wall-normal coordinate for a target $\operatorname{Re}_{\tau}=180 \ldots \ldots \ldots \ldots \ldots \ldots \ldots \ldots$

5-10 Non-dimensionalized RMS of the spanwise vorticity versus the innerscaled (a) and the outer-scaled (b) wall-normal coordinate for a target

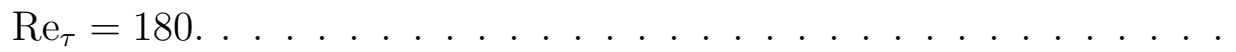

5-11 TKE budget non-dimensionalized by $u_{\tau}$ and $\nu$ for the transport by viscous diffusion $\mathcal{T}^{(\nu)}$ (a), velocity-pressure gradient $\mathcal{T}^{(u p)}$ (b) and turbulence $\mathcal{T}^{(u u)}(\mathrm{c})$ and the production $\mathcal{P}_{k}$ (d) and viscous dissipation $\varepsilon_{k}$

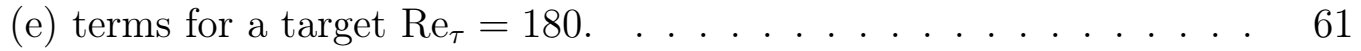

5-12 Non-dimensionalized skewness of the streamwise velocity versus the inner-scaled (a) and the outer-scaled (b) wall-normal coordinate for a

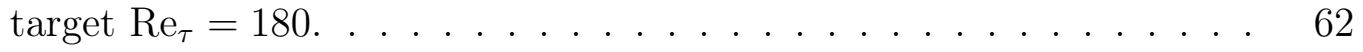

5-13 Non-dimensionalized skewness of the wall-normal velocity versus the inner-scaled (a) and the outer-scaled (b) wall-normal coordinate for a

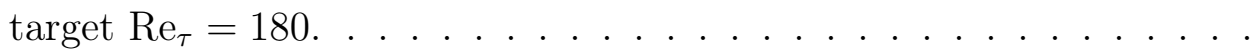

5-14 Non-dimensionalized skewness of the spanwise velocity versus the innerscaled (a) and the outer-scaled (b) wall-normal coordinate for a target

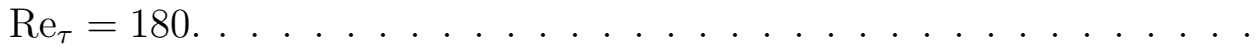

5-15 Non-dimensionalized kurtosis of the streamwise velocity versus the inner-scaled (a) and the outer-scaled (b) wall-normal coordinate for a target $\operatorname{Re}_{\tau}=180 \ldots \ldots \ldots \ldots \ldots \ldots \ldots \ldots \ldots \ldots \ldots \ldots \ldots \ldots$ 
5-16 Non-dimensionalized kurtosis of the wall-normal velocity versus the inner-scaled (a) and the outer-scaled (b) wall-normal coordinate for a target $\operatorname{Re}_{\tau}=180 \ldots \ldots \ldots \ldots$. . . . . . . . . . . .

5-17 Non-dimensionalized kurtosis of the spanwise velocity versus the innerscaled (a) and the outer-scaled (b) wall-normal coordinate for a target $\operatorname{Re}_{\tau}=180 \ldots \ldots \ldots \ldots \ldots$

5-18 Inner-scaled grid resolution in the wall-normal direction up to $\delta$ for a target $\operatorname{Re}_{\tau}=300 \ldots \ldots \ldots \ldots \ldots \ldots$

5-19 Inner-scaled (a) and outer-scaled (b) mean streamwise velocity profile for a target $\mathrm{Re}_{\tau}=300$ using mesh M1-C, M1-I and M1-F. . . . . .

5-20 Inner-scaled $u_{r m s}^{\prime}$ using mesh M1-C (a), M1-I (c) and M1-F (e) and outer-scaled using mesh M1-C (b), M1-I (d) and M1-F (f) for a target $\operatorname{Re}_{\tau}=300 \ldots \ldots \ldots \ldots \ldots \ldots \ldots$

5-21 Inner-scaled $\left\langle u^{\prime} v^{\prime}\right\rangle$ using mesh M1-C (a), M1-I (c) and M1-F (e) and outer-scaled using mesh M1-C (b), M1-I (d) and M1-F (f) for a target $\operatorname{Re}_{\tau}=300 \ldots \ldots \ldots \ldots \ldots \ldots \ldots$

5-22 Inner-scaled $v_{r m s}^{\prime}$ using mesh M1-C (a), M1-I (c) and M1-F (e) and outer-scaled using mesh M1-C (b), M1-I (d) and M1-F (f) for a target $\operatorname{Re}_{\tau}=300 \ldots \ldots \ldots \ldots$. . . . . . . . . . . . . . . . . . . .

5-23 Inner-scaled $w_{r m s}^{\prime}$ using mesh M1-C (a), M1-I (c) and M1-F (e) and outer-scaled using mesh M1-C (b), M1-I (d) and M1-F (f) for a target $\mathrm{Re}_{\tau}=300 \ldots \ldots \ldots \ldots \ldots \ldots \ldots$

5-24 Inner-scaled grid resolutions M1 and M3 displayed in the wall-normal direction up to $\delta$ for a target $\operatorname{Re}_{\tau}=300$.

5-25 Inner-scaled (a) and outer-scaled (b) mean streamwise velocity profile for a target $\operatorname{Re}_{\tau}=300$ using mesh M1-I and M3-I. . . . . . . . . .

5-26 Inner-scaled $u_{r m s}^{\prime}(\mathrm{a}),-\left\langle u^{\prime} v^{\prime}\right\rangle(\mathrm{b}), v_{r m s}^{\prime}(\mathrm{c})$ and $w_{r m s}^{\prime}(\mathrm{d})$ for a target $\operatorname{Re}_{\tau}=300$ using mesh M1-I and M3-I. . . . . . . . . . . . . 76

5-27 Outer-scaled $u_{r m s}^{\prime}(\mathrm{a}),-\left\langle u^{\prime} v^{\prime}\right\rangle(\mathrm{b}), v_{r m s}^{\prime}$ (c) and $w_{r m s}^{\prime}(\mathrm{d})$ for a target $\operatorname{Re}_{\tau}=300$ using mesh M1-I and M3-I. 
5-28 Normalized mean SGS viscosity versus the inner-scaled (a) and the outer-scaled (b) wall-normal coordinate for a target $\operatorname{Re}_{\tau}=300$ using mesh M1-I and M3-I. . . . . . . . . . . . . . . .

5-29 Inner-scaled one-dimensional power spectral density in the streamwise direction for the $u$-velocity component computed with $N_{x}=64$ cells (i.e. $\Delta x^{+} \approx 42$ ) and at wall-normal planes $y^{+} \approx 4.5$ or $y / \delta \approx 0.015$ (a), $y^{+} \approx 14.7$ or $y / \delta \approx 0.05(\mathrm{~b}), y^{+} \approx 150$ or $y / \delta \approx 0.5$ (c) and $y^{+} \approx 294$ or $y / \delta \approx 0.98(\mathrm{~d})$ for a target $\operatorname{Re}_{\tau}=300 . \ldots . . .$.

5-30 Inner-scaled one-dimensional power spectral density in the streamwise direction for the $v$-velocity component computed with $N_{x}=64$ cells (i.e. $\Delta x^{+} \approx 42$ ) and at wall-normal planes $y^{+} \approx 4.5$ or $y / \delta \approx 0.015$ (a), $y^{+} \approx 14.7$ or $y / \delta \approx 0.05$ (b), $y^{+} \approx 150$ or $y / \delta \approx 0.5$ (c) and $y^{+} \approx 294$ or $y / \delta \approx 0.98(\mathrm{~d})$ for a target $\operatorname{Re}_{\tau}=300$. . . . . . . . .

5-31 Inner-scaled one-dimensional power spectral density in the streamwise direction for the $w$-velocity component computed with $N_{x}=64$ cells (i.e. $\Delta x^{+} \approx 42$ ) and at wall-normal planes $y^{+} \approx 4.5$ or $y / \delta \approx 0.015$ (a), $y^{+} \approx 14.7$ or $y / \delta \approx 0.05$ (b), $y^{+} \approx 150$ or $y / \delta \approx 0.5$ (c) and $y^{+} \approx 294$ or $y / \delta \approx 0.98(\mathrm{~d})$ for a target $\operatorname{Re}_{\tau}=300 . \ldots . . . .$.

5-32 Inner-scaled one-dimensional power spectral density in the streamwise direction for the $u$-velocity component computed with $N_{x}=96$ cells (i.e. $\Delta x^{+} \approx 28$ ) and at wall-normal planes $y^{+} \approx 4.5$ or $y / \delta \approx 0.015$ (a), $y^{+} \approx 14.7$ or $y / \delta \approx 0.05$ (b), $y^{+} \approx 150$ or $y / \delta \approx 0.5$ (c) and $y^{+} \approx 294$ or $y / \delta \approx 0.98(\mathrm{~d})$ for a target $\operatorname{Re}_{\tau}=300 . \ldots . . . .$.

5-33 Inner-scaled one-dimensional power spectral density in the streamwise direction for the $v$-velocity component computed with $N_{x}=96$ cells (i.e. $\Delta x^{+} \approx 28$ ) and at wall-normal planes $y^{+} \approx 4.5$ or $y / \delta \approx 0.015$ (a), $y^{+} \approx 14.7$ or $y / \delta \approx 0.05$ (b), $y^{+} \approx 150$ or $y / \delta \approx 0.5$ (c) and $y^{+} \approx 294$ or $y / \delta \approx 0.98(\mathrm{~d})$ for a target $\operatorname{Re}_{\tau}=300 . \ldots . . . .$. 
5-34 Inner-scaled one-dimensional power spectral density in the streamwise direction for the $w$-velocity component computed with $N_{x}=96$ cells (i.e. $\Delta x^{+} \approx 28$ ) and at wall-normal planes $y^{+} \approx 4.5$ or $y / \delta \approx 0.015$ (a), $y^{+} \approx 14.7$ or $y / \delta \approx 0.05$ (b), $y^{+} \approx 150$ or $y / \delta \approx 0.5$ (c) and $y^{+} \approx 294$ or $y / \delta \approx 0.98(\mathrm{~d})$ for a target $\operatorname{Re}_{\tau}=300 . \ldots . . . .$.

5-35 Inner-scaled one-dimensional power spectral density in the streamwise direction for the $u$-velocity component computed with $N_{x}=128$ cells (i.e. $\Delta x^{+} \approx 21$ ) and at wall-normal planes $y^{+} \approx 4.5$ or $y / \delta \approx 0.015$ (a), $y^{+} \approx 14.7$ or $y / \delta \approx 0.05$ (b), $y^{+} \approx 150$ or $y / \delta \approx 0.5$ (c) and $y^{+} \approx 294$ or $y / \delta \approx 0.98(\mathrm{~d})$ for a target $\operatorname{Re}_{\tau}=300$. . . . . . . . . .

5-36 Inner-scaled one-dimensional power spectral density in the streamwise direction for the $v$-velocity component computed with $N_{x}=128$ cells (i.e. $\Delta x^{+} \approx 21$ ) and at wall-normal planes $y^{+} \approx 4.5$ or $y / \delta \approx 0.015$ (a), $y^{+} \approx 14.7$ or $y / \delta \approx 0.05$ (b), $y^{+} \approx 150$ or $y / \delta \approx 0.5$ (c) and

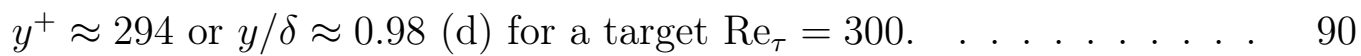

5-37 Inner-scaled one-dimensional power spectral density in the streamwise direction for the $w$-velocity component computed with $N_{x}=128$ cells (i.e. $\Delta x^{+} \approx 21$ ) and at wall-normal planes $y^{+} \approx 4.5$ or $y / \delta \approx 0.015$ (a), $y^{+} \approx 14.7$ or $y / \delta \approx 0.05$ (b), $y^{+} \approx 150$ or $y / \delta \approx 0.5$ (c) and $y^{+} \approx 294$ or $y / \delta \approx 0.98(\mathrm{~d})$ for a target $\operatorname{Re}_{\tau}=300 . \ldots . . .$.

5-38 Inner-scaled one-dimensional power spectral density in the spanwise direction for the $u$-velocity component computed with $N_{x}=64$ cells (i.e. $\Delta x^{+} \approx 42$ ) and at wall-normal planes $y^{+} \approx 4.5$ or $y / \delta \approx 0.015$ (a), $y^{+} \approx 14.7$ or $y / \delta \approx 0.05$ (b), $y^{+} \approx 150$ or $y / \delta \approx 0.5$ (c) and $y^{+} \approx 294$ or $y / \delta \approx 0.98(\mathrm{~d})$ for a target $\operatorname{Re}_{\tau}=300 . \quad$. . . . . . . .

5-39 Inner-scaled one-dimensional power spectral density in the spanwise direction for the $v$-velocity component computed with $N_{x}=64$ cells (i.e. $\Delta x^{+} \approx 42$ ) and at wall-normal planes $y^{+} \approx 4.5$ or $y / \delta \approx 0.015$ (a), $y^{+} \approx 14.7$ or $y / \delta \approx 0.05$ (b), $y^{+} \approx 150$ or $y / \delta \approx 0.5$ (c) and $y^{+} \approx 294$ or $y / \delta \approx 0.98(\mathrm{~d})$ for a target $\operatorname{Re}_{\tau}=300 . \ldots . . .$. 
5-40 Inner-scaled one-dimensional power spectral density in the spanwise direction for the $w$-velocity component computed with $N_{x}=64$ cells (i.e. $\Delta x^{+} \approx 42$ ) and at wall-normal planes $y^{+} \approx 4.5$ or $y / \delta \approx 0.015$ (a), $y^{+} \approx 14.7$ or $y / \delta \approx 0.05$ (b), $y^{+} \approx 150$ or $y / \delta \approx 0.5$ (c) and $y^{+} \approx 294$ or $y / \delta \approx 0.98(\mathrm{~d})$ for a target $\operatorname{Re}_{\tau}=300 . \ldots . . . .$.

5-41 Inner-scaled one-dimensional power spectral density in the spanwise direction for the $u$-velocity component computed with $N_{x}=96$ cells (i.e. $\Delta x^{+} \approx 28$ ) and at wall-normal planes $y^{+} \approx 4.5$ or $y / \delta \approx 0.015$ (a), $y^{+} \approx 14.7$ or $y / \delta \approx 0.05$ (b), $y^{+} \approx 150$ or $y / \delta \approx 0.5$ (c) and $y^{+} \approx 294$ or $y / \delta \approx 0.98(\mathrm{~d})$ for a target $\operatorname{Re}_{\tau}=300 . \ldots . . .$.

5-42 Inner-scaled one-dimensional power spectral density in the spanwise direction for the $v$-velocity component computed with $N_{x}=96$ cells (i.e. $\Delta x^{+} \approx 28$ ) and at wall-normal planes $y^{+} \approx 4.5$ or $y / \delta \approx 0.015$ (a), $y^{+} \approx 14.7$ or $y / \delta \approx 0.05$ (b), $y^{+} \approx 150$ or $y / \delta \approx 0.5$ (c) and $y^{+} \approx 294$ or $y / \delta \approx 0.98(\mathrm{~d})$ for a target $\operatorname{Re}_{\tau}=300$. . . . . . . . . .

5-43 Inner-scaled one-dimensional power spectral density in the spanwise direction for the $w$-velocity component computed with $N_{x}=96$ cells (i.e. $\Delta x^{+} \approx 28$ ) and at wall-normal planes $y^{+} \approx 4.5$ or $y / \delta \approx 0.015$ (a), $y^{+} \approx 14.7$ or $y / \delta \approx 0.05$ (b), $y^{+} \approx 150$ or $y / \delta \approx 0.5$ (c) and $y^{+} \approx 294$ or $y / \delta \approx 0.98(\mathrm{~d})$ for a target $\operatorname{Re}_{\tau}=300 . \ldots . . .$.

5-44 Inner-scaled one-dimensional power spectral density in the spanwise direction for the $u$-velocity component computed with $N_{x}=128$ cells (i.e. $\Delta x^{+} \approx 21$ ) and at wall-normal planes $y^{+} \approx 4.5$ or $y / \delta \approx 0.015$ (a), $y^{+} \approx 14.7$ or $y / \delta \approx 0.05$ (b), $y^{+} \approx 150$ or $y / \delta \approx 0.5$ (c) and

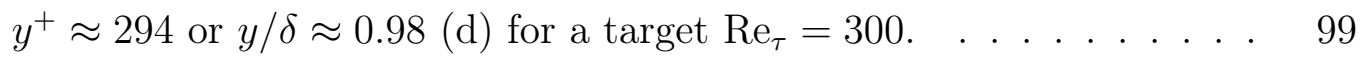

5-45 Inner-scaled one-dimensional power spectral density in the spanwise direction for the $v$-velocity component computed with $N_{x}=128$ cells (i.e. $\Delta x^{+} \approx 21$ ) and at wall-normal planes $y^{+} \approx 4.5$ or $y / \delta \approx 0.015$ (a), $y^{+} \approx 14.7$ or $y / \delta \approx 0.05$ (b), $y^{+} \approx 150$ or $y / \delta \approx 0.5$ (c) and $y^{+} \approx 294$ or $y / \delta \approx 0.98(\mathrm{~d})$ for a target $\operatorname{Re}_{\tau}=300$. . . . . . . . . . 100 
5-46 Inner-scaled one-dimensional power spectral density in the spanwise direction for the $w$-velocity component computed with $N_{x}=128$ cells (i.e. $\Delta x^{+} \approx 21$ ) and at wall-normal planes $y^{+} \approx 4.5$ or $y / \delta \approx 0.015$ (a), $y^{+} \approx 14.7$ or $y / \delta \approx 0.05$ (b), $y^{+} \approx 150$ or $y / \delta \approx 0.5$ (c) and

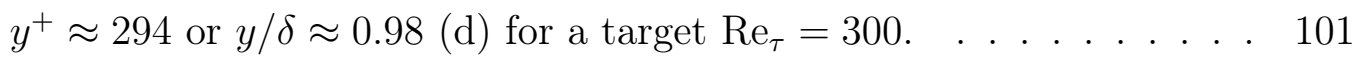

5-47 Relative error of the difference between the converged $u_{\tau}$ of the LES and that of the DNS per grid for a SGS-free (a), a Smagorinsky (b) and a WALE (c) SGS model for a target $\operatorname{Re}_{\tau}=300 . \ldots$. . . . . . . . 102

6-1 Connection between the precursor channel flow and the main BFS flow simulations (a) and expected TBL development along the streamwise direction in the main simulation $(\mathrm{b}) . \ldots . . . . . . . . . .111$

6-2 Backward-facing step geometry with boundary faces. . . . . . . . . 113

6-3 Coarse mesh depiction of BFS in $x y$-plane with a zoom-in near the step.114

6-4 BFS grid resolution (m) of the wall-normal cells versus the wall-normal coordinate (a) and the streamwise cells versus the streamwise coordinate $(\mathrm{b}) \ldots \ldots \ldots \ldots \ldots \ldots$

6-5 Inner-scaled mean streamwise velocity profiles versus the wall-normal coordinate taken at $x / h$ of approximately -3 (a) and 19 (b). . . . . 118

6-6 Outer-scaled mean streamwise velocity profiles taken at six distinct locations in the streamwise direction in the flow over a backward-facing step. . . . . . . . . . . . . . . . . . . . 119

6-7 Outer-scaled $u_{r m s}^{\prime}$ profiles taken at six distinct locations in the streamwise direction in the flow over a backward-facing step. . . . . . . . . . 120

6-8 Outer-scaled $v_{r m s}^{\prime}$ profiles taken at six distinct locations in the streamwise direction in the flow over a backward-facing step. . . . . . . . . . 120

6-9 Outer-scaled $-\left\langle u^{\prime} v^{\prime}\right\rangle$ profiles taken at six distinct locations in the streamwise direction in the flow over a backward-facing step. . . . . . . . . . 121

6-10 Pressure (a) and skin-friction (b) coefficients along the lower wall in the flow over a backward-facing step. . . . . . . . . . . . . . 122 
6-11 Distribution of $\operatorname{Re}_{\tau}$ (a) and $u_{\tau}$ (b) along the streamwise direction over the wall in the flow over a backward-facing step. . . . . . . . . . .

6-12 Inner-scaled mean streamwise velocity profiles versus the wall-normal coordinate taken at $x / h$ of approximately -3 (a) and 19 (b) . . . . .

6-13 Outer-scaled mean streamwise velocity profiles taken at six distinct locations in the streamwise direction in the flow over a backward-facing step. . . . . . . . . . . . . . . . . . . 125

6-14 Outer-scaled $u_{r m s}^{\prime}$ profiles taken at six distinct locations in the streamwise direction in the flow over a backward-facing step. . . . . . . . . 126

6-15 Outer-scaled $v_{r m s}^{\prime}$ profiles taken at six distinct locations in the streamwise direction in the flow over a backward-facing step. . . . . . . . . . 127

6-16 Outer-scaled $-\left\langle u^{\prime} v^{\prime}\right\rangle$ profiles taken at six distinct locations in the streamwise direction in the flow over a backward-facing step. . . . . . . . . . 127

6-17 Pressure (a) and skin-friction (b) coefficients along the lower wall in the flow over a backward-facing step. . . . . . . . . . . . . . . 128

6-18 Distribution of $\operatorname{Re}_{\tau}$ (a) and $u_{\tau}$ (b) along the streamwise direction over the wall in the flow over a backward-facing step. . . . . . . . . . . . . 129

6-19 Inner-scaled mean streamwise velocity profiles versus the wall-normal coordinate taken at $x / h$ of approximately -3 (a) and 19 (b). . . . . 130

6-20 Outer-scaled mean streamwise velocity profiles taken at six distinct locations in the streamwise direction in the flow over a backward-facing

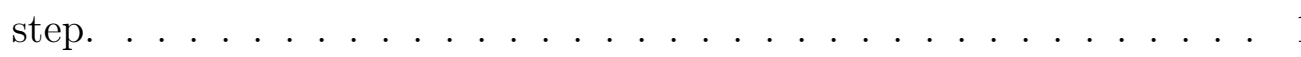

6-21 Outer-scaled $u_{r m s}^{\prime}$ profiles taken at six distinct locations in the streamwise direction in the flow over a backward-facing step. . . . . . . . . .

6-22 Outer-scaled $v_{r m s}^{\prime}$ profiles taken at six distinct locations in the streamwise direction in the flow over a backward-facing step. . . . . . . . . . 132

6-23 Outer-scaled $-\left\langle u^{\prime} v^{\prime}\right\rangle$ profiles taken at six distinct locations in the streamwise direction in the flow over a backward-facing step. . . . . . . . . . 132

6-24 Pressure (a) and skin-friction (b) coefficients along the lower wall in the flow over a backward-facing step. . . . . . . . . . . . . . 133 
6-25 Distribution of $\operatorname{Re}_{\tau}$ (a) and $u_{\tau}$ (b) along the streamwise direction over the wall in the flow over a backward-facing step. . . . . . . . . . .

6-26 Mean pressure for the flow over a backward-facing step using a SGSfree (a) and a WALE (b) model. . . . . . . . . . . . . . . . . . . . 134

6-27 Inner-scaled mean streamwise velocity versus the wall-normal coordinate at all three recovery regions. Profiles include SGS-free (red) and WALE (blue) models including DNS (black) along with the expected log-law and viscous sublayer scaling. . . . . . . . . . . . . . 136

6-28 Outer-scaled mean wall-normal velocity profiles taken at six distinct locations in the streamwise direction in the flow over a backward-facing

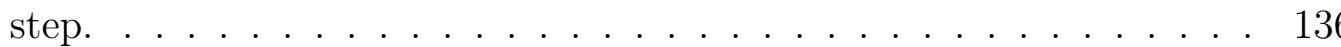

6-29 Inner-scaled mean wall-normal velocity profiles versus the wall-normal coordinate taken at dimensionless streamwise locations $x / h$ of approximately -3 (a), 4 (b), 6 (c), 10 (d), 15 (e) and 19 (f). . . . . . . 137

6-30 Mean velocity components $(\mathrm{m} / \mathrm{s})$ averaged in the spanwise direction. Left and right columns correspond to the SGS-free and the WALE models, respectively; top and bottom rows correspond to the streamwise and wall-normal velocities, respectively. . . . . . . . . . . . 138

6-31 Mean velocity magnitude (m/s) for the SGS-free (a) and WALE (b) models. . . . . . . . . . . . . . . . . . . . . . 138

6-32 Outer-scaled $w_{r m s}^{\prime}$ profiles taken at six distinct locations in the streamwise direction in the flow over a backward-facing step. . . . . . . . .

6-33 Inner-scaled $w_{r m s}^{\prime}$ versus the wall-normal coordinate taken at dimensionless streamwise locations $x / h$ of approximately -3 (a), 4 (b), 6 (c), $10(\mathrm{~d}), 15$ (e) and 19 (f)

6-34 Reynolds stress components $\left(\mathrm{m}^{2} / \mathrm{s}^{2}\right)$ for an SGS-free model in the first column and WALE model in the second column with distinct components per row: $u_{r m s}^{\prime}, v_{r m s}^{\prime}, w_{r m s}^{\prime}$ and $\left\langle u^{\prime} v^{\prime}\right\rangle$ for the first, second, third and fourth row, respectively. . . . . . . . . . . . . . 
6-35 Turbulent kinetic energy $\left(\mathrm{m}^{2} / \mathrm{s}^{2}\right)$ for the SGS-free (a) and WALE (b) models. . . . . . . . . . . . . . . . . .

6-36 Vorticity fluctuation components non-dimensionalized using $\nu / u_{\tau}^{2}$ for SGS-free (red) and WALE (blue) models; $\omega_{x}^{\prime r m s}, \omega_{y}^{\prime r m s}$ and $\omega_{z}^{\prime r m s}$ are denoted using straight lines, triangles and dashed lines, respectively; profiles are taken at $x / h$ approximately -3 (a), 4 (b), 6 (c), 10 (d), 15 (e) and 19 (f) versus the inner-scaled wall-normal coordinate. . . . . . 143

6-37 Vorticity fluctuation diagonal components for the SGS-free and WALE models illustrated in the first and second columns, respectively; $\omega_{x}^{\prime r m s}$, $\omega_{y}^{\prime r m s}$ and $\omega_{z}^{\prime r m s}$ are presented in the first, second and third row, respectively. . . . . . . . . . . . . . . . .

6-38 Vorticity fluctuation off-diagonal components for the SGS-free and WALE models illustrated in the first and second columns, respectively; $\left\langle\omega_{x}^{\prime} \omega_{y}^{\prime}\right\rangle,\left\langle\omega_{x}^{\prime} \omega_{z}^{\prime}\right\rangle$ and $\left\langle\omega_{y}^{\prime} \omega_{z}^{\prime}\right\rangle$ are presented in the first, second and third

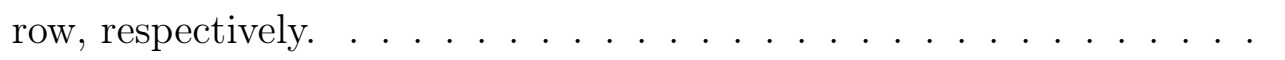

6-39 Mean vorticity components for the SGS-free and WALE models illustrated in the first and second columns, respectively; $\left\langle\omega_{x}\right\rangle,\left\langle\omega_{y}\right\rangle$ and $\left\langle\omega_{z}\right\rangle$ are presented in the first, second and third row, respectively. . .

6-40 Normalized high-order central statistical moments versus outer-scaled wall-normal coordinates at $x / h \approx-3$ for an SGS-free model (red) and WALE model (blue); the first, second and third columns correspond to the streamwise, wall-normal and spanwise components and the first, second and third rows correspond to the third, fourth and fifth moment.148

6-41 Normalized high-order central statistical moments versus inner-scaled wall-normal coordinates at $x / h \approx-3$ for an SGS-free model (red) and WALE model (blue); the first, second and third columns correspond to the streamwise, wall-normal and spanwise components and the first, second and third rows correspond to the third, fourth and fifth moment.149 
6-42 Normalized high-order central statistical moments versus outer-scaled wall-normal coordinates at $x / h \approx 4$ for an SGS-free model (red) and WALE model (blue); the first, second and third columns correspond to the streamwise, wall-normal and spanwise components and the first, second and third rows correspond to the third, fourth and fifth moment.150

6-43 Normalized high-order central statistical moments versus inner-scaled wall-normal coordinates at $x / h \approx 4$ for an SGS-free model (red) and WALE model (blue); the first, second and third columns correspond to the streamwise, wall-normal and spanwise components and the first, second and third rows correspond to the third, fourth and fifth moment.151

6-44 Normalized high-order central statistical moments versus outer-scaled wall-normal coordinates at $x / h \approx 6$ for an SGS-free model (red) and WALE model (blue); the first, second and third columns correspond to the streamwise, wall-normal and spanwise components and the first, second and third rows correspond to the third, fourth and fifth moment.152

6-45 Normalized high-order central statistical moments versus inner-scaled wall-normal coordinates at $x / h \approx 6$ for an SGS-free model (red) and WALE model (blue); the first, second and third columns correspond to the streamwise, wall-normal and spanwise components and the first, second and third rows correspond to the third, fourth and fifth moment.153

6-46 Normalized high-order central statistical moments versus outer-scaled wall-normal coordinates at $x / h \approx 10$ for an SGS-free model (red) and WALE model (blue); the first, second and third columns correspond to the streamwise, wall-normal and spanwise components and the first, second and third rows correspond to the third, fourth and fifth moment.154

6-47 Normalized high-order central statistical moments versus inner-scaled wall-normal coordinates at $x / h \approx 10$ for an SGS-free model (red) and WALE model (blue); the first, second and third columns correspond to the streamwise, wall-normal and spanwise components and the first, second and third rows correspond to the third, fourth and fifth moment.155 
6-48 Normalized high-order central statistical moments versus outer-scaled wall-normal coordinates at $x / h \approx 15$ for an SGS-free model (red) and WALE model (blue); the first, second and third columns correspond to the streamwise, wall-normal and spanwise components and the first, second and third rows correspond to the third, fourth and fifth moment.156

6-49 Normalized high-order central statistical moments versus inner-scaled wall-normal coordinates at $x / h \approx 15$ for an SGS-free model (red) and WALE model (blue); the first, second and third columns correspond to the streamwise, wall-normal and spanwise components and the first, second and third rows correspond to the third, fourth and fifth moment.157

6-50 Normalized high-order central statistical moments versus outer-scaled wall-normal coordinates at $x / h \approx 19$ for an SGS-free model (red) and WALE model (blue); the first, second and third columns correspond to the streamwise, wall-normal and spanwise components and the first, second and third rows correspond to the third, fourth and fifth moment.158

6-51 Normalized high-order central statistical moments versus inner-scaled wall-normal coordinates at $x / h \approx 19$ for an SGS-free model (red) and WALE model (blue); the first, second and third columns correspond to the streamwise, wall-normal and spanwise components and the first, second and third rows correspond to the third, fourth and fifth moment.159

6-52 Outer-scaled TKE budget for the non-transport components $\varepsilon_{k}, \mathcal{P}_{k}$ and $\mathcal{C}_{k}$ versus the wall-normal coordinate for the SGS-free (red) and WALE (blue) models along with the DNS (black) taken at dimensionless streamwise locations $x / h$ of approximately -3 (a), 4 (b), 6 (c), 10 (d), 15 (e) and 19 (f) . . . . . . . . . . . . . . . . 161

6-53 Inner-scaled TKE budget for the non-transport components $\varepsilon_{k}, \mathcal{P}_{k}$ and $\mathcal{C}_{k}$ versus the wall-normal coordinate for the SGS-free (red) and WALE (blue) models along with the DNS (black) taken at dimensionless streamwise locations $x / h$ of approximately -3 (a), 4 (b), 6 (c), 10 (d), 15 (e) and 19 (f) . . . . . . . . . . . . . . . 162 
6-54 Outer-scaled TKE budget for the transport components $\mathcal{T}^{(\nu)}, \mathcal{T}^{(u p)}$ and $\mathcal{T}^{(u u)}$ versus the wall-normal coordinate near the wall for the SGSfree (red) and WALE (blue) models along with the DNS (black) taken at dimensionless streamwise locations $x / h$ of approximately -3 (a), 4 (b), 6 (c), 10 (d), 15 (e) and 19 (f) . . . . . . . . . . .

6-55 Inner-scaled TKE budget for the transport components $\mathcal{T}^{(\nu)}, \mathcal{T}^{(u p)}$ and $\mathcal{T}^{(u u)}$ versus the wall-normal coordinate for the SGS-free (red) and WALE (blue) models along with the DNS (black) taken at dimensionless streamwise locations $x / h$ of approximately -3 (a), 4 (b), 6 (c),

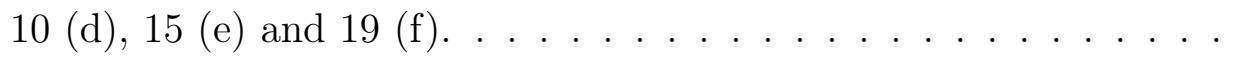

6-56 Non-transport components of the TKE budget for the SGS-free and WALE models in the first and second columns, respectively; $\mathcal{P}_{k}, \mathcal{C}_{k}$ and $\varepsilon$ in the first, second and third row, respectively. . . . . . . . . 165

6-57 Transport components of the TKE budget for the SGS-free and WALE models in the first and second columns, respectively; $\mathcal{T}^{(\nu)}, \mathcal{T}^{(u p)}$ and $\mathcal{T}^{(u u)}$ in the first, second and third row, respectively. . . . . . . . . . . 166

6-58 Inner-scaled 1D spanwise PSD of the velocity versus $\kappa_{z}^{+}$at $x / h \approx 15$ with $y^{+} \approx 193$ (top row) and $x / h \approx 19$ with $y^{+} \approx 5.5$ (bottom row) for the SGS-free (red) and WALE (blue) models; $E_{u u}^{+}\left(\kappa_{z}\right), E_{v v}^{+}\left(\kappa_{z}\right)$ and $E_{w w}^{+}\left(\kappa_{z}\right)$ correspond to the first, second and third columns, respectively. 167

6-59 Inner-scaled 1D spanwise PSD of the velocity versus $\kappa_{z}^{+}$at $x / h \approx 10$ for the SGS-free (red) and WALE (blue) models; $E_{u u}^{+}\left(\kappa_{z}\right), E_{v v}^{+}\left(\kappa_{z}\right)$ and $E_{w w}^{+}\left(\kappa_{z}\right)$ correspond to the first, second and third columns, respectively; $y^{+} \approx 1, y^{+} \approx 5.5, y^{+} \approx 193$ and $y^{+} \approx 376$ correspond to the first, second, third and fourth row, respectively. . . . . . . . . . . . 168

6-60 Inner-scaled 1D spanwise PSD of the velocity versus $\kappa_{z}^{+}$at $x / h \approx 6$ for the SGS-free (red) and WALE (blue) models; $E_{u u}^{+}\left(\kappa_{z}\right), E_{v v}^{+}\left(\kappa_{z}\right)$ and $E_{w w}^{+}\left(\kappa_{z}\right)$ correspond to the first, second and third columns, respectively; $y^{+} \approx 1, y^{+} \approx 5.5, y^{+} \approx 193$ and $y^{+} \approx 376$ correspond to the first, second, third and fourth row, respectively. . . . . . . . . 
6-61 Inner-scaled $1 \mathrm{D}$ spanwise PSD of the velocity versus $\kappa_{z}^{+}$at $x / h \approx 4$ for the SGS-free (red) and WALE (blue) models; $E_{u u}^{+}\left(\kappa_{z}\right), E_{v v}^{+}\left(\kappa_{z}\right)$ and $E_{w w}^{+}\left(\kappa_{z}\right)$ correspond to the first, second and third columns, respectively; $y^{+} \approx 1, y^{+} \approx 193$ and $y^{+} \approx 376$ correspond to the first, second and third row, respectively. . . . . . . . . . . . . . . . 170

6-62 Inner-scaled 1D spanwise PSD of the velocity versus $\kappa_{z}^{+}$at $x / h \approx-3$ for the SGS-free (red) and WALE (blue) models; $E_{u u}^{+}\left(\kappa_{z}\right), E_{v v}^{+}\left(\kappa_{z}\right)$ and $E_{w w}^{+}\left(\kappa_{z}\right)$ correspond to the first, second and third columns, respectively; $y^{+} \approx 2.9, y^{+} \approx 22.8, y^{+} \approx 202$ and $y^{+} \approx 367$ correspond to the first, second, third and fourth row, respectively. . . . . . . . . . .

6-63 ACC of the velocity versus $z / h$ at $x / h \approx 15$ with $y^{+} \approx 193$ (top row) and $x / h \approx 19$ with $y^{+} \approx 5.5$ (bottom row) for the SGS-free (red) and WALE (blue) models; $\rho_{u}\left(r_{z}\right), \rho_{v}\left(r_{z}\right)$ and $\rho_{w}\left(r_{z}\right)$ correspond to the first, second and third columns, respectively. . . . . . . . . . . . .

6-64 ACC of the velocity versus $z / h$ at $x / h \approx 10$ for the SGS-free (red) and WALE (blue) models; $\rho_{u}\left(r_{z}\right), \rho_{v}\left(r_{z}\right)$ and $\rho_{w}\left(r_{z}\right)$ correspond to the first, second and third columns, respectively; $y^{+} \approx 1, y^{+} \approx 5.5, y^{+} \approx 193$ and $y^{+} \approx 376$ correspond to the first, second, third and fourth row,

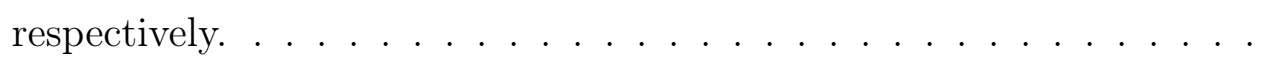

6-65 ACC of the velocity versus $z / h$ at $x / h \approx 6$ for the SGS-free (red) and WALE (blue) models; $\rho_{u}\left(r_{z}\right), \rho_{v}\left(r_{z}\right)$ and $\rho_{w}\left(r_{z}\right)$ correspond to the first, second and third columns, respectively; $y^{+} \approx 1, y^{+} \approx 5.5, y^{+} \approx 193$ and $y^{+} \approx 376$ correspond to the first, second, third and fourth row, respectively. . . . . . . . . . . . . . . . .

6-66 ACC of the velocity versus $z / h$ at $x / h \approx 4$ for the SGS-free (red) and WALE (blue) models; $\rho_{u}\left(r_{z}\right), \rho_{v}\left(r_{z}\right)$ and $\rho_{w}\left(r_{z}\right)$ correspond to the first, second and third columns, respectively; $y^{+} \approx 1, y^{+} \approx 193$ and $y^{+} \approx 376$ correspond to the first, second and third row, respectively. . 176 
6-67 ACC of the velocity versus $z / h$ at $x / h \approx-3$ for the SGS-free (red) and WALE (blue) models; $\rho_{u}\left(r_{z}\right), \rho_{v}\left(r_{z}\right)$ and $\rho_{w}\left(r_{z}\right)$ correspond to the first, second and third columns, respectively; $y^{+} \approx 2.9, y^{+} \approx 22.8, y^{+} \approx 202$ and $y^{+} \approx 367$ correspond to the first, second, third and fourth row, respectively. . . . . . . . . . . . . . . . . . . 177

6-68 Probability of zero streamwise velocity (a) and reverse streamwise velocity (b) versus the streamwise coordinate. . . . . . . . . . . . 178

6-69 Non-dimensionalized first cell from the wall using inner scaling (a) and at the estimated TBL's edge using $\theta(\mathrm{b})$. Also, inner-scaled streamwise (c) and spanwise $(\mathrm{d})$ cell sizes. . . . . . . . . . . . . . . . .

6-70 Normalized mean SGS viscosity profiles taken at six distinct locations using the WALE model along the streamwise direction in the flow over a backward-facing step. . . . . . . . . . . . . . . . . . . . . 181

6-71 Inner-scaled mean streamwise velocity profiles versus the wall-normal coordinate taken at streamwise locations $x / h$ of approximately -3 (a) and 19 (f) . . . . . . . . . . . . . . . . . .

6-72 Outer-scaled $v_{r m s}^{\prime}$ profiles taken at six distinct locations in the streamwise direction in the flow over a backward-facing step. . . . . . . . .

A-1 Visual illustration of transformation of region of interest. . . . . . . . 206

E-1 Inner-scaled mean streamwise velocity profiles versus the wall-normal coordinate taken at dimensionless streamwise locations $x / h$ of approximately -3 (a), 4 (b), 6 (c), 10 (d), 15 (e) and 19 (f) using the coarse-size BFS grid resolution. . . . . . . . . . . . . . . . .

E-2 Inner-scaled $u_{r m s}^{\prime}$ versus the wall-normal coordinate taken at dimensionless streamwise locations $x / h$ of approximately -3 (a), 4 (b), 6 (c), 10 (d), 15 (e) and 19 (f) using the coarse-size BFS grid resolution. . . 223

E-3 Inner-scaled $v_{r m s}^{\prime}$ versus the wall-normal coordinate taken at dimensionless streamwise locations $x / h$ of approximately -3 (a), 4 (b), 6 (c), 10 (d), 15 (e) and 19 (f) using the coarse-size BFS grid resolution. 
E-4 Inner-scaled $-\left\langle u^{\prime} v^{\prime}\right\rangle$ versus the wall-normal coordinate taken at dimensionless streamwise locations $x / h$ of approximately -3 (a), 4 (b), 6 (c), 10 (d), 15 (e) and 19 (f) using the coarse-size BFS grid resolution. . . 225

E-5 Inner-scaled mean streamwise velocity profiles versus the wall-normal coordinate taken at dimensionless streamwise locations $x / h$ of approximately -3 (a), 4 (b), 6 (c), 10 (d), 15 (e) and 19 (f) using the intermediate-size BFS grid resolution. . . . . . . . . . . . . 226

E-6 Inner-scaled $u_{r m s}^{\prime}$ versus the wall-normal coordinate taken at dimensionless streamwise locations $x / h$ of approximately -3 (a), 4 (b), 6 (c), 10 (d), 15 (e) and 19 (f) using the intermediate-size BFS grid resolution.227

E-7 Inner-scaled $v_{r m s}^{\prime}$ versus the wall-normal coordinate taken at dimensionless streamwise locations $x / h$ of approximately -3 (a), 4 (b), 6 (c), 10 (d), 15 (e) and 19 (f) using the intermediate-size BFS grid resolution. . . . . . . . . . . . . . . . . . . 228

E-8 Inner-scaled $-\left\langle u^{\prime} v^{\prime}\right\rangle$ versus the wall-normal coordinate taken at dimensionless streamwise locations $x / h$ of approximately -3 (a), 4 (b), 6 (c), 10 (d), 15 (e) and 19 (f) using the intermediate-size BFS grid resolution.229

E-9 Inner-scaled mean streamwise velocity profiles versus the wall-normal coordinate taken at dimensionless streamwise locations $x / h$ of approximately -3 (a), 4 (b), 6 (c), 10 (d), 15 (e) and 19 (f) using the fine-size BFS grid resolution. . . . . . . . . . . . . . . . . 230

E-10 Inner-scaled $u_{r m s}^{\prime}$ versus the wall-normal coordinate taken at dimensionless streamwise locations $x / h$ of approximately -3 (a), 4 (b), 6 (c), 10 (d), 15 (e) and 19 (f) using the fine-size BFS grid resolution. . . . 231

E-11 Inner-scaled $v_{r m s}^{\prime}$ versus the wall-normal coordinate taken at dimensionless streamwise locations $x / h$ of approximately -3 (a), 4 (b), 6 (c), 10 (d), 15 (e) and 19 (f) using the fine-size BFS grid resolution. . 232

E-12 Inner-scaled $-\left\langle u^{\prime} v^{\prime}\right\rangle$ versus the wall-normal coordinate taken at dimensionless streamwise locations $x / h$ of approximately -3 (a), 4 (b), 6 (c), 10 (d), 15 (e) and 19 (f) using the fine-size BFS grid resolution. . . . 233 


\section{List of Tables}

5.1 Channel flow initial and boundary conditions. . . . . . . . . . 51

5.2 Summary of the simulation parameters in two different LES as compared to the reference benchmark DNS data for a (target) $\operatorname{Re}_{\tau}$ of 180 .

5.3 Summary of the simulation parameters in all nine different LES as compared to the reference benchmark DNS data for a (target) $\operatorname{Re}_{\tau}$ of

5.4 Summary of the simulation parameters in all six different LES as compared to the reference benchmark DNS data for a (target) $\operatorname{Re}_{\tau}$ of 300 .

5.5 Summary of the simulation parameters in all twenty-seven different LES as compared to the reference benchmark DNS data for a (target)

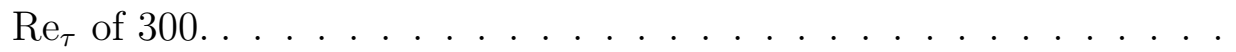

6.1 Backward-facing step initial and boundary conditions. . . . . . . . . 115

6.2 Summary for the parameters used in a coarse-size grid resolution for the flow over a BFS simulations using LES as compared to the reference benchmark DNS data for a (target) $\operatorname{Re}_{h}=5100 \ldots \ldots \ldots \ldots$

6.3 Summary for the parameters used in an intermediate-size grid resolution for flow over a BFS simulations using LES as compared to the reference benchmark DNS data for a (target) $\mathrm{Re}_{h}=5100 . \ldots$. . .

6.4 Summary for the parameters used in a fine-size grid resolution for a flow over a BFS simulations using LES as compared to the reference benchmark DNS data for a (target) $\operatorname{Re}_{h}=5100 \ldots \ldots \ldots \ldots$

6.5 Reattachment locations and their relative absolute error. . . . . . . 183 
Xxx 


\title{
Nomenclature
}

\author{
Acronyms \\ ACC Auto-Correlation Coefficient \\ ACF Auto-Correlation Function \\ BC Boundary Condition \\ BD Backward Difference \\ BFS Backward-Facing Step \\ CD Central Difference \\ CFD Computational Fluid Dynamics \\ CV Control Volume \\ DFT Discrete Fourier Transform \\ DIC Diagonal Incomplete-Cholesky \\ DILU Diagonal Incomplete-LU \\ DNS Direct Numerical Simulation \\ ESD Energy Spectral Density \\ FFT Fast Fourier Transform
}


FVM Finite Volume Method

GAMG Geometric-Algebraic Multi-Grid

HPC High Performance Computing

IC Initial Condition

ILES Implicit Large Eddy Simulation

LES Large Eddy Simulation

NS Navier-Stokes

PBiCG Preconditioned Bi-Conjugate Gradient

PDE Partial Differential Equation

PSD Power Spectral Density

RANS Reynolds Averaged Navier-Stokes

RMS Root Mean Square

SGS Subgrid Scale

SIMPLE Semi-Implicit Method for Pressure-Linked Equations

TBL Turbulent Boundary Layer

TKE Turbulent Kinetic Energy

WALE Wall-Adapting Local Eddy-viscosity

ZPG Zero-Pressure-Gradient

\section{Dimensionless Numbers}

CFL Courant-Friedrichs-Lewy condition number 


$\begin{array}{ll}\operatorname{Re} & \text { Reynolds number } \\ \operatorname{Re}_{\tau} & \text { Friction Reynolds number } \\ \operatorname{Re}_{\theta} & \text { Momentum thickness Reynolds number } \\ \operatorname{Re}_{b} & \text { Bulk Reynolds number } \\ \operatorname{Re}_{h} & \text { Step-height Reynolds number } \\ \operatorname{Re}_{\delta_{99}} & \text { Boundary layer thickness Reynolds number } \\ \Pi & \text { Hyperskewness (5th central moment) } \\ K & \text { Kurtosis (4th central moment) } \\ S & \text { Skewness (3rd central moment) } \\ \text { Greek } & \text { Symbols }\end{array}$

$\omega$

$\Delta$

$\delta$

$\kappa$

$\mathcal{C}_{k}$

$\mathcal{P}_{k}$

$\mathcal{T}^{(\nu)}$

$\mathcal{T}^{(u p)}$

$\mathcal{T}^{(u u)}$

$\mu$
Vorticity vector

Filter cutoff width

Channel half-height

Wavenumber

TKE convection

TKE production

TKE transport by viscous diffusion

TKE transport by velocity-pressure gradient

TKE transport by turbulence

Dynamic viscosity
$1 / \mathrm{s}$

m

$\mathrm{m}$

$1 / \mathrm{m}$

$\mathrm{m}^{2} / \mathrm{s}^{3}$

$\mathrm{m}^{2} / \mathrm{s}^{3}$

$\mathrm{m}^{2} / \mathrm{s}^{3}$

$\mathrm{m}^{2} / \mathrm{s}^{3}$

$\mathrm{m}^{2} / \mathrm{s}^{3}$

$\mathrm{Pa} \cdot \mathrm{s}$ 


$\begin{array}{llr}\nu & \text { Kinematic viscosity } & \mathrm{m}^{2} / \mathrm{s} \\ \nu_{t} & \text { Turbulent eddy-viscosity } & \mathrm{m}^{2} / \mathrm{s} \\ \nu_{s g s} & \text { Subgrid scale viscosity } & \mathrm{m}^{2} / \mathrm{s} \\ \rho & \text { Density } & \mathrm{kg} / \mathrm{m}^{3} \\ \tau_{i j}^{s g s} & \text { Subgrid scale stress tensor } & \mathrm{m}^{2} \cdot \mathrm{s}^{2} \\ \varepsilon_{k} & \text { TKE dissipation } & \mathrm{m}^{2} / \mathrm{s}^{3}\end{array}$

\section{Roman Symbols}

$\begin{array}{llr}\mathbf{u} & \text { Velocity vector } & \mathrm{m} / \mathrm{s} \\ k & \text { Turbulent kinetic energy } & \mathrm{m}^{2} / \mathrm{s}^{2} \\ p & \text { Pressure } & \mathrm{Pa} \\ S_{i j} & \text { Strain-rate tensor } & 1 / \mathrm{s} \\ U_{\infty} & \text { Free-stream velocity component } & \mathrm{m} / \mathrm{s}\end{array}$

\section{Superscripts}

$\bar{\phi} \quad$ Resolved/filtered quantity

$\phi^{\prime} \quad$ Fluctuating quantity

$\phi^{\prime \prime} \quad$ Unresolved quantity

$\phi^{+} \quad$ Inner-scaled dimensionless quantity

\section{Other Symbols}

$\delta_{i j} \quad$ Kronecker delta

$\frac{D}{D t} \quad$ Material/substantial derivative 
\langle\rangle$\quad$ Averaging in time and homogeneous directions

E Expected value

$\mathcal{O} \quad$ Order of computational complexity or higher-order terms

$\mathscr{F} \quad$ Fourier transform

$\nabla \quad$ Gradient operator

$\nabla^{2} \quad$ Laplacian operator 
THIS PAGE IS INTENTIONALLY LEFT BLANK 


\section{Chapter 1}

\section{Introduction}

Turbulence has long captured the attention of observers and scientists alike. This phenomenon has fascinated us due to its enticing nature, aesthetic structure, intricate behavior and omnipresence. Whether this event is taking place in the surrounding air, the nearby oceans, the skies above or even microfluidic environments that occupy most of space, turbulence plays a very important role. Some go so far and dispute that turbulence played a significant role in art as well. For instance, a relatively recent study investigated the mathematical similarities (e.g. probability density functions) of turbulence structures and the patterns of luminescence taking place in some of van Gogh's famous paintings [5]. Scientifically, however, records show that as early as the dawn of the sixteenth century Leonardo da Vinci started empirically investigating this phenomenon. In fact, da Vinci's experiments and later drawings of fluid motion were among the earliest to describe the "eddying" motion involved in a turbulent flow.

From a general overview, turbulence is a state of fluid behavior that is characterized by the seemingly random and chaotic motion. Another important and obligatory property is that for turbulence to take place, there must be three-dimensional vorticities and hence, in reality, there is no actual turbulence below three (spatial) dimensions. Additionally, turbulent flow is comprised of fluid particles moving in a highly irregular motion that is characterized by so-called 'eddies.' 
During the 1880s, Osbourne Reynolds initiated experiments to better study the relation between laminar and turbulent flow. The outcome was the widely used dimensionless Reynolds number which is used to classify the state of the fluid flow according to its inertial-to-viscous forces ratio. Afterwards, Lewis Fry Richardson played a prominent role in motivating further studies about turbulence, especially regarding the multi-scale nature of it (i.e. energy cascade). The idea of characterizing turbulence as a multi-scale fluid behavior that starts from large scales and gets dissipated eventually at the molecular level was put forth so graciously via the below rhyming verse ${ }^{1}$ :

"Big whirls have little whirls

Which feed on their velocity, And little whirls have lesser whirls And so on to viscosity."

- Lewis Fry Richardson (1922)

Technically speaking, this paints a picture that depicts the energy cascade (in a given turbulent flow) as a process starting at large scales of motions (via production of kinetic energy) and is then broken down into smaller scales until it reaches the molecular level and gets dissipated by the dominating viscous action.

Later, a Russian mathematician by the name of Andrey Kolmogorov gave concrete evidence for such a process. This was carried out by Kolmogorov's work in 1941 in which he both identified and quantified this behavior.

Unlike laminar flow, the state of a turbulent flow is a much more dominating and sought after occurrence. During turbulence, the fluid flow experiences more energy dissipation and mixing becomes much more efficient and hence heat transfer is greatly increased. These are some of the major reasons on why more research and investments are being put in understanding this cumbersome and elusive phenomenon.

\footnotetext{
${ }^{1}$ This verse was a clever play on words inspired by Augustus de Morgan's poem in De Morgan [16] which was a paraphrasing by itself taken from Jonathan Swifts' original Swift [76].
} 
Nowadays, turbulence has been incorporated as a core concept across a wide range of disciplines such as, but not restricted to, aerodynamics, meteorology, combustion, hydrodynamics, biomedical engineering and chemical process engineering. Furthermore, the study of turbulence is mainly composed of two distinct yet complementary approaches: experimentation and numerical modeling. These, in turn, help advance the theoretical framework involved in turbulence research, since analytical approaches are seldom applicable in real-life engineering applications. In this thesis, numerical methods are utilized to study certain turbulent flow phenomena while complementing some of the material with available experimental data for benchmark purposes.

One of many intriguing behaviors observed in turbulent flows across surfaces is boundary layer separation, recirculation and reattachment. Examples of such manifestations are abundant. For instance flow around airfoils is one primary example in which the flow's boundary separation is directly linked to the stability of airplanes along with extra fuel consumption due to drag forces. Another example lies in combustion chambers where the diffuser plays a prominent role. In such scenarios, the separated flow can form large unsteady eddies that might greatly reduce the flow inside the diffuser or even lead to inflow distortions (e.g. to the compressors). This will inevitably impact the engine's performance negatively. A third example of boundary layer separation, recirculation and reattachment is one that takes place about buildings. In such cases, studies are conducted to explain how influential and durable certain building designs are with regard to local wind loads.

Usually, such a phenomenon (boundary layer separation) is undesired in most applications that can lead to instabilities or unnecessary additional energy costs. This paves the way towards the need for a solid foundation in better understanding such an utterly crucial phenomenon that takes place in our everyday lives. On such basis, studies of turbulent flows over backward-facing step (BFS) geometries are amongst the most classical procedures investigated. In reality, these cases are a simplified version of (symmetrical) flows through sudden expansion chambers. During such studies, 
boundary separation, recirculation and later reattachment is replicated (to a certain extent) with relative ease due to the simplistic geometry thereof. Nowadays, most flow over a BFS studies are implemented to validate and compare different solvers, accuracy of discretization methods, meshing strategies, other numerical procedures along with the plethora of available turbulence modeling techniques. Furthermore, this is especially true due to the great deal of publicly available statistical datasets reported from both numerical and experimental investigations.

In this work, turbulent flow over a BFS is explored numerically for a step-height Reynolds (Re-)number of 5100 using OpenFOAM². Turbulence modeling is applied through large eddy simulation (LES) using different subgrid scale (SGS) models and grid resolutions. The results are compared to and validated against high-fidelity direct numerical simulations (DNS) as well as available experimental studies done by means of laser-doppler velocimetry. Furthermore, in order to specify an adequate inlet velocity condition for the flow over a BFS, turbulent channel flow simulations with matching characteristics are studied extensively. After settling on suitable target parameters, relatively coarser turbulent channel flow simulations are used to generate an adequate inlet velocity profile for later use in the flow over a BFS simulations. The study in this work covers different grid resolutions and SGS model variants. This would, optimistically, assist the interested reader to gain a detailed understanding about what is going on per model and grid used on each of the flow problems considered (i.e. turbulent channel flow and flow over a BFS). Perhaps, this would serve as a guide for users to comprehend approximately which areas are worth further investing time and computational resources into and which ones definitely are not.

The work in this thesis will be spread across six chapters, excluding this introductory one. Chapter 2 will first highlight the relevant governing equations for fluid flow and then expand on them by introducing turbulence models whereby more emphasis will be placed on LES and the SGS models used. In Chapter 3, the equations will be

\footnotetext{
${ }^{2}$ https://openfoam.org/
} 
discretized using the finite volume method (FVM) and the solution strategy will be covered. Chapter 4 will delve into wall-bounded turbulence for turbulent boundary layers (TBL) and turbulent channel flows. Last, but not least, Chapters 5 and 6 will detail and present the results of several numerical simulations for the turbulent channel flow and the flow over a BFS, respectively. In these studies, the most important parameters addressed are the effects of different grid resolutions and SGS models on the flow. Finally, Chapter 7 will present the conclusion with possible future work. 
THIS PAGE IS INTENTIONALLY LEFT BLANK 


\section{Chapter 2}

\section{Turbulence Modeling}

After starting with a concise introduction about the governing equations describing fluid flow, the narrative in this chapter will focus on turbulence modeling from a numerical standpoint. When addressing turbulent flow, three widespread numerical methods are used: direct numerical simulation, Reynolds averaged Navier-Stokes (RANS) and large eddy simulation. The need for such procedures are based on account of the multi-scale nature of turbulence and the need to effectively resolve it.

A concise description of the above first two (DNS and RANS) commonly used turbulence modeling procedures will be portrayed. Significantly more emphasis will be placed on the latter third technique (LES) with plenty of description about the SGS models used in the scope of this project. Before proceeding, however, the relevant governing equations for fluid flow are first presented. These are known as the NavierStokes (NS) equations and for the current thesis, they will specifically address viscous, incompressible and Newtonian fluids.

\subsection{Navier-Stokes Equations}

The Navier-Stokes equations were derived, independently and originally in the first half of the nineteenth century by Claude-Louis Navier and George Gabriel Stokes [83]. Nonetheless, this achievement was a result of a cumulative process that stretched 
across several decades with the involvement of Navier, Poisson, Saint-Venant, Euler and Stokes - some even argue that there should be even more names involved. Furthermore, for these statements to hold, the fluid particles are assumed to be at sufficient macroscopic length scales such that their molecular structure may be ignored. These set of equations are presented below:

$$
\begin{aligned}
\frac{\partial u}{\partial t}+\nabla \cdot(u \mathbf{u}) & =-\frac{1}{\rho} \frac{\partial p}{\partial x}+\nabla \cdot(\mu \nabla u)+\mathcal{S}_{x} \\
\frac{\partial v}{\partial t}+\nabla \cdot(v \mathbf{u}) & =-\frac{1}{\rho} \frac{\partial p}{\partial y}+\nabla \cdot(\mu \nabla v)+\mathcal{S}_{y} \\
\frac{\partial w}{\partial t}+\nabla \cdot(w \mathbf{u}) & =-\frac{1}{\rho} \frac{\partial p}{\partial z}+\nabla \cdot(\mu \nabla w)+\mathcal{S}_{z} \\
\nabla \cdot \mathbf{u} & =0
\end{aligned}
$$

where $\rho$ is the density, $p$ is the pressure, $\mu$ is the dynamic viscosity, $\mathcal{S}_{i}$ is a source term acting in the $i$ th direction, $u, v$ and $w$ are the velocity components per Cartesian direction in three-dimensional space (i.e. $x, y$ and $z$, respectively).

In Equation 2.1, the first three expressions are referred to as the momentum equations and the the fourth one is the continuity equation. Concerning this project, the flow is restricted to a Newtonian, incompressible, viscous flow without any source terms present. Therefore, the above set of equations may be further simplified and written in either vector or Einstein notation ${ }^{1}$, respectively, as:

- In vector notation:

$$
\begin{gathered}
\frac{\partial \mathbf{u}}{\partial t}+(\mathbf{u} \cdot \nabla) \mathbf{u}=-\frac{1}{\rho} \nabla p+\nu \nabla^{2} \mathbf{u} \\
\nabla \cdot \mathbf{u}=0
\end{gathered}
$$

where $\nabla$ is the gradient operator, $\nabla^{2}=\nabla \cdot \nabla=\Delta$ is the Laplace operator and

\footnotetext{
${ }^{1}$ In order to simplify expressions involving tensors, Albert Einstein introduced a new notation convention in Einstein [25] which is conveniently named after him, although other names exist such as: tensor notation or index/indecial notation.
} 
$\nu=\mu / \rho$ is the kinematic viscosity.

- In tensor notation:

$$
\begin{aligned}
\frac{\partial u_{i}}{\partial t}+u_{j} \frac{\partial u_{i}}{\partial x_{j}} & =-\frac{1}{\rho} \frac{\partial p}{\partial x_{i}}+\nu \frac{\partial^{2} u_{i}}{\partial x_{j} \partial x_{j}} \\
\frac{\partial u_{i}}{\partial x_{i}} & =0
\end{aligned}
$$

where $u_{i}$ (similarly $u_{j}$ ) is the $i$ th velocity component.

In either notation used, on the left-hand side of the equation, the first term is the local temporal rate of change of the velocity, the second term represents the advection/convection of velocity. On the right-hand side of the equality, the first term denotes the pressure gradient term which more-or-less drives the flow and the last term is that of viscous diffusion. Moreover, for a more robust and illustrative description on the NS equations, their derivation and different forms, the interested reader is referred to Anderson [4]. Hereon, unless instructed otherwise, Einstein notation will be adopted due to its simplicity and common use in computational fluid dynamics (CFD) notation. Finally, to be consistent with the software package's notation, the density is implicitly included in the pressure term. Such inclusion is valid for incompressible flow, since density is assumed to be a constant.

\subsection{Direct Numerical Simulation}

As the name suggests, DNS directly solves for the actual NS equations. This means that no additional component or modification is introduced when solving Equation 2.3. For clarity, the NS equations referenced are in the form of Equation 2.3.

The downside to DNS is that, in order to effectively resolve all scales, the computational grid needs to be constructed in such a way that it is able to resolve all length scales. This means the computational grid needs to capture the integral length scales, which are associated with the largest eddies that contain the most kinetic energy, all 
the way down to the most dissipative scales termed the Kolmogorov scales. These scales are formed based on a Reynolds number of unity (i.e. $\eta u_{\eta} / \nu=1$ ) and are characterized by [67]:

$$
\begin{aligned}
\eta & \equiv\left(\nu^{3} / \varepsilon\right)^{1 / 4} \\
u_{\eta} & \equiv(\varepsilon \nu)^{1 / 4} \\
\tau_{\eta} & \equiv(\nu / \varepsilon)^{1 / 2}
\end{aligned}
$$

where $\varepsilon$ is the viscous dissipation and $\eta, u_{\eta}$ and $\tau_{\eta}$ correspond to the length, velocity and time scales, respectively.

Using the viscous dissipation analogy for the largest eddies (i.e. $\varepsilon_{0} \sim u_{0}^{3} / l_{0}$, with the 0 subscript denoting the associated length scale), one is able to determine the ratios of the largest-to-smallest scales (i.e. integral-to-Kolmogorov length scales) as follows [67]:

$$
\begin{aligned}
l_{0} / \eta & \sim \operatorname{Re}^{3 / 4} \\
u_{0} / u_{\eta} & \sim \operatorname{Re}^{1 / 4} \\
\tau_{0} / \tau_{\eta} & \sim \operatorname{Re}^{1 / 2}
\end{aligned}
$$

where the quantities $\varepsilon_{0}, u_{0}, l_{0}$ and $\tau_{0}$ are associated with the largest eddy-containing scales.

The above are described for only a length unit in a single dimension. Accounting for the remaining two, the ratio of largest-to-smallest scales for a three-dimensional flow - which is proportional to the number of required grid points - approximately increases as: $N \sim \mathcal{O}\left(\operatorname{Re}^{9 / 4}\right)$, where $N$ is the total number of grid points.

Taking into consideration the scaling required for time advancement (e.g. CFL condition number), this becomes an operation that scales as Re-number cubed, $\mathcal{O}\left(\mathrm{Re}^{3}\right)$. This becomes an intensive computational process to realize, needless to 
mention the memory requirements for storing such large datasets.

To give the reader some perspective, for a turbulent flow of relatively mediocre Renumber, say $R e=10000$, the required number of grid points on the mesh used in DNS would be of the order of a billion. Moreover, the total computational requirement to simulate such a scenario would exorbitantly cost in the range of $\mathcal{O}\left(10^{12}\right)$ floating-point operations per second (FLOPS). For a detailed explanation of these scales and their importance, the reader is encouraged to read Pope [67, chap. 6].

\subsection{Reynolds Averaged Navier-Stokes}

After establishing the cumbersome, and for most practical cases impossible, procedure with directly solving the NS equations in the presence of turbulence, a more convenient alternative is to use RANS. The philosophy behind this method is to study the timeaveraged solution, instead of the instantaneous field. This reduces the computational expense drastically when compared to DNS. In fact, such a method is heavily used in industry and most, if not all, commercial CFD solvers. This, however, introduces a restraint on the data under study, especially whenever instantaneous behavior is required.

The methodology in formulating RANS stems from decomposing the velocity and pressure quantities into a mean and fluctuating components as such:

$$
\begin{aligned}
& \mathbf{u}=\langle\mathbf{u}\rangle+\mathbf{u}^{\prime} \\
& p=\langle p\rangle+p^{\prime},
\end{aligned}
$$

where the operator $\langle\cdot\rangle$ denotes the time averaging while $p^{\prime}$ and $\mathbf{u}^{\prime}$ are the fluctuating counterparts that, by definition, fluctuate about a zero mean.

Taking Equation 2.4 and inserting it into the temporal average of Equation 2.3 
yields:

$$
\begin{aligned}
\frac{\partial\left\langle\left\langle u_{i}\right\rangle+u_{i}^{\prime}\right\rangle}{\partial t}+\left\langle\left(\left\langle u_{j}\right\rangle+u_{j}^{\prime}\right) \frac{\partial\left(\left\langle u_{i}\right\rangle+u_{i}^{\prime}\right)}{\partial x_{j}}\right\rangle & =-\frac{1}{\rho} \frac{\partial\left\langle\langle p\rangle+p^{\prime}\right\rangle}{\partial x_{i}}+\nu \frac{\partial^{2}\left\langle\left\langle u_{i}\right\rangle+u_{i}^{\prime}\right\rangle}{\partial x_{j} \partial x_{j}} \\
\frac{\partial\left\langle\left\langle u_{i}\right\rangle+u_{i}^{\prime}\right\rangle}{\partial x_{i}} & =0 .
\end{aligned}
$$

Before further expanding the terms, some algebraic properties relevant to the temporal average are used, such as: $\langle\langle u\rangle\rangle=\langle u\rangle,\left\langle u^{\prime}\right\rangle=0,\left\langle u^{\prime} v^{\prime}\right\rangle \neq 0,\left\langle\langle u\rangle v^{\prime}\right\rangle=0$ and $\langle u\langle v\rangle\rangle=\langle u\rangle\langle v\rangle$. Making use of these properties in Equation 2.5 while expanding and re-arranging, the RANS equations boils down to:

$$
\begin{aligned}
\frac{\partial\left\langle u_{i}\right\rangle}{\partial t}+\left\langle u_{j}\right\rangle \frac{\partial\left\langle u_{i}\right\rangle}{\partial x_{j}} & =-\frac{1}{\rho} \frac{\partial\langle p\rangle}{\partial x_{i}}+\frac{\partial}{\partial x_{j}}\left(\nu \frac{\partial\left\langle u_{i}\right\rangle}{\partial x_{j}}-\left\langle u_{i}^{\prime} u_{j}^{\prime}\right\rangle\right) \\
\frac{\partial\left\langle u_{i}\right\rangle}{\partial x_{i}}=\frac{\partial u_{i}^{\prime}}{\partial x_{i}} & =0
\end{aligned}
$$

The above procedure is referred to as Reynolds decomposition. In it, as a result of the convective term, a new term emerges. This term is defined as the Reynolds stress tensor and is expressed as: $-\left\langle u_{i}^{\prime} u_{j}^{\prime}\right\rangle$.

This results in a new dilemma in solving these equations, by adding six additional unknowns (3 diagonal and 3 off-diagonal due to symmetry). This is referred to as the infamous turbulence closure problem since we are solving for ten unknowns (3 for the velocity, 1 for the pressure and 6 for the Reynolds stress components) in four equations ( 3 for the momentum equations and 1 for the continuity equation). Thus in actuality there are more unknowns than equations. Achieving a solution, or trying to at least, via different modeling approaches in order to remedy this problem is what is known as RANS turbulence models.

Some of the commonly used RANS turbulence models make use of what is known as the Boussinesq hypothesis. This hypothesis was proposed by Joseph Valentin Boussinesq in 1877 [10]. It was of the earliest procedures that tried to address the closure problem by stating that the Re-stresses are proportional to the mean rate-of- 
strain as follows:

$$
\begin{aligned}
-\left\langle u_{i}^{\prime} u_{j}^{\prime}\right\rangle & =\nu_{t}\left(\frac{\partial\left\langle u_{i}\right\rangle}{\partial x_{j}}+\frac{\partial\left\langle u_{j}\right\rangle}{\partial x_{i}}\right)-\frac{2}{3} k \delta_{i j} \\
& =2 \nu_{t} S_{i j}-\frac{2}{3} k \delta_{i j},
\end{aligned}
$$

where the last line follows from the incompressibility condition, $S_{i j}=\left(\partial\left\langle u_{i}\right\rangle / \partial x_{j}+\right.$ $\left.\partial\left\langle u_{j}\right\rangle / \partial x_{i}\right) / 2$ is the mean rate-of-strain tensor, $k=\left\langle u_{i}^{\prime} u_{i}^{\prime}\right\rangle / 2$ is the turbulent kinetic energy and $\nu_{t}$ is the turbulent eddy-viscosity which is estimated via different models.

The interested reader is referred to Pope [67, chap. 10-11] for a more satisfying discussion about RANS.

\subsection{Large Eddy Simulation}

So far, from what has been illustrated, when it comes to turbulent flow, one either is confined to obtaining a time-averaged solution (RANS) or a very accurate instantaneous solution (DNS) with the restriction to relatively low Re-number. For many cases, neither of the two is sufficient. For instance, in gas turbine simulations, the combustor part responsible for combustion needs more information than the temporally averaged outcome of the fields therein. On the other hand, due to the nature of the flow, the high value of the Re-number and the complicated geometry involved, DNS is prohibitively expensive for such use. Somewhere in between the two methods, with more inclination towards DNS, lies LES.

The principal idea behind LES is applying a low-pass spatial filter to Equation 2.3. In doing so, the scales up to a certain cutoff threshold are resolved and the remaining ones are discarded. In doing so, the filtering procedure separates the fields describing the flow. This is done by including (resolving) the ones associated with larger eddies and excluding the ones associated with smaller eddies. Somewhere in between the eddy spectrum that incorporates all sizes of eddies, a predetermined threshold that separates the two is specified. This allows the computational grid to become coarser 
than that of a pure DNS. This also allows for relatively larger time-steps to be taken while still taking the CFL number into consideration. As for the unresolved fields, a so-called SGS model is applied to try and recover their effects as much as possible. The (spatial) filtering operation may be expressed as a convolution applied on the quantity of interest as follows:

$$
\bar{\phi}(\mathbf{x}, t) \equiv \int_{-\infty}^{\infty} \int_{-\infty}^{\infty} \int_{-\infty}^{\infty} \mathcal{G}\left(\mathbf{x}, \mathbf{x}^{\prime}, \Delta\right) \phi\left(\mathbf{x}^{\prime}, t\right) d \mathbf{x}^{\prime}
$$

where the over-bar represents a filtered field, $\phi$ is the original (unfiltered) function, $\Delta$ is the filter cutoff width and $\mathcal{G}$ is the filter kernel.

There are many different filters that could be considered for LES. The most renowned of these are the top-hat, Gaussian and Spectral filters. The latter two filters are usually used in research, nonetheless for this project and in conjunction with the discretization method used, the top-hat filter ${ }^{2}$ is adopted (this is also true for $\Delta$, since there are many other choices as well). The top-hat filter kernel along with its cutoff width, respectfully, are defined as follows:

$$
\begin{aligned}
\mathcal{G}\left(\mathbf{x}, \mathbf{x}^{\prime}, \Delta\right) & = \begin{cases}1 / \Delta^{3}, & \text { if }\left|\mathbf{x}-\mathbf{x}^{\prime}\right| \leq \Delta / 2 \\
0, & \text { otherwise }\end{cases} \\
\Delta & =\sqrt[3]{\Delta x \Delta y \Delta z} .
\end{aligned}
$$

The motivation behind selecting such filter kernel and cutoff width will become clear in the following chapter. Meanwhile, the expression for the unresolved part, $\phi^{\prime \prime}$, of $\phi$ may be written in the form:

$$
\phi^{\prime \prime}(\mathbf{x}, t)=\phi(\mathbf{x}, t)-\bar{\phi}(\mathbf{x}, t) .
$$

Taking the spatial filtering operation and applying it on the NS equations while

\footnotetext{
${ }^{2}$ Also known as the box filter.
} 
assuming filtering commutes with differentiation, Equation 2.3 eventually becomes:

$$
\begin{aligned}
\frac{\partial \bar{u}_{i}}{\partial t}+\frac{\partial}{\partial x_{j}}\left(\overline{u_{i} u_{j}}\right) & =-\frac{1}{\rho} \frac{\partial \bar{p}}{\partial x_{i}}+\nu \frac{\partial^{2} \bar{u}_{i}}{\partial x_{j} \partial x_{j}} \\
\frac{\partial \bar{u}_{i}}{\partial x_{i}} & =0 .
\end{aligned}
$$

An important observation to be made is that in Equation 2.10 the convective term $\partial\left(\overline{u_{i} u_{j}}\right) / \partial x_{j}$ is unknown since the only available quantities are the filtered data $\overline{\mathbf{u}}$ and $\bar{p}$. To remedy this, the following mathematical trick is utilized:

$$
\overline{u_{i} u_{j}}=\underbrace{\overline{u_{i} u_{j}}-\bar{u}_{i} \bar{u}_{j}}_{\tau_{i j}^{g g s}}+\bar{u}_{i} \bar{u}_{j},
$$

where $\tau_{i j}^{s g s}$ is the subgrid scale (SGS) stress tensor.

Next, manipulating the momentum equations in Equation 2.10 via the relation in Equation 2.11 while including the above SGS stress tensor, the resulting LES momentum and continuity equations are:

$$
\begin{aligned}
\frac{\partial \bar{u}_{i}}{\partial t}+\frac{\partial}{\partial x_{j}}\left(\bar{u}_{i} \bar{u}_{j}\right) & =-\frac{1}{\rho} \frac{\partial \bar{p}}{\partial x_{i}}-\frac{\partial \tau_{i j}^{s g s}}{\partial x_{j}}+\nu \frac{\partial^{2} \bar{u}_{j}}{\partial x_{j} \partial x_{j}} \\
\frac{\partial \bar{u}_{i}}{\partial x_{i}} & =0 .
\end{aligned}
$$

It is interesting to note how similar Equation 2.12 is in comparison to Equation 2.6. Although, one must keep in mind that each of these equations were derived using completely different methodologies. Therefore, despite being similar in structure, nevertheless they differ in nature and corresponding derivation process.

Therefore, in order to proceed, the SGS stress tensor, $\tau_{i j}^{s g s}$, has to be effectively modeled since, just like in RANS ${ }^{3}$, this tensor gives rise to a possible closure model. In order to achieve this, several different propositions are available with each having

\footnotetext{
${ }^{3}$ Tensors arising from LES and RANS models, though similar structurally, are completely different.
} 
its own merits and flaws. For a more thorough discussion on LES and the research therein, the interested reader is referred to the comprehensive work of Sagaut [71] and Pope [67, chap. 13].

All SGS models used throughout this thesis are portrayed next. All in all, they are composed of four different explicit models and an additional implicit model. The narrative in this section is chiefly concerned in properly presenting sufficient theory about each of these models while capitalizing on some of their advantages or downfalls. Recall, the SGS stress tensor's expression: $\tau_{i j}^{s g s}=\overline{u_{i} u_{j}}-\bar{u}_{i} \bar{u}_{j}$.

In the following five subsections, all the models adopted use the Boussinesq assumption and in doing so, they are eddy-viscosity based models. These type of models calculate the deviatoric part of the SGS stress tensor by relating it, in a linear correspondence, to the rate-of-strain tensor. By doing so, the tensor may be thought of being decomposed into an isotropic and an anisotropic part. The first is also known as the normal stresses and is directly related to the turbulent kinetic energy while the latter measures the deviations from isotropy. Hence, the eddy-viscosity, based on the Boussinesq hypothesis, is expressed as:

$$
\tau_{i j}^{s g s}-\frac{1}{3} \tau_{k k}^{s g s} \delta_{i j}=-2 \nu_{s g s} \bar{S}_{i j},
$$

where $\delta_{i j}$ is the Kronecker delta, $\bar{S}_{i j}=\left(\partial \bar{u}_{i} / \partial x_{j}+\partial \bar{u}_{j} / \partial x_{i}\right) / 2$ is the filtered rateof-strain tensor and $\nu_{s g s}$ is the SGS viscosity which is being estimated via different models in the subsequent subsections.

\subsubsection{Smagorinsky Model}

The Smagorinsky, or Smagorinsky-Lilly, model was first proposed by Joseph Smagorinsky, a meteorologist, around 1963 and later Douglas Lilly - a colleague of Smagorinsky's - modified it [73]. At first, this model was designed for use in meteorological applications. The nature of such flows is mainly about free-shear flows in which wallboundaries has little-to-no consideration. Later on, it was implemented in the first 
LES for turbulent channel flow in [19].

Smagorinsky postulated that since for the smallest of scales the eddies may be estimated to be isotropic, the Boussinesq assumption becomes valid. This follows from Kolmogorov's hypotheses. Moreover, this model assumes that there is local equilibrium between the production and dissipation of SGS TKE. Hence, the SGS viscosity for the Smagorinsky model is defined as:

$$
\begin{aligned}
\nu_{s g s} & =\ell_{s}^{2}\left(2 \bar{S}_{i j} \bar{S}_{i j}\right)^{1 / 2} \\
& =\left(C_{s} \Delta\right)^{2}\left(2 \bar{S}_{i j} \bar{S}_{i j}\right)^{1 / 2},
\end{aligned}
$$

where $\ell_{s}=C_{s} \Delta$ is the Smagorinsky length scale ${ }^{4}$ and $C_{s}$ is the Smagorsinky constant.

Some of the evident drawbacks in using the Smagorinsky model are i) it is very dissipative, ii) there is no backscatter of energy, since $\nu_{s g s}>0$ and the SGS TKE production $\mathcal{P} \equiv 2 \nu_{s g s} \bar{S}_{i j} \bar{S}_{i j}>0$, iii) poor behavior in near-wall regions, and iv) the Smagorinsky constant, $C_{s}$, is treated as a universal constant, regardless of the local flow.

One main inconvenience in the Smagorinsky model arises when trying to resolve the near-wall region. In the viscous wall region, the specified characteristic length, $\ell_{s}=C_{s} \Delta$, does not vanish as is anticipated. A simple fix is to adjust the SGS viscosity by including a van Driest damping function [22]:

$$
\nu_{s g s}=\left(C_{s} \Delta f\right)^{2}\left(2 \bar{S}_{i j} \bar{S}_{i j}\right)^{1 / 2},
$$

where $f=1-\exp \left(-y^{+} / A\right)$ is the van Driest damping function and $y^{+}=y u_{\tau} / \nu$ is the inner-scaled - using the viscosity $\nu$ and the friction velocity $u_{\tau}$ - wall-normal

\footnotetext{
${ }^{4}$ The characteristic length scale associated with the Smagorinsky model is analogous to Prandtl's mixing length hypothesis.
} 
coordinate. The friction velocity is defined using the below relationship:

$$
u_{\tau} \equiv \sqrt{\tau_{w} / \rho},
$$

where $\tau_{w}=\left.\mu \frac{\partial u}{\partial y}\right|_{\text {wall }}$ is the wall shear stress.

For more intricate details about this model, implementation-wise, with respect to OpenFOAM, the open-source finite-volume-based CFD platform, refer to Appendix C.

\subsubsection{SGS Turbulent Kinetic Energy Equation Model}

The SGS turbulent kinetic energy (TKE) equation, commonly referred to as the $k$ equation, starts off very similarly in construction as that of the Smagorinsky model. The eddy-viscosity assumption is used for computing the SGS stress model through Equation C.2. Also, the subgrid viscosity is computed using the same expression as in Equation C.1. That said, the similarities between the two models end here. In the $k$-equation, instead of employing local equilibrium for the computation of the SGS TKE, a transport equation is solved [84].

The main reason for using a transport equation for the SGS TKE instead of algebraically evaluating it such as in Equation C.5, is to avoid the assumption that local isotropy exists. Thus, the resulting transport equation is defined as:

$$
\frac{\partial\left(\rho k_{s g s}\right)}{\partial t}+\frac{\partial\left(\rho \bar{u}_{j} k_{s g s}\right)}{\partial x_{j}}-\frac{\partial}{\partial x_{j}}\left[\rho\left(\nu+\nu_{s g s}\right) \frac{\partial k_{s g s}}{\partial x_{j}}\right]=-\rho \tau_{i j}^{s g s} \bar{S}_{i j}-C_{\varepsilon} \frac{\rho k_{s g s}^{3 / 2}}{\Delta}
$$

where the terms from left to right correspond to the temporal derivative, advection, diffusion, production and dissipation of the SGS TKE, respectively, and $k_{s g s}=\left(\overline{u_{k} u_{k}}-\right.$ $\left.\bar{u}_{k} \bar{u}_{k}\right) / 2$ is the SGS TKE.

For additional implementation details in OpenFOAM, refer to the relevant section in Appendix C. 


\subsubsection{Dynamic SGS TKE Equation Model}

The main motivation behind constructing the dynamic $k$-equation model was to better account for the local flow constants. For instance, in the previous SGS models the constants were assumed universal. This, unfortunately does not always hold. Furthermore, different sources recommend different values. Despite the small difference in these values, fine-tuning such constants could have a prominent role on the simulation results. The calibration process can be cumbersome due to many reasons (e.g. nature of the flow, range of Re-number... etc.), therefore it is greatly rewarding to adapt these constants dynamically as the flow evolves.

One of the first and most commonly used dynamic procedures in SGS modeling was proposed in Germano et al. [27] and later modified by Lilly [48]. These class of dynamic models are derived by applying an additional so-called 'test' filter on the solution field in order to construct a relation to compute a model's coefficient. Initially, the Germano identity was used to determine a dynamic coefficient for the Smagorinsky model, however it is straightforward to apply to other SGS models as well. One issue with this formulation was stabilization - mainly due to zero denominators. Lilly later modified this nuisance by advocating the use of a least-squares approach for computing the model's coefficient. The test filter used is usually taken twice the original filter:

$$
\widetilde{\Delta}=2 \Delta,
$$

where the tilde-bar symbol, , denotes the explicit 'test' filtering operation.

The dynamic $k$-equation SGS model is based on the work of Kim and Menon [42]. The expression for the subgrid viscosity maintains similarity with Equation C.1, except that the model's coefficient is now evaluated as:

$$
C_{k}=\frac{1}{2} \frac{\mathcal{L}_{i j} \mathcal{M}_{i j}}{\mathcal{M}_{i j} \mathcal{M}_{i j}},
$$


where

$$
\begin{aligned}
\mathcal{L}_{i j} & =L_{i j}-\frac{1}{3} L_{k k} \delta_{i j} \\
\mathcal{M}_{i j} & =-\widetilde{\Delta} k_{\text {test }}^{1 / 2} \widetilde{S}_{i j},
\end{aligned}
$$

with $L_{i j}=\widetilde{\bar{u}}_{i} \bar{u}_{j}-\widetilde{\bar{u}}_{i} \widetilde{\bar{u}}_{j}$ and $k_{\text {test }}=L_{i i} / 2$. Moreover, taking a closer look at $\mathcal{L}_{i j}$ after replacing its $L_{i j}$ component, it becomes evident how identical and hence analogous to the deviatoric part of the SGS tensor as depicted in Equation 2.13. In fact, this may be understood as a wider filtered tensor and in doing so, it is modeled as:

$$
\mathcal{L}_{i j}=-2 \mathcal{M}_{i j}+\frac{1}{3} L_{k k} \delta_{i j}
$$

The transport equation used in this model is exactly the same as that of Equation 2.16. The difference lies in that the constant $C_{\varepsilon}$ is now dynamically evaluated:

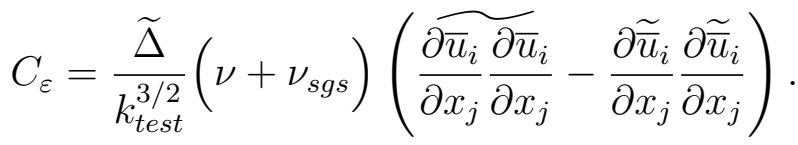

One of the most intriguing prospects of this model is its ability to predict backscatter. Moreover, the cost associated with the involved TKE transport equation is somewhat tolerable, because larger time steps may be used. Also, unlike in Germano et al. [27], averaging in the homogeneous direction is not something to worry about. Unfortunately, some drawbacks are also present such as a slight increase in memory for solving the extra transport equation and the lack of accurate initial data to start the procedure - although, one may consider starting from a Smagorinsky SGS model.

\subsubsection{Wall-Adapting Local Eddy-viscosity (WALE) Model}

The WALE model, short for wall-adapting local eddy-viscosity, is another more recent proposal introduced in 1999 by Franck Nicoud and Frédéric Ducros for capturing the correct behavior of subgrid viscosity in the vicinity of solid walls [63]. 
The main incentive for developing such a model was motivated by the vast majority of SGS models failing to effectively mimic the correct SGS viscosity behavior near wall boundaries. Incorrect SGS viscosity profile near the walls, in turn, results in an incorrect skin-friction coefficient due to the excess damping of fluctuations [71]. This model addresses such an issue without resorting to a transport-like equation or adding a wall- and skin-friction-dependent damping function - both of which require more sophisticated implementations and hence rendering the process either an ad hoc or a more computationally demanding one.

The WALE model, on the other hand, considers a more general algebraic approach. This method allows obtaining a subgrid viscosity proportional to the wall-normal component cubed $\left(\nu_{s g s} \propto y^{3}\right)$, as ought to be [67]. Additionally, this allows the SGS viscosity to vanish near the solid walls.

To start off, consider taking the traceless symmetric part of the square of the velocity gradient tensor as follows:

$$
S_{i j}^{d}=\frac{1}{2}\left(\bar{g}_{i j}^{2}+\bar{g}_{j i}^{2}\right)-\frac{1}{3} \delta_{i j} \bar{g}_{k k}^{2}
$$

with $\bar{g}_{i j}^{2}=\bar{g}_{i k} \bar{g}_{k j}$ and $\bar{g}_{i j}=\partial \bar{u}_{i} / \partial x_{j}$ being the filtered velocity gradient tensor. To further simplify things, denote the deviatoric part of the filtered velocity gradient tensor, the vorticity tensor, as:

$$
\bar{\Omega}_{i j}=\frac{1}{2}\left(\bar{g}_{i j}-\bar{g}_{j i}\right)
$$

Then, Equation 2.17, by means of some tedious algebra, is rearranged into:

$$
S_{i j}^{d}=\bar{S}_{i k} \bar{S}_{k j}+\bar{\Omega}_{i k} \bar{\Omega}_{k j}-\frac{1}{3} \delta_{i j}\left(\bar{S}_{m n} \bar{S}_{m n}-\bar{\Omega}_{m n} \bar{\Omega}_{m n}\right)
$$

It can be noted that $S_{i j}^{d}$ has a zero trace and its second invariant remains finite and proportional to $S_{i j}^{d} S_{i j}^{d}$. Next, further manipulation is introduced to Equation 2.19 
mathematically via the Cayley-Hamilton theorem while maintaining the initial assumption of incompressible flow [23]. This yields:

$$
S_{i j}^{d} S_{i j}^{d}=\frac{1}{6}\left(S^{2} S^{2}+\Omega^{2} \Omega^{2}\right)+\frac{2}{3} S^{2} \Omega^{2}+2 \Gamma,
$$

where $S^{2}=\bar{S}_{i j} \bar{S}_{i j}, \Omega^{2}=\bar{\Omega}_{i j} \bar{\Omega}_{i j}$ and $\Gamma=\bar{S}_{i k} \bar{S}_{k j} \bar{\Omega}_{j l} \bar{\Omega}_{l i}$.

Therefore, a LES model that uses Equation 2.20 is able to detect turbulence structures with either (large) strain or rotation rate, or even both [23]. Hence, in the event of pure shear flow, with a velocity gradient present only in the $x y$-plane ${ }^{5}, \bar{g}_{12}$, the eddy-viscosity will nearly vanish near the solid boundaries during laminar flow ${ }^{6}$.

The SGS tensor in the limit of a wall-normal approaching zero behaves as:

$$
\lim _{y \rightarrow 0} \tau_{i j}^{s g s} \propto \mathcal{O}\left(y^{3}\right)
$$

where the Taylor series expansion for the $\tau_{13}^{\text {sgs }}$ (the most important SGS tensor component to ensure proper scaling) component behaves proportional to the wall-normal cubed $\left(y^{3}\right)$. This is demonstrated in Sagaut [71, p. 159-160].

In turn, given that $\bar{S}_{i j}$ is of order $\mathcal{O}(1)$, this allows the eddy-viscosity to scale in the order of $\mathcal{O}\left(y^{3}\right)$. Ultimately, the WALE model may be defined via the below relation:

$$
\nu_{s g s}=\left(C_{w} \Delta\right)^{2} \frac{\left(S_{i j}^{d} S_{i j}^{d}\right)^{3 / 2}}{\left(\bar{S}_{i j} \bar{S}_{i j}\right)^{5 / 2}+\left(S_{i j}^{d} S_{i j}^{d}\right)^{5 / 4}},
$$

with $C_{w}=0.55-0.60$ being the WALE model constant.

Pertaining to the current version used of OpenFOAM, the WALE model is implemented in a straightforward fashion as Equation 2.21. Also, the model constant is specified with a default value of $C_{w}=0.325$.

\footnotetext{
${ }^{5}$ In this formulation, $y$ is the wall-normal direction and the remaining ones are statistically homogeneous flow directions.

${ }^{6}$ The eddy-viscosity is dependent on Equation 2.20. In this particular case, $S_{i j}^{d} S_{i j}^{d}=0$ since $S^{2}=\Omega^{2}=4 \bar{S}_{12}$ while $\Gamma=-S^{2} S^{2} / 2$.
} 


\subsubsection{Subgrid-Scale (SGS)-Free Model}

So far, the SGS models presented rely on injecting artificial dissipation through turbulence modeling. In this approach, the numerical scheme's dissipative nature is considered to be solely responsible for providing this effect. In the scope of this thesis, given no additional numerical adjustment is performed, this is denoted as the SGS-free model.

In the event of carefully tuned numerical schemes that account for a target numerical dissipation (by means of the truncation error), then this is referred to as implicit large eddy simulation (ILES). In technical terms, ILES relies on carefully constructed numerical schemes to best mimic artificial diffusion, e.g. deconvolution methods or CPR schemes. One of the earliest pioneers to suggest such school of thought were Boris et al. [9]. In their argument, they advocate not using an explicit filtering or subgrid modeling approach. Instead, they stress the importance of relying on an appropriately selected discretization technique in conjunction with a relatively coarse grid. That way, the effect of applying both a filter and modeling the subgrid stresses is arguably included through the numerical method's dissipation. For a more fulfilling explanation about ILES, the reader is encouraged to look at Adams and Hickel [2]

The main idea in an SGS-free model is rather simple. In fact, for this case, one may think of it as a coarse DNS since not all the scales are effectively resolved (as should be in the case of a DNS); the truncated terms used in the discretization method act as a subgrid stress. In the scope of this project, given that the numerical method used to discretize the computational domain is second-order accurate, the SGS viscosity behaves nominally proportional to the square of each grid's cell. More on the numerical scheme's accuracy shall be demonstrated in the following chapter.

The main advantage in such a model-free technique is the relatively cheap computational cost involved. Unlike previous models, this has no explicit modeling whatsoever and, as a matter of fact, one is solving for the NS equations directly. However, the actual solution is not a DNS for the reasons mentioned above. 
THIS PAGE IS INTENTIONALLY LEFT BLANK 


\section{Chapter 3}

\section{Numerical Implementation}

So far, only the computational aspects involved in the physics of incompressible turbulent flow, especially modeling via LES, has been covered. In this chapter, the numerical method and its implementation in the employed software, OpenFOAM, will be discussed. The FVM is used to discretize a generic partial differential equation (PDE) modeling a convective-diffusive transport equation. This is similar in structure to the governing fluid equations without going into the tedious details thereabout. The theoretical foundation and the implementation thereof is presented first. Afterwards, a numerical solution strategy is discussed that solves for the discretized set of equations using certain solver algorithms. Note that vector notation will be used in this chapter since it conveys a more intuitive approach.

\subsection{The Finite Volume Method}

The discretization method used in this framework is the FVM. Such a discretization technique is very common in the CFD industry, mainly due to its ability in handling complex geometries with relative ease. Additionally, another attractive characteristic is effectively capturing the fluid control volume (CV) while maintaining the governing PDE's conservation form without relying on ancillary steps. Besides, OpenFOAM is mostly based on FVM. Generally speaking, the FVM entails the following sequential 
steps: i) mesh a region into CVs, ii) integrate governing PDEs per CV, iii) choose a numerical integration technique, iv) select interpolation scheme, v) assemble discretized PDEs into an algebraic system, and vi) solve resulting system of equations.

Consider the general form of a second-order three-dimensional transient transport equation for a scalar value $\phi$ and with $\mathbf{u}$ being a vector field:

$$
\underbrace{\frac{\partial \phi}{\partial t}}_{\mathrm{I}}+\underbrace{\nabla \cdot(\phi \mathbf{u})}_{\mathrm{III}}-\underbrace{\nabla \cdot(\Gamma \nabla \phi)}_{\mathrm{III}}=\underbrace{S(\phi)}_{\mathrm{IV}},
$$

where $\Gamma$ is the diffusion coefficient and the four terms correspond to the temporal derivative (I), convection term (II), diffusion term (III) and the source term (IV).

In order to effectively approximate Equation 3.1, the numerical accuracy should be at least of the same order of the original PDE's order. In this case, it is sufficient for the numerical accuracy of the discretization to be second-order accurate; the FVM used henceforth is of second-order accuracy. This means that all spatial terms having an order of two and beyond are truncated when Taylor expanding.

In the following, and in accordance with later simulations, a structured grid is utilized. Moreover, only internal cells, or CVs, that share six neighboring cells are addressed. In order to implement the FVM on Equation 3.1 acting about an arbitrary control volume $V_{p}$ with centroid $P$, the following integral form must be satisfied:

$$
\begin{aligned}
\int_{t}^{t+\Delta t}\left[\int_{V_{p}} S(\phi) d V\right] d t & \\
\int_{t}^{t+\Delta t} & {\left[\int_{V_{p}} \frac{\partial \phi}{\partial t} d V+\int_{V_{p}} \nabla \cdot(\phi \mathbf{u}) d V-\int_{V_{p}} \nabla \cdot(\Gamma \nabla \phi) d V\right] d t . }
\end{aligned}
$$

The next step is to discretize Equation 3.2 while preserving global accuracy. To accomplish this, each term will be considered separately by starting with the spatial and moving towards the temporal discretization. Note, since no sources are used in the context of this thesis, the source term discretization will be neglected altogether. 


\subsubsection{Spatial Discretization}

Before proceeding, there are two core concepts to heed in discretization procedures. First, maintaining the overall target spatial accuracy in each term. Second, boundedness of the schemes should be considered in order to avoid instabilities.

\subsubsection{Convective Term}

The convective term may be mathematically manipulated, given that this is a closed region in space, and further decomposed into a more intuitive expression:

$$
\begin{aligned}
\int_{V_{p}} \nabla \cdot(\phi \mathbf{u}) d V & =\sum_{f}(\phi \mathbf{u})_{f_{i}} \cdot \mathbf{S}_{f_{i}} \\
& =\sum_{f=\text { owner }}(\phi \mathbf{u})_{f_{i}} \cdot \mathbf{S}_{f_{i}}-\sum_{f=\text { neighbor }}(\phi \mathbf{u})_{f_{i}} \cdot \mathbf{S}_{f_{i}}
\end{aligned}
$$

where the boundary face decomposition is performed such that the current owner cell is related to its neighboring cells and $\mathbf{S}_{f}$ is the outward pointing surface area vector.

In relation to OpenFOAM, the divergence scheme used for this term is known as central difference (CD). The CD scheme is based on the assumption that $\phi$ varies linearly between two neighboring $\mathrm{CVs}$ and is second-order accurate Ferziger and Peric [26]. Mathematically, this translates into:

$$
\phi_{f}=f_{x} \phi_{P}+\left(1-f_{x}\right) \phi_{Q}
$$

where $f_{x}$ is defined as the ratio of distances: $f_{x}=\|f Q\| /\|P Q\|$ with $P$ and $Q$ denoting the centroids of the two CVs and $f$ their common face.

\subsubsection{Diffusive Term}

Similar to the above convection term's discretization procedure, the diffusive term follows very closely. Hence, with some analogous algebraic manipulation, the diffusive term successively becomes: 


$$
\begin{aligned}
\int_{V_{p}} \nabla \cdot(\Gamma \nabla \phi) d V & =\int_{\partial V_{p}}(\Gamma \nabla \phi) \cdot \mathbf{n} d S \\
& =\sum_{f} \Gamma_{f_{i}}(\nabla \phi)_{f_{i}} \cdot \mathbf{S}_{f_{i}} .
\end{aligned}
$$

Examples for discretization schemes for such a term are dependent on the orthogonality of the mesh. In order to secure an inclusive approach, both orthogonal and non-orthogonal contributions need be considered. Fortunately, however, since the mesh at hand is constructed using the blockMesh utility, the resulting grid is orthogonal. The resulting discretized diffusion term becomes:

$$
\mathbf{S} \cdot(\nabla)_{f}=\frac{\left|\mathbf{S}_{f}\right|}{|\mathbf{d}|}\left(\phi_{Q}-\phi_{P}\right)
$$

where $|\mathbf{d}|=\|P Q\|$ is the distance between the two cell centers $(P$ and $Q)$ and $\left|\mathbf{S}_{f}\right|$ is the area of the corresponding face.

Finally, since the grid considered uses an orthogonal mesh, such a scheme is both suitable and second-order accurate.

\subsubsection{Temportal Discretization}

Concerning discretizion in time, the method of choice is the so-called second-order accurate in time backward differencing (BD) scheme. Formulating such a scheme requires the following steps. Consider the following pair of Taylor series expansions of $\phi$ in time about $t$ :

$$
\begin{aligned}
\phi^{n-1} & =\phi^{n}-\frac{\partial \phi}{\partial t} \Delta t+\frac{1}{2} \frac{\partial^{2} \phi}{\partial^{2} t} \Delta t^{2}-\frac{1}{3 !} \frac{\partial^{3} \phi}{\partial^{3} t} \Delta t^{3}+\mathcal{O}\left(\Delta t^{4}\right) \\
\phi^{n-2} & =\phi^{n}-2 \frac{\partial \phi}{\partial t} \Delta t+\frac{4}{2} \frac{\partial^{2} \phi}{\partial^{2} t} \Delta t^{2}-\frac{6}{3 !} \frac{\partial^{3} \phi}{\partial^{3} t} \Delta t^{3}+\mathcal{O}\left(\Delta t^{4}\right)
\end{aligned}
$$

where $\phi^{n-1}=\phi(\mathbf{x}, t-\Delta t)$ and $\phi^{n-2}=\phi(\mathbf{x}, t-2 \Delta t)$. 
Applying some algebra on Equation 3.4 and rearranging, the resulting relation forms what is known as the second-order (in time) BD scheme:

$$
\begin{aligned}
3 \phi^{n}-4 \phi^{n-1}+\phi^{n-2} & =2 \frac{\partial \phi}{\partial t} \Delta t+\frac{1}{3} \frac{\partial^{3} \phi}{\partial^{3} t} \Delta t^{3}+\mathcal{O}\left(\Delta t^{4}\right) \\
\therefore \quad \frac{\partial \phi}{\partial t} & =\frac{3 \phi^{n}-4 \phi^{n-1}+\phi^{n-2}}{2 \Delta t}+\mathcal{O}\left(\Delta t^{2}\right) .
\end{aligned}
$$

As evidenced, constructing such finite difference schemes is tedious, nonetheless for the sake of completion the steps were portrayed.

\subsubsection{System of Algebraic Equation}

After discretizing each of the required terms in Equation 3.1, proper rearrangement needs to be exercised. Taking into consideration all of the resulting discrete forms, the final expression that includes both space and time becomes:

$$
\frac{3 \phi_{P}^{n}-4 \phi_{P}^{n-1}+\phi_{P}^{n-2}}{2 \Delta t} V_{P}+\sum_{f}\left[\mathbf{u} \cdot \mathbf{S} \phi^{n}\right]_{f_{i}}-\sum_{f}\left[\Gamma \mathbf{S} \cdot \nabla \phi^{n}\right]_{f_{i}}=0
$$

This in turn is used to formulate a system of linear equations of the form:

$$
\underbrace{\left(\begin{array}{cccc}
a_{1,1} & a_{1,2} & \cdots & a_{1, N} \\
a_{2,1} & a_{2,2} & \cdots & a_{2, N} \\
\vdots & \vdots & \ddots & \vdots \\
a_{M, 1} & a_{M, 2} & \cdots & a_{M, N}
\end{array}\right)}_{\mathbf{A}} \underbrace{\left(\begin{array}{c}
\phi_{1}^{n} \\
\phi_{2}^{n} \\
\vdots \\
\phi_{N}^{n}
\end{array}\right)}_{\mathbf{x}}=\underbrace{\left(\begin{array}{c}
b_{1} \\
b_{2} \\
\vdots \\
b_{N}
\end{array}\right)}_{\mathbf{b}}
$$

where $\mathbf{A}$ is a $M$-by- $N$ matrix with the coefficients of $\phi^{n}, \mathbf{x}$ is a 1 -by- $N$ vector representing the solution being iterated for at time $n$ and $\mathbf{b}$ is also a 1-by- $N$ vector holding all the known data including that of the previous time steps. Also, $N$ and $M$ correspond to the local cell stencil and the global cell positions on the grid, respectively.

Solving such a system of equations can be done in either explicit of implicit form. The first is relatively faster to iterate through and requires less memory storage than 
the latter. However, the time increments required may be ridiculously small to ensure numerical stability. This means that the Courant-Friedrichs-Lewy (CFL) condition number should not be violated:

$$
\mathrm{CFL} \approx \Delta t \sum_{i=1}^{3} \frac{\phi_{i}}{\Delta x_{i}} \leq 1,
$$

where $i=1,2,3$ correspond to the three degrees of freedom, or dimensions, and consequently $\phi_{i}$ and $\Delta x_{i}$ denote the relevant terms corresponding to the solution and cell size, respectively.

Regarding the CFL number, below unity translates to a stable solution, a CFL equal to one is termed weakly stable while above that the solution blows up. On the other hand, an implicit scheme is not that sensitive to this condition number and strict fulfillment is not obligatory. On such basis, Equation 3.5 is solved using an implicit procedure, because of the temporal discretization scheme. This implies that the system of algebraic equations is unconditionally stable. Unfortunately, this uses more memory for storing current values as opposed to an explicit scheme. For a detailed comparison and error analysis with respect to different schemes either in time or space, refer to Jasak [35, chap. 3].

Finally, in an effort to connect the accumulated explanation thus far, the link in between the filtering done in LES and the FVM will be stressed. Recall, in the previous chapter, the filtering operation selected was that of a top-hat filter as depicted in Equation 2.8. The motivation behind selecting such a filter kernel and cutoff width is solely based on the finite volume discretization method used. In fact, Equation 2.8 reduces into a simple averaging procedure per traversed cell. In other words, this implies that the FVM, by construction, employs a top-hat filter. Therefore, the filtering operation for LES is implicitly applied by the discretization method. Any other filtering choice, such as Gaussian or spectral filters, would in essence, when used in conjunction with the FVM, mean that two filters are being applied at once: 
an implicit top-hat filter and an additional explicit one. Moreover, the filter's cutoff width - which is responsible for the size of eddies retained - may be chosen to theoretically have any value. Nonetheless, due to the nature of the cell definitions in FVM, any lesser or larger choice than the one used would be pointless since each cell is assumed an averaged quantity anyway. For more details on the relation between the discretization method and the filtering operation in LES, the reader is advised to read Versteeg and Malalasekera [81, chap. 3].

\subsection{Pressure-velocity Coupling}

After setting up the computational grid and effectively ensuring the target accuracy in both space and time is met, the solution method will now be addressed. For the sake of brevity, only the used solver in OpenFOAM will be discussed. This solver is based on a combination of two algorithms and is conveniently named pimpleFoam. To describe it, the SIMPLE and PISO algorithms will be covered first since the solver is comprised of the combination of these two (as the name hints). Accordingly, each of the SIMPLE and PISO algorithms will be discussed succinctly and separately before moving towards the implementation in the mentioned solver.

\subsubsection{SIMPLE Algorithm}

The SIMPLE algorithm, short for Semi-Implicit Method for Pressure Linked Equations, is an iterative technique first introduced in [65]. This method may be thought of as a guess-and-correct approach for the pressure on a given grid. The SIMPLE algorithm, briefly, may be summarized as starting from a tentative pressure field. Then, the discretized momentum equations are solved using such pressure field. Since this pressure field guess does not necessarily satisfy the continuity equation, a correction (Poisson equation) is then introduced to obtain the corrected pressure field and consequently the continuity-satisfying velocity field. 


\subsubsection{PISO Algorithm}

The PISO algorithm, short for Pressure Implicit with Splitting of Operators, is a technique, used as an extension to the SIMPLE algorithm to compute the pressurevelocity procedure [32]. The idea behind it is to solve for an unsteady solution using two steps: one predictor and, usually, two corrector steps.

The way it works is by adding an extra corrector step similar to the corrector step in the SIMPLE algorithm above. This also takes place right after it; in other words, two corrector steps are performed inside the above SIMPLE algorithm instead of just one. This is what is referred to the PISO algorithm and by now it is clear why it is thought of as an extension to the SIMPLE algorithm. In turn, performing two corrector steps instead of one, in general, takes less CPU time and returns a more stable solution.

\subsubsection{PIMPLE}

As previously stated, the PIMPLE algorithm is a mix between the previous SIMPLE and PISO algorithms. It is a transient solver and the way it works is by performing two loops. One for the pressure-velocity coupling. This is in an outer loop and the other is for the pressure- and velocity-corrections situated in an inner loop. These are illustratively displayed in Algorithm 1.

The interpretation of this pseudo-code formally starts after deciding on the simulation parameters, time, boundary and initial conditions as well as setting up the mesh and other transport equations. The pimpleFoam solver starts by first solving the discretized momentum equations using a starting guess at $t=t_{0}$. Then, a pressurecorrection loop is accessed. In this loop, an equation is solved to find an estimate for the amount by which the pressure needs correction. Consequently, this pressure correction factor is used to correct for the actual pressure value and in turn the latter is used to improve the velocity fields. If the pressure-correction loop occurs a single time, then this solver is reduced into the SIMPLE solver, else this is a PISO solver. 


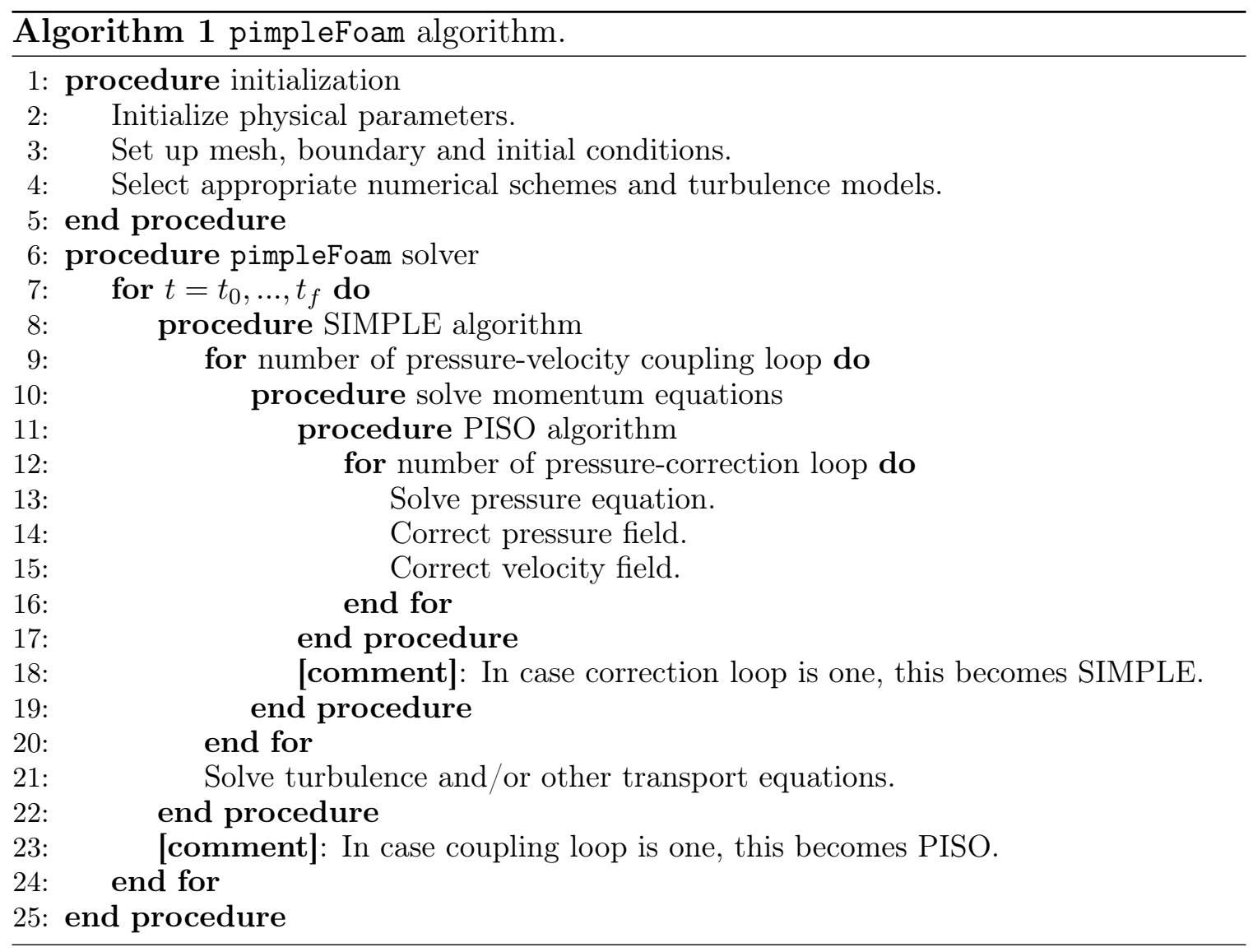

Finally, the entire remaining transport equations and turbulence models (if present) are solved. In the likelihood that convergence has not been achieved yet, then the entire processes is repeated all over again depending on the amount of repetitions the pressure-velocity loop is specified. If no repetition is involved, then this algorithm reduces into the PISO algorithm, otherwise this is the SIMPLE solver.

Pertaining to the implementation in OpenFOAM, the pressure-correction and the pressure-velocity coupling loops are specified inside the fvSolution file using the variables nCorrectors and nOuterCorrectors, respectively. In relation to the current project, the first is selected to yield a value of three while the latter has a value of one. Technically, this means that the pimpleFoam solver is actually implementing the PISO algorithm while performing three correction iterations per time step. Ideally, the pressure-correction loop is selected to have a value either two or three. Three 
was selected after checking the residuals in OpenFOAM and although after two iterations the residuals do drop down significantly, nonetheless an extra third iteration is used. After all, the overall computational expense does not outweigh the possible advantages in convergence time.

As for the linear solvers responsible for computing the velocities and pressure, they were selected such that convergence is attained relatively faster. The pressure solver uses the GAMG with the smoother DIC which correspond to the generalized geometric-algebraic multi-grid and the diagonal incomplete-Cholesky, respectively. The GAMG is used to obtain a faster convergence rate by taking advantage of multiple grids, hence the name. It starts from the finest level and reaches to the max level which is selected to have a maximum number of ten cells. This coarsening and refining process takes place in stages. The DIC is chosen as a smoother since the pressure matrix is symmetric and so this works well on it. For this case, only one sweep of the smoother is employed in conjunction with the GAMG. On the other hand, the velocity uses the PBiCG solver along with the DILU preconditioner which correspond to the preconditioned bi-conjugate gradient and the diagonal incomplete-LU. The velocity matrix is asymmetric in nature and hence both these methods take advantage of that fact to yield relatively better, more efficient results. Also, the turbulent kinetic energy is solved with the same settings as that of the velocity, whenever applicable. There are lots of literature that cover different methods when it comes to solvers and preconditions, one highly-esteemed reference is Saad [70]. 


\section{Chapter 4}

\section{Wall-bounded Turbulence}

Up to this point, the mathematics describing turbulence has been presented and different turbulence modeling procedures, specifically LES, were outlined. No elaborate consideration has been stressed on the intrinsic role certain boundaries play nor the expected behavior that arise thence. The presence of solid surfaces greatly affects how turbulent flow behaves. It is in the local vicinity of these solid walls that most of the cumbersome procedure in dealing with turbulence takes place. In turn, properly understanding turbulent flow near such regions is of utter importance.

Recall, the Reynolds number is defined as the ratio of inertial-to-viscous forces. Correspondingly, the closer the flow is to a wall the more dominant the viscous forces become.

In this chapter, two types of wall-bounded turbulent flows shall be addressed: channel and boundary layer. The importance of these with regard to the overall project will be observed in subsequent chapters. Note, the analysis will depict how the flow changes from an initial laminar phase until reaching turbulence. This is used only to serve as a complete picture of the flow's development. Emphasis is solely focused on the turbulent regime. Also, the analysis here shall take place with the assumption of smooth flat surfaces, a zero-pressure-gradient (ZPG) and no-slip velocity at the boundaries. 


\subsection{Wall-bounded Flows}

Flows moving across a flat surface with a uniform velocity and a ZPG are subject to the friction forces arising from the solid boundaries. These physical walls dictate a no-slip velocity boundary condition. This imposes that the fluid molecules adjacent to the stationary walls to stick on them. In turn, this creates a sort of chain-like behavior that results in slowing down the molecules directly above them. This goes on all the way until a certain threshold is reached whereby above it, the flow is smooth and negligibly affected by the viscous effects.

Below, two particular boundary layer flows will be examined separately. In each of these sections the flow characteristics will be detailed.

\subsubsection{Turbulent Boundary Layer Flow}

Turbulent boundary layer (TBL) flows may be described by considering an infinitely flat surface with flow moving across it in the streamwise direction. At its wall boundary, no-slip velocity conditions are imposed. Above it, a fictitious-like boundary exists where the free-stream velocity is reached and is referred to as the boundary thickness.

The procedure described above starts from a laminar flow with the fluid moving in smooth and organized layers. At some point the velocity profile, due to the noslip boundary condition and its magnitude, gets 'tripped'1. By doing so, the flow regime enters a transient phase and it loses its coherent structure and smooth layer property. After some critical distance, the flow becomes fully disordered and is then rendered as a statistically developed TBL. Refer to Figure 4-1a for an illustration of the flow regime changes. The velocity profiles in the laminar and turbulent regions are showcased in red and they correspond to the averaged values in the homogeneous directions and time. As opposed to the laminar, in the turbulent region the streamwise velocity profile has a steeper gradient near the wall. An instantaneous TBL taken

\footnotetext{
${ }^{1}$ This is not to be confused by artificially tripping a flow using a rough surface or discontinuous object to speed up the process of reaching turbulent flow.
} 


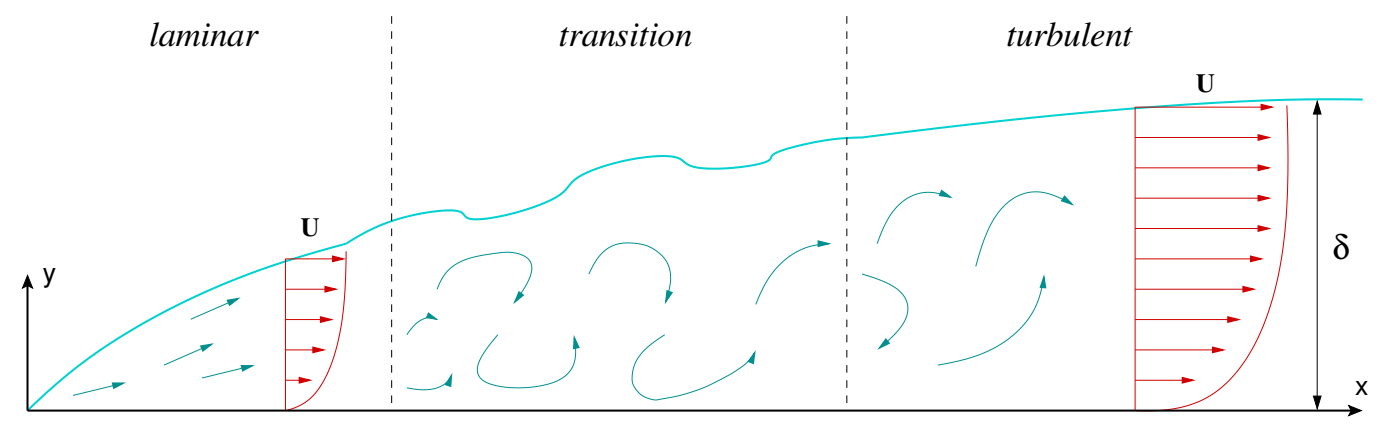

(a)

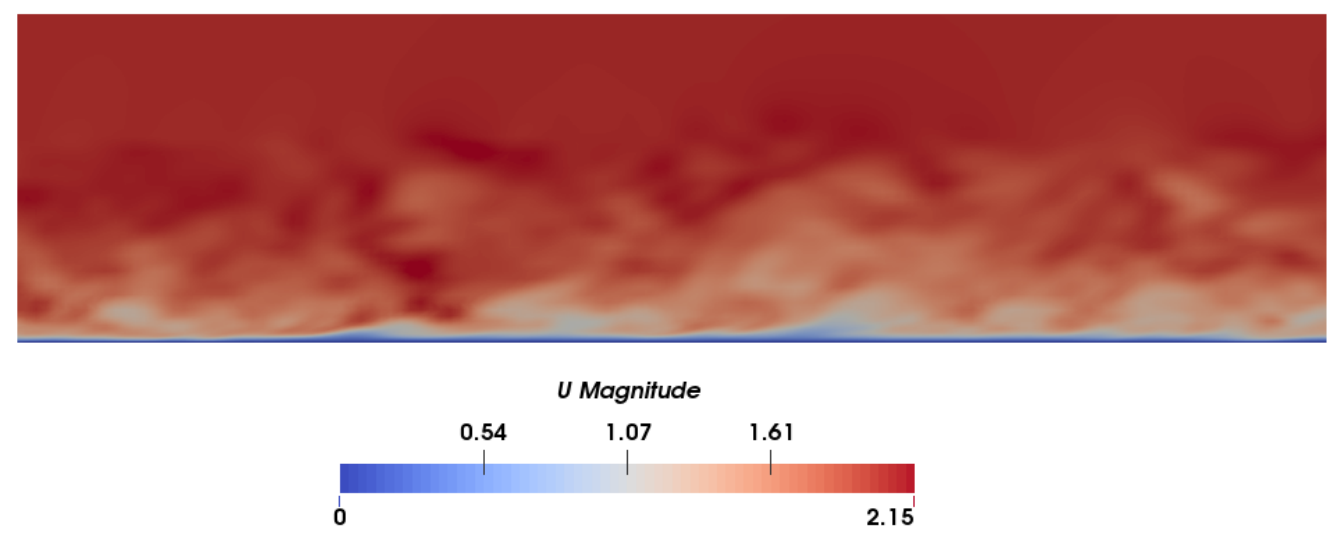

(b)

Figure 4-1: Sketch illustrating how the mean flow develops over a boundary layer (a) and a 2D slice taken from a LES depicting the instantaneous velocity magnitude $(\mathrm{m} / \mathrm{s})$ of a developed TBL (b).

from a LES is presented in Figure 4-1b. The TBL is said to be further developing in the wall-normal direction and is statistically independent of the spanwise direction. This implies that the boundary layer thickens as the flow proceeds. This is a direct result of the loss of momentum due to friction.

One important aspect in characterizing a TBL is its corresponding boundary layer thickness $\delta$. This is a theoretical threshold that separates the inertially-dominated from the viscous-dominated turbulent flow. There are three common ways to approximate the boundary layer's length scale: using ninety-nine percent of the free-stream velocity, the momentum thickness and the displacement thickness. Each of these terms are calculated (assuming incompressible flow) using: 


$$
\begin{aligned}
\delta_{99} & =\left.y\right|_{\langle u\rangle=0.99 U_{\infty}} \\
\theta & =\int_{0}^{\infty} \frac{\langle u(y)\rangle}{U_{\infty}}\left(1-\frac{\langle u(y)\rangle}{U_{\infty}}\right) d y \\
\delta^{*} & =\int_{0}^{\infty}\left(1-\frac{\langle u(y)\rangle}{U_{\infty}}\right) d y,
\end{aligned}
$$

where $U_{\infty}=\langle u(\delta)\rangle$ denotes the free-stream streamwise velocity, $y$ is the wall-normal coordinate, $\theta$ is the momentum thickness, $\delta^{*}$ is the displacement thickness and all the velocity terms are homogeneously averaged quantities via the $\langle\cdot\rangle$ operator. More emphasis will be placed on the usage of the first two in later chapters, since they are more frequently used.

Consequently, for each of these length scales a corresponding characteristic Reynolds number is defined as such:

$$
\operatorname{Re}_{\delta_{99}}=\frac{U_{\infty} \delta_{99}}{\nu}, \quad \operatorname{Re}_{\theta}=\frac{U_{\infty} \theta}{\nu}, \quad \operatorname{Re}_{\delta^{*}}=\frac{U_{\infty} \delta^{*}}{\nu}
$$

\subsubsection{Turbulent Channel Flow}

Turbulent channel flows may be described as having a pressure-driven flow in between two infinitely flat plates. Similarly to the TBL above, the flow starts from a uniform laminar flow. Then due to the no-slip boundary conditions and given sufficient magnitude, the velocity profiles become unstable in the transient phase. Finally, a statistically fully-developed turbulent flow takes place. Also, similar to the previous TBL, the turbulent velocity profile maintains a steeper gradient in the vicinity of the walls as opposed to the laminar region. This is illustrated in Figure 4-2a. Bear in mind that the velocity profiles depicted are averaged in both time and the spanwise directions. In this case, the flow is considered statistically independent of these directions and hence they are referred to as homogeneous. In Figure 4-2b, an illustration 


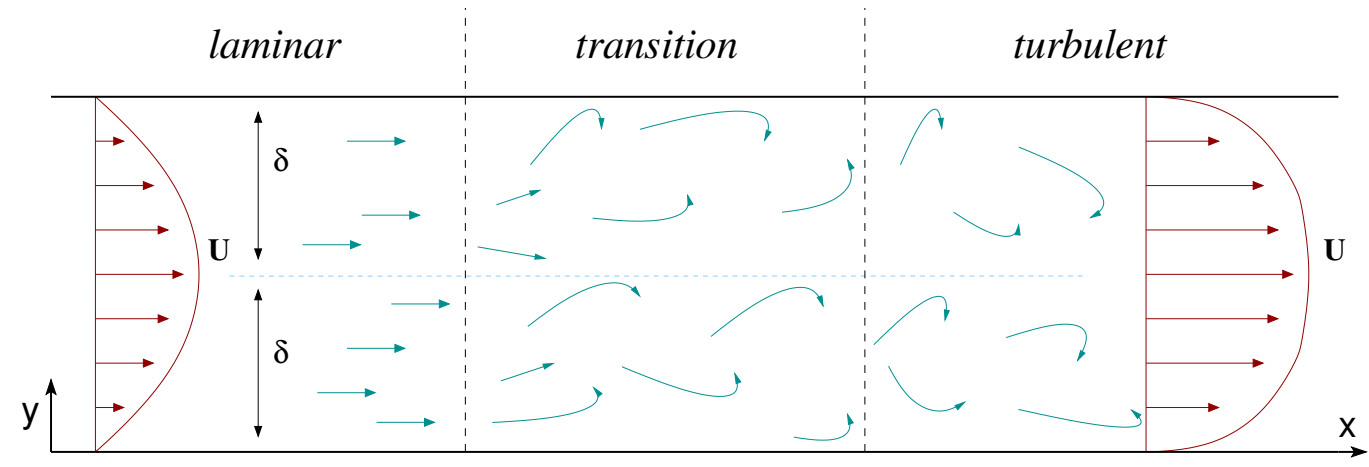

(a)
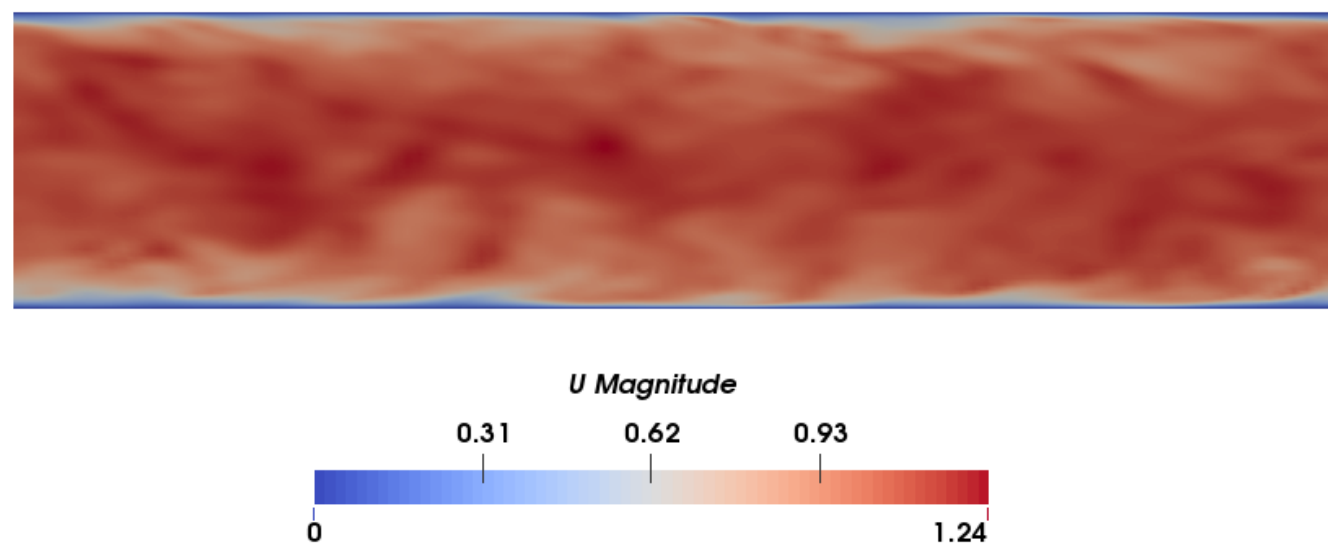

(b)

Figure 4-2: Sketch illustrating how the mean flow develops over a channel flow (a) and a 2D slice taken from a LES depicting the instantaneous velocity magnitude $(\mathrm{m} / \mathrm{s})$ of a fully-developed turbulent channel flow (b).

of the instantaneous velocity magnitude of a fully-developed channel flow using LES is presented.

Moreover, statistically symmetric conditions apply in channel flows about their half-height. Hence, the boundary layer thickness may be taken equal to be the channel's half-height $\delta$. To further characterize the flow, there are two common Reynolds numbers used based on the friction, or shear, velocity and the bulk velocity. They are defined as:

$$
\operatorname{Re}_{b}=\frac{U_{b} \delta}{\nu}, \quad \operatorname{Re}_{\tau}=\frac{u_{\tau} \delta}{\nu},
$$


where $u_{\tau}$ is defined in Equation 2.15 and $U_{b}$ has the following expression:

$$
U_{b}=\frac{1}{\delta} \int_{0}^{\delta}\langle u\rangle d y
$$

In an effort to correlate the skin friction coefficient to the bulk Reynolds number, Dean [18] conducted a series of experiments. In his effort, a relation conforming to a power-law between these two was found to be:

$$
C_{f}=0.073 \mathrm{Re}_{b}^{-1 / 4}
$$

where the skin-friction coefficient is defined as $C_{f}=\tau_{w} /\left(\rho U_{b}^{2} / 2\right)$.

This allows for a direct correlation between the friction and bulk Reynolds numbers which is very practical in later applications with regard to this project. The correlation used is [85]:

$$
\operatorname{Re}_{\tau} \approx 0.1751 \operatorname{Re}_{b}^{0.875}
$$

\subsection{Near-wall Flow Characteristics}

In either of the two cases of wall-bounded flows described above, there's a common behavior with respect to the velocity profiles. To proceed, this section will address two crucial properties. The first will introduce the concept of dimensionless scaling to better study the data relative to the wall. The second will tackle the theoretical expectation of how certain quantities behave near the wall. For a more thorough explanation, the interested reader is referred to Pope [67, chap. 7].

\subsubsection{Wall Regions}

Wall-bounded flows may be studied using two scales: a so-called 'inner' and 'outer' scale. They are intrinsically related to the Reynolds number definition. Near the wall, inner-scaled units are used. Inner scaling, commonly referred to as wall units, depend on the friction velocity and kinematic viscosity. Sufficiently far from the wall, viscous 


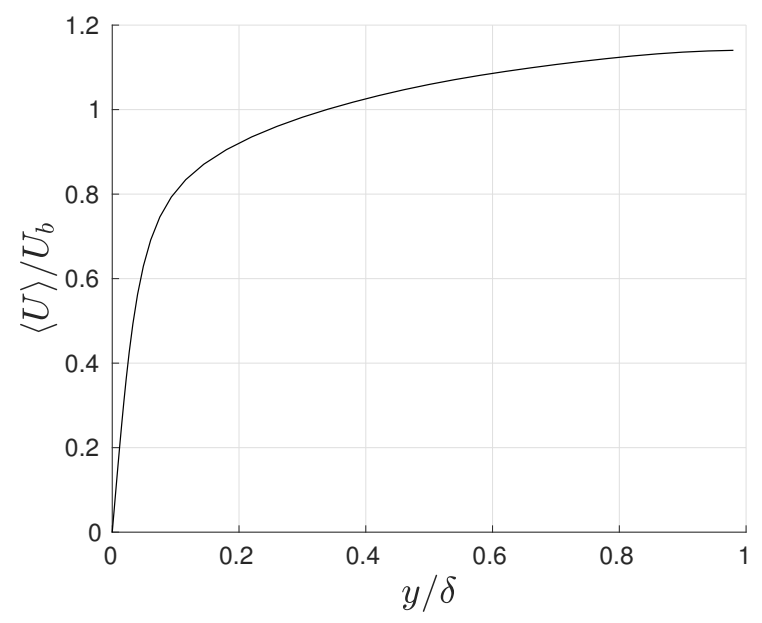

(a)

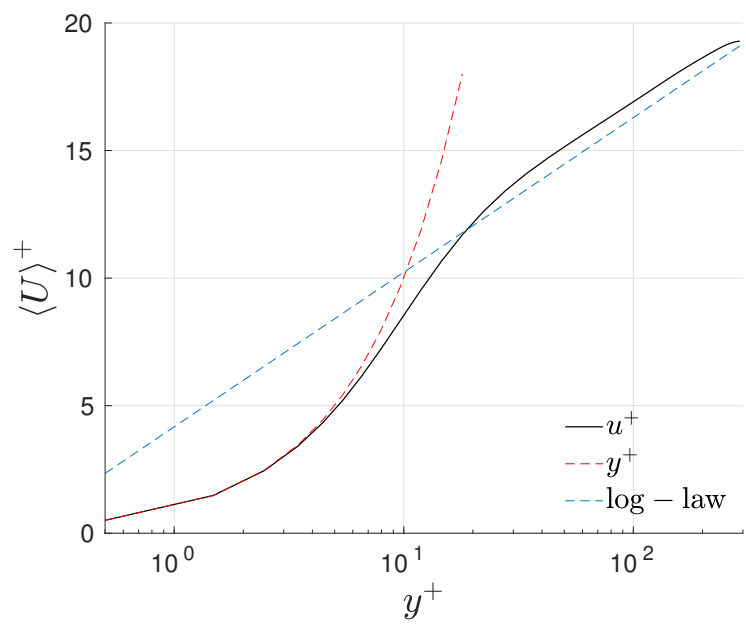

(b)

Figure 4-3: Mean streamwise velocity component versus the wall-normal coordinate taken from a fully-developed turbulent channel flow with a target $\operatorname{Re}_{\tau}$ of 300 using LES, expressed in outer scaling (a) and inner scaling (b).

forces are of negligible effect, hence the scaling used is dependent on a boundary layer's thickness and bulk velocity.

In Figure 4-3, the same averaged streamwise velocity component is expressed in two separate scalings that non-dimensionalize the data. It is evident how poor the plots are near the wall in conjunction with outer scaling. Thus, the velocity is expressed in wall units for more illustrative purposes in the vicinity of the no-slip boundaries. These wall units are denoted by a plus sign superscript and are defined as $u^{+} \equiv u / u_{\tau}$ and $y^{+} \equiv y \delta / \nu$. Recall, for channel flows $\delta$ is the half-height.

In the inner-scaled plot, separate regions in each of the outer and inner layers are discussed next ${ }^{2}$. The inner layer covers roughly $0 \leq y^{+}<100$ while the outer layer stretches above it. Nonetheless, these two layers share a common region. For $y^{+}<5$, this is the viscous sublayer. In this region, the viscous forces greatly dominate the flow while the velocity scales linearly. This is illustrated by the red-dotted line. For $30<y^{+}<200$, this is the log-law region, whereby it is widely accepted that the

\footnotetext{
${ }^{2}$ All intervals presented and discussed are based on a rough estimation from the inner- and outer-scaled panels in Figure 4-3 and for this particular case only - i.e. a target $\operatorname{Re}_{\tau}=300$.
} 
velocity follows an arguably universal curve of logarithmic nature expressed as:

$$
u^{+}=\frac{1}{\kappa} \log y^{+}+B
$$

where $B$ is a constant and $\kappa$ is the von Karman constant with values $\kappa=0.38$ and $B=4.17$ [62]. These are denoted by the blue-dotted line.

Between $5<y^{+}<30$, this interval is considered as the buffer layer and in it the velocity deviates from both linear and log-law scaling. Last but not least, in between $100<y^{+}<200$, this is the overlap region where, as the name suggests, the velocity is considered as belonging to both inner and outer layers. Finally, in the outer layer and especially for $y^{+}>200$ the inner-scaled figure is abandoned and, conveniently, an outer-scaled one is used instead.

While discussing the above wall-normal intervals and their relation to the velocity profile, the reader ought to know that these are neither precise nor universal intervals. Presently, the intervals used were interpreted for a target $\operatorname{Re}_{\tau}$ of 300 as depicted in Figure 4-3 - i.e. a specific case. Moreover, these intervals are more recognizable at higher Re-numbers; refer to Pope [67, chap. 7]. That being said, although the intervals are subject to slight variations, nonetheless there is some consistent behavior. These consistencies give evidence of a log-law region in the far inner and near outer layers as well as a viscous sublayer nearest to the wall. Observe, these regions/layers stretch and contract proportional to the Reynolds number characterizing the flow. Additionally, some useful properties by definition are $y^{+} \approx 1$ means the viscous forces are equivalent to that of the inertial forces and when $y=\delta$ then $y^{+} \approx \operatorname{Re}_{\tau}$.

\subsubsection{Velocity Fluctuation Behavior}

In order to effectively study the fluid motion in the immediate vicinity of a wall, one needs to take a deeper look at the inner region with respect to turbulent quantities. More specifically, the velocity fluctuations. To do so, the relevant terms need to be expanded in the limit of a wall and for a very small wall-normal coordinate. Moreover, 
an assumption of statistical homogeneity in the streamwise and spanwise directions is employed as was the case in the types of wall-flows considered earlier. The following is based on Pope [67, p. 283-284]. Thus, a Taylor series expansion is performed up to second order on each of the velocity fluctuations at the limit of the wall $(y \rightarrow 0)$ :

$$
\begin{gathered}
u^{\prime}=a_{1}+b_{1} y+c_{1} y^{2}+\mathcal{O}\left(y^{3}\right) \\
v^{\prime}=a_{2}+b_{2} y+c_{2} y^{2}+\mathcal{O}\left(y^{3}\right) \\
w^{\prime}=a_{3}+b_{3} y+c_{3} y^{2}+\mathcal{O}\left(y^{3}\right),
\end{gathered}
$$

where $u_{i}^{\prime}=u_{i}-\left\langle u_{i}\right\rangle$ is the velocity fluctuations and the coefficients $a_{i}, b_{i}$ and $c_{i}$ correspond to the point about which the expansion is taking place, its first derivative and its second derivative, respectively. Also, the subscript $i=1,2,3$ denotes the streamwise, wall-normal and spanwise directions, respectively.

Using the no-slip boundary condition at the wall, the velocity fluctuations are zeroed out $u_{i}^{\prime}=0$ and as a result $a_{i}=0$. Next, using the continuity equation, the coefficient $b_{2}=\partial v^{\prime} / \partial y=0$ as $y \rightarrow 0$. The resulting Taylor expanded terms are:

$$
\begin{array}{rrrr}
u^{\prime} & = & b_{1} y+c_{1} y^{2}+ & \mathcal{O}\left(y^{3}\right) \\
v^{\prime}= & c_{2} y^{2}+ & \mathcal{O}\left(y^{3}\right) \\
w^{\prime}= & b_{3} y+c_{3} y^{2}+ & \mathcal{O}\left(y^{3}\right) .
\end{array}
$$

Taking the expansion in Equation 4.4 and applying it onto the temporal and spatial averaged Reynolds stress quantities, the final output becomes:

$$
\begin{aligned}
\left\langle u^{\prime} u^{\prime}\right\rangle & =\left\langle b_{1} b_{1}\right\rangle y^{2}+\mathcal{O}\left(y^{4}\right) \\
\left\langle v^{\prime} v^{\prime}\right\rangle & =\left\langle c_{2} c_{2}\right\rangle y^{4}+\mathcal{O}\left(y^{6}\right) \\
\left\langle w^{\prime} w^{\prime}\right\rangle & =\left\langle b_{3} b_{3}\right\rangle y^{2}+\mathcal{O}\left(y^{4}\right) \\
\left\langle u^{\prime} v^{\prime}\right\rangle & =\left\langle b_{1} c_{2}\right\rangle y^{3}+\mathcal{O}\left(y^{4}\right) .
\end{aligned}
$$




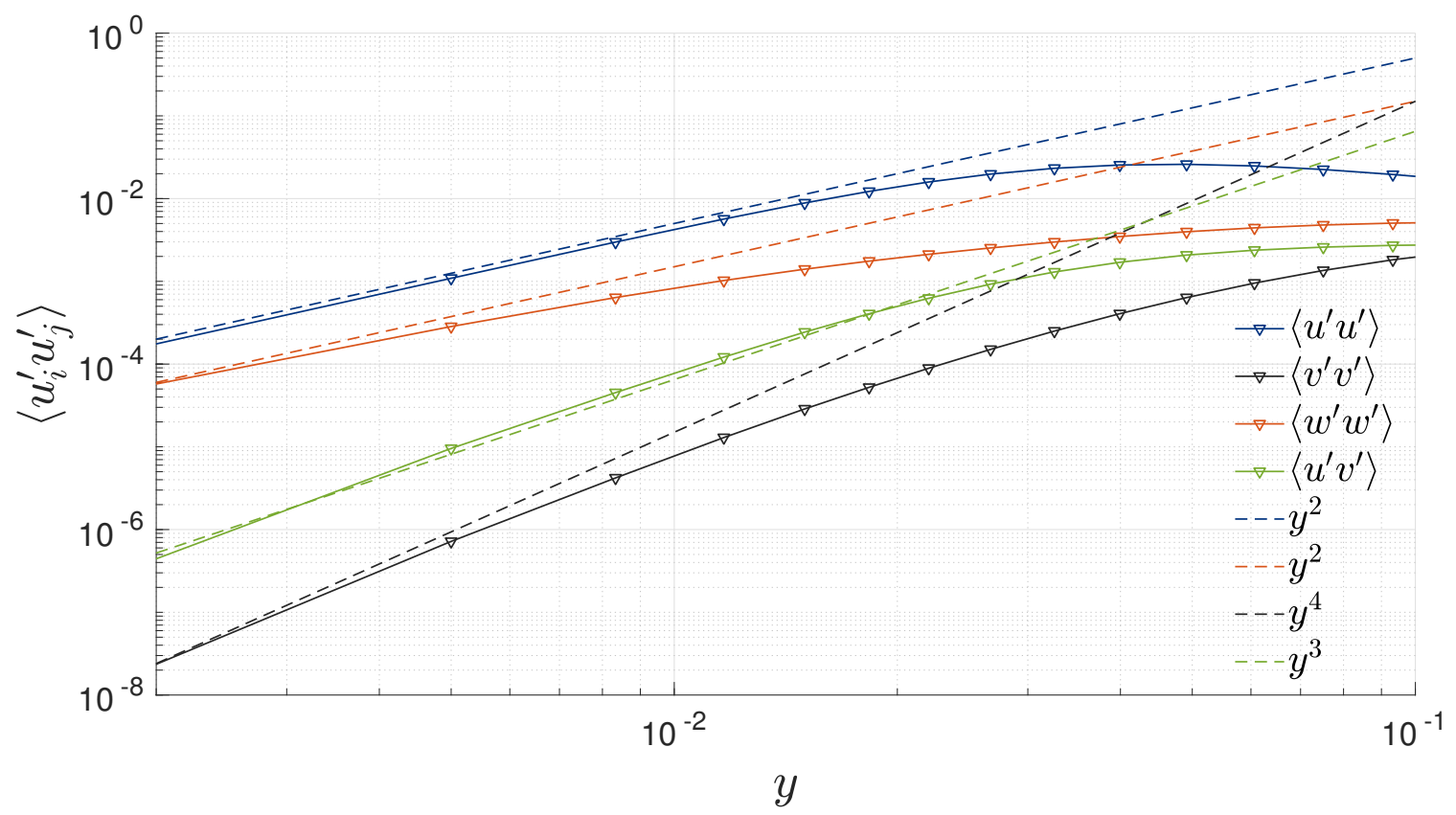

Figure 4-4: Scaling of Reynolds stress components as a function of the wall-normal coordinate near the wall. These are taken from a LES of fully-developed turbulent channel flow for a target $\operatorname{Re}_{\tau}=300$.

The results indicate that the turbulent quantities (i.e. Reynolds stresses) scale differently near the wall. This is illustrated in Figure 4-4 with the Reynolds stresses plotted in solid-triangular lines and their respective scale behavior in dashed lines with matching colors. The $\left\langle u^{\prime} u^{\prime}\right\rangle$ and $\left\langle w^{\prime} w^{\prime}\right\rangle$ scale as $y^{2}$ while the $\left\langle v^{\prime} v^{\prime}\right\rangle$ scale as $y^{4}$. Additionally, the shear stress $\left\langle u^{\prime} v^{\prime}\right\rangle$ scales as $y^{3}$.

The scalings discussed are based on $y$, however, near the wall it is more appropriate to use inner scaling $y^{+}$. Replacing $y$ by $y^{+}$, more universality is gained in correctly capturing the near-wall behavior of the components of the Reynolds stress tensor.

A careful observation to note is that the plots in Figure 4-4 are that of a large eddy simulation and not a direct numerical simulation. In the case of the latter, the quantities will be more resolved nearer to the wall and hence further alignment with their respective scalings will be made than with the current simulation. Despite this fact, the profiles using LES demonstrate acceptable scaling; this is because several points in the viscous sublayer were used to capture the linear behavior. 


\section{Chapter 5}

\section{Turbulent Channel Flow}

After establishing the theory and numerical procedure required, and before moving towards the backward-facing step problem, a detour about turbulent channel flow is essential. To begin with, channel flows are amongst what is considered as canonical turbulent flows. This is especially true when considering statistically fully-developed turbulent channel flows in which wall-bounded turbulence plays a prominent role. Additionally, the nature of such simulations is relatively straightforward to set-up and run. Consequently, this enables a more transparent comparison and validation procedure of numerical results.

In this chapter, the underlying sections will first discuss its purpose, next survey existing relevant literature, afterwards highlight the numerical set-up, then present results and finally discuss them.

\subsection{Objective}

The main purpose of this chapter is two-fold. First, a study about a relatively low target $\operatorname{Re}_{\tau}=180$ is conducted. This serves as a cornerstone for later advancements as well as validation purposes. Second, a relatively higher target $\mathrm{Re}_{\tau}=300$ is investigated in a more rigorous fashion. The motivation behind using the latter Re-number is because of its relative proximity with that at the backward-facing step's inlet. Both 
spatial resolution and different SGS models are examined. The results are compared to DNS benchmark data with similar target Re-number in both cases.

\subsection{Literature Review}

The progression in turbulent channel flow research started gaining momentum at the beginning of the twentieth century. Laufer [45] was amongst the first to conduct a series of experiments pertaining to both mean and fluctuating flow components and document the results. In his study, three different Re-numbers were investigated and by observing the energy spectra, near isotropic behavior was witnessed. This confirmed Kolmogorov's hypothesis [43] which postulated the existence of statistical isotropy in small-scale turbulent motions at sufficiently high Re-numbers. Then, Comte-Bellot [15] conducted a series of experiments with a wide range of Re-numbers and reported data for high-order statistics. Afterwards, more experiments were carried out that placed more emphasis on certain criteria such as the inner layer region [13] and laminar-turbulent transition [66].

At this point in time, the first published LES of a three-dimensional channel flow was conducted in Deardorff [19] using the SGS model developed in Smagorinsky [73]. Although the grid employed was too coarse for such Re-number and despite artificial boundary conditions ${ }^{1}$ being specified at the walls, the results, arguably, did manage to capture some of the profile shapes of the flow components. This rendered LES an interesting option to further evolve for wall-bounded flows ${ }^{2}$. Accordingly, Schumann [72] extended the LES model by utilizing a transport equation for the SGS model and a finer mesh while also maintaining artificial boundary conditions at the walls. Nonetheless, his results did not prove to be significantly better. In the meantime, experiments were still being carried out in order to better extract data [31, 24, 36].

As of yet, there was no reliable agreement between reported numerical simulations and experimental results. Moin et al. [56] performed a LES with $\mathrm{Re}_{\tau}=640$ and

\footnotetext{
${ }^{1}$ Near-wall modeling was used instead of the wall-resolving no-slip velocity BC.

${ }^{2}$ At that instant, LES was mainly developed with free-shear flows in mind.
} 
managed to obtain relatively better results than prior LES studies without relying on artificial boundary conditions at the walls. The SGS model used was a combination of the Smagorinsky far from the walls and a modified SGS model that is used to better imitate the viscosity via Prandtl's mixing length hypothesis near the walls. The main hindrance in their simulation was the grid resolution; the total grid points in both homogeneous directions (spanwise and streamwise) was insufficient due to computational and memory availability at the time. Another LES was carried out in Moin and Kim [54] for a Re-number of 13800 (based on the centerline velocity and channel half-height) and in turn yielded good results. In it, a Smagorinsky SGS model was used alongside a van Driest damping function near the walls [80]. In their simulation, the grid constructed did manage to capture efficiently the viscous sublayer via non-uniformly placed points and was also much finer than earlier studies. This further advocated the possibility for LES to attain good results in wall-bounded turbulent flows. Afterwards, LES was gradually becoming more used, especially using the Smagorinsky model $[29,52]$.

Up until this instant, there was no simulation of a fully-developed turbulent channel flow capable of providing extensive data about all the scales of motion. Most comprehensive numerical simulations conducted thus far were that of LES. While the outcome, as compared with experimental results, was relatively good, yet there was still a noticeable discrepancy at the vicinity of the walls. This rendered the current LES inadequate for extracting further information to complement fluid experimentation. Kim et al. [41] accomplished the first DNS of a fully-developed turbulent channel flow for $\mathrm{Re}=3300$ (based on the centerline velocity and channel half-height) using a fully spectral method. Also, for the first time, complementary statistical data and correlations were available in conjunction with the experimental data. The outcome was in good agreement when compared to its experimental counterpart, however discrepancies were still evident, though mostly restricted close to the wall. Many studies were performed on such an exhaustive simulation with abounding data that 
had significant and complementary insights $[51,55]$. This demonstrates how much information may be extracted from a carefully resolved numerical simulation.

During this time, numerical simulation of fully-developed turbulent channel flows started becoming more popular. This was greatly facilitated by the advancements in computing technology that rendered such computations plausible. Many other DNS simulations began taking place and some even incorporated heat transfer studies too [50, 69]. Later, Moser et al. [58] performed a DNS for $\operatorname{Re}_{\tau}=180,395,590$ using a spectral method. The results displayed good agreement with experimental studies. Accordingly, an exhaustive set of statistical flow data was made available for public use afterwards.

Shortly thereafter, Iwamoto et al. [34] also conducted a successful DNS in order to evaluate the effectiveness of feedback control algorithms that reduce the skinfriction coefficient. The simulations addressed $\operatorname{Re}_{\tau}=110,150,300,400,650$ using a spectral method. In this study, the interaction between the flow structures near and far from the wall was of great interest. For the time being, numerical simulations - even DNS - were becoming more-or-less the trend in studying channel flows, see e.g. $[28,20,30,53,79,49,60]$. This has, ever since, further picked up the pace by performing DNS reaching up to an astounding $\operatorname{Re}_{\tau}=5200$ in Lee and Moser [47].

As for the present study, grid resolution and SGS models are compared extensively for two $\operatorname{Re}_{\tau}$. The results give a detailed insight on how the different combinations of grid and SGS models used impact the outcome. Moreover, all results are presented as a database for turbulence modeling purposes.

\subsection{Simulation Set-up}

This section will detail the simulation set-up in OpenFOAM. First, setting up the computational grid will be discussed along with the logic behind it. Next, initial and boundary conditions specified will be addressed. Finally, the physical parameters involved as well as the flow specifications will be covered. 


\subsubsection{Computational Domain}

The channel geometry is simple to mesh in a structured manner. Three-dimensional rectangular blocks are utilized during domain decomposition via the blockMesh mesh generation utility available in OpenFOAM. The blocks cover both of the channel's homogeneous directions entirely and depend only on the wall-normal meshing method used. Refer to Figure 5-1. The channel dimensions specified are set as a function of its half-height, $\delta$, such that $9 \delta \times 2 \delta \times 4 \delta$ correspond to $L_{x}, L_{y}$ and $L_{z}$, respectively. The reason behind selecting such $\delta$ coefficients goes back to the two-point correlations reported in Comte-Bellot [15]; this dictates that sufficient separation length must be applied for the velocity components to become independent. Moreover, the assumption of periodic boundary conditions (i.e. homogeneity) in both spanwise and streamwise directions require twice the thresholds used to account for the repercussions of applying artificial periodic conditions [54]. A channel half-height value of $\delta=1 \mathrm{~m}$ is chosen.

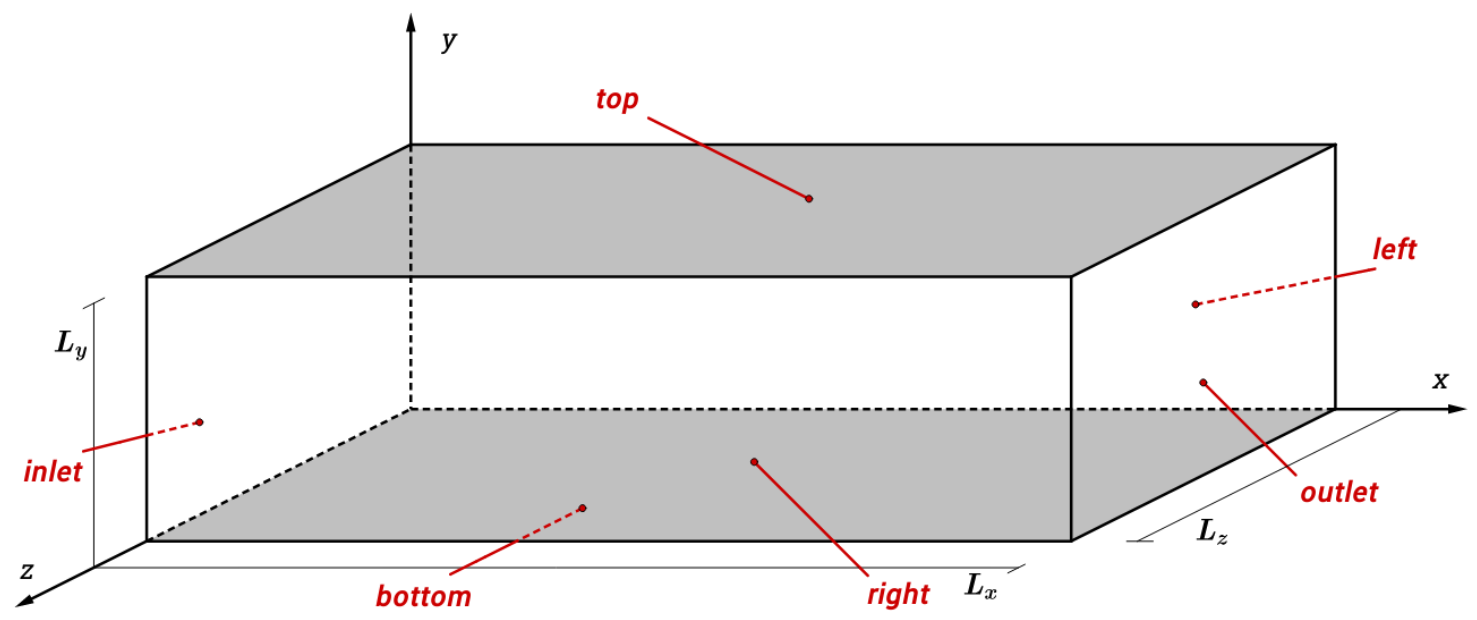

Figure 5-1: Channel computational domain with respect to the global Cartesian coordinate system (dark surfaces correspond to walls).

In subsequent simulations, two different wall-normal meshing approaches are used. In both of them, the cells in the homogeneous directions are kept equidistant while in the wall-normal direction they vary via a geometric expansion that is dependent on 
the height of the first and last cell. The flow is symmetric about the channel's center in the wall-normal direction, hence all resulting explanation will cover one portion (e.g. $0 \leq y \leq \delta)$.

The first wall-normal meshing approach, referred to as M1 thereon, divides the wall-normal direction, up to $\delta$, into three layers. This was initially inspired by Pope [67, chap. 13] and later calibrated in Rezaeiravesh and Liefvendahl [68]. These layers correspond to the viscous sublayer, the overlap region and the outer layer. Their corresponding cell sizes may be expressed by:

$$
\Delta y=\min \left[\max \left(\frac{y_{1}^{+}}{\operatorname{Re}_{\tau}}, \eta_{i n} \eta\right), \frac{1}{M}\right] \delta,
$$

where $\Delta y$ is the cell size in the wall-normal direction, $\eta=y / \delta$, the two constants ${ }^{3}$ $\eta_{\text {in }}=0.2, M=20$ and the first cell-center distance (in wall units) from the wall is $y_{1}^{+}=y_{1} \operatorname{Re}_{\tau} / \delta$.

The interpretation of Equation 5.1 will follow next. Starting from the viscous sublayer $\left(0 \leq y^{+} \leq 5\right)$, the cell size is set to a constant of $\Delta y=y_{1}^{+} \delta / \operatorname{Re}_{\tau}$. For the overlap region $\left(5<y^{+} \text {and } y / \delta<\eta_{i n}\right)^{4}$, the cell size is geometrically expanded from the previous size until it reaches $\delta / M$. Finally, for the remaining region, the outer layer $\left(\eta_{i n} \leq y / \delta \leq 1\right)$, the cell size is kept constant at $\Delta y=\delta / M$.

The second wall-normal meshing approach, referred to as M3 thereon ${ }^{5}$, uses a single block that stretches all the way up to the channel's half-height. In this technique, the cell size is expanded geometrically starting from $\Delta y=y_{1}^{+} \delta / \operatorname{Re}_{\tau}$ in the first cell from the wall and grows until it reaches $\Delta y=\delta / M$ at the channel center for $y=\delta$.

\footnotetext{
${ }^{3}$ These values are used by default. However, the reader is instructed to take a look at the summarizing simulation table in each section. In case of discrepancy between values reported here and in the table, values in the tables supersede the ones provided here.

${ }^{4}$ Commonly, as defined in [67, chap. 7], the overlap region ends at $\eta_{i n} \approx 0.1$. In this work, however, a value of 0.2 was used instead. This is justified based on the number of cells expanded being better fit to cover this small region with $\eta_{i n}=0.2$ than for $\eta_{i n}=0.1$.

${ }^{5}$ The author intentionally uses the naming convention M1 and M3. This is chosen for consistency with Rezaeiravesh and Liefvendahl [68] in which these are referred to as distribution strategies.
} 
For further details about each grid structure per simulation, the reader is instructed to read the summarizing tables in the results section. Finally, in all the simulations presented in this chapter, a total of five cells are placed in the viscous sublayer.

\subsubsection{Initial and Boundary Conditions}

Statistically fully-developed channel flow studies dictate that a fluid flows between two infinitely long parallel walls. In order to achieve that, periodic boundary conditions in the spanwise and streamwise directions are prescribed. Refer to Figure 5-1. This is done by pairing the outlet with the inlet for the streamwise direction and the left with the right for the spanwise direction. As for the top and bottom, a no-slip boundary condition and a zero gradient are prescribed for the velocity and pressure, respectively.

In the case of utilizing different SGS models, some extra parameters are also introduced, such as $k_{s g s}$ and $\nu_{s g s}$ which are the SGS kinetic energy and SGS viscosity, respectively. A summary detailing all variables' boundary conditions (BC) on the top and bottom walls with their corresponding values as well as their respective initial conditions (IC) is showcased in Table 5.1. Note, ICs are prescribed for the nonboundary quantities. The remaining portion of BCs, as stated earlier, are periodic.

Table 5.1: Channel flow initial and boundary conditions.

\begin{tabular}{l|lc|c} 
& \multicolumn{2}{|c|}{ BC } & IC \\
Field & Type & Value & Value \\
\hline$\overline{\mathbf{u}}$ & Dirichlet & 0 & - \\
$\nu_{s g s}$ & Dirichlet & 0 & 0 \\
$k_{s g s}$ & Dirichlet & 0 & 0.01 \\
$\bar{p}$ & Neumann & 0 & 0
\end{tabular}

The ICs specified for the velocity need modification in such a way that it becomes attainable and much faster to reach a fully-developed turbulent state. To accomplish 
this, the velocity field is perturbed to better mimic turbulence via the perturbU utility which is based on the work in De Villiers [17].

\subsubsection{Physical and Flow Parameters}

The flow is characterized by its friction Re-number, $\operatorname{Re}_{\tau}$, as defined in Equation 4.1. For an a priori friction velocity and channel half-height, $\mathrm{Re}_{\tau}$ may be adjusted using its kinematic viscosity only. The viscosities used are $\nu=3.5 \cdot 10^{-4} \mathrm{~m}^{2} / \mathrm{s}$ and $\nu=$ $1.9922 \cdot 10^{-4} \mathrm{~m}^{2} / \mathrm{s}$ for a target $\operatorname{Re}_{\tau}=180$ and $\mathrm{Re}_{\tau}=300$, respectively. The bulk velocity is $U_{b}=1 \mathrm{~m} / \mathrm{s}$. In order to drive the flow in the streamwise direction, an artificial force is required. Such a force is treated as a source function based on the bulk velocity. Accordingly, the source function is adjusted after each time step (using the bulk velocity) and inserted into the momentum equations to consistently drive the flow while maintaining the target $\operatorname{Re}_{\tau}$. This is all done via the fvOption functionality in OpenFOAM.

\subsection{Results and Discussion}

In the remaining part of this section, two distinct simulations for statistically fullydeveloped turbulent channel flows are carried. These simulations correspond to a different target Re-number. The results are grouped according to the computed quantities (e.g. velocity, Re-stresses, energy spectra... etc).

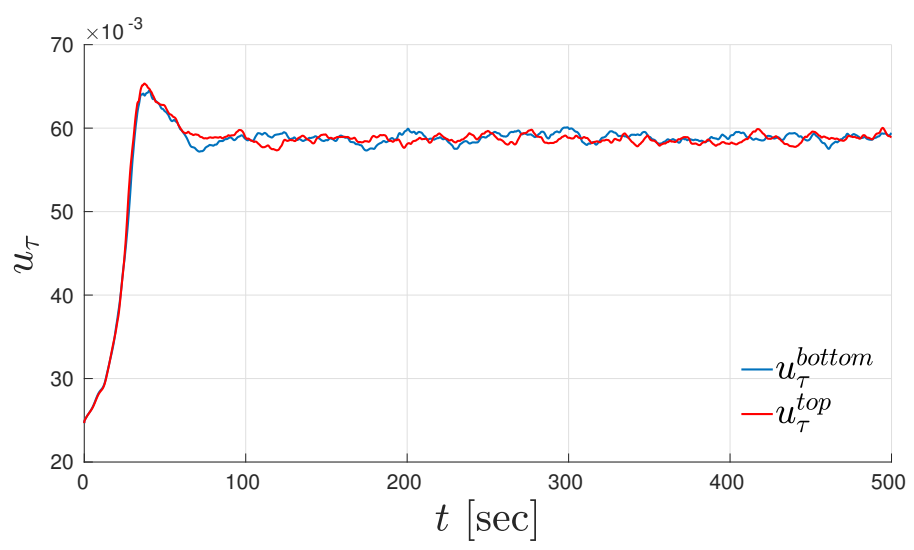

Figure 5-2: Transient phase for $u_{\tau}$ taken from a LES with target $\operatorname{Re}_{\tau}=300$. 
Each simulation is run until there were no significant oscillations in the $u_{\tau}$ values. Refer to Figure 5-2 for an illustration. For the remaining simulations in this section, a transient period of $\Delta T^{t r} \approx 55 L_{x} / U_{b}$ is found suitable for attaining a statistically fully-developed turbulent flow. Afterwards, the simulation starts averaging in time for later post-processing. The total simulation time is $\Delta T^{t o t} \approx 83 L_{x} / U_{b}$ of which approximately 27 out of 83 'flow-through' times are reserved for statistical data sampling. A 'flow-through' time is defined based on the bulk velocity and the time it takes to cover the entire streamwise direction.

The friction velocity values are computed by averaging the values on the top and bottom walls. Note, the primitive terms (e.g. velocity, pressure) in LES figures do not use their filtering symbol (e.g. $\left\langle\overline{u^{\prime} u^{\prime}}\right\rangle / u_{\tau}$ or $\langle\bar{U}\rangle^{+}$); the filtering sign is neglected in order to remain relevant to the DNS data (which do not use a filter).

\subsubsection{Numerical Simulations for a Target $\operatorname{Re}_{\tau}$ of 180}

In Figure 5-3, the M1 grid resolution is illustrated. Such a non-DNS resolution is considered amply fine for a target $\mathrm{Re}_{\tau}=180$.

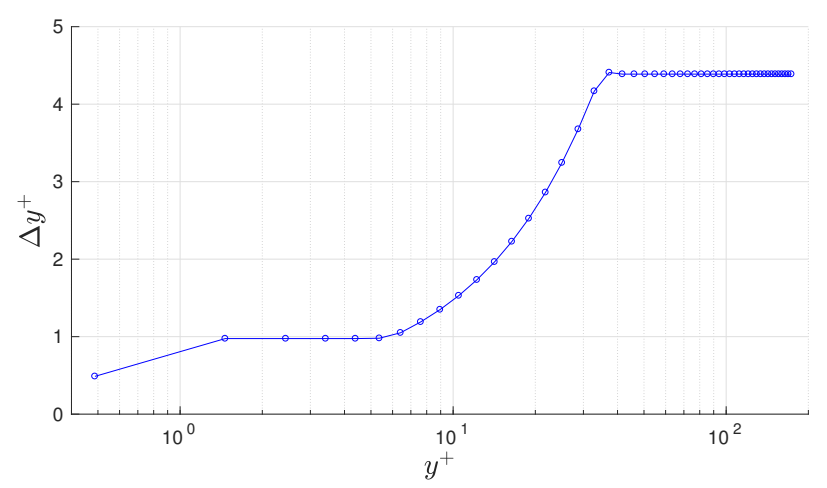

Figure 5-3: Inner-scaled grid resolution of the wall-normal direction up to $\delta$ for a target $\operatorname{Re}_{\tau}=180$.

The details of the simulations are presented in Table 5.2. The SGS models considered are the WALE [63] and SGS-free models. All DNS data is taken from Lee and Moser [47] except the high-order statistics; these quantities are validated against the DNS data of Moser et al. [58]. 
Table 5.2: Summary of the simulation parameters in two different LES as compared to the reference benchmark DNS data for a (target) $\operatorname{Re}_{\tau}$ of 180 .

\begin{tabular}{cccc}
\hline \multirow{2}{*}{ parameters } & \multicolumn{2}{c}{ LES model } & \multirow{2}{*}{ DNS $\dagger$} \\
\cline { 2 - 3 } & SGS-free & WALE & \\
\hline$\Delta x^{+}$ & 12.46 & 12.46 & - \\
$\Delta y^{+}$ & $0.5-4.5$ & $0.5-4.5$ & - \\
$\Delta z^{+}$ & 9 & 9 & - \\
$y_{1}^{+}$ & 0.5 & 0.5 & $1.0546 \cdot 10^{-2}$ \\
$\#$ cells & $1,040,000$ & $1,040,000$ & - \\
\cline { 2 - 4 }$u_{\tau}$ & $6.143 \cdot 10^{-2}$ & $6.101 \cdot 10^{-2}$ & $6.37309 \cdot 10^{-2}$ \\
$\operatorname{Re}_{\tau}$ & 175.514 & 174.314 & 182.088 \\
\hline \hline \multirow{2}{*}{ dimensions } & \multicolumn{2}{c}{$(9 \delta \times 2 \delta \times 4 \delta)$} & $(8 \pi \times 2 \delta \times 3 \pi)$ \\
\hline \hline
\end{tabular}

${ }^{\dagger}$ DNS data are obtained from Lee and Moser [47].

\subsubsection{Mean Velocity Profiles}

The mean velocity profiles display good agreement between the DNS and the LES. This is evident in Figure 5-4. The left and right panels correspond to the inner-scaled and outer-scaled mean velocity up to the channel half-height, respectively.

Starting from the left panel, the inner-scaled velocities demonstrate excellent agreement with the DNS data for the viscous sublayer and part of the buffer region $\left(y^{+} \leq 10\right)$. In the buffer region, specifically for $y^{+}>10$, the LES velocity profiles begin to deviate and over-predict that of the DNS. When compared with the logarithmic law (displayed with the dotted line) with expression: $y^{+}=(1 / 0.38) \log y^{+}+4.17$, the results display a parallel profile in the log-law region. In the right panel, using the outer-scaled velocity, the results depict very good agreement with that of the DNS, although there exists a slight under-prediction for $y / \delta<0.25$ and an over-prediction above it. All in all, though barely showing, the SGS-free model exhibits slightly better approximation for the mean velocity, compared to the WALE SGS model.

The mean spanwise velocity profiles are reported in Figure 5-5. These, ideally, should be zero due to the homogeneity in the corresponding direction. Nonetheless, 


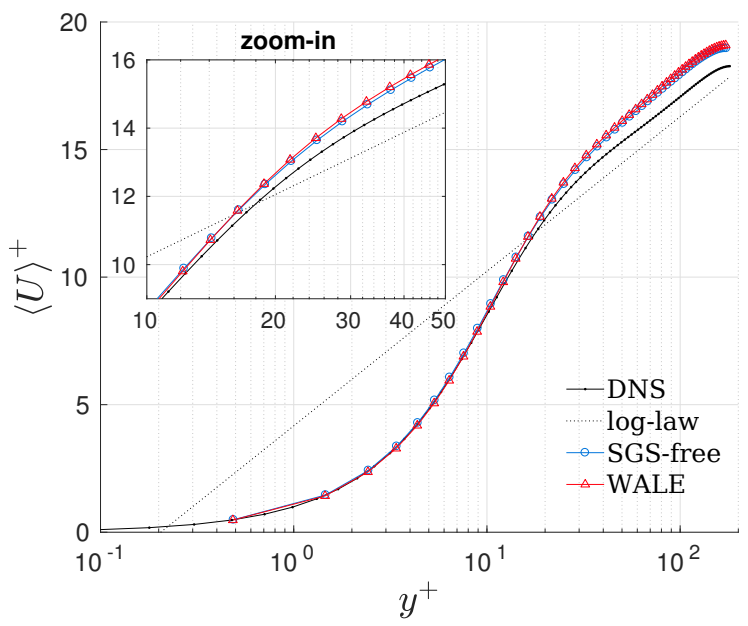

(a)

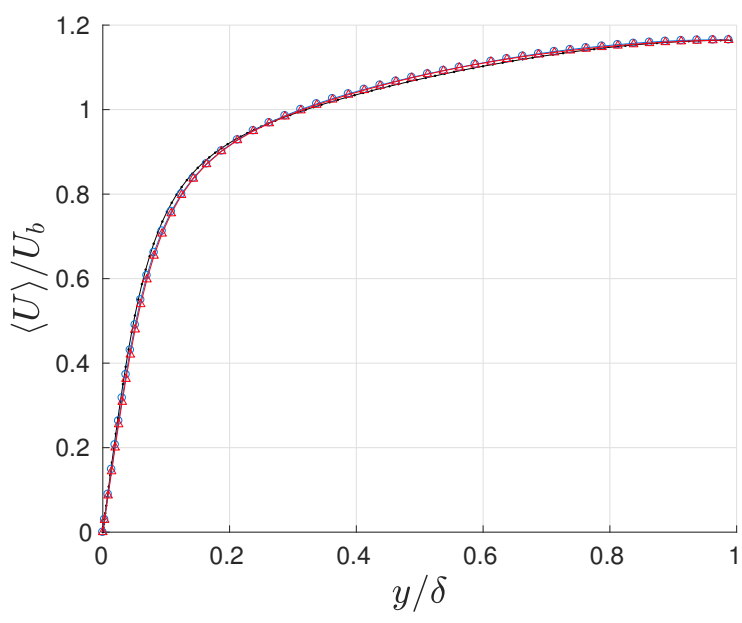

(b)

Figure 5-4: Inner-scaled (a) and outer-scaled (b) mean streamwise velocity profile for a target $\operatorname{Re}_{\tau}=180$.

they are presented here as a statistical quality indicator. In both inner- and outerscaled profiles, the data seem to be noticeably worse than that of the DNS. That said, the magnitude of the components is, at max, in the order of $10^{-2}$ and $10^{-3}$ in the inner and outer scaling, respectively. Hence, these may be deemed acceptable.

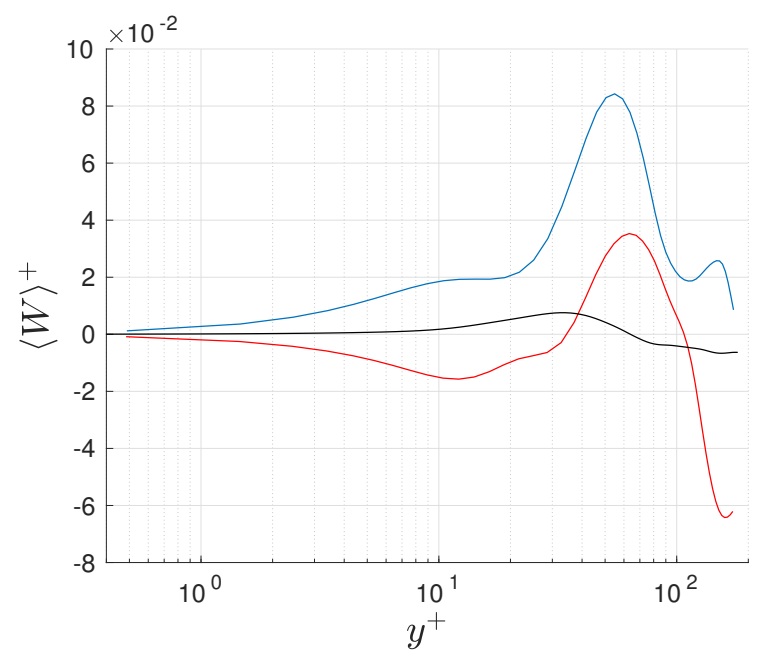

(a)

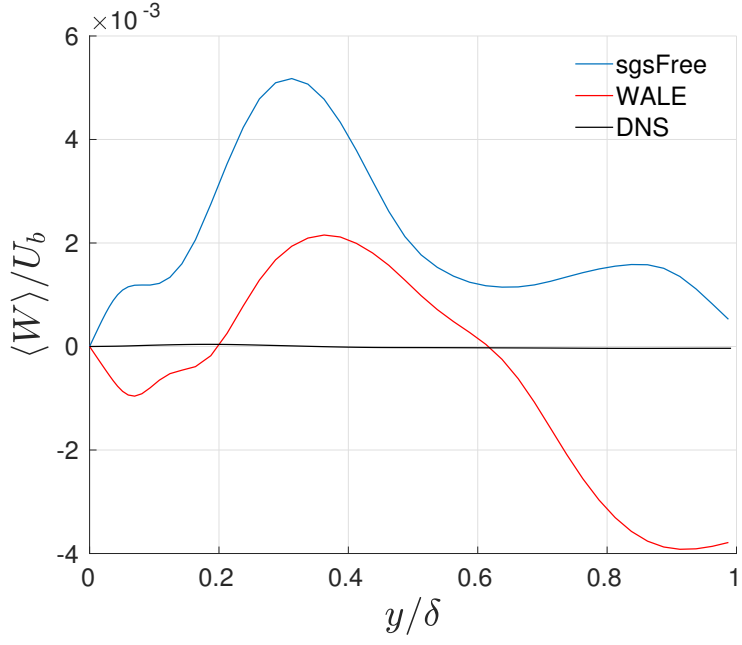

(b)

Figure 5-5: Inner-scaled (a) and outer-scaled (b) mean spanwise velocity profile for a target $\operatorname{Re}_{\tau}=180$. 


\subsubsection{Reynolds Stress Components}

The Re-stress results, in both inner and outer scaling, for either model under-predict the DNS values. Refer to Figures 5-6 and 5-7. Also, one observes that the SGS-free model is more accurate than the WALE model in all figures except that of the $u_{r m s}^{\prime}$ and, to a lesser extent, the $\left\langle u^{\prime} v^{\prime}\right\rangle$. On the other hand, in both Figures 5-6a and 5-7a, the SGS-free model displays slightly better agreement near the wall while the WALE model is better off far from it.

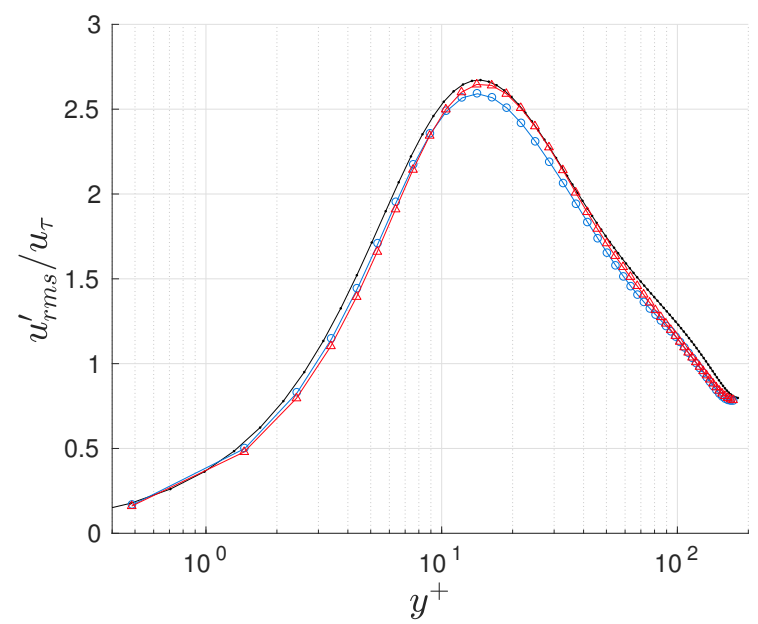

(a)

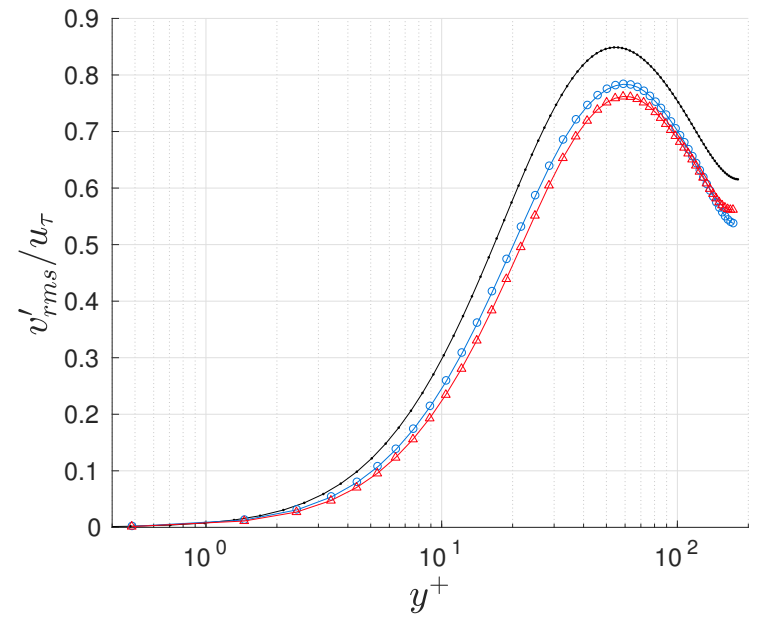

(c)

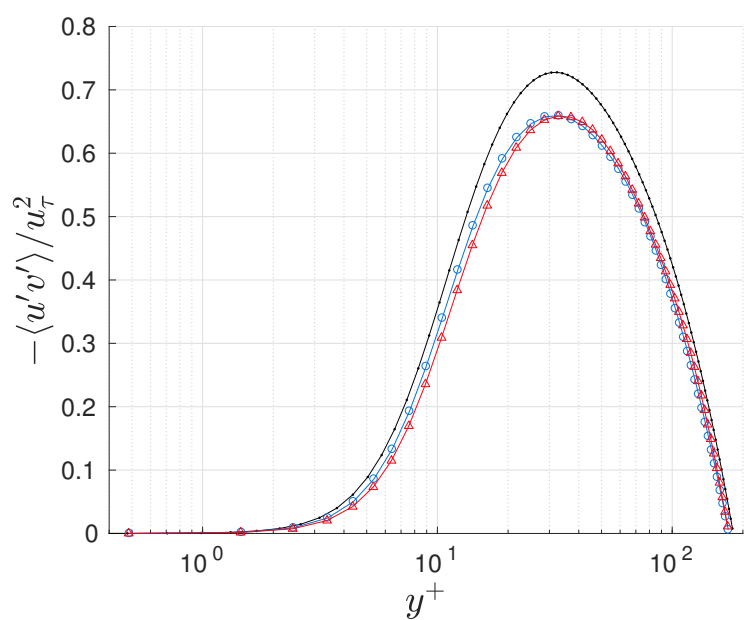

(b)

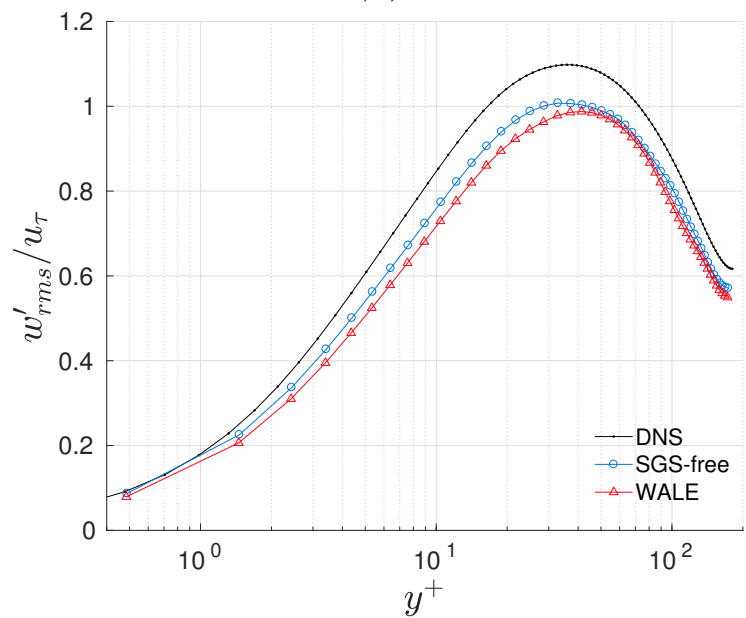

(d)

Figure 5-6: Inner-scaled $u_{r m s}^{\prime}$ (a), $-\left\langle u^{\prime} v^{\prime}\right\rangle$ (b), $v_{r m s}^{\prime}$ (c) and $w_{r m s}^{\prime}$ (d) for a target $\operatorname{Re}_{\tau}=180$. 


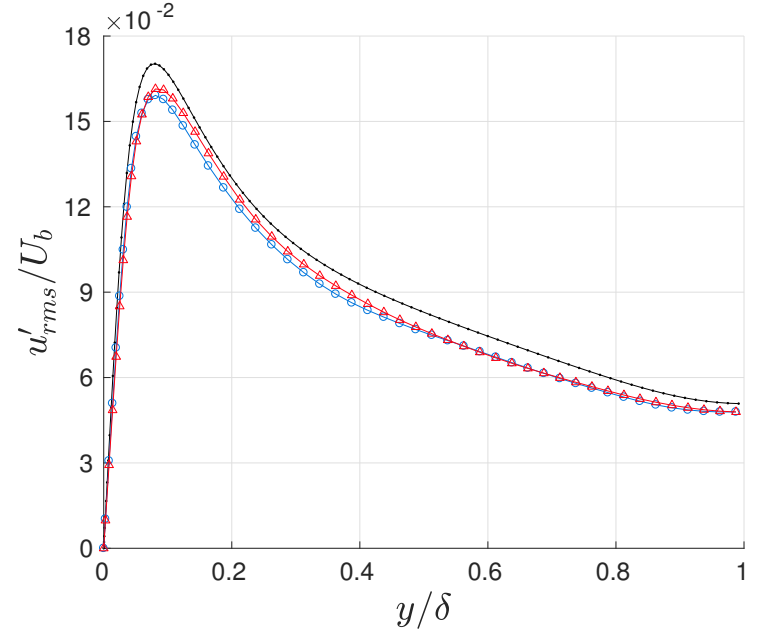

(a)

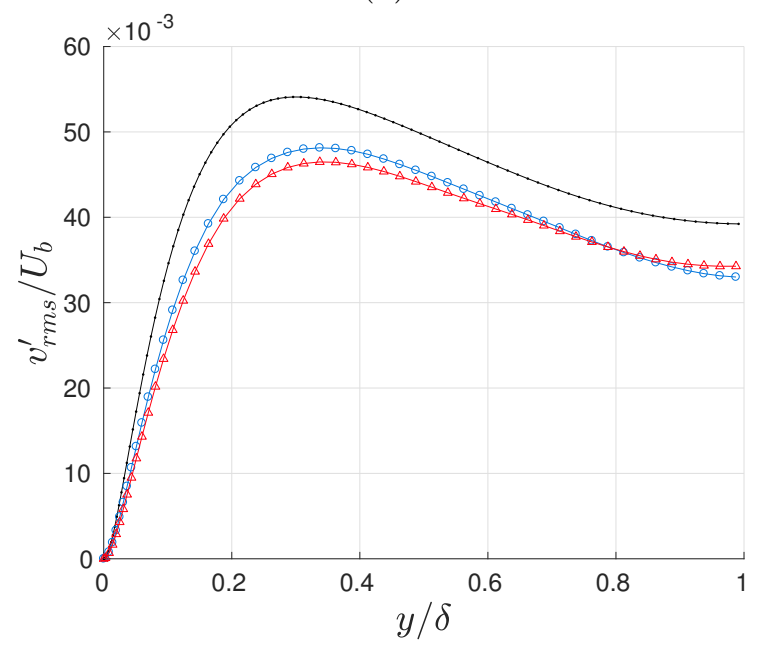

(c)

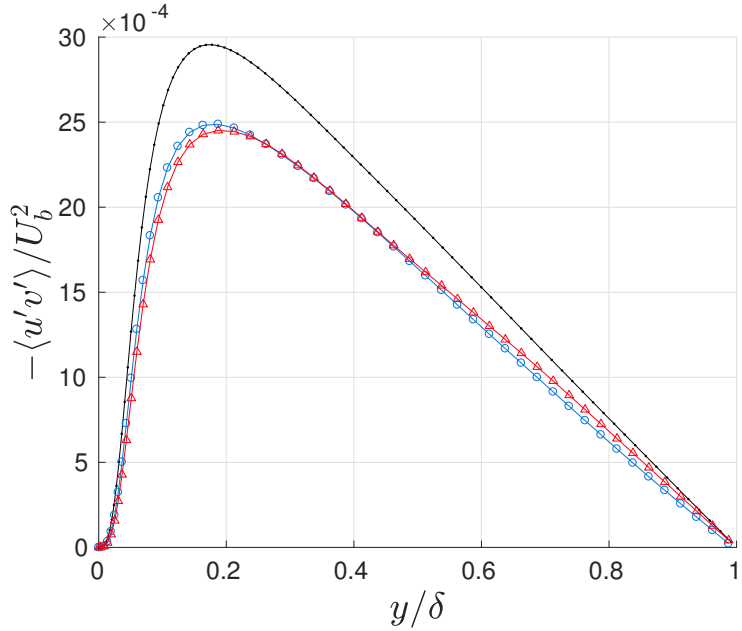

(b)

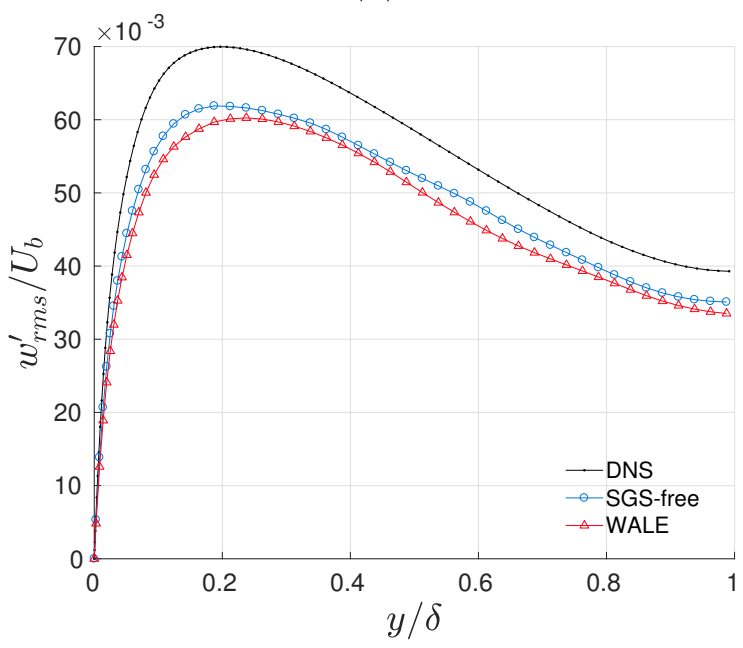

(d)

Figure 5-7: Outer-scaled $u_{r m s}^{\prime}(\mathrm{a}),-\left\langle u^{\prime} v^{\prime}\right\rangle$ (b), $v_{r m s}^{\prime}$ (c) and $w_{r m s}^{\prime}$ (d) for a target $\operatorname{Re}_{\tau}=180$. 


\subsubsection{Vorticity Fluctuations}

The vorticity (denoted by $\omega$ ) is the tendency of a fluid particle to rotate at a given location. Mathematically, it is defined as the curl of the velocity vector. Refer to Equation 2.18 for tensor notation and in vector notation, it is expressed as:

$$
\boldsymbol{\omega} \equiv \nabla \times \mathbf{u}
$$

The vorticity presented here is normalized by $\nu / u_{\tau}^{2}$. The RMS of each of its components in the streamwise, wall-normal and spanwise directions are presented (inner- and outer-scaled) in Figures 5-8 to 5-10, respectively.

The LES profiles agree well with the DNS data, although under-prediction is witnessed; the under-prediction is mostly evident for $\omega_{x}^{\prime r m s}$ and $\omega_{y}^{\prime r m s}$. In the case of $\omega_{z}^{\prime r m s}$, the agreement between the DNS and both SGS-free and WALE SGS model is very good in both inner and outer scaling.

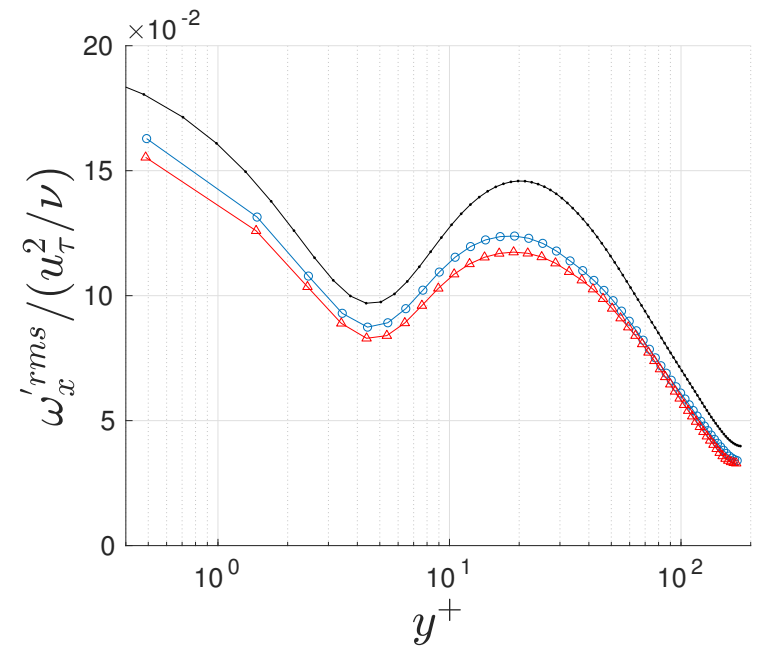

(a)

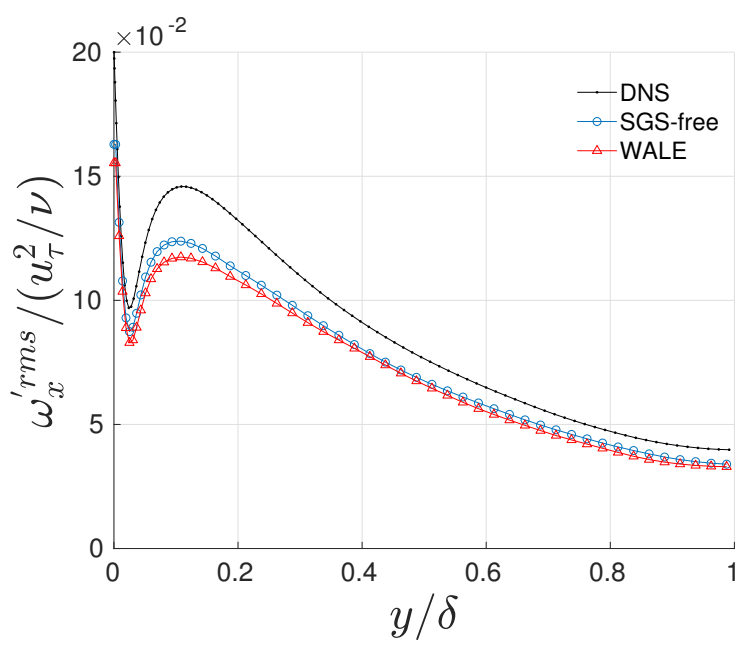

(b)

Figure 5-8: Non-dimensionalized RMS of the streamwise vorticity versus the innerscaled (a) and the outer-scaled (b) wall-normal coordinate for a target $\operatorname{Re}_{\tau}=180$. 


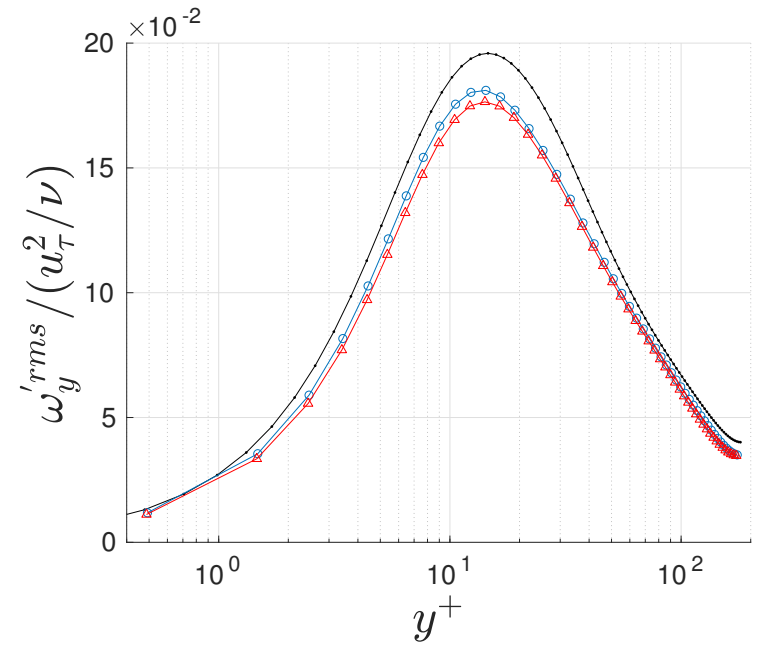

(a)

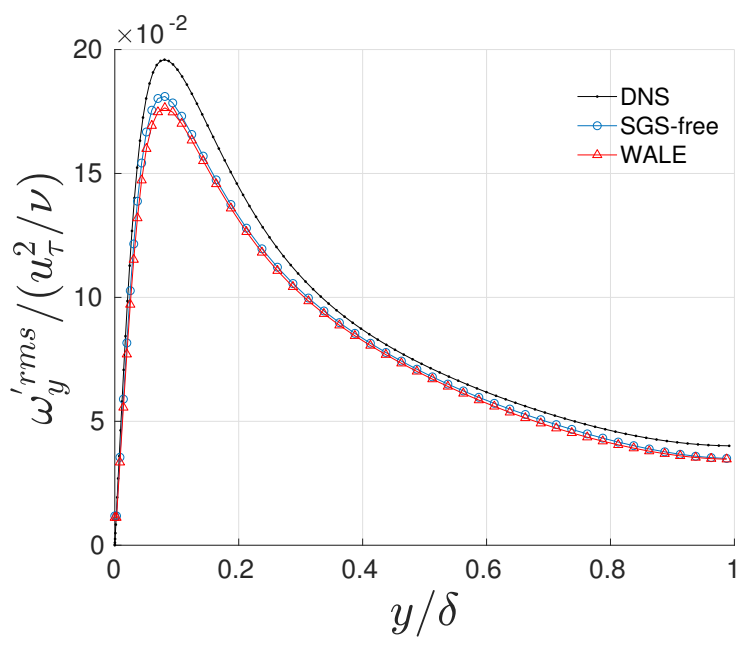

(b)

Figure 5-9: Non-dimensionalized RMS of the wall-normal vorticity versus the innerscaled (a) and the outer-scaled (b) wall-normal coordinate for a target $\operatorname{Re}_{\tau}=180$.

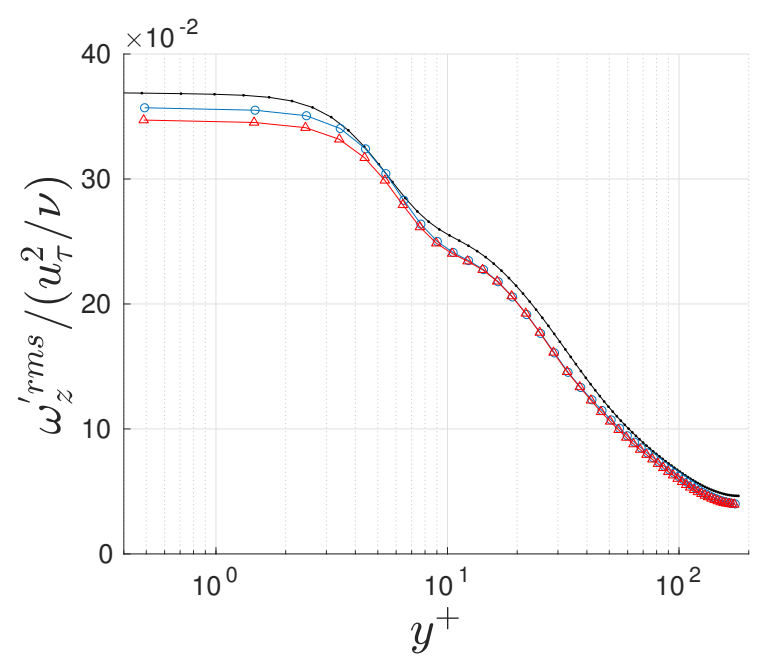

(a)

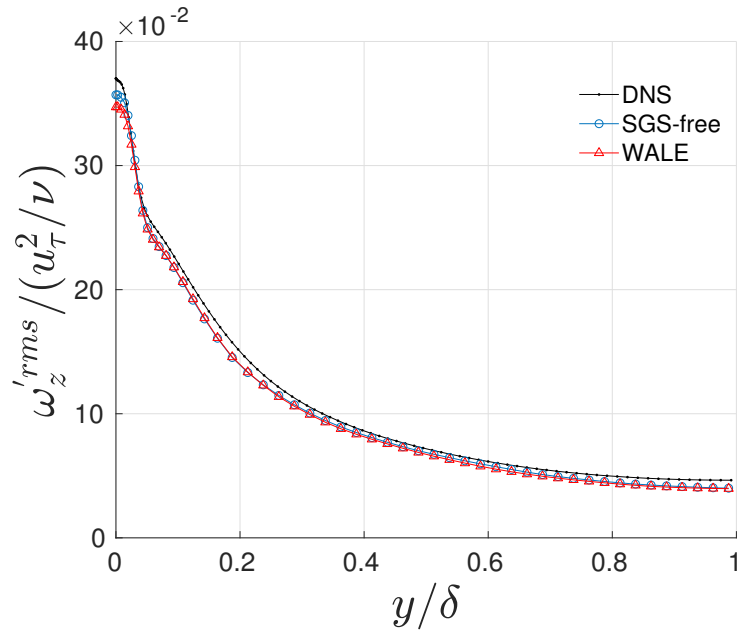

(b)

Figure 5-10: Non-dimensionalized RMS of the spanwise vorticity versus the innerscaled (a) and the outer-scaled (b) wall-normal coordinate for a target $\operatorname{Re}_{\tau}=180$. 


\subsubsection{Turbulent Kinetic Energy Budget}

An additional quantity of interest in studying turbulence is the turbulent kinetic energy (TKE) budget and the quantities constituting it. For a more thorough approach that addresses computing these quantities in OpenFOAM, refer to Appendix B. In the current study, the swak4Foam and its funkySetFields utilities are used to write and compute the required expressions. The TKE budget is given by:

$$
\frac{D k}{D t}=-\underbrace{\left\langle u_{i}^{\prime} u_{j}^{\prime}\right\rangle \frac{\partial\left\langle u_{i}\right\rangle}{\partial x_{j}}}_{\mathrm{I}}-\underbrace{\nu\left\langle\frac{\partial u_{i}^{\prime}}{\partial x_{j}} \frac{\partial u_{i}^{\prime}}{\partial x_{j}}\right\rangle}_{\mathrm{II}}+\underbrace{\nu \frac{\partial^{2}\langle k\rangle}{\partial x_{j} \partial x_{j}}}_{\mathrm{III}}-\underbrace{\frac{1}{\rho} \frac{\left\langle u_{i}^{\prime} p^{\prime}\right\rangle}{\partial x_{j}}}_{\mathrm{IV}}-\underbrace{\frac{\partial\left\langle u_{j}^{\prime} k\right\rangle}{\partial x_{j}}}_{\mathrm{V}},
$$

where $k=u_{i}^{\prime} u_{i}^{\prime} / 2$ and the material, or substantial, derivative is defined as:

$$
\frac{D}{D t}=\frac{\partial}{\partial t}\langle\cdot\rangle+\left\langle u_{j}\right\rangle \frac{\partial}{\partial x_{j}}\langle\cdot\rangle .
$$

The quantities on the right-hand-side of the equality in Equation 5.2 are individually referred to as production $\mathcal{P}_{k}$ (I), viscous dissipation $\varepsilon_{k}$ (II), transport by viscous diffusion $\mathcal{T}^{(\nu)}$ (III), velocity-pressure gradient $\mathcal{T}^{(u p)}(\mathrm{IV})$ and turbulence $\mathcal{T}^{(u u)}(\mathrm{V})$ of TKE. Additionally, the material derivative's constituting terms are referred to as the local derivative and convection/advection operators as taken from left to right. The TKE convection $\mathcal{C}_{k}$ term is the convection operator applied to $k$.

The different terms in Equation 5.2 are computed by LES and compared to DNS in Figure 5-11. Note, these terms are non-dimensionalized by the friction velocity and kinematic viscosity.

The TKE budget terms exhibit satisfactory behavior with that of the DNS data. This is specially true for $\mathcal{P}_{k}$ and $\mathcal{T}^{(\nu)}$, while deviations are evident for the $\mathcal{T}^{(u u)}$, $\mathcal{T}^{(u p)}$ and $\varepsilon_{k}$ terms. Nonetheless, the SGS-free model demonstrates a slightly better outcome than the WALE model. 


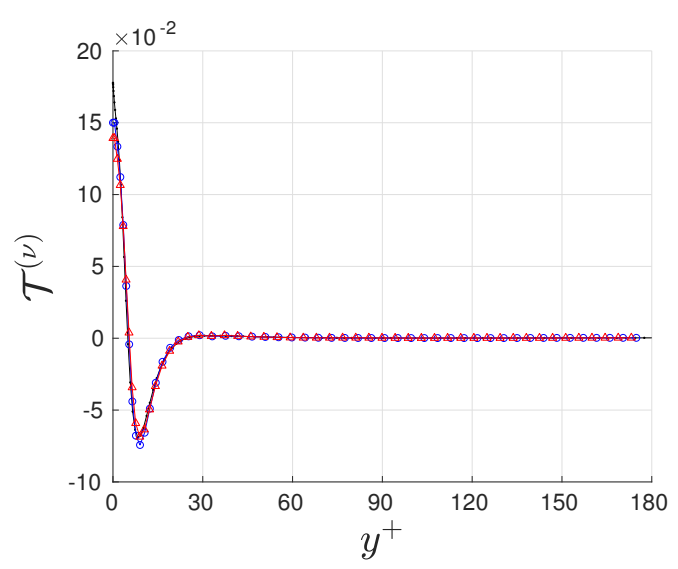

(a)

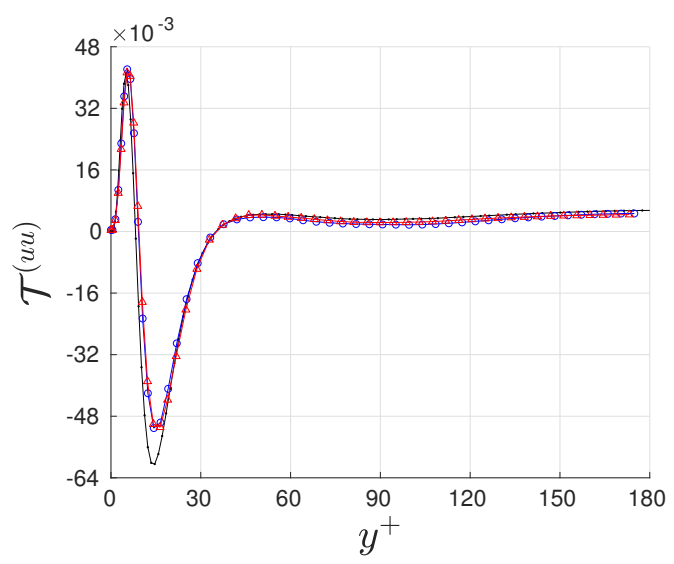

(c)

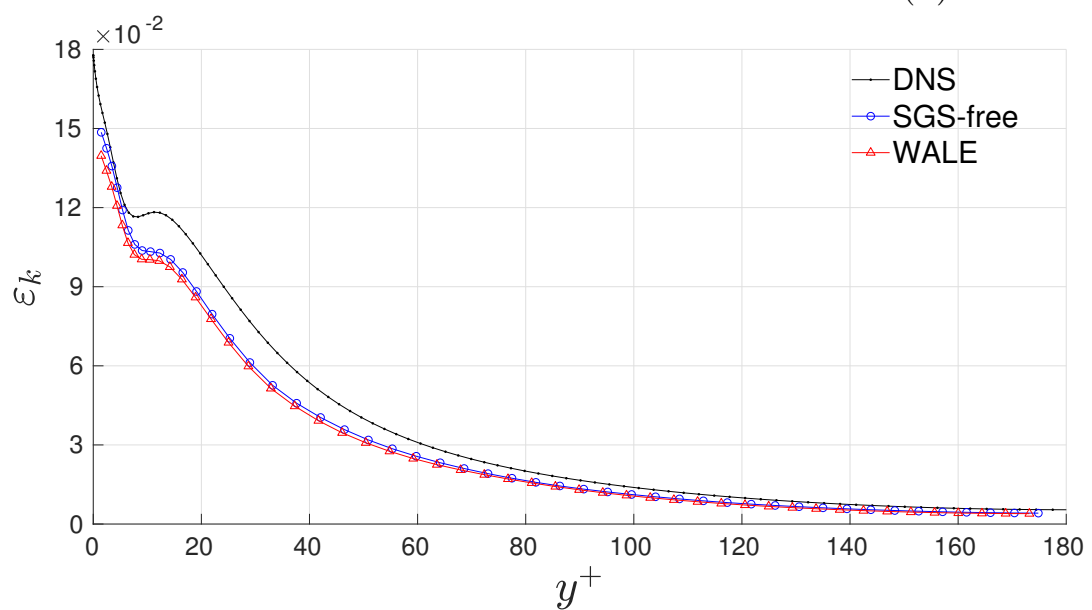

(e)

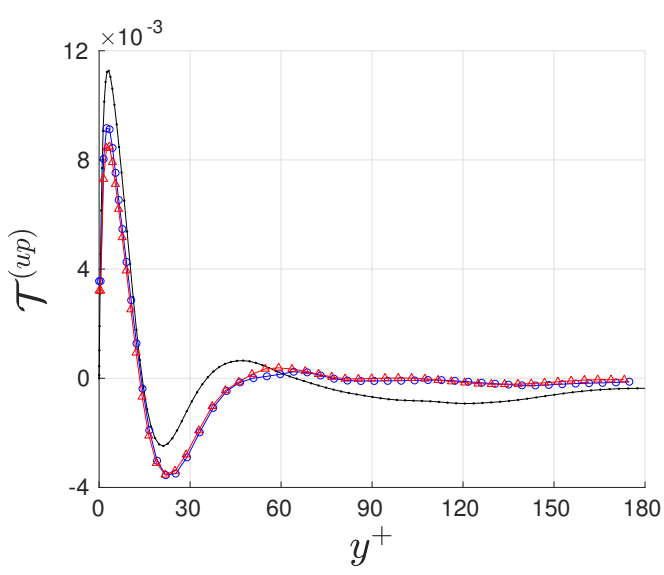

(b)

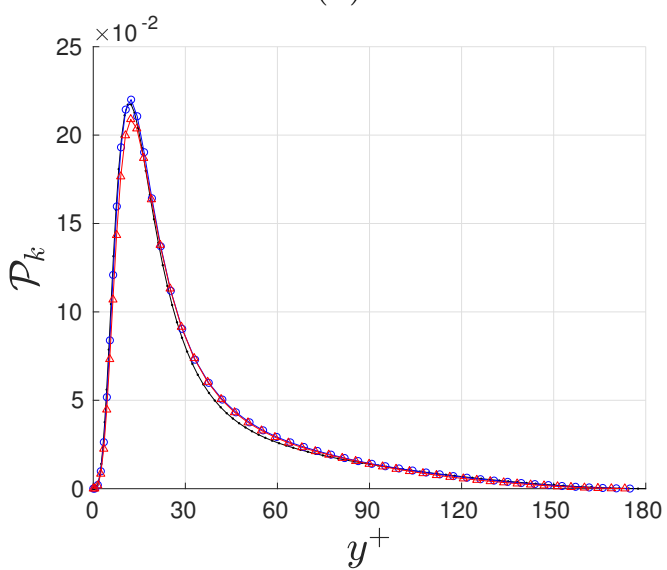

(d) 


\subsubsection{High-order Central Statistical Moments}

Another important quantity of interest is the velocity's central statistical moments. Two high-order statistical moments are studied: the third- and fourth-order central moments. These are also termed the skewness and kurtosis (or flatness), respectively. Their mathematical representation is expressed in Appendix D.

The skewness, $S$, and kurtosis, $K$, are sampled for a relatively longer period of time (as opposed to prior reported data). This is explained by the very slow convergence rate of the high-order statistics. Hence, for $S$ and $K$, the convergence time required is much longer than that of the earlier plots which, at best, had second-order moments (Re-stresses). The total time used for temporal averaging is approximately 160 'flowthrough' times. The total number of temporal samples used are just above 144000 .

The LES results convey a slightly better converged statistics than that of the DNS. This is especially evident in Figure 5-14 in which the skewness of the $w$-velocity component is closer to zero (as should be). Nonetheless, the kurtosis of the $u$-velocity component, near the channel's half-height, displays minor oscillations. To remedy this, more samples should be gathered and averaged altogether.

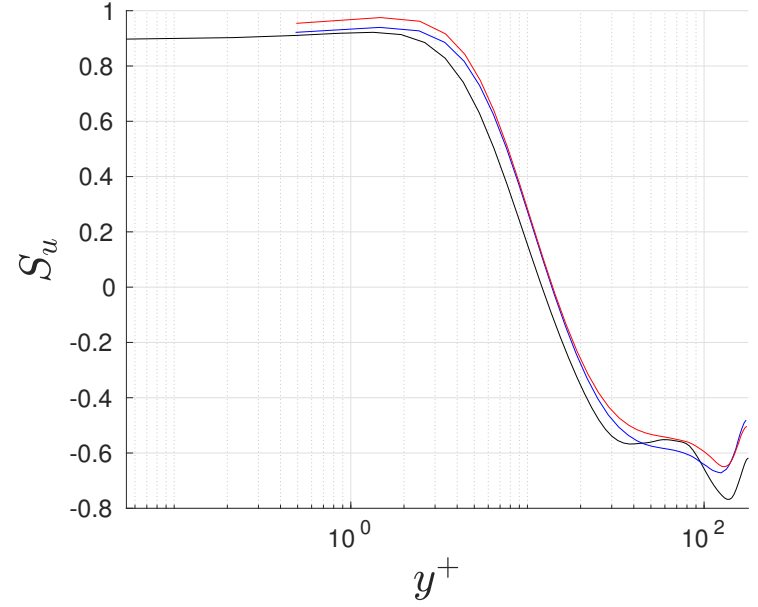

(a)

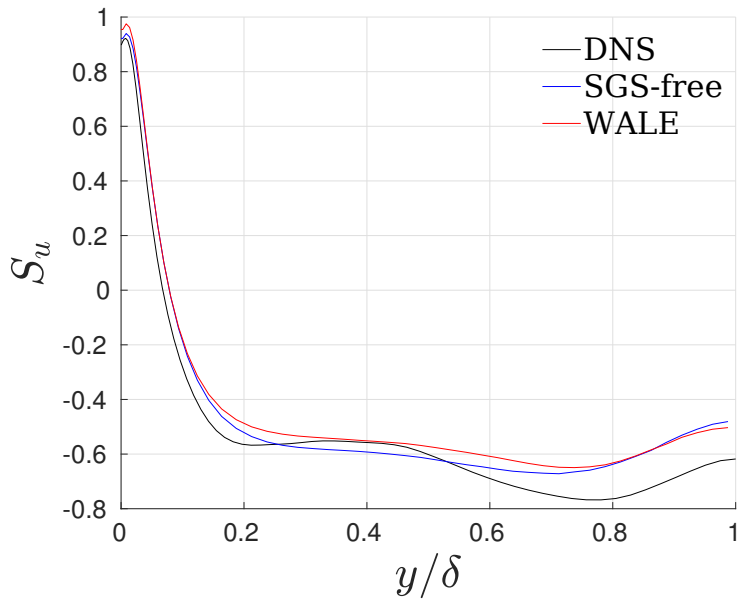

(b)

Figure 5-12: Non-dimensionalized skewness of the streamwise velocity versus the inner-scaled (a) and the outer-scaled (b) wall-normal coordinate for a target $\operatorname{Re}_{\tau}=$ 180. 


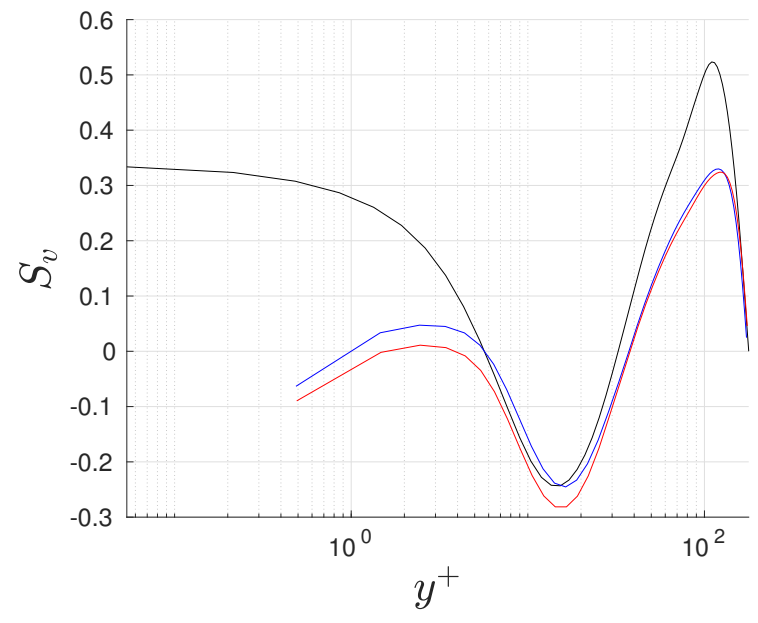

(a)

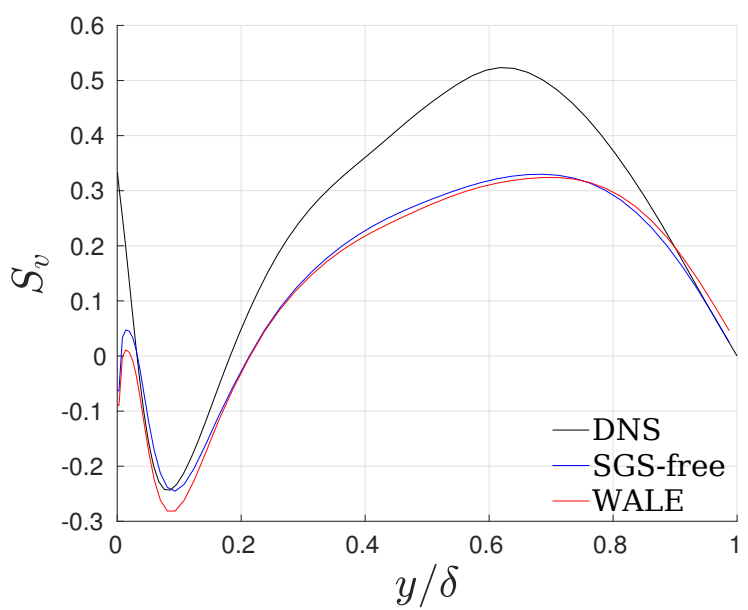

(b)

Figure 5-13: Non-dimensionalized skewness of the wall-normal velocity versus the inner-scaled (a) and the outer-scaled (b) wall-normal coordinate for a target $\operatorname{Re}_{\tau}=$ 180.

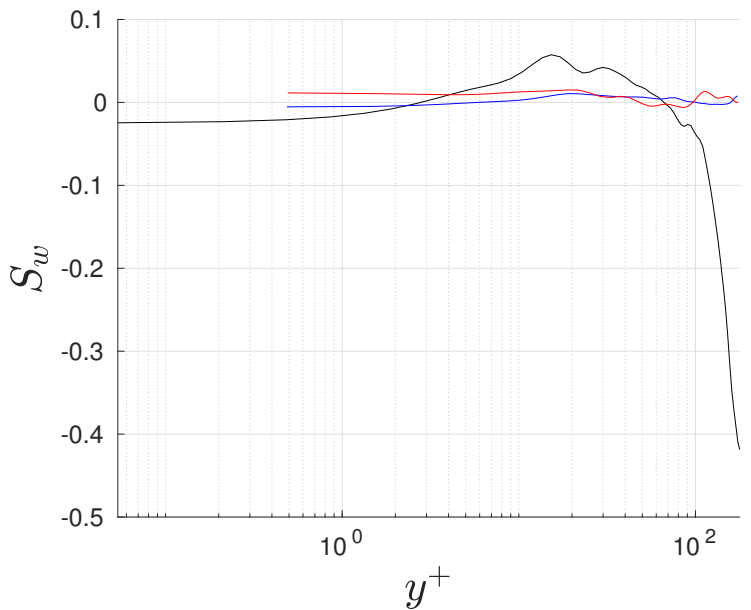

(a)

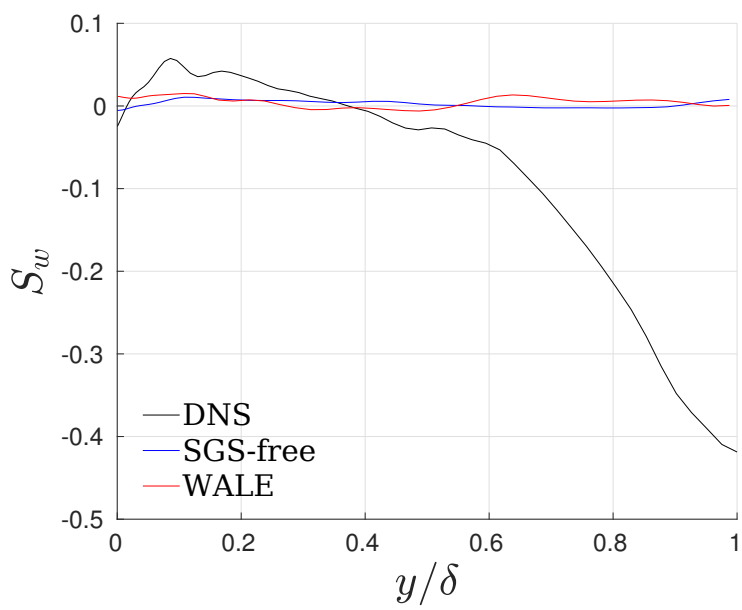

(b)

Figure 5-14: Non-dimensionalized skewness of the spanwise velocity versus the innerscaled (a) and the outer-scaled (b) wall-normal coordinate for a target $\operatorname{Re}_{\tau}=180$. 


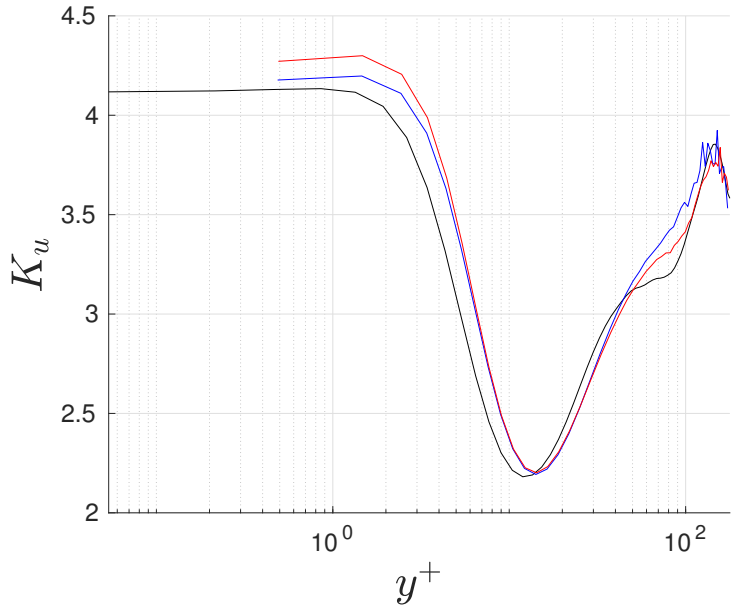

(a)

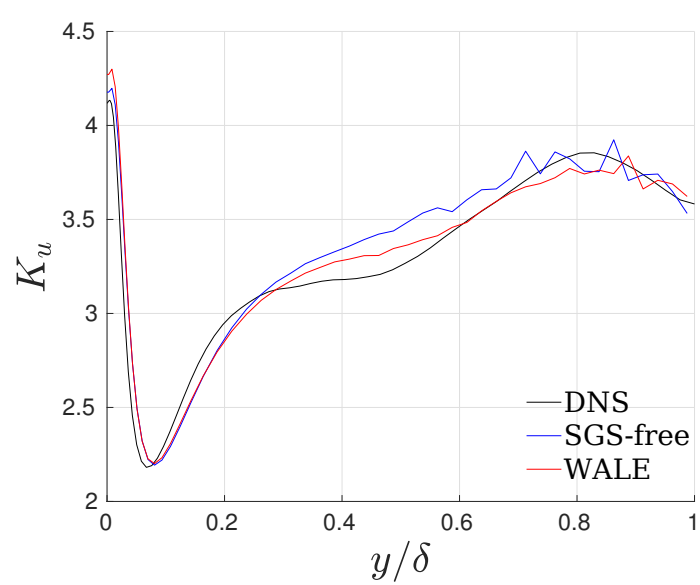

(b)

Figure 5-15: Non-dimensionalized kurtosis of the streamwise velocity versus the innerscaled (a) and the outer-scaled (b) wall-normal coordinate for a target $\operatorname{Re}_{\tau}=180$.

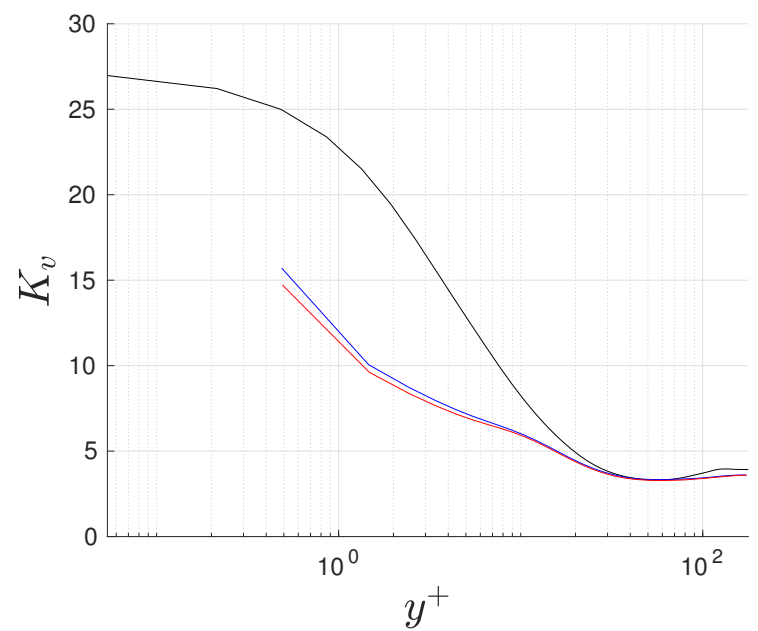

(a)

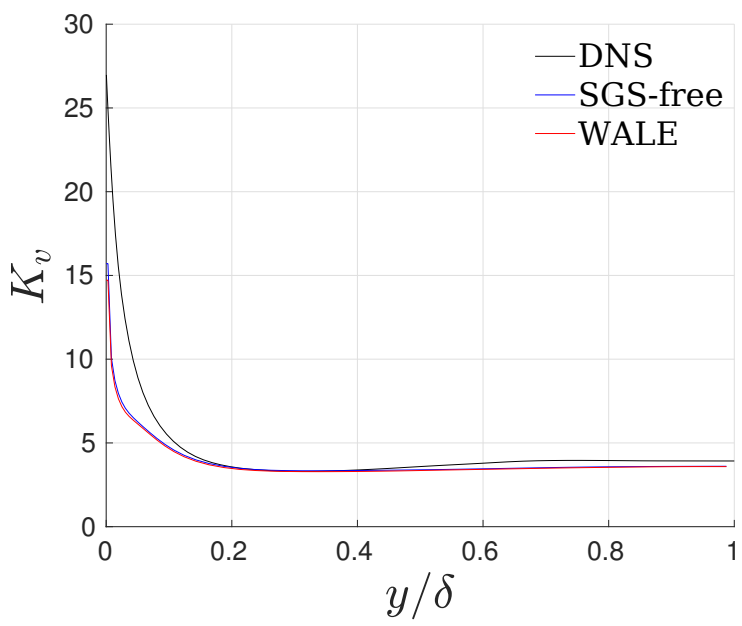

(b)

Figure 5-16: Non-dimensionalized kurtosis of the wall-normal velocity versus the inner-scaled (a) and the outer-scaled (b) wall-normal coordinate for a target $\operatorname{Re}_{\tau}=$ 180. 


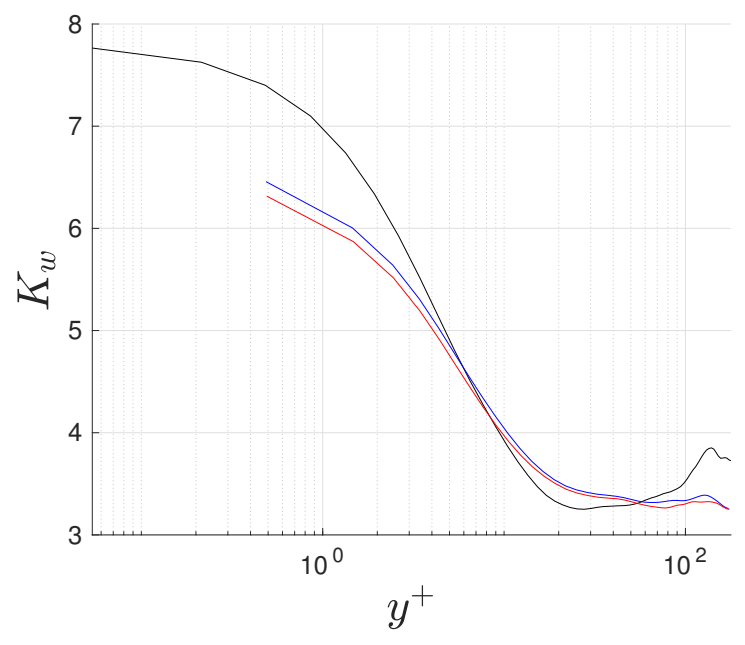

(a)

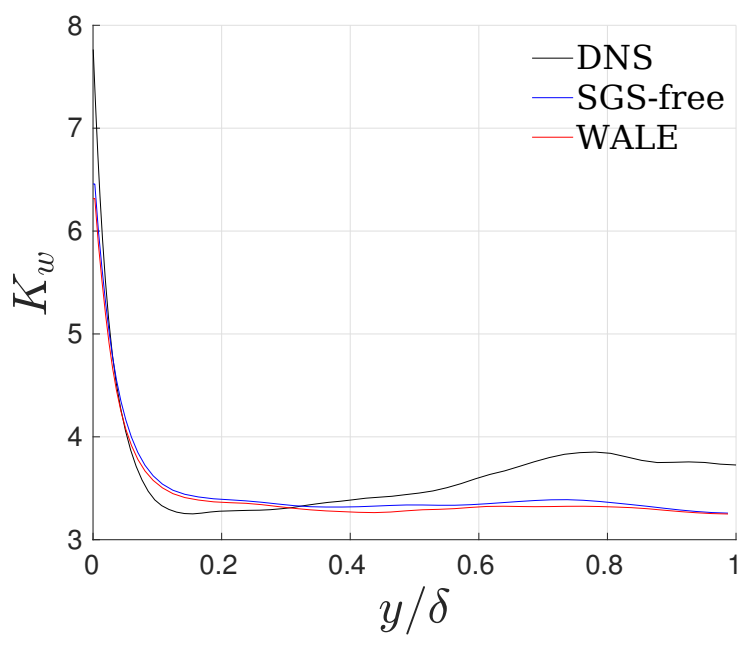

(b)

Figure 5-17: Non-dimensionalized kurtosis of the spanwise velocity versus the innerscaled (a) and the outer-scaled (b) wall-normal coordinate for a target $\operatorname{Re}_{\tau}=180$.

\subsubsection{SGS Study on the Same Wall-normal Grid Resolution}

So far, turbulent channel flow simulations addressed a target $\operatorname{Re}_{\tau}=180$. Now, the simulations will address a higher target $\operatorname{Re}_{\tau}=300$. In turn, this study will include more SGS models and different homogeneous grid resolutions.

The wall-normal grid resolution used here is displayed in Figure 5-24. Mesh M1 is assumed to be employed by default, unless stated otherwise.

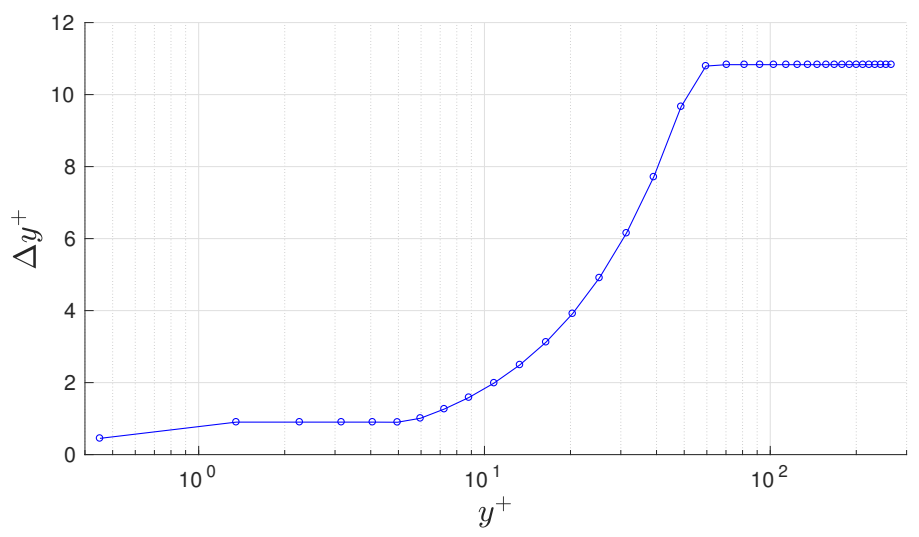

Figure 5-18: Inner-scaled grid resolution in the wall-normal direction up to $\delta$ for a target $\operatorname{Re}_{\tau}=300$. 
The wall-normal grid resolution (based on the target $\mathrm{Re}_{\tau}$ ) has its first cell placed at $y_{1}^{+} \approx 0.5$ away from the wall. The last cell is about 11 wall units near the channel's half-height. Three separate grid resolutions are tested with variations restricted to the homogeneous directions only. They are referred to as coarse, intermediate and fine meshes whereby the cell sizes in wall units are 45, 20 and 25 in the streamwise direction and 20, 20 and 10 in the spanwise direction, respectively. Three SGS models are used: the Smagorinsky [73], the WALE [63] and the SGS-free model. Refer to Table 5.3.

All simulations under-predict the target $\mathrm{Re}_{\tau}$, except that of the intermediate grid which over-predicts it. As for the remaining two grid resolutions, the WALE model seems to exhibit the best outcome. In fact, the WALE model, in all but the intermediate grid, yields the largest converged $\operatorname{Re}_{\tau}$ while the Smagorinsky model consistently has the lowest among all simulations.

Table 5.3: Summary of the simulation parameters in all nine different LES as compared to the reference benchmark DNS data for a (target) $\operatorname{Re}_{\tau}$ of 300 .

\begin{tabular}{clcccc}
\hline \multirow{2}{*}{ parameters } & & \multicolumn{3}{c}{ LES mesh type } & \multirow{2}{*}{ DNS $\dagger$} \\
\cline { 3 - 5 } & model & coarse & intermediate & fine & \\
\hline$\Delta x^{+}$ & & 45 & 20 & 25 & 18.4 \\
$\Delta y^{+}$ & & $0.5-11$ & $0.5-11$ & $0.5-11$ & $0.04-4.91$ \\
$\Delta z^{+}$ & & 20 & 20 & 10 & 7.36 \\
$y_{1}^{+}$ & & 0.5 & 0.5 & 0.5 & $9.96952 \cdot 10^{-3}$ \\
$\#$ cells & & 266,400 & 599,400 & 959,040 & - \\
& SGS-free & $5.629 \cdot 10^{-2}$ & $6.498 \cdot 10^{-2}$ & $5.503 \cdot 10^{-2}$ & \\
$u_{\tau}$ & Smagorinsky & $5.448 \cdot 10^{-2}$ & $6.090 \cdot 10^{-2}$ & $5.385 \cdot 10^{-2}$ & $5.9347 \cdot 10^{-2}$ \\
& WALE & $5.911 \cdot 10^{-2}$ & $6.223 \cdot 10^{-2}$ & $5.811 \cdot 10^{-2}$ & \\
\cline { 2 - 5 } $\operatorname{Re}_{\tau}$ & SGS-free & 282.552 & 326.172 & 276.227 & \\
& Smagorinsky & 273.467 & 305.692 & 270.304 & 297.899 \\
& WALE & 296.707 & 312.368 & 291.687 & \\
\hline \hline \multirow{2}{*}{ abbreviation } & SGS-free & C0 & I0 & F0 & \\
& Smagorinsky & $\mathrm{C} 1$ & $\mathrm{I} 1$ & $\mathrm{~F} 1$ & \\
\hline \multirow{2}{*}{ dimensions } & WALE & $\mathrm{C} 2$ & $\mathrm{I} 2$ & $\mathrm{~F} 2$ & \\
\hline \hline
\end{tabular}

${ }^{\dagger}$ DNS data are obtained from the THTLAB - [33] and [34]. 


\subsubsection{Mean Velocity Profiles}

The velocity profiles are illustrated in both scalings in Figure 5-19. The Smagorinsky model, independent of the grid used, displays great under-prediction in inner scaling. In the right panel, in outer scaling, the Smagorinsky model starts by under-predicting the DNS close to the wall, whereas near the start of the outer-region it over-predicts it. On the other hand, the WALE and SGS-free models both display excellent agreement in the viscous sublayer, although they begin to deviate in the buffer zone. It is surprising to observe in the case of these two models in inner scaling, the coarse mesh has somewhat better results than the intermediate one. Only results using the fine mesh are in close proximity with the DNS velocity profile in the log-law and outer region as displayed in the left panel of the figure.

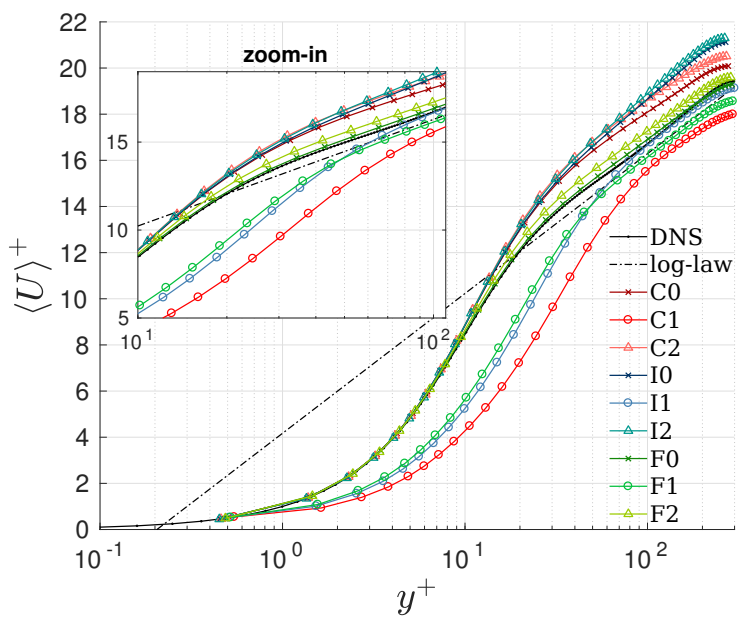

(a)

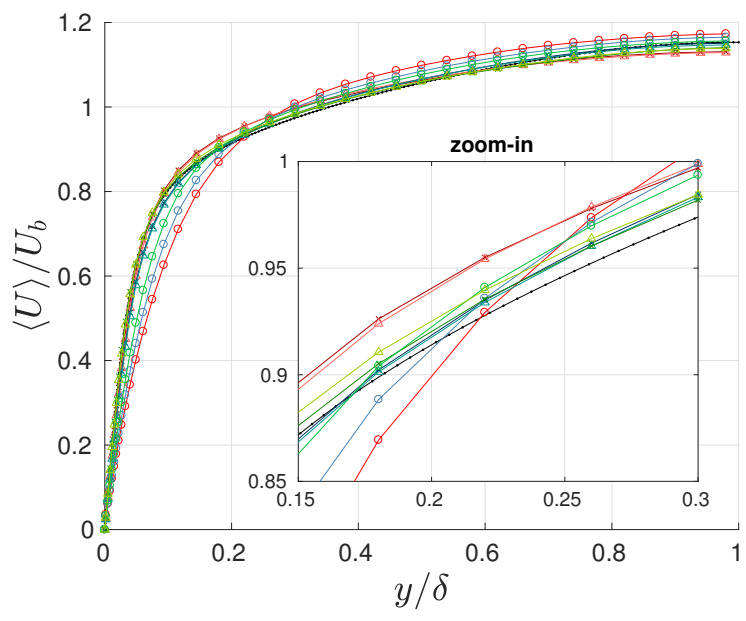

(b)

Figure 5-19: Inner-scaled (a) and outer-scaled (b) mean streamwise velocity profile for a target $\mathrm{Re}_{\tau}=300$ using mesh M1-C, M1-I and M1-F.

\subsubsection{Reynolds Stress Components}

The Re-stress components are presented separately. In each of the figures, the left panels depict inner scaling whereas the right panels correspond to outer scaling. Moreover, each row corresponds to a different grid resolution; from top to bottom, these rows correspond to the coarse (M1-C), intermediate (M1-I) and fine (M1-F) grids, 
respectively (see Table 5.3).

The $u_{r m s}^{\prime}$ component is depicted in Figure 5-20. Not much gain in accuracy is witnessed between M1-C and M1-I, but for M1-F excellent agreement between the WALE, SGS-free and DNS data are observed. Similar behavior is deduced from the $v_{r m s}^{\prime}$ component in Figure 5-22. As evidenced in Figure 5-21, the $\left\langle u^{\prime} v^{\prime}\right\rangle$ component also shows good agreement using the M1-F mesh, nonetheless M1-C is surprisingly slightly better than the M1-I grid. In Figure 5-23, the trend describing the $w_{r m s}^{\prime}$ component seems to be logical; the difference between LES and DNS decreases as the mesh is further refined. In all cases, the Smagorinsky model consistently displays incorrect behavior. Of all three models, the SGS-free revealed the best agreement with the DNS data. 


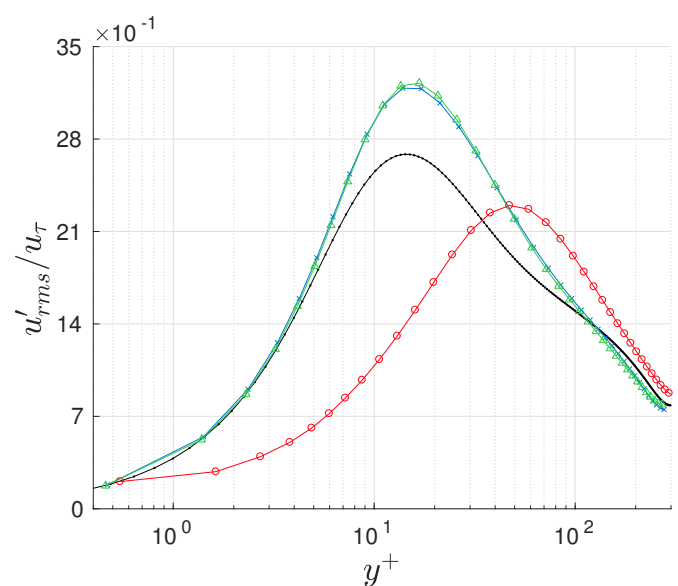

(a)

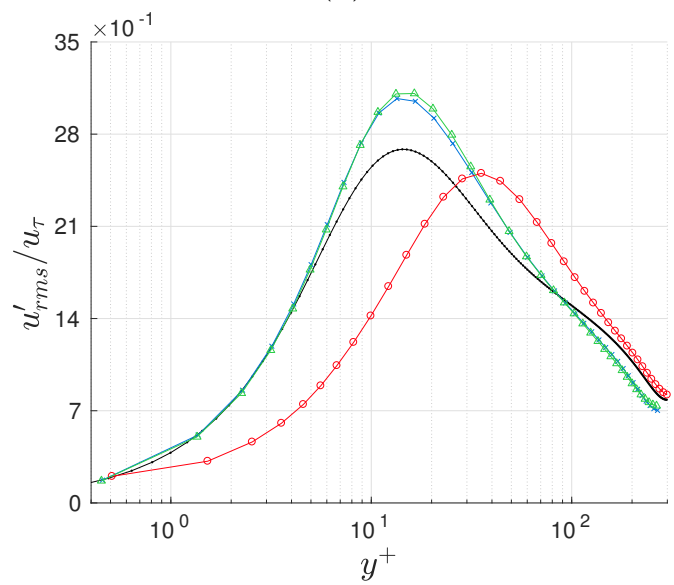

(c)

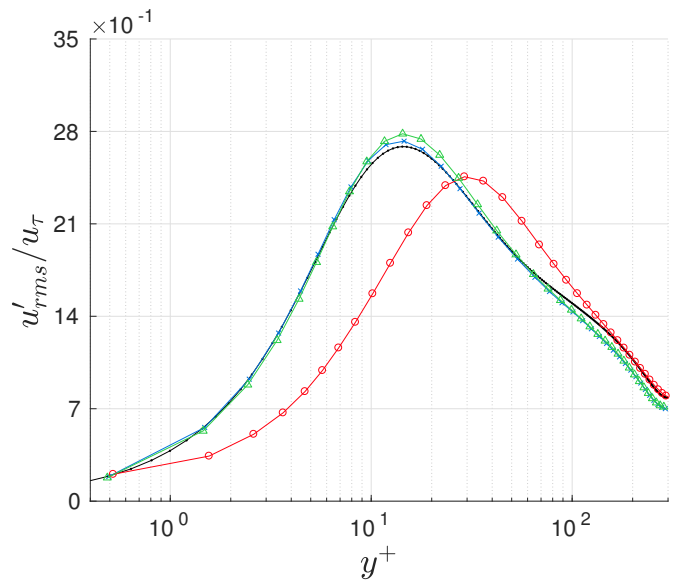

(e)

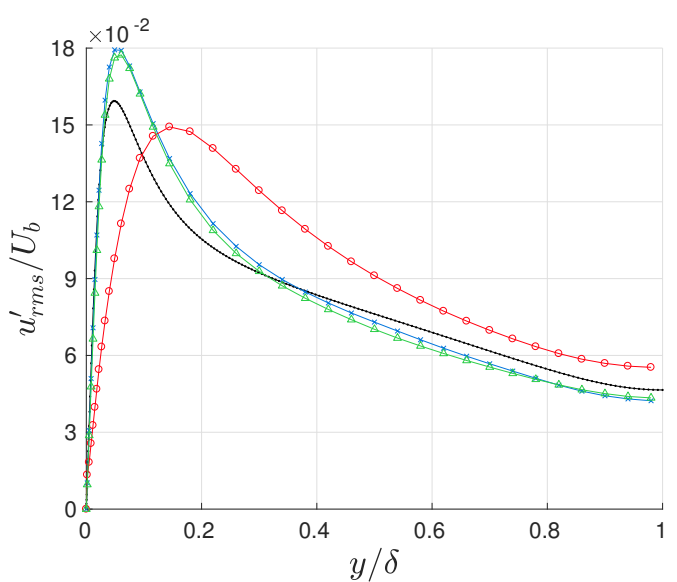

(b)

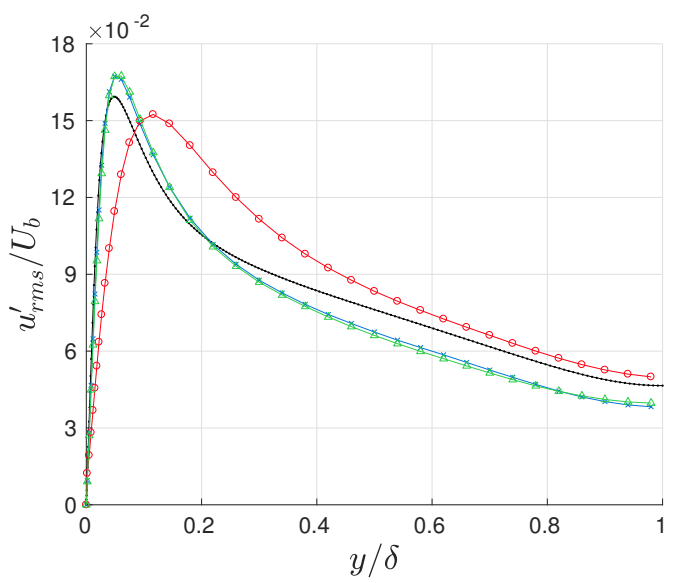

(d)

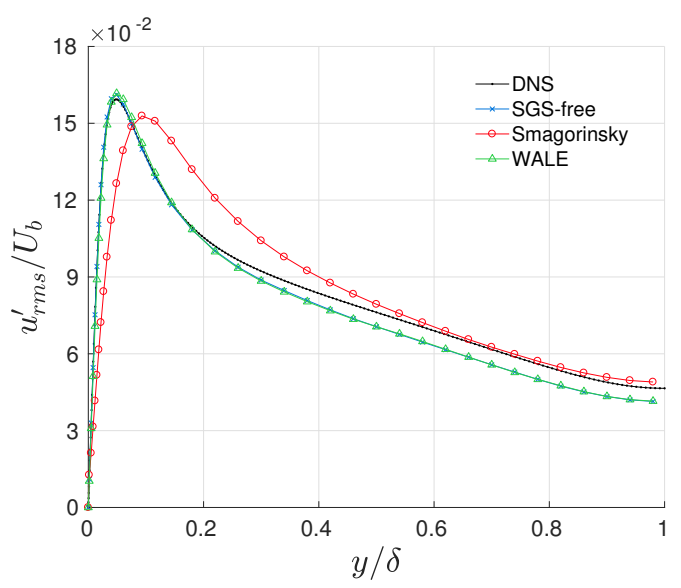

(f)

Figure 5-20: Inner-scaled $u_{r m s}^{\prime}$ using mesh M1-C (a), M1-I (c) and M1-F (e) and outer-scaled using mesh M1-C (b), M1-I (d) and M1-F (f) for a target $\operatorname{Re}_{\tau}=300$. 


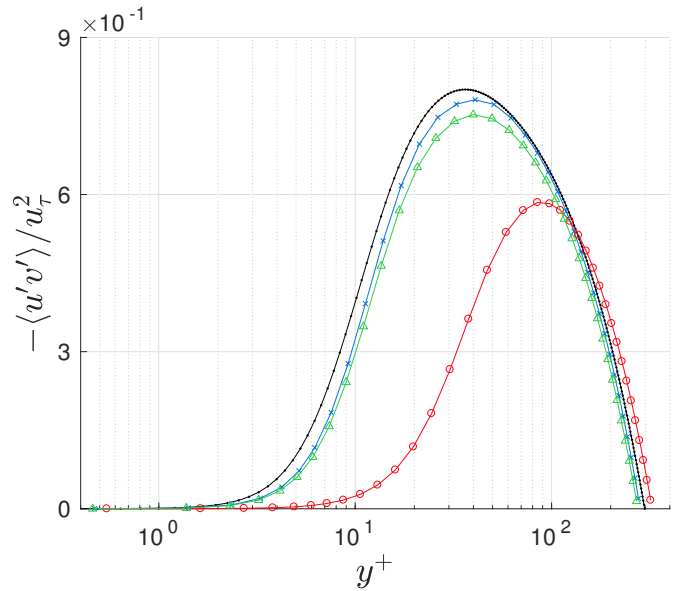

(a)

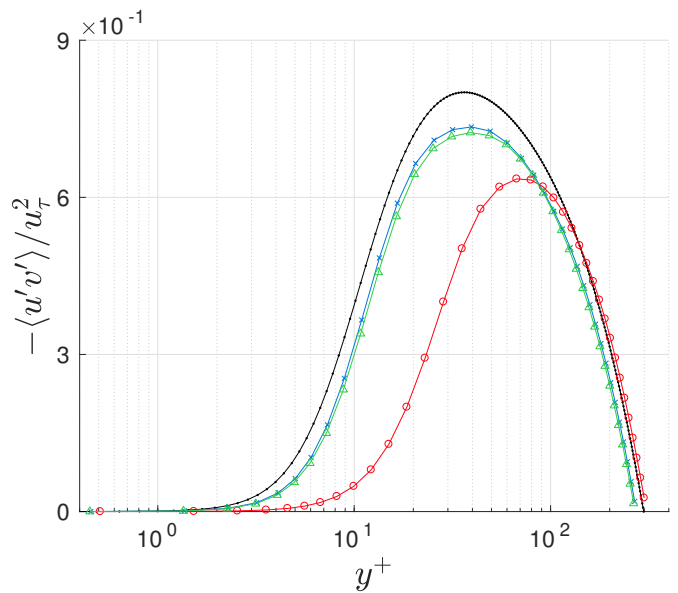

(c)

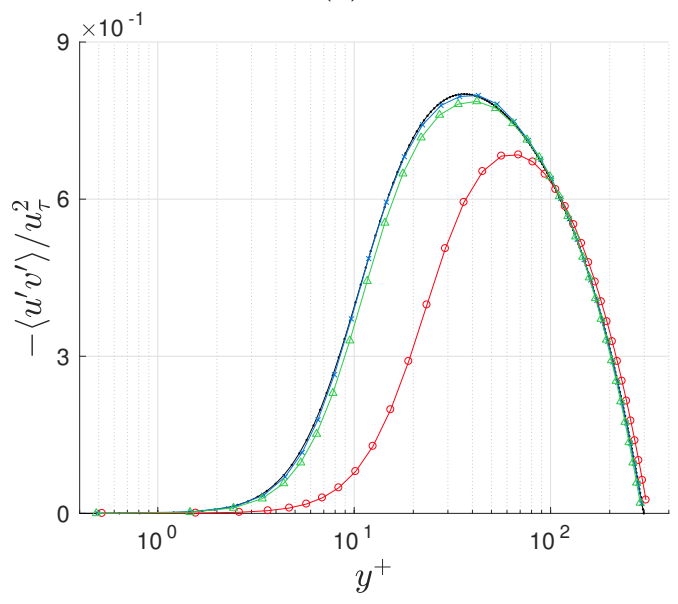

(e)

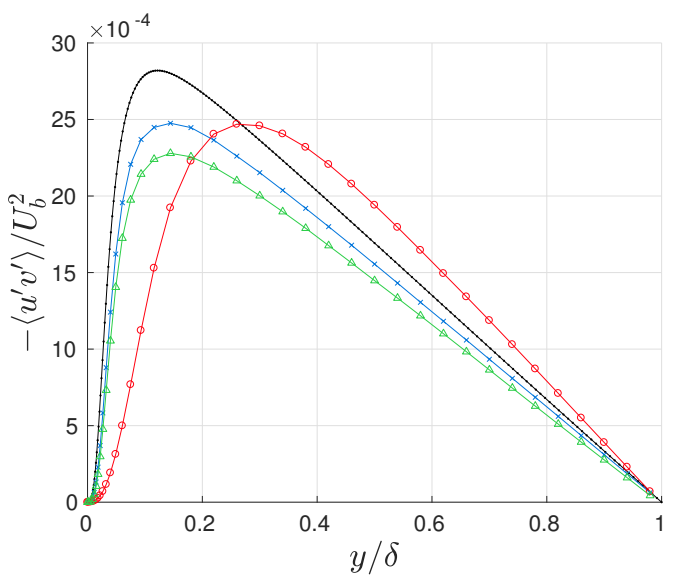

(b)

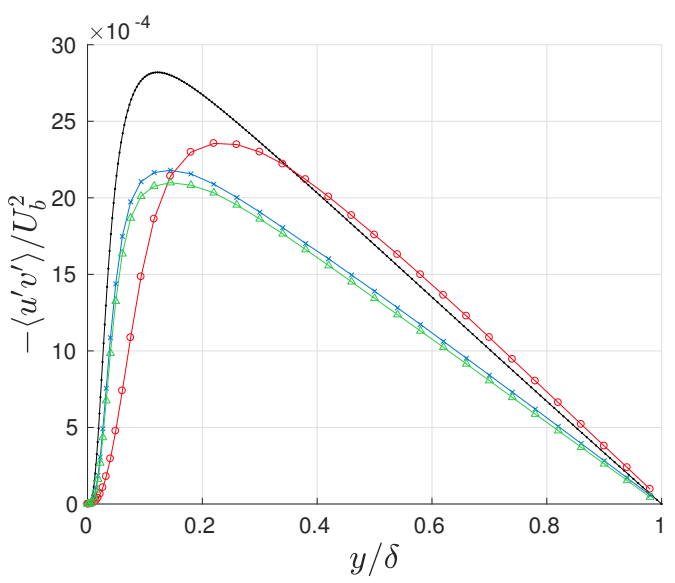

(d)

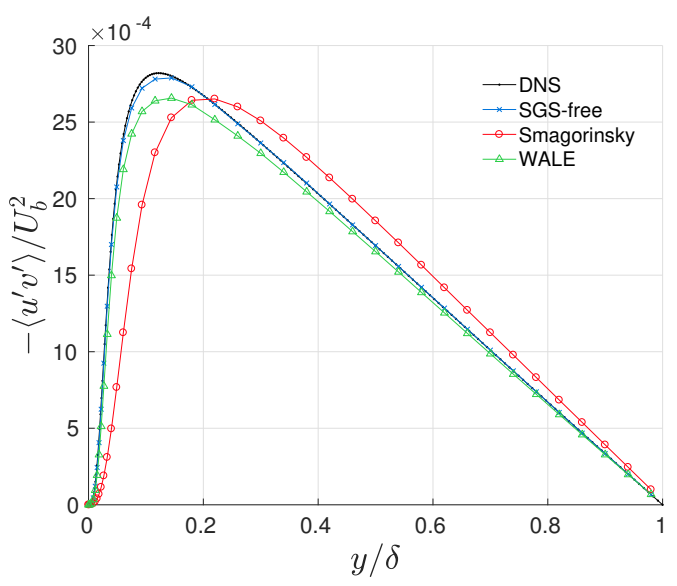

(f)

Figure 5-21: Inner-scaled $\left\langle u^{\prime} v^{\prime}\right\rangle$ using mesh M1-C (a), M1-I (c) and M1-F (e) and outer-scaled using mesh M1-C (b), M1-I (d) and M1-F (f) for a target $\operatorname{Re}_{\tau}=300$. 


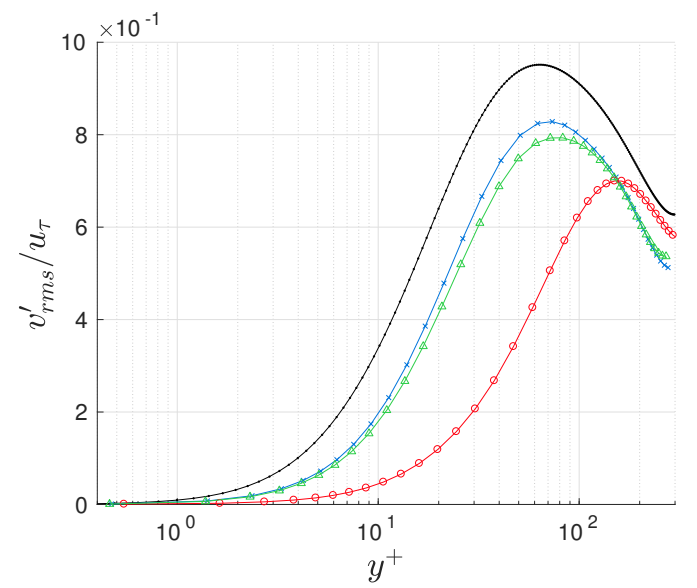

(a)

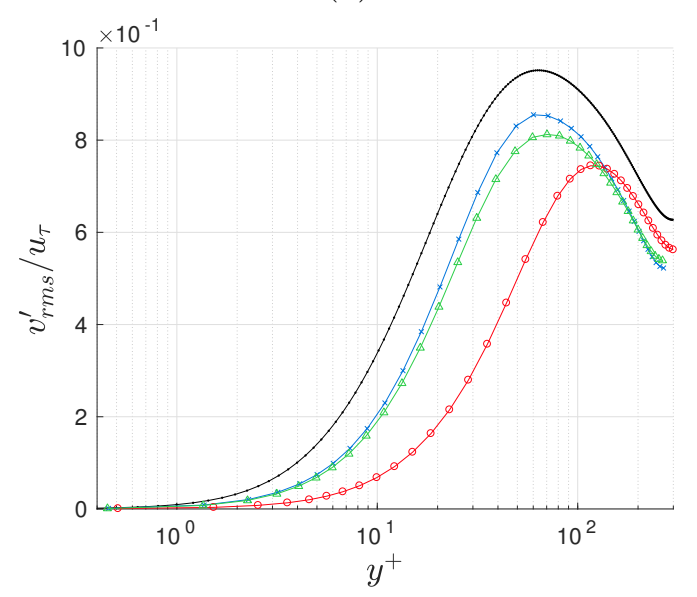

(c)

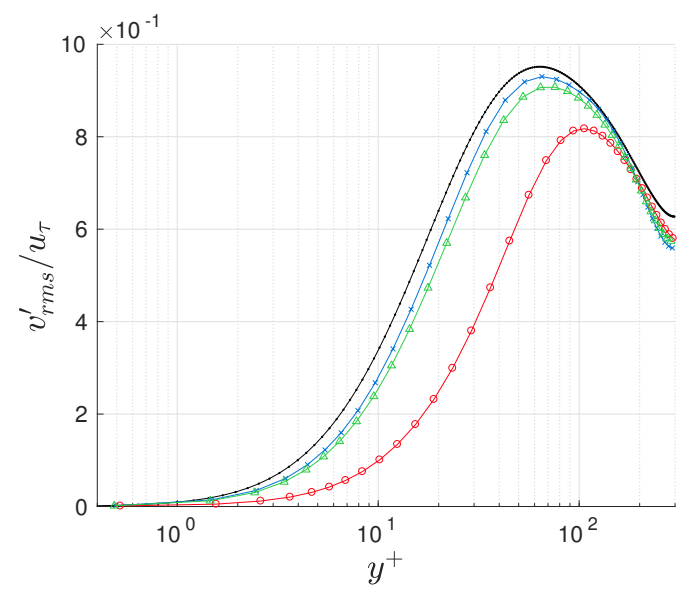

(e)

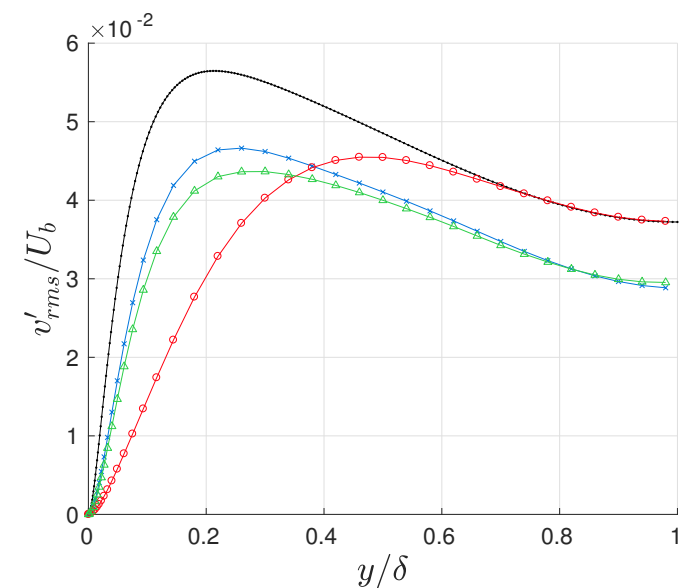

(b)

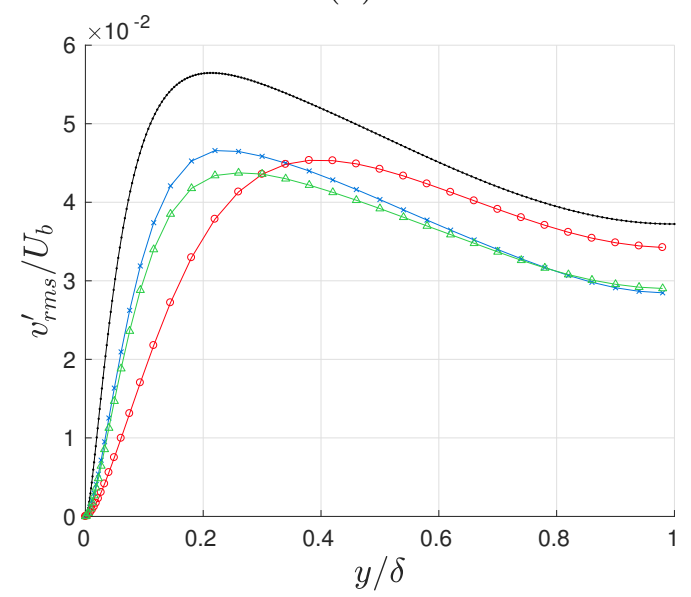

(d)

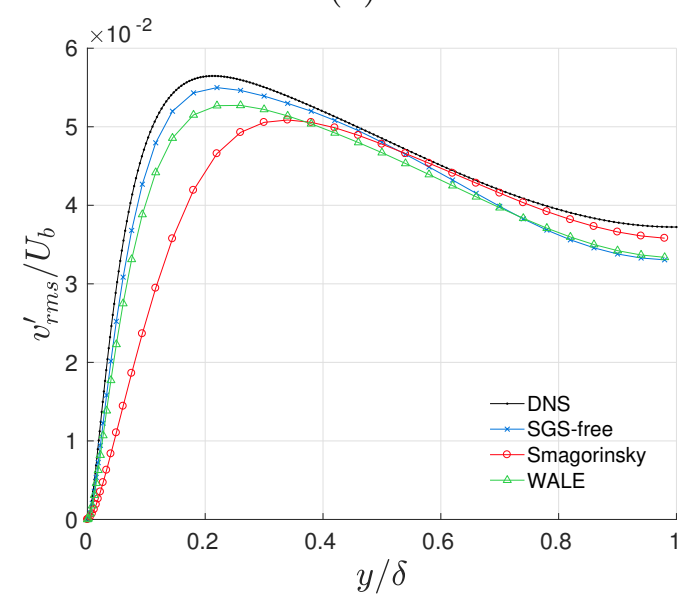

(f)

Figure 5-22: Inner-scaled $v_{r m s}^{\prime}$ using mesh M1-C (a), M1-I (c) and M1-F (e) and outer-scaled using mesh M1-C (b), M1-I (d) and M1-F (f) for a target $\operatorname{Re}_{\tau}=300$. 


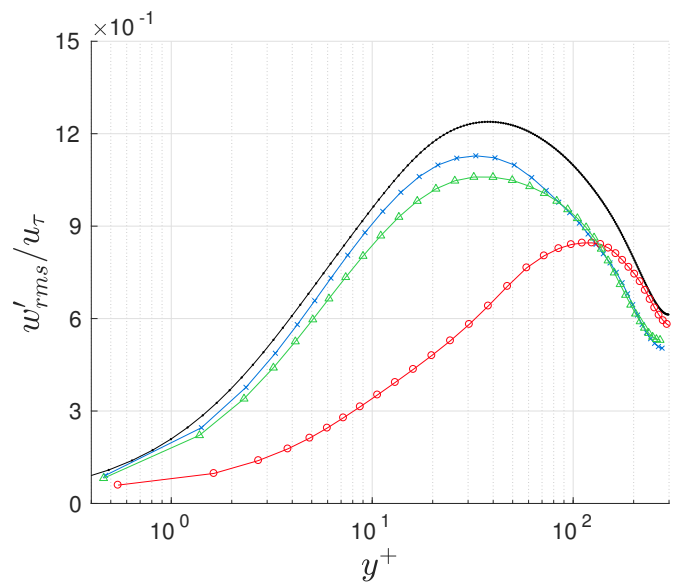

(a)

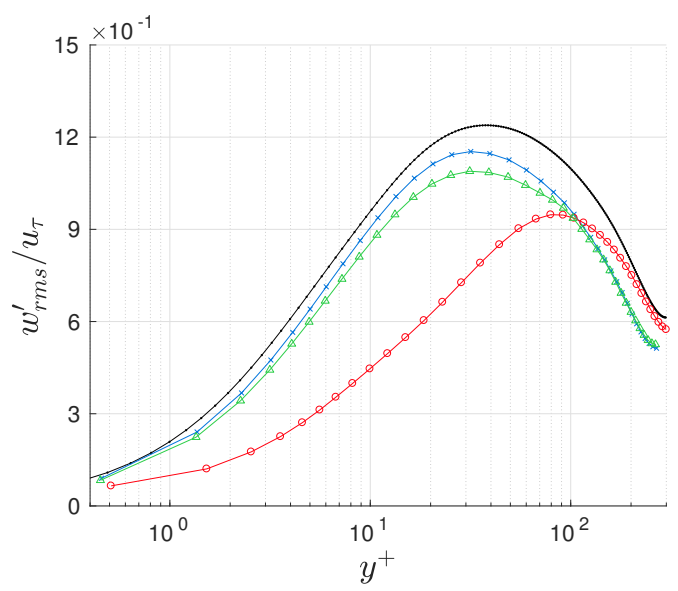

(c)

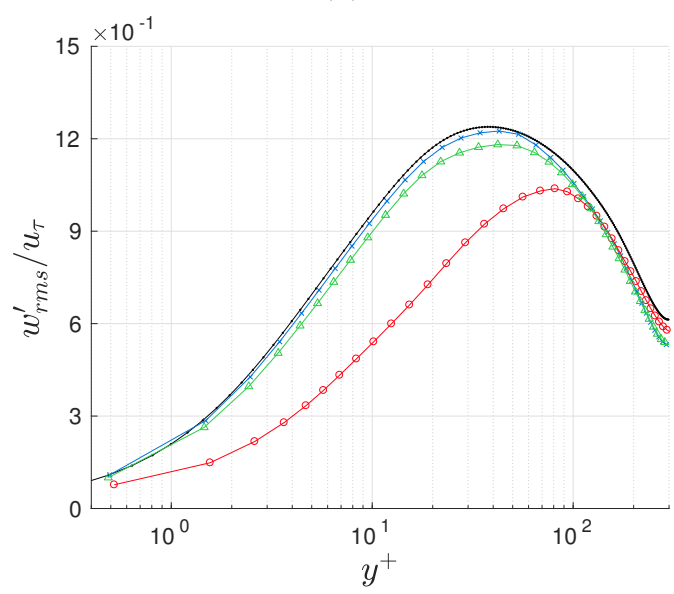

(e)

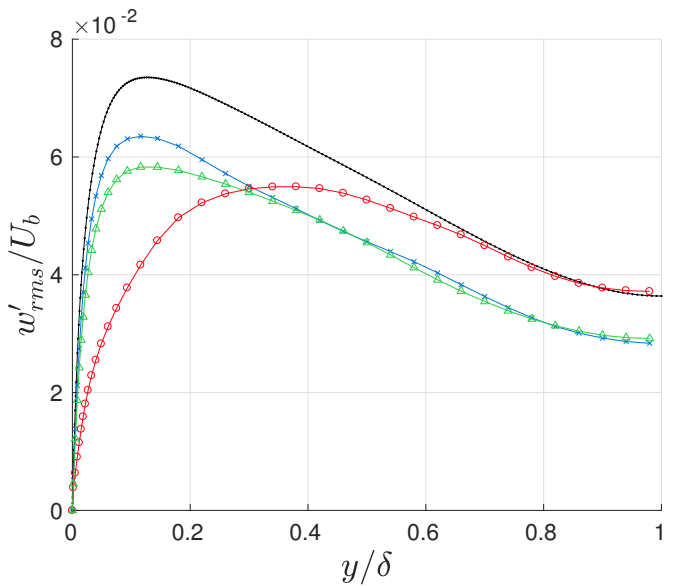

(b)

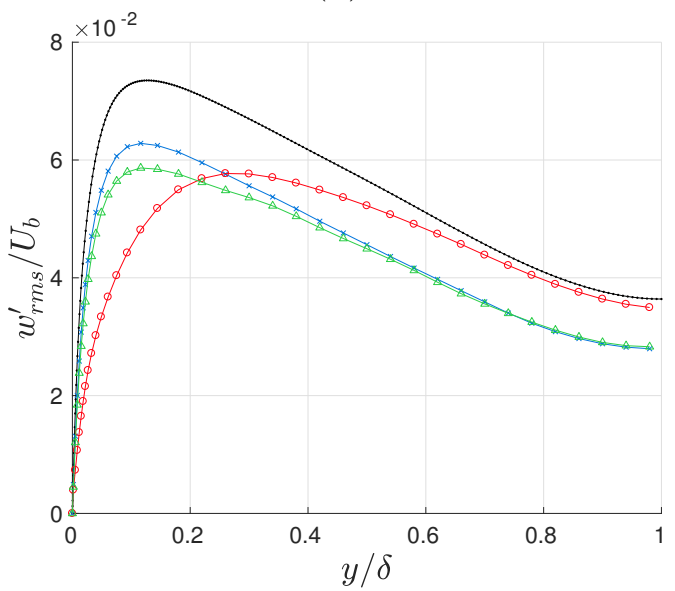

(d)

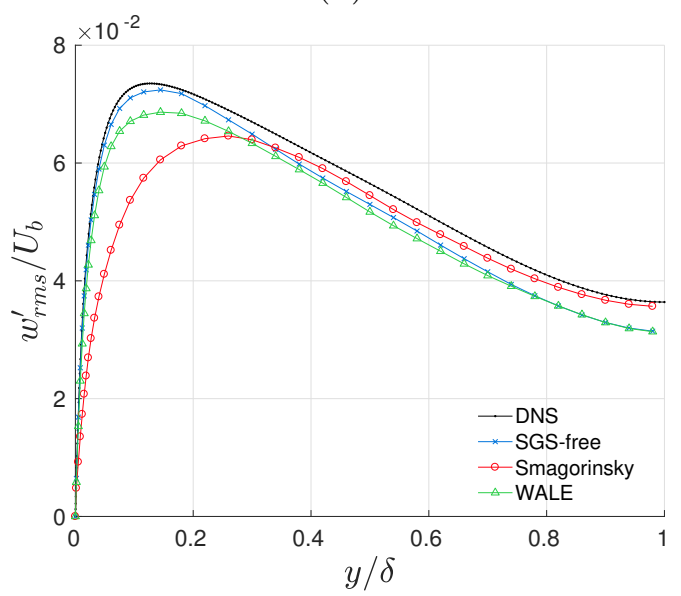

(f)

Figure 5-23: Inner-scaled $w_{r m s}^{\prime}$ using mesh M1-C (a), M1-I (c) and M1-F (e) and outer-scaled using mesh M1-C (b), M1-I (d) and M1-F (f) for a target $\operatorname{Re}_{\tau}=300$. 


\subsubsection{SGS Study on Different Wall-normal Grid Resolutions}

In this study, the cell size in both homogeneous directions is retained as that of the intermediate mesh from prior simulations. This means that a uniform cell size of 20 wall units (based on a target $\operatorname{Re}_{\tau}=300$ ) is enforced for the streamwise and spanwise directions. Two wall-normal grids are used: M1 and M3. Refer to Figure 5-24 for illustration. In both grids, the cell sizes, in wall units, vary starting from $y_{1}^{+} \approx 0.5$ to $y_{N}^{+} \approx 11$ in the viscous sublayer and the channel half-height, respectively. This results with an approximate increase of $80 \%$ of cells from M1-I to M3-I.

As for the SGS models, only the Smagorinsky [73], the WALE [63] and the Smagorinsky with a van Driest damping function [22] near the wall are used. For the sake of clear distinction in between the first and latter, when referencing the van Driest model, this implies the Smagorinsky model with a van Driest damping function. Conversely, whenever the author discusses the Smagorinsky model, this refers to the typical SGS model without near wall damping.

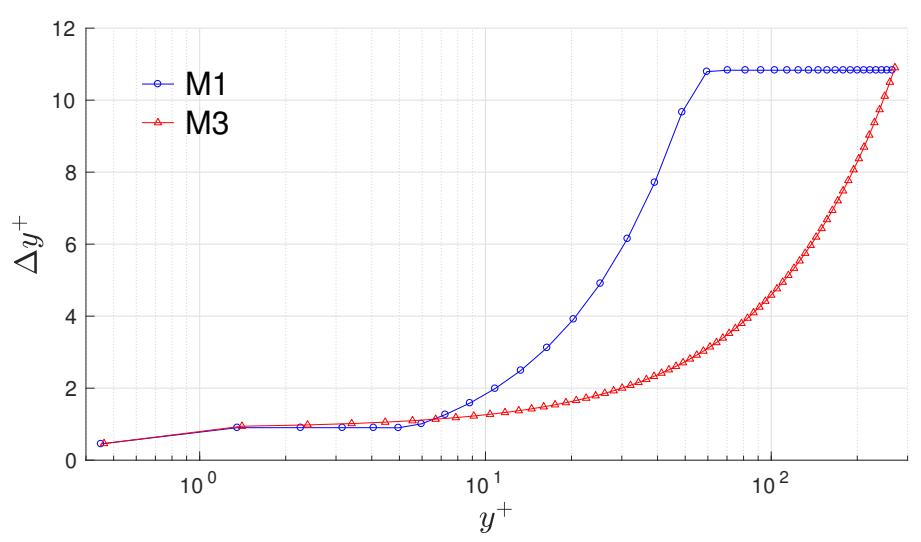

Figure 5-24: Inner-scaled grid resolutions M1 and M3 displayed in the wall-normal direction up to $\delta$ for a target $\operatorname{Re}_{\tau}=300$.

Further details about these simulations are presented in Table 5.4. According to the statistically converged $\operatorname{Re}_{\tau}$, the Smagorinsky model over-predicts in both grid cases while the remaining SGS models consistently under-predict the target $\operatorname{Re}_{\tau}$. 
Table 5.4: Summary of the simulation parameters in all six different LES as compared to the reference benchmark DNS data for a (target) $\operatorname{Re}_{\tau}$ of 300 .

\begin{tabular}{|c|c|c|c|c|c|}
\hline \multirow{2}{*}{ parameters } & \multirow[b]{2}{*}{ mesh-type } & \multicolumn{3}{|c|}{ LES SGS model } & \multirow{2}{*}{$\mathrm{DNS} \dagger$} \\
\hline & & Smagorinsky & WALE & van Driest & \\
\hline$\Delta x^{+}$ & & 20 & 20 & 20 & 18.4 \\
\hline$\Delta y^{+}$ & & $0.5-11$ & $0.5-11$ & $0.5-11$ & $0.04-4.91$ \\
\hline$\Delta z^{+}$ & & 20 & 20 & 20 & 7.36 \\
\hline$y_{1}^{+}$ & & 0.5 & 0.5 & 0.5 & $9.96952 \cdot 10^{-3}$ \\
\hline \multirow{2}{*}{$\#$ cells } & M1-I & 599,400 & 599,400 & 599,400 & \multirow{2}{*}{ - } \\
\hline & M3-I & $1,085,400$ & $1,085,400$ & $1,085,400$ & \\
\hline \multirow[b]{2}{*}{$u_{\tau}$} & M1-I & $6.076 \cdot 10^{-2}$ & $5.395 \cdot 10^{-2}$ & $5.474 \cdot 10^{-2}$ & \multirow{2}{*}{$5.9347 \cdot 10^{-2}$} \\
\hline & M3-I & $6.297 \cdot 10^{-2}$ & $5.508 \cdot 10^{-2}$ & $5.618 \cdot 10^{-2}$ & \\
\hline \multirow{2}{*}{$\operatorname{Re}_{\tau}$} & M1-I & 304.989 & 270.806 & 274.771 & \multirow{2}{*}{297.899} \\
\hline & M3-I & 316.083 & 276.478 & 281.999 & \\
\hline \multirow{2}{*}{ abbreviation } & \multirow{2}{*}{ intermediate } & S-M1 & W-M1 & SvD-M1 & \multirow{2}{*}{ - } \\
\hline & & S-M3 & W-M3 & SvD-M3 & \\
\hline dimensions & & & $\delta \times 2 \delta \times 4 \delta)$ & & $(2.5 \pi \delta \times 2 \delta \times \pi \delta)$ \\
\hline
\end{tabular}

${ }^{\dagger}$ DNS data are obtained from the THTLAB - [33] and [34].

\subsubsection{Mean Velocity Profiles}

Observing the velocity profiles, the difference between both wall-normal grids is relatively small. This is mostly evident in the inner-scaled quantities represented in the left panel of Figure 5-25. The Smagorinsky model falls beneath the DNS outcome by a considerable amount. The remaining WALE and van Driest models, however, prove much more reliable. Despite displaying excellent agreement in the viscous sublayer, deviations essentially initiate in the buffer zone and increase progressively farther from the wall. It is interesting to see that these deviations take place with an overprediction with the WALE model while an under-prediction is noticed in the case of the van Driest model. The latter manages to over-predict the DNS profile at the outer-layer. This behavior is similar in either grid type. Eventually, both WALE SGS model and the SGS-free over-predict the DNS profile by a fair amount (as displayed in inner scaling). 


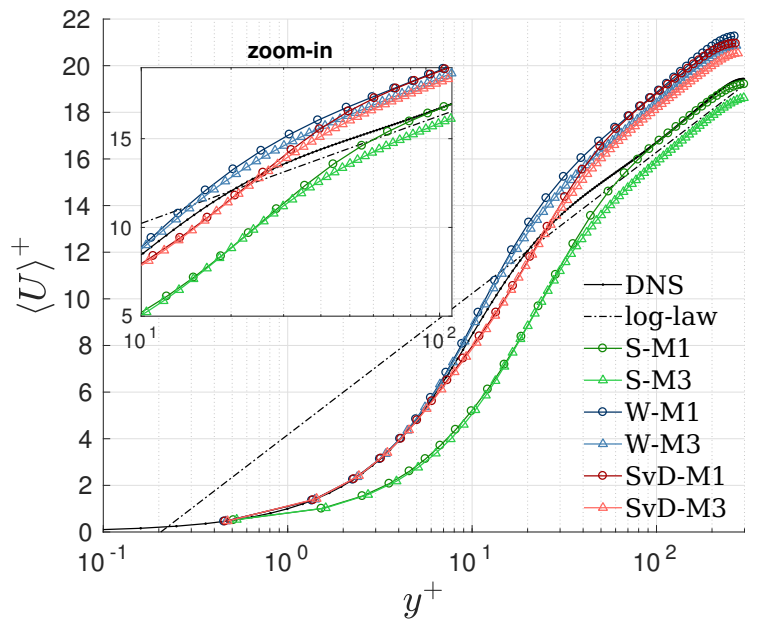

(a)

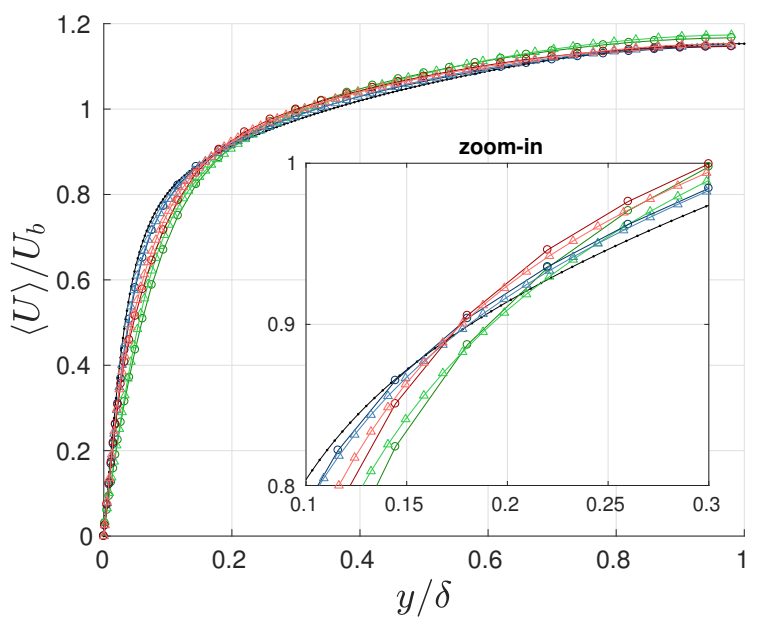

(b)

Figure 5-25: Inner-scaled (a) and outer-scaled (b) mean streamwise velocity profile for a target $\operatorname{Re}_{\tau}=300$ using mesh M1-I and M3-I.

\subsubsection{Reynolds Stress Components}

The Re-stress components are displayed in inner and outer scaling in Figures 526 and 5-27, respectively. The WALE model in either scaling exhibits insignificant changes when compared with M1-I and M3-I mesh results. The most pronounced difference is however observed in the $\left\langle u^{\prime} v^{\prime}\right\rangle$ component. Taking all cases into consideration, the WALE model displays an overall better agreement with the DNS data. It is also observed that the outer-scaled quantities, aside from the $u_{r m s}^{\prime}$, are moreor-less under-predicting the benchmark data by an appreciable amount. On that basis, the $u_{r m s}^{\prime}$ component demonstrates almost no sensitivity towards variations in the wall-normal grids used. This is evident in both wall units and outer scaling.

Furthermore, the van Driest model seems to present an inconsistent behavior in between the outer-scaled and inner-scaled figures. For instance, in Figure 5-27, using outer scaling, the Smagorinsky model displays a much better profile with respect to the DNS data than its damped counterpart. In inner scaling, however, the van Driest model shows similar behavior if not slightly better than the Smagorinsky model. 


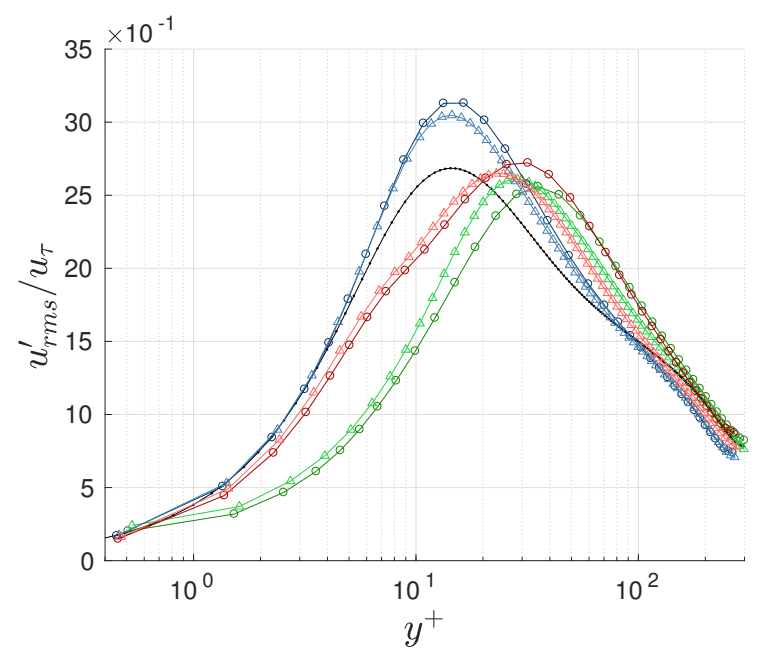

(a)

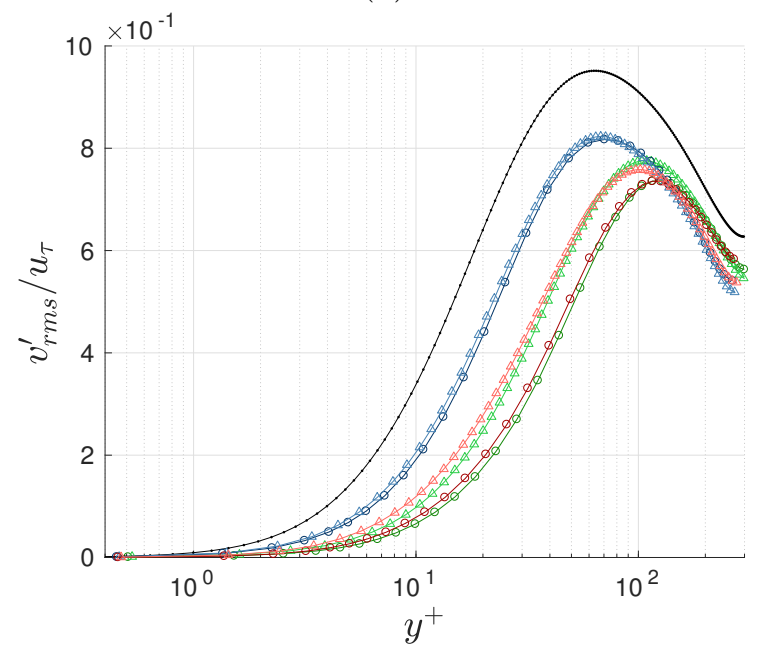

(c)

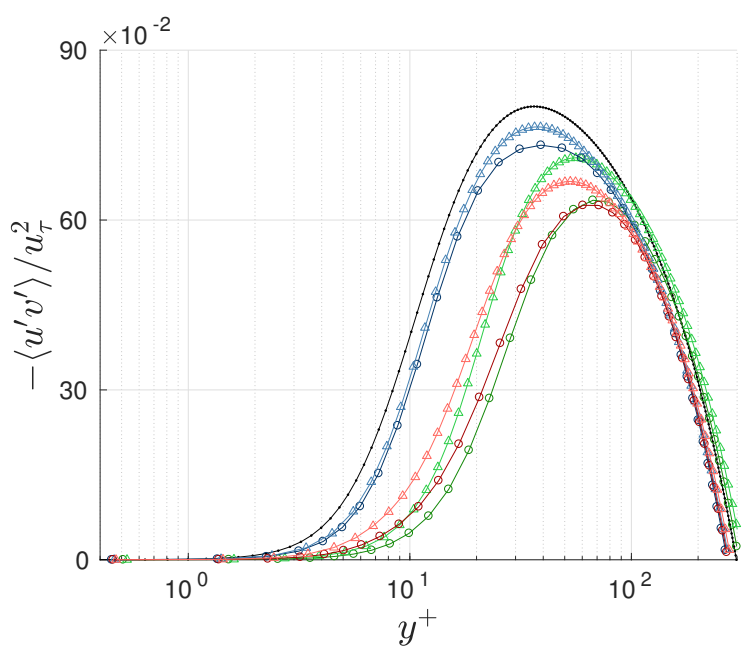

(b)

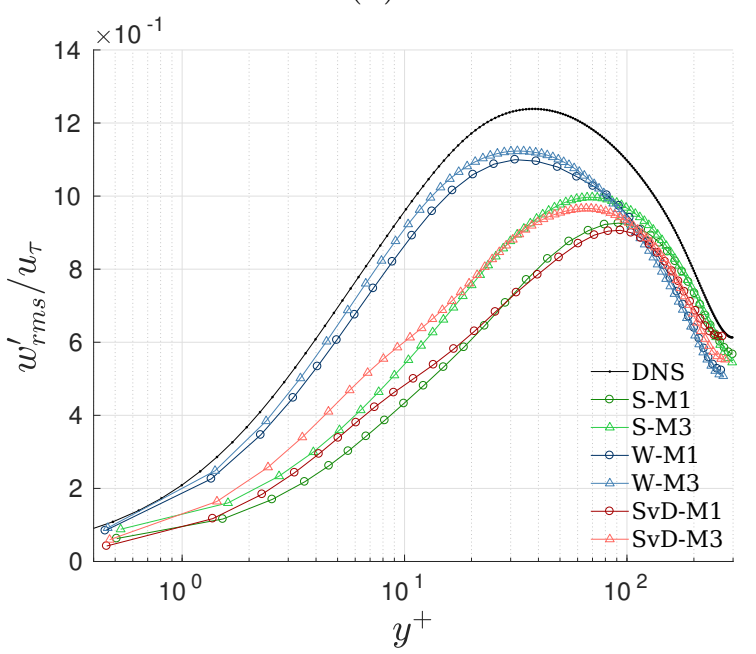

(d)

Figure 5-26: Inner-scaled $u_{r m s}^{\prime}(\mathrm{a}),-\left\langle u^{\prime} v^{\prime}\right\rangle(\mathrm{b}), v_{r m s}^{\prime}$ (c) and $w_{r m s}^{\prime}$ (d) for a target $\operatorname{Re}_{\tau}=300$ using mesh M1-I and M3-I. 


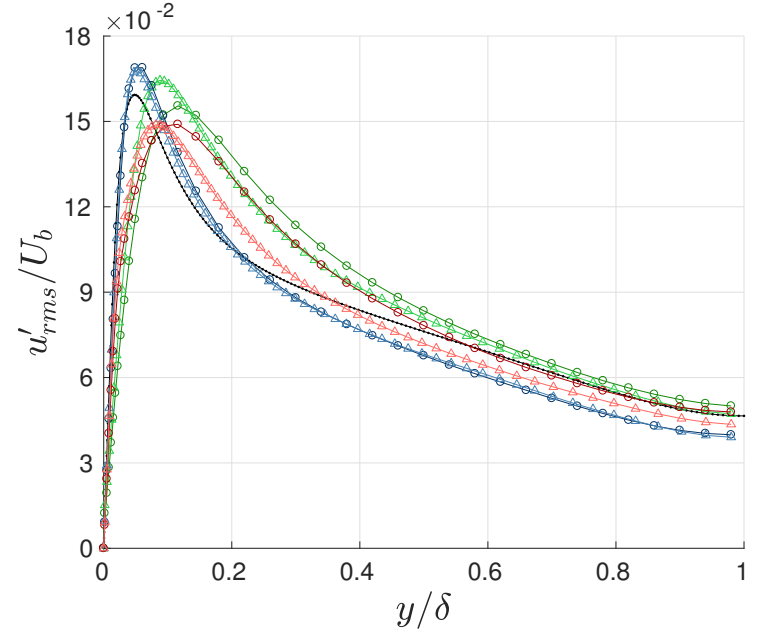

(a)

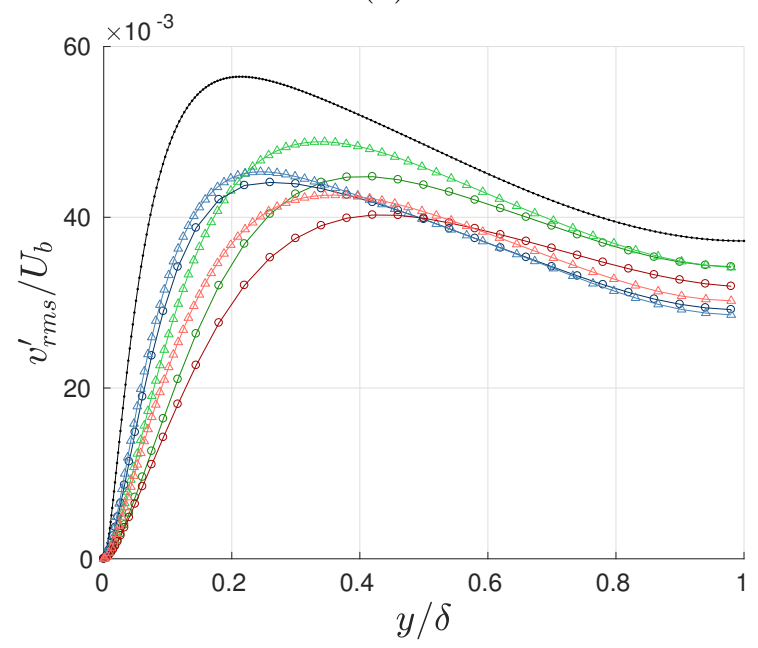

(c)

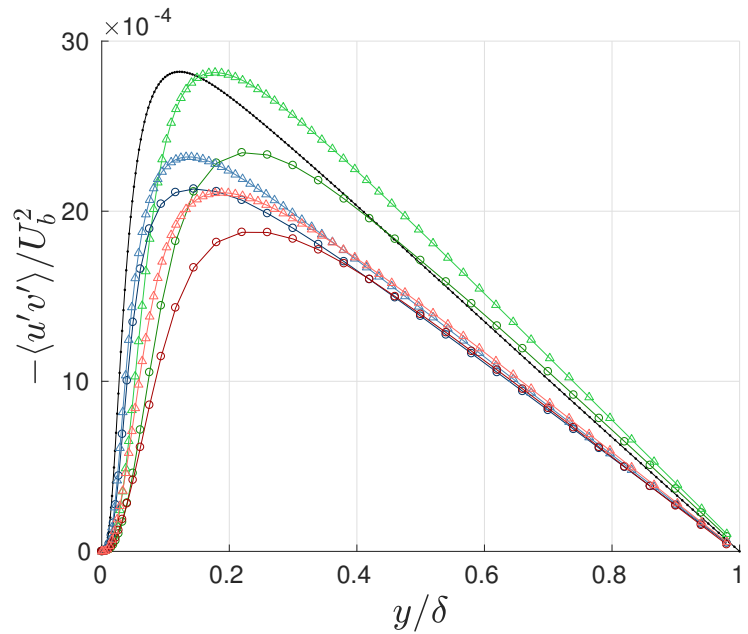

(b)

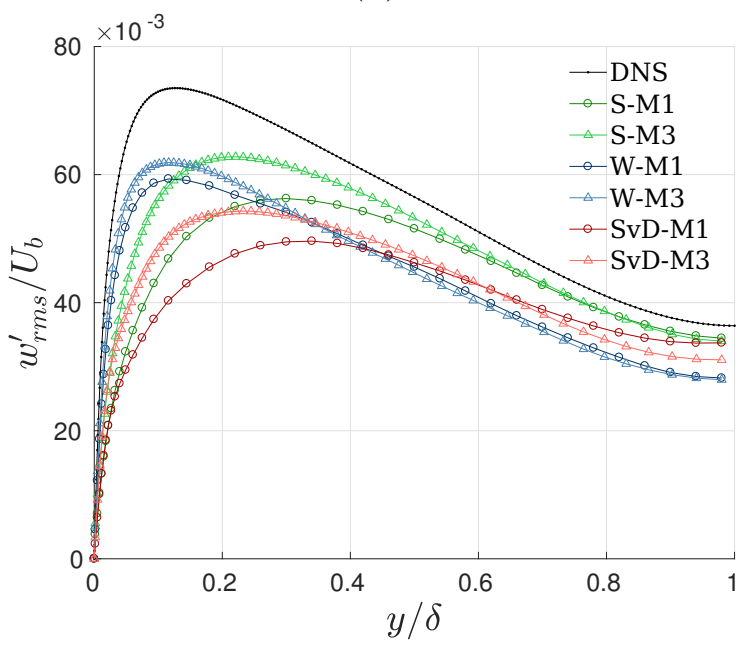

(d)

Figure 5-27: Outer-scaled $u_{r m s}^{\prime}(\mathrm{a}),-\left\langle u^{\prime} v^{\prime}\right\rangle$ (b), $v_{r m s}^{\prime}$ (c) and $w_{r m s}^{\prime}$ (d) for a target $\mathrm{Re}_{\tau}=300$ using mesh M1-I and M3-I. 


\subsubsection{Subgrid Scale Viscosity}

To effectively assess the grid's key role in each of the SGS models used, the normalized SGS viscosity is presented versus inner and outer scaling in Figure 5-28. The normalization is done by taking the ratio of the subgrid-to-kinematic viscosities. Unlike the WALE and SGS-free models, the Smagorinsky depicts extremely faulty behavior in the vicinity of the wall. This is one of the reasons that necessitates the need of a damping function for the regular Smagorinsky model in wall-bounded flows. Gridrefined areas in the wall-normal direction reduce the influence of the SGS viscosity.

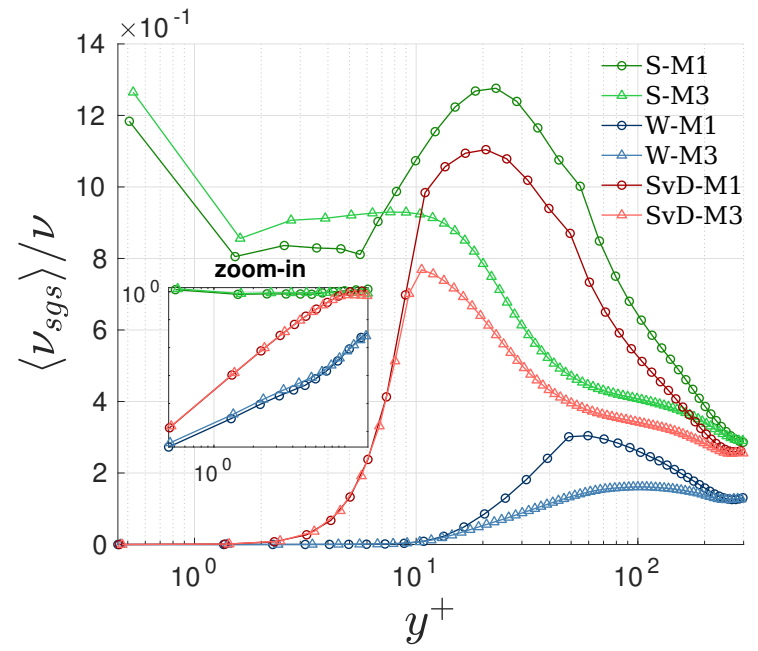

(a)

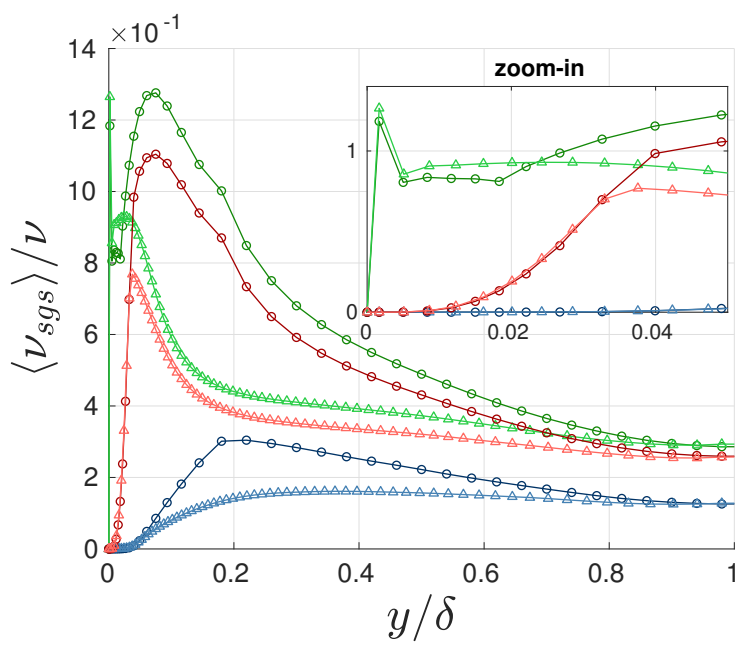

(b)

Figure 5-28: Normalized mean SGS viscosity versus the inner-scaled (a) and the outer-scaled (b) wall-normal coordinate for a target $\mathrm{Re}_{\tau}=300$ using mesh M1-I and M3-I.

\subsubsection{Spectral Analysis of SGS Models and Grid Resolutions}

In the following, a focus on the grid resolution for a selected number of SGS models is studied. The analysis revolves about one-dimensional energy spectra computations in the homogeneous directions.

The LES study is restricted to the SGS-free, the Smagorinsky [73] and the WALE [63] model. As for the wall-normal grid employed, the M1 meshing strategy is adopted. 
The homogeneous direction's cell spacings are kept uniform. In wall units and based on a target $\operatorname{Re}_{\tau}=300$, this equates to $\Delta x^{+} \approx 42,28,21$ and $\Delta z^{+} \approx 18,12,9$ for the streamwise and spanwise cell sizes, respectively. These generated a total number of cells ranging from 266400 all the way to 959 040. For more details, refer to Table 5.5.

Table 5.5: Summary of the simulation parameters in all twenty-seven different LES as compared to the reference benchmark DNS data for a (target) $\operatorname{Re}_{\tau}$ of 300 .

\begin{tabular}{|c|c|c|c|c|c|c|}
\hline \multirow{2}{*}{ parameters } & \multicolumn{2}{|c|}{ mesh } & \multicolumn{3}{|c|}{ LES SGS model } & \multirow{2}{*}{ DNS $\dagger$} \\
\hline & $\overline{\Delta x^{+}}$ & $\Delta z^{+}$ & SGS-free & Smagorinsky & WALE & \\
\hline \multirow{5}{*}{$\begin{array}{c}\Delta y^{+} \\
y_{1}^{+}\end{array}$} & & & $0.5-11$ & $0.5-11$ & $0.5-11$ & $0.04-4.91$ \\
\hline & & & 0.5 & 0.5 & 0.5 & $9.96952 \cdot 10^{-3}$ \\
\hline & & 18 & $5.727 \cdot 10^{-2}$ & $6.491 \cdot 10^{-2}$ & $5.575 \cdot 10^{-2}$ & \multirow{9}{*}{$5.9347 \cdot 10^{-2}$} \\
\hline & 42 & 12 & $5.951 \cdot 10^{-2}$ & $6.521 \cdot 10^{-2}$ & $5.816 \cdot 10^{-2}$ & \\
\hline & & 9 & $6.060 \cdot 10^{-2}$ & $6.553 \cdot 10^{-2}$ & $5.941 \cdot 10^{-2}$ & \\
\hline \multirow{9}{*}{$u_{\tau}$} & & $\overline{18}$ & $5.603 \cdot 10^{-2}$ & $6.310 \cdot 10^{-2}$ & $5.490 \cdot 10^{-2}$ & \\
\hline & 28 & 12 & $5.862 \cdot 10^{-2}$ & $6.300 \cdot 10^{-2}$ & $5.748 \cdot 10^{-2}$ & \\
\hline & & 9 & $5.969 \cdot 10^{-2}$ & $6.330 \cdot 10^{-2}$ & $5.872 \cdot 10^{-2}$ & \\
\hline & & 18 & $5.525 \cdot 10^{-2}$ & $6.124 \cdot 10^{-2}$ & $5.450 \cdot 10^{-2}$ & \\
\hline & 21 & 12 & $5.757 \cdot 10^{-2}$ & $6.161 \cdot 10^{-2}$ & $5.691 \cdot 10^{-2}$ & \\
\hline & & 9 & $5.870 \cdot 10^{-2}$ & $6.146 \cdot 10^{-2}$ & $5.787 \cdot 10^{-2}$ & \\
\hline & & 18 & 287.471 & 325.821 & 279.841 & \multirow{9}{*}{297.899} \\
\hline & 42 & 12 & 298.715 & 327.327 & 291.939 & \\
\hline & & 9 & 304.186 & 328.933 & 298.213 & \\
\hline \multirow{6}{*}{$\operatorname{Re}_{\tau}$} & & $\overline{18}$ & 281.247 & 316.735 & 275.575 & \\
\hline & 28 & 12 & 294.248 & 316.233 & 288.525 & \\
\hline & & 9 & 299.619 & 317.739 & 294.750 & \\
\hline & & 18 & 277.332 & 307.399 & 273.567 & \\
\hline & 21 & 12 & 288.977 & 309.256 & 285.664 & \\
\hline & & 9 & 294.649 & 308.503 & 290.483 & \\
\hline dimensions & & & \multicolumn{3}{|c|}{$(9 \delta \times 2 \delta \times 4 \delta)$} & $(2.5 \pi \delta \times 2 \delta$ \\
\hline
\end{tabular}

${ }^{\dagger}$ DNS data are obtained from the THTLAB $-[33]$ and [34].

During spectral analysis of turbulent flow, the energy cascade process is directly and heavily involved. In brief terms, this process may be described by alluding to three dominant localities: intergral length scale, Taylor microscale and Kolmogorov length scale which are associated with the energy-containing range, inertial subrange and dissipation range, respectively. These correspond to the highest to lowest energy- 
containing eddies and accordingly from the lowest to highest wavenumbers. The key point of a spectra study, amongst other things, is to prove a resulting simulation is at least able to resolve most of the inertial subrange. Simultaneously, it gives perspicacity to how much TKE is contained in the eddies associated with wavenumber $\kappa$. Since in this thesis the energy spectra is restrained to only one-dimension (due to lack of isotropy), then this addresses only the corresponding portion of the turbulent kinetic energy - i.e. either of the three fluctuating components of velocity and not their summation as implied by the definition of TKE. Moreover, the aim is to study how much energy the selected combination of grid and SGS model are able to recover in comparison to the benchmark DNS data. For a more in-depth explanation about the power spectral density (PSD), refer to Appendix A.

The total number of flow-through times used for averaging and collecting data is approximately 22 times. As for the transient phase, a total of 55 flow-through times (same as previous sections in this chapter) is considered. The overall number of temporal samples, after attaining statistically fully-developed turbulent channel flow, collected are 20000 . Moreover, in order to yield a better statistical result, instead of sampling data per line, the data are collected from planes parallel to the wall. The idea is to sample across four different wall-normal planes and then collapse the data in the required direction in order to output the desired spectral density. The four planes sampled are taken in outer scaling at $y / \delta \approx 0.015,0.05,0.5,0.98$ or in inner scaling at $y^{+} \approx 4.5,14.7,150,294$. These selected wall-normal positions mitigate the study of PSD in the viscous sublayer, buffer zone, channel quarter-height and channel half-height, respectively. Correspondingly, the DNS data, taken from the THTLAB $^{6}$, are also sampled at similar wall-normal intervals and they correspond to $y / \delta \approx 0.018,0.05,0.5,1$ in outer scaling, or $y^{+} \approx 5.2,15,149,297.9$ in inner scaling.

In the following series of spectra figures, an abbreviation for ease of notation is introduced for the legends therein. This is composed of two parts separated by a

\footnotetext{
${ }^{6}$ http: //www.thtlab.jp/
} 
dash in between: a capital letter on the left and a number on the right. The letters denote the SGS models used, in this case they are: F, S and W, which correspond to the SGS-free, the Smagorinsky and the WALE model, respectively. The numbers to the right of the dash symbol in the abbreviation denote the cell size in the spanwise direction expressed in wall units (based on the target $\operatorname{Re}_{\tau}$ ).

The resulting set of figures illustrate the inner-scaled spanwise and streamwise one-dimensional PSD of each of the fluctuating velocity components, SGS models and grid resolution used. In each figure, the corresponding data is displayed at each of the four distinct wall-normal planes in fittingly separate panels.

\subsubsection{One-dimensional Streamwise Power Spectral Density}

At the highest wavenumbers, most of the streamwise energy recovered is in the planes corresponding to the viscous sublayer and the buffer zone. This is evident for all three velocity components. A possible explanation is by how the wall-normal meshing strategy works; refinement is done near the wall while gradual coarsening is used farther away. The $v$ - and $w$-velocity components demonstrate a steeper gradient than the streamwise velocity's inner-scaled PSD. Hence, in comparison with the streamwise velocities PSD, lower energies are recovered for the wall-normal and spanwise velocities. Both WALE and SGS-free models display similar satisfactory profiles with the DNS data, nonetheless the latter is slightly better in most planes.

Moving from $\Delta x^{+} \approx 42$ to 28 and 21 , the PSD curves further converge towards the reported DNS data. Evidently, a spanwise resolution of either $\Delta z^{+} \approx 9$ or 12 yields barely differentiable results. Despite the streamwise resolution used, the wall-normal PSD corresponding to the viscous sublayer plane displays, relatively, the highest discrepancy. This is demonstrated in Figures 5-30, 5-33 and 5-36. Additionally, for $\kappa_{x}^{+}<0.03$ in those three figures, almost no change takes place in spite of the different streamwise resolutions used.

By increasing the number of cells per streamwise direction, the energies recovered manage to include smaller eddies at higher wavenumbers. The Smagorinsky model 
under-predicts the reported DNS curves the most. This is especially evident in both viscous sublayer and buffer zone. Away from the wall, the sampling planes corresponding to the channel's quarter- and half-height both fail to lessen the discrepancy in a similar manner as that witnessed in the two inner layer planes. This possibly indicates that, despite the refinement in the homogeneous directions, the wall-normal resolution in the outer region is still coarse with respect to that used in the DNS. 


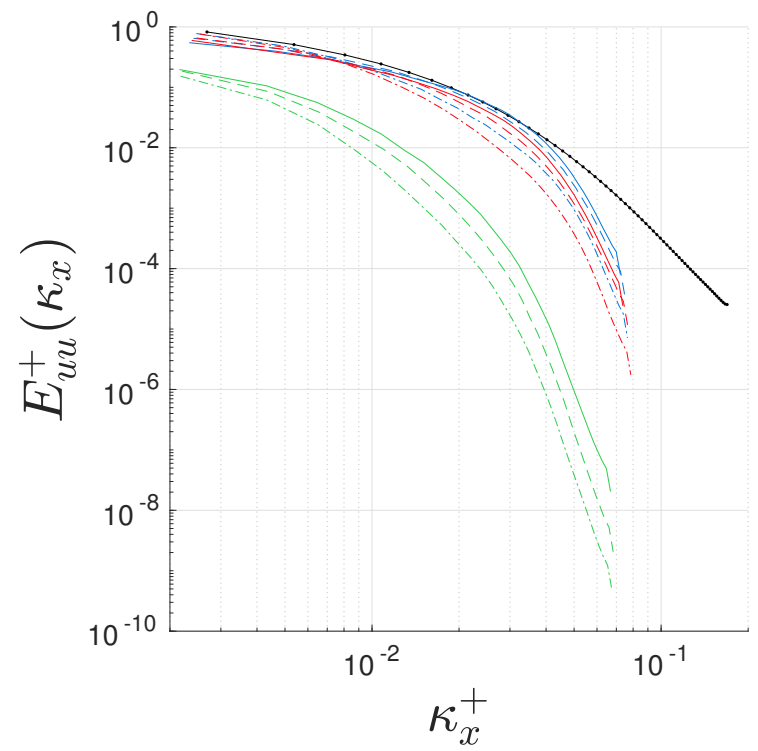

(a)

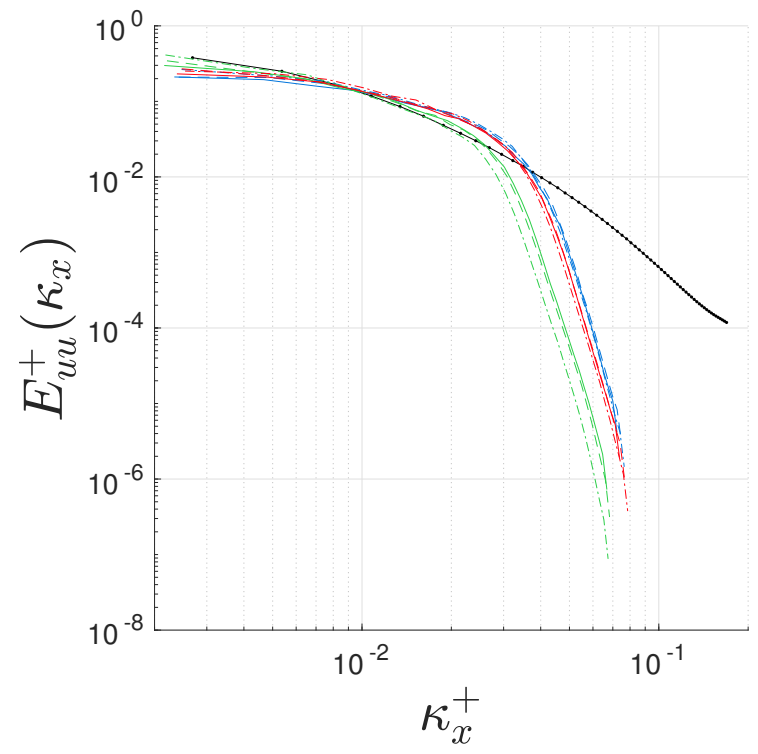

(c)

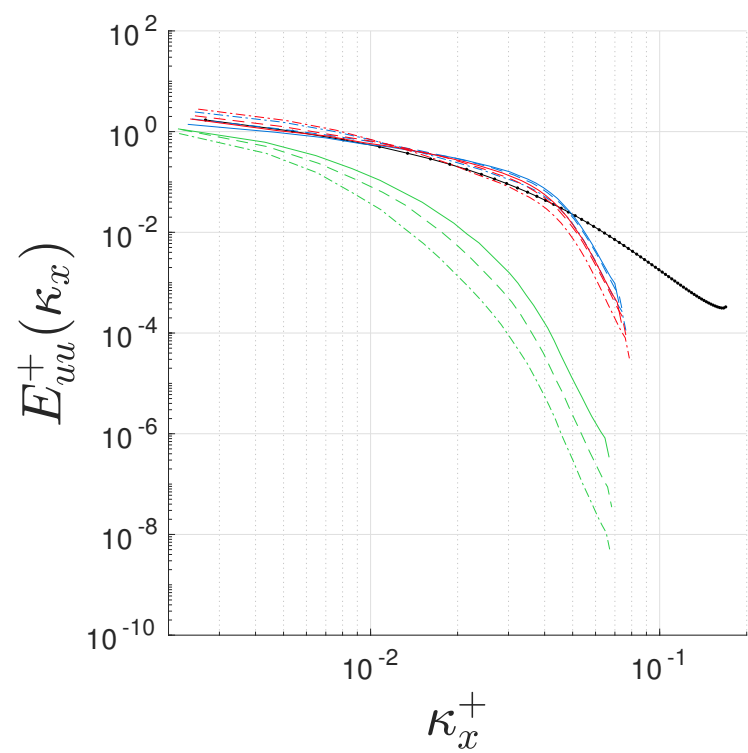

(b)

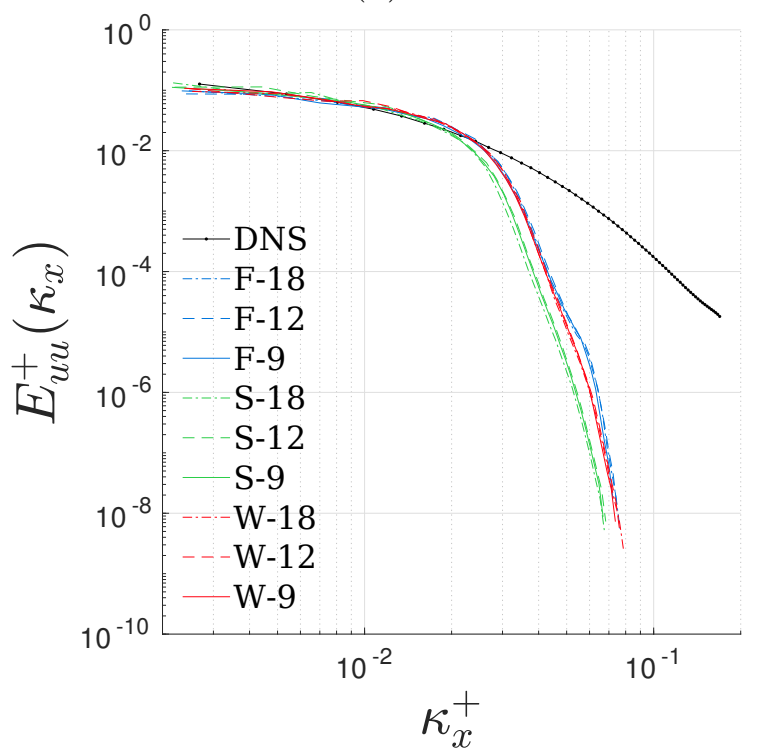

(d)

Figure 5-29: Inner-scaled one-dimensional power spectral density in the streamwise direction for the $u$-velocity component computed with $N_{x}=64$ cells (i.e. $\Delta x^{+} \approx 42$ ) and at wall-normal planes $y^{+} \approx 4.5$ or $y / \delta \approx 0.015(\mathrm{a}), y^{+} \approx 14.7$ or $y / \delta \approx 0.05(\mathrm{~b})$, $y^{+} \approx 150$ or $y / \delta \approx 0.5$ (c) and $y^{+} \approx 294$ or $y / \delta \approx 0.98(\mathrm{~d})$ for a target $\operatorname{Re}_{\tau}=300$. 


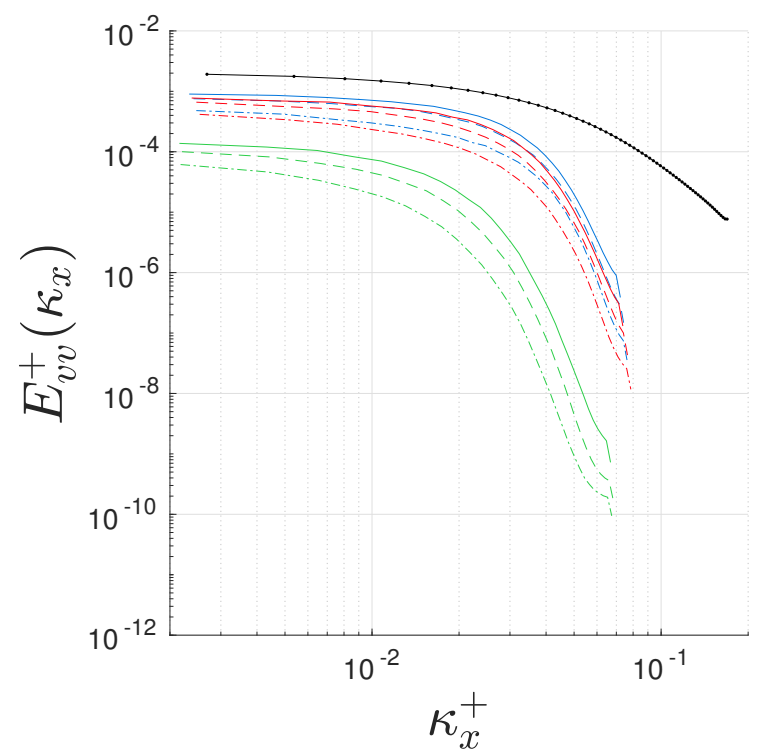

(a)

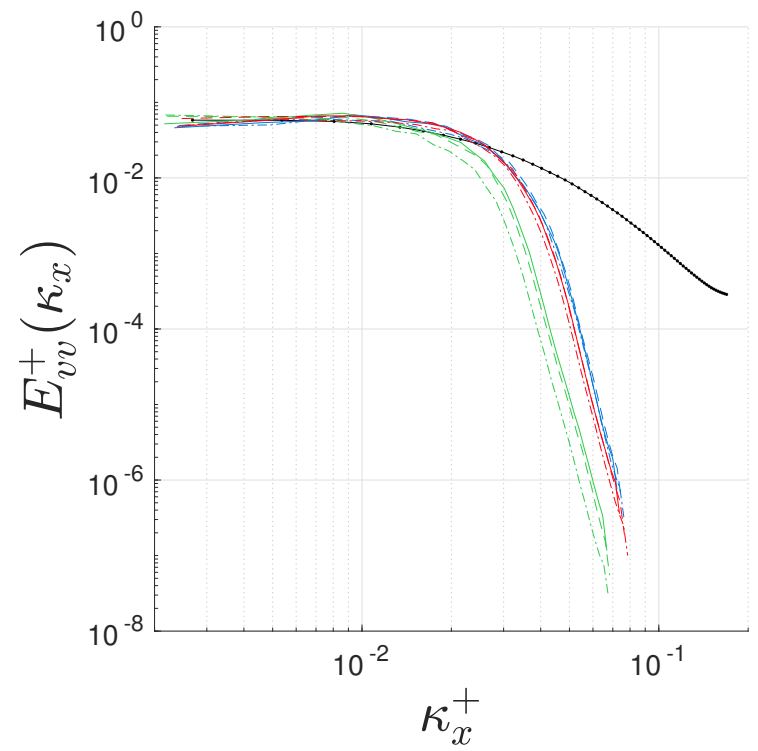

(c)

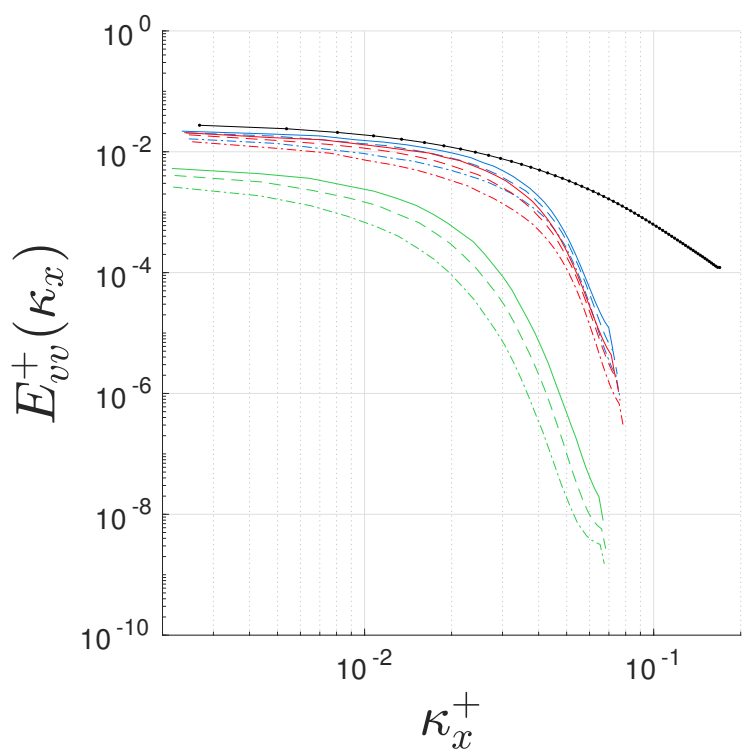

(b)

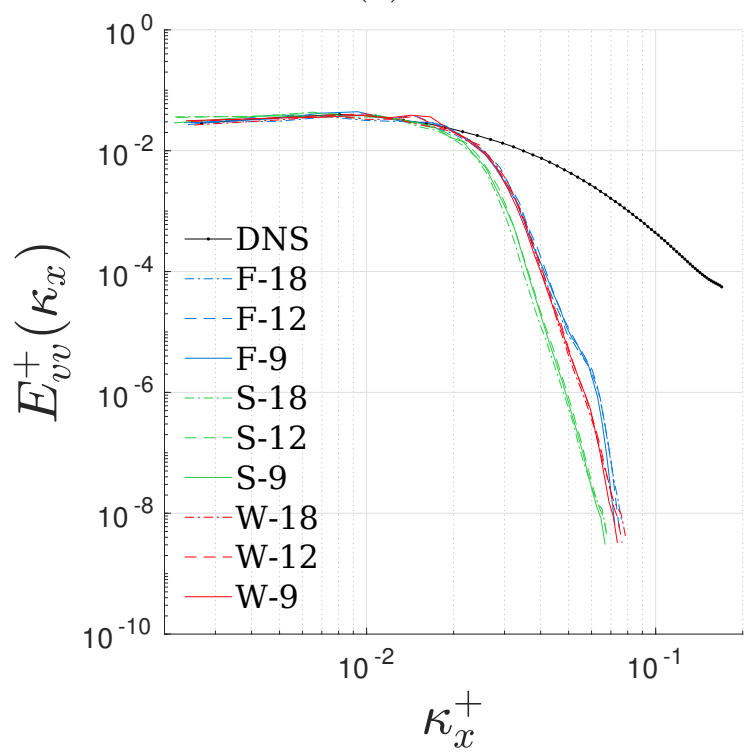

(d)

Figure 5-30: Inner-scaled one-dimensional power spectral density in the streamwise direction for the $v$-velocity component computed with $N_{x}=64$ cells (i.e. $\Delta x^{+} \approx 42$ ) and at wall-normal planes $y^{+} \approx 4.5$ or $y / \delta \approx 0.015(\mathrm{a}), y^{+} \approx 14.7$ or $y / \delta \approx 0.05(\mathrm{~b})$, $y^{+} \approx 150$ or $y / \delta \approx 0.5$ (c) and $y^{+} \approx 294$ or $y / \delta \approx 0.98(\mathrm{~d})$ for a target $\operatorname{Re}_{\tau}=300$. 


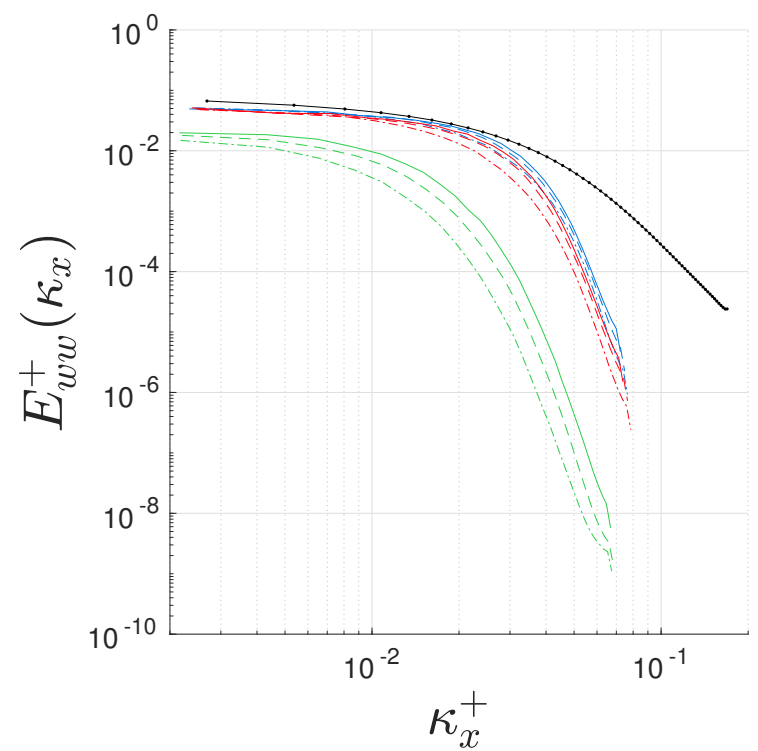

(a)

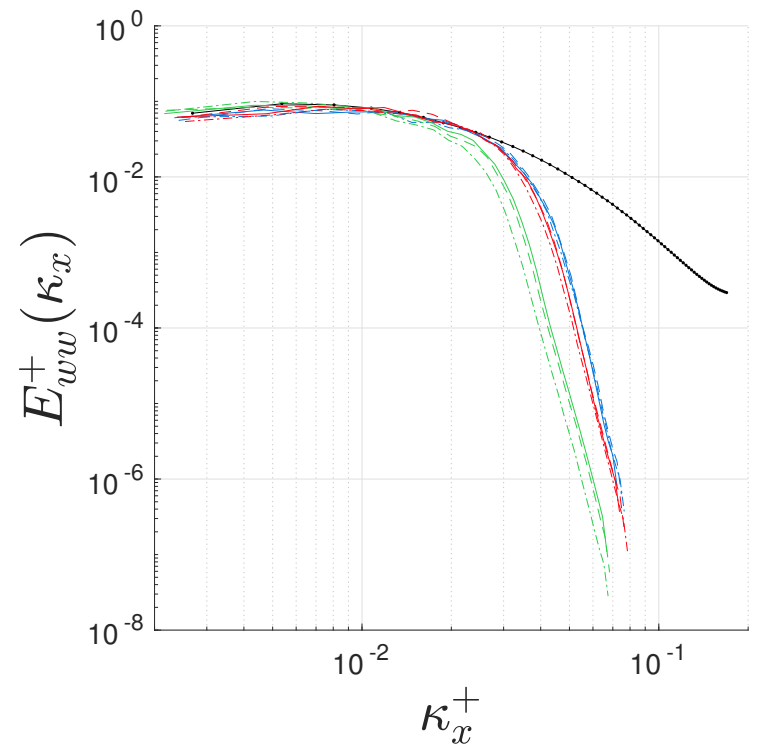

(c)

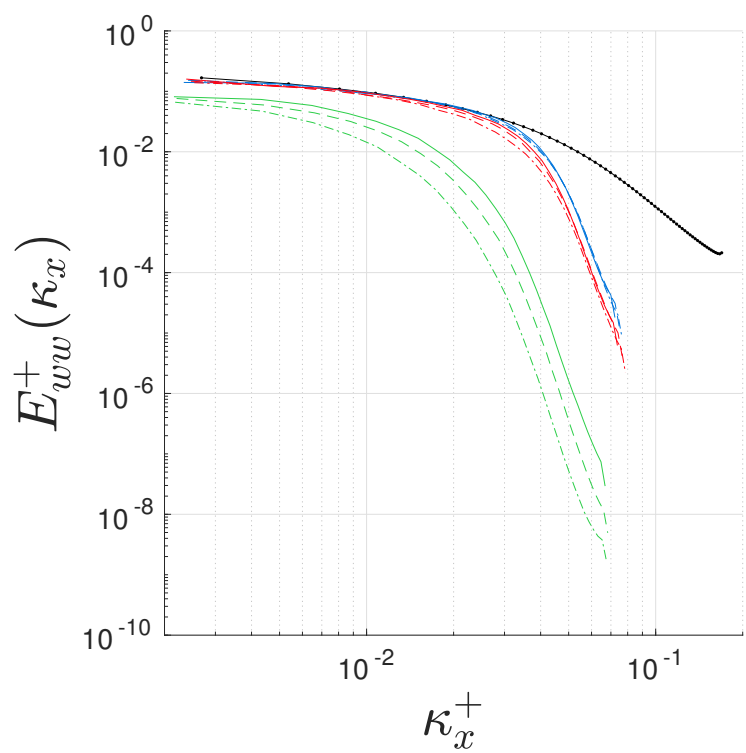

(b)

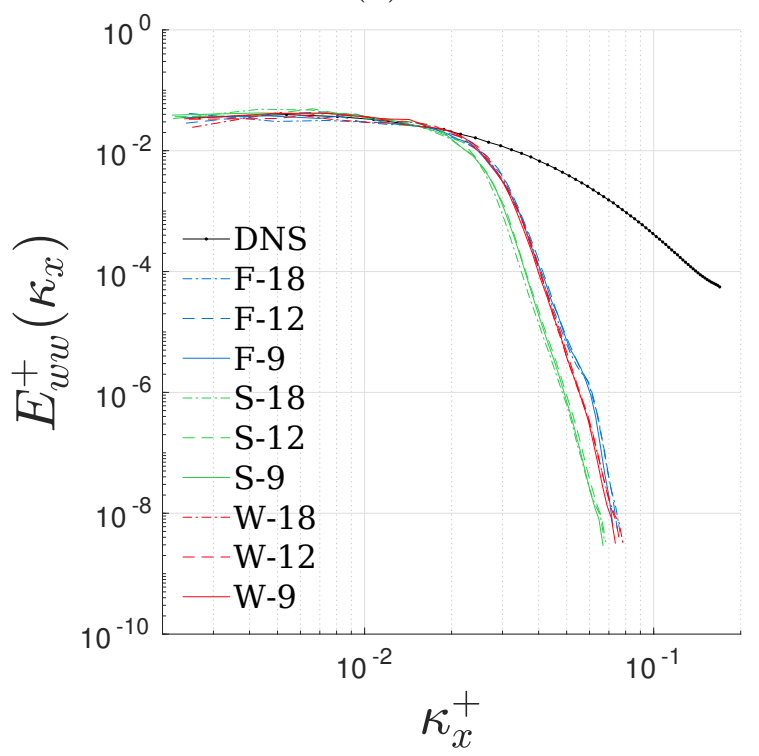

(d)

Figure 5-31: Inner-scaled one-dimensional power spectral density in the streamwise direction for the $w$-velocity component computed with $N_{x}=64$ cells (i.e. $\Delta x^{+} \approx 42$ ) and at wall-normal planes $y^{+} \approx 4.5$ or $y / \delta \approx 0.015$ (a), $y^{+} \approx 14.7$ or $y / \delta \approx 0.05$ (b), $y^{+} \approx 150$ or $y / \delta \approx 0.5$ (c) and $y^{+} \approx 294$ or $y / \delta \approx 0.98$ (d) for a target $\operatorname{Re}_{\tau}=300$. 


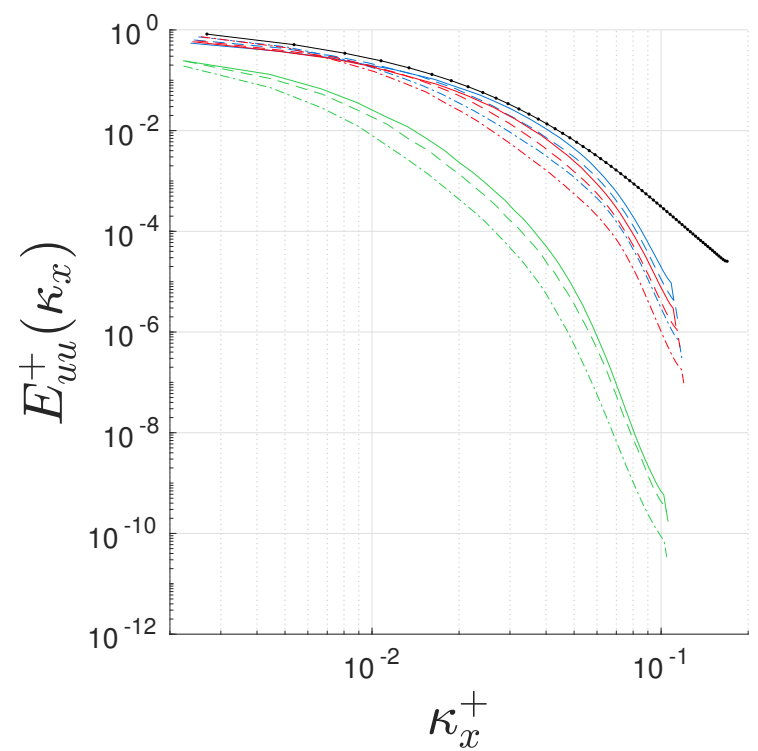

(a)

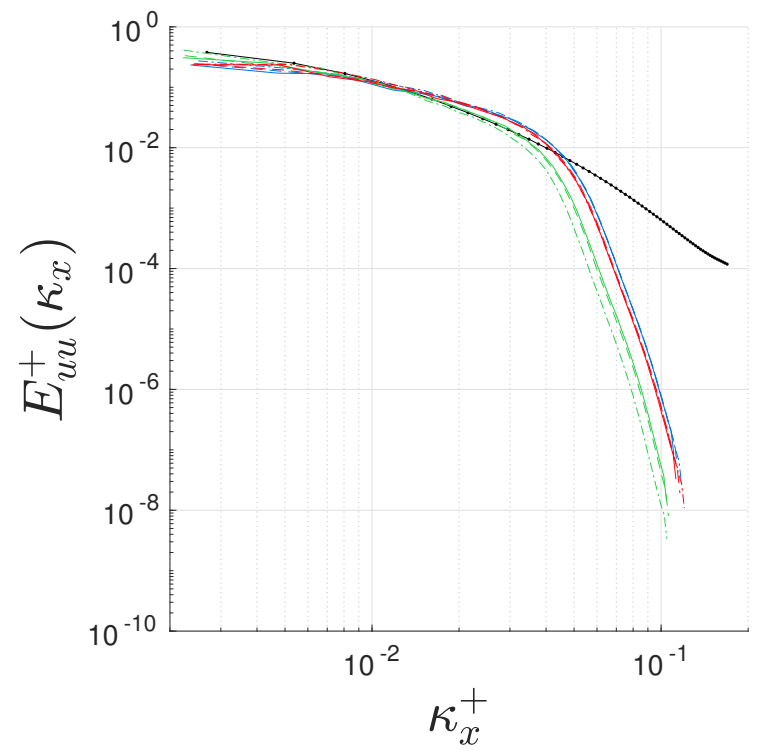

(c)

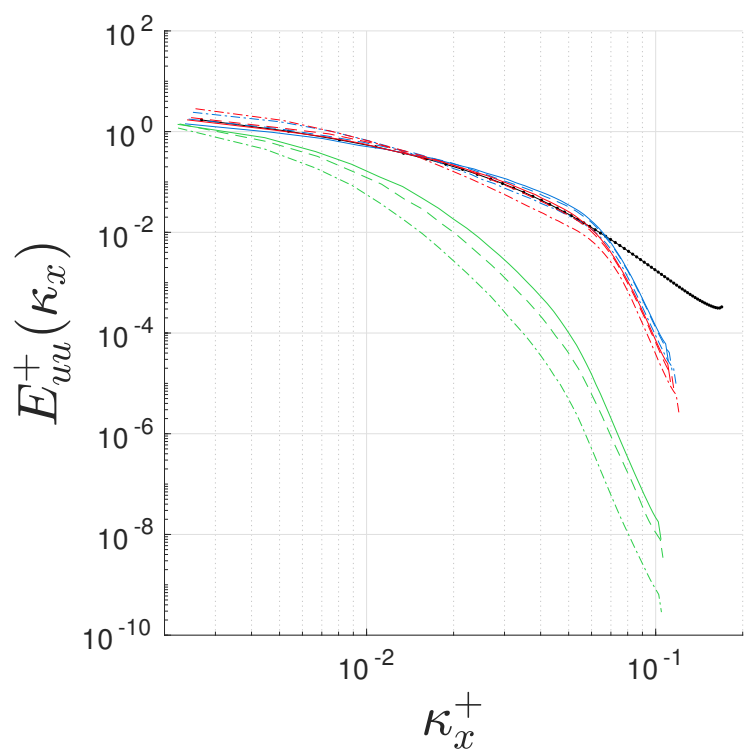

(b)

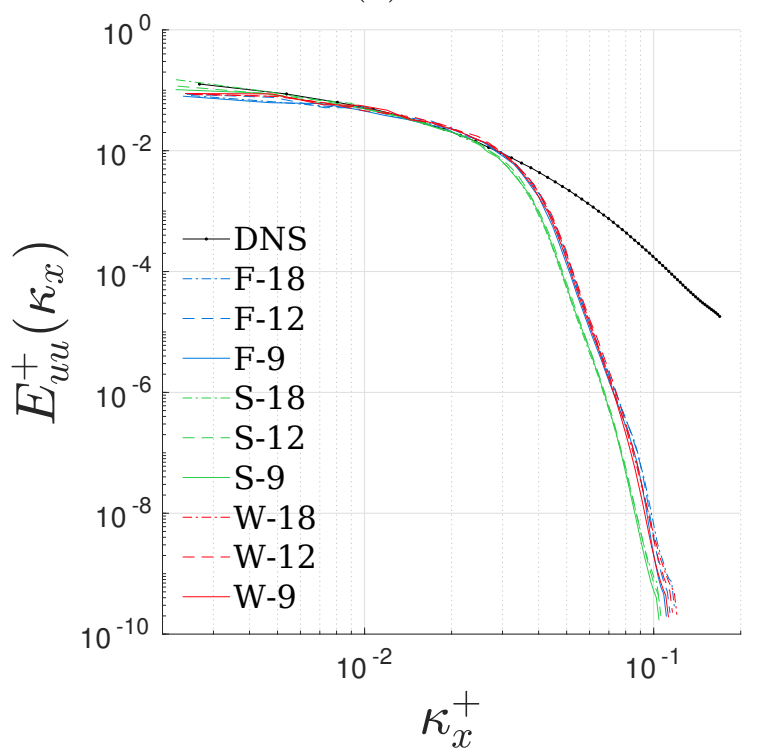

(d)

Figure 5-32: Inner-scaled one-dimensional power spectral density in the streamwise direction for the $u$-velocity component computed with $N_{x}=96$ cells (i.e. $\Delta x^{+} \approx 28$ ) and at wall-normal planes $y^{+} \approx 4.5$ or $y / \delta \approx 0.015(\mathrm{a}), y^{+} \approx 14.7$ or $y / \delta \approx 0.05(\mathrm{~b})$, $y^{+} \approx 150$ or $y / \delta \approx 0.5$ (c) and $y^{+} \approx 294$ or $y / \delta \approx 0.98(\mathrm{~d})$ for a target $\operatorname{Re}_{\tau}=300$. 


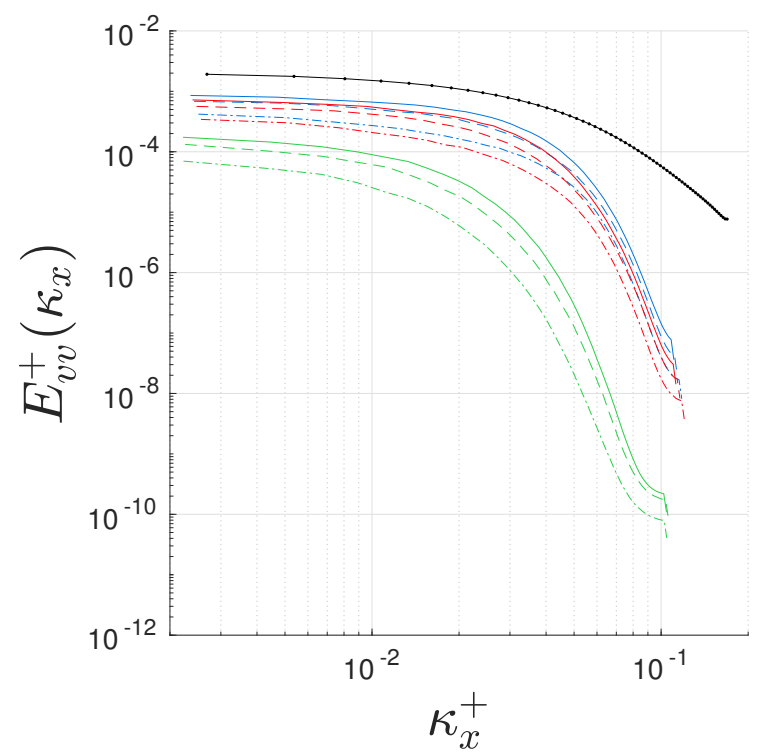

(a)

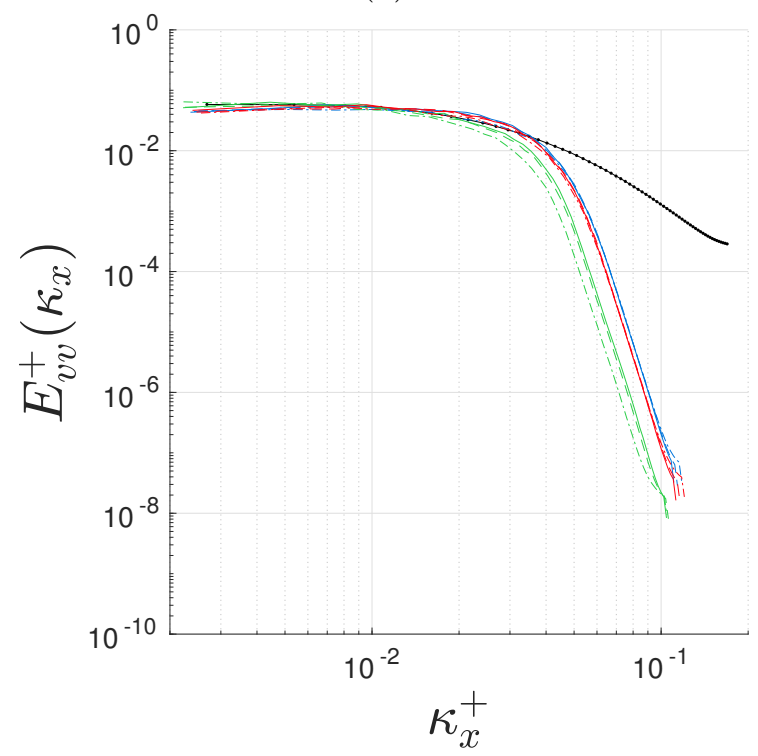

(c)

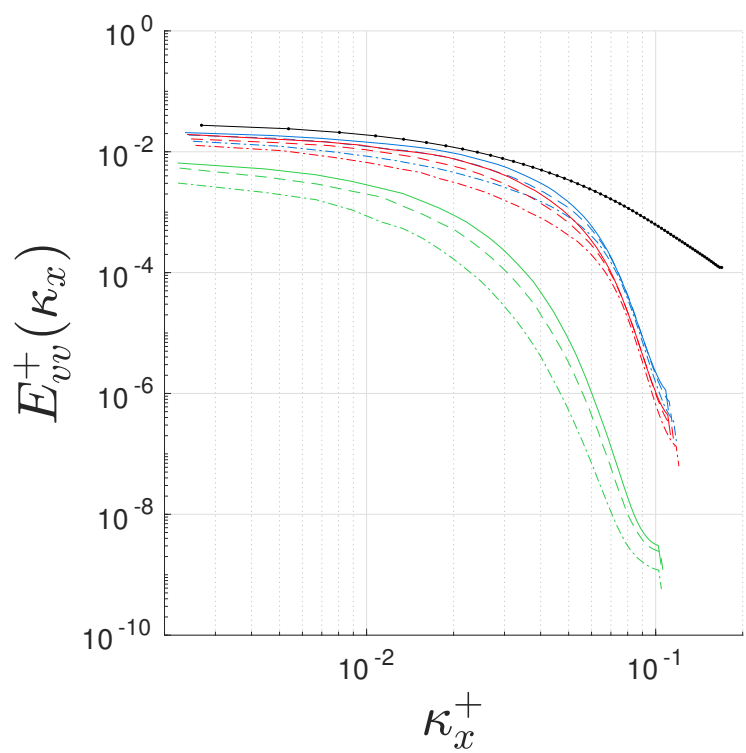

(b)

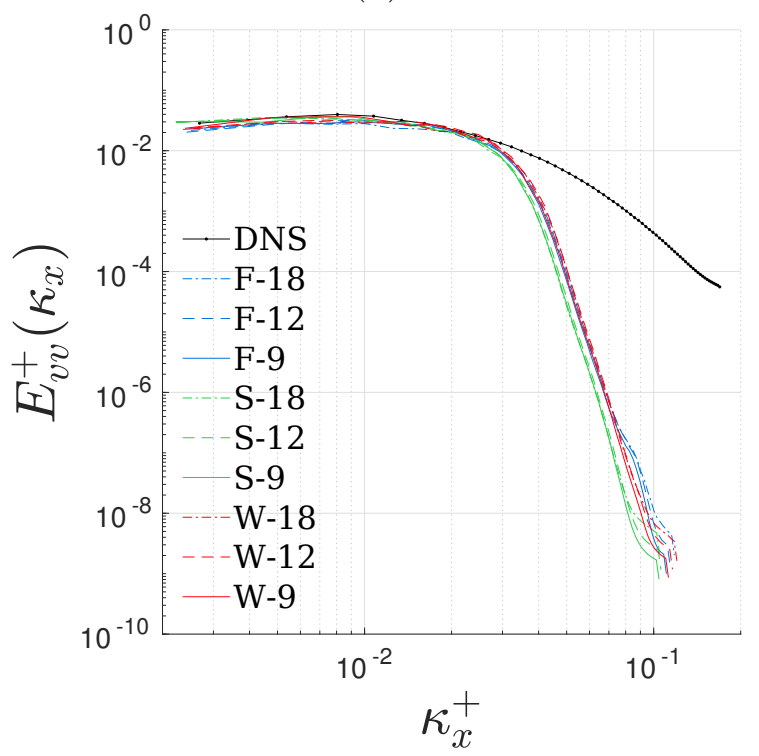

(d)

Figure 5-33: Inner-scaled one-dimensional power spectral density in the streamwise direction for the $v$-velocity component computed with $N_{x}=96$ cells (i.e. $\Delta x^{+} \approx 28$ ) and at wall-normal planes $y^{+} \approx 4.5$ or $y / \delta \approx 0.015$ (a), $y^{+} \approx 14.7$ or $y / \delta \approx 0.05$ (b), $y^{+} \approx 150$ or $y / \delta \approx 0.5$ (c) and $y^{+} \approx 294$ or $y / \delta \approx 0.98$ (d) for a target $\operatorname{Re}_{\tau}=300$. 


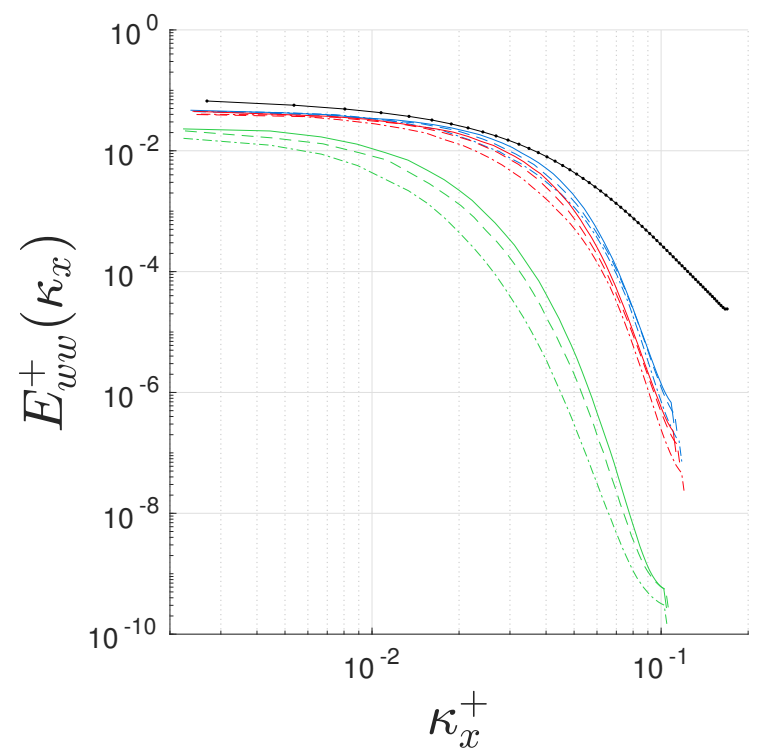

(a)

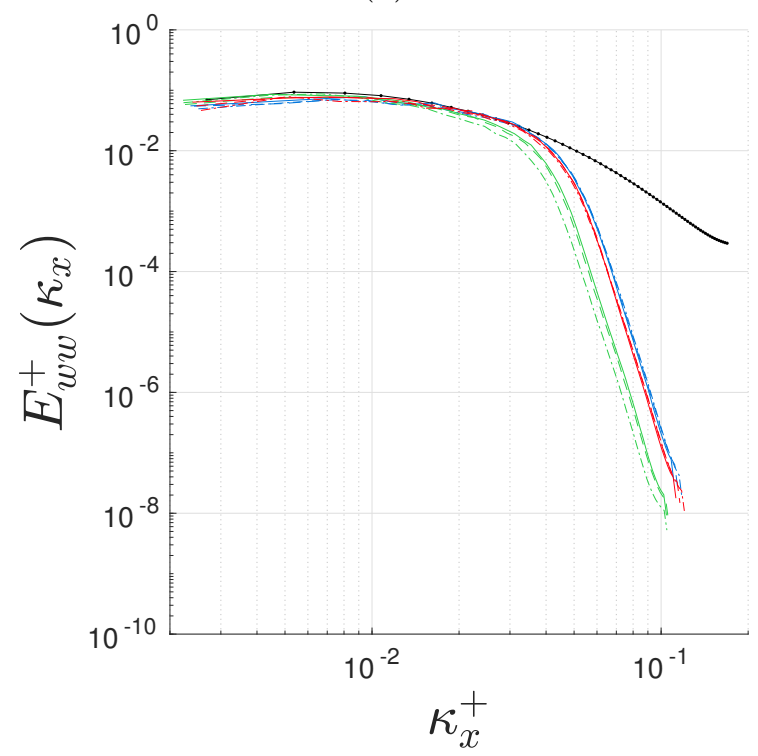

(c)

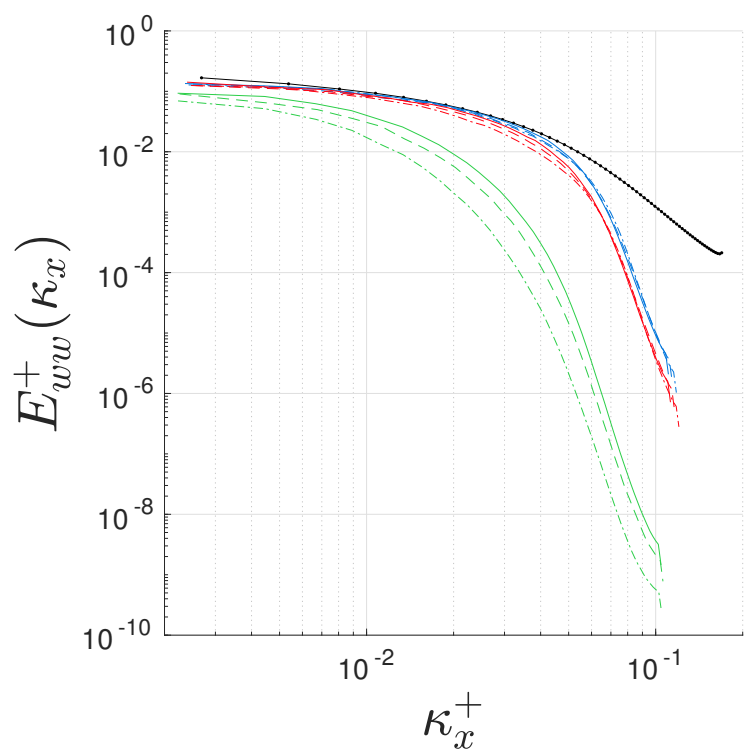

(b)

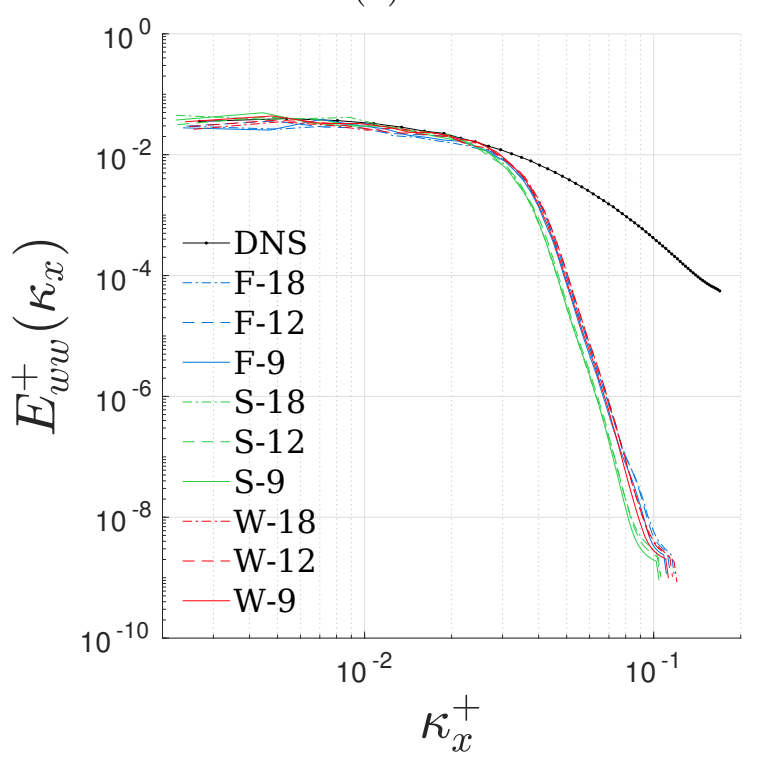

(d)

Figure 5-34: Inner-scaled one-dimensional power spectral density in the streamwise direction for the $w$-velocity component computed with $N_{x}=96$ cells (i.e. $\Delta x^{+} \approx 28$ ) and at wall-normal planes $y^{+} \approx 4.5$ or $y / \delta \approx 0.015(\mathrm{a}), y^{+} \approx 14.7$ or $y / \delta \approx 0.05(\mathrm{~b})$, $y^{+} \approx 150$ or $y / \delta \approx 0.5$ (c) and $y^{+} \approx 294$ or $y / \delta \approx 0.98$ (d) for a target $\operatorname{Re}_{\tau}=300$. 


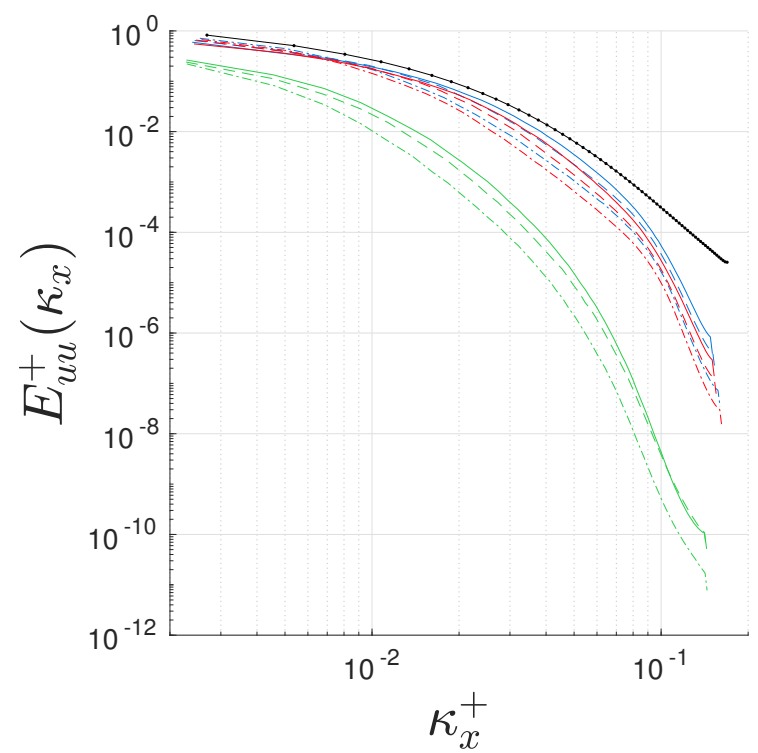

(a)

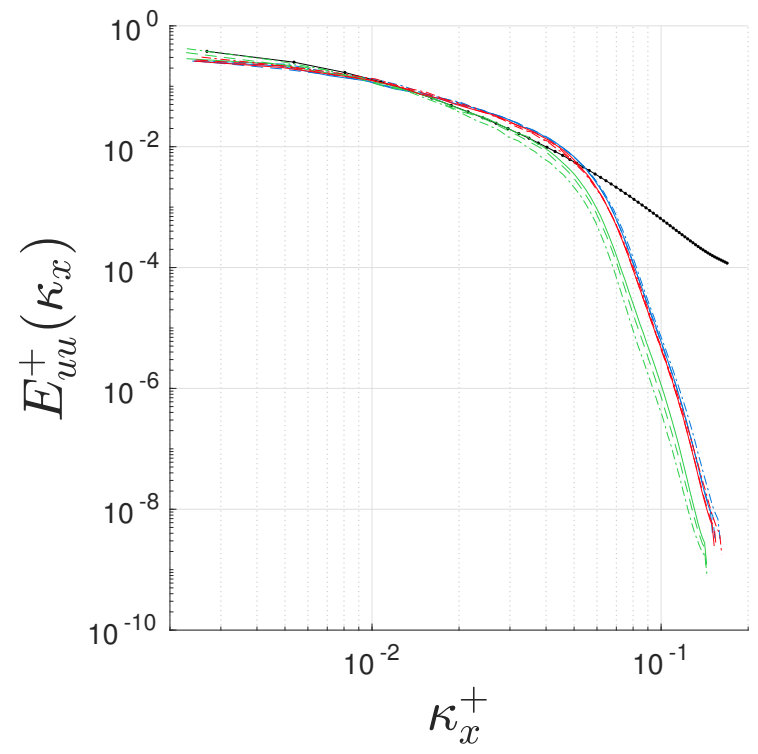

(c)

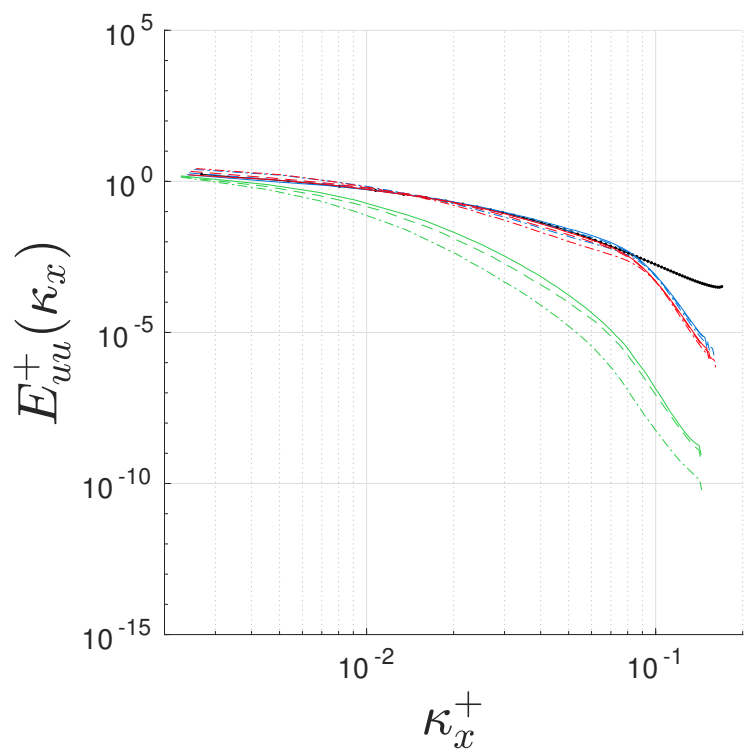

(b)

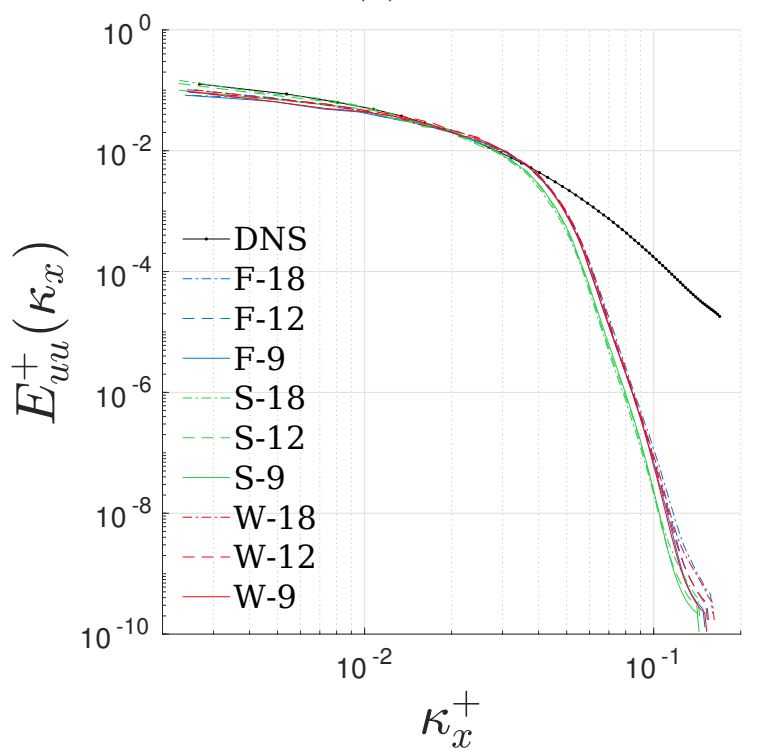

(d)

Figure 5-35: Inner-scaled one-dimensional power spectral density in the streamwise direction for the $u$-velocity component computed with $N_{x}=128$ cells (i.e. $\Delta x^{+} \approx 21$ ) and at wall-normal planes $y^{+} \approx 4.5$ or $y / \delta \approx 0.015(\mathrm{a}), y^{+} \approx 14.7$ or $y / \delta \approx 0.05(\mathrm{~b})$, $y^{+} \approx 150$ or $y / \delta \approx 0.5$ (c) and $y^{+} \approx 294$ or $y / \delta \approx 0.98$ (d) for a target $\operatorname{Re}_{\tau}=300$. 


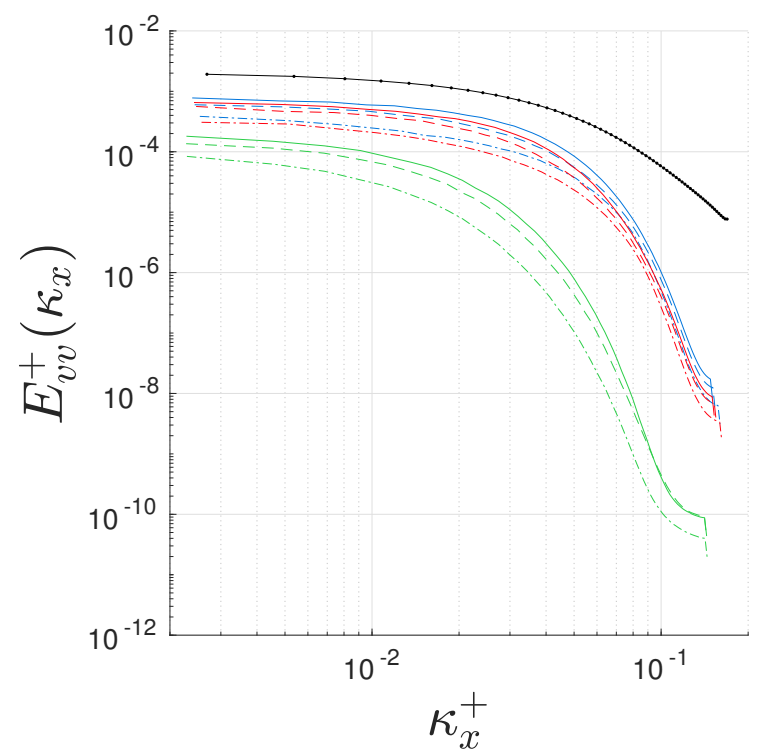

(a)

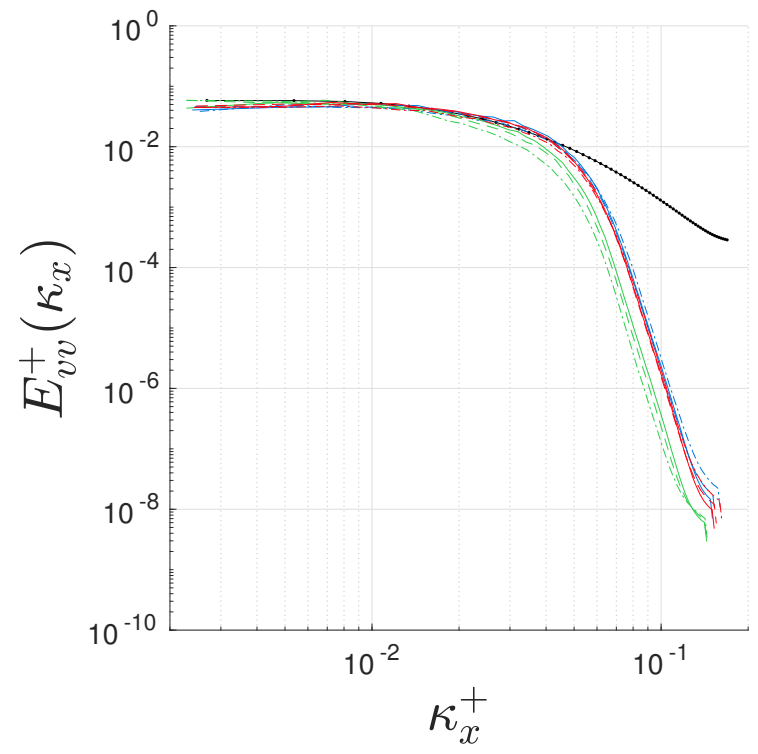

(c)

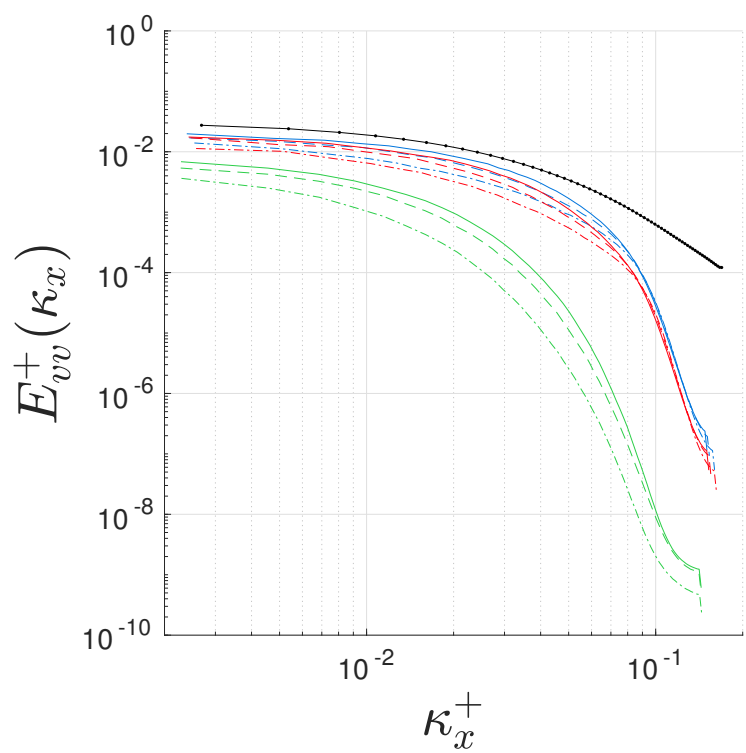

(b)

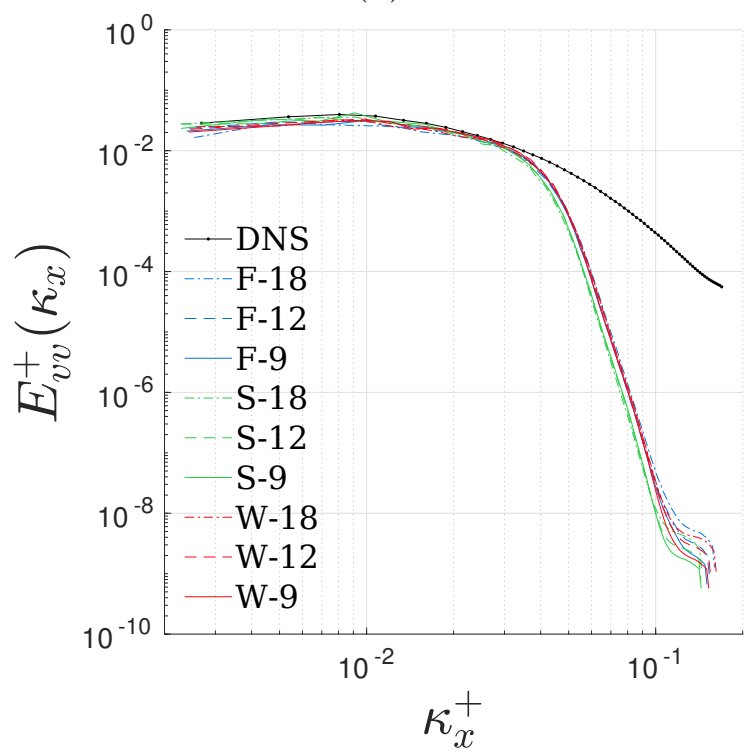

(d)

Figure 5-36: Inner-scaled one-dimensional power spectral density in the streamwise direction for the $v$-velocity component computed with $N_{x}=128$ cells (i.e. $\Delta x^{+} \approx 21$ ) and at wall-normal planes $y^{+} \approx 4.5$ or $y / \delta \approx 0.015(\mathrm{a}), y^{+} \approx 14.7$ or $y / \delta \approx 0.05(\mathrm{~b})$, $y^{+} \approx 150$ or $y / \delta \approx 0.5$ (c) and $y^{+} \approx 294$ or $y / \delta \approx 0.98$ (d) for a target $\operatorname{Re}_{\tau}=300$. 


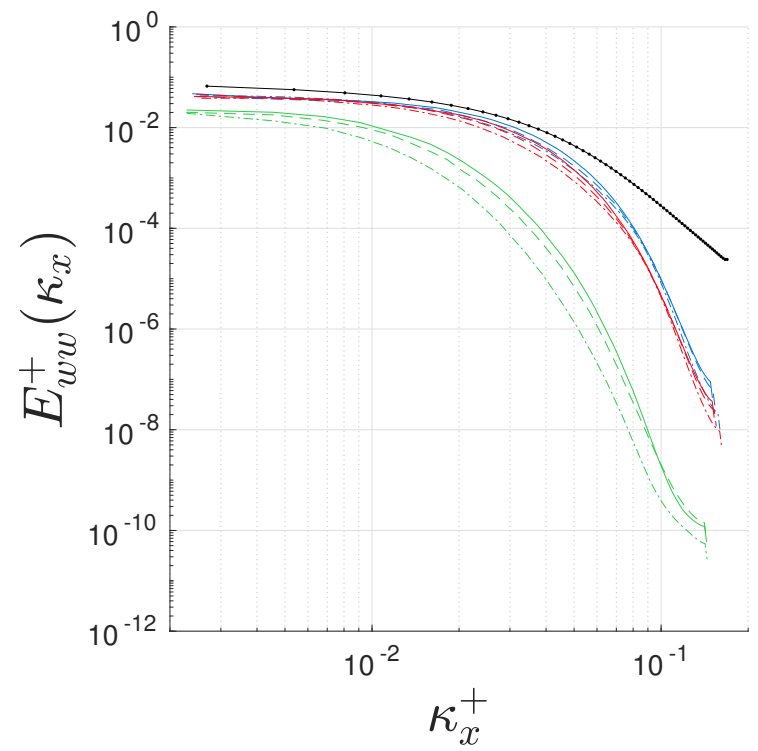

(a)

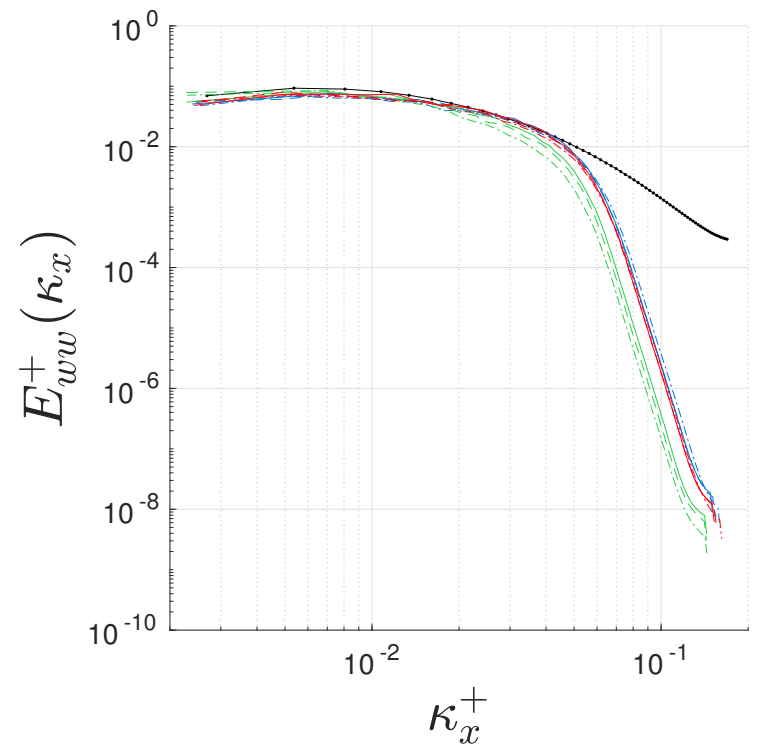

(c)

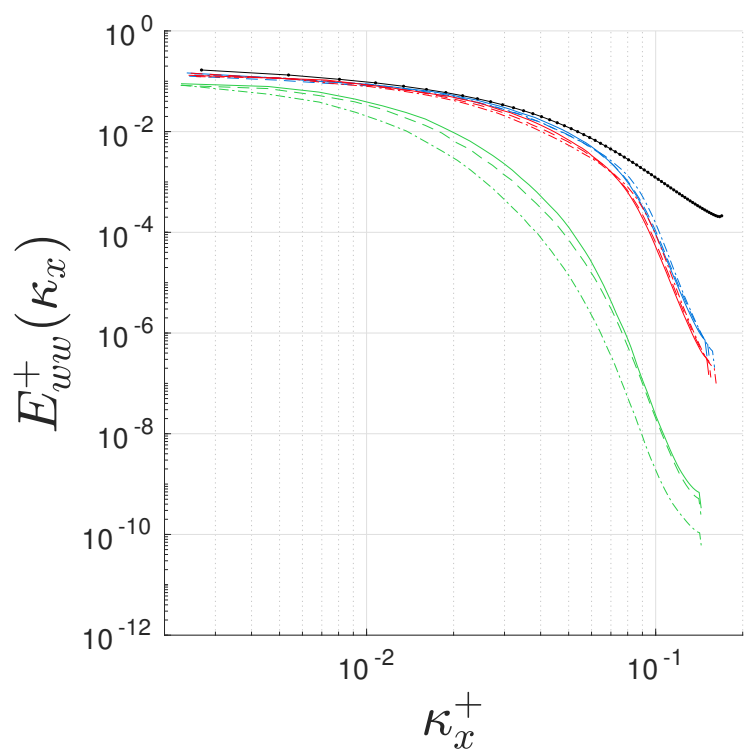

(b)

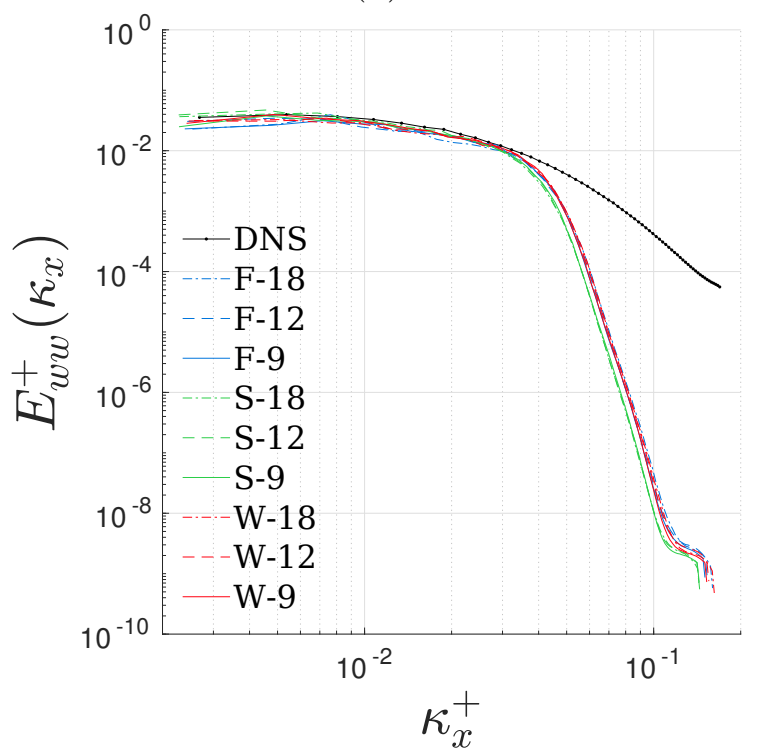

(d)

Figure 5-37: Inner-scaled one-dimensional power spectral density in the streamwise direction for the $w$-velocity component computed with $N_{x}=128$ cells (i.e. $\Delta x^{+} \approx 21$ ) and at wall-normal planes $y^{+} \approx 4.5$ or $y / \delta \approx 0.015(\mathrm{a}), y^{+} \approx 14.7$ or $y / \delta \approx 0.05(\mathrm{~b})$, $y^{+} \approx 150$ or $y / \delta \approx 0.5$ (c) and $y^{+} \approx 294$ or $y / \delta \approx 0.98(\mathrm{~d})$ for a target $\operatorname{Re}_{\tau}=300$. 


\subsubsection{One-dimensional Spanwise Power Spectral Density}

At the coarsest streamwise resolution, few oscillations show up in the $u$-velocity PSD in the viscous sublayer and the buffer zone wall-normal planes. For demonstration, see Figures 5-38, 5-41 and 5-44. The Smagorinsky model maintains the most underprediction of all three SGS models, despite the grid resolution. The WALE and SGS-free models sustain close proximity with each other.

Some poor agreement is still evident, similar to the streamwise PSD, for the $v$-velocity PSD at the first plane sampled from the wall. For instance, check Figures 5-39, 5-42 and 5-45. The remaining three wall-normal planes yield a very good outcome. The $u$-velocity PSD reports almost excellent agreement with the DNS data even at coarse streamwise grid resolutions. The spanwise velocities, on the other hand, although frequently agreeing very well with the DNS profiles, display some unanticipated behavior. At certain sampling plane locations, specifically in between the viscous sublayer and channel center, the $w$-velocity PSD profiles at high wavenumbers display a flat profile instead of the descending shape. Such behavior is mostly evident for $\Delta z^{+} \approx 18$ and, to a lesser extent, 12. An explanation could be related to the convergence of the $w$-velocity statistics. Strikingly, the Smagorinsky model, although it under-predicts the DNS data the most, does not demonstrate such a profile. A possible explanation for such an outcome might be related to how dissipative the Smagorinsky model is; the excessive numerical dissipation introduced by such a model might interact with the statistical convergence of the $w$-velocity - though it won't lead to a correct outcome, it might manage to 'dampen' the profiles' oscillations. 


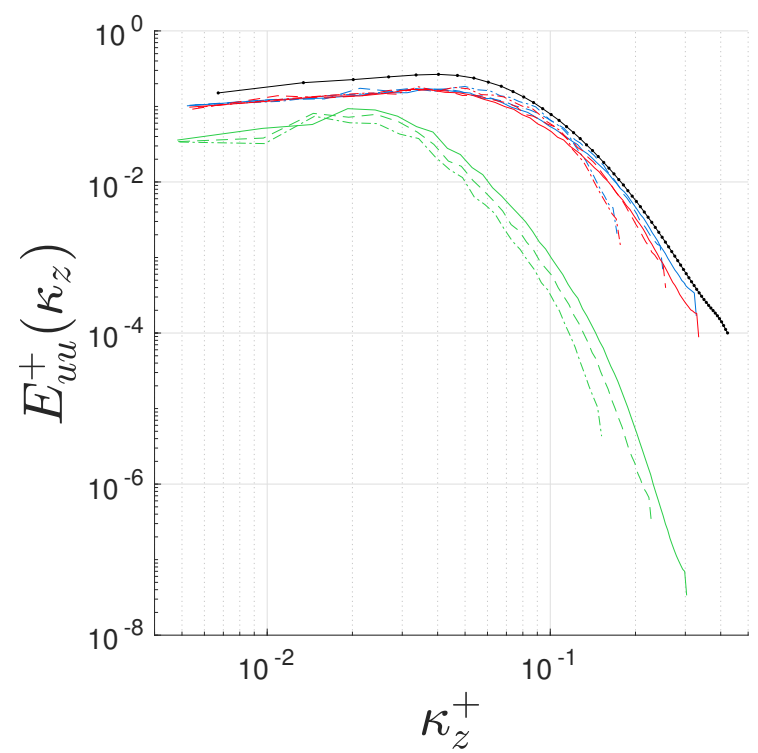

(a)

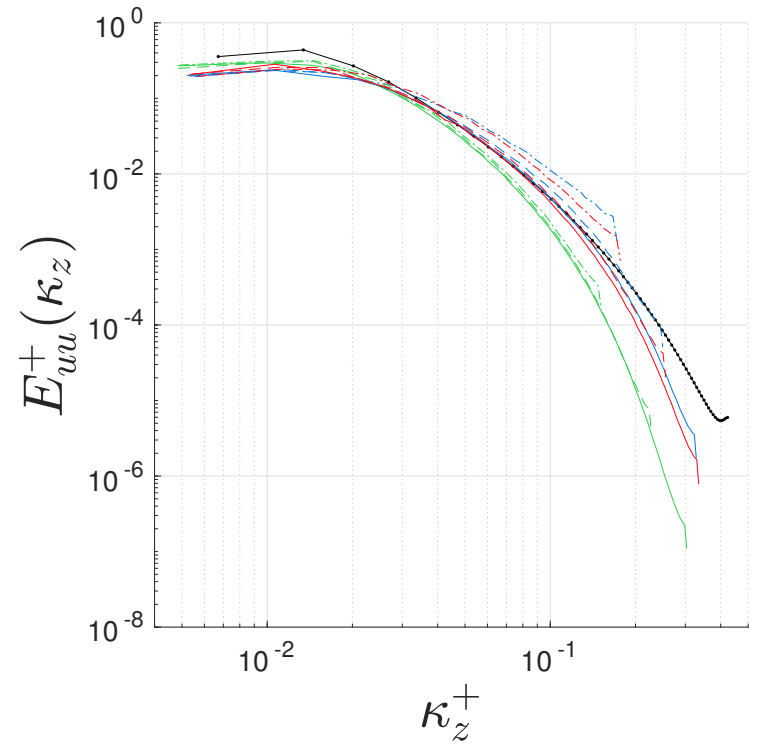

(c)

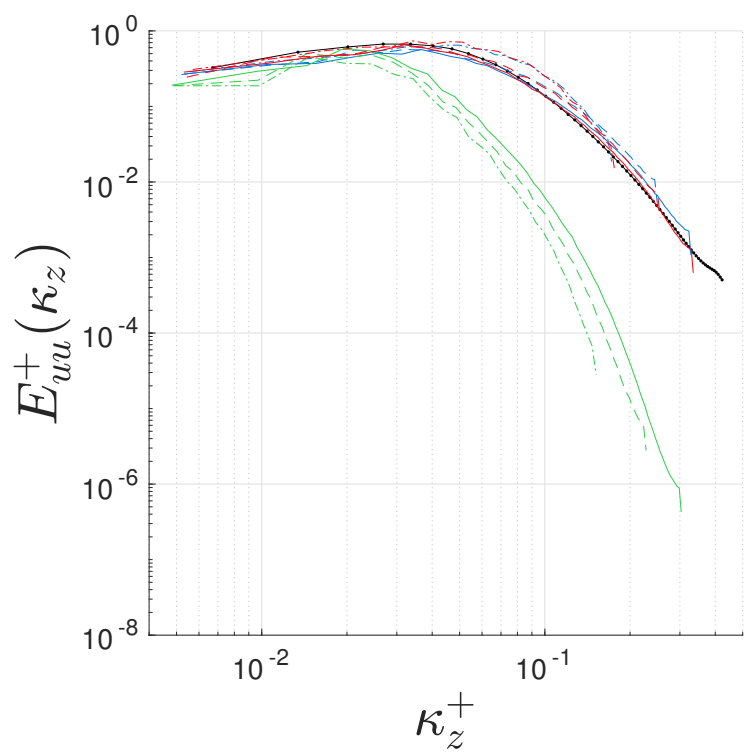

(b)

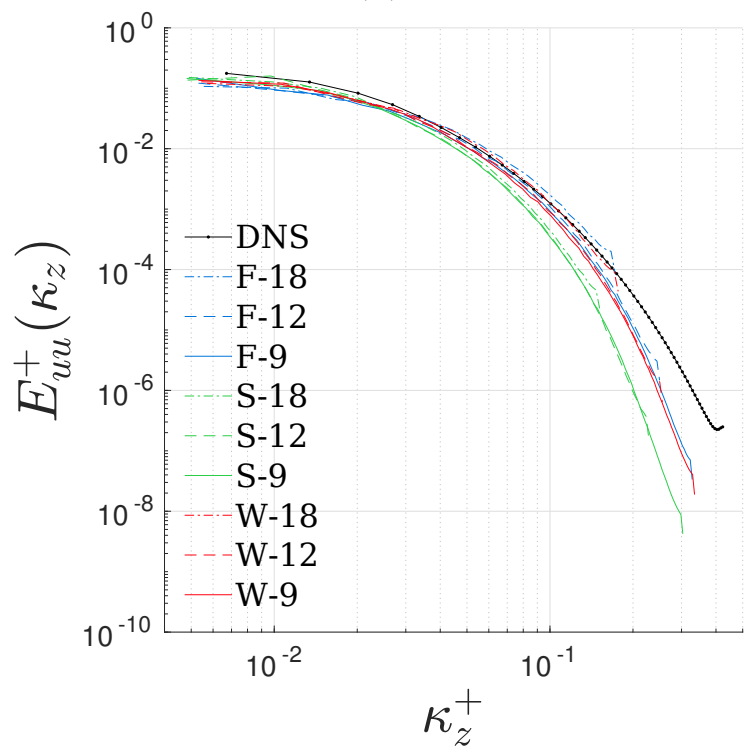

(d)

Figure 5-38: Inner-scaled one-dimensional power spectral density in the spanwise direction for the $u$-velocity component computed with $N_{x}=64$ cells (i.e. $\Delta x^{+} \approx 42$ ) and at wall-normal planes $y^{+} \approx 4.5$ or $y / \delta \approx 0.015(\mathrm{a}), y^{+} \approx 14.7$ or $y / \delta \approx 0.05(\mathrm{~b})$, $y^{+} \approx 150$ or $y / \delta \approx 0.5$ (c) and $y^{+} \approx 294$ or $y / \delta \approx 0.98(\mathrm{~d})$ for a target $\operatorname{Re}_{\tau}=300$. 


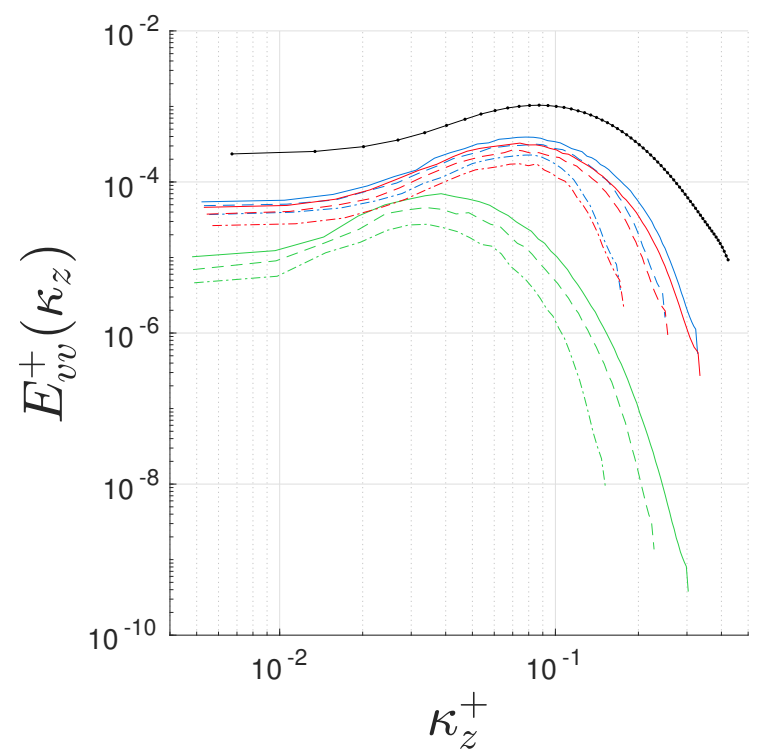

(a)

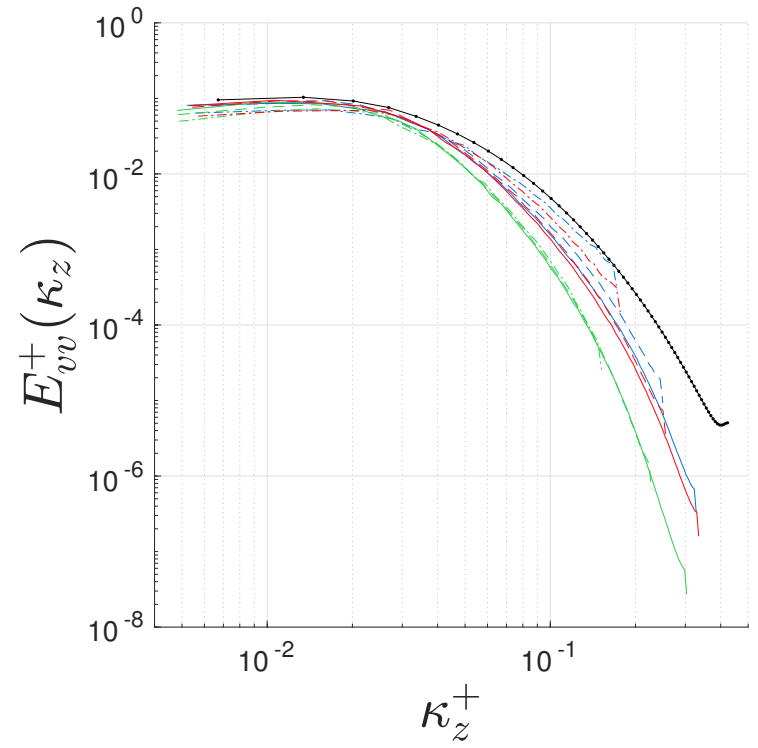

(c)

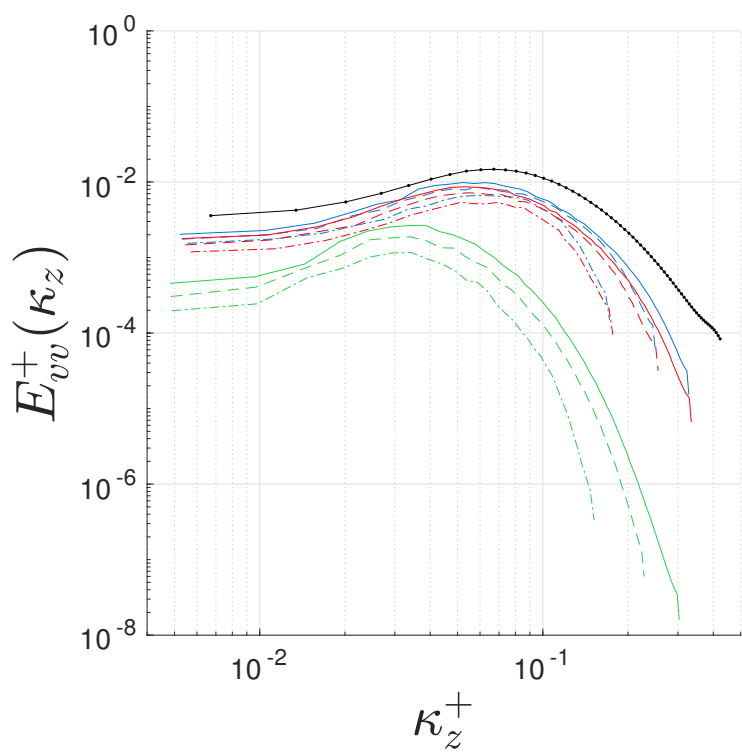

(b)

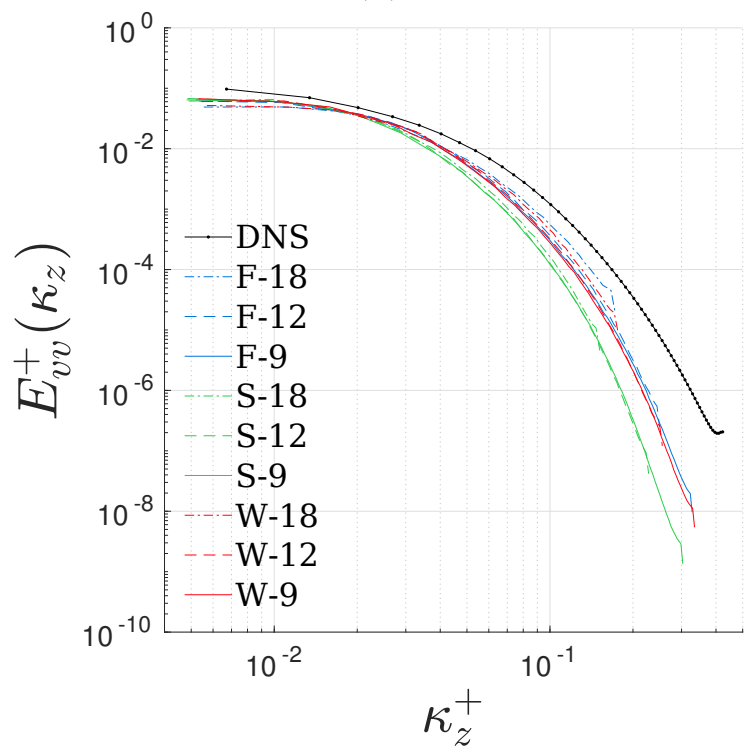

(d)

Figure 5-39: Inner-scaled one-dimensional power spectral density in the spanwise direction for the $v$-velocity component computed with $N_{x}=64$ cells (i.e. $\Delta x^{+} \approx 42$ ) and at wall-normal planes $y^{+} \approx 4.5$ or $y / \delta \approx 0.015(\mathrm{a}), y^{+} \approx 14.7$ or $y / \delta \approx 0.05(\mathrm{~b})$, $y^{+} \approx 150$ or $y / \delta \approx 0.5$ (c) and $y^{+} \approx 294$ or $y / \delta \approx 0.98(\mathrm{~d})$ for a target $\operatorname{Re}_{\tau}=300$. 


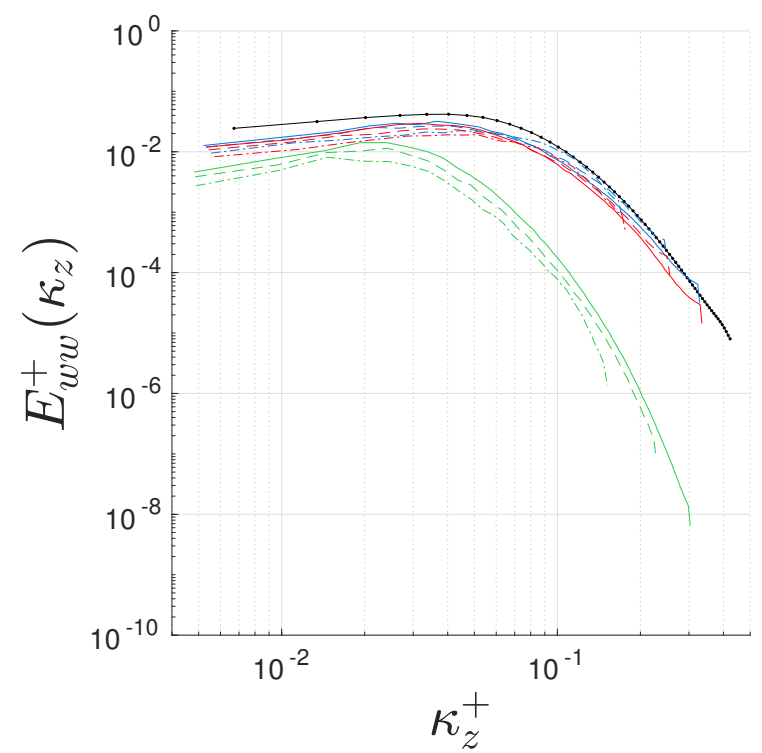

(a)

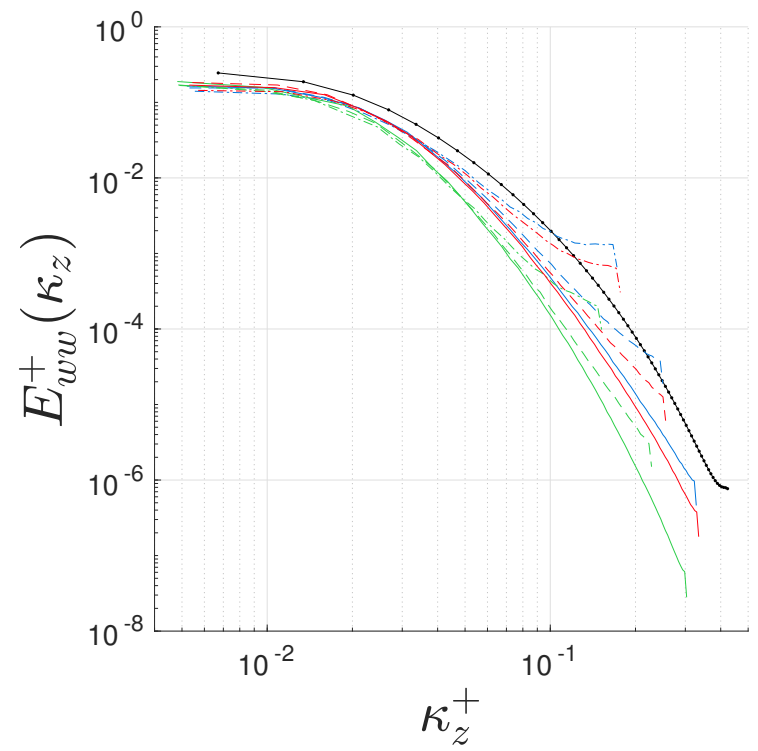

(c)

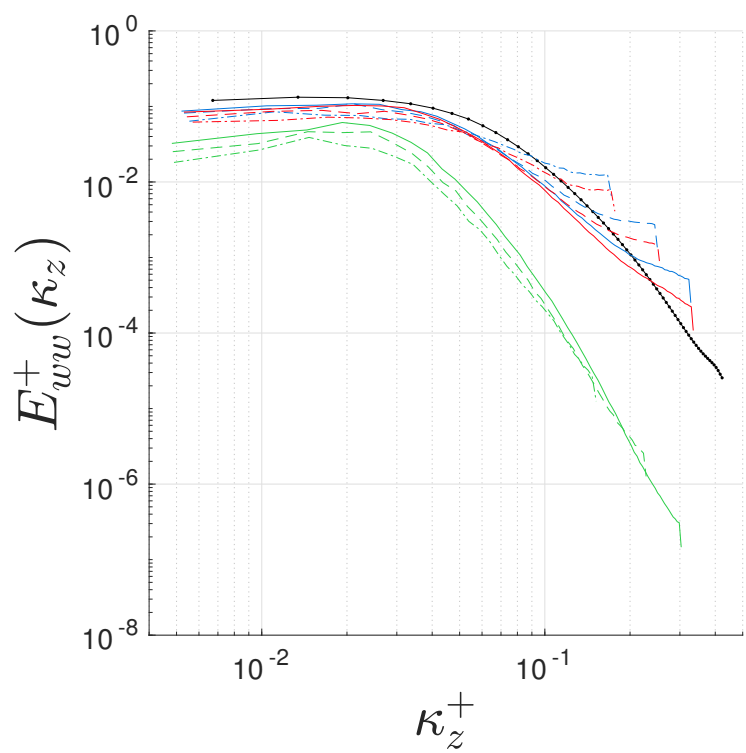

(b)

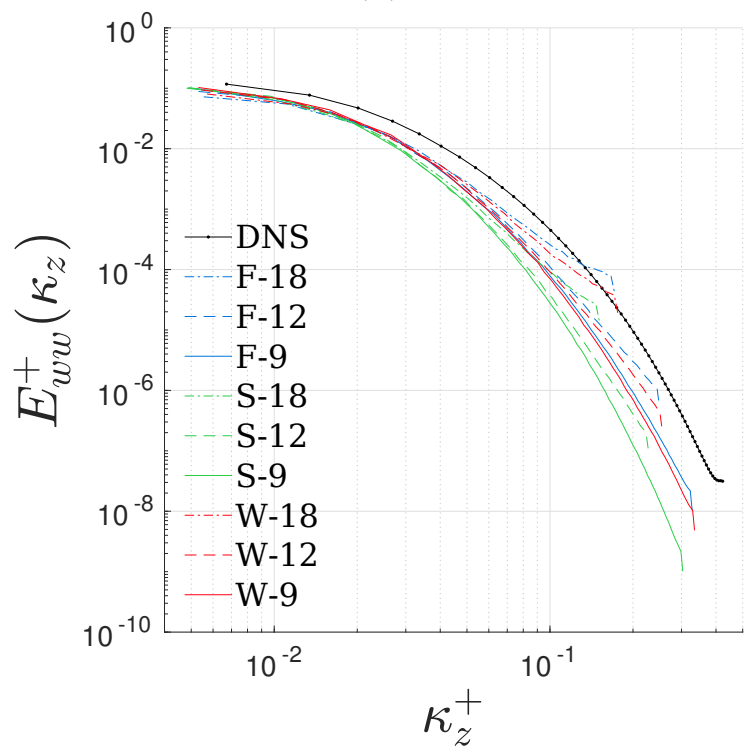

(d)

Figure 5-40: Inner-scaled one-dimensional power spectral density in the spanwise direction for the $w$-velocity component computed with $N_{x}=64$ cells (i.e. $\Delta x^{+} \approx 42$ ) and at wall-normal planes $y^{+} \approx 4.5$ or $y / \delta \approx 0.015(\mathrm{a}), y^{+} \approx 14.7$ or $y / \delta \approx 0.05(\mathrm{~b})$, $y^{+} \approx 150$ or $y / \delta \approx 0.5$ (c) and $y^{+} \approx 294$ or $y / \delta \approx 0.98$ (d) for a target $\operatorname{Re}_{\tau}=300$. 


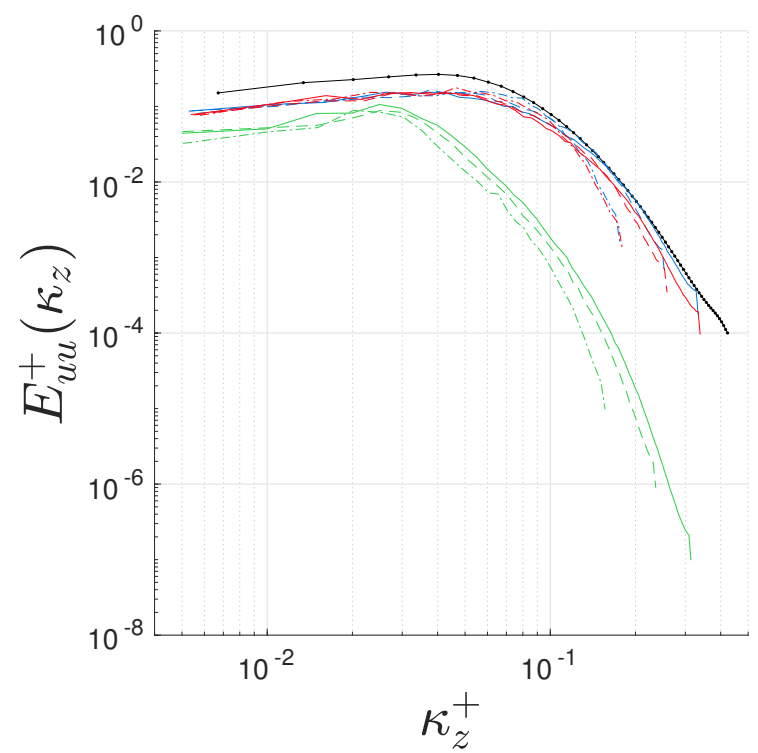

(a)

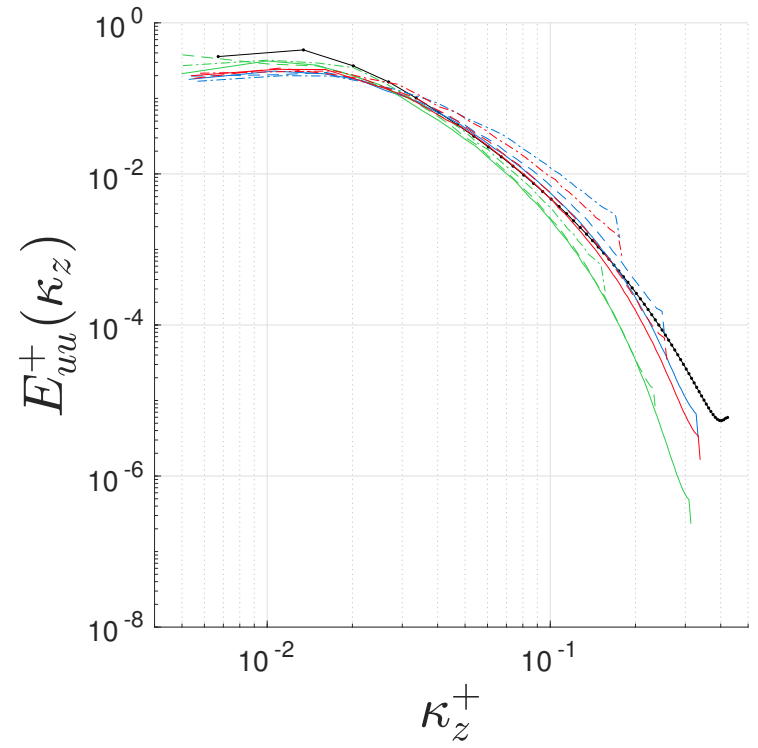

(c)

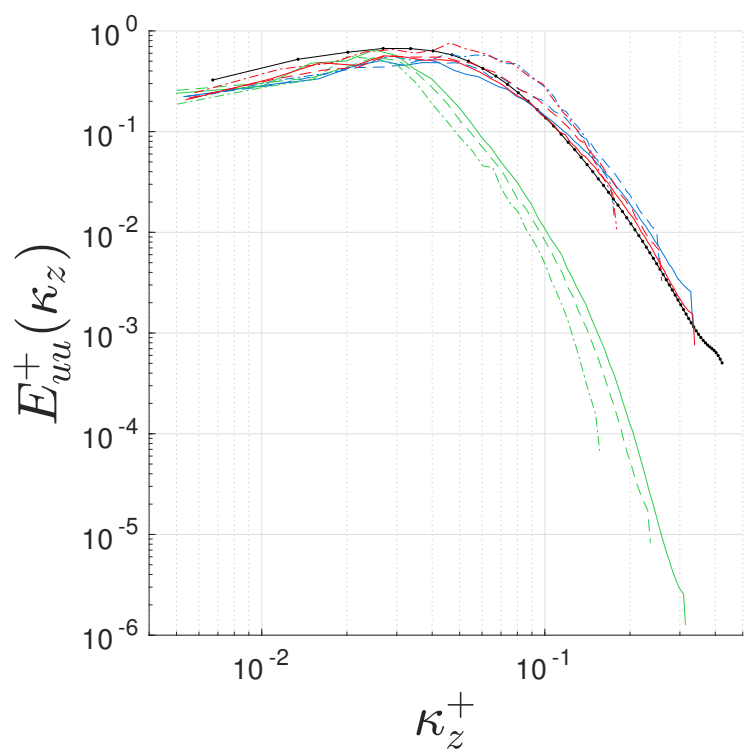

(b)

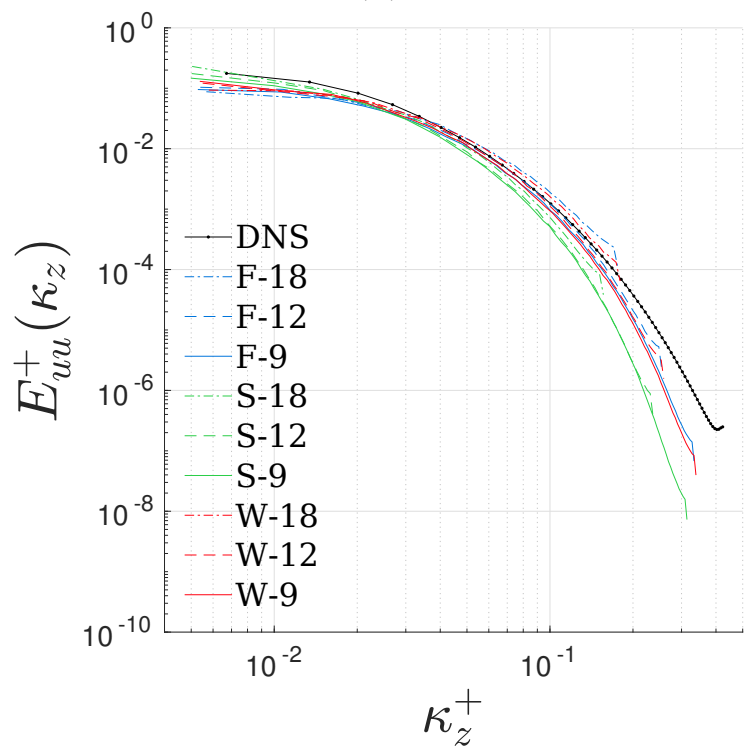

(d)

Figure 5-41: Inner-scaled one-dimensional power spectral density in the spanwise direction for the $u$-velocity component computed with $N_{x}=96$ cells (i.e. $\Delta x^{+} \approx 28$ ) and at wall-normal planes $y^{+} \approx 4.5$ or $y / \delta \approx 0.015(\mathrm{a}), y^{+} \approx 14.7$ or $y / \delta \approx 0.05(\mathrm{~b})$, $y^{+} \approx 150$ or $y / \delta \approx 0.5$ (c) and $y^{+} \approx 294$ or $y / \delta \approx 0.98(\mathrm{~d})$ for a target $\operatorname{Re}_{\tau}=300$. 


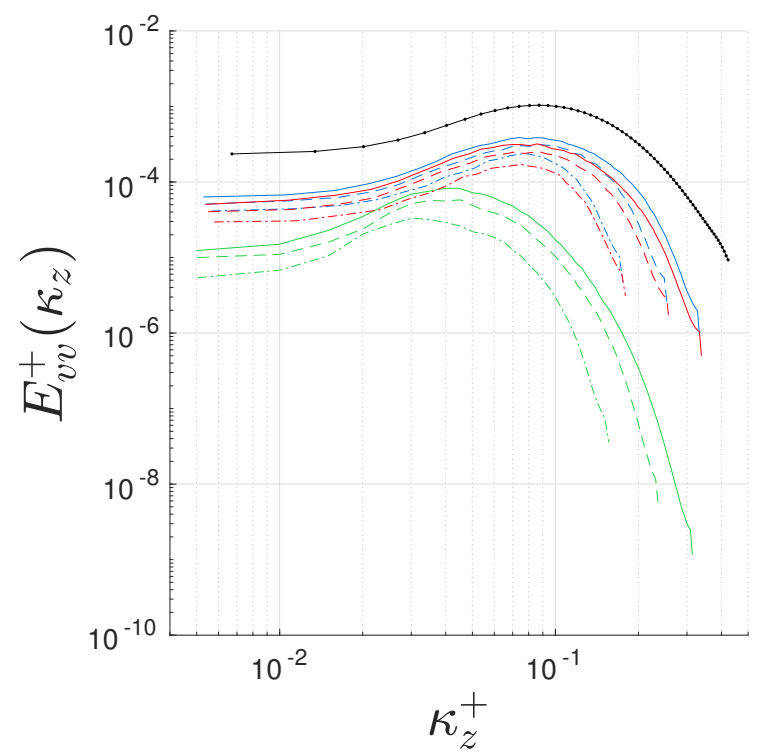

(a)

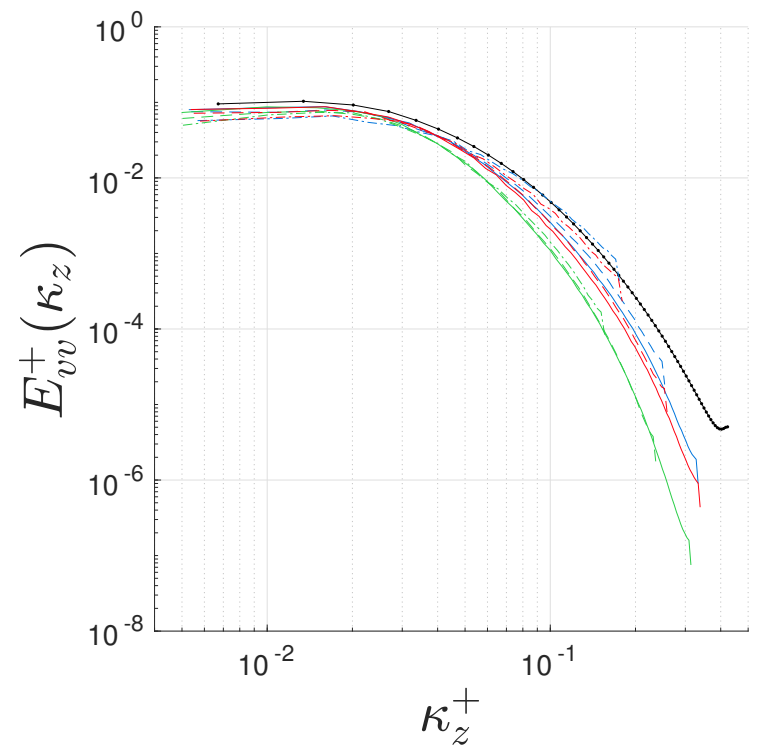

(c)

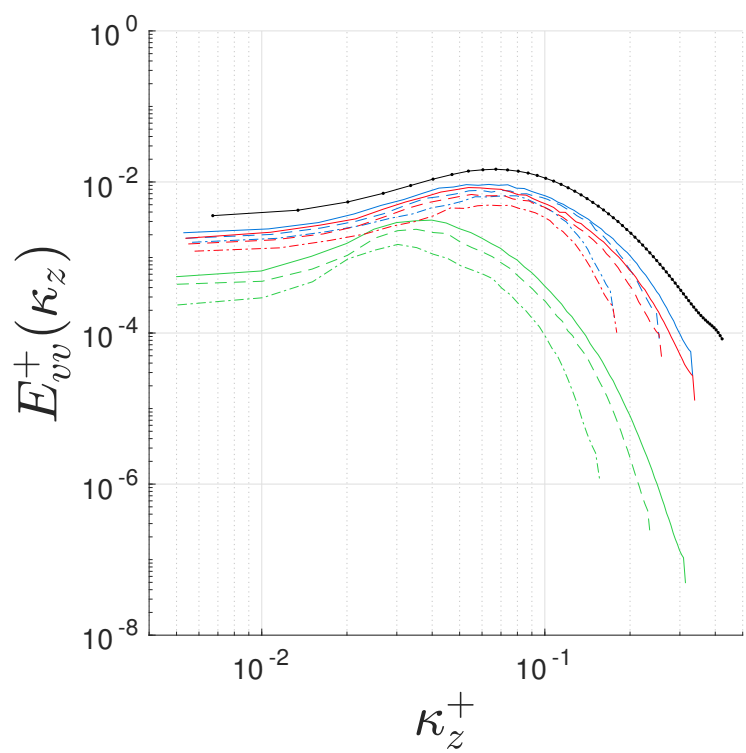

(b)

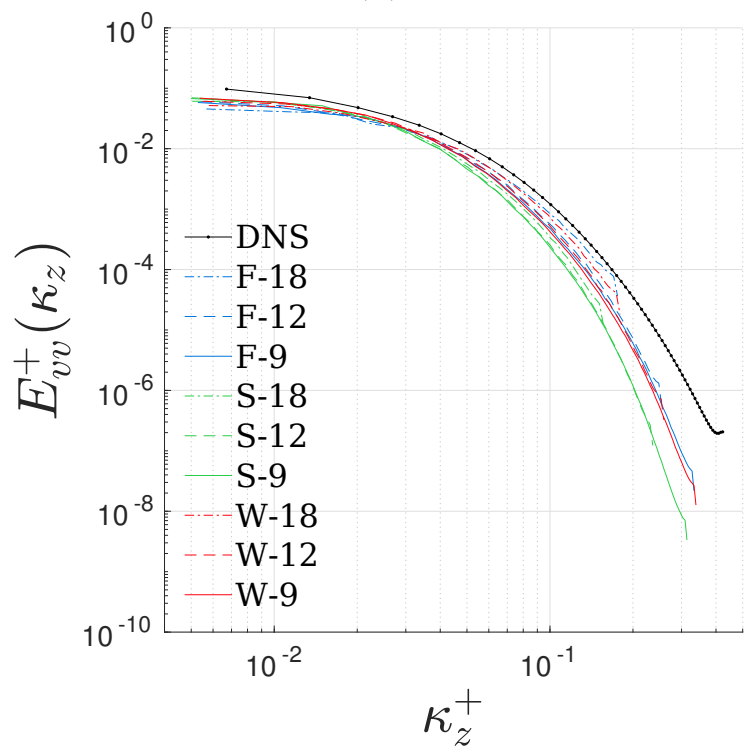

(d)

Figure 5-42: Inner-scaled one-dimensional power spectral density in the spanwise direction for the $v$-velocity component computed with $N_{x}=96$ cells (i.e. $\Delta x^{+} \approx 28$ ) and at wall-normal planes $y^{+} \approx 4.5$ or $y / \delta \approx 0.015(\mathrm{a}), y^{+} \approx 14.7$ or $y / \delta \approx 0.05(\mathrm{~b})$, $y^{+} \approx 150$ or $y / \delta \approx 0.5$ (c) and $y^{+} \approx 294$ or $y / \delta \approx 0.98(\mathrm{~d})$ for a target $\operatorname{Re}_{\tau}=300$. 


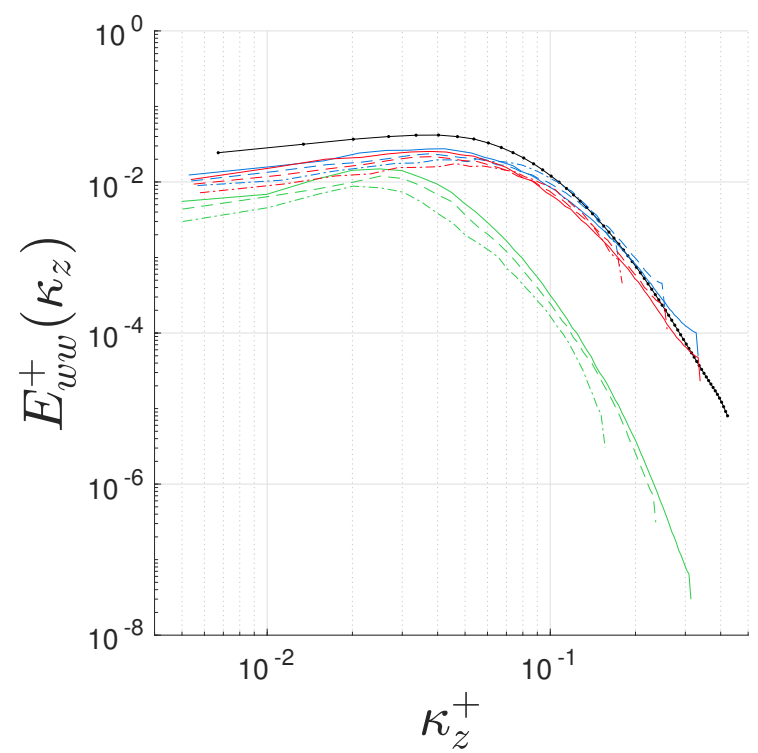

(a)

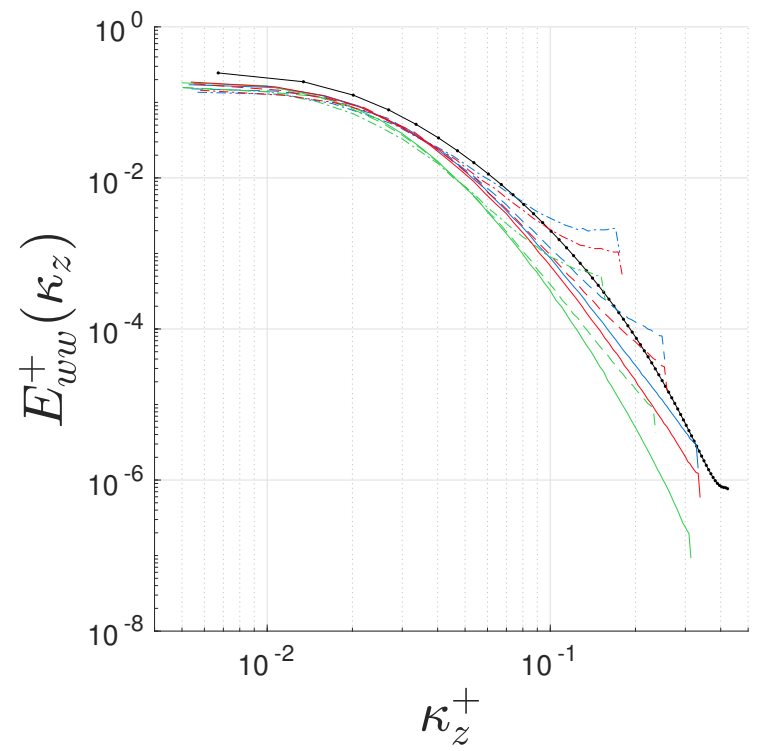

(c)

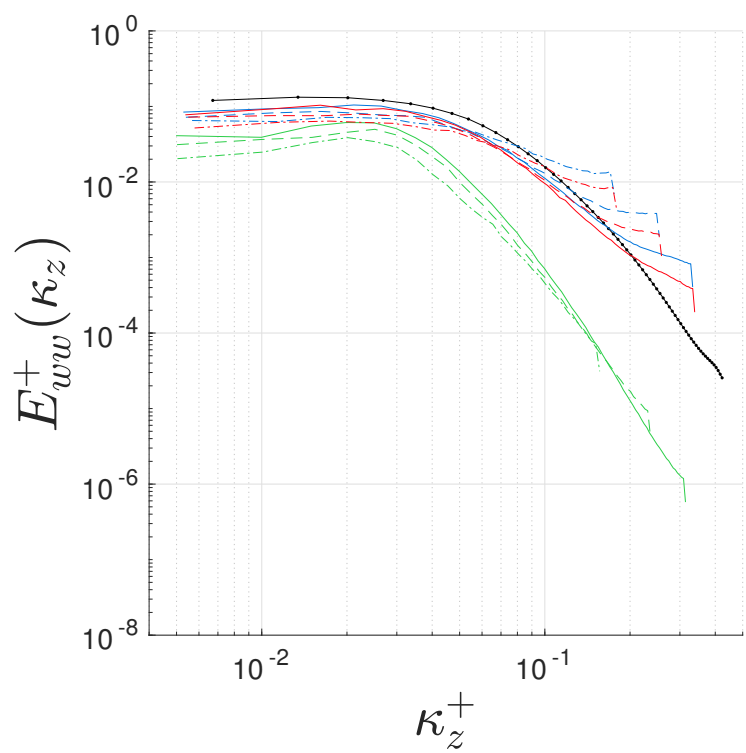

(b)

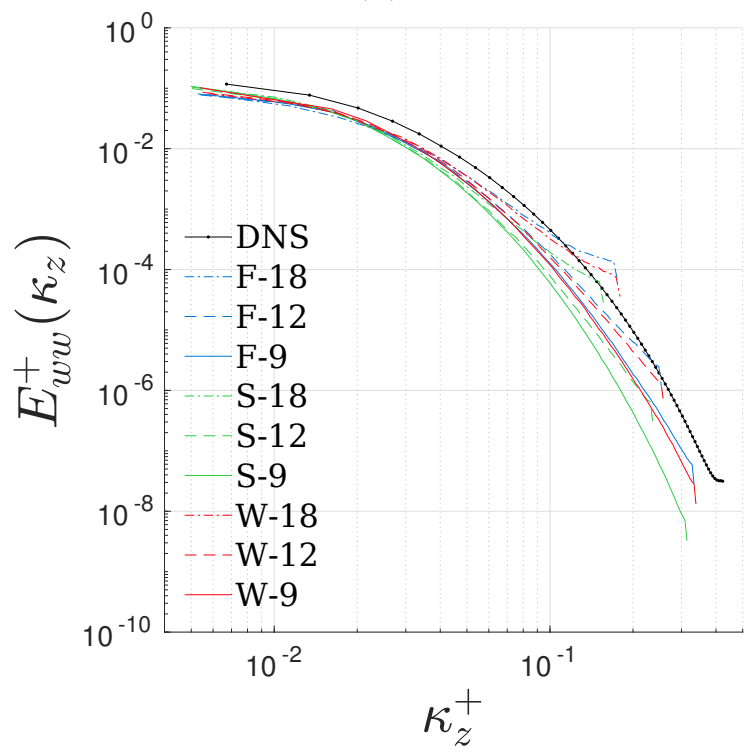

(d)

Figure 5-43: Inner-scaled one-dimensional power spectral density in the spanwise direction for the $w$-velocity component computed with $N_{x}=96$ cells (i.e. $\Delta x^{+} \approx 28$ ) and at wall-normal planes $y^{+} \approx 4.5$ or $y / \delta \approx 0.015$ (a), $y^{+} \approx 14.7$ or $y / \delta \approx 0.05(\mathrm{~b})$, $y^{+} \approx 150$ or $y / \delta \approx 0.5$ (c) and $y^{+} \approx 294$ or $y / \delta \approx 0.98(\mathrm{~d})$ for a target $\operatorname{Re}_{\tau}=300$. 


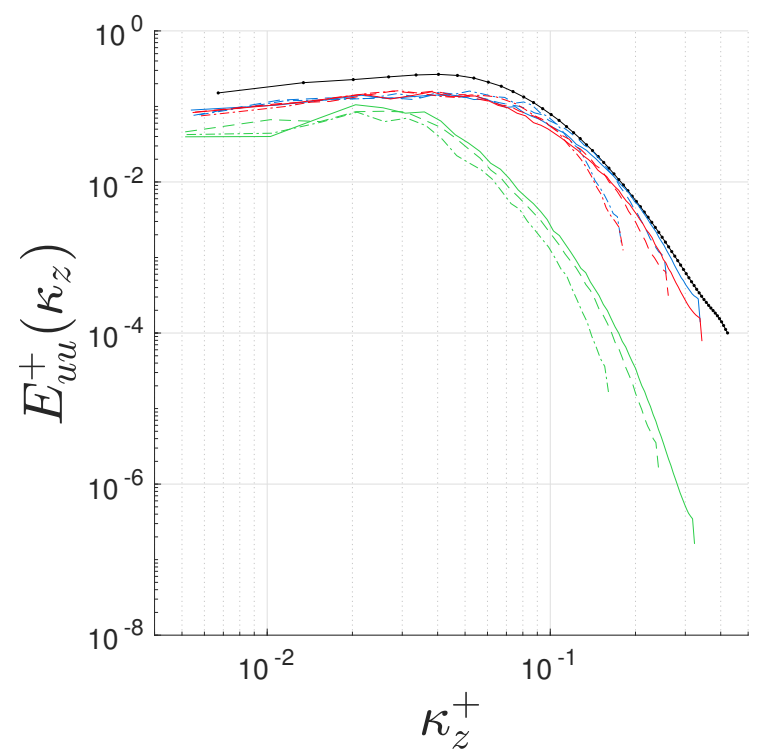

(a)

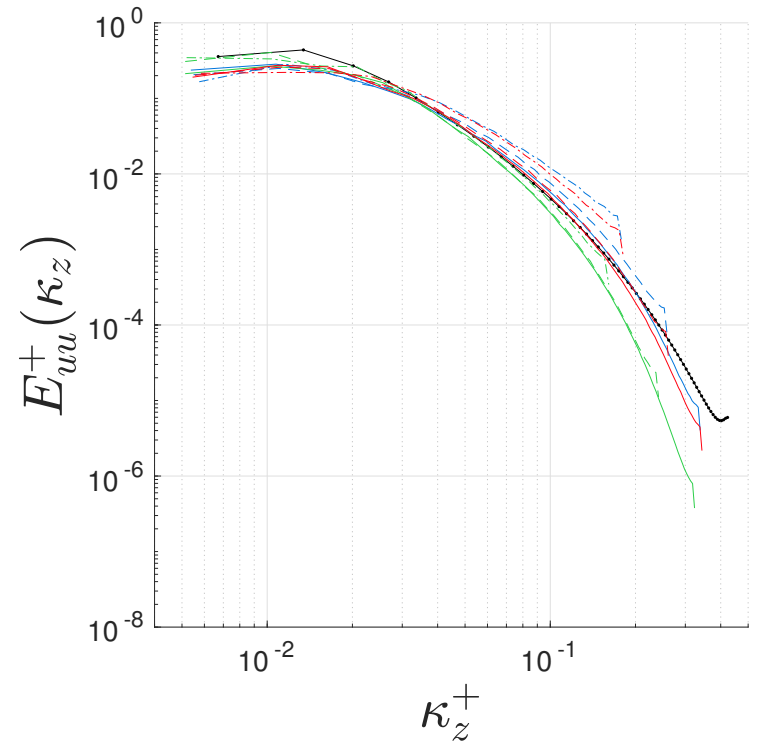

(c)

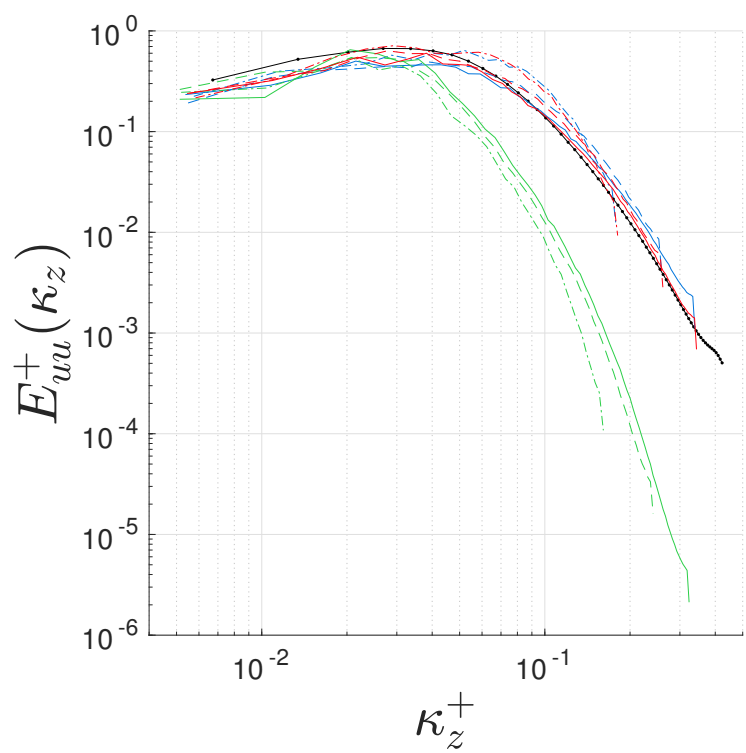

(b)

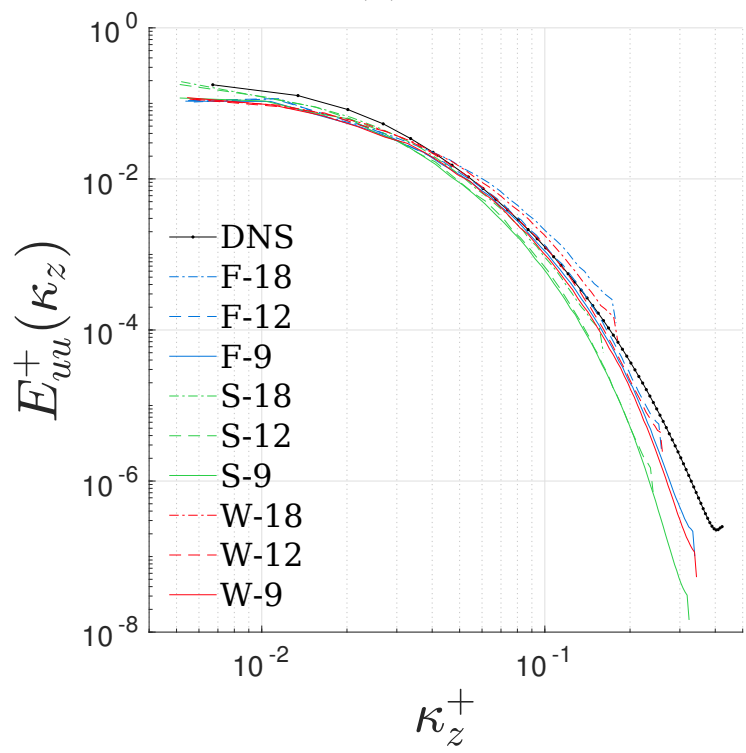

(d)

Figure 5-44: Inner-scaled one-dimensional power spectral density in the spanwise direction for the $u$-velocity component computed with $N_{x}=128$ cells (i.e. $\Delta x^{+} \approx 21$ ) and at wall-normal planes $y^{+} \approx 4.5$ or $y / \delta \approx 0.015(\mathrm{a}), y^{+} \approx 14.7$ or $y / \delta \approx 0.05(\mathrm{~b})$, $y^{+} \approx 150$ or $y / \delta \approx 0.5$ (c) and $y^{+} \approx 294$ or $y / \delta \approx 0.98(\mathrm{~d})$ for a target $\operatorname{Re}_{\tau}=300$. 


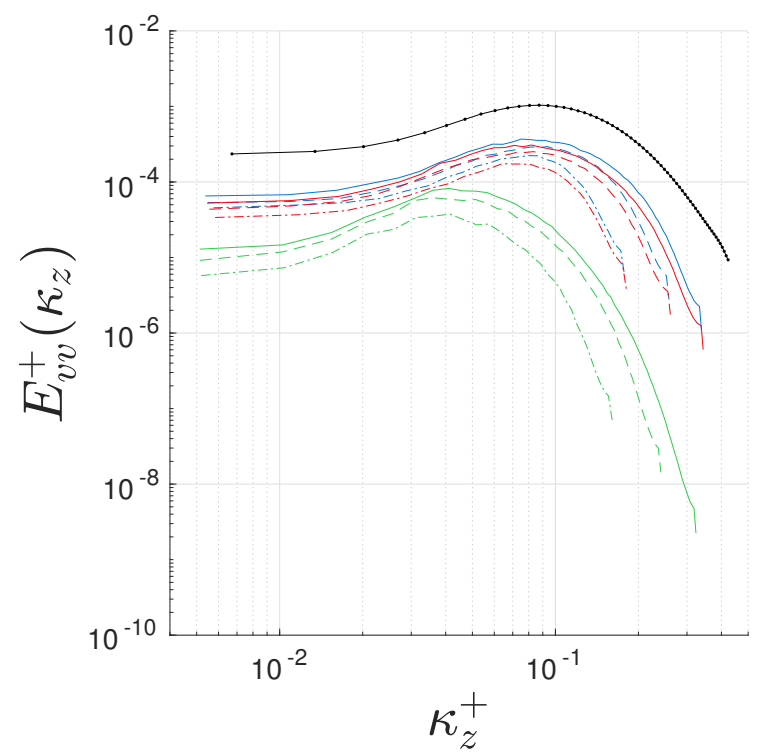

(a)

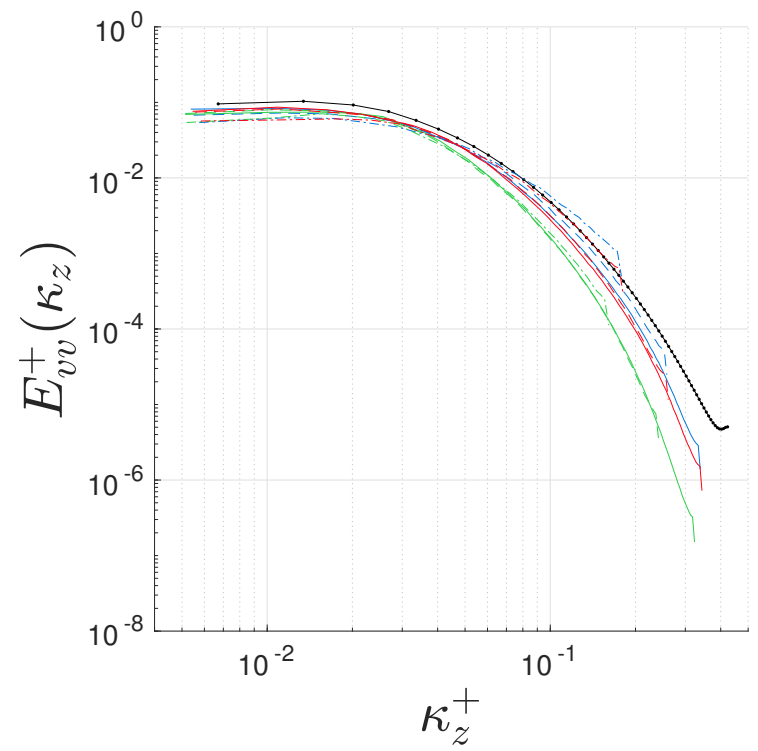

(c)

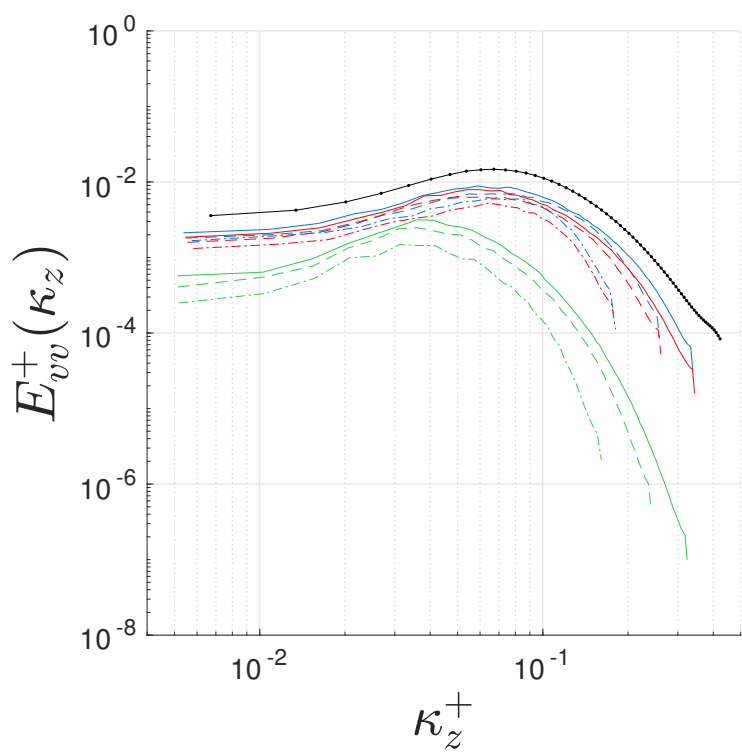

(b)

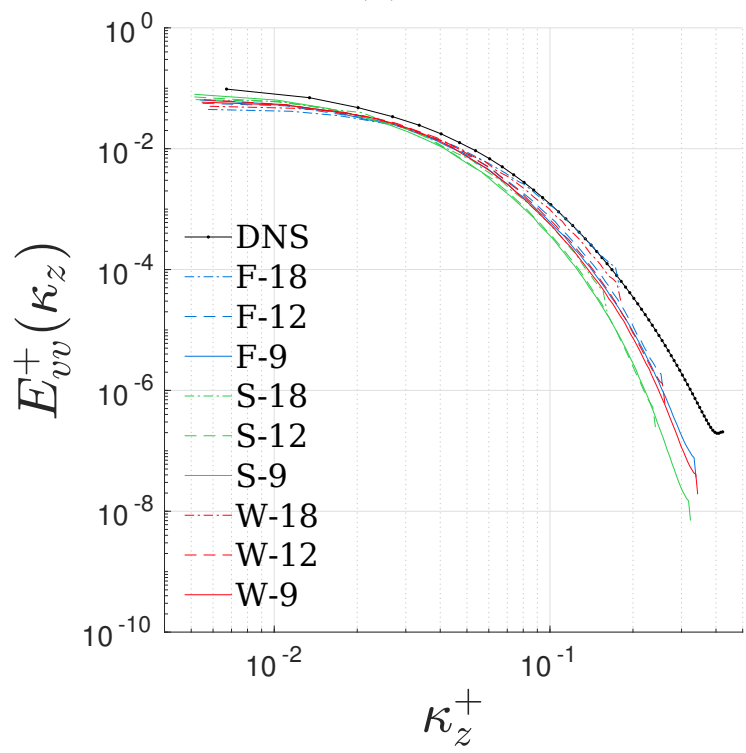

(d)

Figure 5-45: Inner-scaled one-dimensional power spectral density in the spanwise direction for the $v$-velocity component computed with $N_{x}=128$ cells (i.e. $\Delta x^{+} \approx 21$ ) and at wall-normal planes $y^{+} \approx 4.5$ or $y / \delta \approx 0.015(\mathrm{a}), y^{+} \approx 14.7$ or $y / \delta \approx 0.05(\mathrm{~b})$, $y^{+} \approx 150$ or $y / \delta \approx 0.5$ (c) and $y^{+} \approx 294$ or $y / \delta \approx 0.98(\mathrm{~d})$ for a target $\operatorname{Re}_{\tau}=300$. 


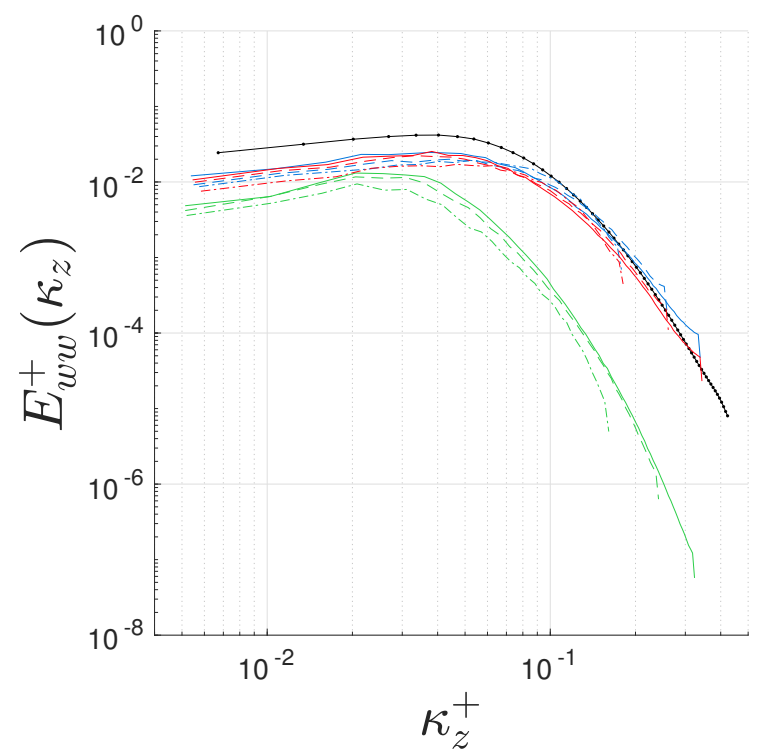

(a)

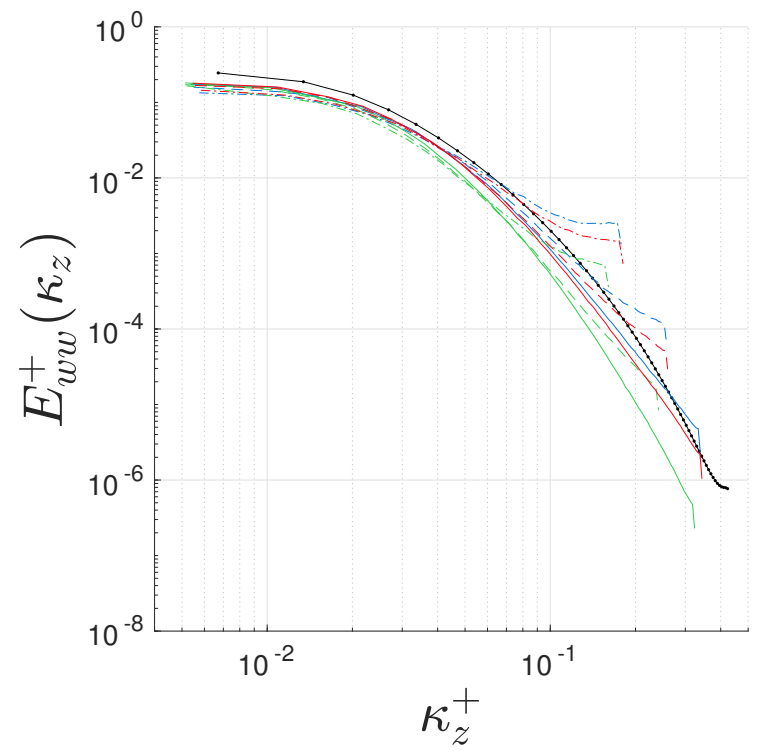

(c)

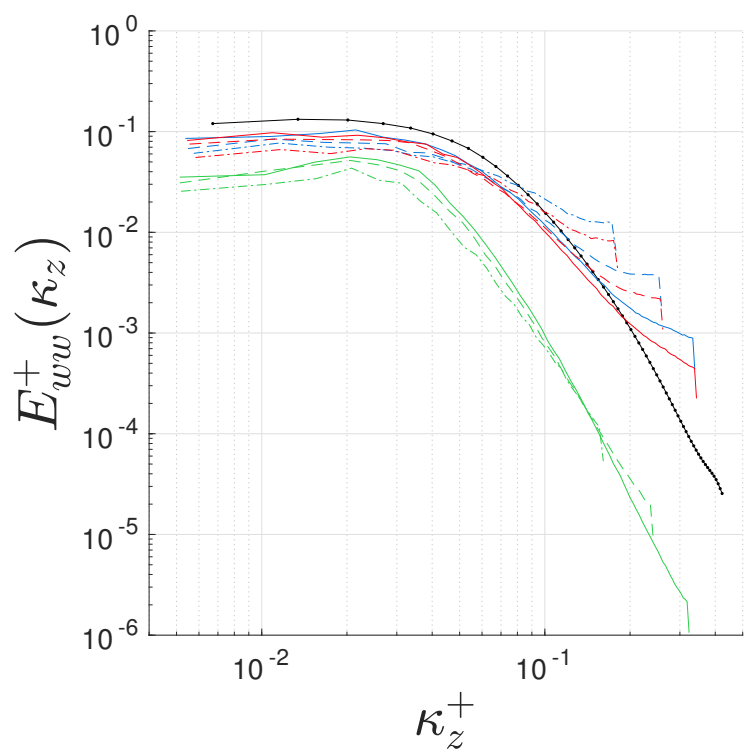

(b)

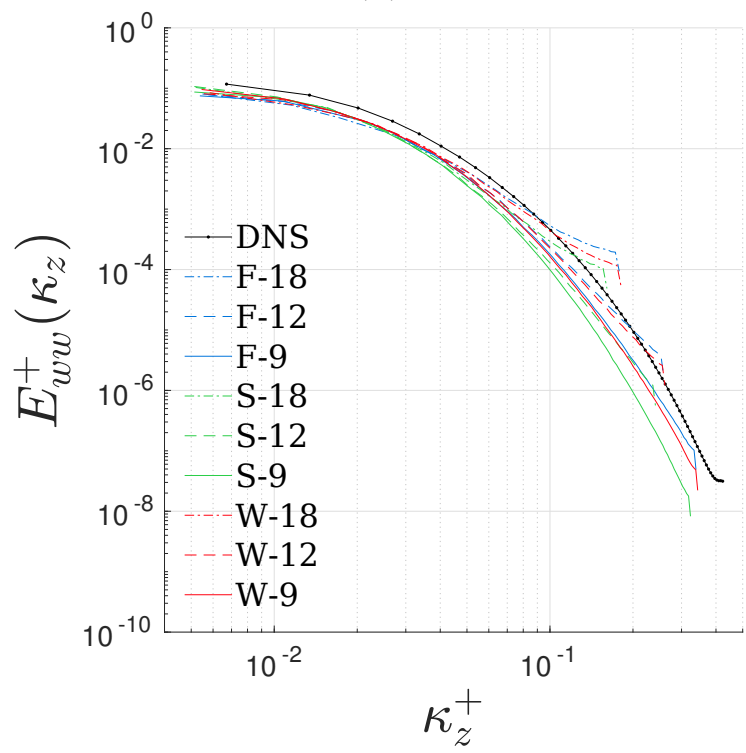

(d)

Figure 5-46: Inner-scaled one-dimensional power spectral density in the spanwise direction for the $w$-velocity component computed with $N_{x}=128$ cells (i.e. $\Delta x^{+} \approx 21$ ) and at wall-normal planes $y^{+} \approx 4.5$ or $y / \delta \approx 0.015(\mathrm{a}), y^{+} \approx 14.7$ or $y / \delta \approx 0.05(\mathrm{~b})$, $y^{+} \approx 150$ or $y / \delta \approx 0.5$ (c) and $y^{+} \approx 294$ or $y / \delta \approx 0.98(\mathrm{~d})$ for a target $\operatorname{Re}_{\tau}=300$. 


\subsubsection{Convergence of the Friction Velocity}

Another compelling quantity to survey is the convergence of $u_{\tau}$ per grid for each of the three SGS models. The results are presented in Figure 5-47. The absolute of the relative error, with respect to the DNS data, in percentage form for the friction velocity is computed using the below expression:

$$
e^{r e l}=100\left|\frac{u_{\tau}-u_{\tau}^{D N S}}{u_{\tau}^{D N S}}\right|,
$$

where $u_{\tau}^{D N S}$ is the converged DNS friction velocity and $u_{\tau}$ is the LES friction velocity.

In Figure 5-47, the relative differences in absolute are reported using the gray-scale color range. White colors correspond to large deviations from the DNS data and vice versa for the darker colors. It is clear that of all three models, the Smagorinsky displays most deviations from the DNS result. This is illustrated in the figure by the amount of white color present in contrast to the other models. The remaining WALE and SGS-free models display somewhat similar and relatively better agreement with the DNS than does the Smagorinsky. Arguably, however, the SGS-free model seems to yield a slightly better overall converged outcome.

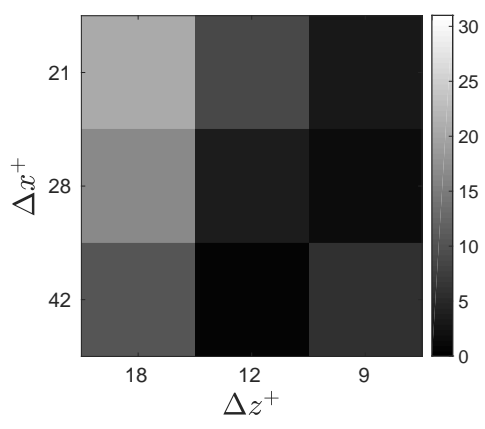

(a)

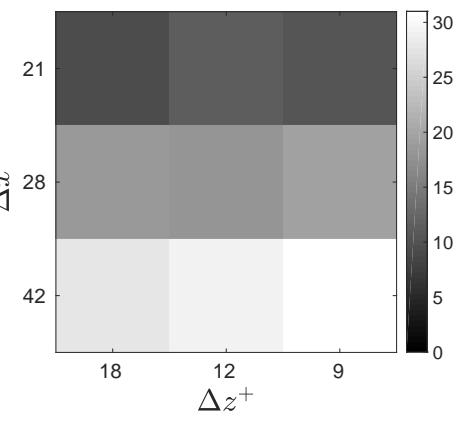

(b)

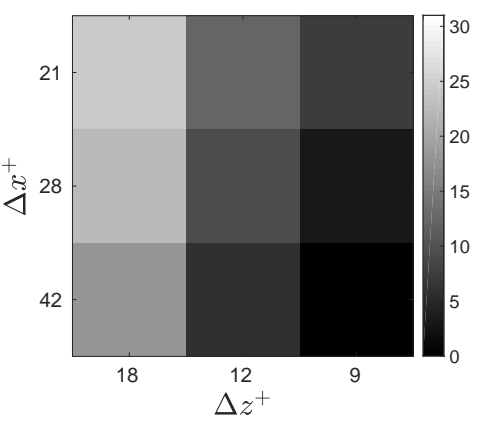

(c)

Figure 5-47: Relative error of the difference between the converged $u_{\tau}$ of the LES and that of the DNS per grid for a SGS-free (a), a Smagorinsky (b) and a WALE (c) SGS model for a target $\mathrm{Re}_{\tau}=300$. 


\subsection{Concluding Remarks}

Four distinct studies are conducted for statistically fully-developed turbulence in a channel flow. Two target $\operatorname{Re}_{\tau}$ are used: 180 and 300. In both cases, the first cell center from the wall is at $y_{1}^{+} \approx 0.5$. The lower $\operatorname{Re}_{\tau}$ is used mainly to validate the TKE budget and high-order statistics along with proper case set-up in OpenFOAM. After all, the mesh requirements for such a relatively low Re-number are less demanding than higher ones. As for the higher $\mathrm{Re}_{\tau}$, it is studied in a more meticulous manner. The motivation behind selecting a $\operatorname{Re}_{\tau}$ of 300 is based on how adjacent it is to next chapter's inlet target Re-number as well as the extensive publicly available DNS database for it.

In subsection 5.4.1, an M1 wall-normal mesh is used with a relatively fine grid for a target $\operatorname{Re}_{\tau}=180$. Refer to Figure 5-3 for illustration of the M1 mesh. The results are in very good agreement with the DNS when it comes to the mean and fluctuating velocities. This also applies to the TKE budget terms in Figure 5-11. According to Figure 5-5, however, in comparison to DNS, the current LES mean spanwise velocity has a noticeably worse quality of statistics. This prompted the need to sample for about six times more (in time) when computing third and fourth central statistical moments. Eventually, the high-order statistics demonstrate good agreement, if not slightly better, than the DNS data (e.g. Figure 5-14).

In subsection 5.4.2, three different grids and SGS models are explored for a target $\operatorname{Re}_{\tau}=300$. The grids have different resolutions in homogeneous directions but maintain the M1 method in the wall-normal direction. Refer to Table 5.3 for clarification about the different grid resolutions. The resulting data suggest the Smagorinsky model having the least agreement in most, if not all, quantities. As for the remaining WALE and SGS-free models, they both depict increasing agreement with the DNS data moving from mesh M1-C to M1-F. Remarkably, for these two models, mesh M1-I proves even less efficient than M1-C as evidenced in some quantities such as in 
Figure 5-21. In between the WALE and SGS-free models, the latter demonstrates slightly better agreement with the DNS data. Another important observation is that the quantities are much more sensitive in the spanwise direction than in the streamwise direction. This is especially evident for M1-I and M1-F grid resolutions; despite M1-I having the finer streamwise resolution, M1-F exhibits the best outcome as compared with the DNS data. This also suggests that refinement until reaching $\Delta x^{+} \approx 25$ yields excellent results.

In subsection 5.4.3, the effectiveness of SGS models near the wall are particularly addressed. Two wall-normal meshing strategies are used: M1 and M3; refer to Figure 5-24. The target $\operatorname{Re}_{\tau}$ selected is 300. As for the homogeneous directions, the intermediate mesh from earlier simulations is adopted (i.e. $\Delta x^{+}=\Delta z^{+}=20$ ). The fine wall-normal resolution of the M3 mesh did display additional accuracy gains in the overall solution as witnessed in Figure 5-26. In retrospect, however, taking into consideration that refinement from M1 to M3 requires almost twice the number of cells, the compromise is not justifiable. The Smagorinsky model maintains its poor behavior in comparison to the DNS data. A variant of this model using a van Driest damping function is also considered along with the WALE model. The Smagorinsky model's SGS viscosity exhibits highly unsatisfactory behavior in the vicinity of the walls. Using a van Driest damping function, however, alleviates such poor performance as evidenced in Figure 5-28. The WALE model presents the best agreement with the DNS data for most quantities. As for the Smagorinsky model, even with a damping function, it still performs worse in comparison to the WALE SGS model.

Finally, in subsection 5.4.4, an exhaustive set of simulations targeting the grid resolution in the homogeneous directions is presented. In this investigation, three SGS models are used: Smagorinsky, WALE and SGS-free. The M1 wall-normal grid resolution is utilized. The considered grid spacings, in wall units based on a target $\operatorname{Re}_{\tau}=300$, are selected to yield 18,12 and 9 in the spanwise and 42, 28 and 21 in the streamwise direction, respectively. For more information, refer to Table 5.5. 
The main focus of this study is to monitor the inner-scaled PSD analysis of all three velocity components in all simulations and compare accordingly to the DNS database.

The results show that the inertial subrange, which is situated at the middle of each curve, is mostly resolved for a grid spacing of at least $\Delta x^{+} \approx 28$ and $\Delta z^{+} \approx 12$ for the streamwise and spanwise PSD, respectively. The spanwise PSD for the $w$-velocity component displays some noticeable deviations from the DNS results at the highest wavenumbers. These are particularly observed at sampled planes in the buffer zone and channel quarter-height, see Figures 5-40, 5-43 and 5-46. One speculation suggests that the spanwise PSD for the $w$-velocity requires more than 22 flow-through times for the grid resolutions used. This may be supported by the outcome of Figure 5-5; despite the fine grid at an even lower Re-number, high deviations are acutely apparent.

Under the above circumstances, the WALE and SGS-free models produce similar predictions; for a grid resolution of $\Delta x^{+} \leq 28$ and $\Delta z^{+} \leq 12$, most of the energy dispersed in different sizes of eddies is recovered. The Smagorinsky model considerably under-predicts the expected PSD curve for the first two sampled planes from the wall with respect to the DNS data, even at low wavenumbers. Concerning the grid resolution, two important observations are made. The first is that the LES streamwise PSD curve recovers less energy at the highest wavenumbers whenever the sampled wall-normal plane is farther from the wall. For instance, see Figure 5-35. A possible explanation is the way the M1 mesh is constructed; near the wall, more energy is resolved since finer cells are used while less energy is resolved further away, because the cells become progressively coarser. The second observation is that additional refinement after $\Delta z^{+} \approx 12$ does not produce results that could arguably outweigh the computational complexity involved. As for the statistical convergence of $u_{\tau}$, compared to that of the DNS, the Smagorinsky model generates the most deviations with respect to the WALE and SGS-free models; this is observed in Figure 5-47. The latter two models yield similar error, nonetheless, for increasing grid resolution, the SGS-free model displays slightly better agreement with the $u_{\tau}$ reported by the DNS. 
THIS PAGE IS INTENTIONALLY LEFT BLANK 


\section{Chapter 6}

\section{Turbulent Flow over a BFS}

All theoretical framework and numerical implementation along with prior preliminary channel flow considerations predicated the work's fruition thus far. Turbulent flow over a backward-facing step (BFS) is interesting to study for several reasons. Whether it is the simple geometry, physics behind boundary separation, reattachment location or recovery region, it gives plenty of insight about the flow nature for the curious scientist or pragmatic engineer. Moreover, given its relative simple geometry, this constitutes for an exciting, feasible and fruitful numerical investigation.

In this chapter, the sections herein will first address the main goal of this study. Afterwards, the relevant BFS literature is surveyed. Then, the simulation set-up is discussed. Finally, results and discussions are presented.

\subsection{Objective}

The work in this study starts from five different SGS models and a coarse grid resolution. Then, the grid resolution is progressively refined in both spanwise and streamwise directions while eliminating SGS models with poor agreement. Afterwards, an in-depth study takes place using the best two SGS models on the most refined grid. The step-height Re-number is defined as $\operatorname{Re}_{h} \equiv U_{0} h / \nu$ (based on the free-stream inlet velocity and step-height) and in this study the target is taken to be 5100 . Results are 
compared to two benchmark references: a numerical and an experimental one. The flow development is based on a TBL fed into the BFS's inlet from a fully-developed turbulent channel flow simulation. Eventually, the final results are discussed and proper analysis follows.

\subsection{Literature Review}

One of the earliest attempts to study turbulent flow over a BFS was done experimentally in Abbott and Kline [1]. The results mainly focused on the velocity profiles and the effects of different Re-numbers on certain flow properties. No major changes were found between the Re-number variations to the flow's reattachment zones in fully-developed turbulent flow. In Kim et al. [40], experimental investigation of flow characteristics in both shear and boundary layers were conducted. A normalization modification was suggested to better study data downstream of the flow as well as the implication that the TBL's separation exerts a strong effect on the recovery region. Later, a more detailed study was conducted in Armaly et al. [6]. In their work, both experimental and numerical procedures were carried out for laminar, transient and turbulent incompressible flows. They claim good agreement between experiments and numerical methods were achieved for fully-developed steady laminar flow. In an attempt to understand heat transfer in a BFS flow, Vogel and Eaton [82] conducted an experimental procedure. Their findings correlated the effects of some quantities such as the skin-friction and temperature on heat transfer in different flow regions, especially near the wall.

Later, Jovic and Driver [37] performed experiments using laser doppler velocimetry on turbulent flow over a BFS. Their Re-number was $\mathrm{Re}_{h}=5000$. The simulation was set up such that it might be used to validate data from Le et al. [46]. Another experimental attempt to collect significant statistical data about the flow was done in Kasagi and Matsunaga [38]. Their experimental method was based on $\operatorname{Re}_{h}=5540$ using particle image velocimetry. Some of their interesting findings highlight that 
the normal stress in the spanwise direction played the most dominant role among the remaining normal stresses near the reattachment region. Preliminary optimistic results for LES over a BFS were reported in [12,3]. Their simulations were based on $\operatorname{Re}_{h}=5100$ and they used a Smagorinsky SGS model combined with a van Driest damping function near the wall. The numerical scheme used was a least-squares spectral element method. Another LES was performed in Cabot [11] for $\operatorname{Re}_{h}=28000$, but using near-wall modeling. The results they obtained, however, were not in good agreement with benchmark data especially near the reattachment region due to the wall model.

At this point, more frequent studies of flow over a BFS were being conducted using numerical methods. This is of no surprise since the computational resources were starting to become more powerful and cost-effective at this time. Some of these numerical simulations addressed laminar up to transition phases while others tackled turbulence using LES. Up to this point, there was no publicly available DNS simulation for statistically fully-developed turbulent flow over a BFS. Le et al. [46] performed the most detailed simulation at this time using DNS in order to study turbulent flow over a BFS for $\mathrm{Re}_{h}=5100$. Their discretization method was done based on a finite difference method. Of particular accomplishment in their results are how the skin-friction coefficient and velocity profiles behave in certain regions at such a relatively low Re-number. Moreover, many turbulent quantities of interest were calculated such as the TKE budget terms at several streamwise locations.

Shortly thereafter, Kostas et al. [44] set up a particle image velocimetry experiment for turbulent flow tackling $\mathrm{Re}_{h}=4660$. They employed the proper orthogonal decomposition technique to attain the needed parameters. They found a correlation between how effective the large and small length scales are with respect to different turbulent quantities such as the Re-stresses and the TKE production and at different flow regions. Afterwards, in an effort to better study how the flow over a BFS changes with respect to the expansion ratio and Re-number, Biswas et al. [8] conducted a se- 
ries of numerical studies. Their simulations were based on finite volume methods and they considered several expansion ratios and Re-numbers in their work. Nevertheless, these investigations were restricted to an utmost relatively moderate Re-number and hence no turbulence was involved. Another detailed DNS was conducted by Barri and Andersson [7] in order to study how rotation affects turbulent flow over a BFS.

Many other recent studies using LES on a flow over a BFS were conducted in an attempt to further study how the fully-developed turbulent flow may be better approximated. Some addressed the different subgrid scale models such as in Panjwani et al. [64], while others also included inlet and wall models too Toms [78]. In the case of the latter two, studies were conducted with $\operatorname{Re}_{h}=5100$.

In the present work, a study about grid resolutions and SGS models is presented using LES. The results give extensive insight into how each of the several SGS models perform and how the grid refinement affects them. Additionally, all results are available publicly as a database.

\subsection{Simulation Set-up}

In order to understand the set-up procedure involved in the flow over a BFS simulation, an overall general explanation is given first. Unlike the previous channel simulations, the BFS is only homogeneous in its spanwise direction. This gives rise to the need to use data from a secondary simulation and, after attaining a fullydeveloped turbulent state, use it as the inlet condition for the primary simulation. An overview depicting the holistic approach involved is presented in Figure 6-1.

Hereon, the primary and secondary simulations are referred to as the main and precursor, respectively. The main simulation is the one pertaining to the backwardfacing step while the precursor is a relatively coarser channel flow simulation. The idea is to conduct a simulation in a channel flow that has similar characteristics as that needed for the main simulation. For instance, the same mesh covering the TBL in both spanwise and wall-normal direction is retained as that of the BFS along with 


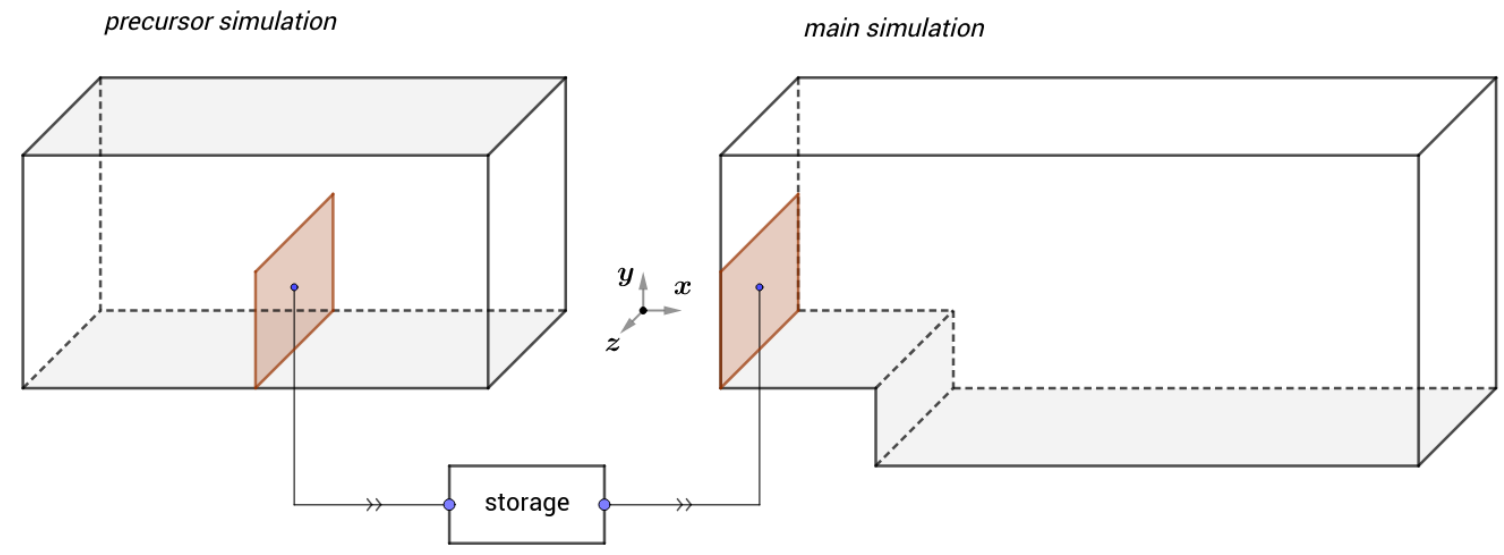

(a)

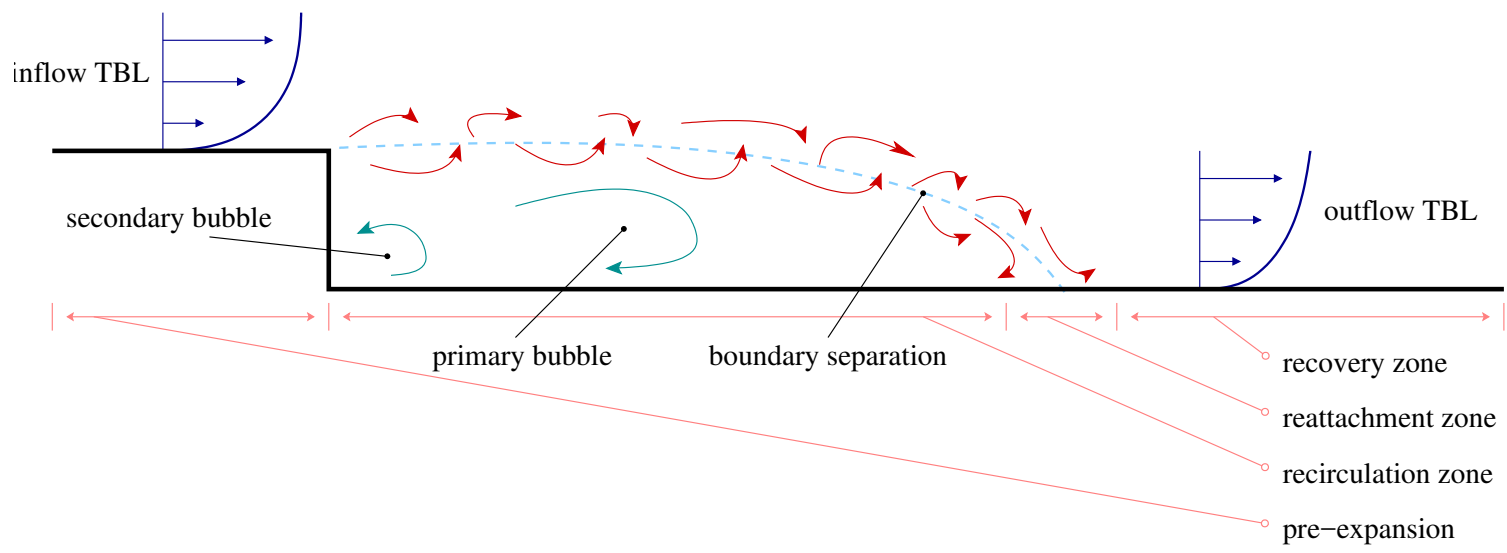

(b)

Figure 6-1: Connection between the precursor channel flow and the main BFS flow simulations (a) and expected TBL development along the streamwise direction in the main simulation (b).

the time increments and other flow characterizations (e.g. viscosity). The channel flow is run until reaching statistically fully-developed turbulent flow.

Afterwards, a wall-normal plane is sampled and stored for later use. Finally, the main simulation is run and the stored velocity profiles act as an inlet condition for the BFS. This is illustrated in Figure 6-1a. The mapping of the wall-normal plane between the two grids is done using eddylicious ${ }^{1}$, a Python library. For a full description about how the mapping is implemented, refer to Mukha [59]. Also, note that for a

\footnotetext{
$1_{\text {https://github.com/timofeymukha/eddylicious }}$
} 
BFS, only the bottom half of the channel's cross-section is required to mimic a TBL as illustrated. As for the remaining portion above the TBL, free-stream velocity is used.

After assigning the inlet condition for the velocity in the main simulation, the flow is then simulated as if it was a TBL. In Figure 6-1b, a depiction of how the inlet TBL develops is sketched. The interpretation of this diagram is that after the flow reaches the edge of the step, it starts to separate until it finally reattaches further down the stream. The separation is due to an adverse pressure gradient (APG) resulting from the sudden expansion. In the recirculation zone, which lies inside the separation boundary, two observable 'bubbles' form. Finally, in the recovery region, after the flow has been reattached, a TBL emerges. These descriptions are based on a statistically fully-developed flow and hence they are described in terms of statistical averaging in the homogeneous direction.

\subsubsection{Computational Domain}

The description of the assigned geometry and meshing procedure is discussed in two categories. The first addresses the main simulation and from it a deduction about the requirements in a precursor simulation will be constructed accordingly. The BFS meshing procedure is performed using a Python script that takes data and maps it onto a block diagram which gets fed into OpenFOAM's native blockMesh utility to generate the desired computational grid. Each of these local blocks are constructed with flexibility in mind. This means that the included cells are subject to stretching, contracting and geometric expansion that depends on the ratios of the first-to-last cell sizes. Also, similar to the channel flow orientation, the BFS uses the streamwise, wall-normal and spanwise notation to denote the $x, y$ and $z$ directions, respectively.

When constructing the BFS grid, certain design parameters are kept in mind. For instance, the wall-normal cells near the wall and close to the step (i.e. $y=0$ ) are placed satisfying an inlet target $\operatorname{Re}_{\tau} \approx 1$. At the top boundary, the wall-normal 


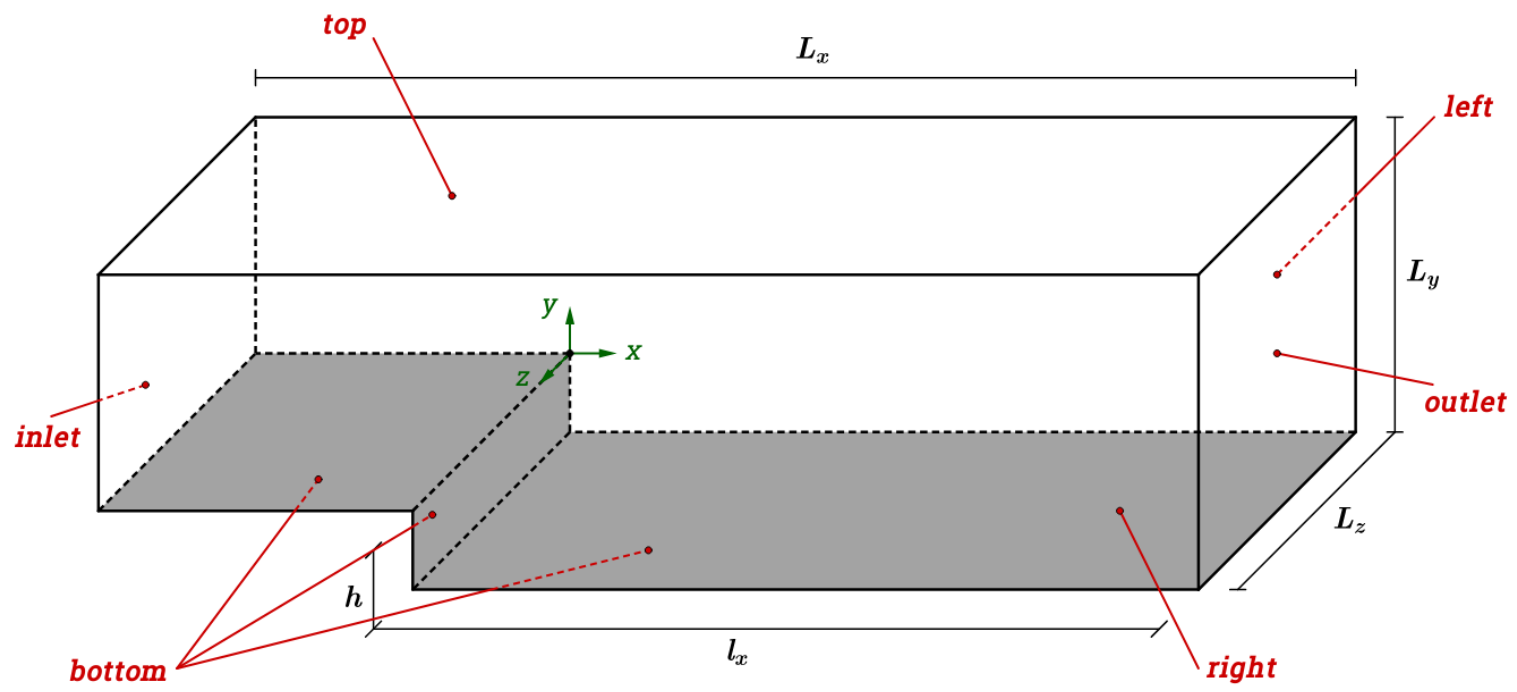

(a)

Figure 6-2: Backward-facing step geometry with boundary faces.

cells are five times as the ones at the inlet's $\delta_{99}$. Between the wall and estimated TBL, the wall-normal grid is the same as that of the lower half of the precursor's (i.e. $0 \leq y \leq \delta$ ). Refer to Figure 6-3. In the streamwise direction, the most notable design arrangement is near the step's edge; streamwise cell sizes become finer near $x=0$. Away from the edge, the streamwise cells expand until reaching a maximum acceptable threshold size taken with previous channel flow simulation results in mind. All dimensionless cell units were based on the converged inlet friction velocity from the precursor simulations.

As for the spanwise direction, due to homogeneity, the cells are kept uniform. The BFS dimensions are selected to match that of Le et al. [46] with values dependent on the step-height $h: L_{x}=30 h, l_{x}=20 h, L_{y}=6 h$ and $L_{z}=4 h$, refer to Figure 6-2.

In the case of the precursor simulation, a target $\mathrm{Re}_{\tau}$ is estimated from a series of correlations and used to construct the underlying channel mesh (see subsection 6.3.3). The cell size variation in the homogeneous directions are kept constant. The wallnormal grid used is that of the M1, taken from Chapter 5. The only difference is instead of five cells, only three are placed in the viscous sublayer. The channel's 

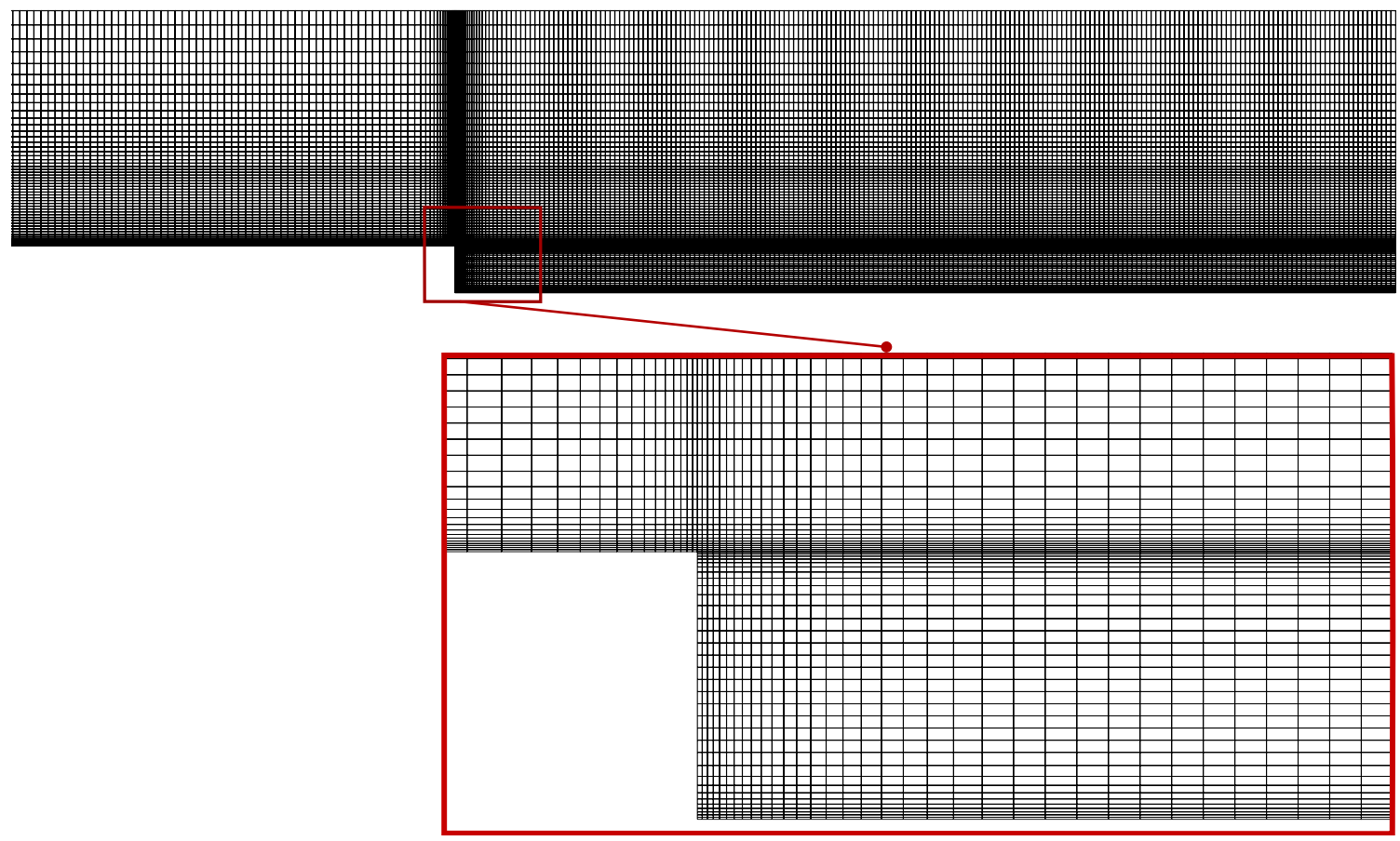

Figure 6-3: Coarse mesh depiction of BFS in $x y$-plane with a zoom-in near the step.

dimension in the spanwise direction is selected to match that of the BFS. As for the wall-normal dimension, the precursor's is at most below that of the BFS's inlet.

The precursor's geometrical dimensions are a bit less straightforward to compute. The spanwise length, as stated earlier, is equivalent to that of the BFS (i.e. $L_{z}=4 h$ ), but the remaining two were dependent on the TBL's approximated thickness. In other words, the channel dimensions are $L_{x}=9 \delta, L_{y}=2 \delta$ and $L_{z}=4 h$ in the streamwise, wall-normal and spanwise directions, respectively.

\subsubsection{Initial and Boundary Conditions}

The ICs and BCs used for the precursor simulation are the same as that used in Chapter 5, refer to Table 5.1. As for the main BFS simulation, a ZPG is prescribed at the inlet. The velocity inlet condition is a time-varying TBL. Refer to Figure 6-1. At the outlet boundary, outletInlet and inletOutet conditions in OpenFOAM are used for the pressure and velocity, respectively. This takes care of any backflow produced 
at the outlet during the simulations which might negatively affect the solution.

The left and right boundaries are coupled via periodic boundary conditions. The top boundary corresponds to a symmetry condition - i.e. zero gradient for all parameters. The bottom shaded region has no-slip velocity and ZPG conditions, respectively.

Table 6.1: Backward-facing step initial and boundary conditions.

\begin{tabular}{l|lc|lc|lc||c} 
& \multicolumn{2}{|c|}{ inlet BC } & \multicolumn{2}{|c|}{ outlet BC } & \multicolumn{2}{c||}{ bottom BC } & IC \\
Field & Type & Value & Type & Value & Type & Value & Value \\
\hline$\overline{\mathbf{u}}$ & Dirichlet & TBL & Dirichlet / Neumann & 0 & Dirichlet & 0 & $(0.5,0,0)$ \\
$\bar{p}$ & Neumann & 0 & Neumann / Dirichlet & 0 & Neumann & 0 & 0 \\
$k_{\text {sgs }}$ & Dirichlet & 0 & Dirichlet & 0 & Dirichlet & 0 & 0.01 \\
$\nu_{\text {sgs }}$ & Dirichlet & 0 & Dirichlet & 0 & Dirichlet & 0 & 0
\end{tabular}

A summary depicting the boundary and initial conditions for the main simulation is showcased in Table 6.1. Note, ICs are prescribed for the non-boundary quantities.

\subsubsection{Physical and Flow Parameters}

Concerning the inlet for the main simulation, the precursor is needed as mentioned previously. However, to proceed, an estimation for the TBL's thickness is required $a$ priori. Thus, the following relation is used:

$$
\delta=\theta f_{2}\left(\operatorname{Re}_{\theta}\right)
$$

where $f_{2}$ is defined in Equation $6.2, \operatorname{Re}_{\theta}$ and $\theta$ are presented in subsection 4.1.1. The latter is calculated as: $\theta=\nu \operatorname{Re}_{\theta} / U_{0}$, with $U_{0}=\nu \operatorname{Re}_{h} / h$ being the max inlet velocity of the BFS.

Moreover, to further proceed with the simulation of a channel flow, a target $\operatorname{Re}_{\tau}$ is required. This follows from using the correlation from Equation 4.2 that states the bulk and friction Re-number are correlated via [85]:

$$
\operatorname{Re}_{\tau} \approx 0.1751 \operatorname{Re}_{b}^{0.875}
$$


where $\operatorname{Re}_{b}=\delta U_{b} / \nu$ and finally the bulk velocity $U_{b}$ is approximated via the following relation: $U_{b}=U_{0} f_{1}\left(\operatorname{Re}_{\theta}\right)$, where $f_{1}$ is a defined in Equation 6.1.

In this procedure, $\operatorname{Re}_{\theta}$ and $\operatorname{Re}_{h}$ are taken to meet that of the ones used in the BFS's inlet in Le et al. [46] - i.e. $\operatorname{Re}_{\theta}=670$ and $\operatorname{Re}_{h}=5100$, respectively. Furthermore, the functions $f_{1}$ and $f_{2}$ are taken from Mukha and Liefvendahl [61] with expressions:

$$
\begin{aligned}
& f_{1}=\gamma_{1}+\alpha_{1} \operatorname{Re}_{\theta}^{\beta_{1}} \\
& f_{2}=\gamma_{2}+\alpha_{2} \operatorname{Re}_{\theta}^{\beta_{2}}
\end{aligned}
$$

where $\alpha_{1}=0.3427, \beta_{1}=0.1287$ and $\gamma_{1}=1$ while $\alpha_{2}=2.603 \cdot 10^{-4}, \beta_{2}=0.9834$ and $\gamma_{2}=11.28$

The kinematic viscosity and step-height are kept constant in all simulations with a value of $\nu=2 \cdot 10^{-4} \mathrm{~m}^{2} / \mathrm{s}$ and $h=0.5 \mathrm{~m}$, respectively. Eventually, this leads to a target $\operatorname{Re}_{\tau} \approx 388$ used in the precursor simulation.

\subsection{Results and Discussion}

In this section, the results pertain to three separate grids hereby referred to as coarse, intermediate and fine. In all three cases, the resolution in the wall-normal direction is kept constant and the only changes are the streamwise and spanwise cells. Refer to Figure 6-4. Also, for the complete set of figures, refer to Appendix E.

A temporal quantity based on the 'flow-through' time is adopted. This is defined as the time taken for the flow to traverse the entirety of the expansion region based on the mean convective velocity. The latter is estimated to be $80 \%$ of the maximum mean inlet velocity [46]. Subsequently, a little over 16 flow-through times are used for the transient phase which allows the residuals to diminish. Afterwards, when the flow is fully-developed, slightly over 5.6 flow-through times are used for timeaveraging. Hence, a total of about 65000 temporal samples are taken (excluding subsection 6.4.4). 


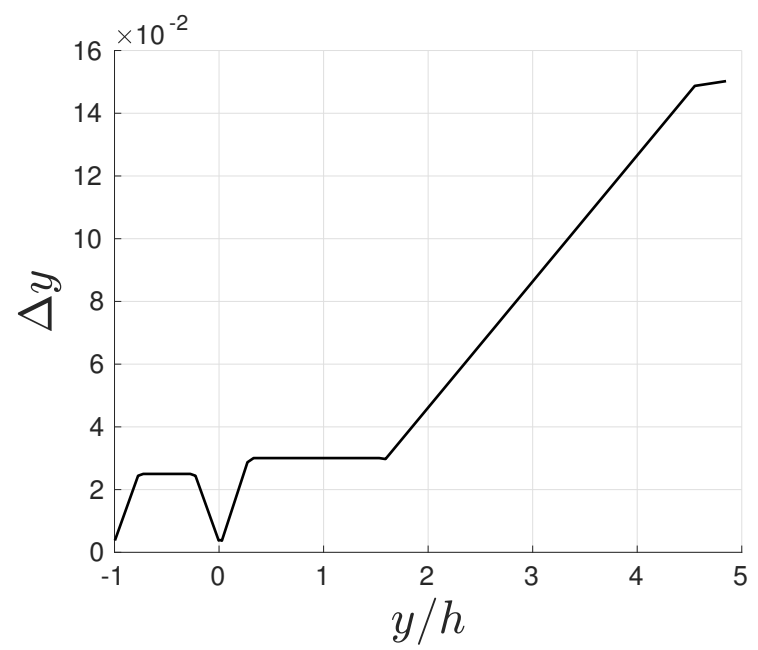

(a)

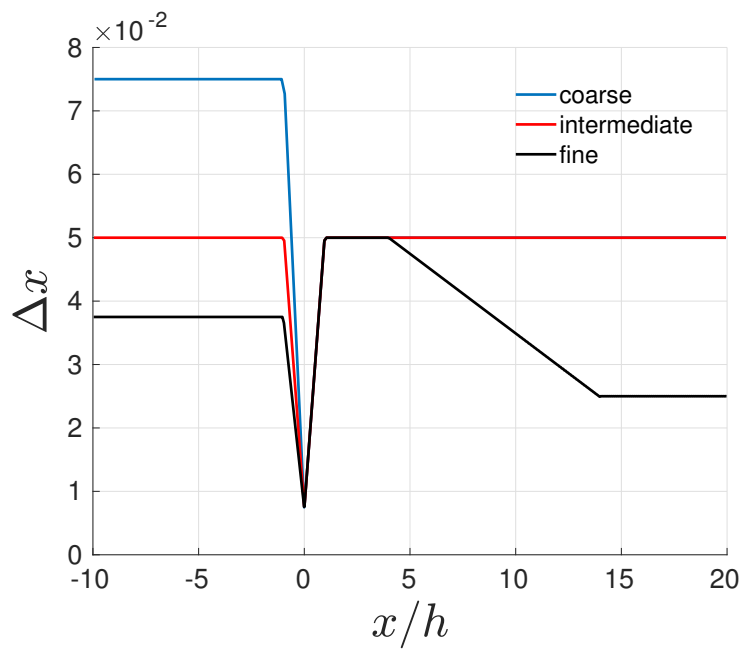

(b)

Figure 6-4: BFS grid resolution $(\mathrm{m})$ of the wall-normal cells versus the wall-normal coordinate (a) and the streamwise cells versus the streamwise coordinate (b).

Additionally, for the sake of brevity, the experimental results validated against are referred to as the JD experiment or simply as 'JD' in the plots. These are taken from Jovic and Driver [37]. Also, in inner-scaled plots, the normalized quantity $y^{+}$ within the expansion region is essentially displayed shifted by $h$ - i.e. $y^{+}=(y+h)^{+}$; in order to compensate for the negative data falling beneath $y=h$, given this is a logarithmic scale. Nonetheless, for intuitive reasons, the scale labeling is kept as $y^{+}$.

\subsubsection{Numerical Simulation: Coarse Mesh}

In the first BFS study, a grid with 704070 cells is used with five different LES SGS models. These models correspond to the Smagorinsky [73], $k$-equation [84], dynamic $k$-equation [42], WALE [63] and SGS-free models. Refer to Table 6.2 for an overview. Additionally, $\operatorname{Re}_{\theta}$ and $\delta_{99}$ in the table are based on the inlet TBL.

The precursor resolution in wall units for both homogeneous directions are $\Delta x^{+} \approx$ 29 and $\Delta z^{+} \approx 34.5$, based on the inlet target $\operatorname{Re}_{\tau}$ (see subsection 6.3.3). This results in a total of 244800 number of cells. Furthermore, the final converged $\operatorname{Re}_{\tau}$ was about 330 and hence under-predicting the target value. 
Table 6.2: Summary for the parameters used in a coarse-size grid resolution for the flow over a BFS simulations using LES as compared to the reference benchmark DNS data for a (target) $\operatorname{Re}_{h}=5100$.

\begin{tabular}{ccc}
\hline parameters & LES & DNS $\dagger$ \\
\hline$\Delta x^{+}$ & $3.3-33$ & 10 \\
$\Delta y^{+}$ & $1.66-66$ & $0.3-31$ \\
$\Delta z^{+}$ & 29 & 15 \\
$\delta_{99}$ & $1.232 h$ & $1.2 h$ \\
$\operatorname{Re}_{\theta}$ & 630 & 670 \\
$u_{\tau}^{\text {inlet }}$ & $8.8 \cdot 10^{-2}$ & - \\
$\Delta t$ & $0.0041 h / U_{0}$ & $0.0018 h / U_{0}$ \\
$\Delta T^{\text {tot }}$ & $548 h / U_{0}$ & $382 h / U_{0}$ \\
$\Delta T^{\text {avg }}$ & $142 h / U_{0}$ & $109 h / U_{0}$ \\
$\#$ cells & 704,070 & - \\
\hline \hline
\end{tabular}

${ }^{\dagger}$ DNS data are obtained from Le et al. [46].

\subsubsection{Mean Streamwise Velocity}

In Figure 6-6, the outer-scaled streamwise velocity displays acceptable behavior for all but the Smagorinsky and $k$-equation models. Nonetheless, as evidenced in Figure 6-5, the inner-scaled velocity profiles did in fact over-predict the benchmark data.

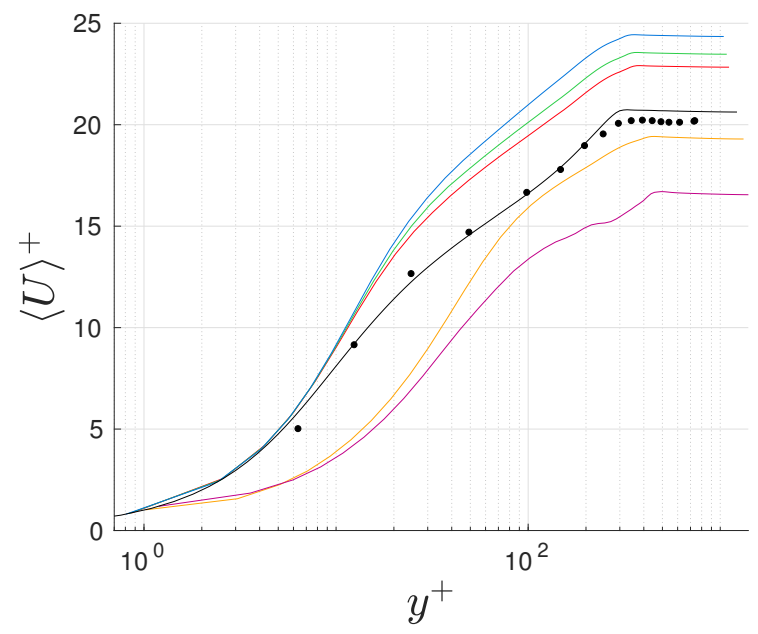

(a)

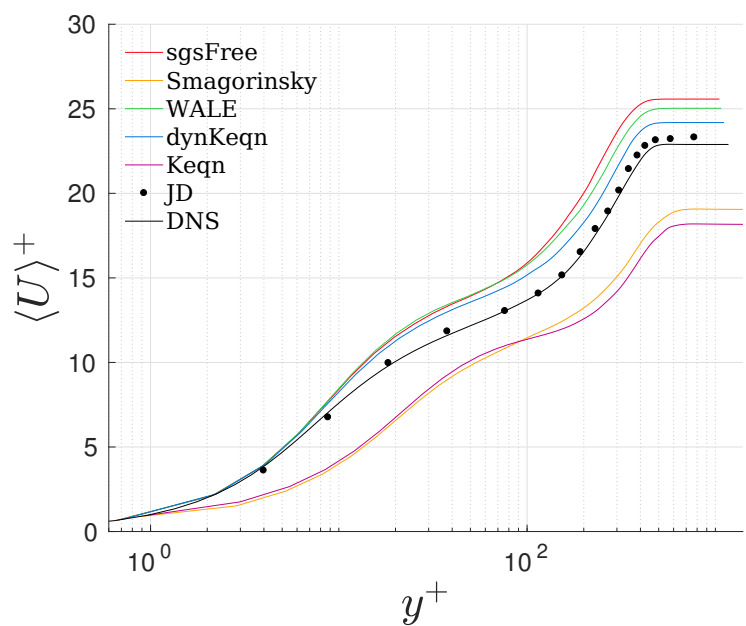

(b)

Figure 6-5: Inner-scaled mean streamwise velocity profiles versus the wall-normal coordinate taken at $x / h$ of approximately -3 (a) and 19 (b). 


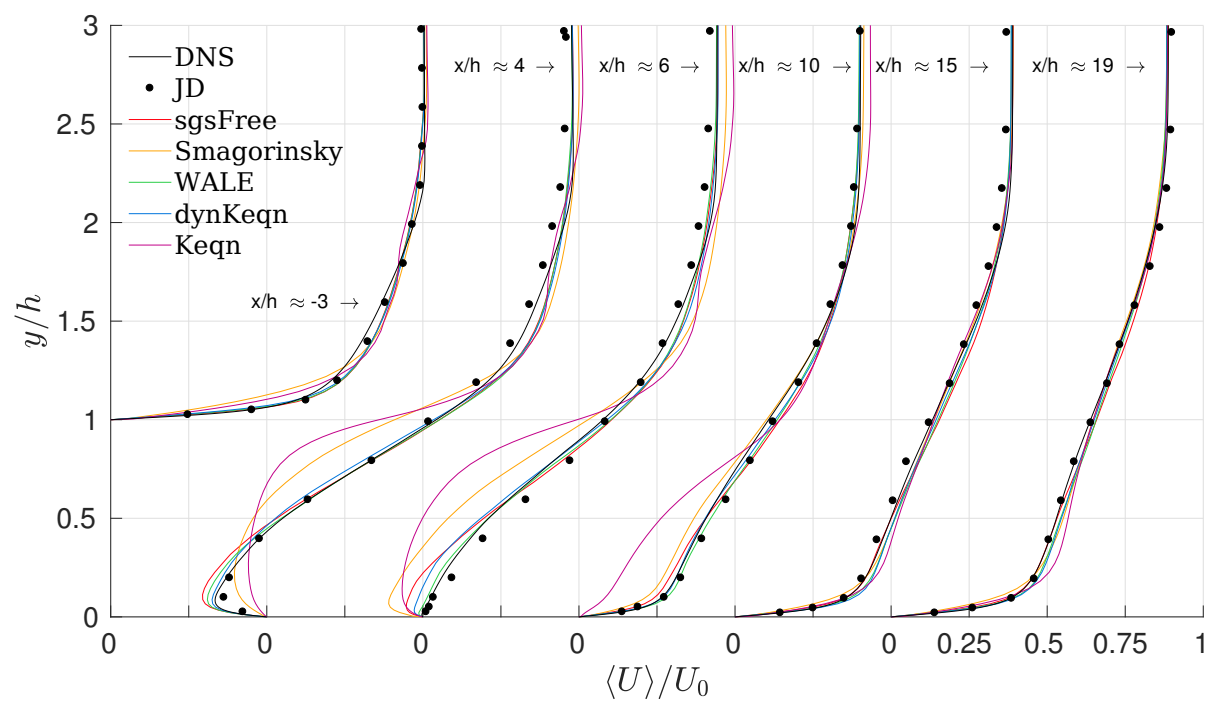

Figure 6-6: Outer-scaled mean streamwise velocity profiles taken at six distinct locations in the streamwise direction in the flow over a backward-facing step.

\subsubsection{Reynolds Stress Components}

Taking a closer look at the Re-stresses, the deviations from the mean streamwise velocity are further amplified in both outer- and inner-scaled plots. Similar to the velocity figures, the Smagorinsky and $k$-equation models display highly dismal outcome which are noticed in all streamwise locations. As for the remaining three SGS models, in outer scaling, they do manage to predict the correct profiles. Of these three, the WALE model in outer-scaled units seem to display relatively better agreement.

Additionally, the SGS-free model seems to consistently have the best approximation to the benchmark data in the pre-expansion. However, more often than not, it under-predicts the most in the remaining expansion regions, refer to Figures 6-7 to 6-9. Analogously, these remarks are also valid for the inner-scaled quantities as evidenced in Figures E-2 to E-4. Consequently, the WALE model is arguably the better overall model when it comes to the variance of the velocity fluctuations. 


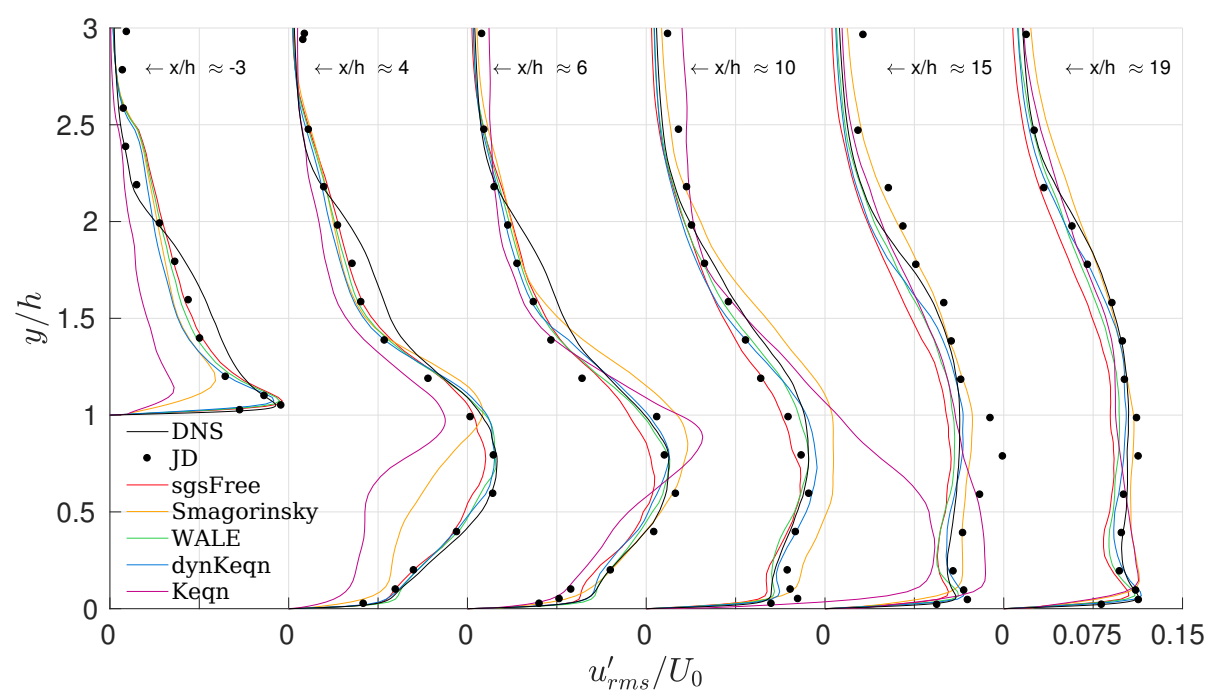

Figure 6-7: Outer-scaled $u_{r m s}^{\prime}$ profiles taken at six distinct locations in the streamwise direction in the flow over a backward-facing step.

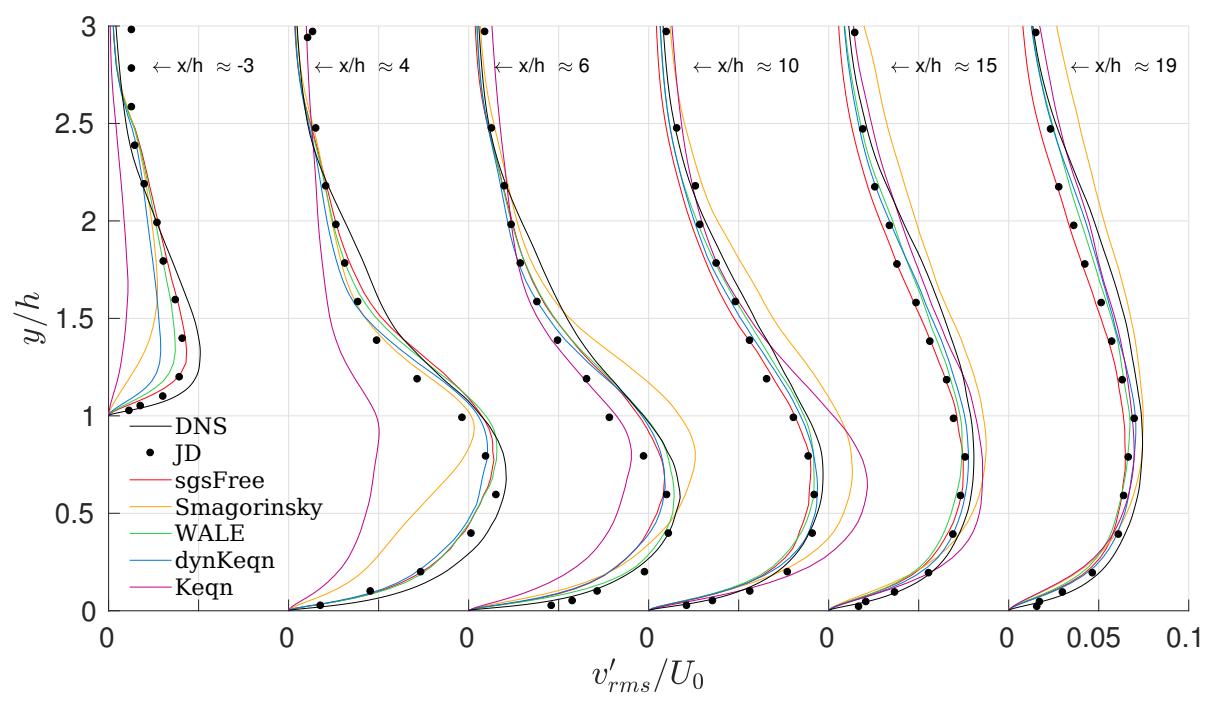

Figure 6-8: Outer-scaled $v_{r m s}^{\prime}$ profiles taken at six distinct locations in the streamwise direction in the flow over a backward-facing step. 


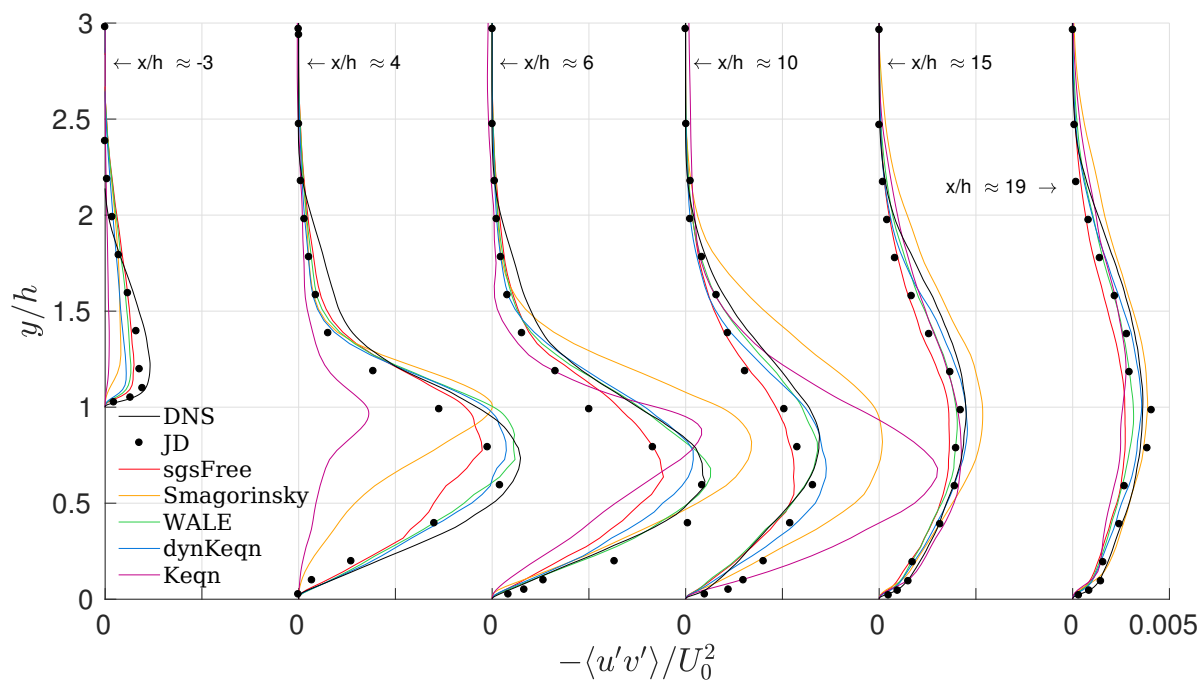

Figure 6-9: Outer-scaled $-\left\langle u^{\prime} v^{\prime}\right\rangle$ profiles taken at six distinct locations in the streamwise direction in the flow over a backward-facing step.

\subsubsection{Pressure and Skin-friction Coefficients}

The skin-friction coefficient is a very crucial parameter to monitor. It can also assist in estimating the reattachment location. It is defined as:

$$
C_{f}=\frac{\tau_{w}}{\frac{1}{2} \rho U_{0}^{2}}
$$

When this coefficient is zero, this means that the friction velocity is zero. This implies the existence of a possible flow reattachment location or the two main bubbles are interfering with one another. Observing Figure 6-10b which shows the predicted $C_{f}$ values, the $k$-equation and Smagorinsky models prove highly inaccurate. The other three SGS models, especially the WALE model, exhibit relatively acceptable prediction, although they all over-estimate the reattachment location. On the other hand, in the pre-expansion region, all models diverge from the data of both DNS and JD (which, to the author's knowledge, is only publicly available via a single point).

The pressure coefficient is yet another quantity of interest which gives insight about the relative pressure distribution along the bottom wall's surface after averaging 
in the spanwise direction. It is defined as:

$$
C_{p}=\frac{\langle\bar{p}\rangle-\langle\bar{p}\rangle_{r e f}}{\frac{1}{2} \rho U_{0}^{2}},
$$

where $\langle\bar{p}\rangle$ is the local pressure at the wall and $\langle\bar{p}\rangle_{r e f}$ is the reference wall pressure taken at $x / h \approx-5$. Note, both wall pressure quantities are averaged in time and the spanwise direction.

Aside from the Smagorinsky and $k$-equation models, according to Figure 6-10, the remaining SGS models yield fairly similar behavior compared to the JD experiment. However, in both the recirculation and recovery regions all models over-predict the JD data. Between both these regions, minor under-prediction takes place. Unfortunately, no concrete remarks may be drawn at the pre-expansion region, due to lack of access to available data.

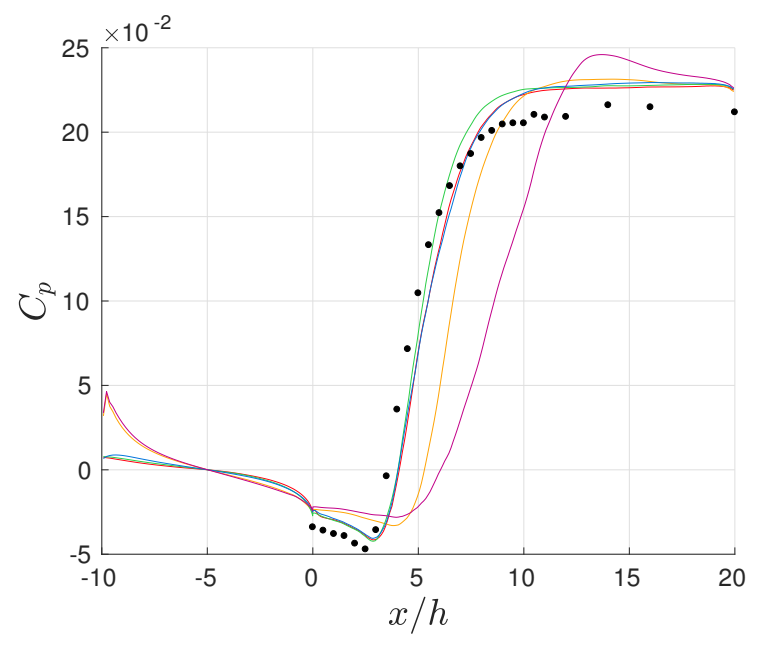

(a)

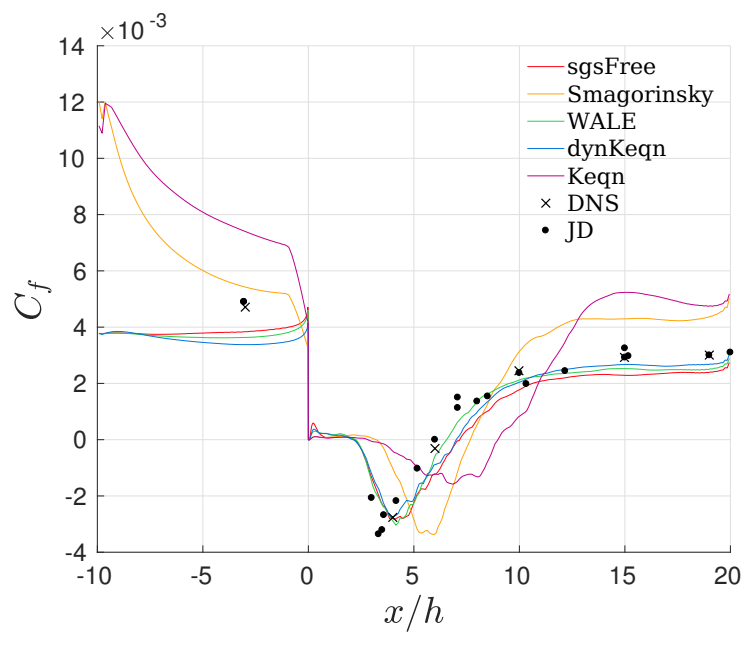

(b)

Figure 6-10: Pressure (a) and skin-friction (b) coefficients along the lower wall in the flow over a backward-facing step.

\subsubsection{Friction Velocity and Friction Reynolds Number}

The local friction velocity and Re-number are computed and displayed in Figure 6-11. The latter, in the case of a TBL, is defined as: $\operatorname{Re}_{\tau} \equiv u_{\tau} \delta_{99} / \nu$. 
In the left panel, $\operatorname{Re}_{\tau}$ is calculated using values for $u_{\tau}$ and $\delta_{99}$ per each cell's corresponding streamwise location. In this figure, certain zigzag-like behavior is noticed. These are explained by the boundary layer thickness' numerical estimation via $\delta_{99}$, since the actual value is theoretical and impossible to determine exactly.

The right panel, illustrating the friction velocity, yields a more meaningful picture regarding the events taking place locally near the wall. For instance, in the expansion region and between $0<x / h<10$, the minor singularity-like local minima correlate with a probable reattachment location while the peaks in between correlate with the reverse flow region. Accordingly, the major and minor peaks each correspond to the centers of the primary and secondary bubbles, respectively. Also evident is the lack of comprehensive and publicly available data samples for the DNS outcome.

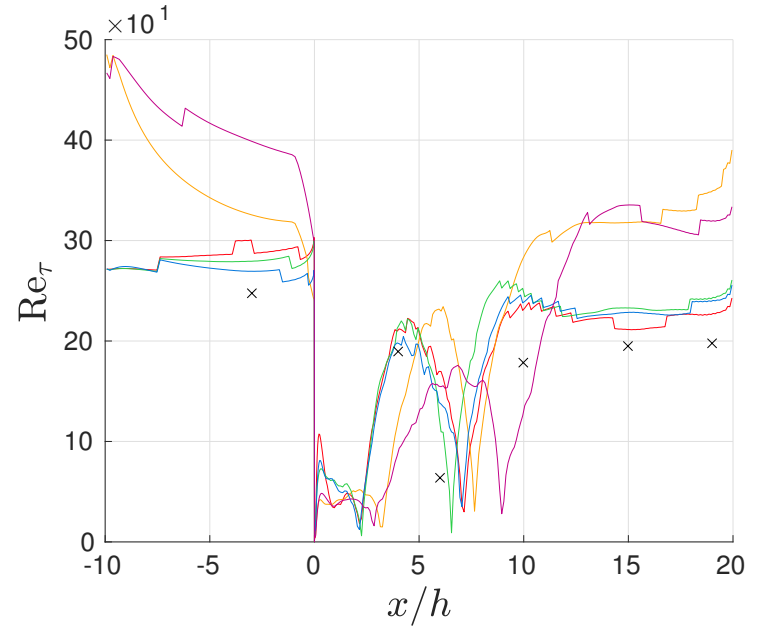

(a)

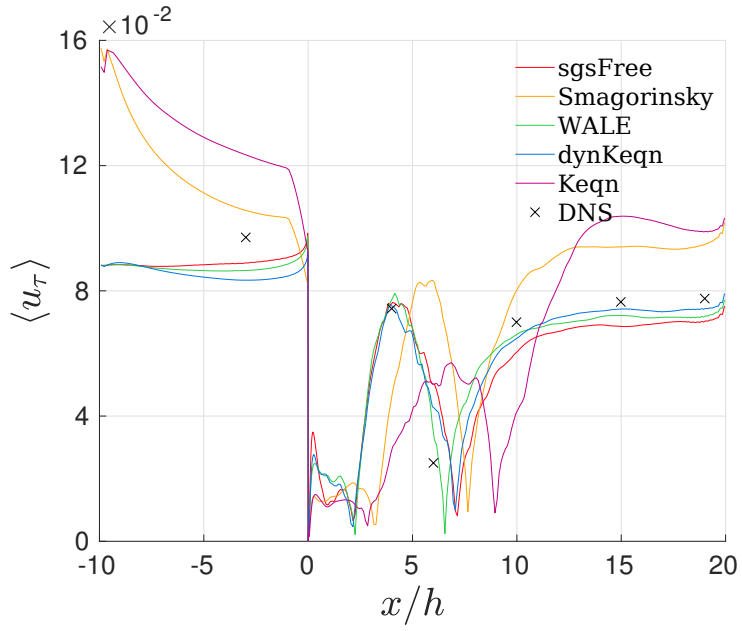

(b)

Figure 6-11: Distribution of $\operatorname{Re}_{\tau}$ (a) and $u_{\tau}$ (b) along the streamwise direction over the wall in the flow over a backward-facing step.

\subsubsection{Numerical Simulation: Intermediate Mesh}

For the second BFS study, a grid with 1893900 cells is used along with three different SGS models. This is motivated by the comparatively unsatisfactory behavior of the $k$ equation and Smagorinsky models from the prior coarser set of simulations. The SGS models used in this study are the dynamic $k$-equation [42], WALE [63] and SGS-free 
models. Table 6.3 illustrates the main details of the simulations (all normalizations are based on the target inlet values).

The precursor grid resolution in both homogeneous directions is $\Delta x^{+} \approx 29$ and $\Delta z^{+} \approx 13.8$, based on the previously computed target $\operatorname{Re}_{\tau}$ at the inlet (see subsection 6.3.3). This results in a total of 612000 number of cells. Furthermore, the final converged $\operatorname{Re}_{\tau}$ is 380 which is relatively close to that of the estimated target.

Table 6.3: Summary for the parameters used in an intermediate-size grid resolution for flow over a BFS simulations using LES as compared to the reference benchmark DNS data for a (target) $\mathrm{Re}_{h}=5100$.

\begin{tabular}{ccc}
\hline parameters & LES & DNS $\dagger$ \\
\hline$\Delta x^{+}$ & $3.87-25.29$ & 10 \\
$\Delta y^{+}$ & $1.87-75.97$ & $0.3-31$ \\
$\Delta z^{+}$ & 13.49 & 15 \\
$\delta_{99}$ & $1.232 h$ & $1.2 h$ \\
$\operatorname{Re}_{\theta}$ & 630 & 670 \\
$u_{\tau}^{\text {inlet }}$ & $10.1 \cdot 10^{-2}$ & - \\
$\Delta t$ & $0.004 h / U_{0}$ & $0.0018 h / U_{0}$ \\
$\Delta T^{\text {tot }}$ & $544 h / U_{0}$ & $382 h / U_{0}$ \\
$\Delta T^{\text {avg }}$ & $141 h / U_{0}$ & $109 h / U_{0}$ \\
$\#$ cells & $1,893,900$ & - \\
\hline \hline
\end{tabular}

${ }^{\dagger}$ DNS data are obtained from Le et al. [46].

\subsubsection{Mean Streamwise Velocity}

The velocity profiles in outer scaling result in good agreement with the benchmark data for both WALE and SGS-free models. This is illustrated in Figure 6-13. The dynamic $k$-equation model appears to lack such success, especially near the reattachment region; at such streamwise location, the dynamic $k$-equation model predicts an APG inducing separation of the TBL as evidenced by the presence of reverse flow. 


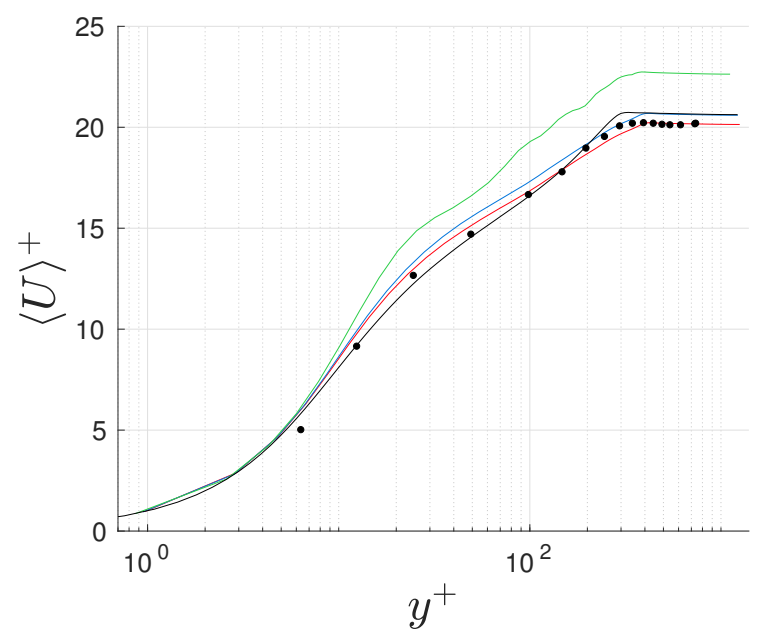

(a)

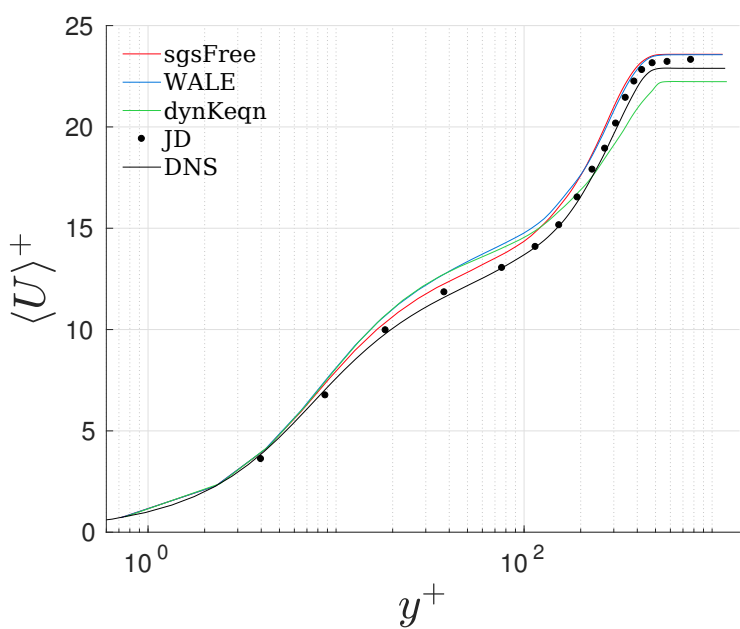

(b)

Figure 6-12: Inner-scaled mean streamwise velocity profiles versus the wall-normal coordinate taken at $x / h$ of approximately -3 (a) and 19 (b).

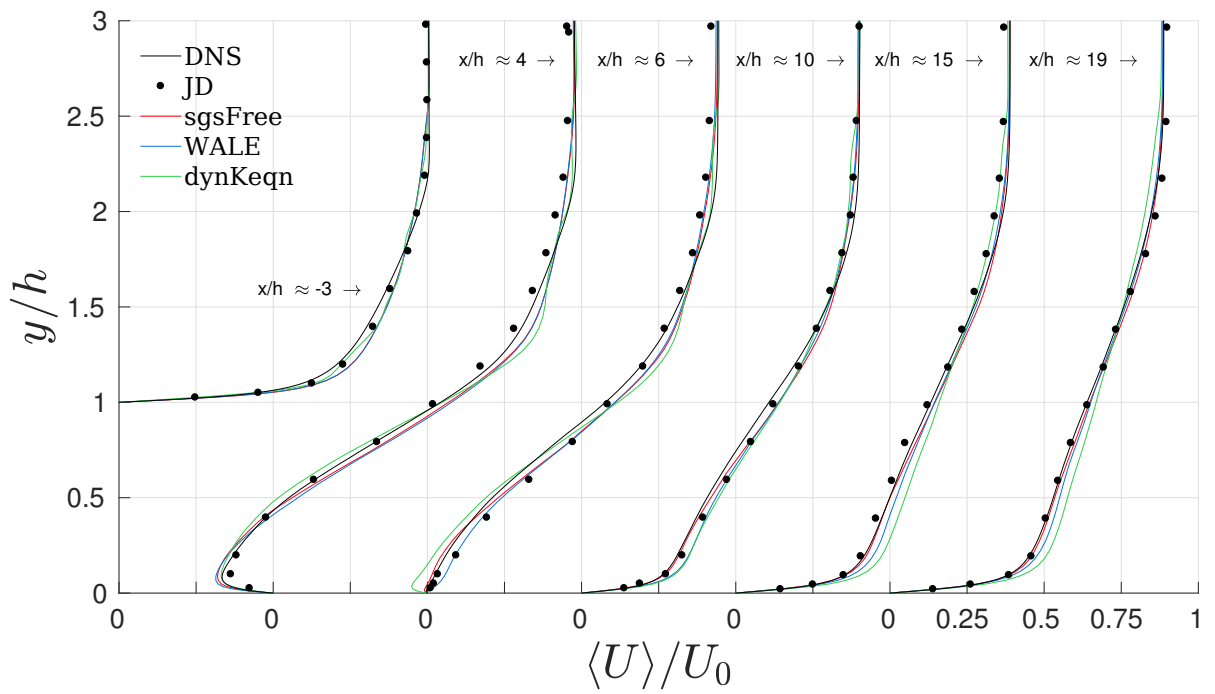

Figure 6-13: Outer-scaled mean streamwise velocity profiles taken at six distinct locations in the streamwise direction in the flow over a backward-facing step. 


\subsubsection{Reynolds Stress Components}

In all the Re-stress components, the pre-expansion profiles of the dynamic $k$-equation greatly under-predict the correct result. This may be shown in inner- and outerscaled quantities in Figures 6-14 to 6-16 and E-6 to E-8. Furthermore, up until the recovery region, all dynamic $k$-equation Re-stress profiles under-predict above the edge of the TBL. At the estimated reattachment point, the dynamic $k$-equation model demonstrates the best outcome in outer scaling within the TBL region in comparison to the other SGS models.

As for the remaining SGS models, both SGS-free and the WALE SGS model agree very well with most benchmark data. However, the WALE model seems to demonstrate reasonably better agreement in most of the regions and for most Restress components.

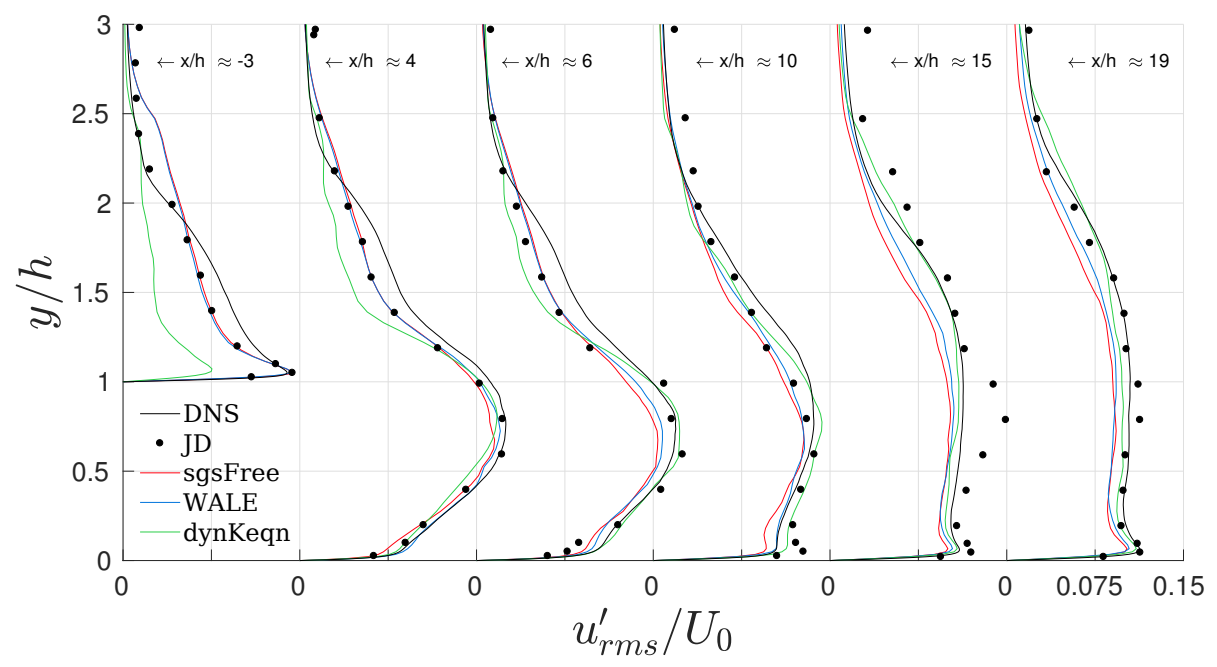

Figure 6-14: Outer-scaled $u_{r m s}^{\prime}$ profiles taken at six distinct locations in the streamwise direction in the flow over a backward-facing step. 


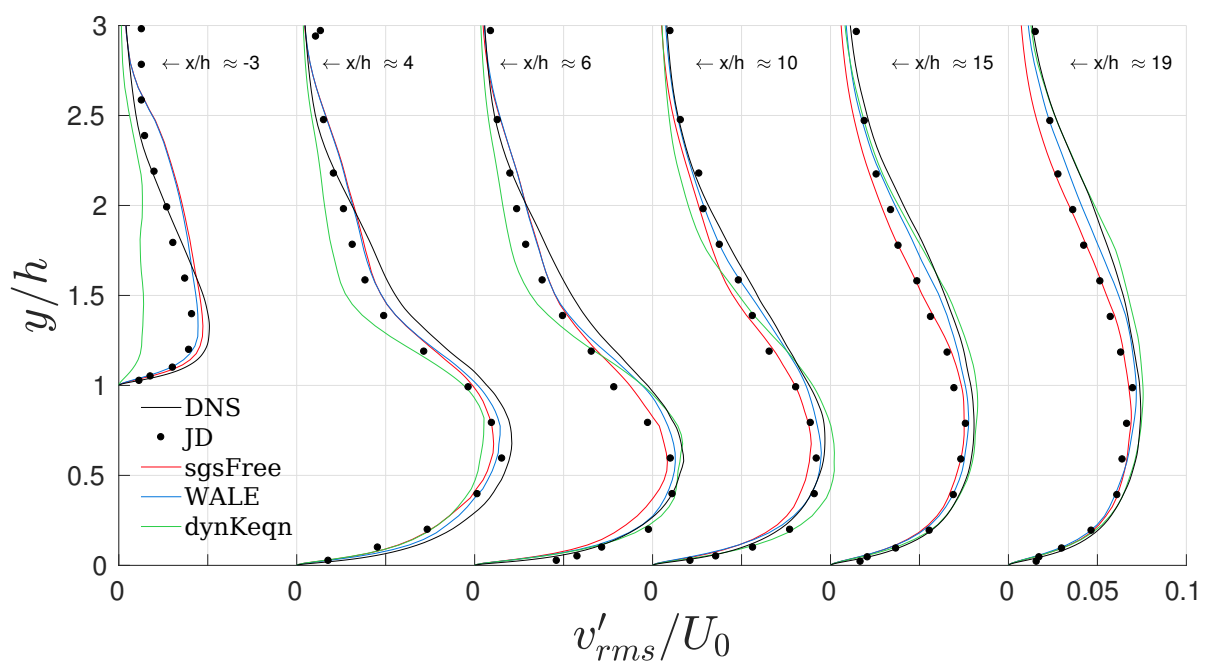

Figure 6-15: Outer-scaled $v_{r m s}^{\prime}$ profiles taken at six distinct locations in the streamwise direction in the flow over a backward-facing step.

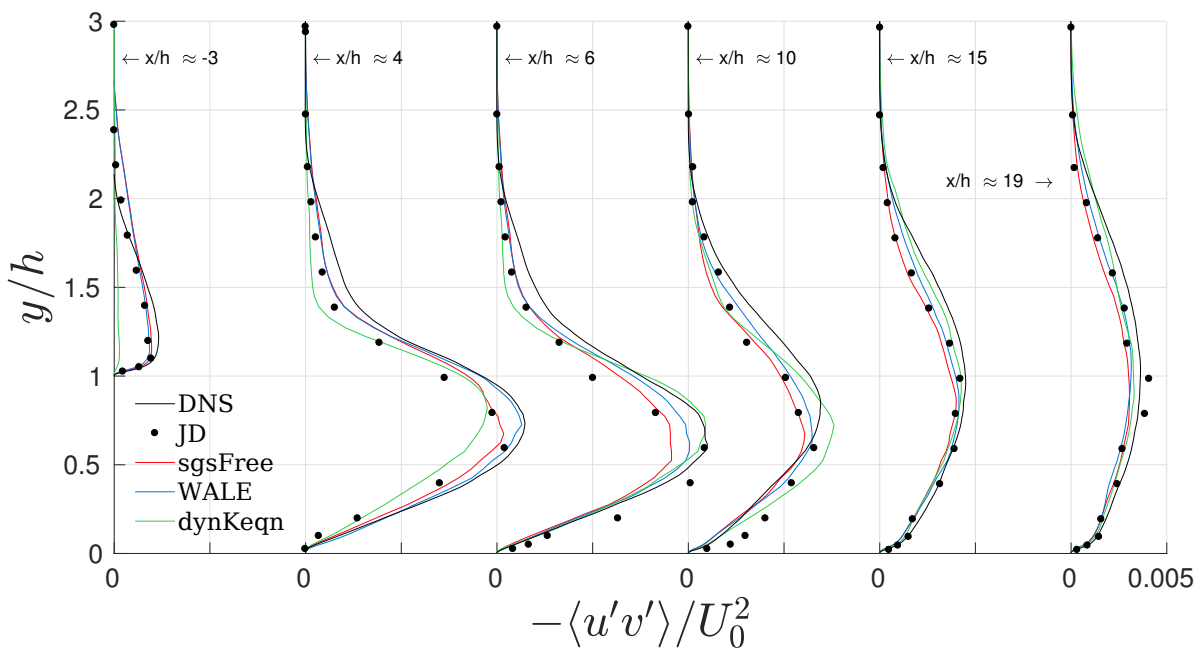

Figure 6-16: Outer-scaled $-\left\langle u^{\prime} v^{\prime}\right\rangle$ profiles taken at six distinct locations in the streamwise direction in the flow over a backward-facing step. 


\subsubsection{Pressure and Skin-friction Coefficients}

Similar to earlier observations, as shown in Figure 6-17, the WALE and SGS-free models result in accurate $C_{p}$ and $C_{f}$ profiles, compared to the benchmark data. The SGSfree model slightly better captures the pressure coefficient curve, while the WALE model manages to predict the reattachment location with better accuracy. Unfortunately, the dynamic $k$-equation has poor results in most streamwise locations and particularly within the pre-expansion region.

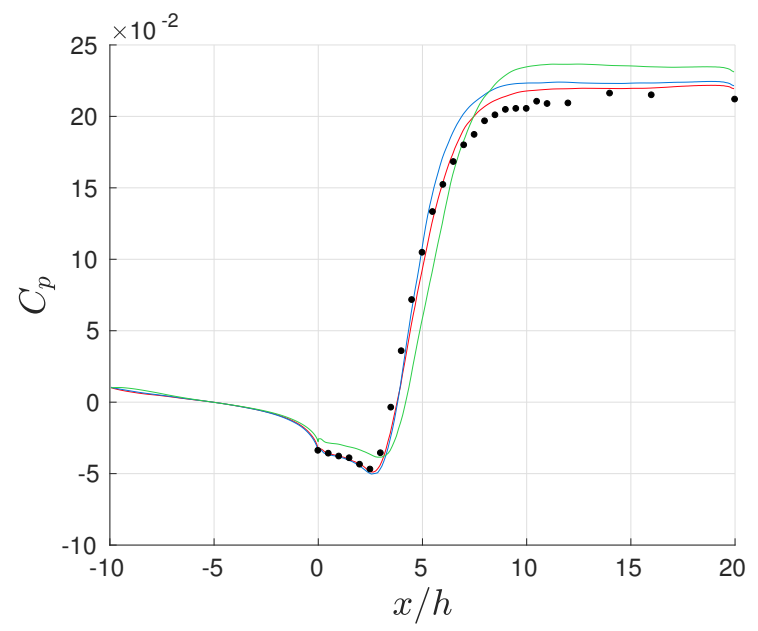

(a)

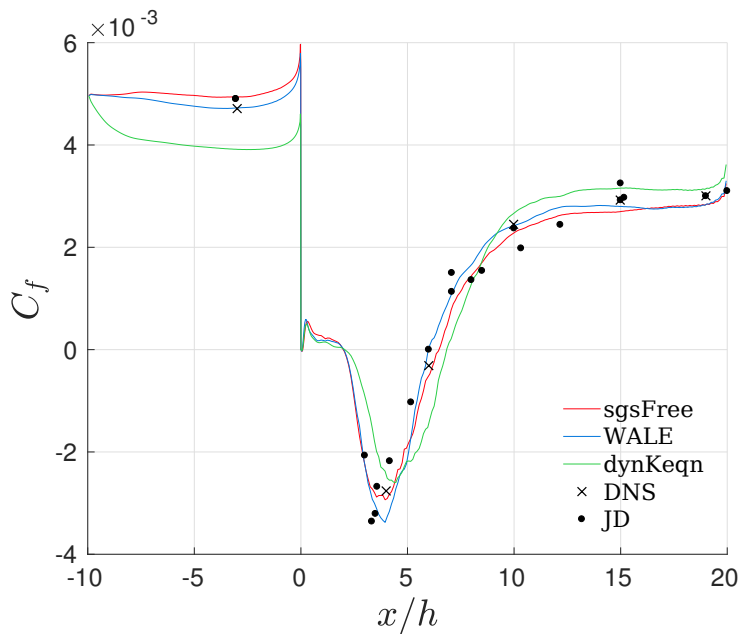

(b)

Figure 6-17: Pressure (a) and skin-friction (b) coefficients along the lower wall in the flow over a backward-facing step.

\subsubsection{Friction Velocity and Friction Reynolds Number}

The local $u_{\tau}$ and $\operatorname{Re}_{\tau}$ in the streamwise direction are presented in Figure 6-18. In both panels, at the pre-expansion region, the dynamic $k$-equation SGS model has a noticeable under-prediction relative to the remaining models. As for $\mathrm{Re}_{\tau}$, a larger difference in between the LES and DNS data is witnessed than in the $u_{\tau}$ figure. This is due to the numerical estimation of $\delta_{99}$ in the LES data. 


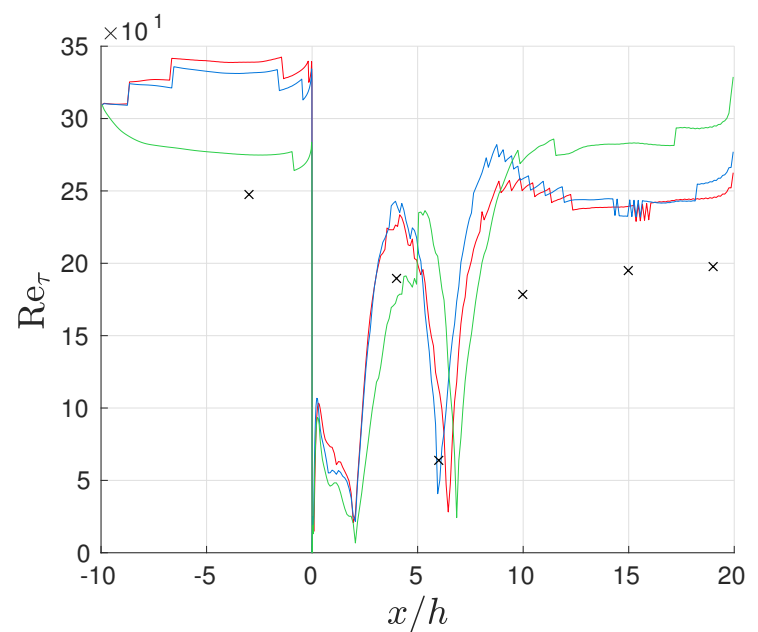

(a)

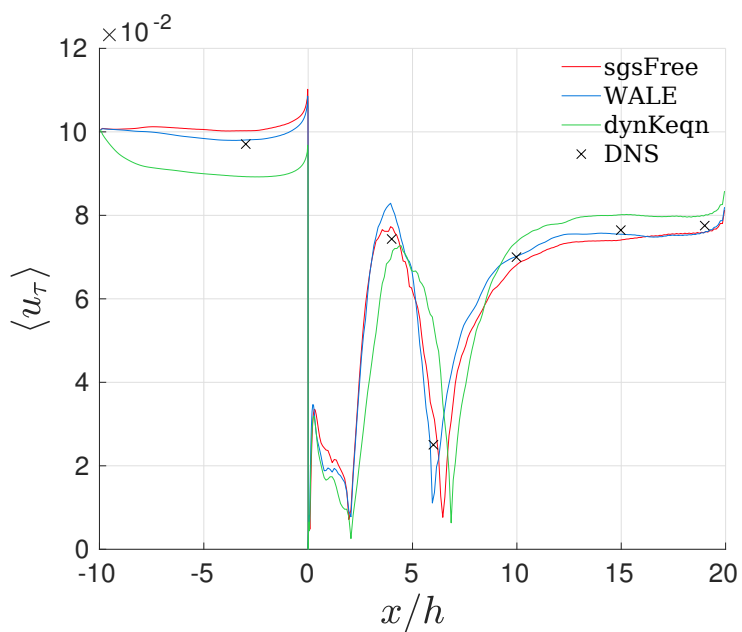

(b)

Figure 6-18: Distribution of $\operatorname{Re}_{\tau}$ (a) and $u_{\tau}$ (b) along the streamwise direction over the wall in the flow over a backward-facing step.

\subsubsection{Numerical Simulation: Fine Mesh}

For the third study, a grid with a total of 7464006 cells is used along with two different SGS models. As illustrated from the previous results, the dynamic $k$-equation model exhibits a relatively poor outcome as opposed to the remaining two (SGS-free and WALE). Consequently, for this fine-size grid simulations the WALE [63] and SGS-free models are used only. The main details are presented in Table 6.4. Similar to prior sections, all normalizations in the table are based on the inlet values.

The precursor simulation's grid resolution in both homogeneous directions is $\Delta x^{+}=10$ and $\Delta z^{+}=5$, based on the previously computed target $\operatorname{Re}_{\tau}$ (see subsection 6.3.3). This results in a total of 4926600 number of cells. The final converged $\operatorname{Re}_{\tau}$ is about 375 . 
Table 6.4: Summary for the parameters used in a fine-size grid resolution for a flow over a BFS simulations using LES as compared to the reference benchmark DNS data for a (target) $\operatorname{Re}_{h}=5100$.

\begin{tabular}{ccc}
\hline parameters & LES & DNS $\dagger$ \\
\hline$\Delta x^{+}$ & $3.76-24.94$ & 10 \\
$\Delta y^{+}$ & $1.84-74.93$ & $0.3-31$ \\
$\Delta z^{+}$ & 4.82 & 15 \\
$\delta_{99}$ & $1.232 h$ & $1.2 h$ \\
$\operatorname{Re}_{\theta}$ & 687 & 670 \\
$u_{\tau}^{\text {inlet }}$ & $9.9 \cdot 10^{-2}$ & - \\
$\Delta t$ & $0.0041 h / U_{0}$ & $0.0018 h / U_{0}$ \\
$\Delta T^{\text {tot }}$ & $552 h / U_{0}$ & $382 h / U_{0}$ \\
$\Delta T^{\text {avg }}$ & $143 h / U_{0}$ & $109 h / U_{0}$ \\
$\#$ cells & $7,464,006$ & - \\
\hline \hline
\end{tabular}

${ }^{\dagger}$ DNS data are obtained from Le et al. [46].

\subsubsection{Mean Streamwise Velocity}

The velocity profiles in inner and outer scaling yield highly satisfactory agreement with the benchmark data. This can be seen in Figures 6-20 and E-9. Nonetheless, in inner-scaling at the recovery region, deviations leading to over-prediction exist.

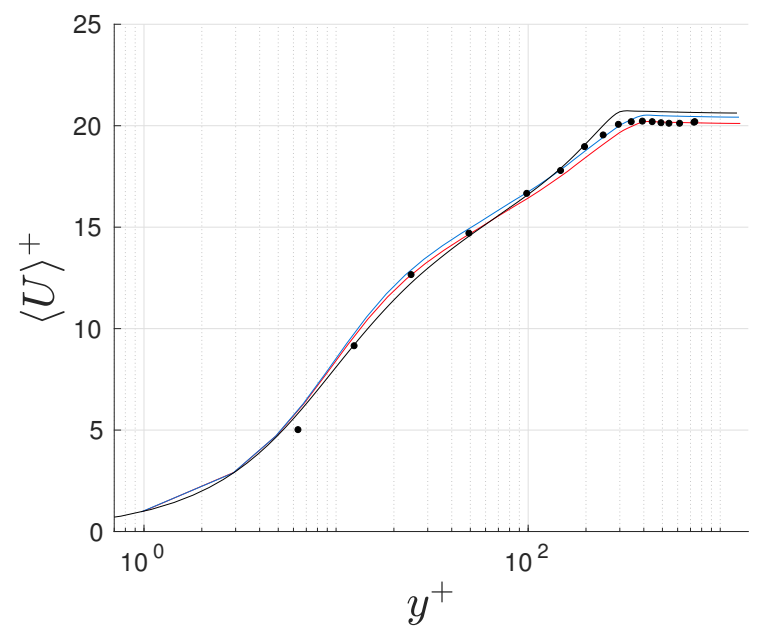

(a)

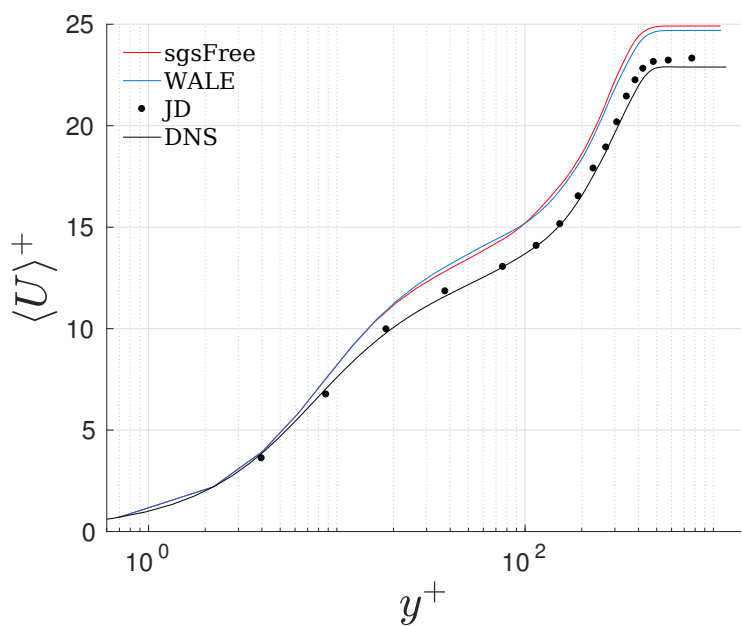

(b)

Figure 6-19: Inner-scaled mean streamwise velocity profiles versus the wall-normal coordinate taken at $x / h$ of approximately -3 (a) and 19 (b). 


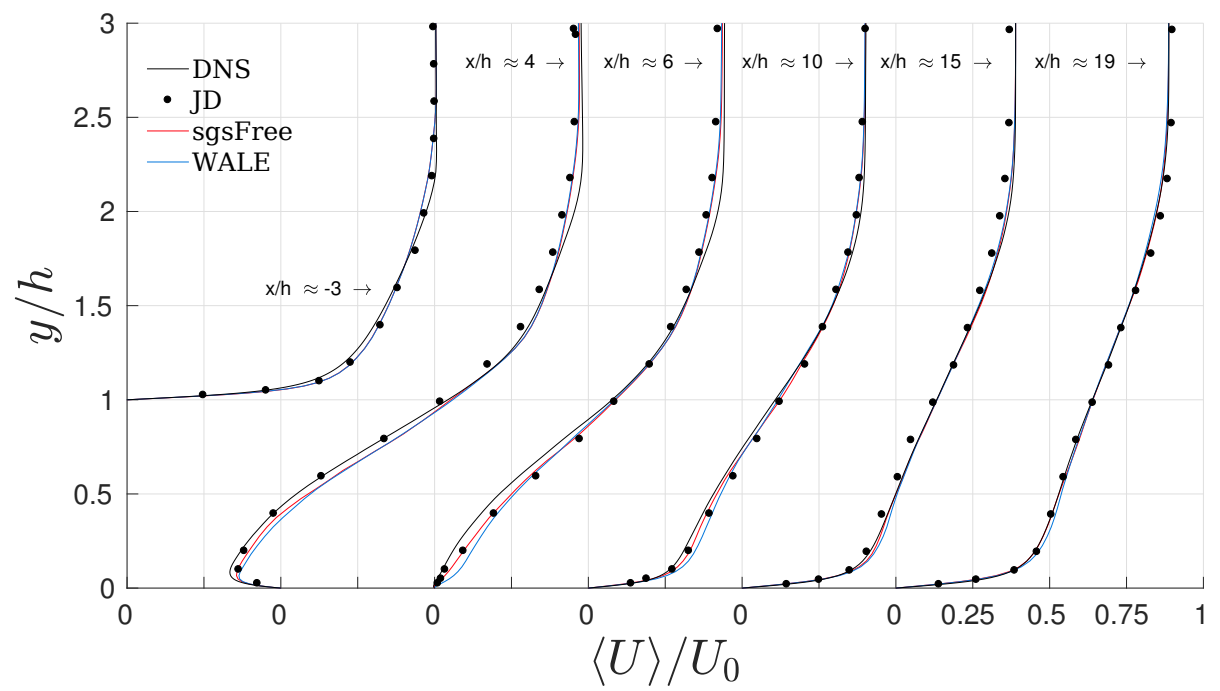

Figure 6-20: Outer-scaled mean streamwise velocity profiles taken at six distinct locations in the streamwise direction in the flow over a backward-facing step.

\subsubsection{Reynolds Stress Components}

The Re-stresses show reasonably good agreement with the benchmark data, despite minor discrepancies present in the $\left\langle u^{\prime} v^{\prime}\right\rangle$ component, as evident in Figure 6-23. The $v_{r m s}^{\prime}$ component outputs better results in Figure 6-22, while Figure 6-21 shows how under-predicting the streamwise Re-stresses are, particularly at the recovery region.

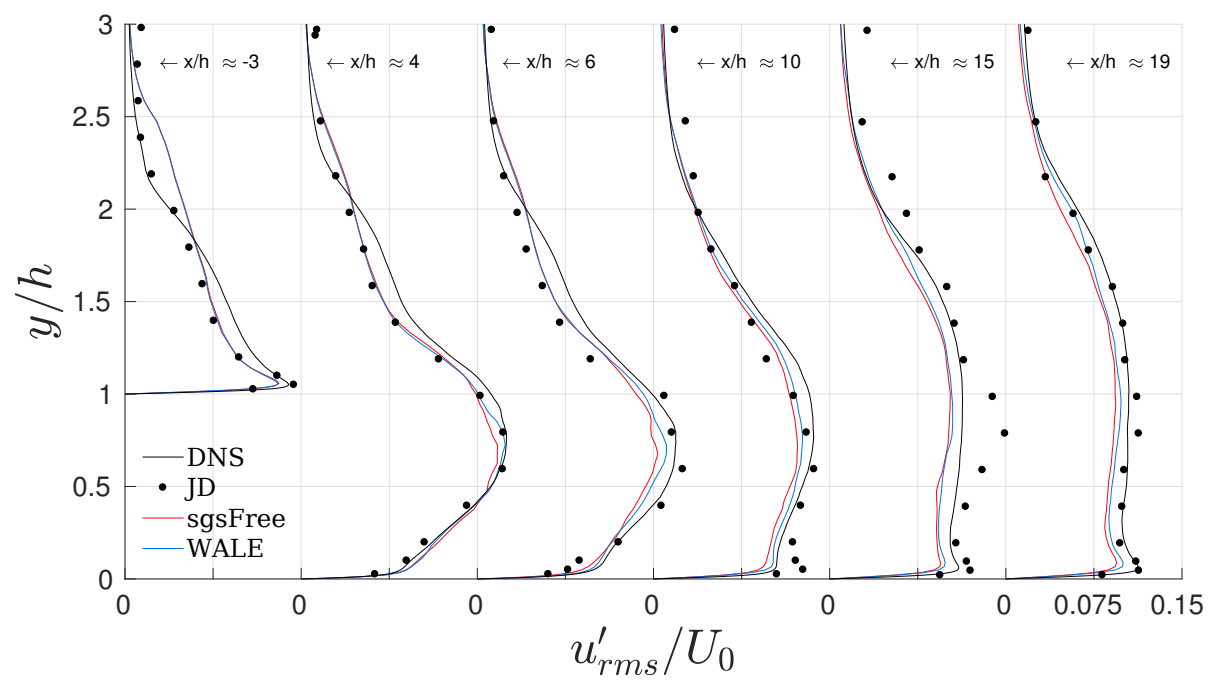

Figure 6-21: Outer-scaled $u_{r m s}^{\prime}$ profiles taken at six distinct locations in the streamwise direction in the flow over a backward-facing step. 


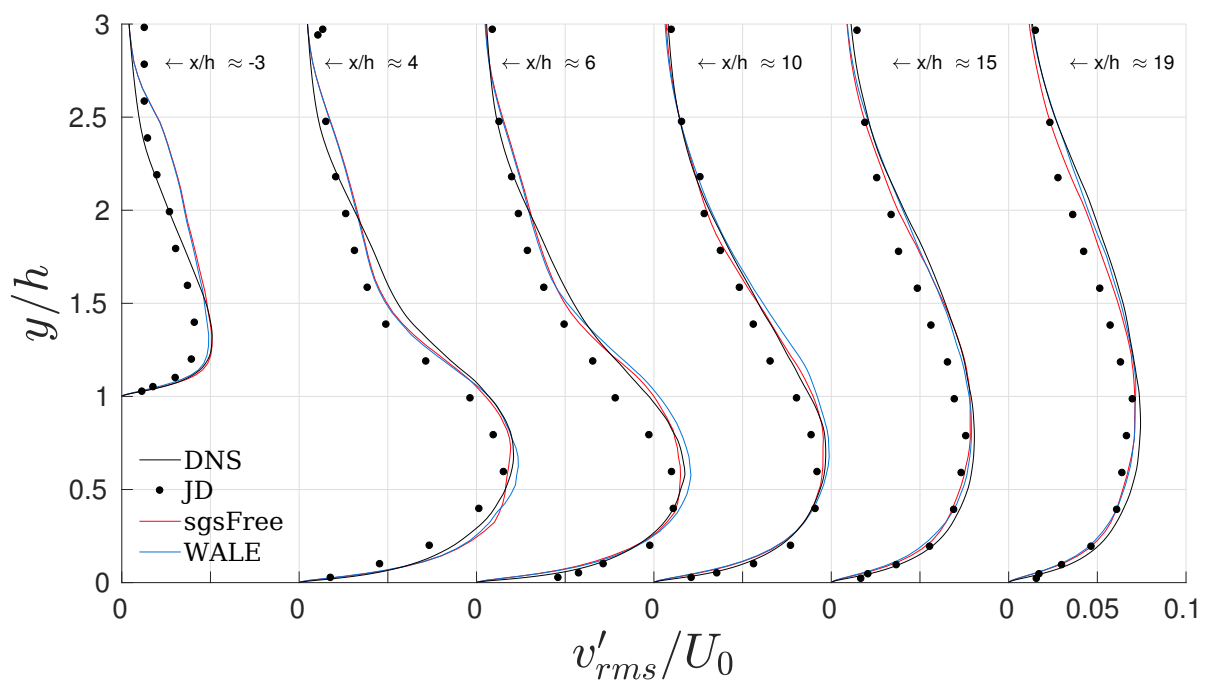

Figure 6-22: Outer-scaled $v_{r m s}^{\prime}$ profiles taken at six distinct locations in the streamwise direction in the flow over a backward-facing step.

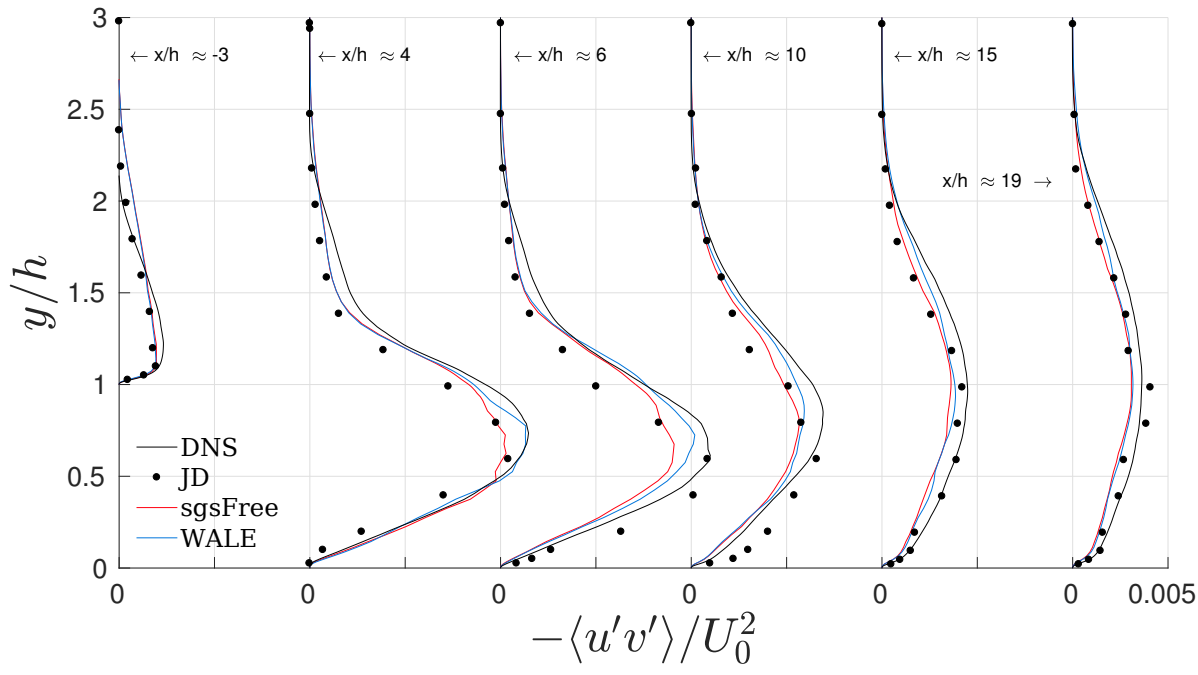

Figure 6-23: Outer-scaled $-\left\langle u^{\prime} v^{\prime}\right\rangle$ profiles taken at six distinct locations in the streamwise direction in the flow over a backward-facing step.

\subsubsection{Pressure and Skin-friction Coefficients}

The pressure and skin-friction coefficients both show good agreement with the DNS and JD data as evidenced in Figure 6-24. Nonetheless, certain minor deviations are evident after the reattachment location. Additionally, according to $C_{f}$, a very good reattachment location is predicted. 


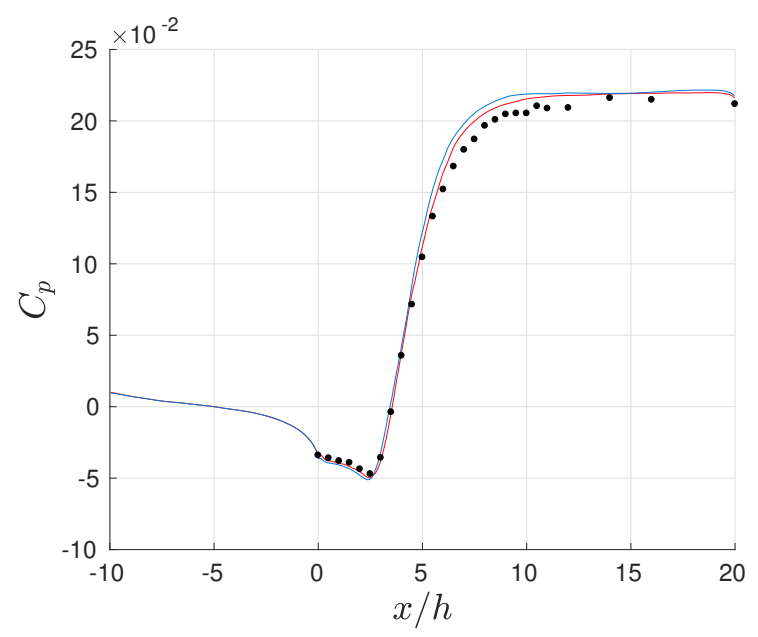

(a)

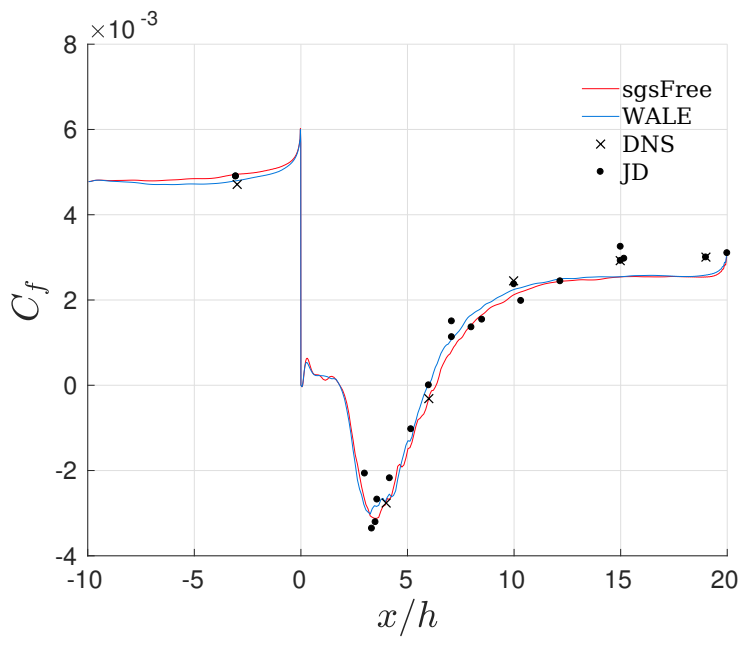

(b)

Figure 6-24: Pressure (a) and skin-friction (b) coefficients along the lower wall in the flow over a backward-facing step.

\subsubsection{Friction Velocity and Friction Reynolds Number}

The local - averaged in time and spanwise direction $-u_{\tau}$ and $\operatorname{Re}_{\tau}$ are presented in Figure 6-25. Though there are slight variations, nevertheless the profiles of both quantities display similar satisfactory performance. Oscillations of $\operatorname{Re}_{\tau}$ are due to $\delta_{99}$.

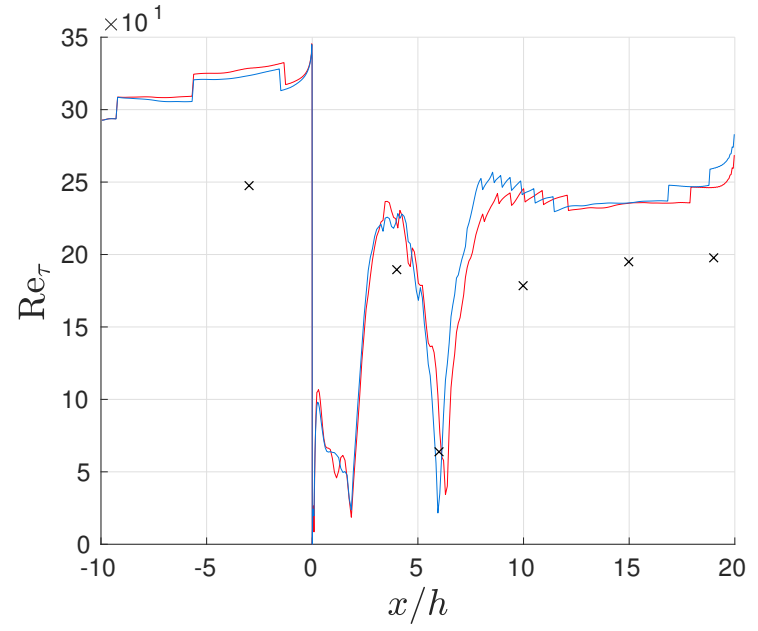

(a)

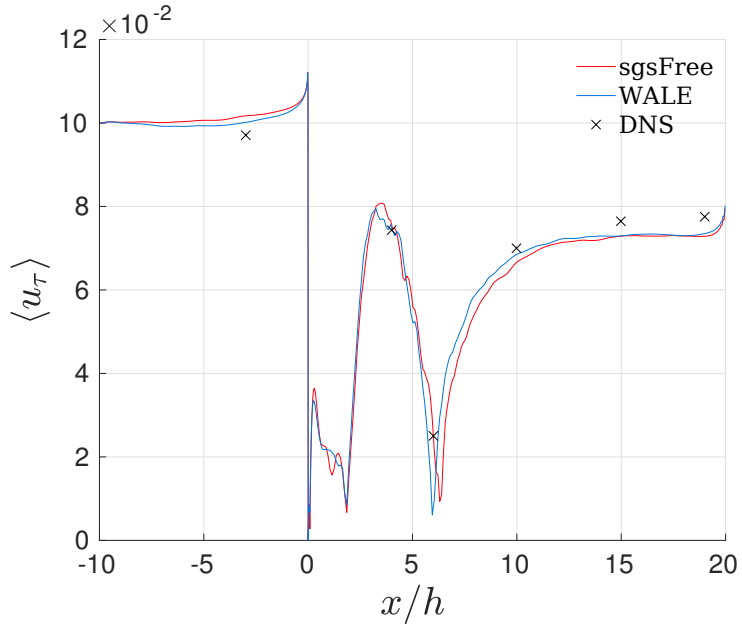

(b)

Figure 6-25: Distribution of $\operatorname{Re}_{\tau}$ (a) and $u_{\tau}$ (b) along the streamwise direction over the wall in the flow over a backward-facing step. 


\subsubsection{Comprehensive Numerical Simulation on a Fine Mesh}

In this study, investigations are carried out using the previously studied fine-size grid resolution in subsection 6.4.3. The time taken for averaging is $\Delta T^{\text {avg }} \approx 674 h / U_{0}$, while the entire simulation is $\Delta T^{\text {tot }} \approx 1226 h / U_{0}$. In flow-through times, this means about 27 and 49 flow-throughs for averaging and the entire simulation, respectively. The number of temporal statistical samples gathered is about 165000 . However, for only the velocity and pressure, these are sampled for approximately 32 flow-through times with the equivalence of about 200000 temporal samples.

\subsubsection{Mean Pressure Profiles}

The pressure distribution is illustrated in Figure 6-26. These contours correspond to the mean pressure fields after averaging in the homogeneous (spanwise) direction. Both SGS models have near identical results.

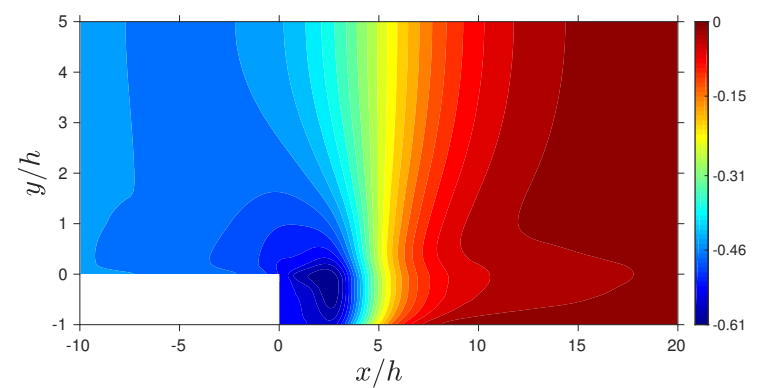

(a)

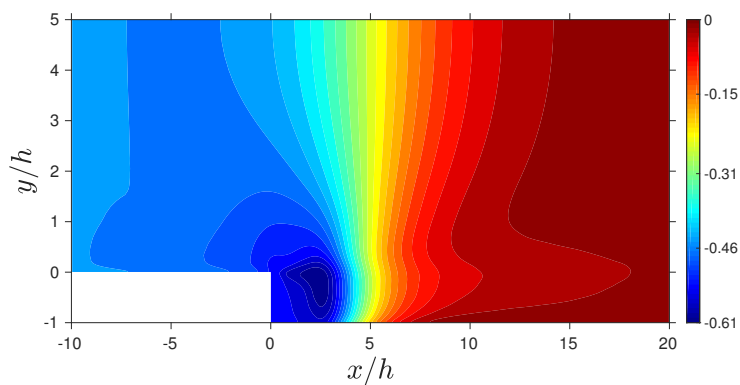

(b)

Figure 6-26: Mean pressure for the flow over a backward-facing step using a SGS-free (a) and a WALE (b) model.

\subsubsection{Mean Velocity Profiles}

The mean velocity profiles are presented for the wall-normal component in outer and inner scaling; see Figures 6-28 and 6-29, respectively. All the mean streamwise velocity components at the recovery region for both WALE and SGS-free models are presented in Figure 6-27. The remaining streamwise velocity components are identical to the ones reported in subsection 6.4.3. Nevertheless, the mean values of both streamwise 
and wall-normal velocities are produced in contour plots in Figure 6-30. Also, the mean velocity magnitude is illustrated in Figure 6-31.

Compared with the expected log-law region's behavior, the streamwise velocity profiles under-predict this area in all the recovery stations as witnessed in Figure 627. This observation was also reported previously in Le et al. [46]. However, with increasing streamwise distance, the velocity profiles further approach the correct scaling in the log-law region. At $x / h \approx 19$, both SGS models are in close proximity to the expected behavior. As for the log-law curve, two separate expressions are utilized, each with coefficients: $\kappa_{1}=0.41, B_{1}=5.0$ [14] and $\kappa_{2}=0.38, B_{2}=4.17$ [62]; refer to Equation 4.3.

As for the mean wall-normal velocity, both benchmark data display large deviations from one another. This is evident in both scalings as demonstrated in Figures 628 and 6-29. In outer scaling, both SGS models show very good agreement with the DNS data at the pre-expansion and recovery regions. In the recirculation region, a noticeable over-prediction is observed between the SGS models and both benchmark data. The remaining contour plots in Figures 6-30 and 6-31, both SGS models are almost indistinguishable. 


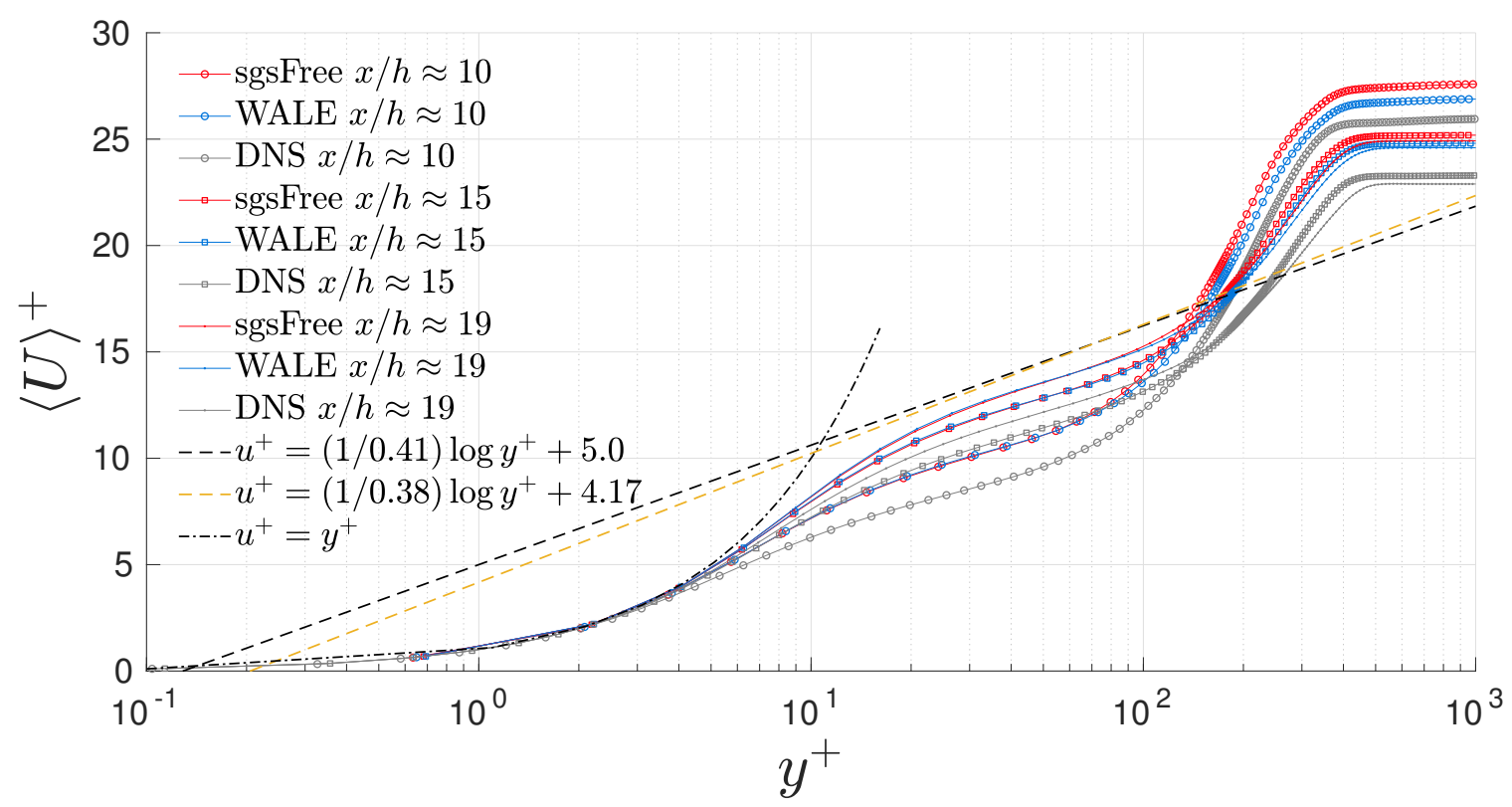

Figure 6-27: Inner-scaled mean streamwise velocity versus the wall-normal coordinate at all three recovery regions. Profiles include SGS-free (red) and WALE (blue) models including DNS (black) along with the expected log-law and viscous sublayer scaling.

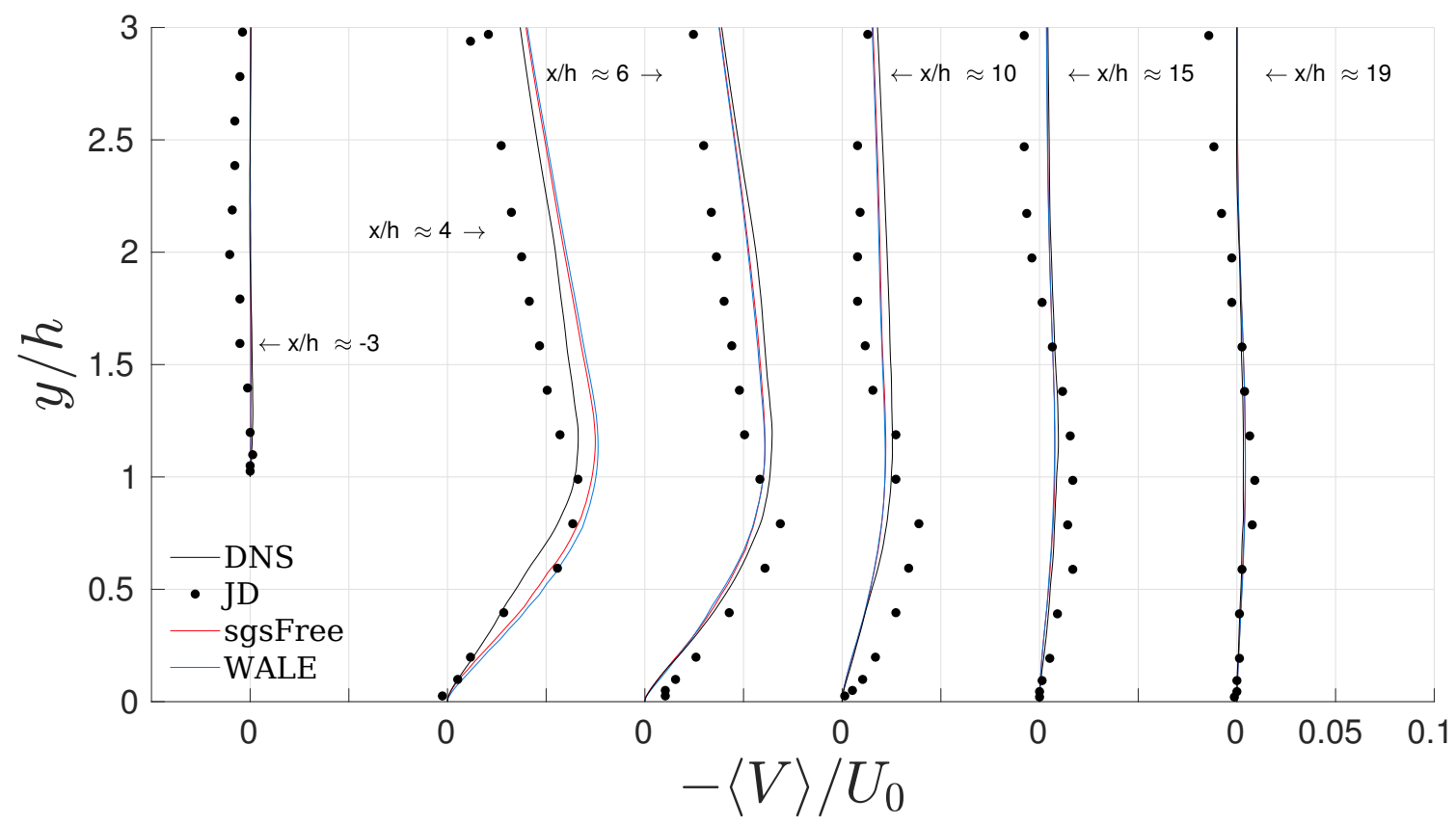

Figure 6-28: Outer-scaled mean wall-normal velocity profiles taken at six distinct locations in the streamwise direction in the flow over a backward-facing step. 


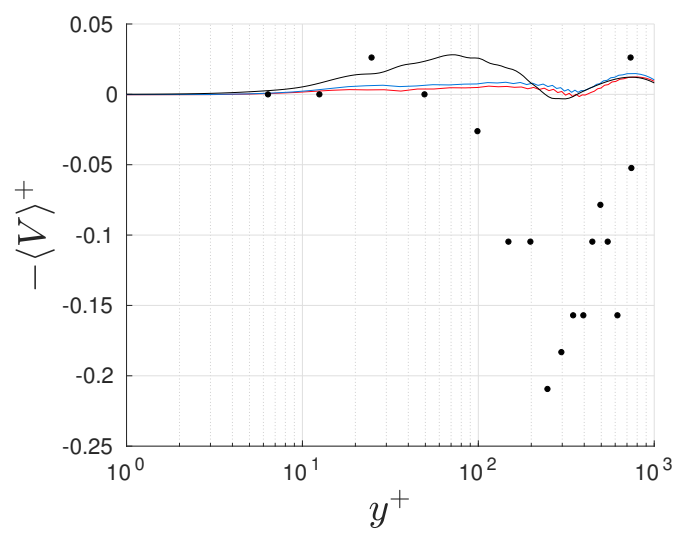

(a)

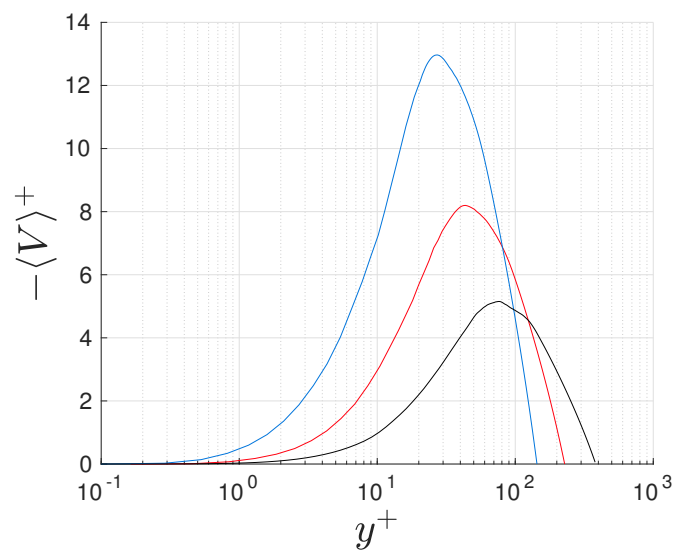

(c)

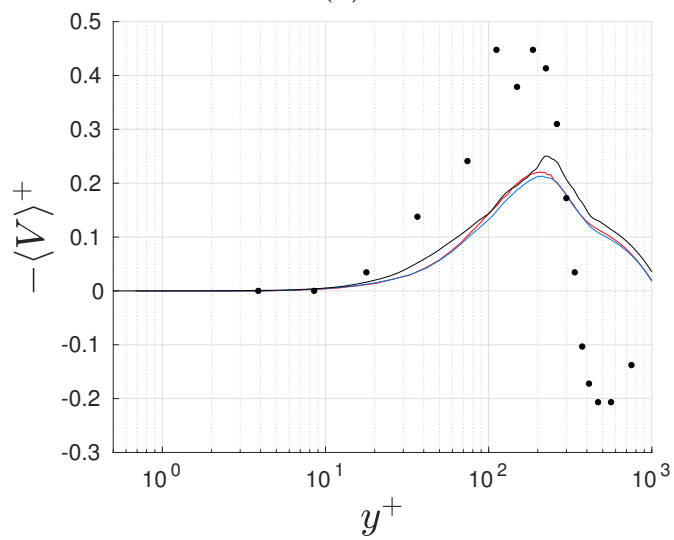

(e)

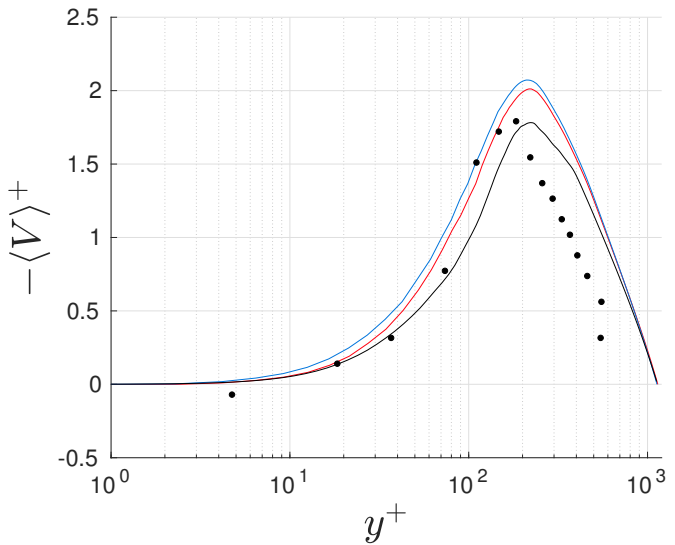

(b)

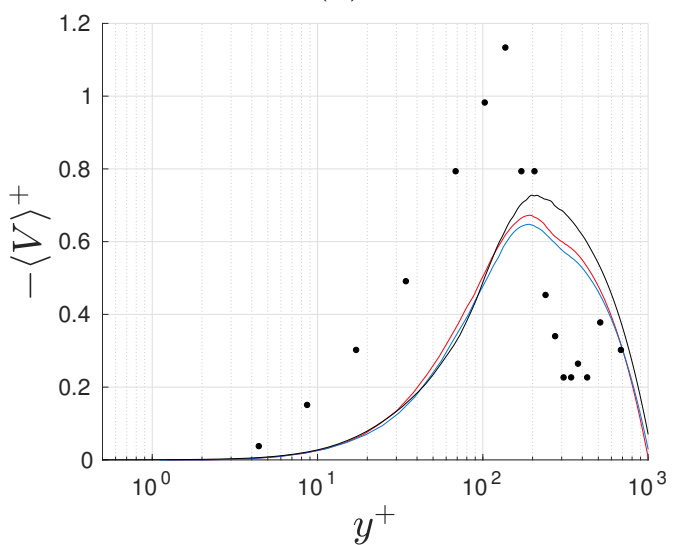

(d)

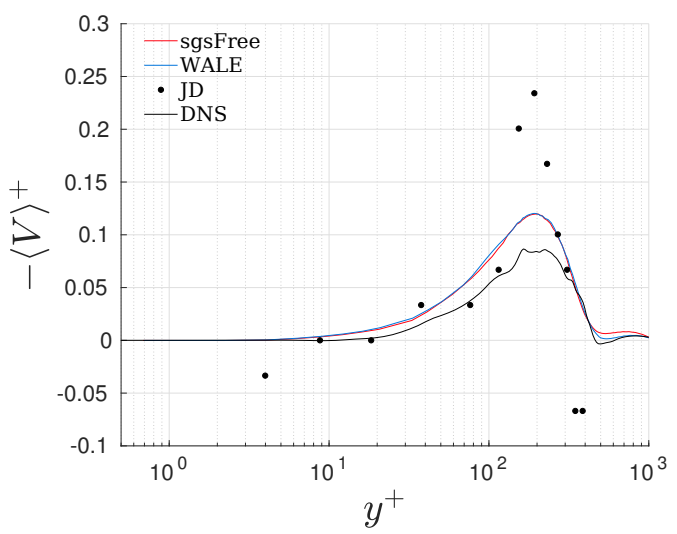

(f)

Figure 6-29: Inner-scaled mean wall-normal velocity profiles versus the wall-normal coordinate taken at dimensionless streamwise locations $x / h$ of approximately -3 (a), 4 (b), 6 (c), 10 (d), 15 (e) and 19 (f). 


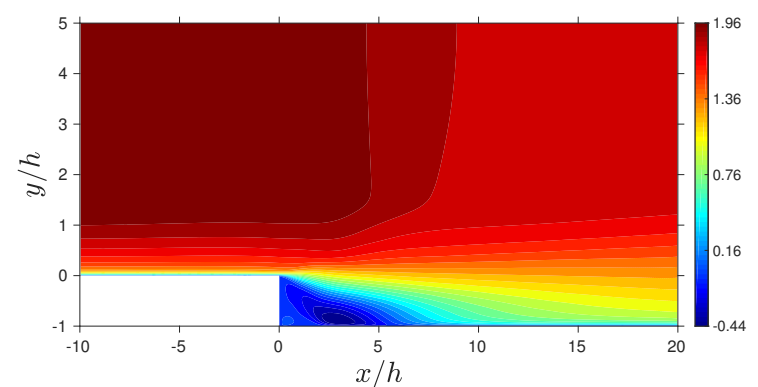

(a)

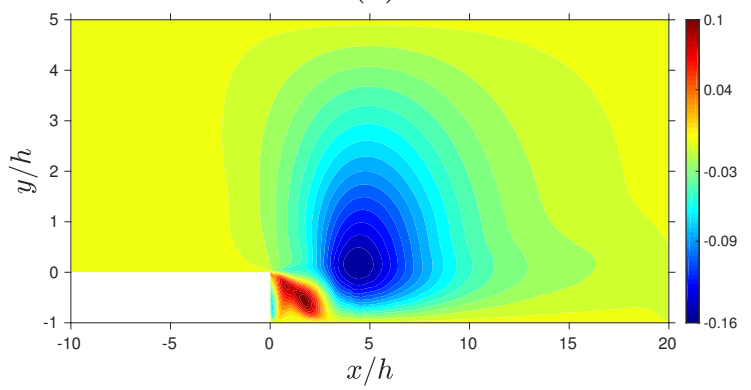

(c)

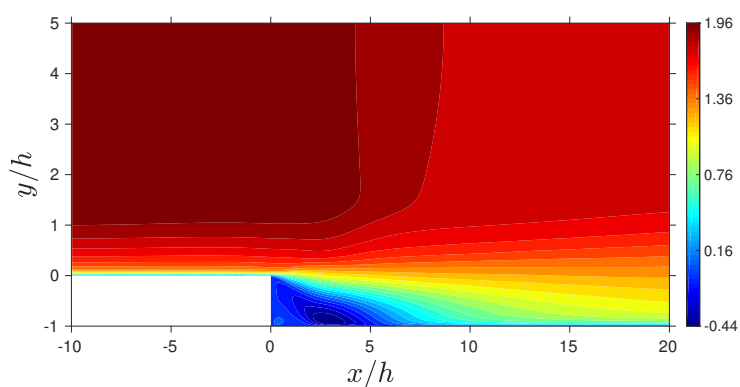

(b)

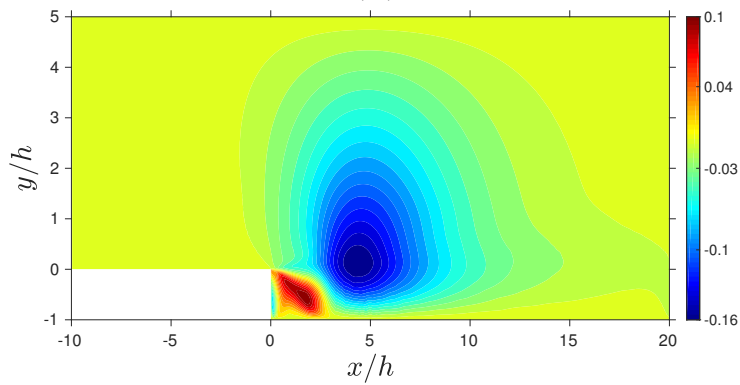

(d)

Figure 6-30: Mean velocity components $(\mathrm{m} / \mathrm{s})$ averaged in the spanwise direction. Left and right columns correspond to the SGS-free and the WALE models, respectively; top and bottom rows correspond to the streamwise and wall-normal velocities, respectively.

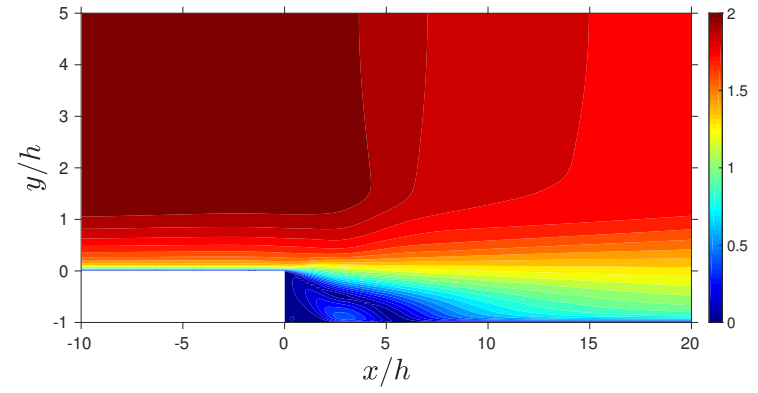

(a)

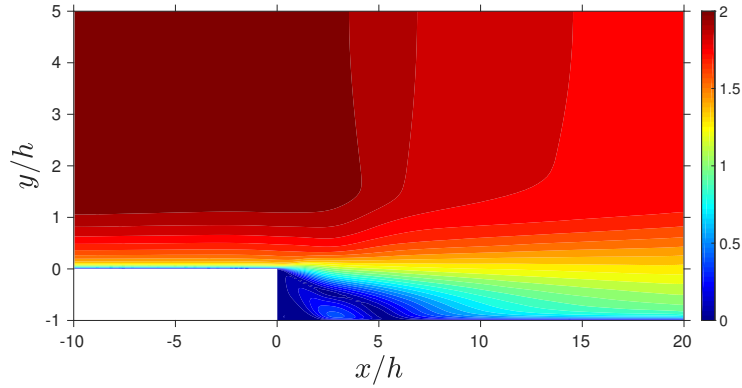

(b)

Figure 6-31: Mean velocity magnitude (m/s) for the SGS-free (a) and WALE (b) models. 


\subsubsection{Reynolds Stress Components}

In order to avoid tedious repetition, only the spanwise Re-stress component is presented; see Figures 6-32 and 6-33 for outer and inner scaling of $w_{r m s}^{\prime}$, respectively. The outer-scaled spanwise stress agree very well with the DNS data, although in inner scaling they over-predict it at all streamwise locations. For the remaining Re-stress components (excluding $\left\langle u^{\prime} w^{\prime}\right\rangle$ and $\left\langle v^{\prime} w^{\prime}\right\rangle$ ), refer to subsection 6.4.3. Additionally, in contour plots averaged in the spanwise direction, Figures 6-34 and 6-35 illustrate the main Re-stress components and TKE, respectively.

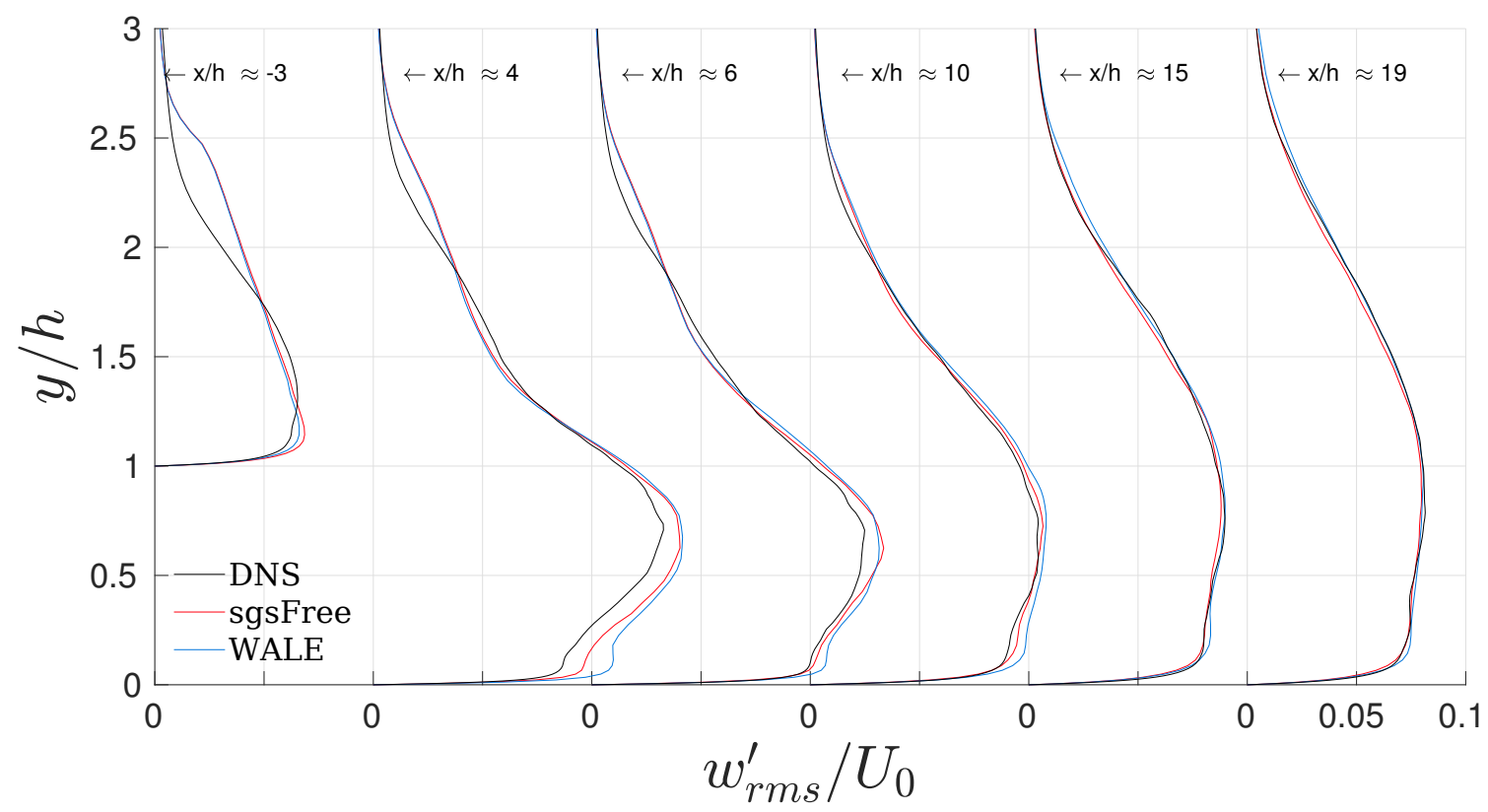

Figure 6-32: Outer-scaled $w_{r m s}^{\prime}$ profiles taken at six distinct locations in the streamwise direction in the flow over a backward-facing step. 


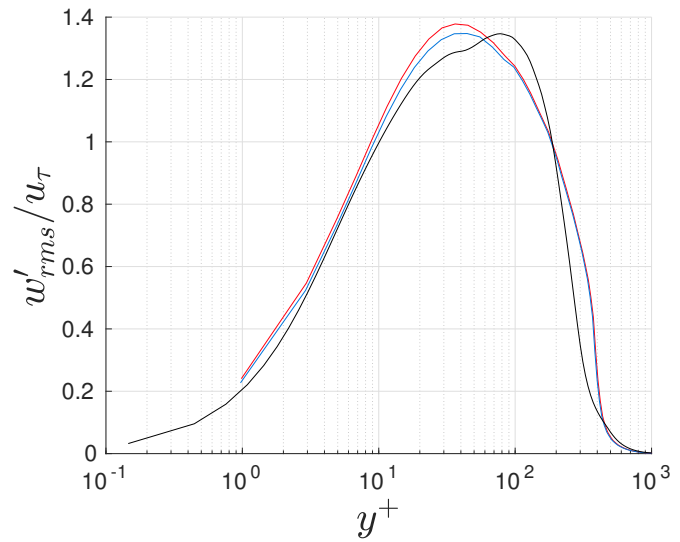

(a)

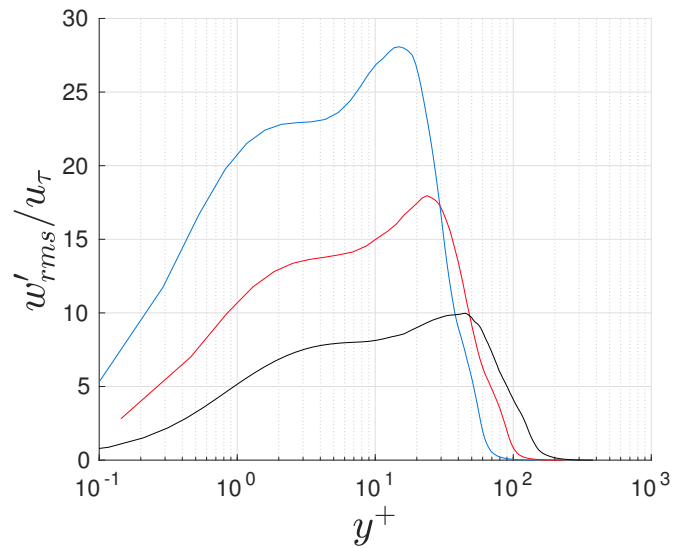

(c)

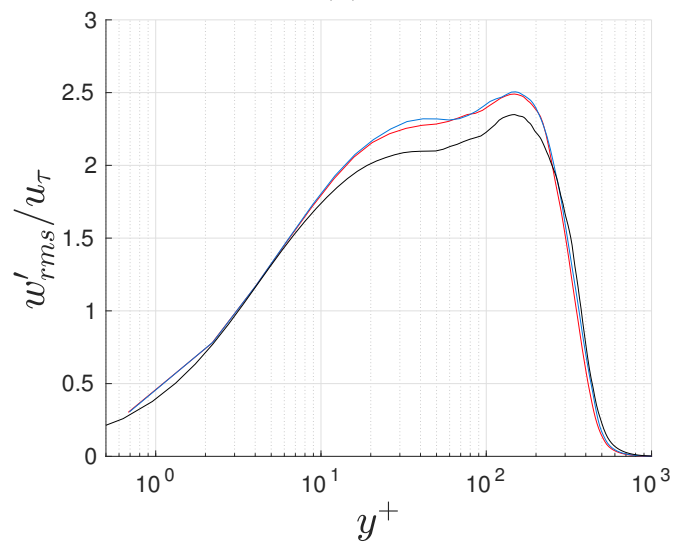

(e)

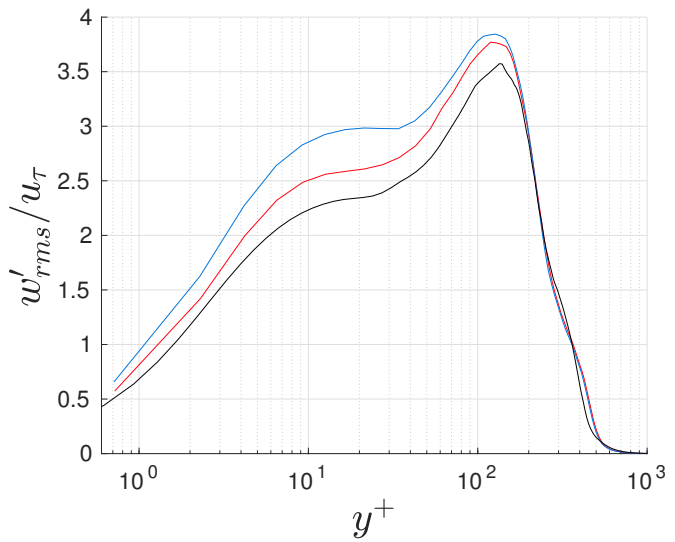

(b)

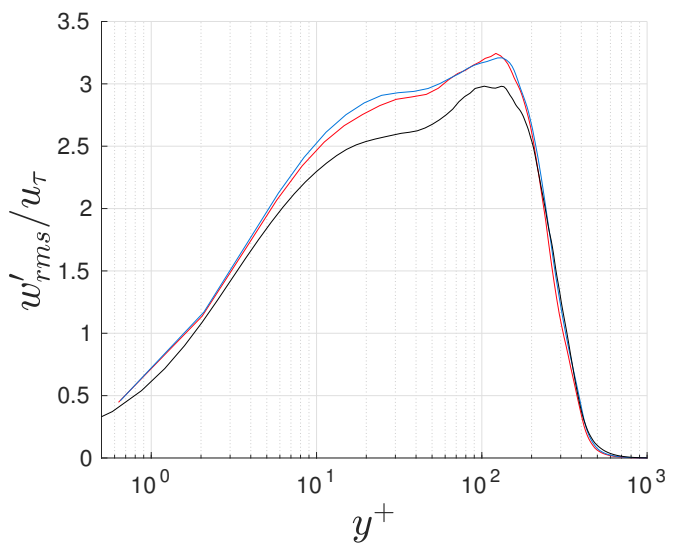

(d)

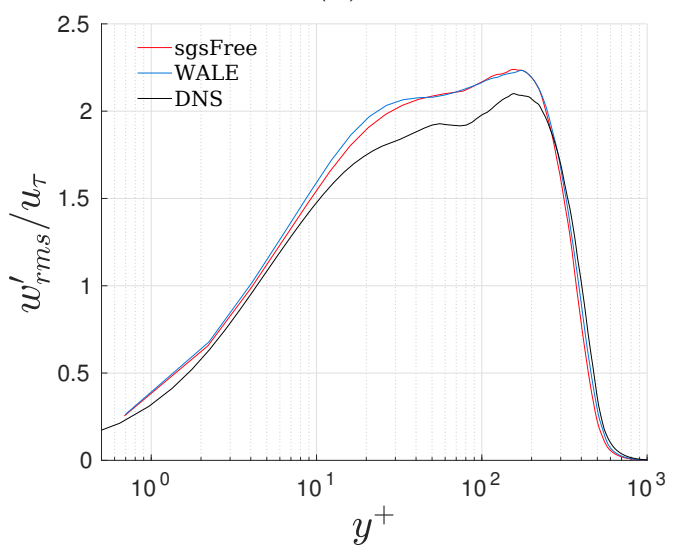

(f)

Figure 6-33: Inner-scaled $w_{r m s}^{\prime}$ versus the wall-normal coordinate taken at dimensionless streamwise locations $x / h$ of approximately -3 (a), 4 (b), 6 (c), 10 (d), 15 (e) and 19 (f). 


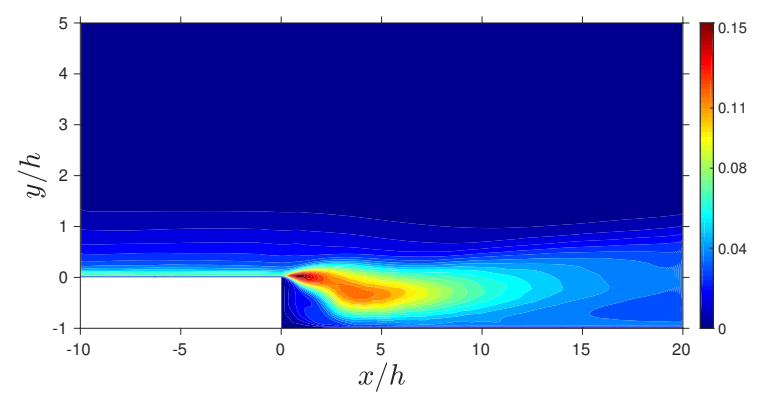

(a)

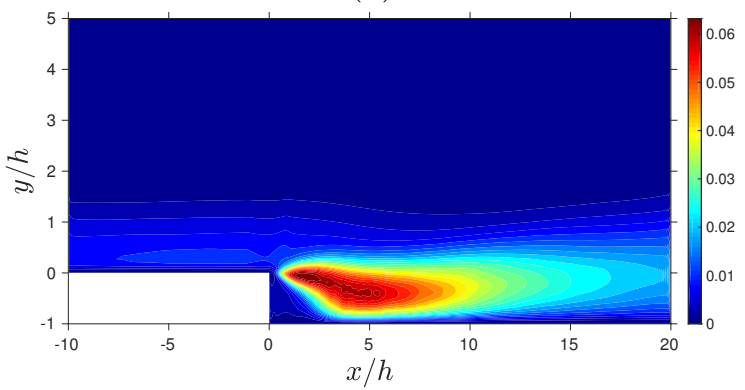

(c)

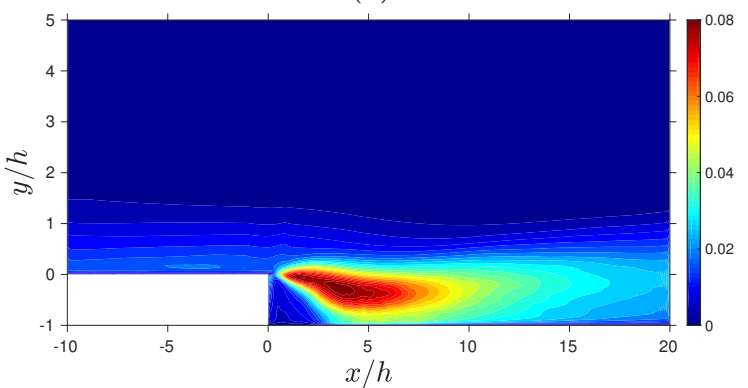

(e)

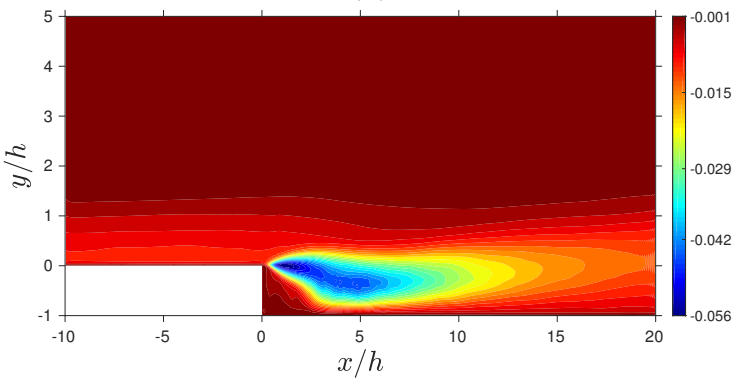

$(\mathrm{g})$

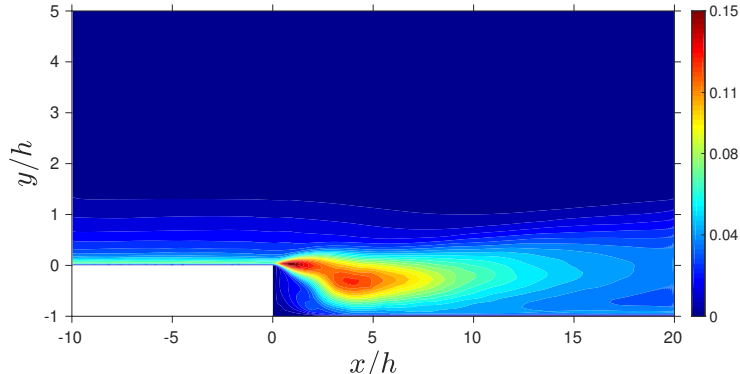

(b)

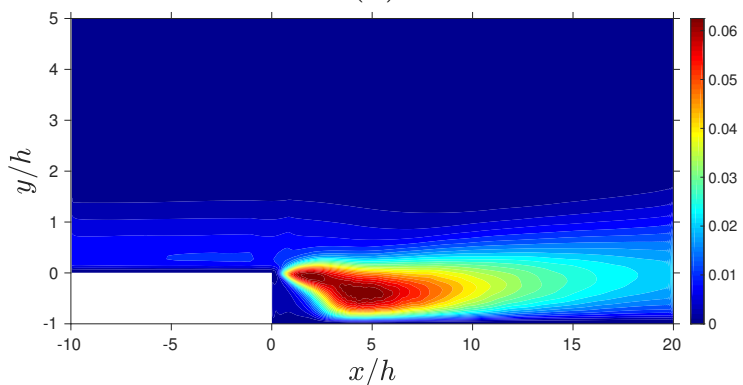

(d)

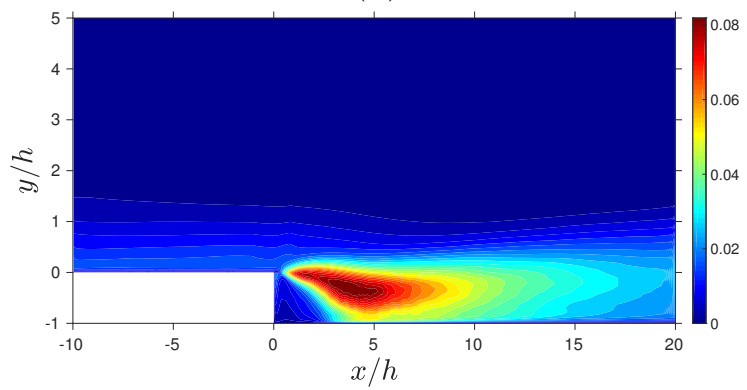

(f)

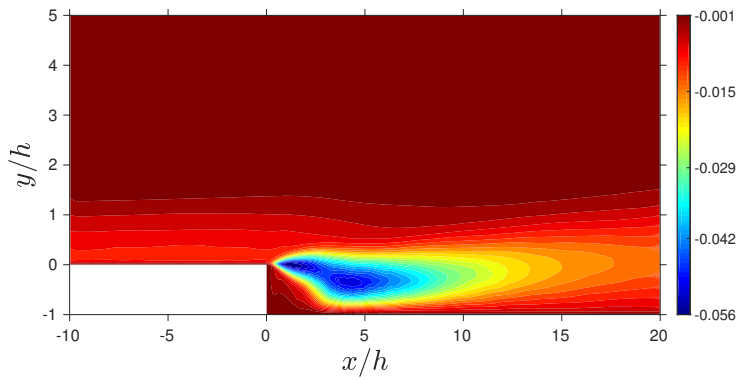

(h)

Figure 6-34: Reynolds stress components $\left(\mathrm{m}^{2} / \mathrm{s}^{2}\right)$ for an SGS-free model in the first column and WALE model in the second column with distinct components per row: $u_{r m s}^{\prime}, v_{r m s}^{\prime}, w_{r m s}^{\prime}$ and $\left\langle u^{\prime} v^{\prime}\right\rangle$ for the first, second, third and fourth row, respectively. 


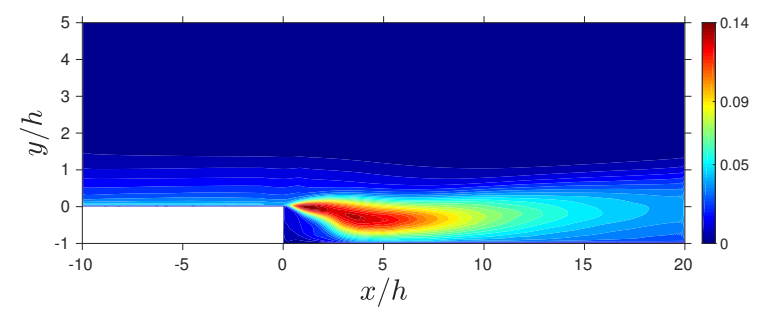

(a)

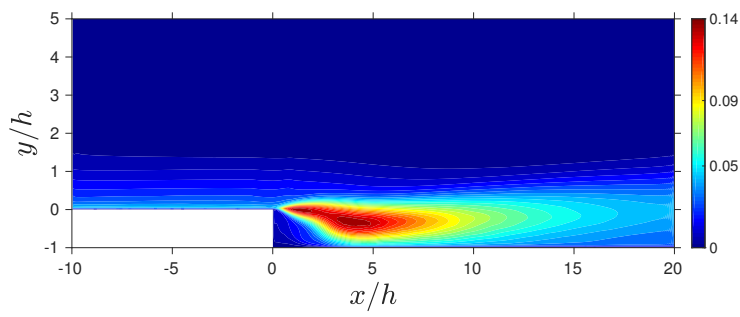

(b)

Figure 6-35: Turbulent kinetic energy $\left(\mathrm{m}^{2} / \mathrm{s}^{2}\right)$ for the SGS-free (a) and WALE (b) models.

\subsubsection{Vorticity}

The vorticity is presented in first- and second-order statistics as the mean and fluctuation tensor (i.e. the variance of the vorticity fluctuation) components, respectively. The RMS of the diagonal components of the vorticity fluctuation tensor are presented in Figure 6-36 for both SGS-free and WALE models. These denote the $\omega_{x}^{\prime r m s}, \omega_{y}^{\prime r m s}$ and $\omega_{z}^{\prime r m s}$ components normalized using $\nu / u_{\tau}^{2}$ versus the inner-scaled wall-normal direction at six different streamwise locations. The only noticeable variation between the two SGS models is at the reattachment location, $x / h \approx 6$; the WALE model over-predicts the SGS-free model in all vorticity components.

In Figure 6-37, the RMS of the diagonal components of the vorticity fluctuation tensor are displayed in contour plots. In these plots, the most observable discrepancy between SGS-free and WALE models is at the recirculation region and for the $\omega_{y}^{\prime r m s}$ component. The vorticity fluctuation's off-diagonal tensor components are presented in Figure 6-38; these consist of $\left\langle\omega_{x}^{\prime} \omega_{y}^{\prime}\right\rangle,\left\langle\omega_{x}^{\prime} \omega_{z}^{\prime}\right\rangle$ and $\left\langle\omega_{y}^{\prime} \omega_{z}^{\prime}\right\rangle$. Similarly, at the recirculation region, a slightly higher values of $\left\langle\omega_{y}^{\prime} \omega_{z}^{\prime}\right\rangle$ are seen in the WALE model as opposed to the SGS-free mode.

Also, the mean vorticity components $\left\langle\omega_{x}\right\rangle,\left\langle\omega_{y}\right\rangle$ and $\left\langle\omega_{z}\right\rangle$ are reported in contour plots in Figure 6-39. No major discrepancy is evident in such components. 


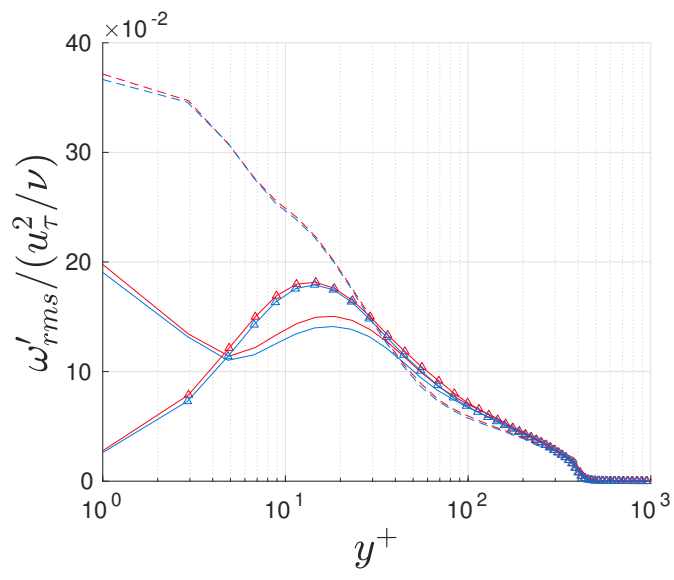

(a)

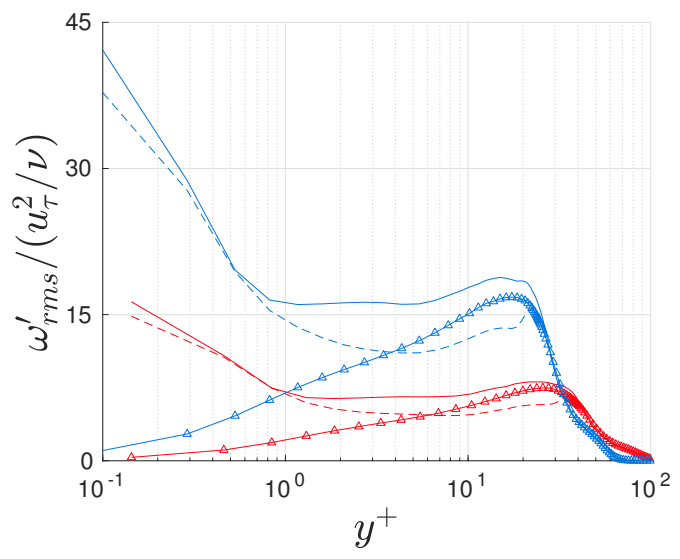

(c)

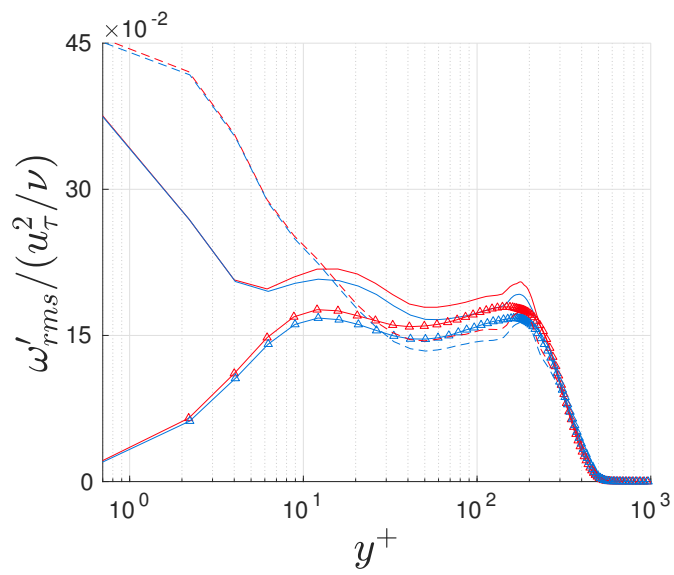

(e)

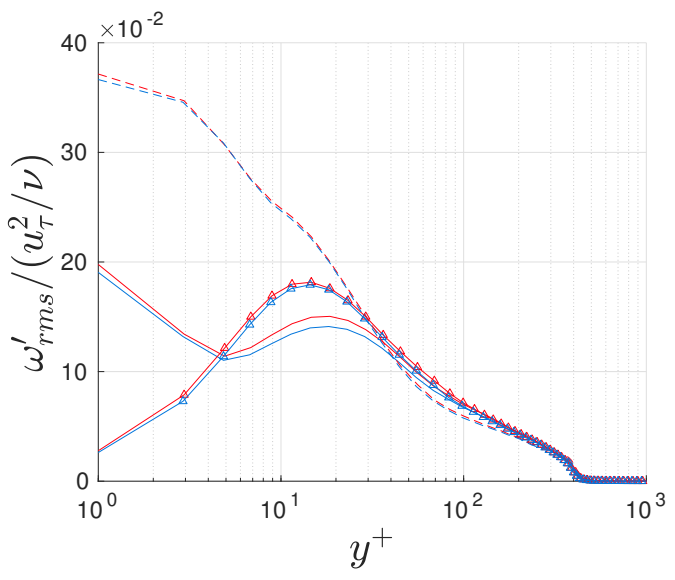

(b)

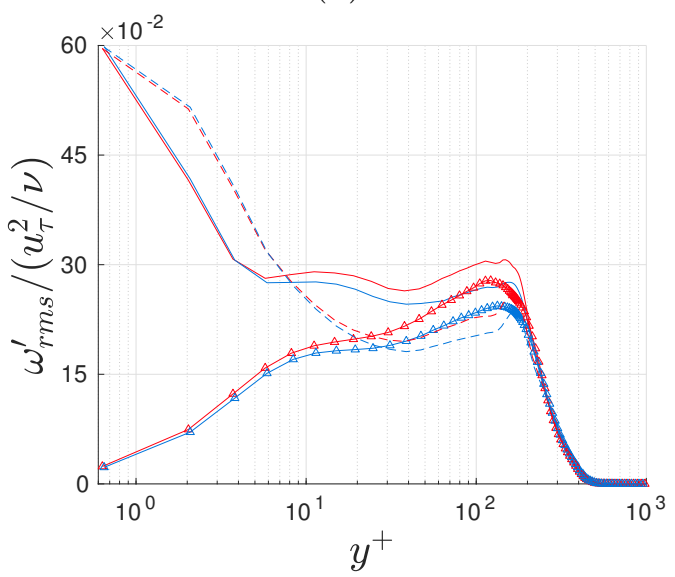

(d)

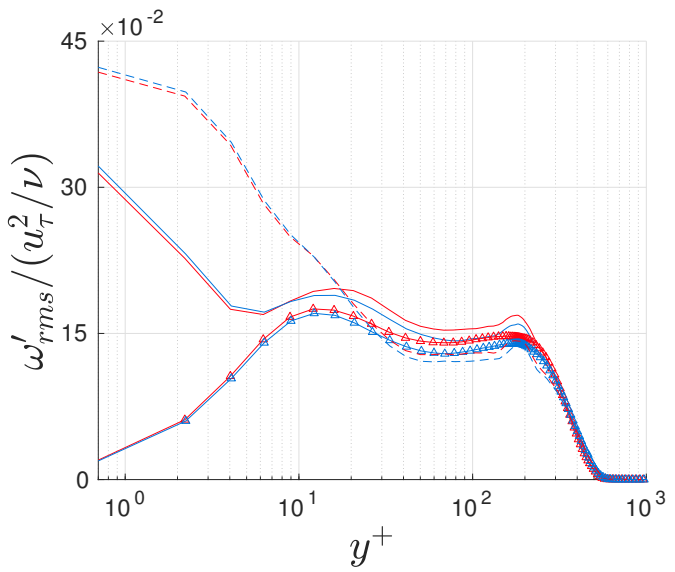

(f)

Figure 6-36: Vorticity fluctuation components non-dimensionalized using $\nu / u_{\tau}^{2}$ for SGS-free (red) and WALE (blue) models; $\omega_{x}^{\prime r m s}, \omega_{y}^{\prime r m s}$ and $\omega_{z}^{\prime r m s}$ are denoted using straight lines, triangles and dashed lines, respectively; profiles are taken at $x / h$ approximately -3 (a), 4 (b), 6 (c), 10 (d), 15 (e) and 19 (f) versus the inner-scaled wall-normal coordinate. 


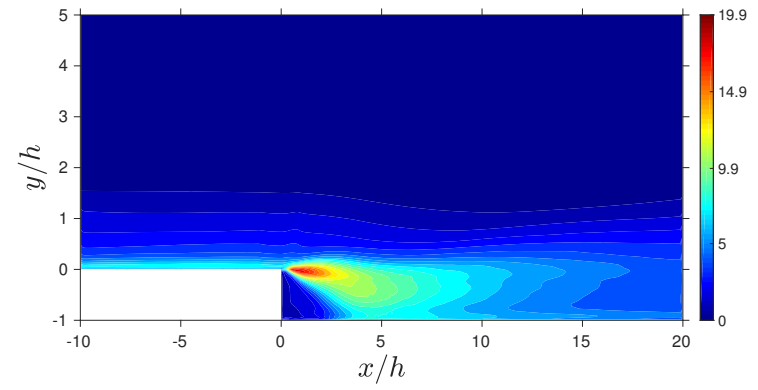

(a)

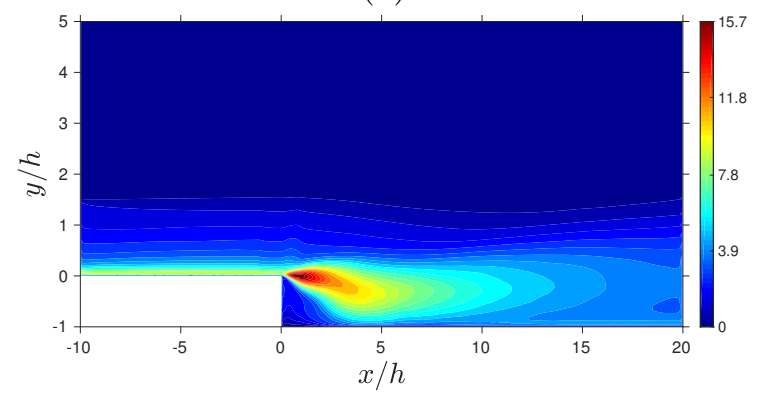

(c)

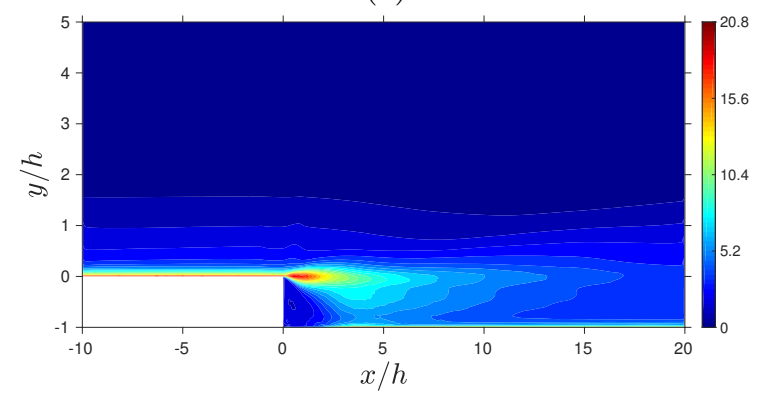

(e)

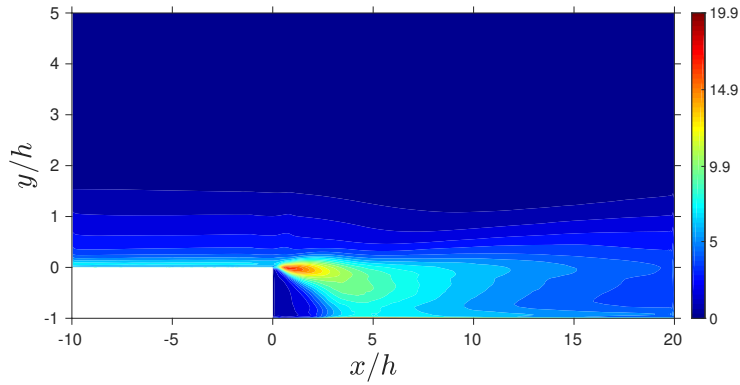

(b)

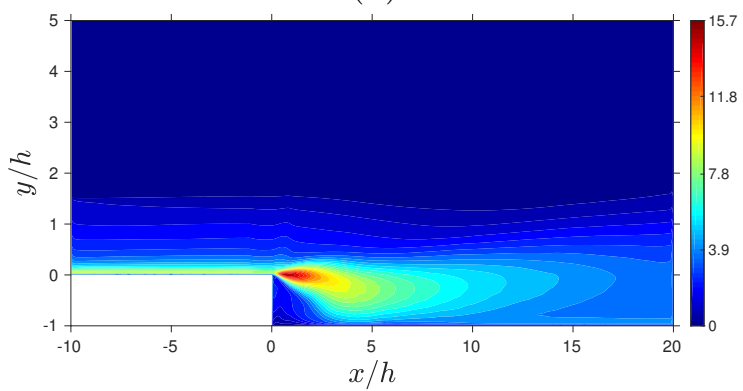

(d)

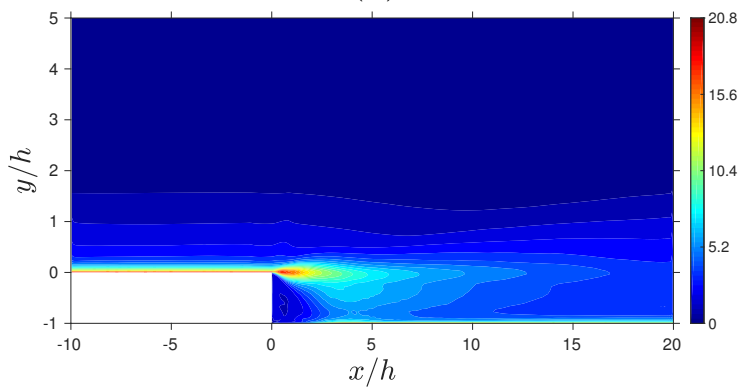

(f)

Figure 6-37: Vorticity fluctuation diagonal components for the SGS-free and WALE models illustrated in the first and second columns, respectively; $\omega_{x}^{\prime r m s}, \omega_{y}^{\prime r m s}$ and $\omega_{z}^{\prime r m s}$ are presented in the first, second and third row, respectively. 


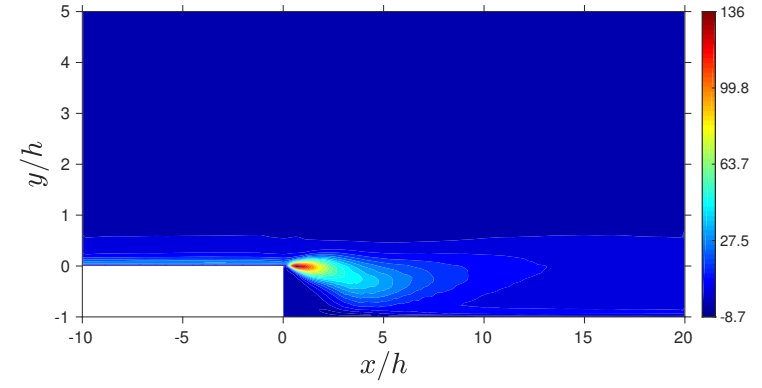

(a)

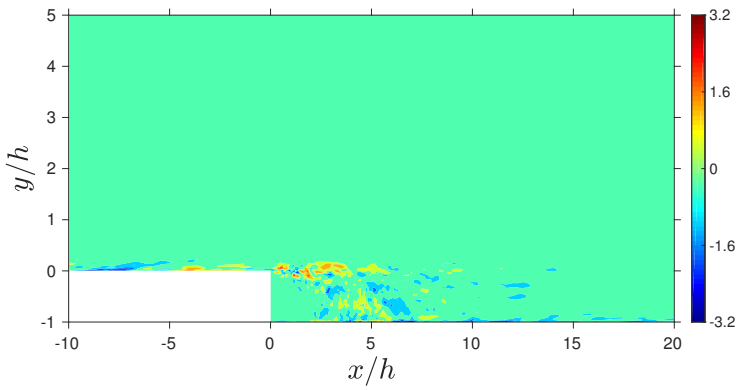

(c)

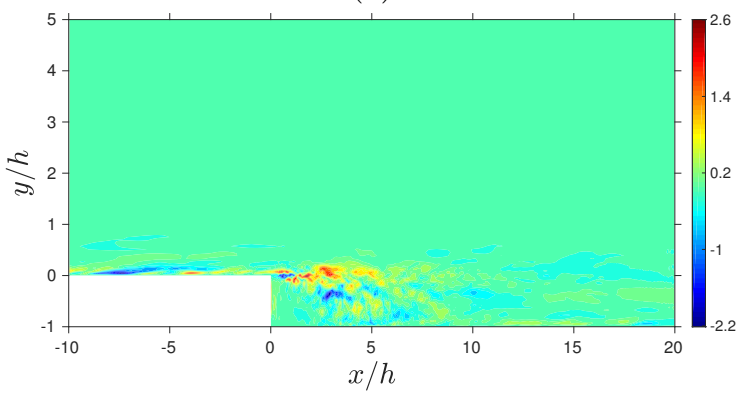

(e)

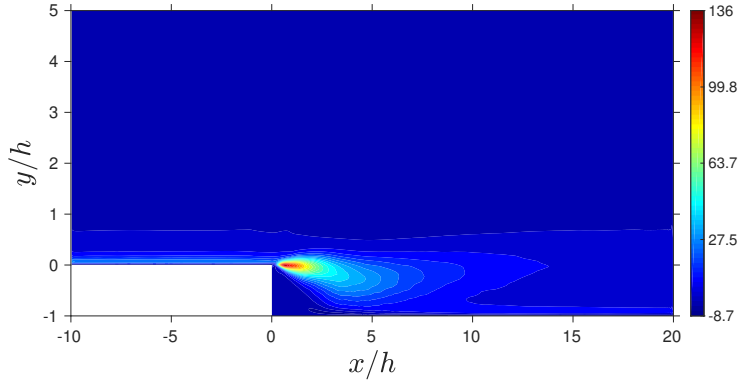

(b)

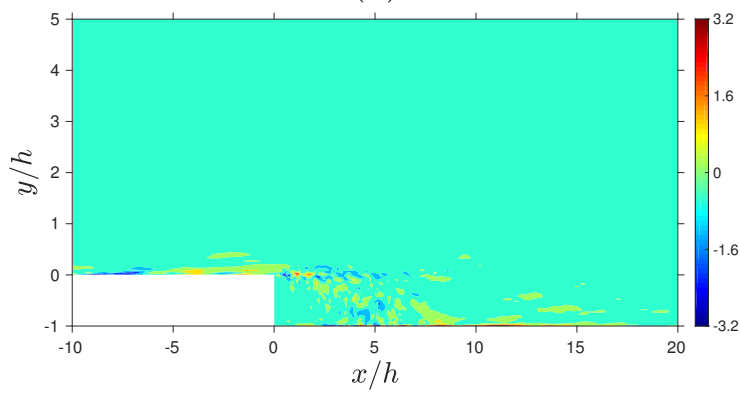

(d)

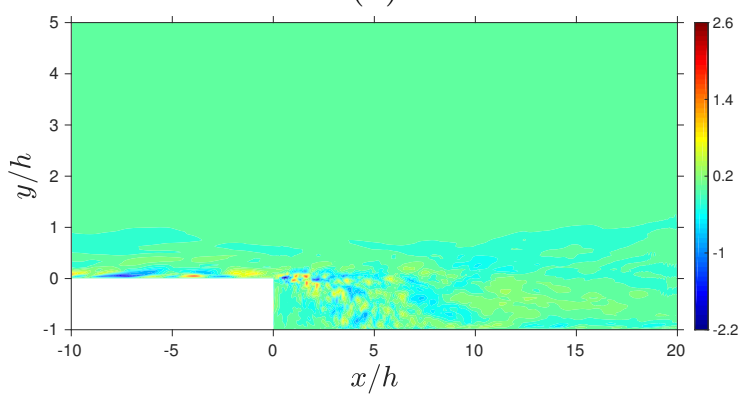

(f)

Figure 6-38: Vorticity fluctuation off-diagonal components for the SGS-free and WALE models illustrated in the first and second columns, respectively; $\left\langle\omega_{x}^{\prime} \omega_{y}^{\prime}\right\rangle,\left\langle\omega_{x}^{\prime} \omega_{z}^{\prime}\right\rangle$ and $\left\langle\omega_{y}^{\prime} \omega_{z}^{\prime}\right\rangle$ are presented in the first, second and third row, respectively. 


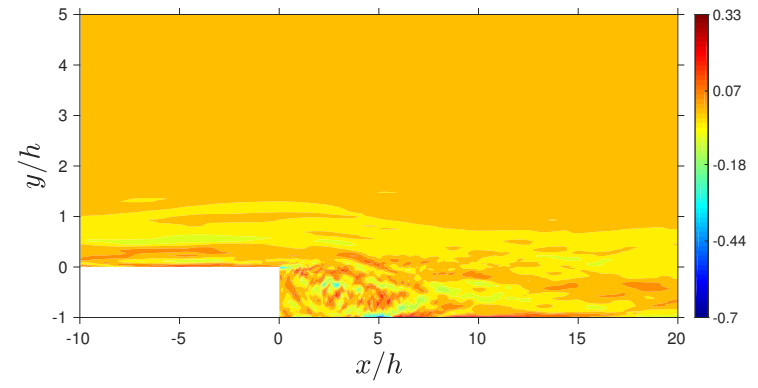

(a)

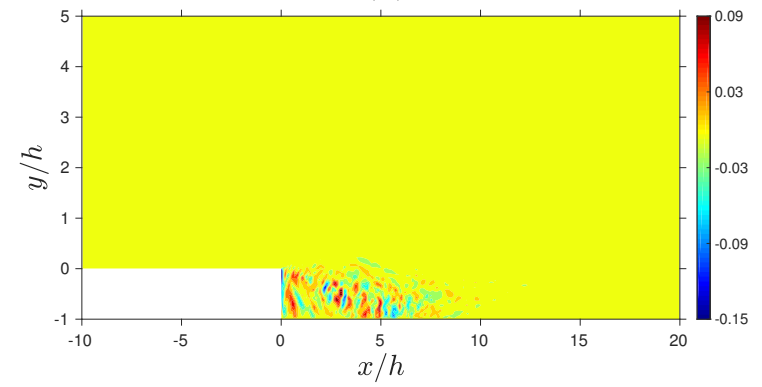

(c)

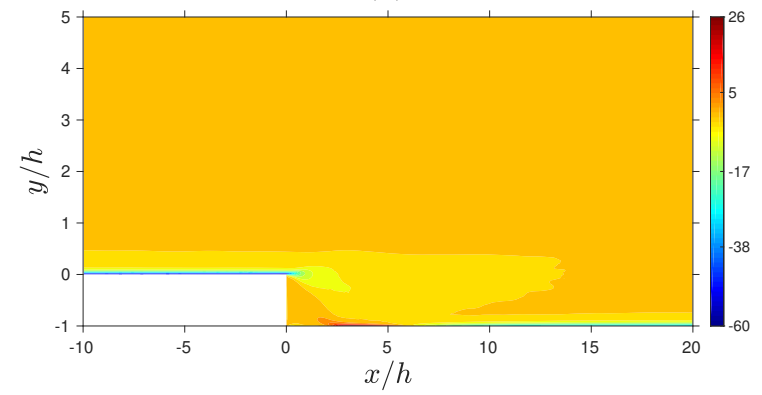

(e)

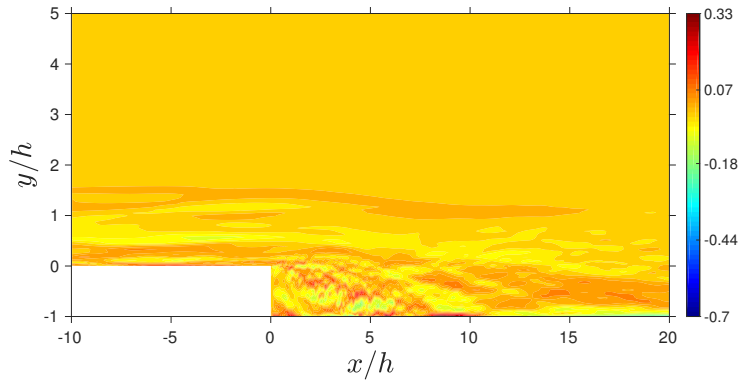

(b)

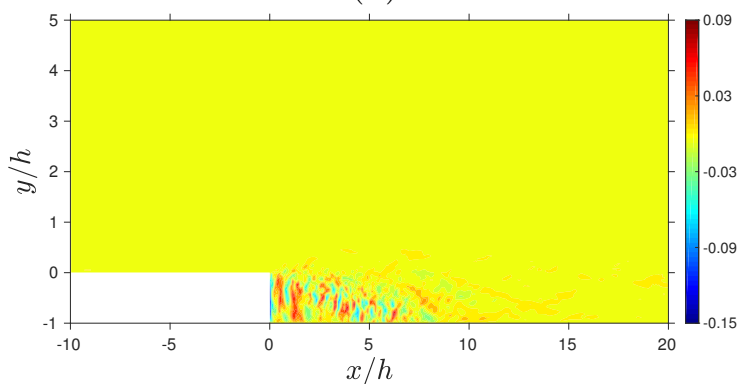

(d)

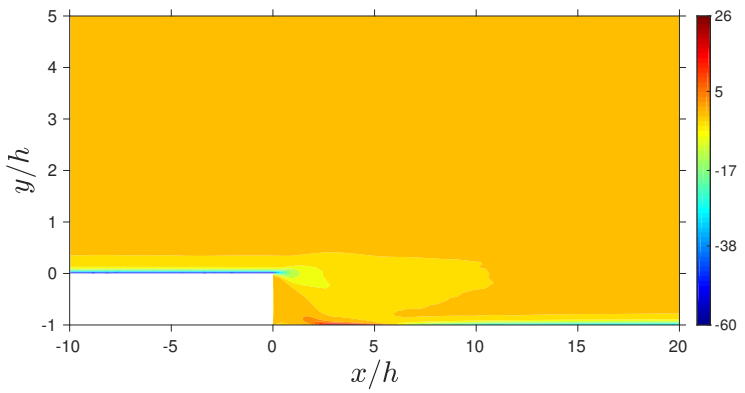

(f)

Figure 6-39: Mean vorticity components for the SGS-free and WALE models illustrated in the first and second columns, respectively; $\left\langle\omega_{x}\right\rangle,\left\langle\omega_{y}\right\rangle$ and $\left\langle\omega_{z}\right\rangle$ are presented in the first, second and third row, respectively. 


\subsubsection{High-order Statistical Moments}

The high-order central statistical moments used in this part are up to fifth order. The skewness, $S$, and kurtosis, $K$, along with their normalizations are predefined in Equation D.2 and Equation D.3. Recall, these correspond to the third and fourth moments, respectively. As for the fifth, its definition follows by using the same expression as that of Equation D.1 with an analogous normalization procedure. The fifth moment, also known as the hyperskewness, is denoted by П. For its mathematical expression, refer to Equation D.4.

The statistics are displayed at six different streamwise locations versus the innerand outer-scaled wall-normal coordinate in Figures 6-40 to 6-51. In each figure, the first, second and third columns correspond to the statistics of the $u, v$ and $w$ velocity components; the first, second and third rows correspond to the skewness, kurtosis and hyperskewness, respectively; the SGS-free and WALE models are denoted by red and blue colors, respectively.

The most notable deviation in between the two SGS models is observed for the streamwise components. This is evident even at the third moment, e.g. see $S_{u}$ in Figure 6-40. Accordingly, for higher moments, the error becomes more pronounced. For instance, this is evident for the $K_{u}$ and $\Pi_{u}$ components in the same figure. The $v$ component statistics display a much better agreement between the SGS-free and WALE models, e.g. see second column in Figure 6-44. 


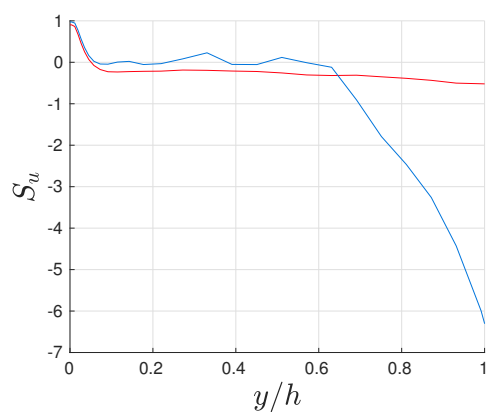

(a)

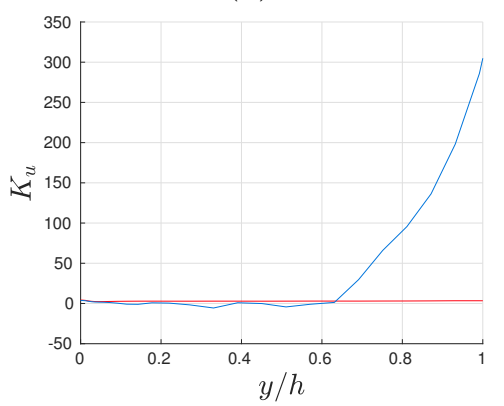

(d)

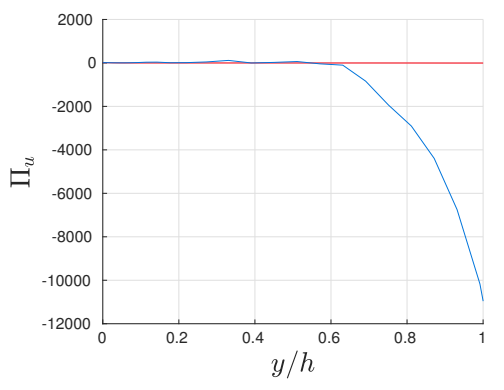

(g)

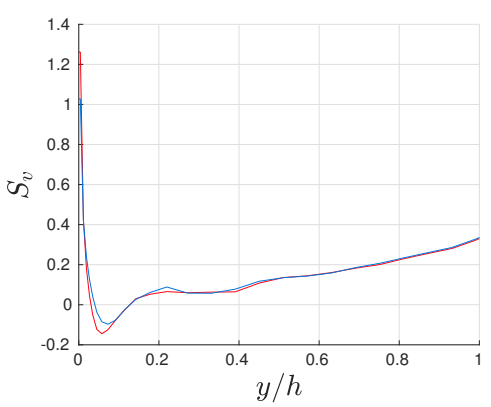

(b)

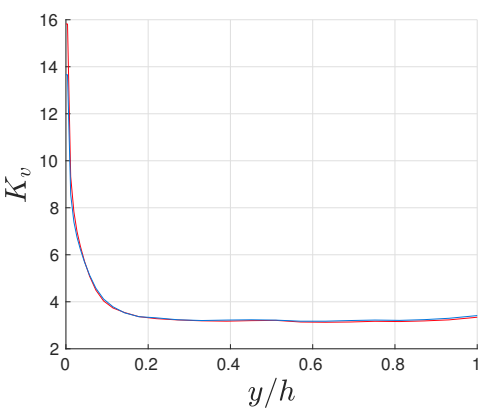

(e)

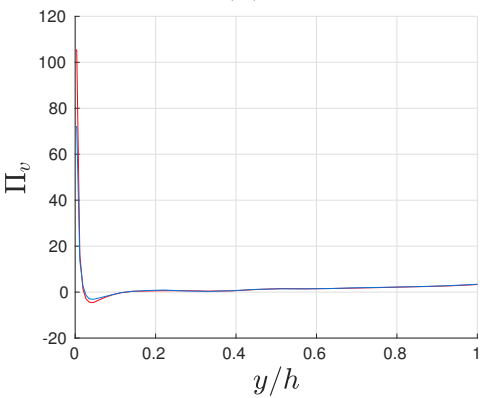

(h)

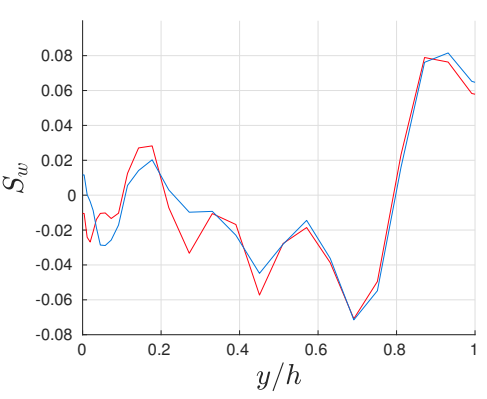

(c)

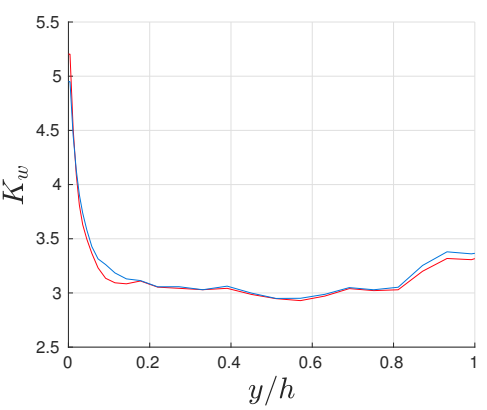

(f)

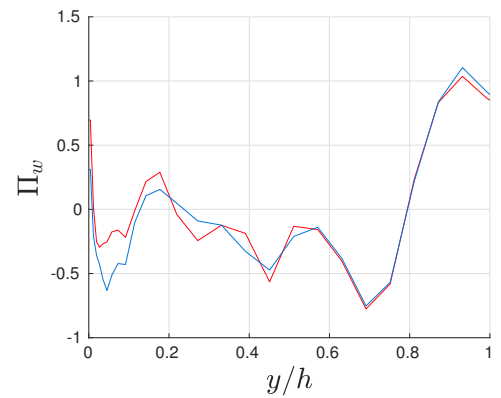

(i)

Figure 6-40: Normalized high-order central statistical moments versus outer-scaled wall-normal coordinates at $x / h \approx-3$ for an SGS-free model (red) and WALE model (blue); the first, second and third columns correspond to the streamwise, wall-normal and spanwise components and the first, second and third rows correspond to the third, fourth and fifth moment. 


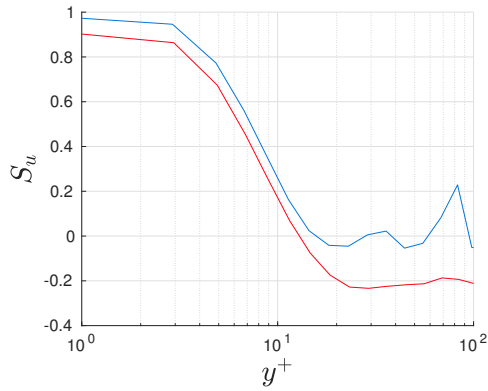

(a)

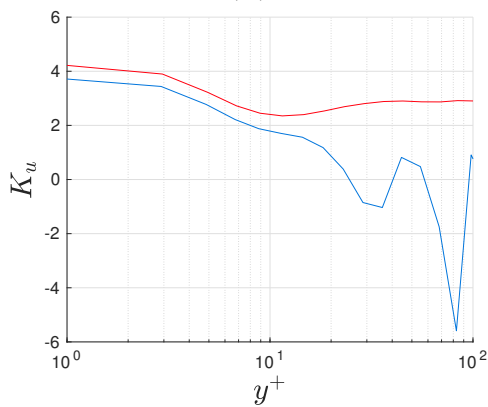

(d)

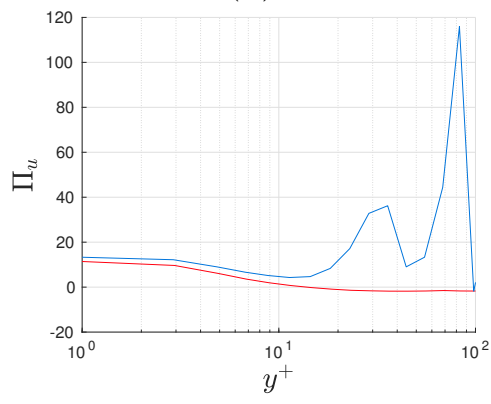

$(\mathrm{g})$

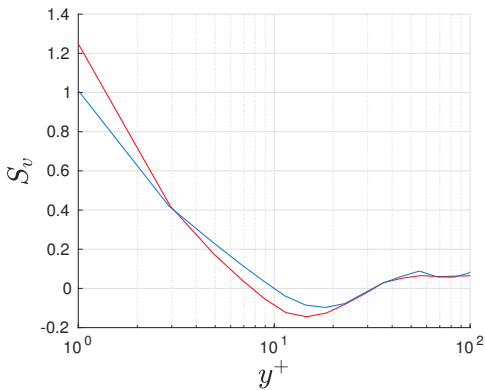

(b)

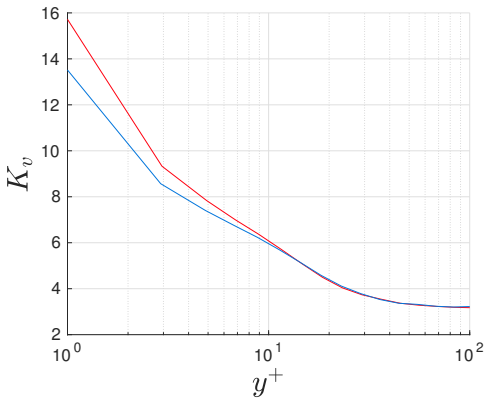

(e)

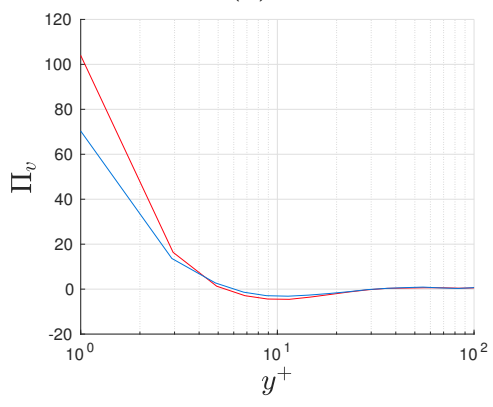

(h)

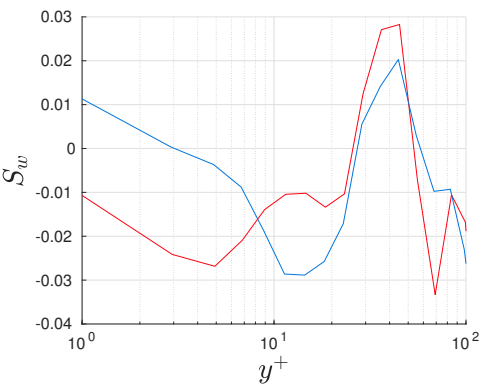

(c)

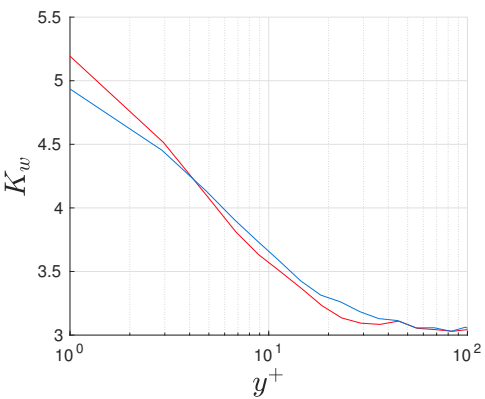

(f)

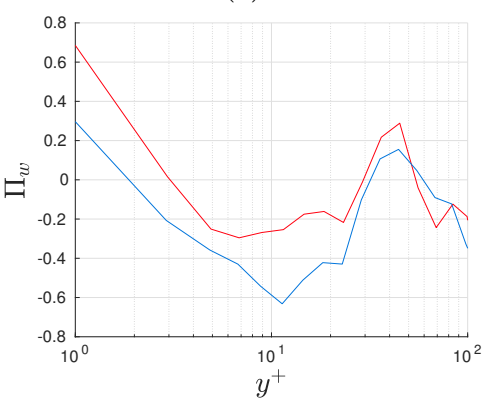

(i)

Figure 6-41: Normalized high-order central statistical moments versus inner-scaled wall-normal coordinates at $x / h \approx-3$ for an SGS-free model (red) and WALE model (blue); the first, second and third columns correspond to the streamwise, wall-normal and spanwise components and the first, second and third rows correspond to the third, fourth and fifth moment. 


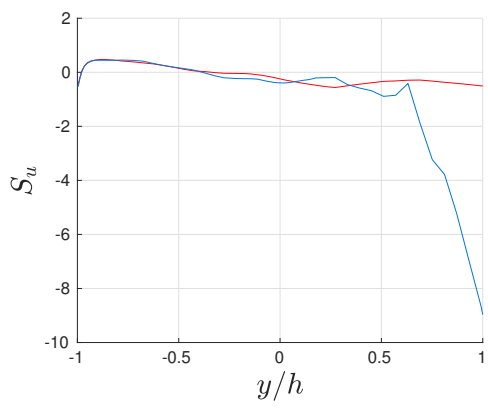

(a)

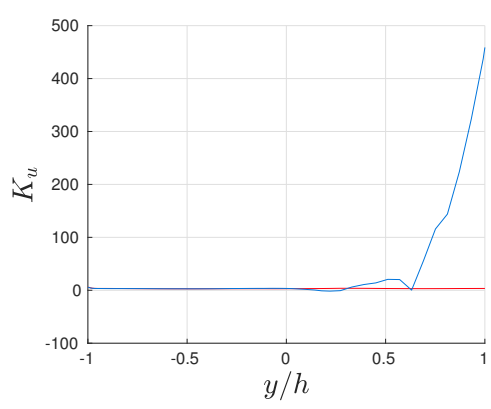

(d)

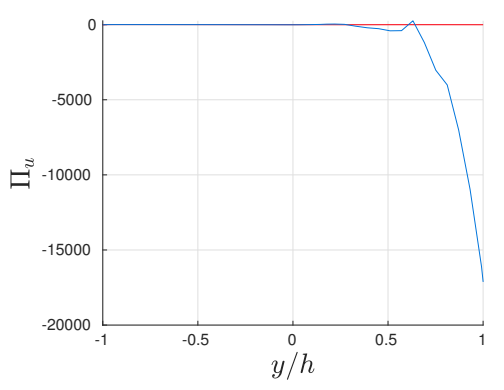

(g)

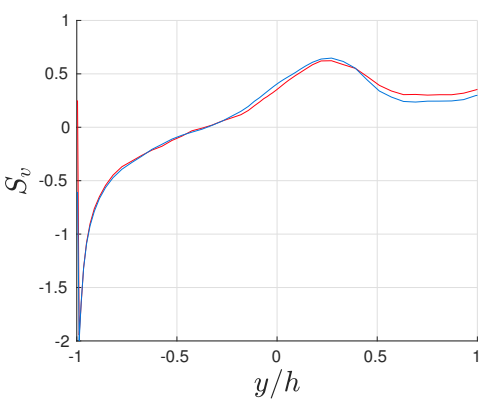

(b)

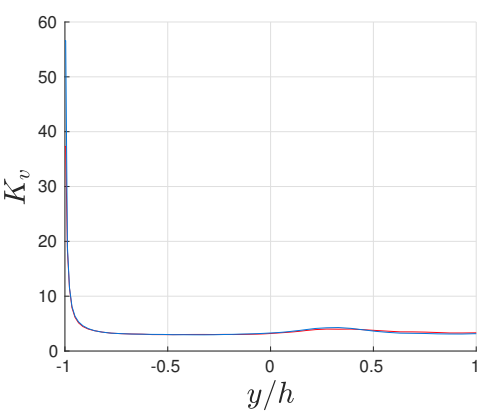

(e)

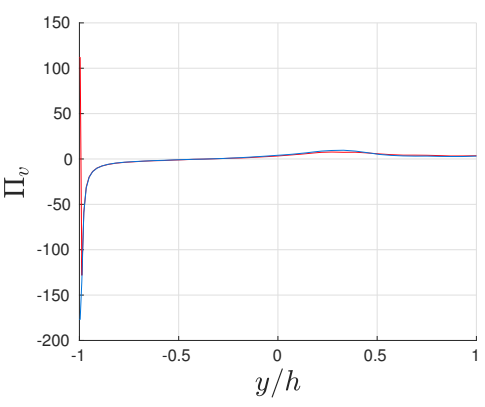

(h)

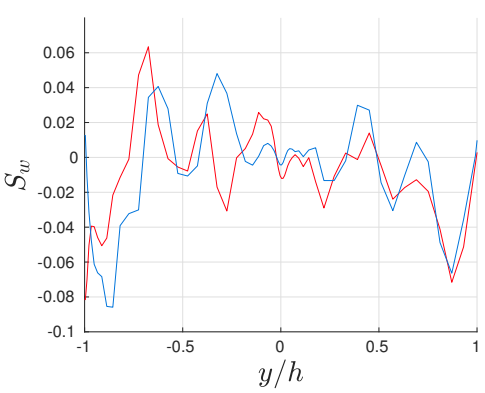

(c)

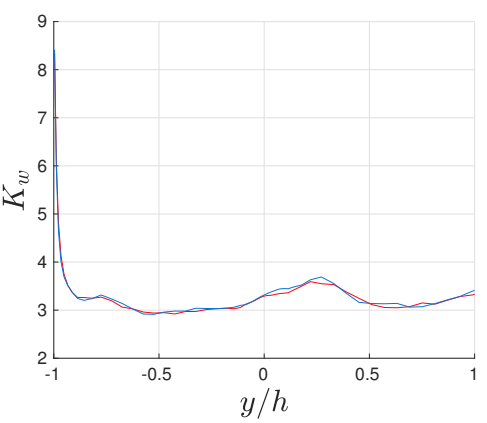

(f)

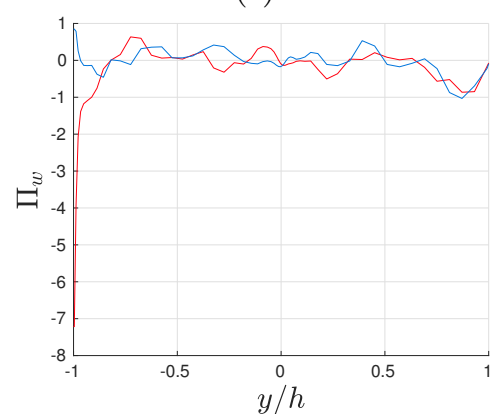

(i)

Figure 6-42: Normalized high-order central statistical moments versus outer-scaled wall-normal coordinates at $x / h \approx 4$ for an SGS-free model (red) and WALE model (blue); the first, second and third columns correspond to the streamwise, wall-normal and spanwise components and the first, second and third rows correspond to the third, fourth and fifth moment. 


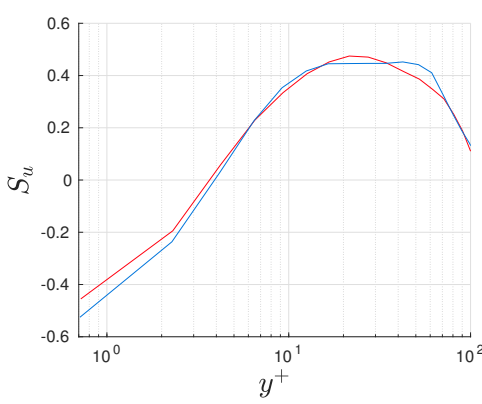

(a)

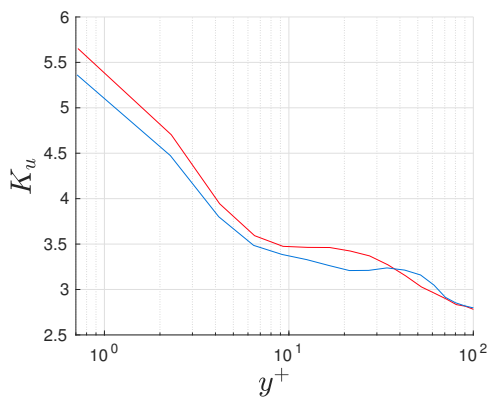

(d)

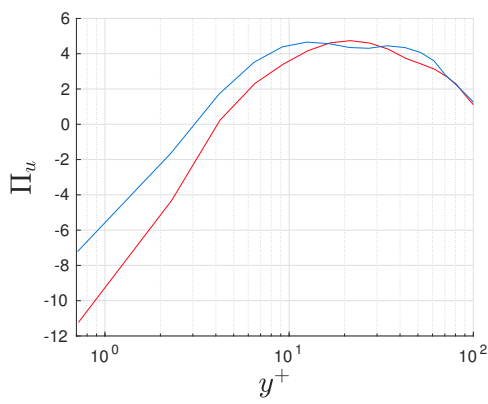

(g)

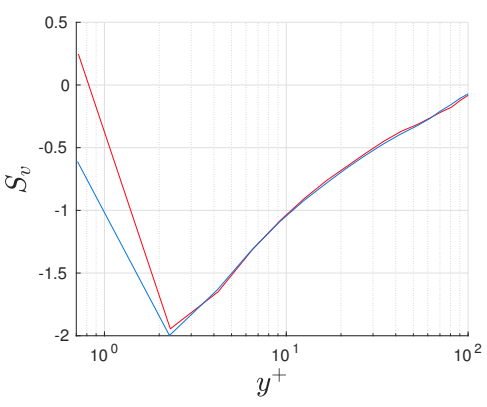

(b)

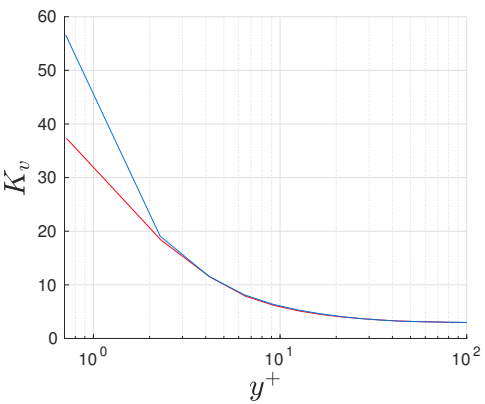

(e)

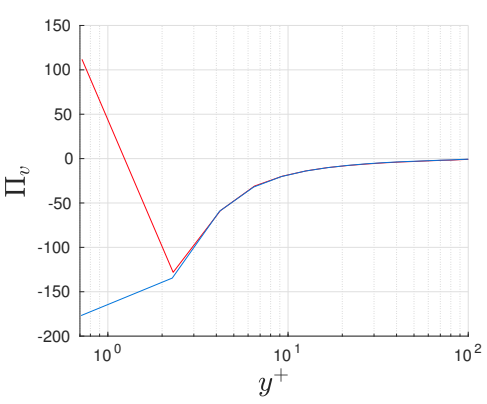

(h)

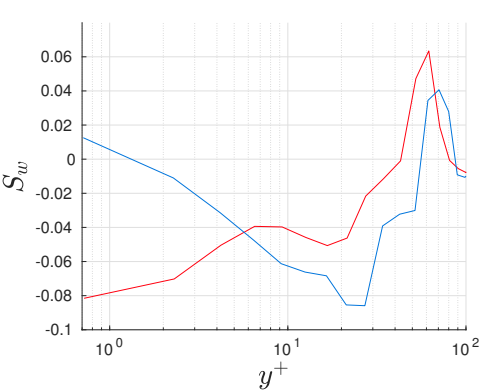

(c)

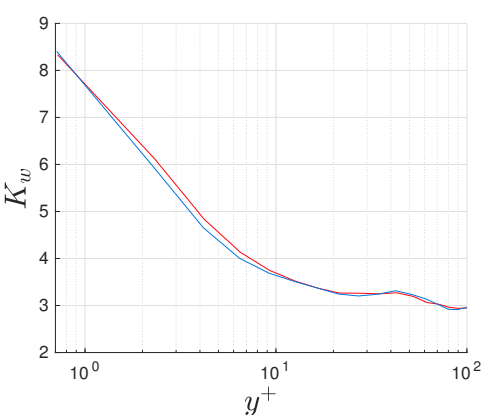

(f)

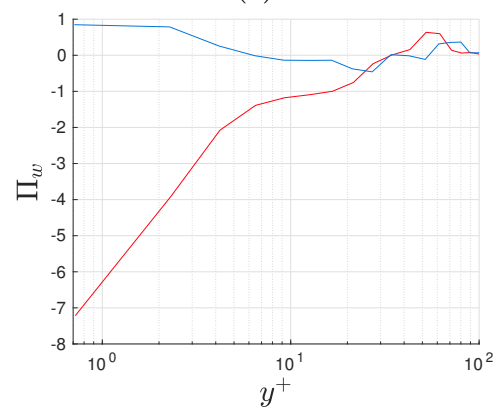

(i)

Figure 6-43: Normalized high-order central statistical moments versus inner-scaled wall-normal coordinates at $x / h \approx 4$ for an SGS-free model (red) and WALE model (blue); the first, second and third columns correspond to the streamwise, wall-normal and spanwise components and the first, second and third rows correspond to the third, fourth and fifth moment. 


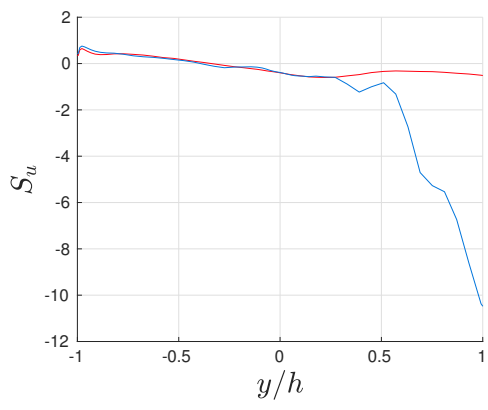

(a)

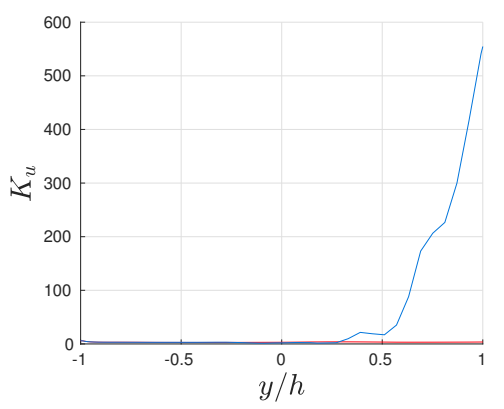

(d)

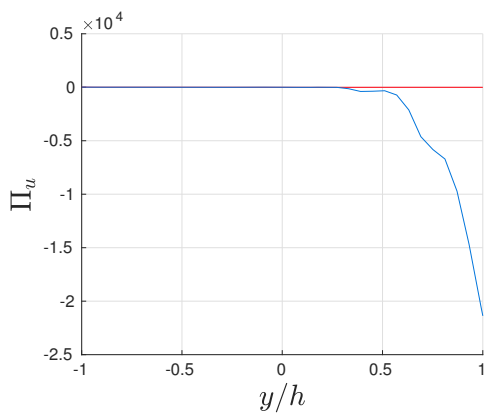

(g)

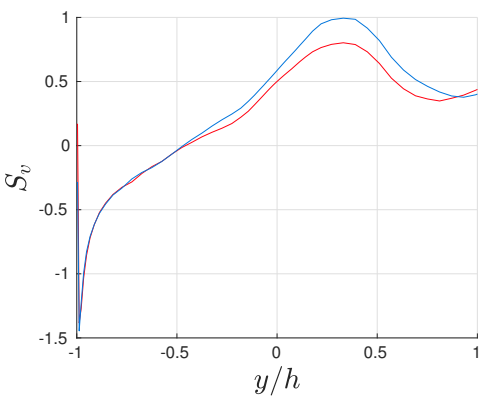

(b)

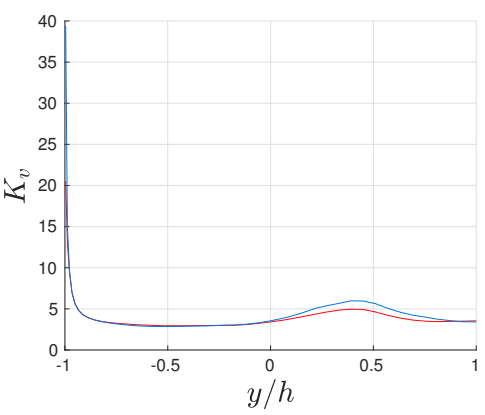

(e)

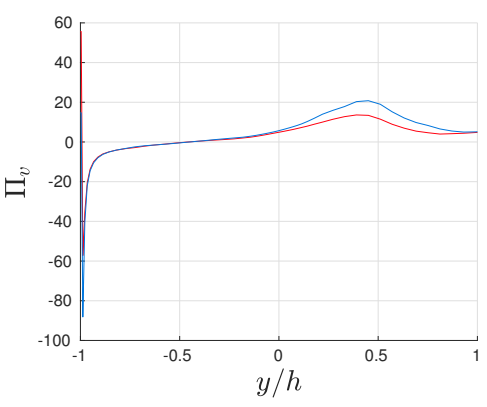

(h)

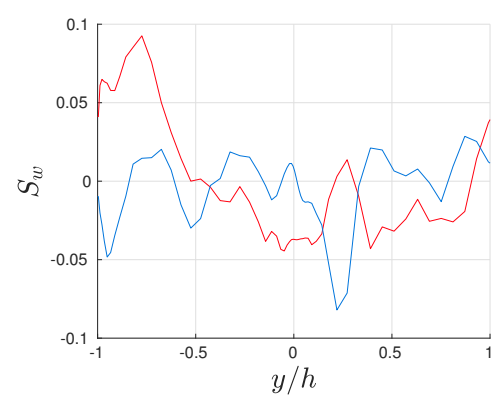

(c)

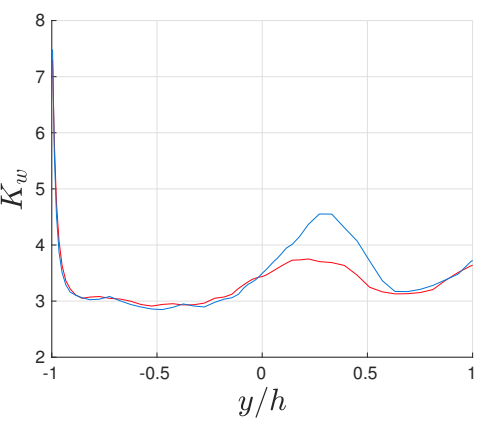

(f)

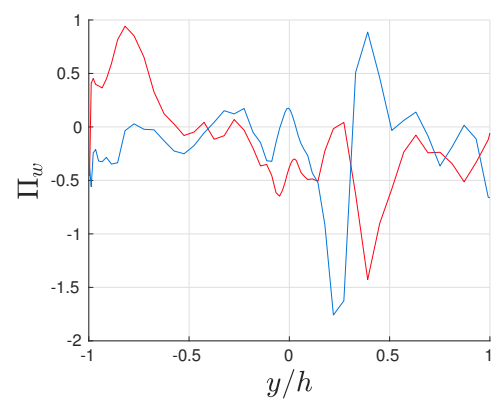

(i)

Figure 6-44: Normalized high-order central statistical moments versus outer-scaled wall-normal coordinates at $x / h \approx 6$ for an SGS-free model (red) and WALE model (blue); the first, second and third columns correspond to the streamwise, wall-normal and spanwise components and the first, second and third rows correspond to the third, fourth and fifth moment. 


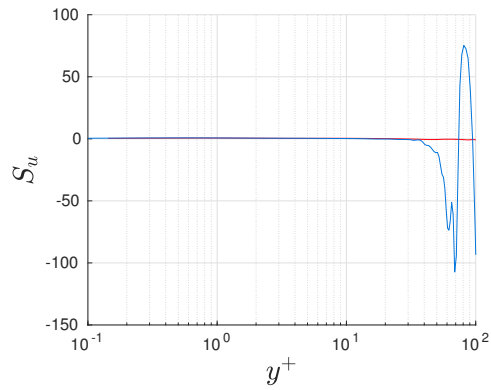

(a)

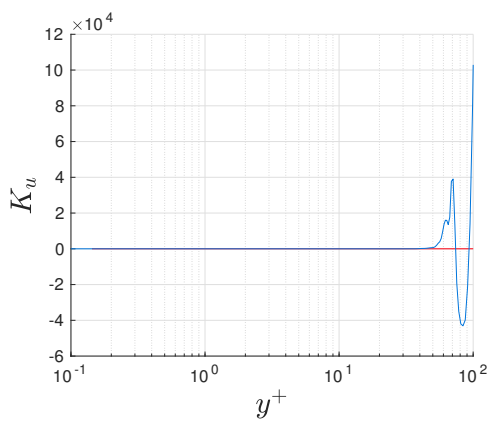

(d)

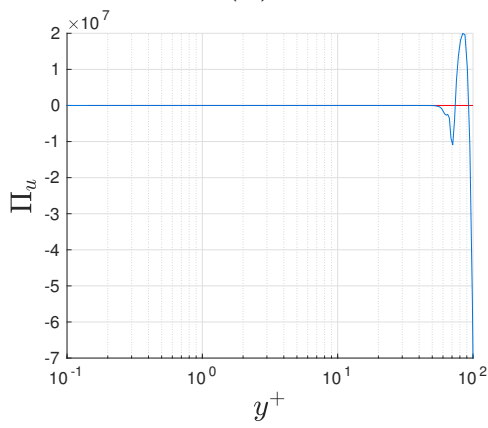

(g)

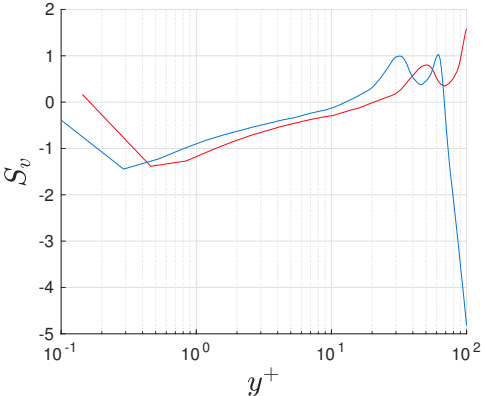

(b)

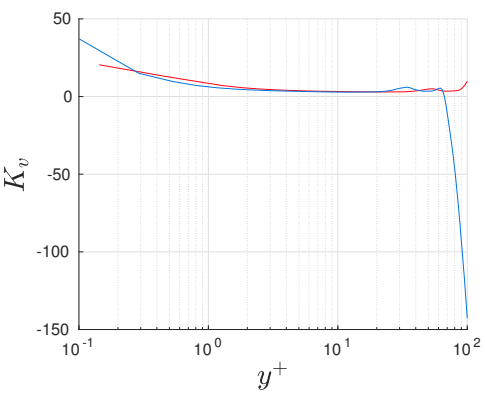

(e)

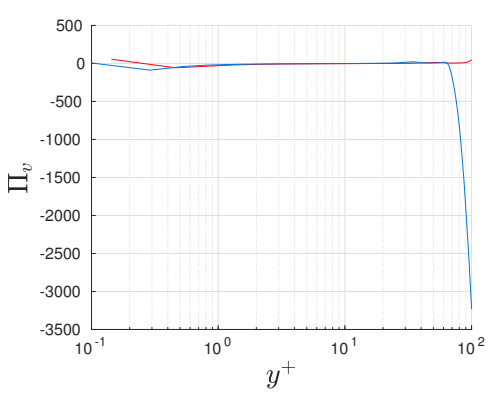

(h)

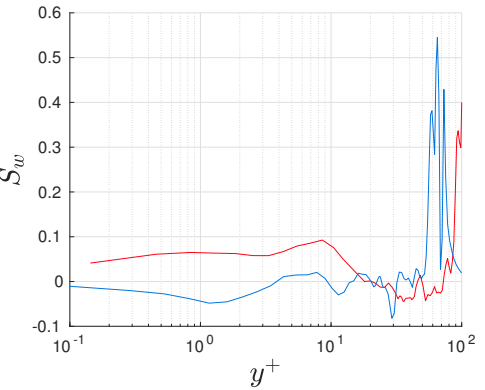

(c)

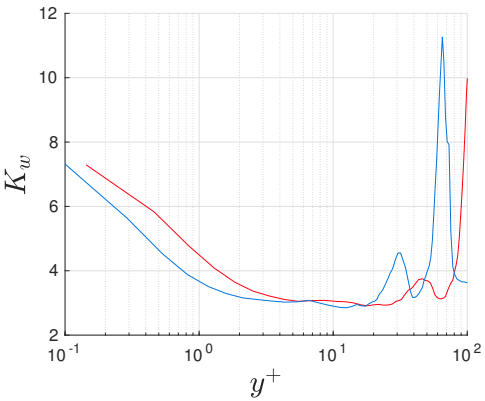

(f)

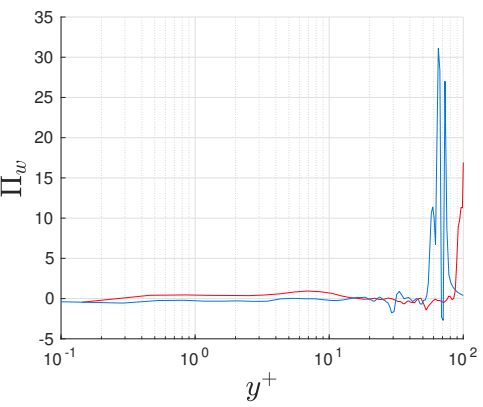

(i)

Figure 6-45: Normalized high-order central statistical moments versus inner-scaled wall-normal coordinates at $x / h \approx 6$ for an SGS-free model (red) and WALE model (blue); the first, second and third columns correspond to the streamwise, wall-normal and spanwise components and the first, second and third rows correspond to the third, fourth and fifth moment. 


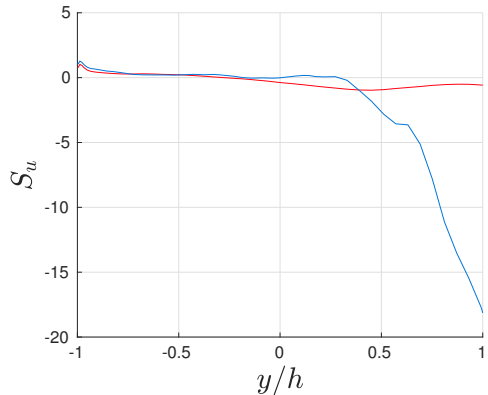

(a)

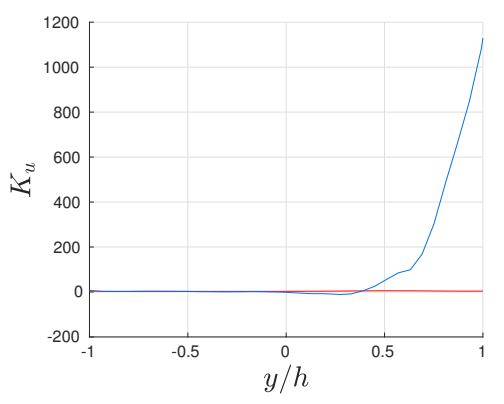

(d)

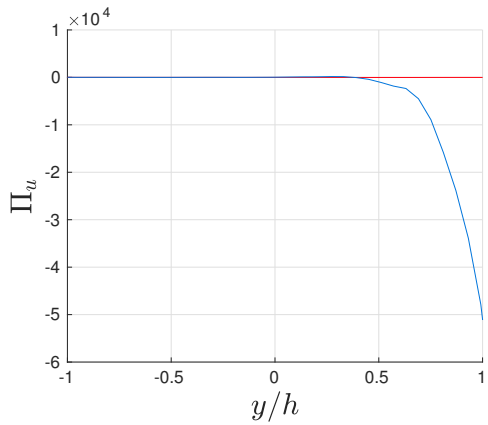

(g)

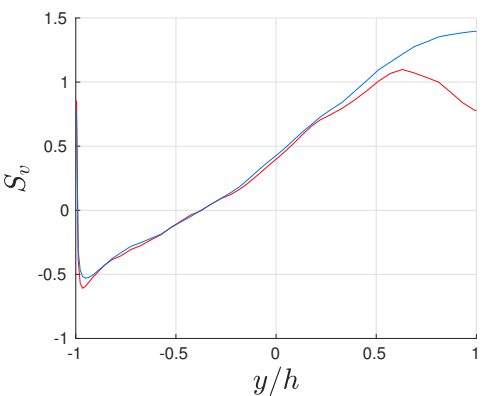

(b)

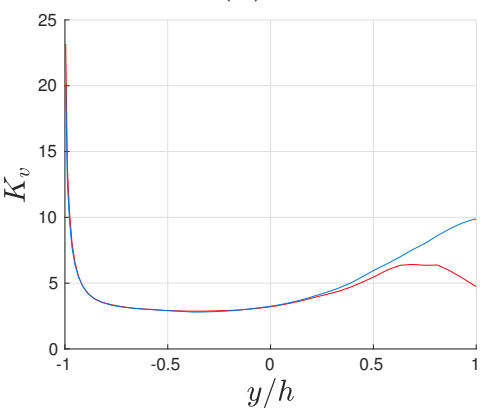

(e)

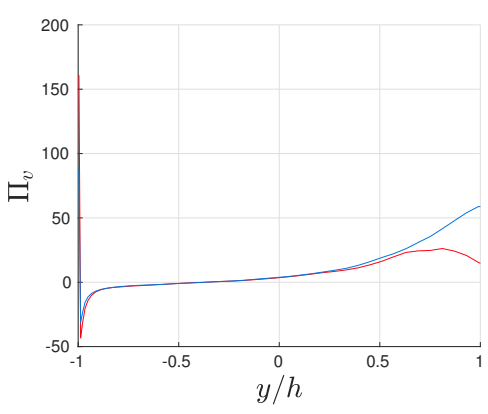

(h)

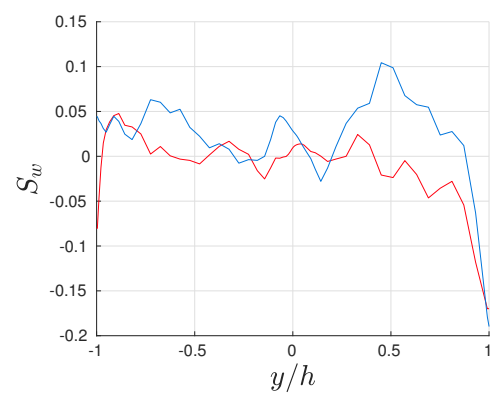

(c)

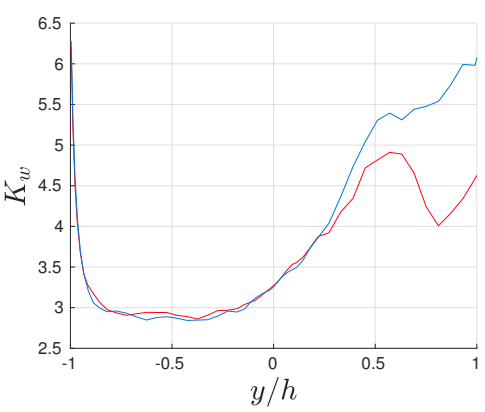

(f)

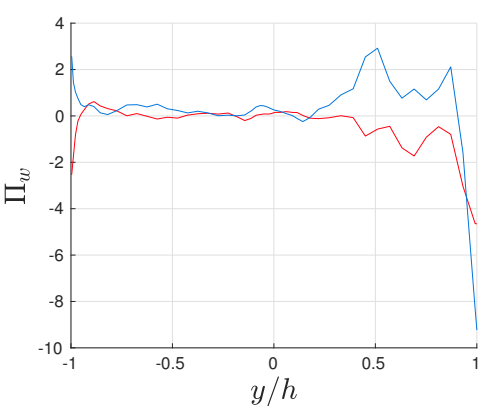

(i)

Figure 6-46: Normalized high-order central statistical moments versus outer-scaled wall-normal coordinates at $x / h \approx 10$ for an SGS-free model (red) and WALE model (blue); the first, second and third columns correspond to the streamwise, wall-normal and spanwise components and the first, second and third rows correspond to the third, fourth and fifth moment. 


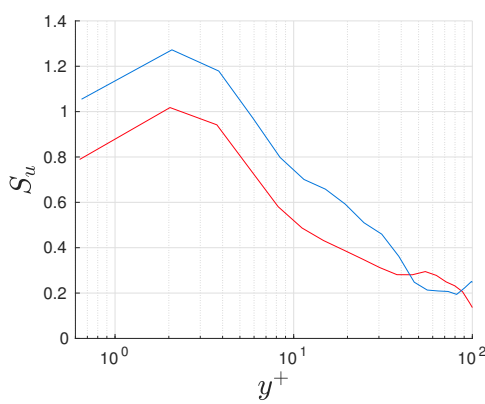

(a)

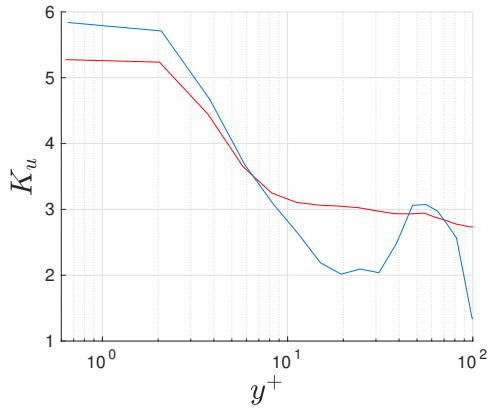

(d)

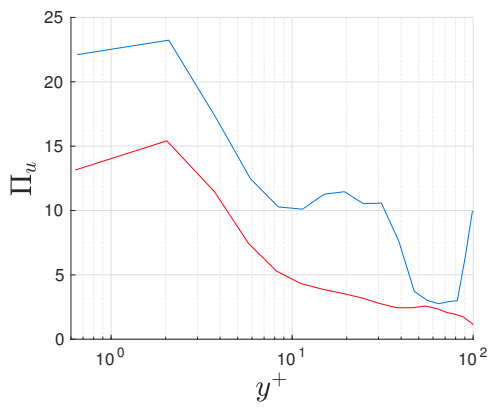

(g)

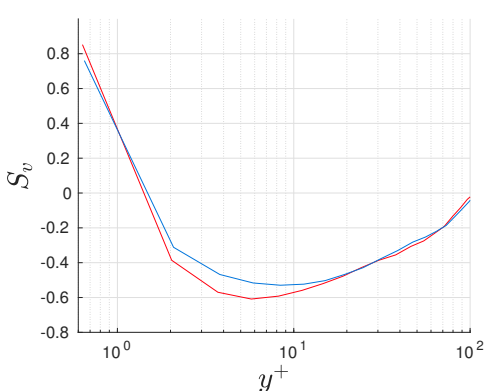

(b)

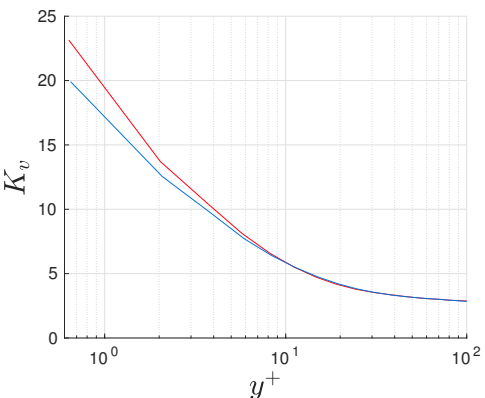

(e)

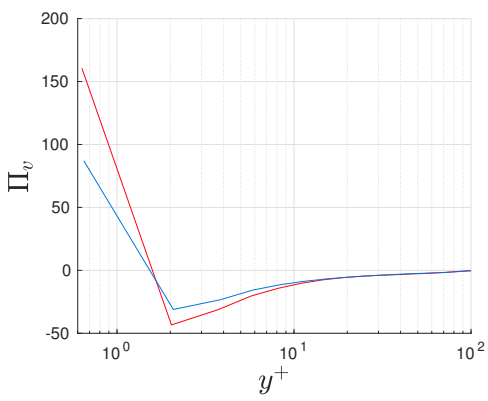

(h)

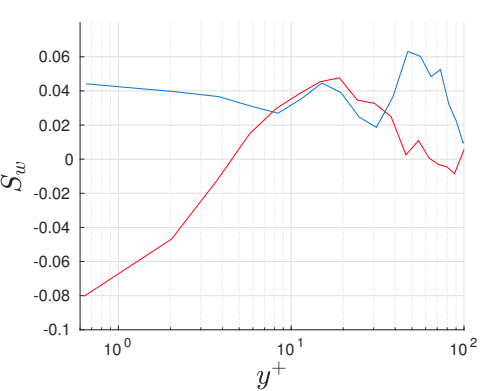

(c)

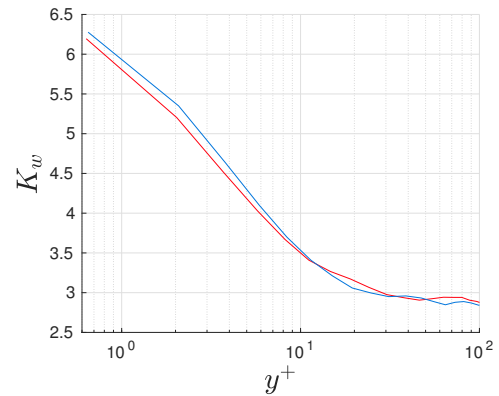

(f)

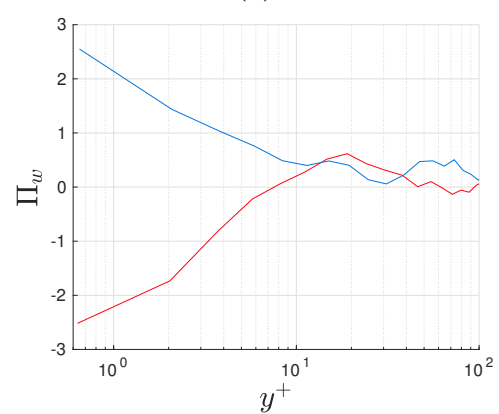

(i)

Figure 6-47: Normalized high-order central statistical moments versus inner-scaled wall-normal coordinates at $x / h \approx 10$ for an SGS-free model (red) and WALE model (blue); the first, second and third columns correspond to the streamwise, wall-normal and spanwise components and the first, second and third rows correspond to the third, fourth and fifth moment. 


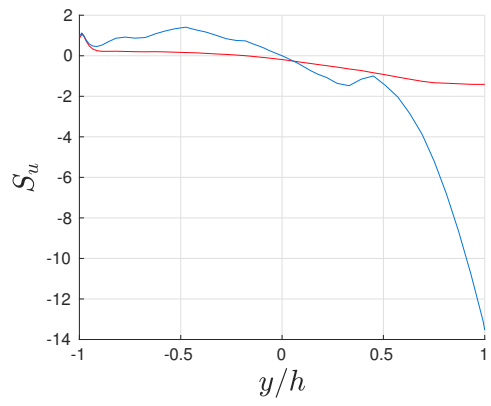

(a)

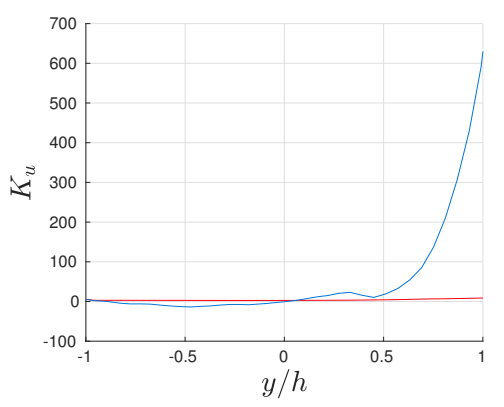

(d)

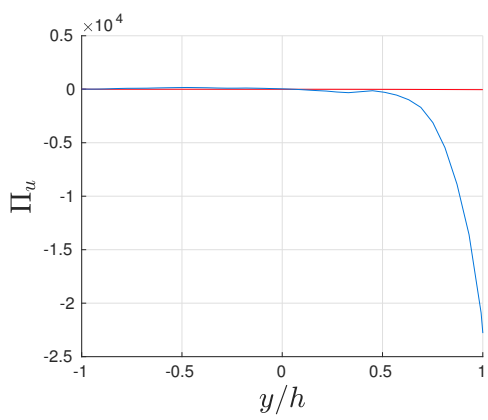

(g)

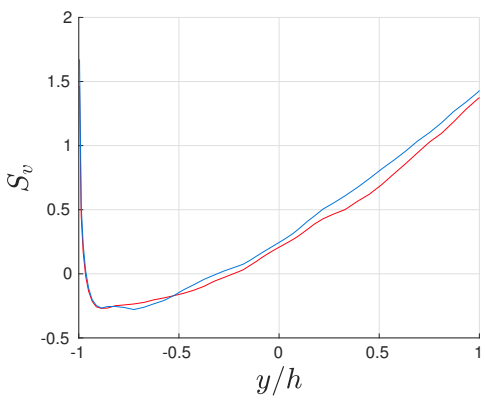

(b)

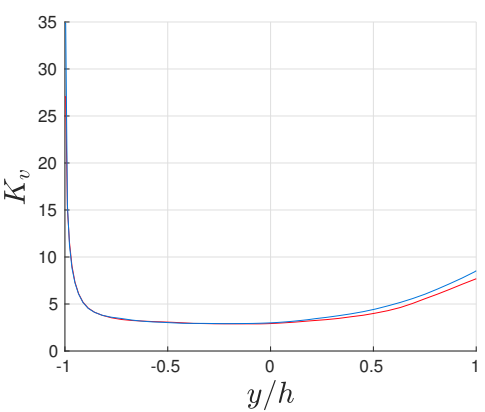

(e)

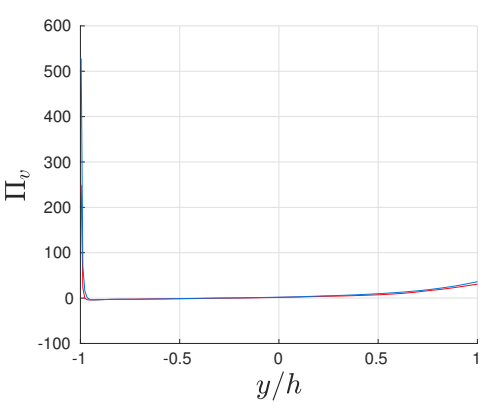

(h)

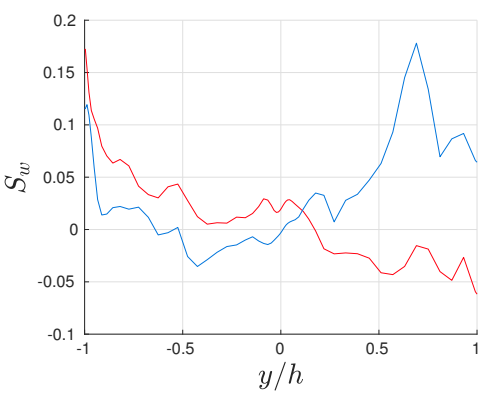

(c)

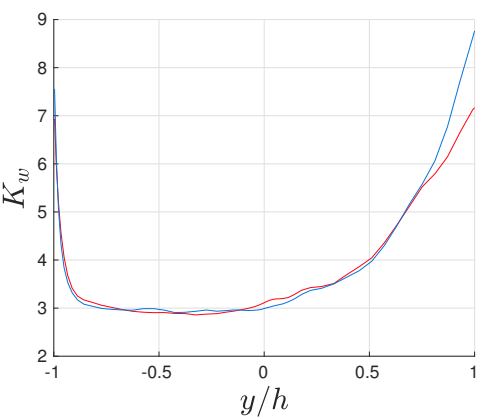

(f)

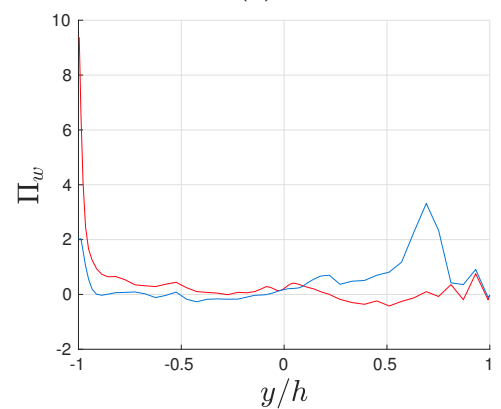

(i)

Figure 6-48: Normalized high-order central statistical moments versus outer-scaled wall-normal coordinates at $x / h \approx 15$ for an SGS-free model (red) and WALE model (blue); the first, second and third columns correspond to the streamwise, wall-normal and spanwise components and the first, second and third rows correspond to the third, fourth and fifth moment. 


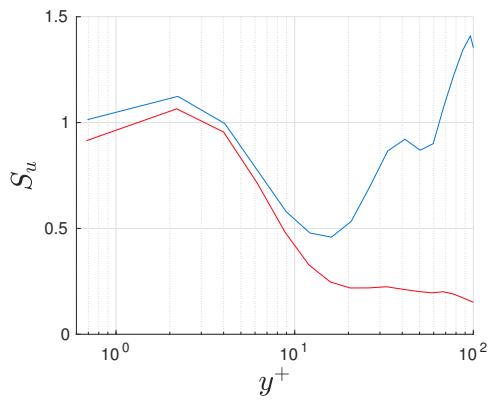

(a)

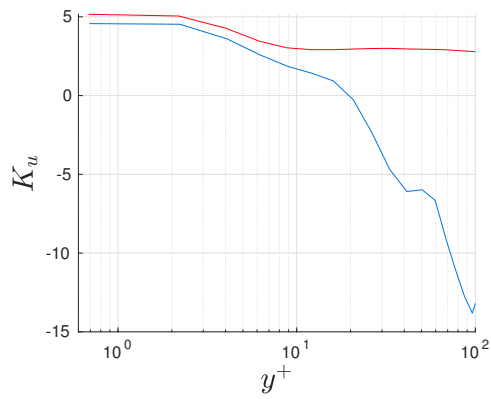

(d)

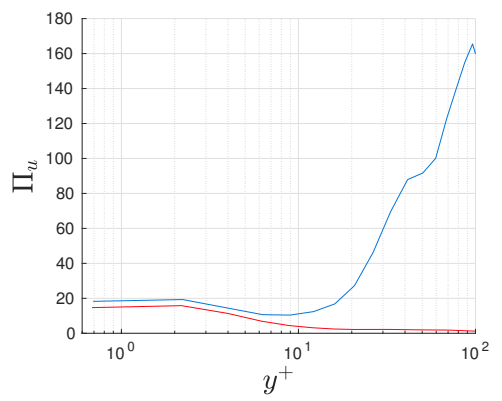

$(\mathrm{g})$

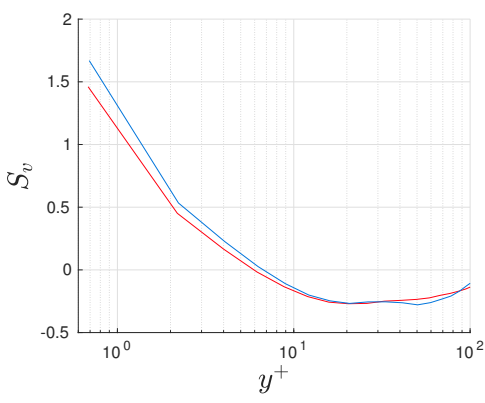

(b)

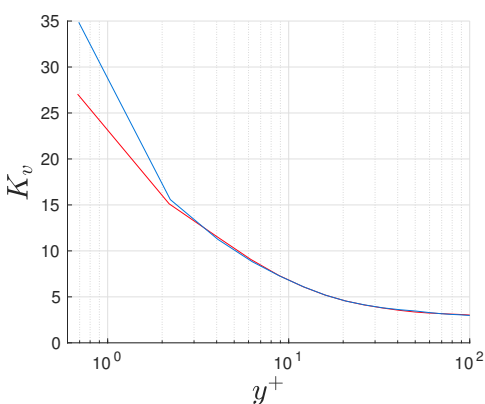

(e)

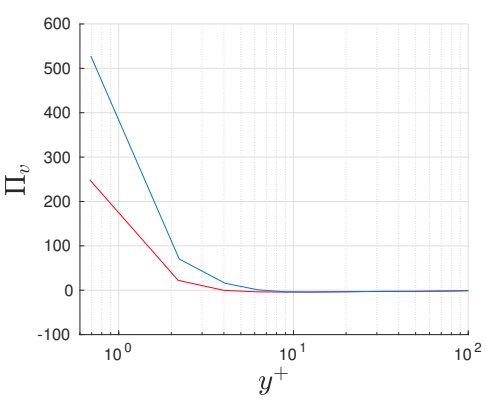

(h)

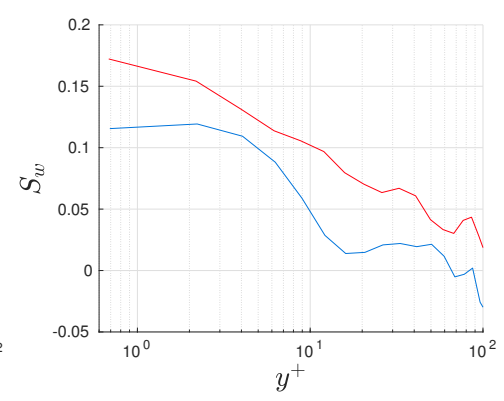

(c)

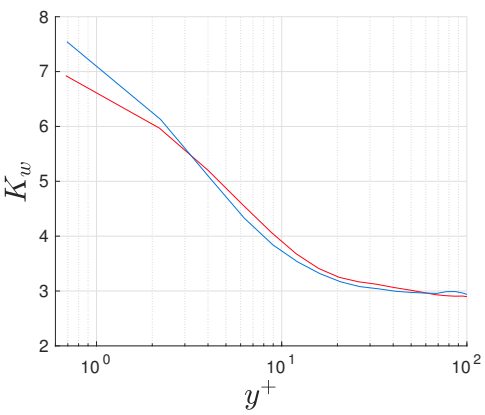

(f)

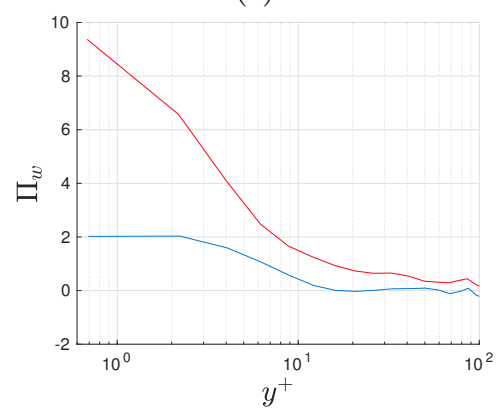

(i)

Figure 6-49: Normalized high-order central statistical moments versus inner-scaled wall-normal coordinates at $x / h \approx 15$ for an SGS-free model (red) and WALE model (blue); the first, second and third columns correspond to the streamwise, wall-normal and spanwise components and the first, second and third rows correspond to the third, fourth and fifth moment. 


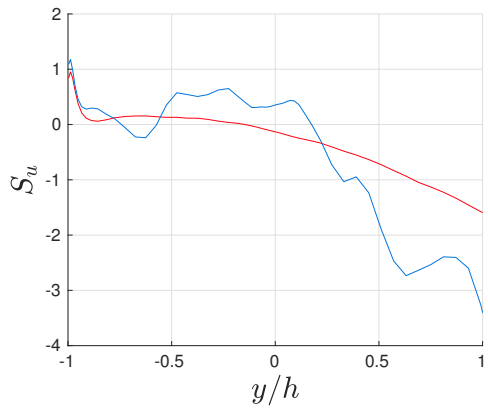

(a)

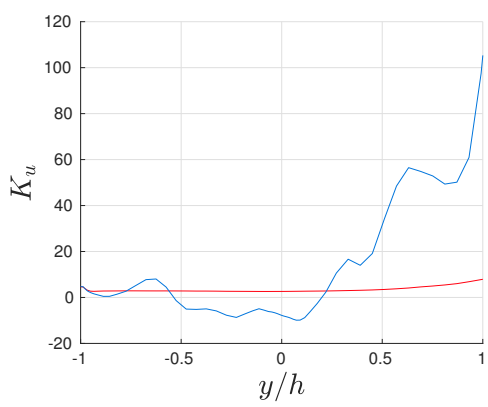

(d)

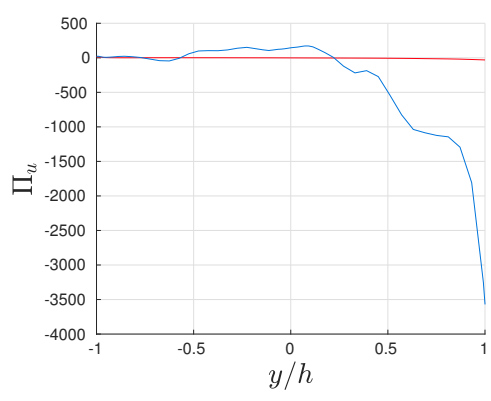

$(\mathrm{g})$

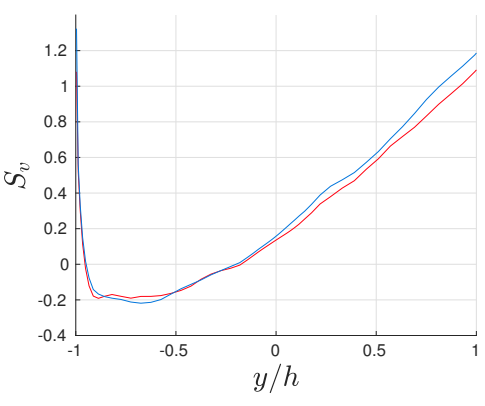

(b)

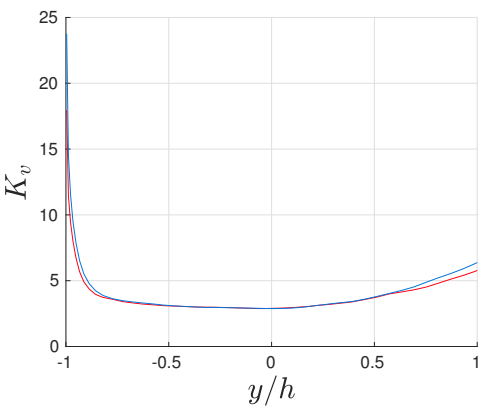

(e)

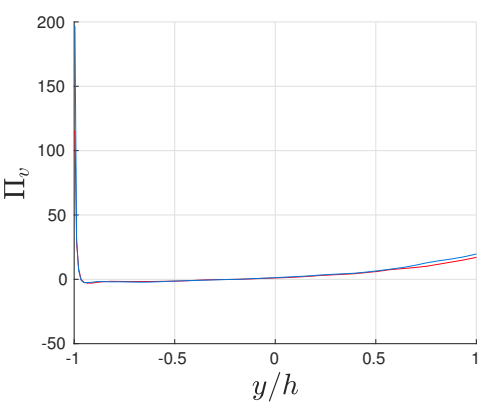

(h)

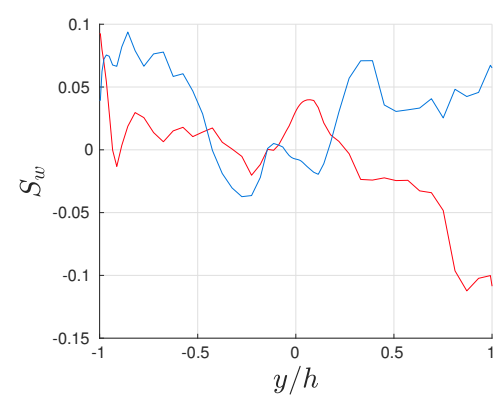

(c)

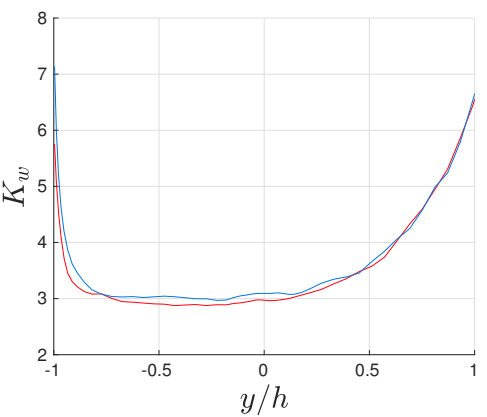

(f)

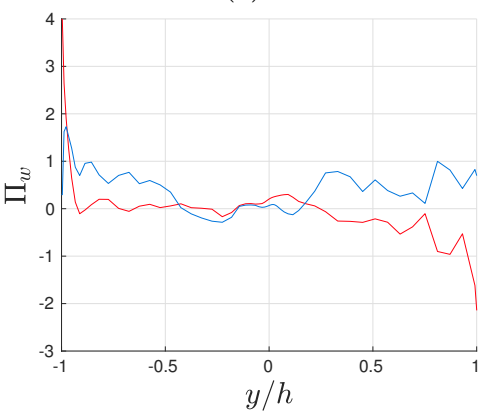

(i)

Figure 6-50: Normalized high-order central statistical moments versus outer-scaled wall-normal coordinates at $x / h \approx 19$ for an SGS-free model (red) and WALE model (blue); the first, second and third columns correspond to the streamwise, wall-normal and spanwise components and the first, second and third rows correspond to the third, fourth and fifth moment. 


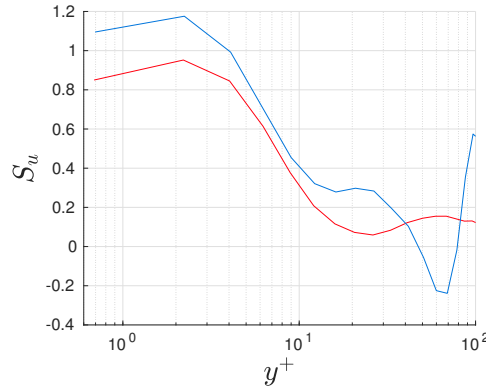

(a)

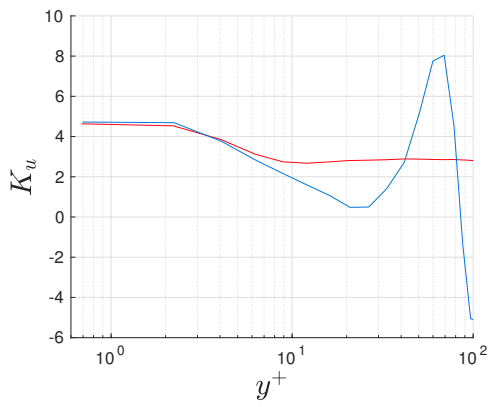

(d)

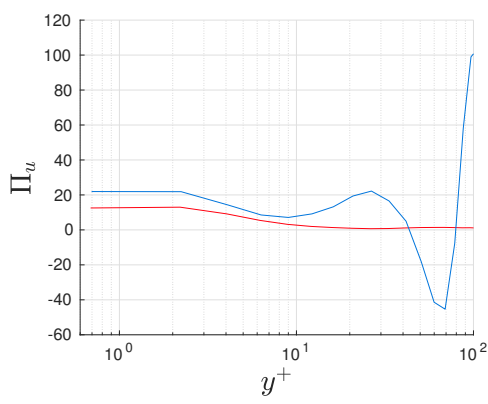

(g)

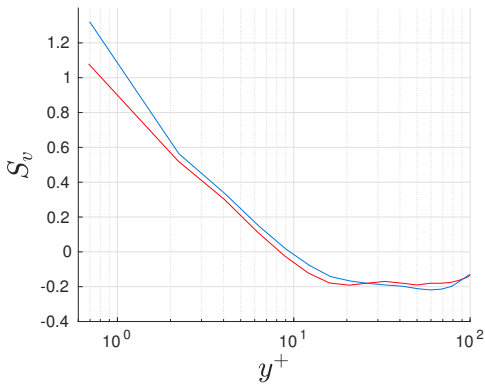

(b)

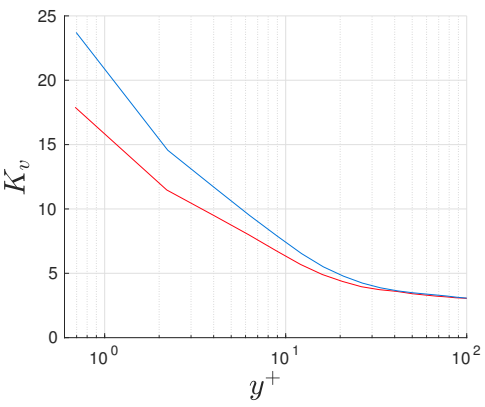

(e)

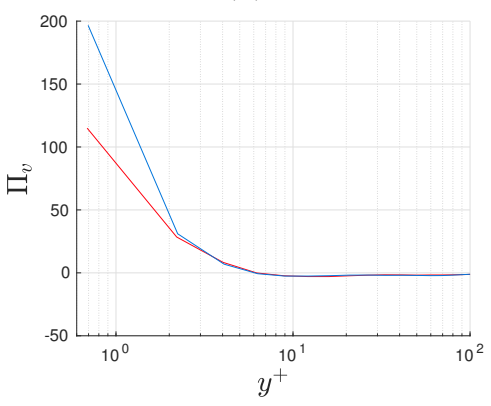

(h)

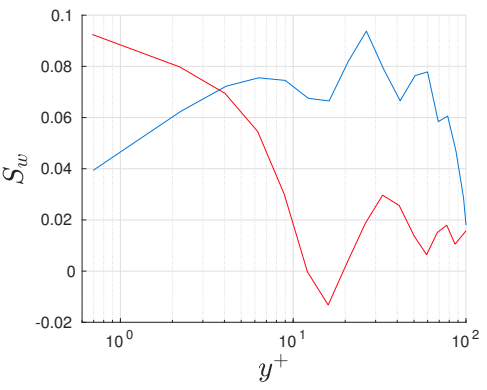

(c)

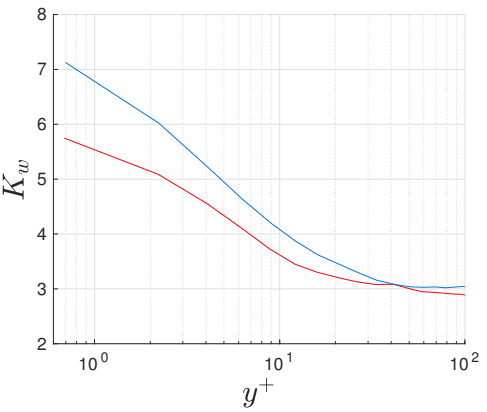

(f)

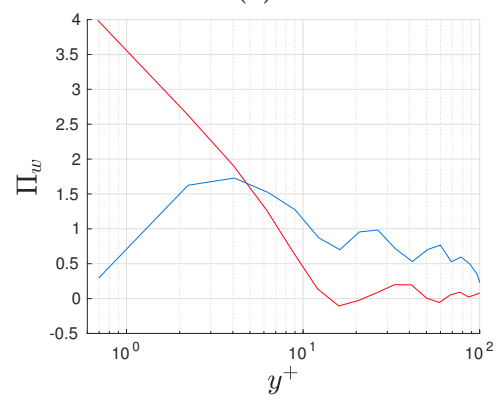

(i)

Figure 6-51: Normalized high-order central statistical moments versus inner-scaled wall-normal coordinates at $x / h \approx 19$ for an SGS-free model (red) and WALE model (blue); the first, second and third columns correspond to the streamwise, wall-normal and spanwise components and the first, second and third rows correspond to the third, fourth and fifth moment. 


\subsubsection{Turbulent Kinetic Energy Budget}

The TKE budget is computed using expression Equation 5.2. Each of the six constituting terms of the budget are presented at different streamwise locations and compared to the DNS benchmark data. The budget terms are separated into two categories: transport and non-transport terms. The first includes production $\left(\mathcal{P}_{k}\right)$, viscous dissipation $\left(\varepsilon_{k}\right)$ and convection $\left(\mathcal{C}_{k}\right)$, while the latter includes transport terms via turbulence $\left(\mathcal{T}^{(u u)}\right)$, viscous diffusion $\left(\mathcal{T}^{(\nu)}\right)$ and velocity-pressure gradient $\left(\mathcal{T}^{(u p)}\right)$. For complete expressions of the TKE budget, refer to Appendix B.

The non-transport terms of the TKE budget are illustrated in Figures 6-52 and 653 in inner and outer scaling, respectively; the transport terms are illustrated in Figures 6-54 and 6-55 in inner and outer scaling, respectively. Additionally, twodimensional contour plots collapsed in the spanwise homogeneous direction are produced in Figures 6-56 and 6-57 for the non-transport and transport TKE budget terms, respectively.

The non-transport terms of the TKE budget (Equation 5.2) follow very closely the DNS data, e.g. see Figure 6-52. This is especially true for $\mathcal{P}_{k}$ and $\mathcal{C}_{k}$. The $\varepsilon$ term consistently over-predicts the DNS curve regardless of the SGS model used. However, the WALE model has slightly better agreement with the DNS benchmark data than does the SGS-free model.

As opposed to the non-transport terms, the transport terms of the TKE budget (Equation 5.2) agree relatively less with the DNS data, e.g. see Figure 6-54. For instance, at all streamwise locations, $\mathcal{T}^{(u u)}$ greatly under-predicts the first peak near the wall. Moreover, sometimes the resulting $\mathcal{T}^{(u u)}$ behavior dismisses the entire first peak (e.g. at $x / h \approx 4$ ) and grows afterwards in a completely different manner than from the DNS data (e.g. at $x / h \approx 6)$.

In inner scaling, near the reattachment location, the SGS models in Figures 653 and 6-53 display much higher values than the DNS. This is explained by how comparatively small $u_{\tau}$ for the LES is at $x / h \approx 6$ in contrast to that of the DNS. 


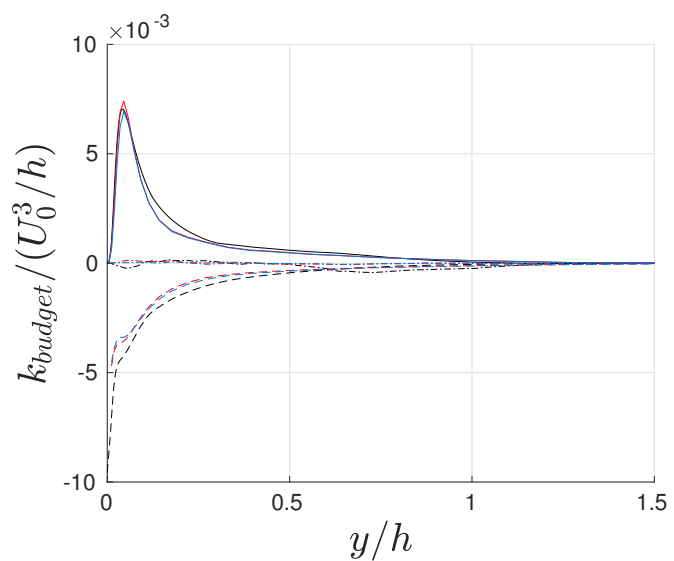

(a)

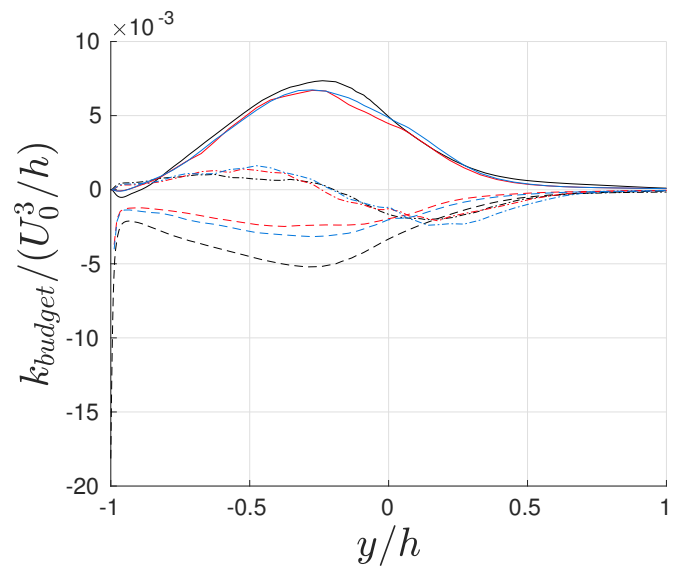

(c)

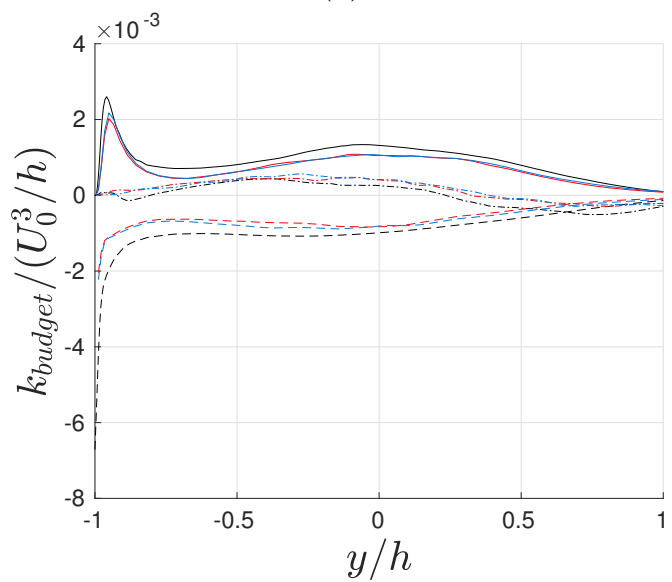

(e)

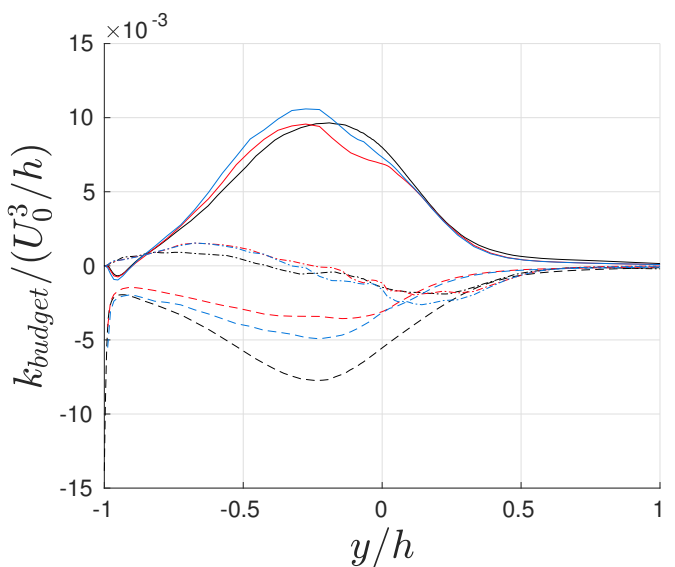

(b)

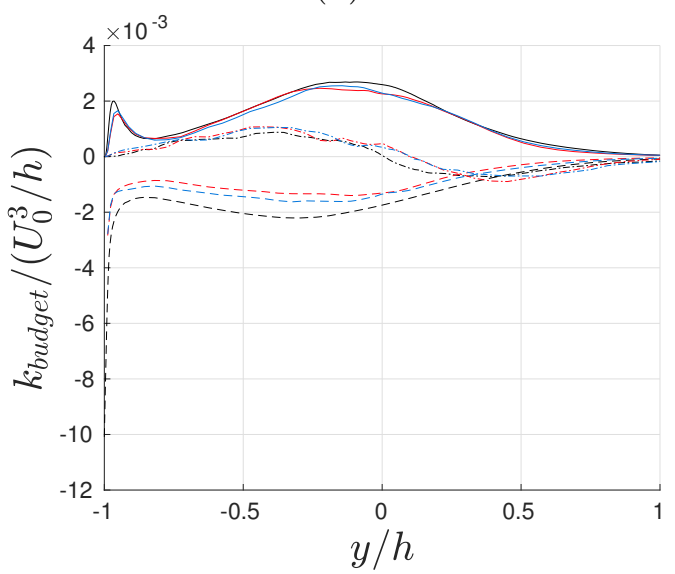

(d)

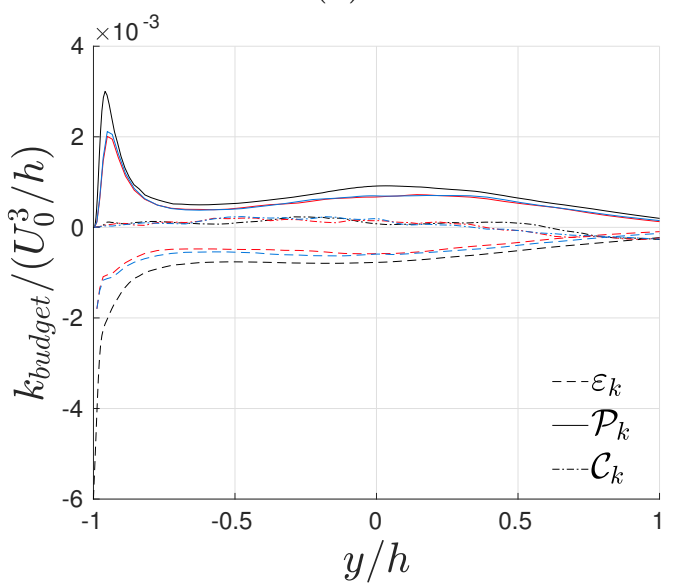

(f)

Figure 6-52: Outer-scaled TKE budget for the non-transport components $\varepsilon_{k}, \mathcal{P}_{k}$ and $\mathcal{C}_{k}$ versus the wall-normal coordinate for the SGS-free (red) and WALE (blue) models along with the DNS (black) taken at dimensionless streamwise locations $x / h$ of approximately -3 (a), 4 (b), 6 (c), 10 (d), 15 (e) and 19 (f). 


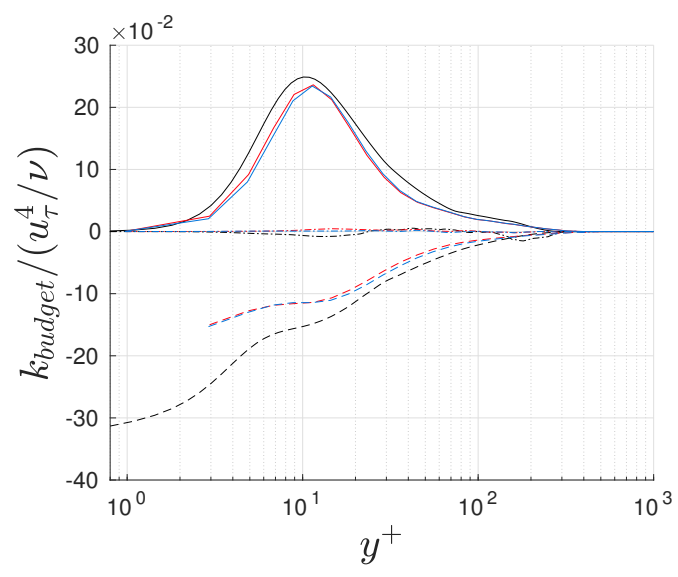

(a)

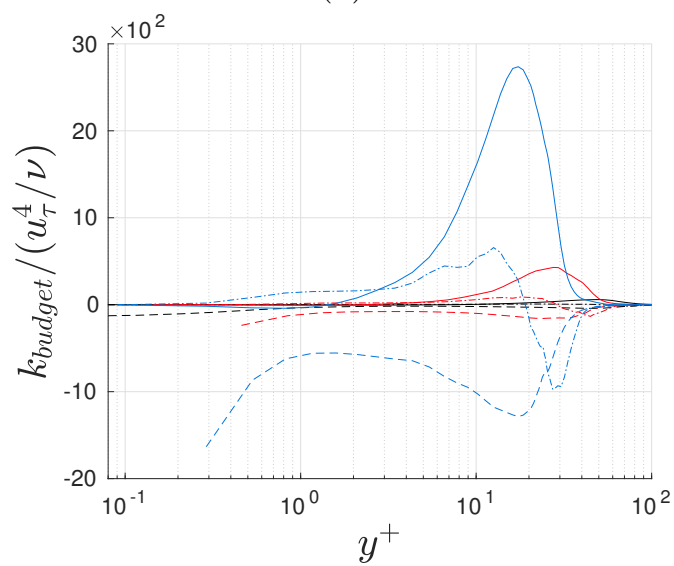

(c)

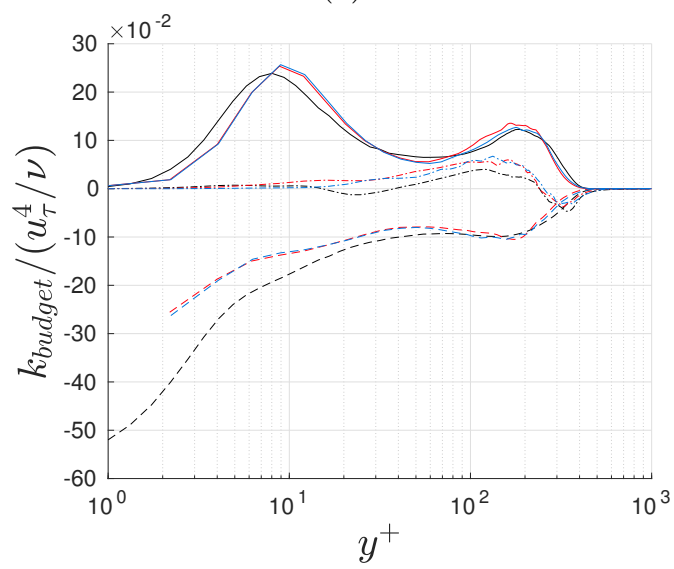

(e)

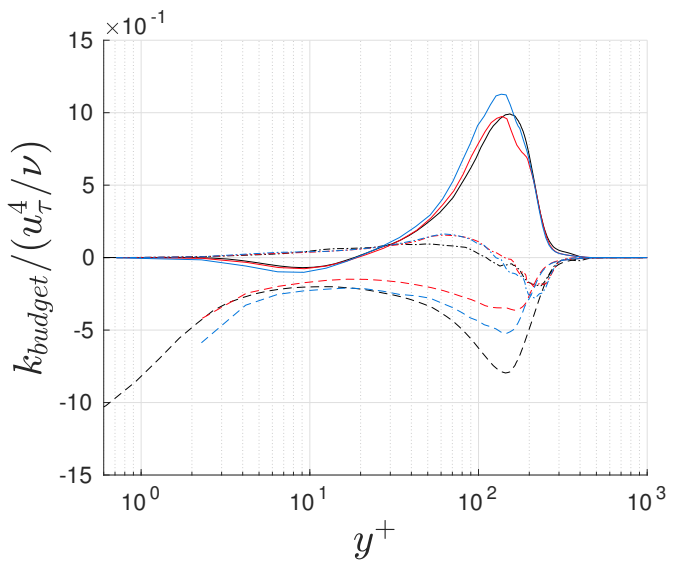

(b)

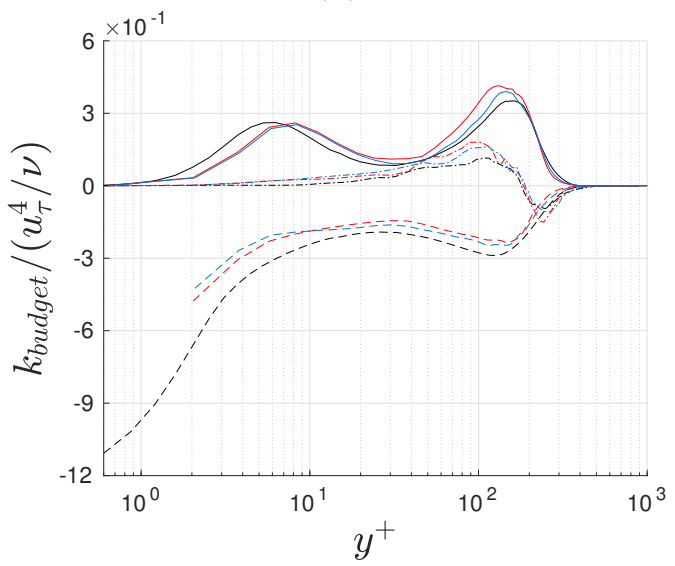

(d)

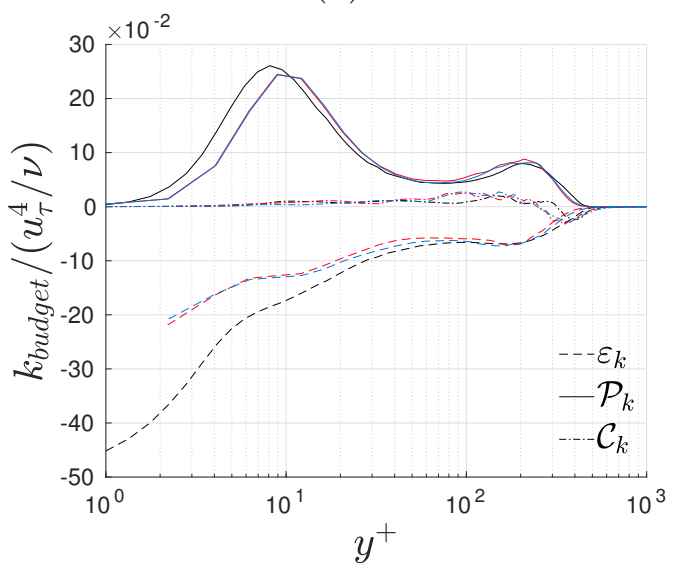

(f)

Figure 6-53: Inner-scaled TKE budget for the non-transport components $\varepsilon_{k}, \mathcal{P}_{k}$ and $\mathcal{C}_{k}$ versus the wall-normal coordinate for the SGS-free (red) and WALE (blue) models along with the DNS (black) taken at dimensionless streamwise locations $x / h$ of approximately -3 (a), 4 (b), 6 (c), 10 (d), 15 (e) and 19 (f). 


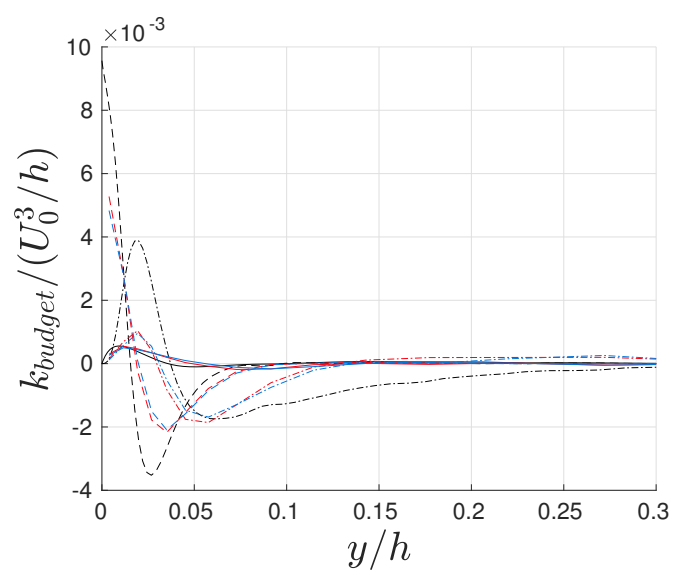

(a)

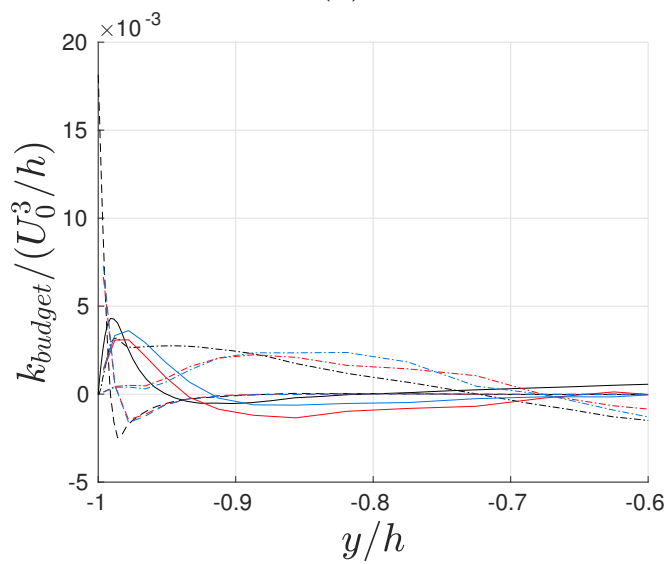

(c)

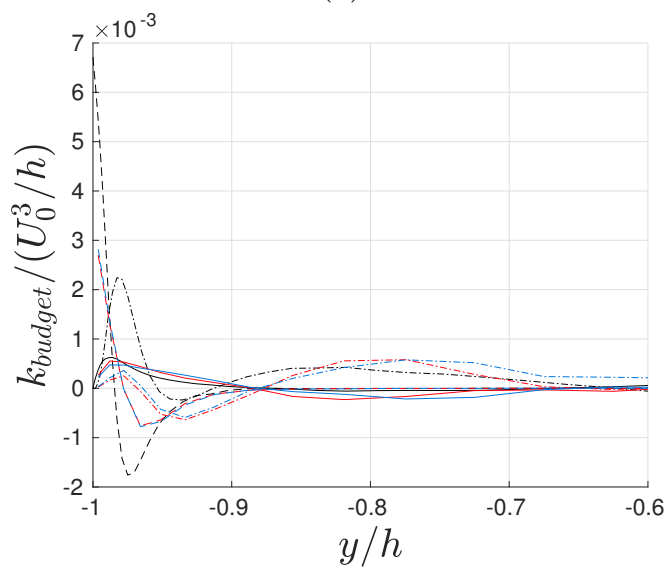

(e)

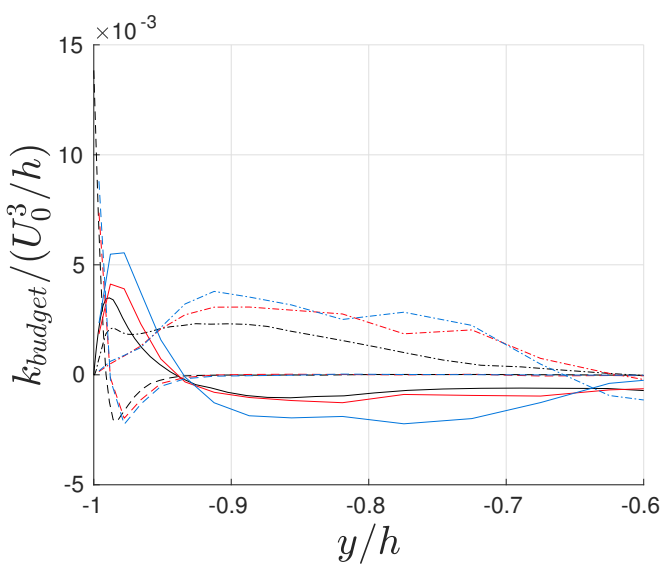

(b)

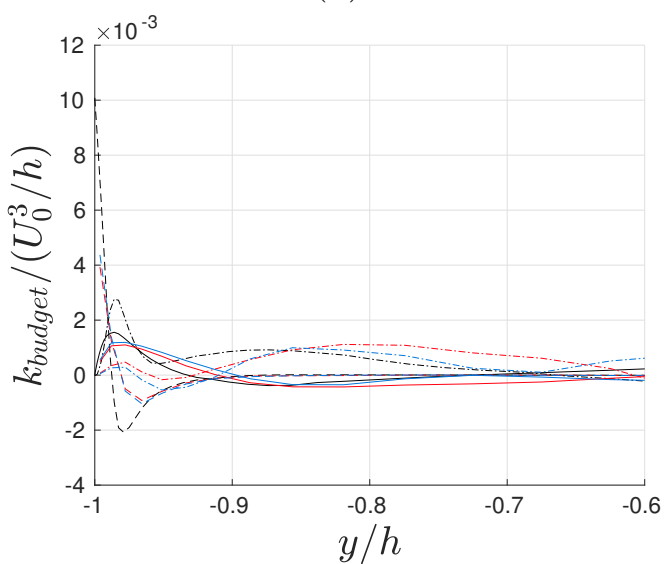

(d)

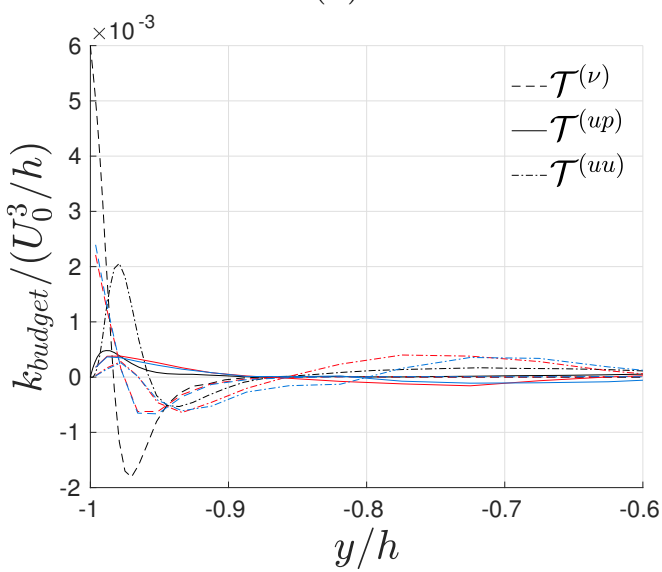

(f)

Figure 6-54: Outer-scaled TKE budget for the transport components $\mathcal{T}^{(\nu)}, \mathcal{T}^{(u p)}$ and $\mathcal{T}^{(u u)}$ versus the wall-normal coordinate near the wall for the SGS-free (red) and WALE (blue) models along with the DNS (black) taken at dimensionless streamwise locations $x / h$ of approximately -3 (a), 4 (b), 6 (c), 10 (d), 15 (e) and 19 (f). 


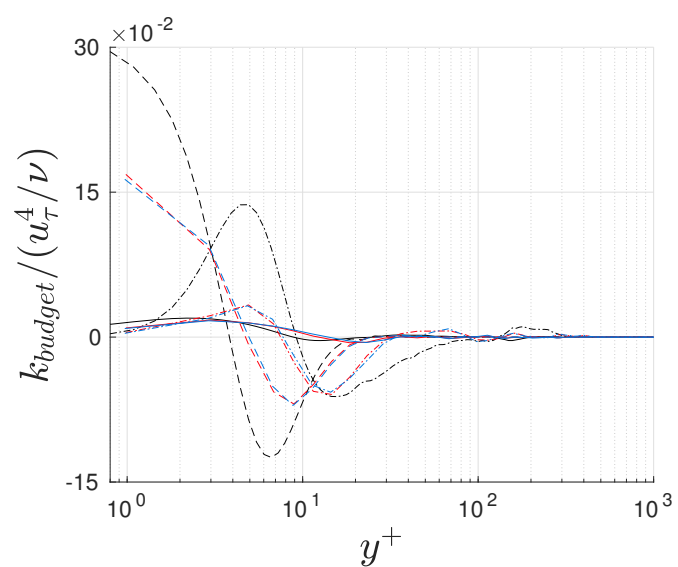

(a)

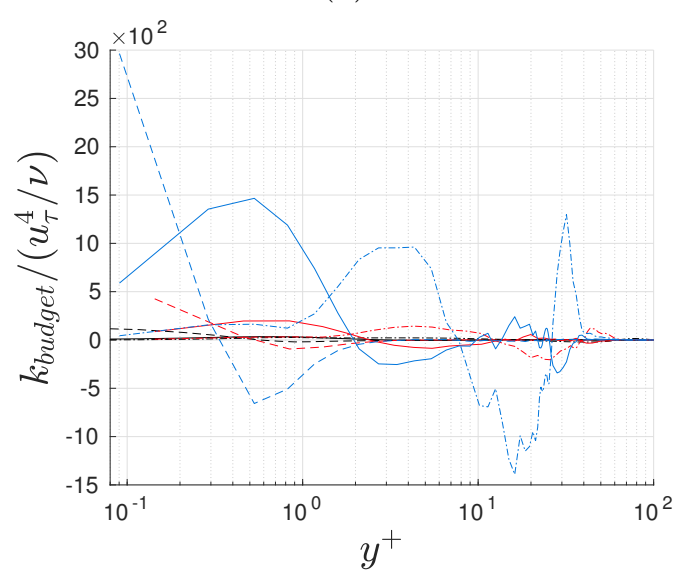

(c)

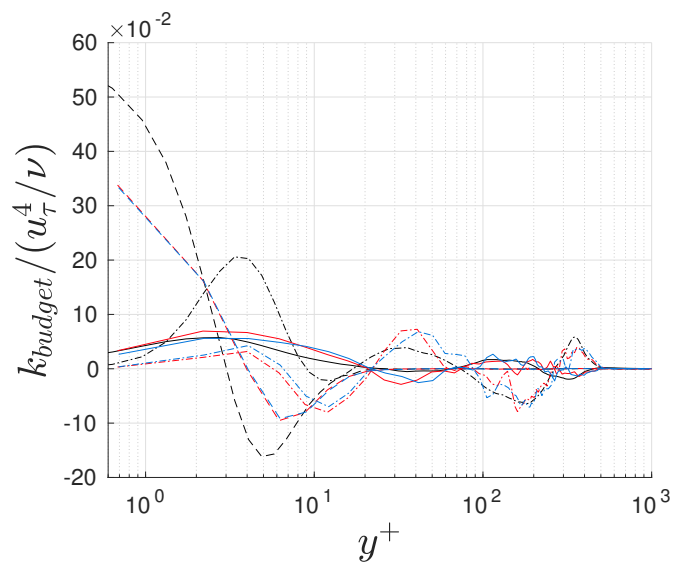

(e)

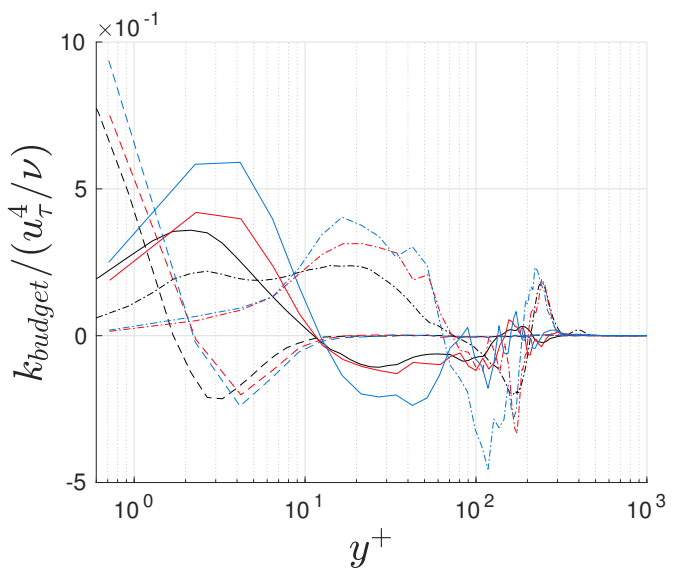

(b)

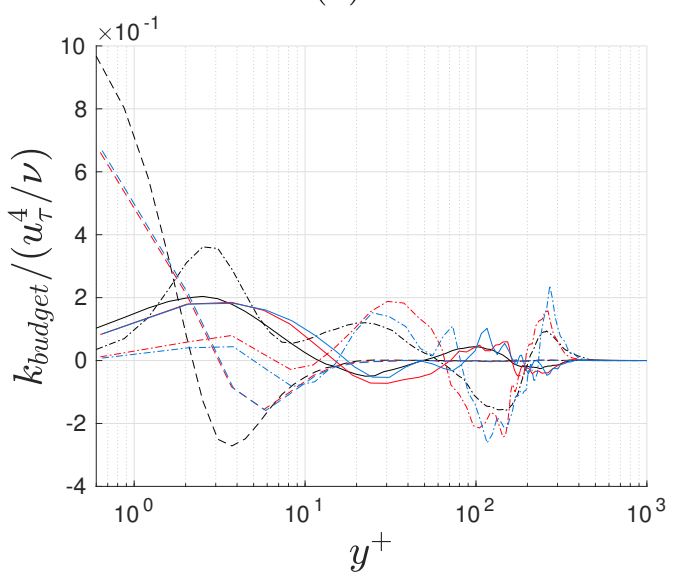

(d)

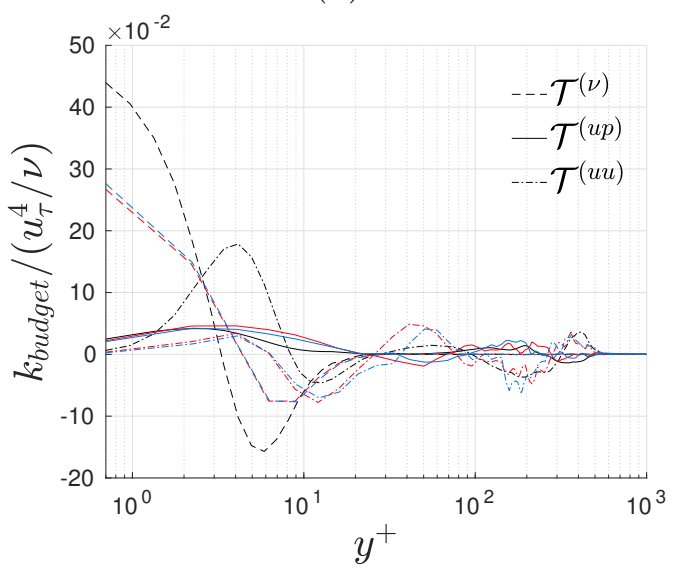

(f)

Figure 6-55: Inner-scaled TKE budget for the transport components $\mathcal{T}^{(\nu)}, \mathcal{T}^{(u p)}$ and $\mathcal{T}^{(u u)}$ versus the wall-normal coordinate for the SGS-free (red) and WALE (blue) models along with the DNS (black) taken at dimensionless streamwise locations $x / h$ of approximately -3 (a), 4 (b), 6 (c), 10 (d), 15 (e) and 19 (f). 


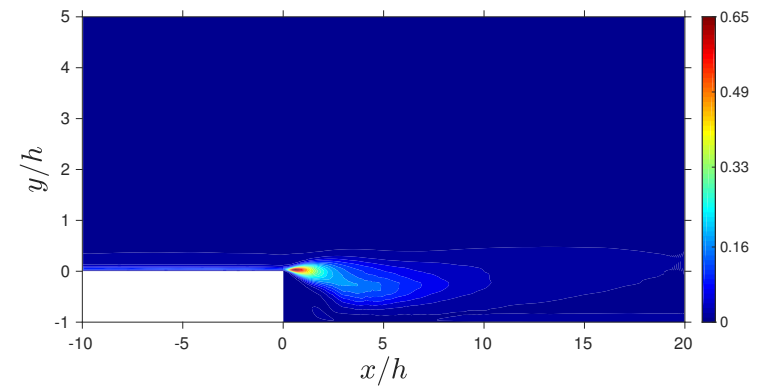

(a)

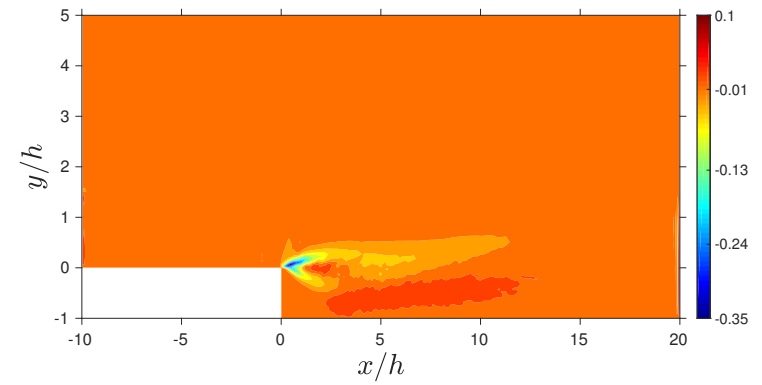

(c)

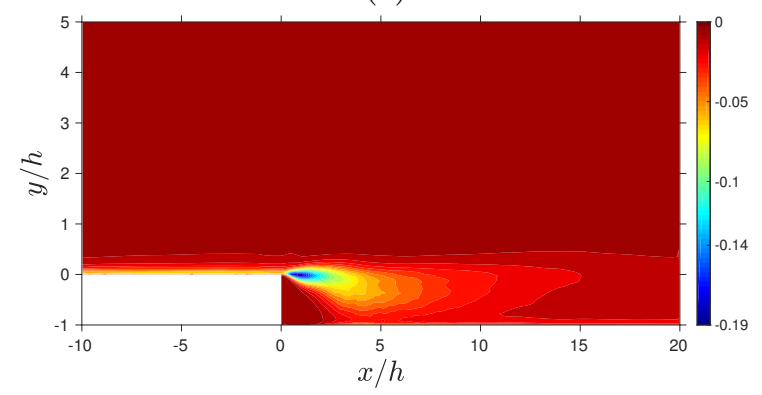

(e)

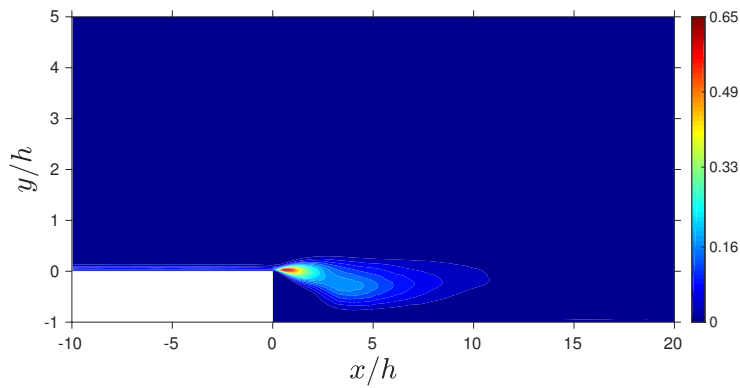

(b)

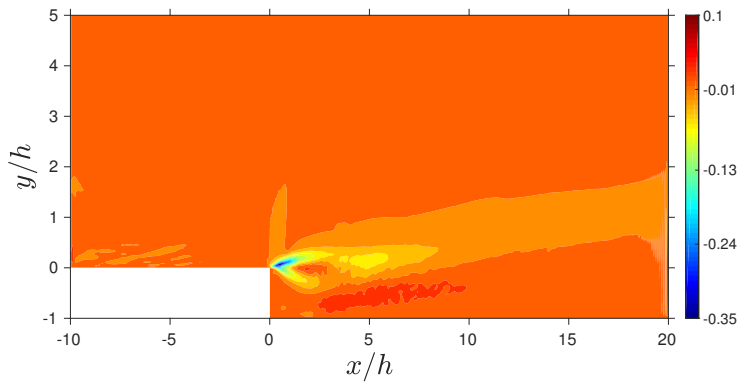

(d)

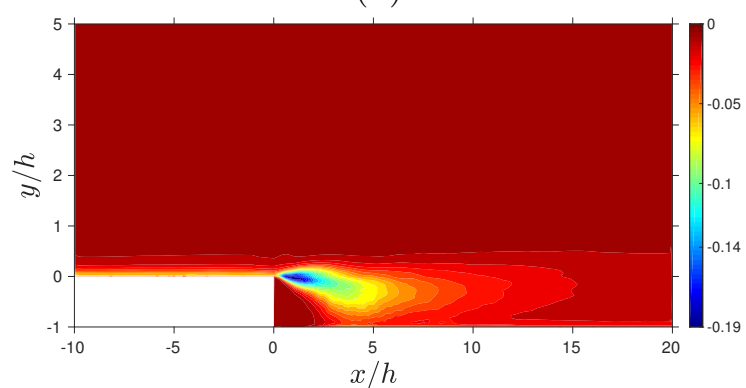

(f)

Figure 6-56: Non-transport components of the TKE budget for the SGS-free and WALE models in the first and second columns, respectively; $\mathcal{P}_{k}, \mathcal{C}_{k}$ and $\varepsilon$ in the first, second and third row, respectively. 


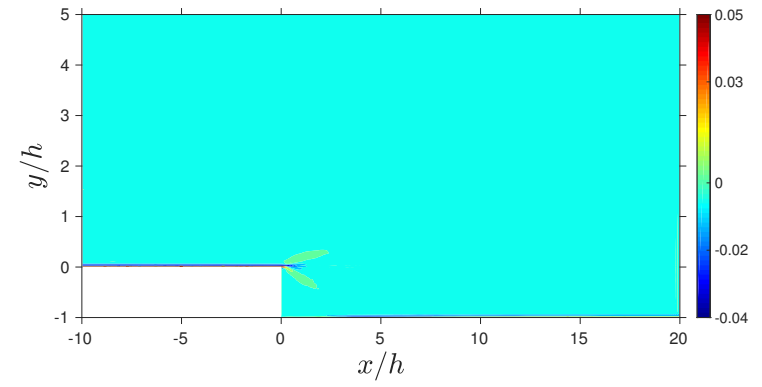

(a)

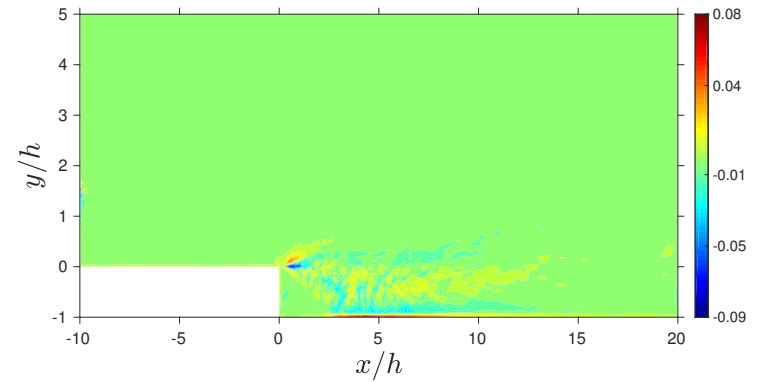

(c)

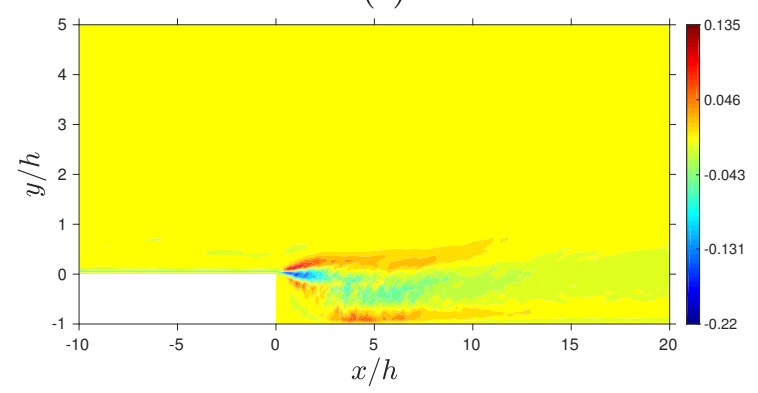

(e)

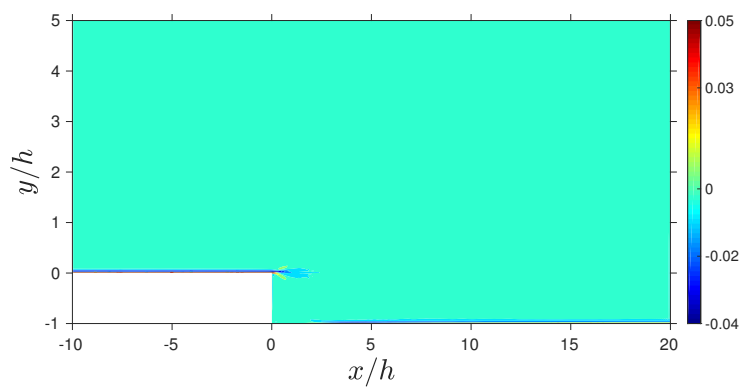

(b)

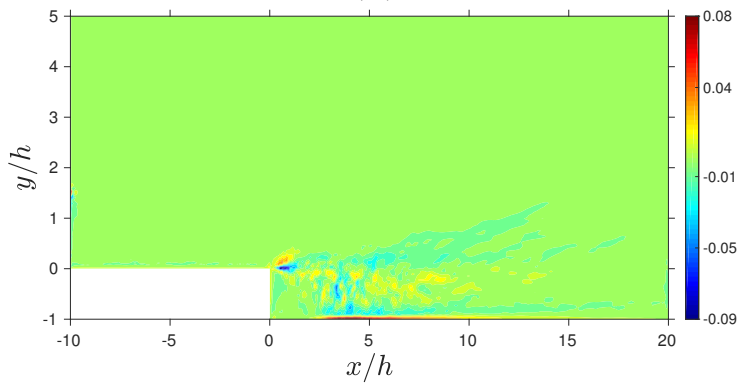

(d)

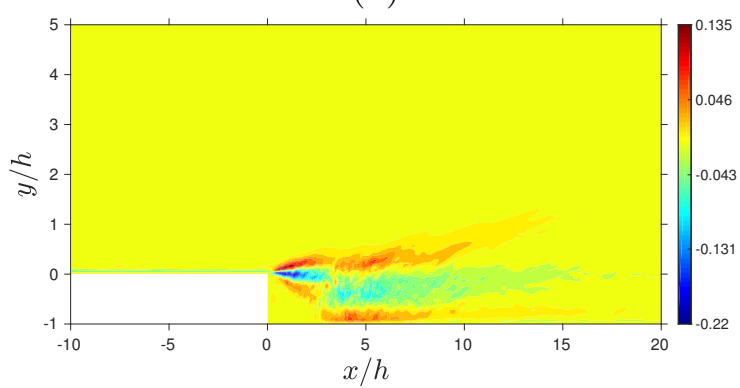

(f)

Figure 6-57: Transport components of the TKE budget for the SGS-free and WALE models in the first and second columns, respectively; $\mathcal{T}^{(\nu)}, \mathcal{T}^{(u p)}$ and $\mathcal{T}^{(u u)}$ in the first, second and third row, respectively. 


\subsubsection{One-dimensional Spanwise Spectra}

The PSD calculations are exactly the same as that used in subsection 5.4.4. The only difference implementation-wise is that, unlike turbulent channel flow simulations, only one homogeneous direction exists in the flow over a BFS. The inner-scaled PSD of the velocity is sampled at six different streamwise locations and presented in Figures 6-58 to 6-62. In each of these locations, the PSD is computed for each of the velocity components only in the spanwise direction, separately. Moreover, up to four distinct wall-normal locations are considered; viscous sublayer, inner-region, TBL half-height and near the TBL edge. For more information about the mathematical expressions involved in PSD computations, refer to Appendix A.

The only major difference in the velocity PSD between the SGS-free (red) and WALE (blue) models is observed near the reattachment location in Figure 6-60.

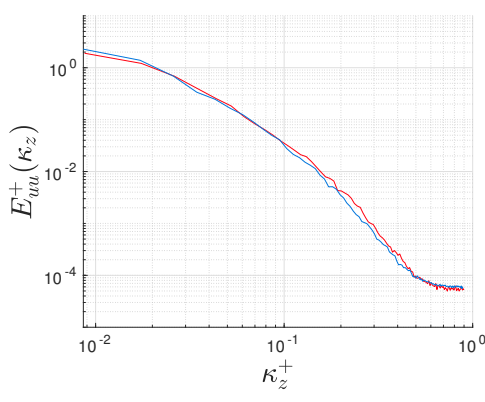

(a)

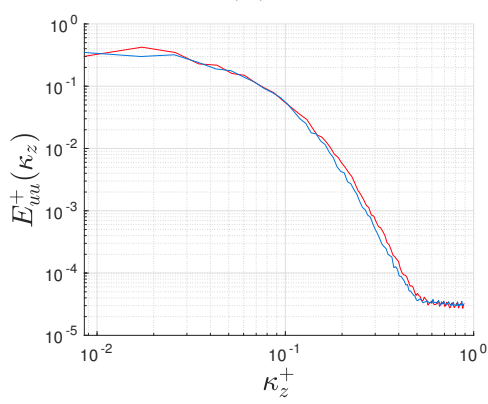

(d)

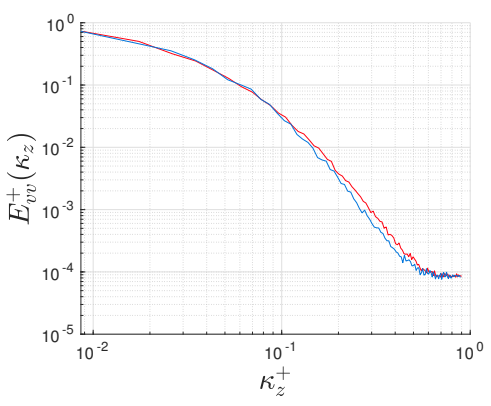

(b)

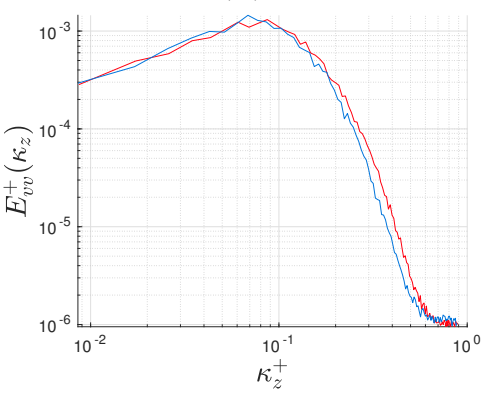

(e)

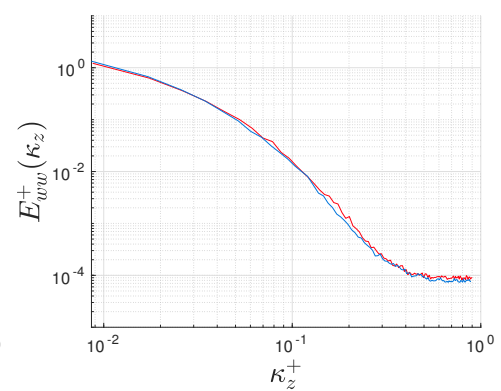

(c)

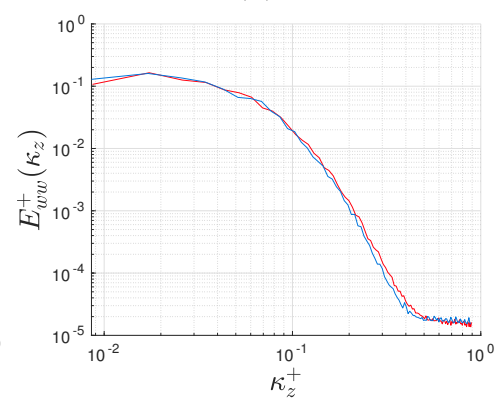

(f)

Figure 6-58: Inner-scaled $1 \mathrm{D}$ spanwise PSD of the velocity versus $\kappa_{z}^{+}$at $x / h \approx 15$ with $y^{+} \approx 193$ (top row) and $x / h \approx 19$ with $y^{+} \approx 5.5$ (bottom row) for the SGS-free (red) and WALE (blue) models; $E_{u u}^{+}\left(\kappa_{z}\right), E_{v v}^{+}\left(\kappa_{z}\right)$ and $E_{w w}^{+}\left(\kappa_{z}\right)$ correspond to the first, second and third columns, respectively. 


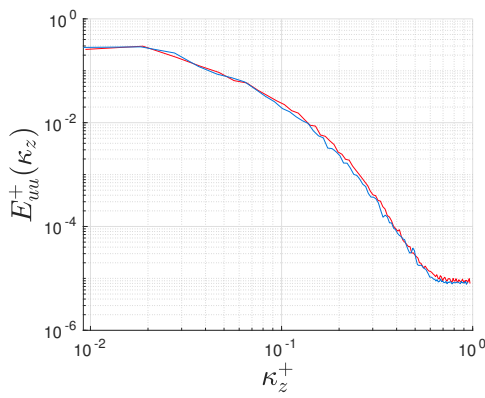

(a)

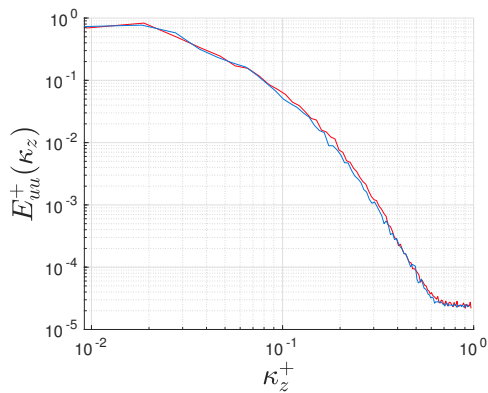

(d)

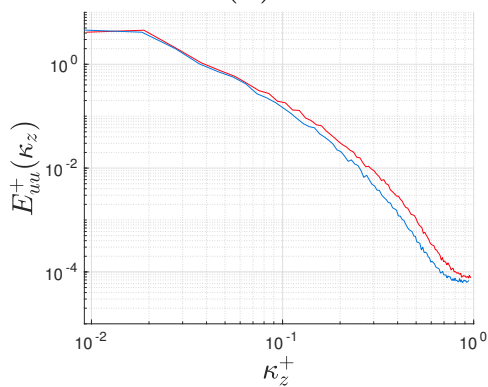

(g)

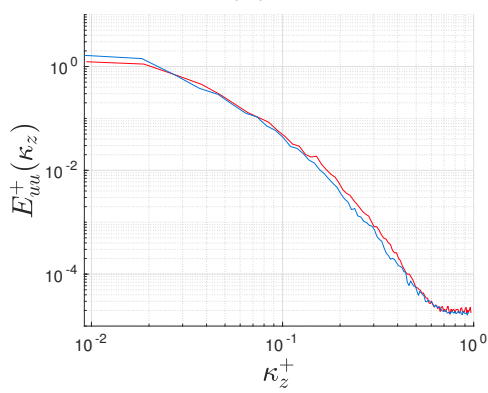

(j)

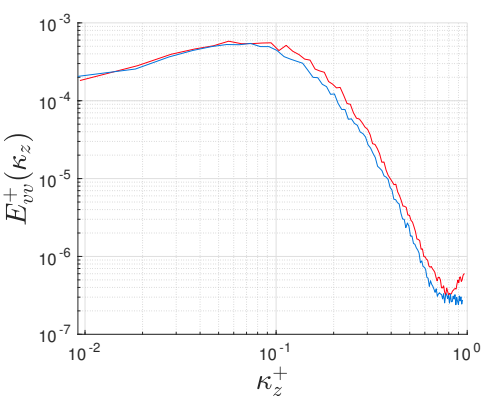

(b)

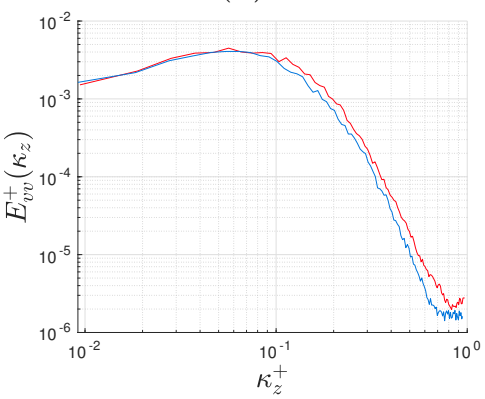

(e)

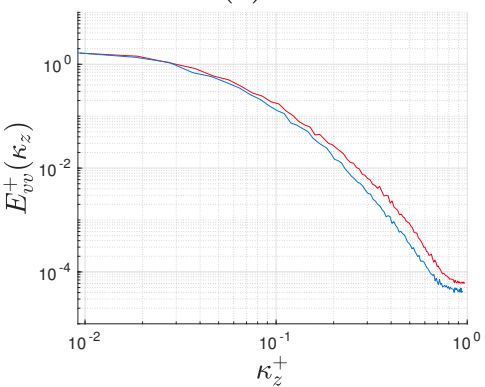

(h)

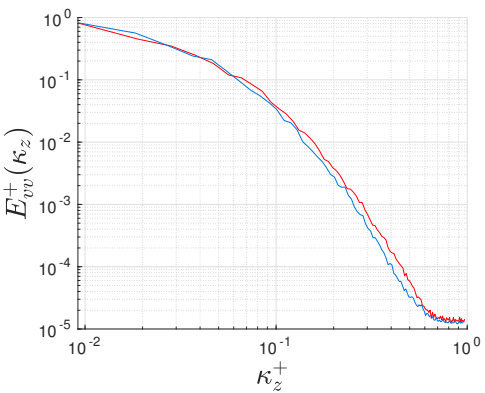

(k)

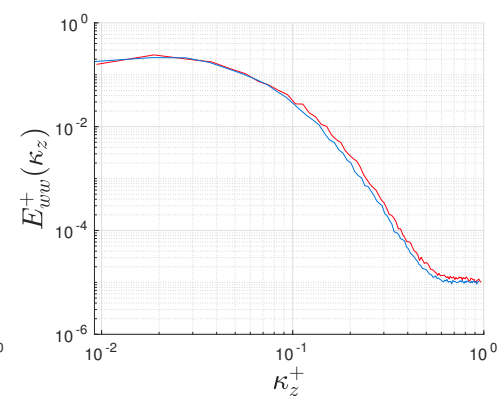

(c)

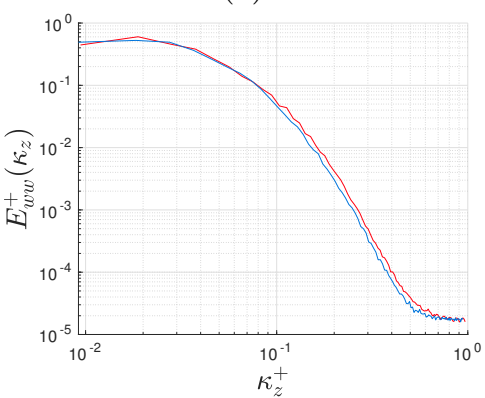

(f)

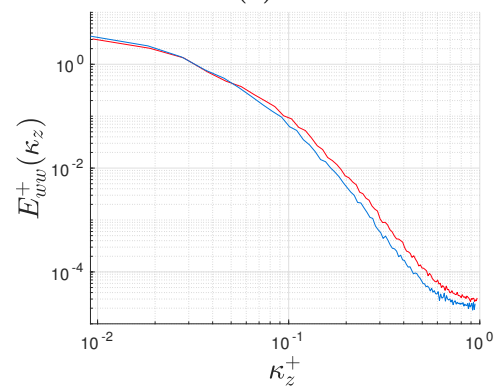

(i)

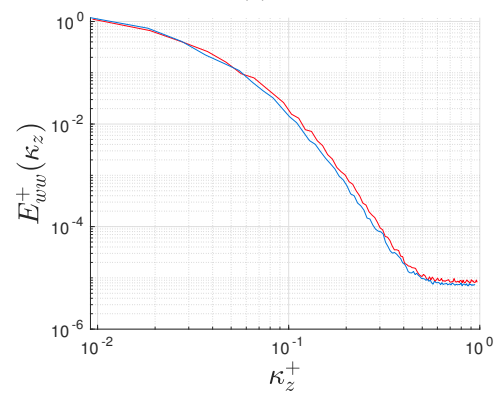

(l)

Figure 6-59: Inner-scaled 1D spanwise PSD of the velocity versus $\kappa_{z}^{+}$at $x / h \approx 10$ for the SGS-free (red) and WALE (blue) models; $E_{u u}^{+}\left(\kappa_{z}\right), E_{v v}^{+}\left(\kappa_{z}\right)$ and $E_{w w}^{+}\left(\kappa_{z}\right)$ correspond to the first, second and third columns, respectively; $y^{+} \approx 1, y^{+} \approx 5.5, y^{+} \approx 193$ and $y^{+} \approx 376$ correspond to the first, second, third and fourth row, respectively. 


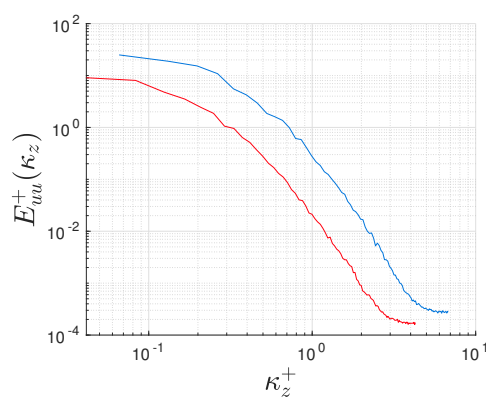

(a)

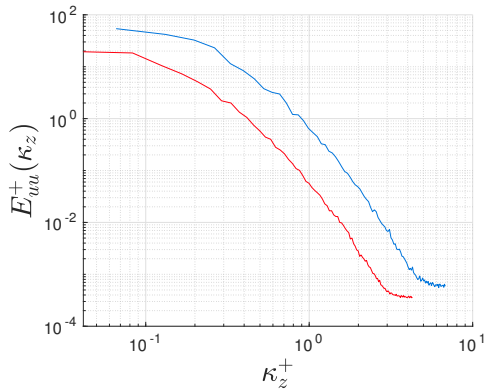

(d)

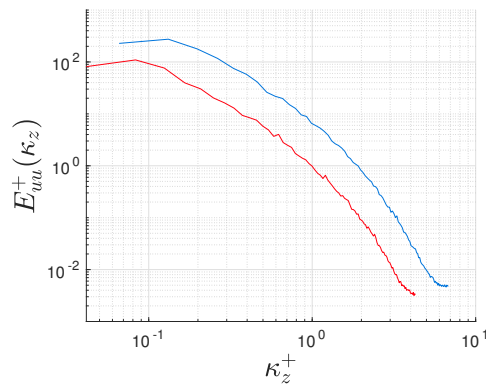

(g)

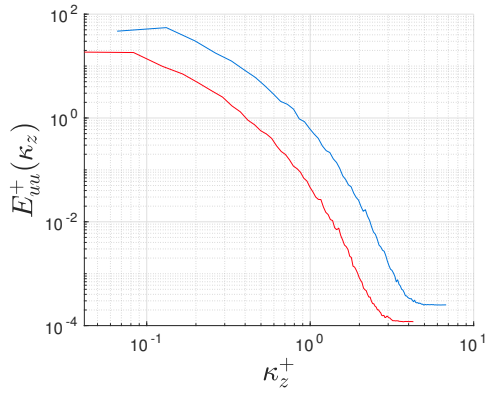

(j)

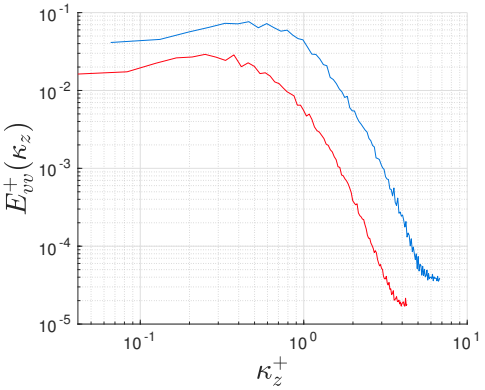

(b)

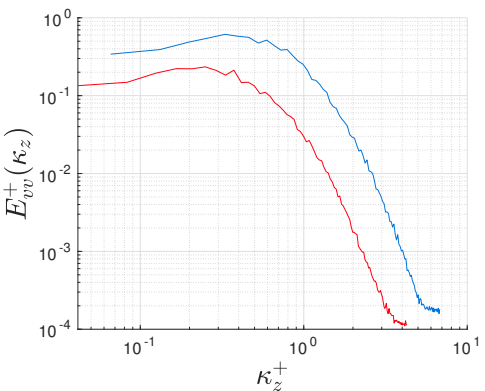

(e)

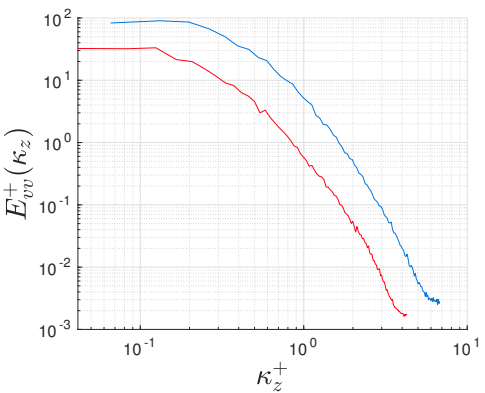

(h)

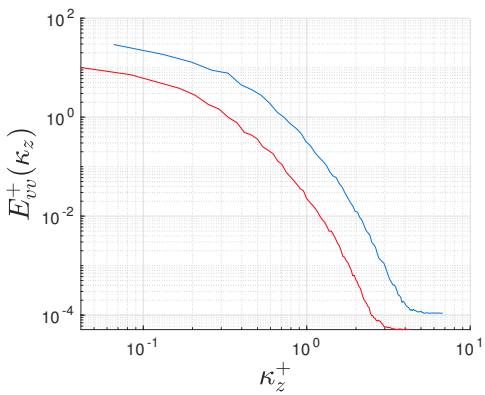

(k)

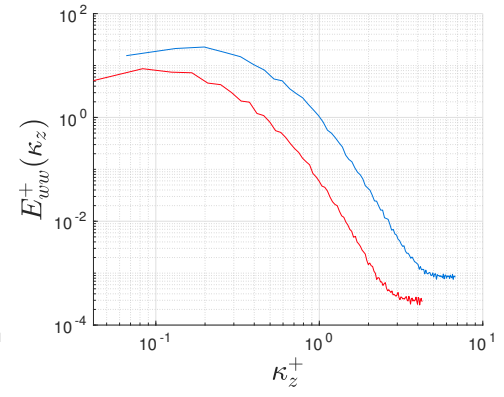

(c)

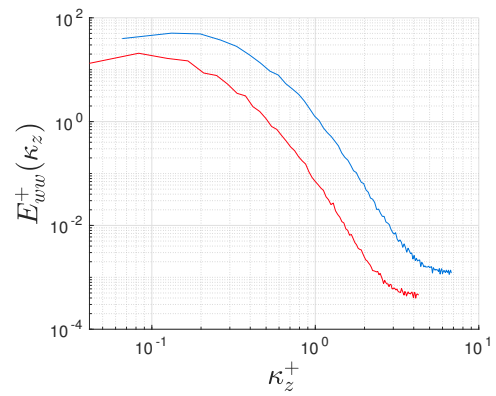

(f)

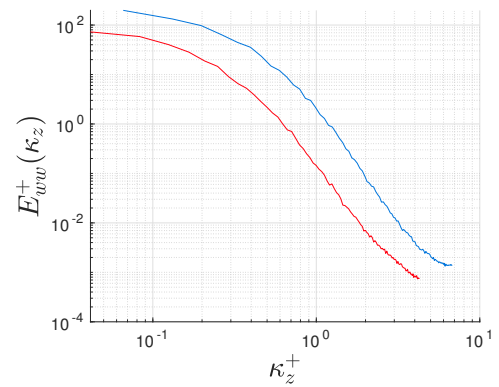

(i)

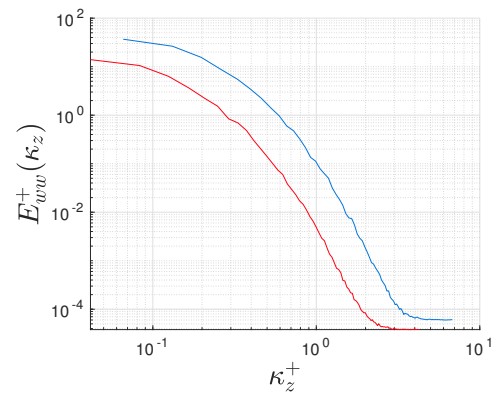

(1)

Figure 6-60: Inner-scaled 1D spanwise PSD of the velocity versus $\kappa_{z}^{+}$at $x / h \approx 6$ for the SGS-free (red) and WALE (blue) models; $E_{u u}^{+}\left(\kappa_{z}\right), E_{v v}^{+}\left(\kappa_{z}\right)$ and $E_{w w}^{+}\left(\kappa_{z}\right)$ correspond to the first, second and third columns, respectively; $y^{+} \approx 1, y^{+} \approx 5.5, y^{+} \approx 193$ and $y^{+} \approx 376$ correspond to the first, second, third and fourth row, respectively. 


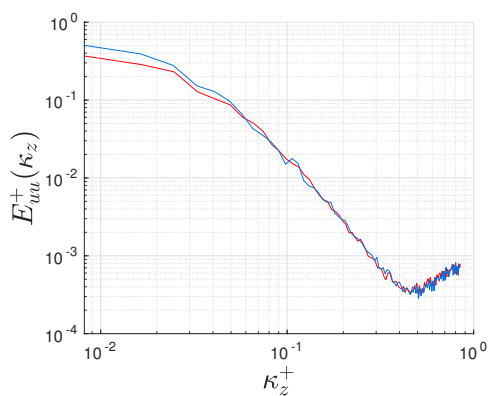

(a)

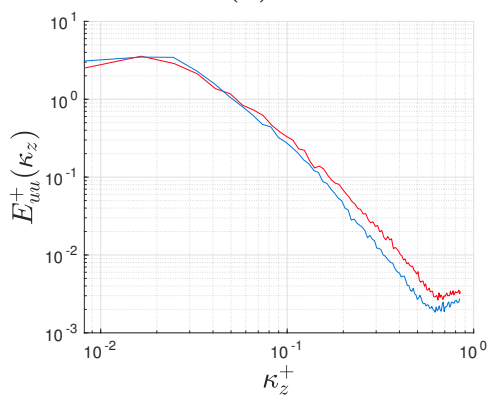

(d)

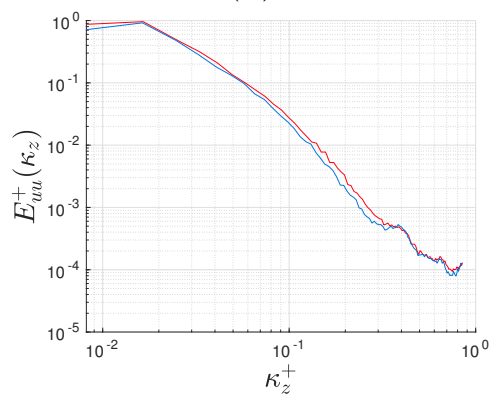

$(\mathrm{g})$

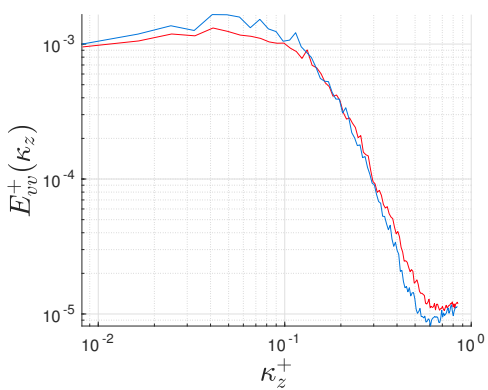

(b)

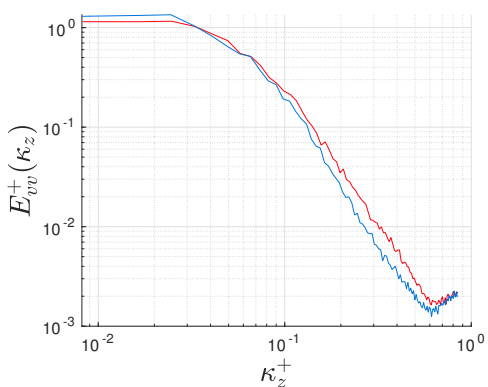

(e)

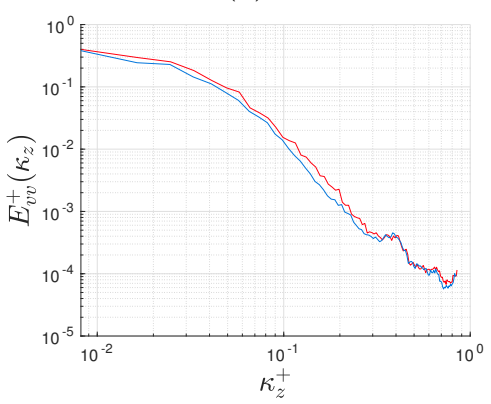

(h)

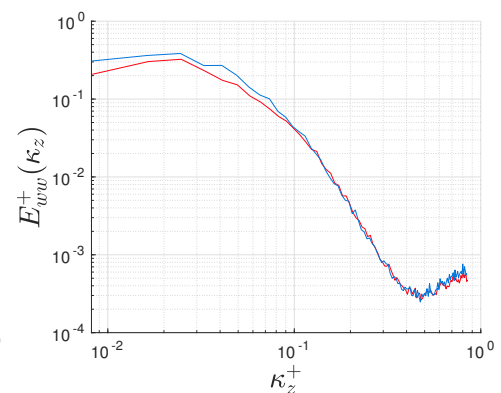

(c)

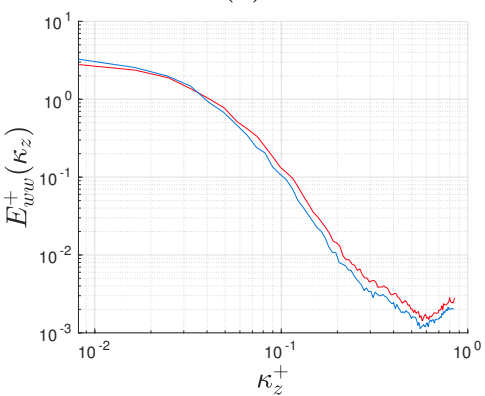

(f)

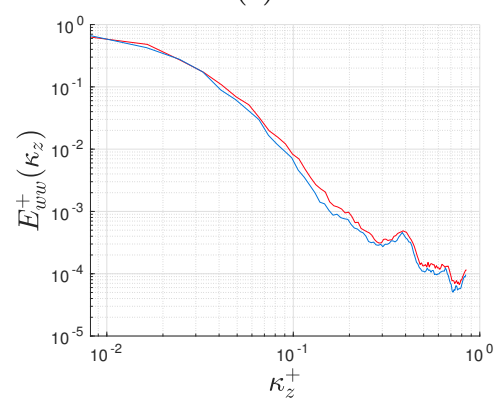

(i)

Figure 6-61: Inner-scaled 1D spanwise PSD of the velocity versus $\kappa_{z}^{+}$at $x / h \approx 4$ for the SGS-free (red) and WALE (blue) models; $E_{u u}^{+}\left(\kappa_{z}\right), E_{v v}^{+}\left(\kappa_{z}\right)$ and $E_{w w}^{+}\left(\kappa_{z}\right)$ correspond to the first, second and third columns, respectively; $y^{+} \approx 1, y^{+} \approx 193$ and $y^{+} \approx 376$ correspond to the first, second and third row, respectively. 


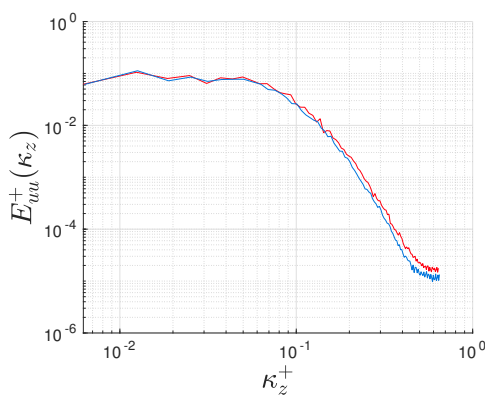

(a)

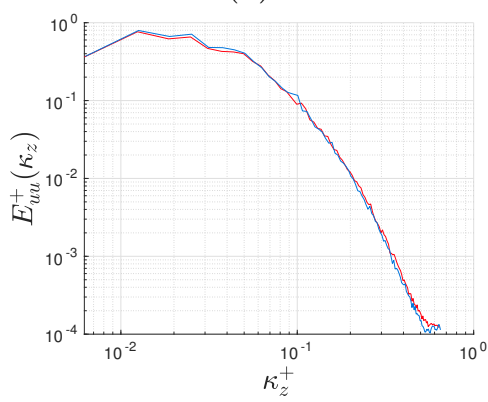

(d)

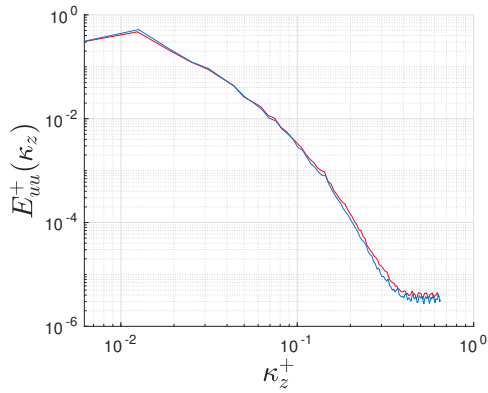

(g)

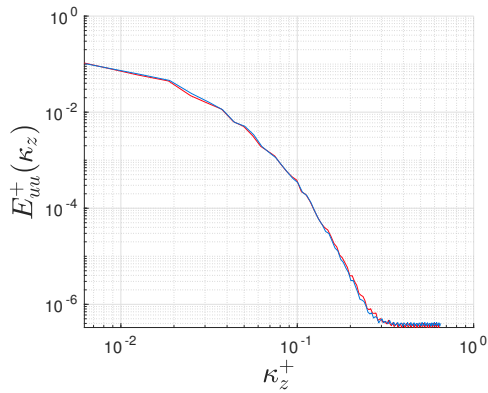

(j)

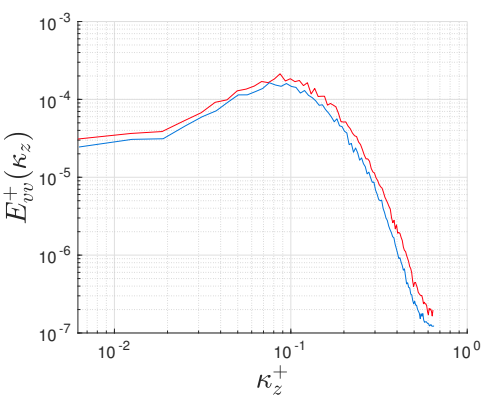

(b)

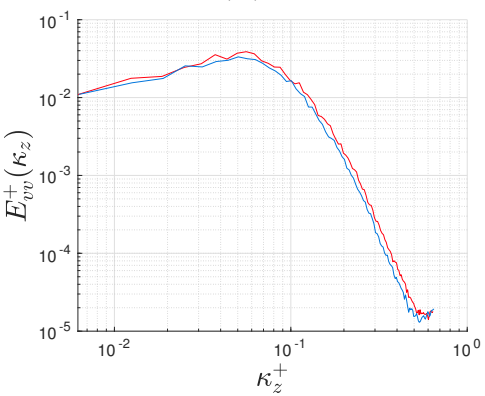

(e)

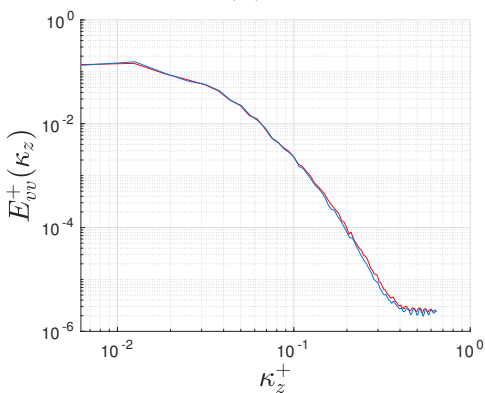

(h)

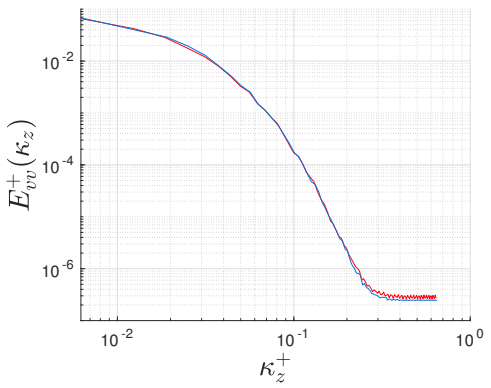

(k)

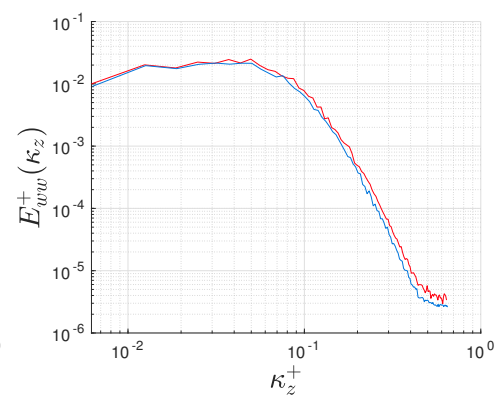

(c)

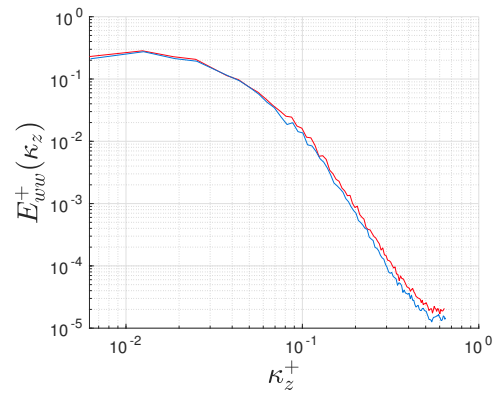

(f)

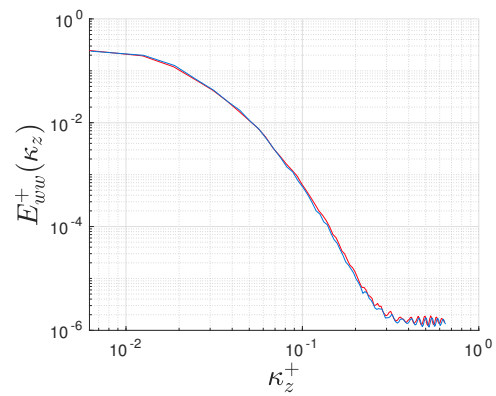

(i)

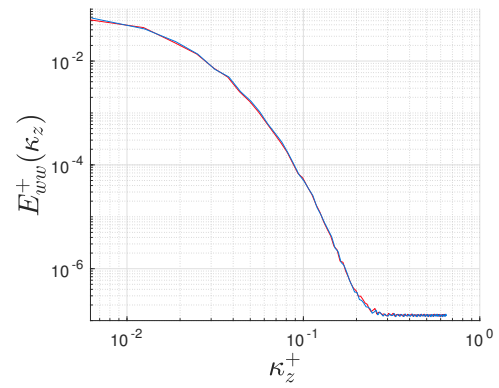

(1)

Figure 6-62: Inner-scaled $1 \mathrm{D}$ spanwise PSD of the velocity versus $\kappa_{z}^{+}$at $x / h \approx-3$ for the SGS-free (red) and WALE (blue) models; $E_{u u}^{+}\left(\kappa_{z}\right), E_{v v}^{+}\left(\kappa_{z}\right)$ and $E_{w w}^{+}\left(\kappa_{z}\right)$ correspond to the first, second and third columns, respectively; $y^{+} \approx 2.9, y^{+} \approx 22.8$, $y^{+} \approx 202$ and $y^{+} \approx 367$ correspond to the first, second, third and fourth row, respectively. 


\subsubsection{Auto-correlation Function in the Spanwise Direction}

The auto-correlation function $(\mathrm{ACF})$ is presented after normalization using the variance in Figures 6-63 to 6-67. This is referred to as the auto-correlation coefficient (ACC) and is denoted as $\rho_{u_{i}}\left(r_{x_{i}}\right)$ in which the $i$ th subscript indicates the velocity, or directional component, used in the ACC. The $r_{x_{i}}$ is the correlation strength or as commonly known, the lag parameter. In this case, since the only statistical homogeneous direction is the spanwise, then all velocity correlations use a lagging parameter that varies via a straight-line in the spanwise direction: $r_{z}$. For additional information about the ACF and ACC, refer to Appendix A. All ACC figures are presented in a similar manner to that used in subsubsection 6.4.4.7.

The ACC of all velocity components display almost identical results between the SGS-free and WALE models. The correlation strength almost diminishes significantly after $r_{z}=h$, e.g. see Figure 6-65. The only exception is the $u$-velocity ACC at $x / h \approx 4$ and 10 , which shows strong correlation up until $r_{z} \approx 3 h$; these are observed in Figures 6-64 and 6-66. This indicates that the BFS domain size in the spanwise direction is sufficient for statistical correlations of the velocities. 


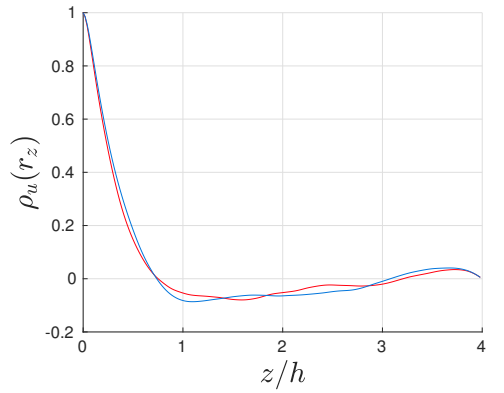

(a)

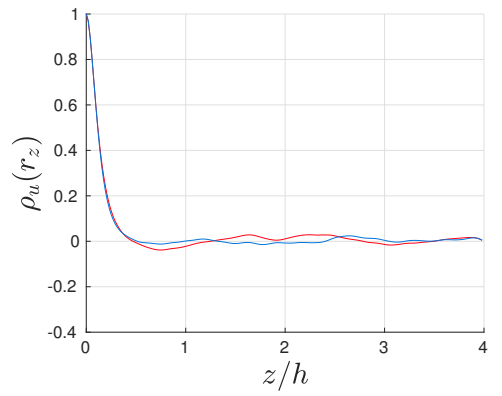

(d)

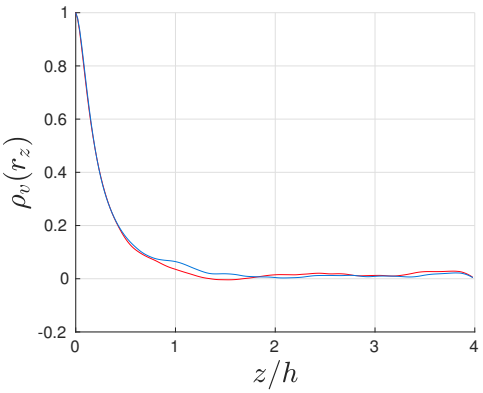

(b)

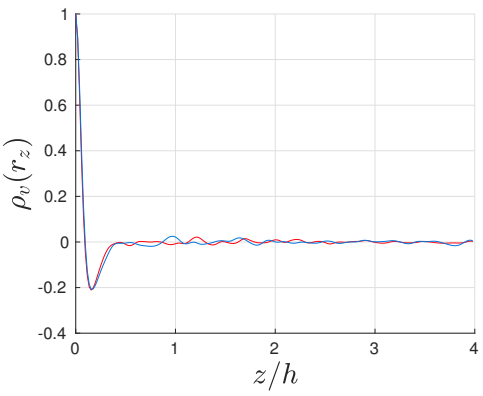

(e)

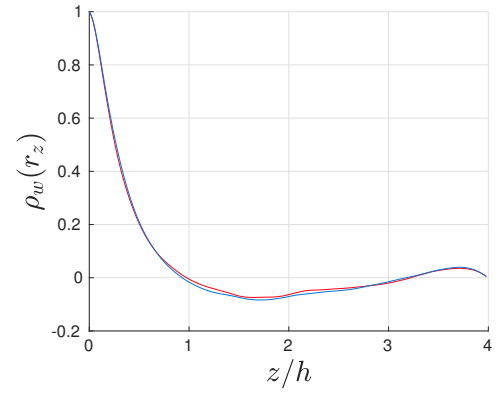

(c)

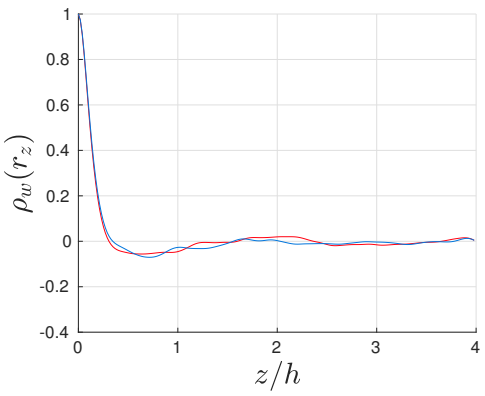

(f)

Figure 6-63: ACC of the velocity versus $z / h$ at $x / h \approx 15$ with $y^{+} \approx 193$ (top row) and $x / h \approx 19$ with $y^{+} \approx 5.5$ (bottom row) for the SGS-free (red) and WALE (blue) models; $\rho_{u}\left(r_{z}\right), \rho_{v}\left(r_{z}\right)$ and $\rho_{w}\left(r_{z}\right)$ correspond to the first, second and third columns, respectively. 


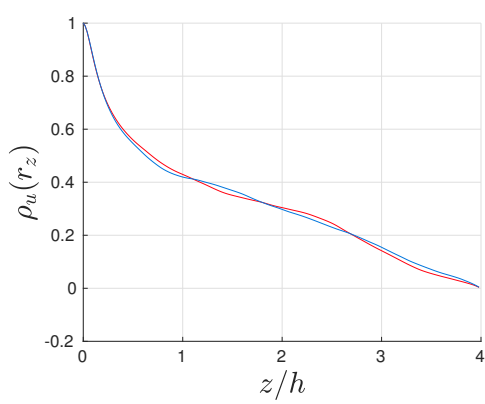

(a)

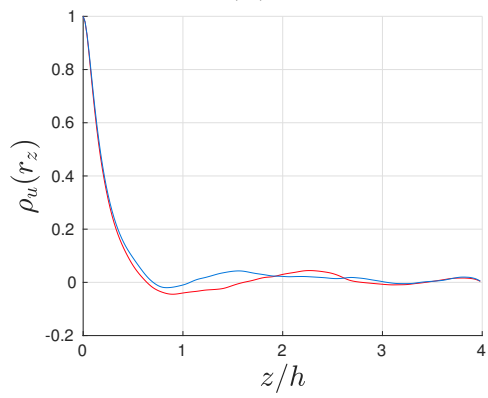

(d)

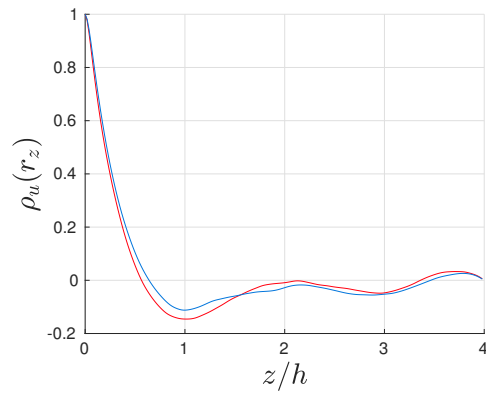

(g)

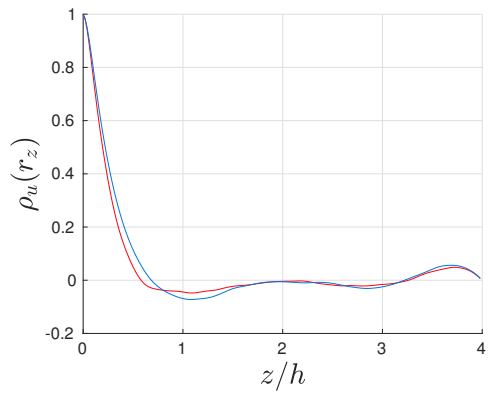

(j)

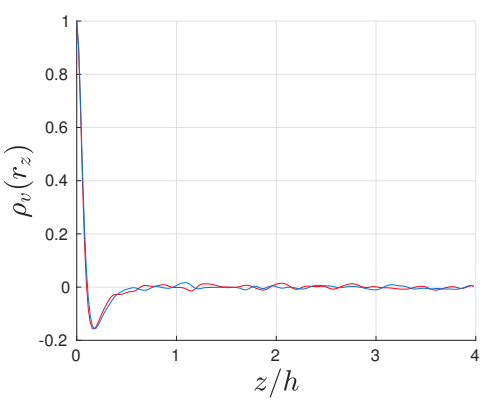

(b)

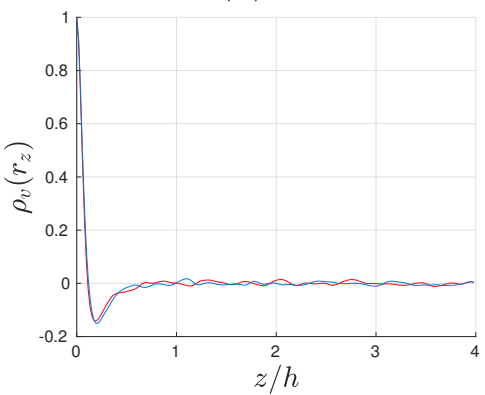

(e)

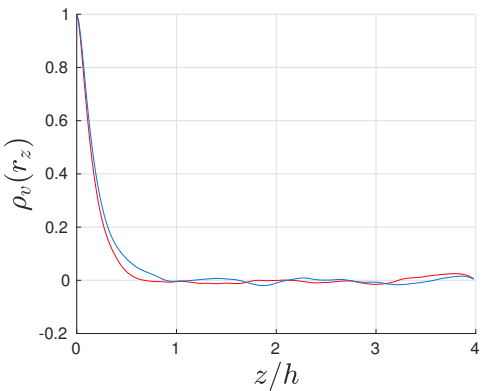

(h)

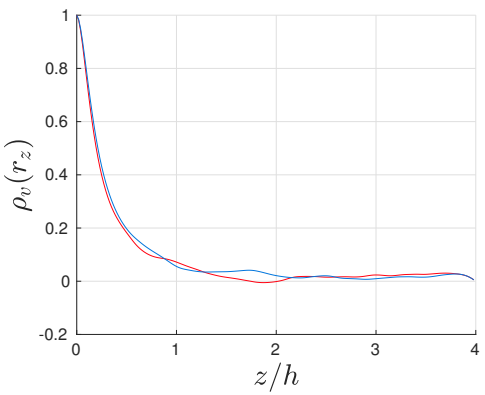

(k)

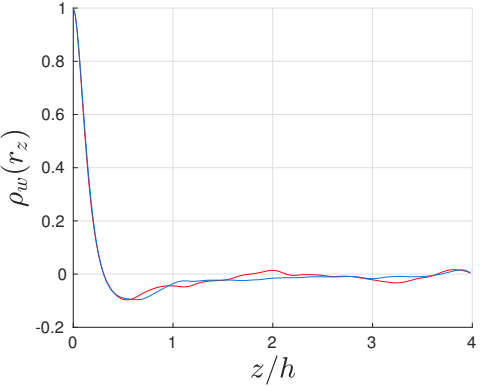

(c)

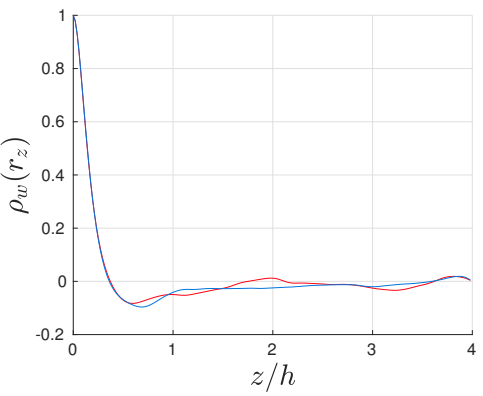

(f)

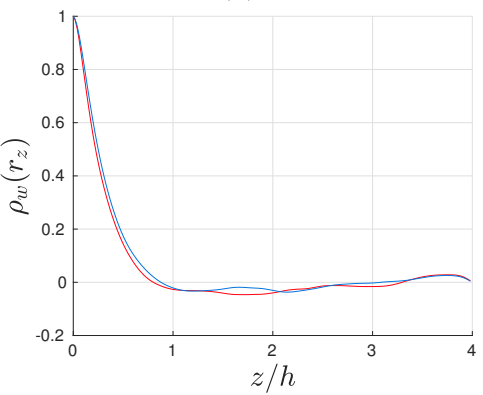

(i)

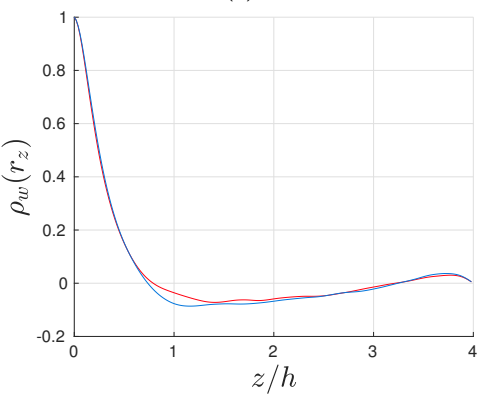

(l)

Figure 6-64: ACC of the velocity versus $z / h$ at $x / h \approx 10$ for the SGS-free (red) and WALE (blue) models; $\rho_{u}\left(r_{z}\right), \rho_{v}\left(r_{z}\right)$ and $\rho_{w}\left(r_{z}\right)$ correspond to the first, second and third columns, respectively; $y^{+} \approx 1, y^{+} \approx 5.5, y^{+} \approx 193$ and $y^{+} \approx 376$ correspond to the first, second, third and fourth row, respectively. 


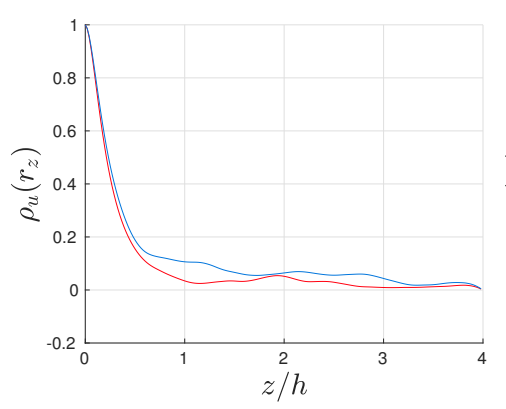

(a)

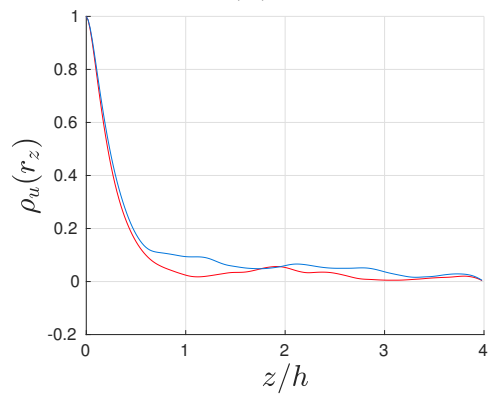

(d)

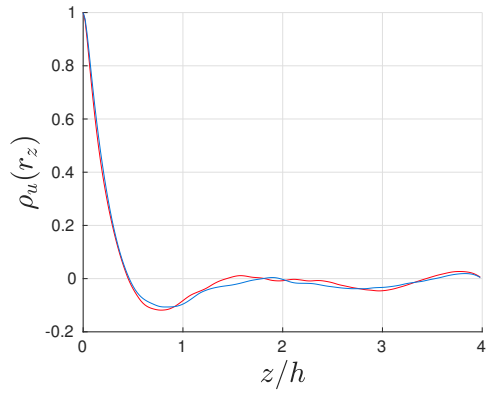

(g)

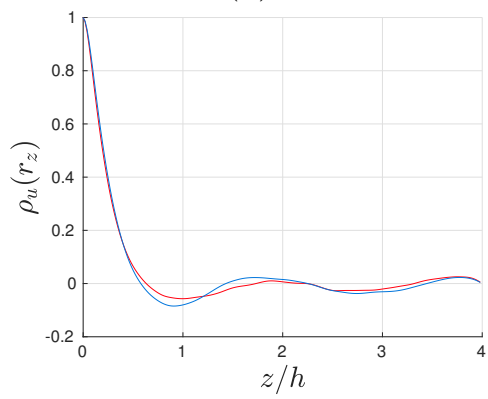

(j)

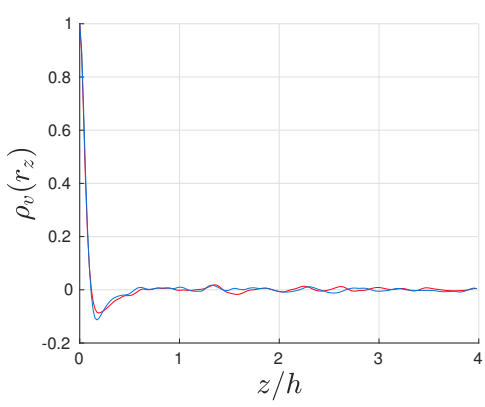

(b)

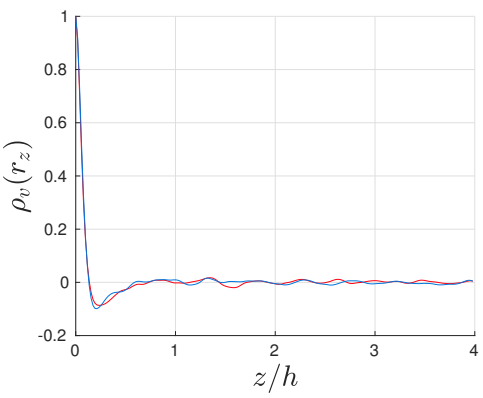

(e)

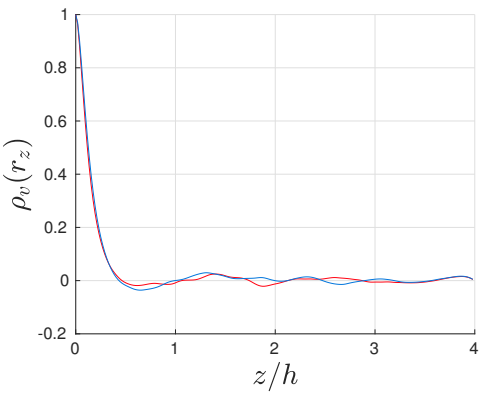

(h)

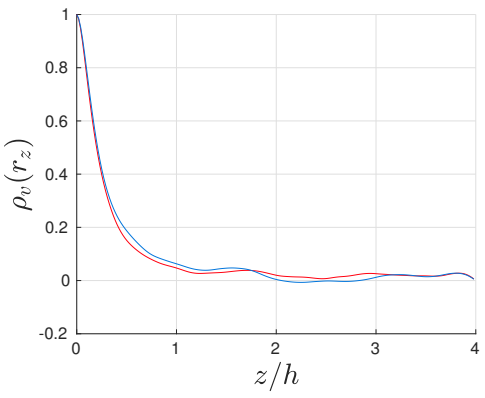

(k)

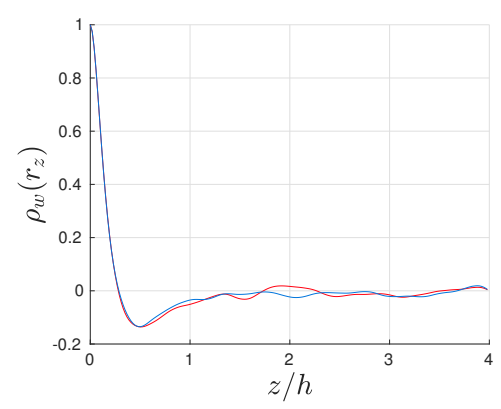

(c)

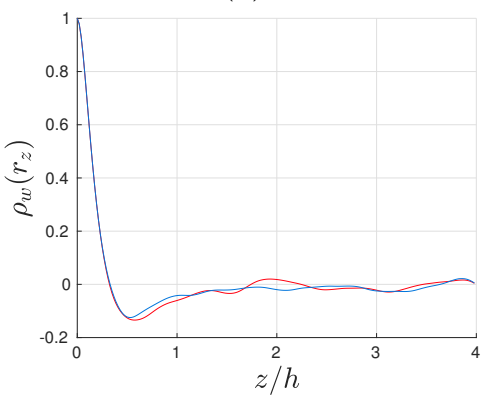

(f)

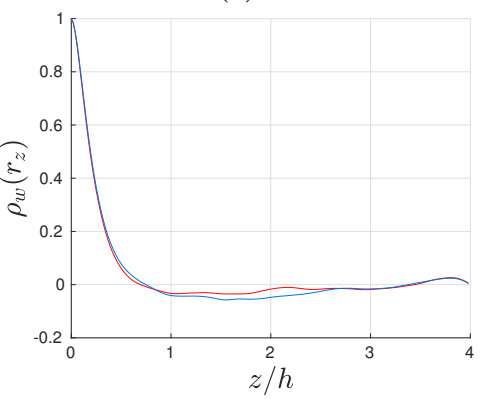

(i)

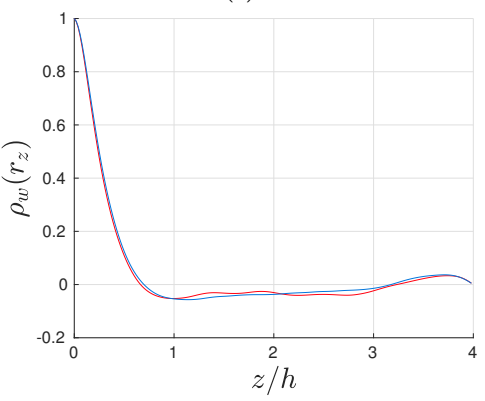

(l)

Figure 6-65: ACC of the velocity versus $z / h$ at $x / h \approx 6$ for the SGS-free (red) and WALE (blue) models; $\rho_{u}\left(r_{z}\right), \rho_{v}\left(r_{z}\right)$ and $\rho_{w}\left(r_{z}\right)$ correspond to the first, second and third columns, respectively; $y^{+} \approx 1, y^{+} \approx 5.5, y^{+} \approx 193$ and $y^{+} \approx 376$ correspond to the first, second, third and fourth row, respectively. 


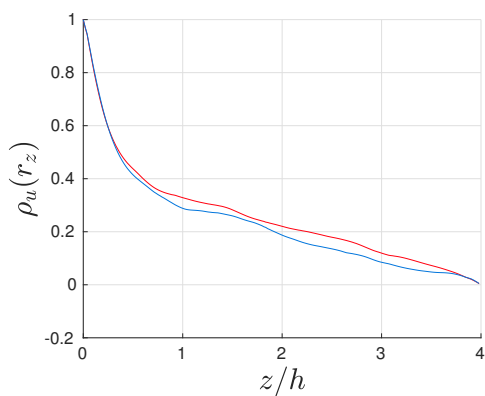

(a)

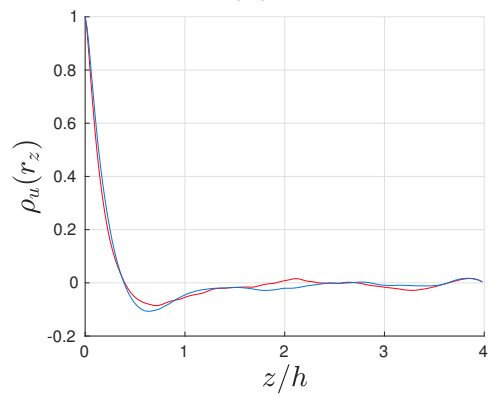

(d)

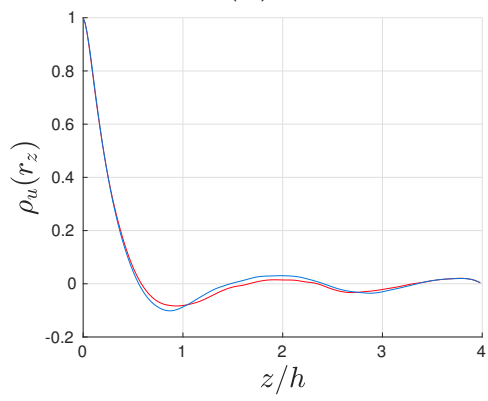

(g)

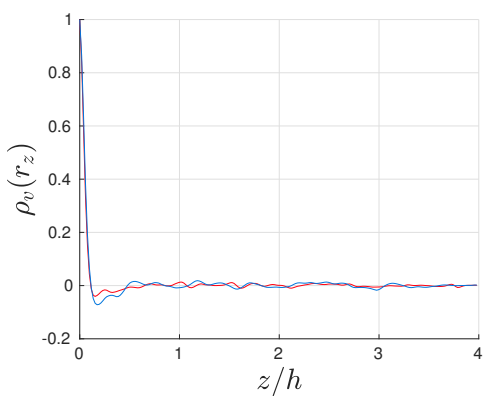

(b)

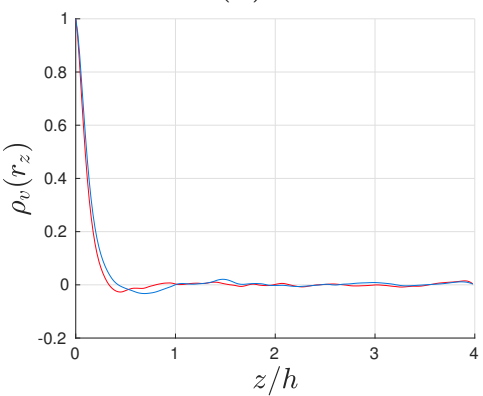

(e)

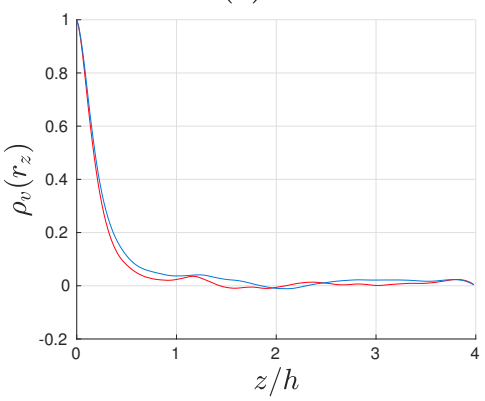

(h)

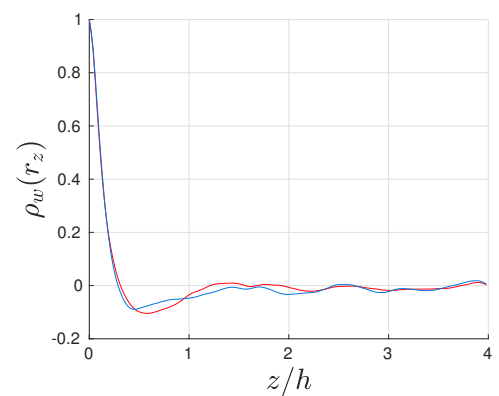

(c)

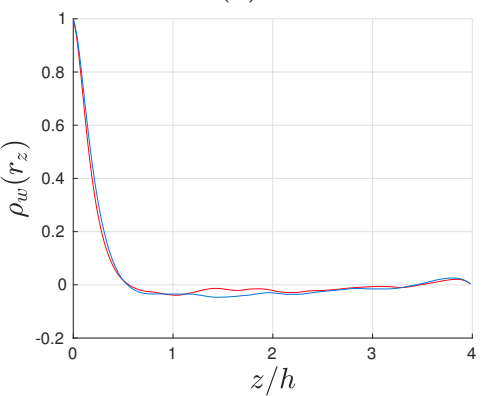

(f)

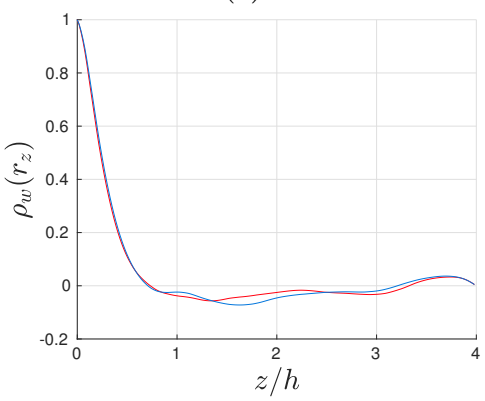

(i)

Figure 6-66: ACC of the velocity versus $z / h$ at $x / h \approx 4$ for the SGS-free (red) and WALE (blue) models; $\rho_{u}\left(r_{z}\right), \rho_{v}\left(r_{z}\right)$ and $\rho_{w}\left(r_{z}\right)$ correspond to the first, second and third columns, respectively; $y^{+} \approx 1, y^{+} \approx 193$ and $y^{+} \approx 376$ correspond to the first, second and third row, respectively. 


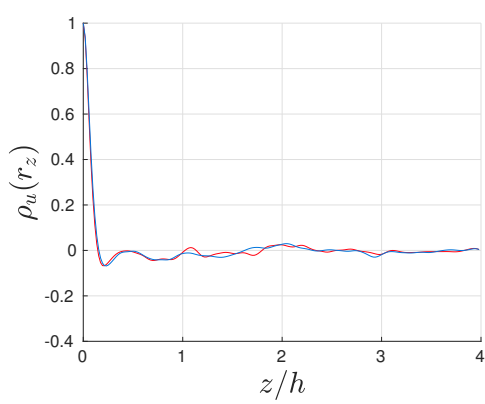

(a)

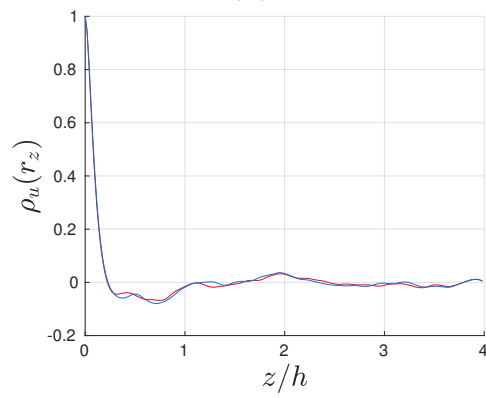

(d)

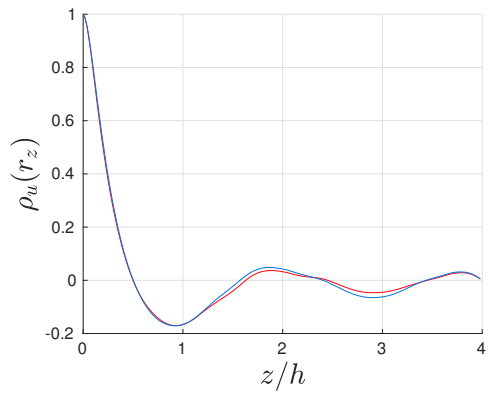

(g)

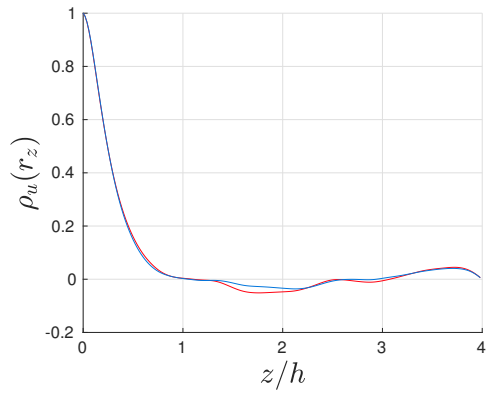

(j)

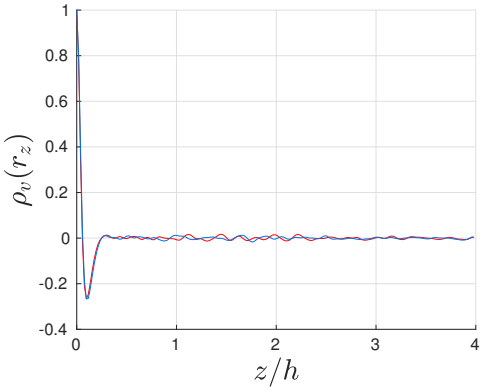

(b)

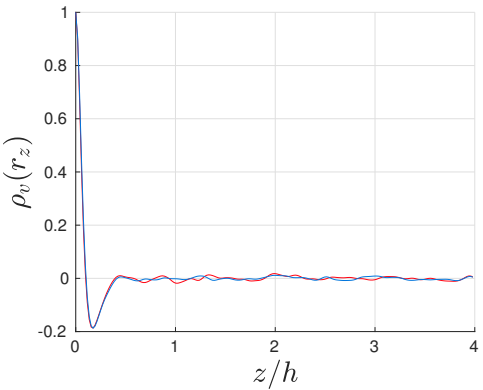

(e)

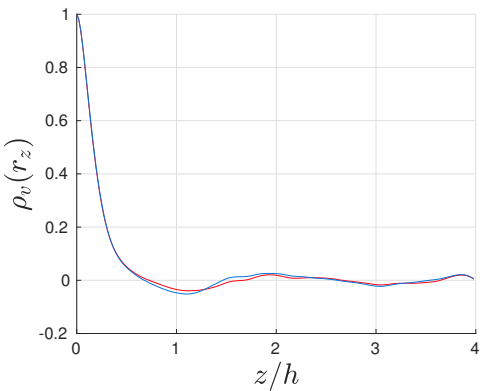

(h)

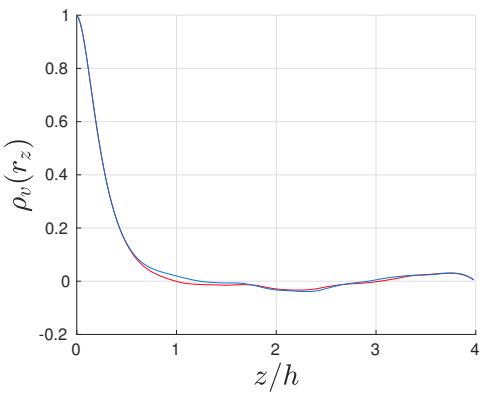

(k)

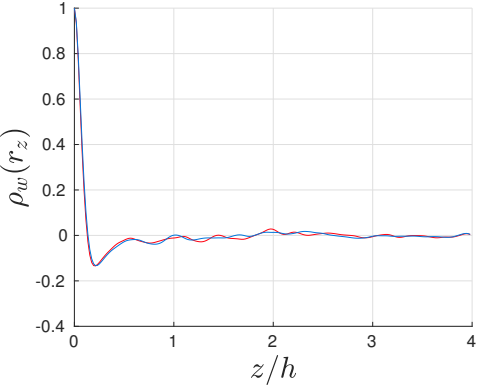

(c)

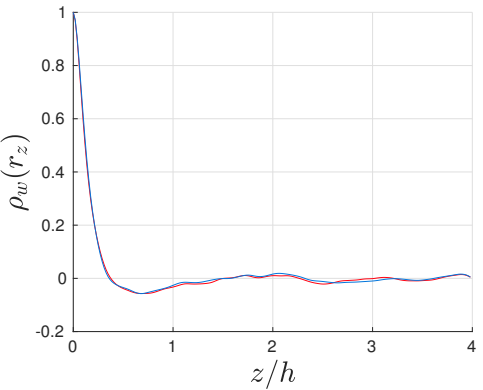

(f)

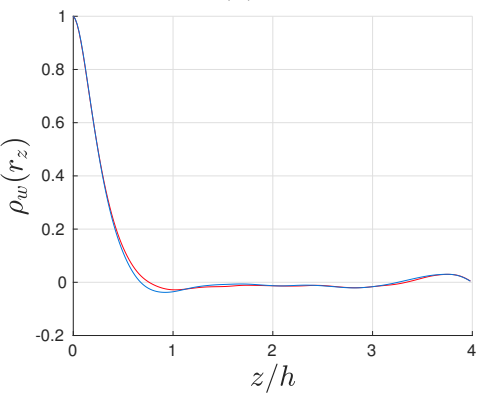

(i)

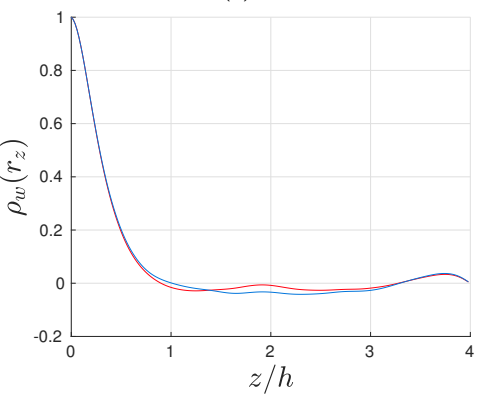

(l)

Figure 6-67: ACC of the velocity versus $z / h$ at $x / h \approx-3$ for the SGS-free (red) and WALE (blue) models; $\rho_{u}\left(r_{z}\right), \rho_{v}\left(r_{z}\right)$ and $\rho_{w}\left(r_{z}\right)$ correspond to the first, second and third columns, respectively; $y^{+} \approx 2.9, y^{+} \approx 22.8, y^{+} \approx 202$ and $y^{+} \approx 367$ correspond to the first, second, third and fourth row, respectively. 


\subsubsection{Probability of Backflow and Reattachment}

In this part, the probability of backflow and reattachment of the streamwise velocity is computed and presented in Figure 6-68. All data are sampled at the first wall-normal plane parallel and adjacent to the wall in the expansion region. Then, the sampled data are normalized using the total time steps used (i.e. 165000) and averaged in the spanwise homogeneous direction.

In the case of both reattachment (left panel) and backflow (right panel) in Figure 668 , the SGS-free and WALE models report very similar values. In $P(u(x)=0)$, two main peaks are observed with a local minimum in between. The first peak is likely to correspond to the interaction between the primary and secondary bubbles, while the latter peak denotes the reattachment zone. For a schematic illustration, refer to Figure 6-1b. In $P(u(x)<0)$, one major peak is observed. This, most likely, represents the recirculation region, especially the primary bubble. Using probability of backflow, the reattachment point may be approximated by $P(u(x)<0)=0.5$, which leads to 6.216 and 6.085 for the SGS-free and WALE models, respectively. These values also lie in the topmost peak of zero-streamwise probability with $P(u(x)=0)>0.2$.

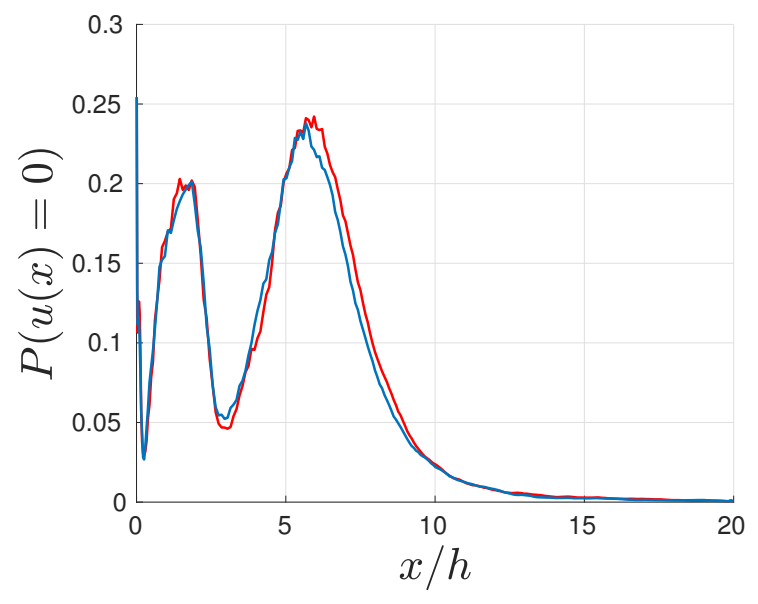

(a)

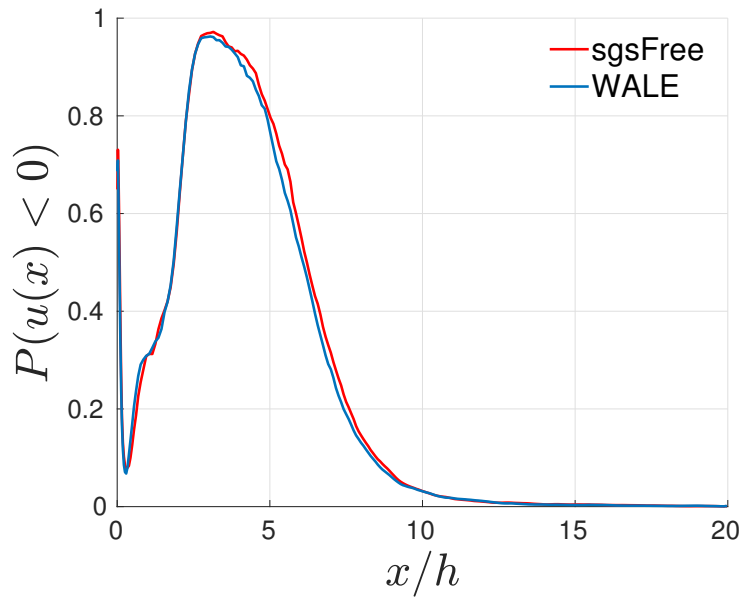

(b)

Figure 6-68: Probability of zero streamwise velocity (a) and reverse streamwise velocity (b) versus the streamwise coordinate. 


\subsubsection{Effects of Grid Refinement}

In this study, the effect of different grid resolutions is investigated. The grid resolutions used are the coarse (subsection 6.4.1), intermediate (subsection 6.4.2) and fine (subsection 6.4.3) from previous subsections; recall, the total number of cells in each of these grids are 704070, 1893900 and 7464006 , respectively. Moreover, for the first three parts, only the WALE SGS model will be subject to scrutiny using all three grids. Afterwards, in the flow reattachment computations, the SGS-free model is included as well ${ }^{2}$, although the coarse grid is excluded.

\subsubsection{Cell Sizes}

In Figure 6-69, four dimensionless cell sizes are displayed along the streamwise direction. The upper two panels correspond to the wall-normal cells directly adjacent to the wall (top-left) and the cells at the estimated TBL edge $\delta_{99}$ (top-right); the first is non-dimensionalized using wall units while the latter uses the momentum thickness $\theta$. The bottom two panels correspond to the cell sizes of the streamwise (bottom-left) and spanwise (bottom-right) directions; non-dimensionalization is done using wall units. Additionally, each panel displays all three grid resolution profiles using the WALE model only.

The wall-normal cell sizes $\Delta y_{1}^{+}$and $\Delta y_{N} / \theta$ show similar behavior along the streamwise direction for all grid resolutions. In inner scaling, all first wall-normal cell sizes retain a value less than unity (excluding the discontinuity at the step). The most notable discrepancy is observed at the pre-expansion region where the coarse-size grid resolution has the least value. As for $\Delta y_{N} / \theta$, slight zigzag-like behavior is observed after the reattachment point (i.e. $x / h>6$ ). This may be due to $\delta_{99}$ at the beginning of the recovery region. Note, in the case of the recirculation region, $0 \leq y / h \lesssim 6$, the momentum thickness is not well-defined.

The inner-scaled spanwise cell sizes of the coarse-size grid (blue) are more than

\footnotetext{
${ }^{2}$ Among the remaining five SGS models, both WALE and SGS-free models demonstrated the best agreement with the DNS and JD data in all three separate grid resolutions.
} 
double that of the intermediate's. In the intermediate-size grid (green), $\Delta z^{+} \approx$ $\Delta x^{+} / 2$. As for the fine-size grid (red), the $\Delta x^{+}$at the pre-expansion region is about twice as large as the ones in the recovery region $(x / h>14)$. Moreover, $\Delta z^{+}$of the fine-size grid sustains below five wall units (i.e. more than five times that of the coarse-size grid). On average, in inner scaling, the ratios of the pre-expansion-torecovery region's cell sizes are roughly 1.8, 1.3 and 2, for the coarse, intermediate and fine grids, respectively.

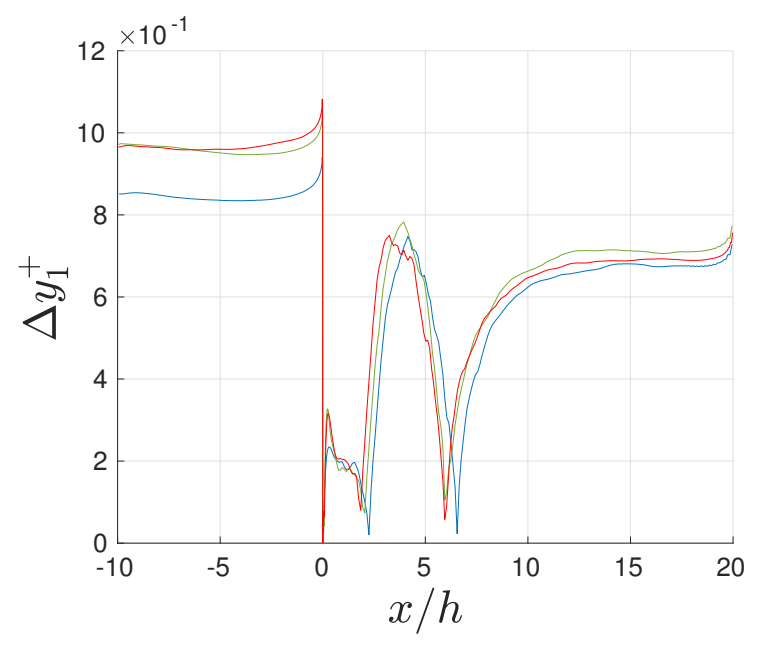

(a)

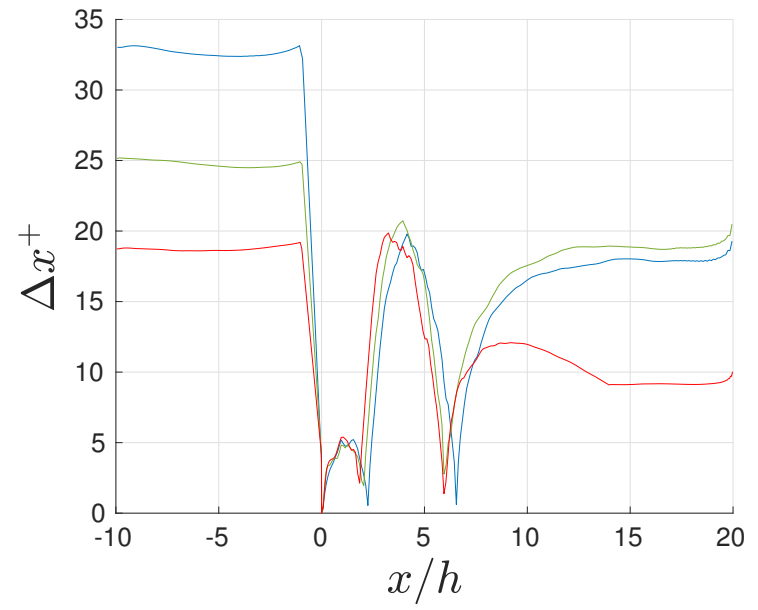

(c)

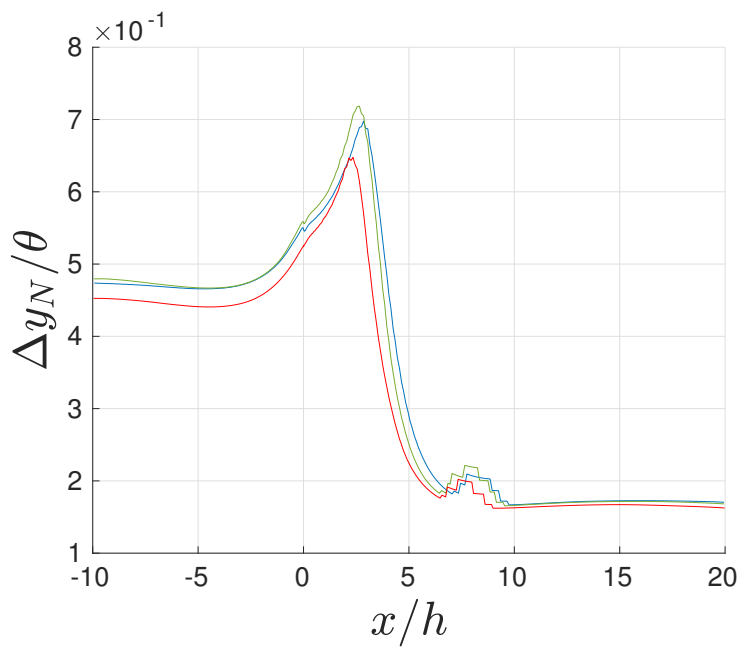

(b)

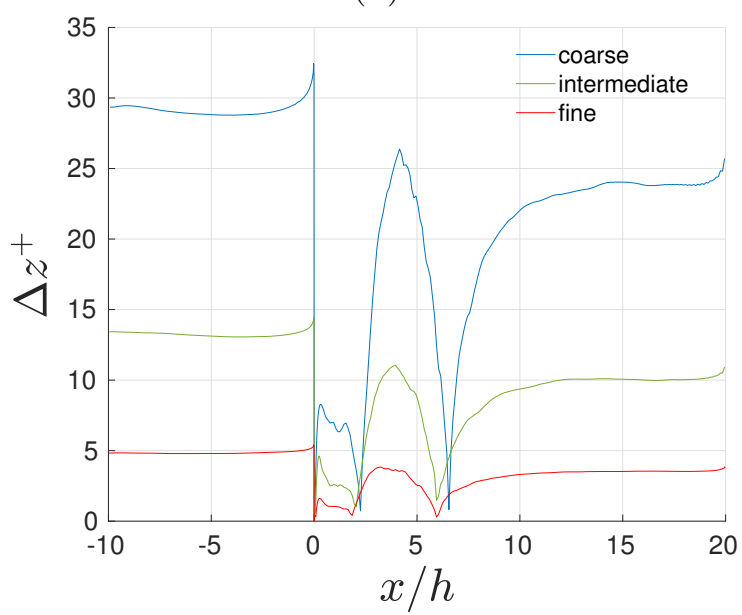

(d)

Figure 6-69: Non-dimensionalized first cell from the wall using inner scaling (a) and at the estimated TBL's edge using $\theta$ (b). Also, inner-scaled streamwise (c) and spanwise (d) cell sizes. 


\subsubsection{Subgrid Scale Viscosity}

The normalizes SGS viscosity is presented in Figure 6-70. The most active SGS viscosity is at the bottom of the expansion region, especially at the recirculation region; in the case of the coarse-size grid, at such region $\left\langle\nu_{s g s}\right\rangle>\nu$. Additionally, with increasing refinement, the effect of the SGS viscosity in the WALE model is further reduced.

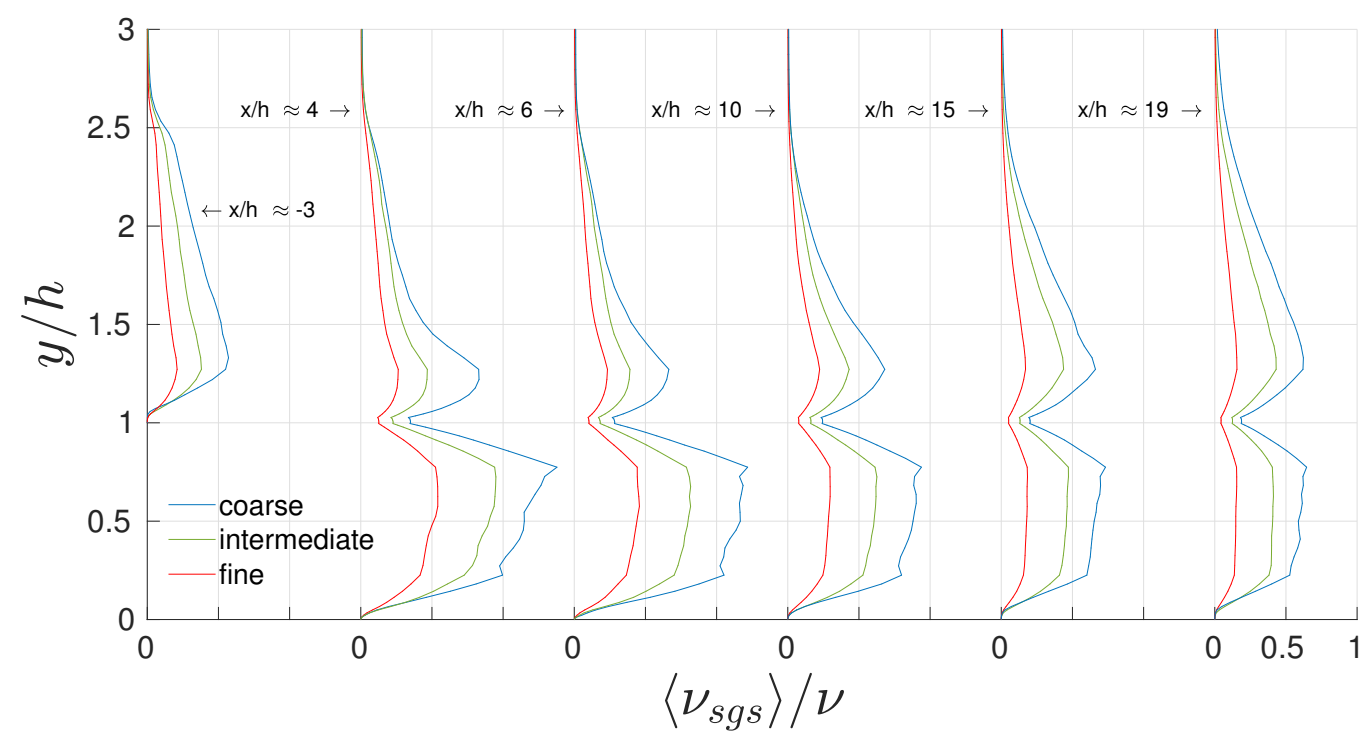

Figure 6-70: Normalized mean SGS viscosity profiles taken at six distinct locations using the WALE model along the streamwise direction in the flow over a backwardfacing step.

\subsubsection{Mean Streamwise Velocity and RMS of Wall-normal Velocity}

The inner-scaled mean streamwise velocity is presented in Figure 6-71, while the outerscaled RMS of the wall-normal velocity is presented in Figure 6-72. More particularly, for the sake of brevity, $\langle u\rangle^{+}$is illustrated at streamwise location $x / h \approx-3$ and 19. However, $v_{r m s}^{\prime}$ is displayed for all six different streamwise locations.

According to both panels of Figure 6-71, unlike the intermediate- and fine-size grids, $\langle u\rangle^{+}$of the coarse-size grid has a poorer agreement with both benchmark data. However, at $x / h \approx-3$, the coarse grid has a much more noticeable deviation from 
the benchmark data than at $x / h \approx 19$. As for Figure $6-72, v_{r m s}^{\prime} / U_{0}$ also confirms that the coarse-size grid has the least favorable agreement with the DNS and JD data at all six streamwise locations. Also, it is interesting to note how very similar both intermediate- and fine-size grid resolution results are.

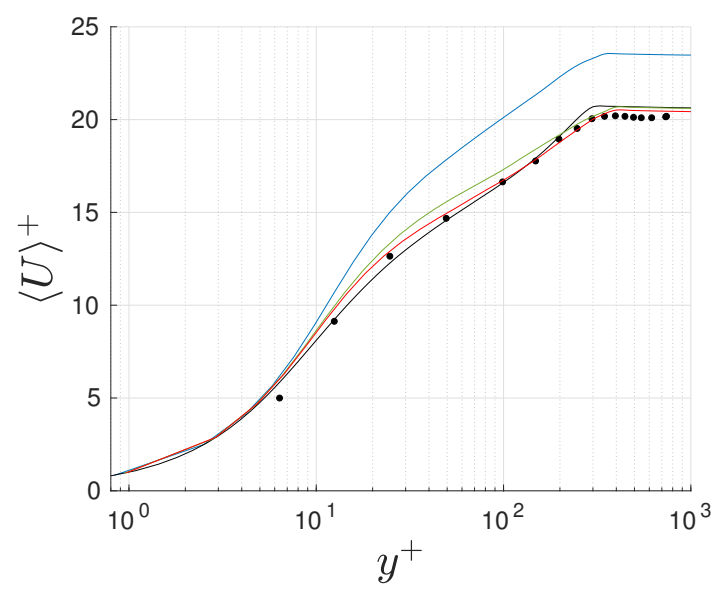

(a)

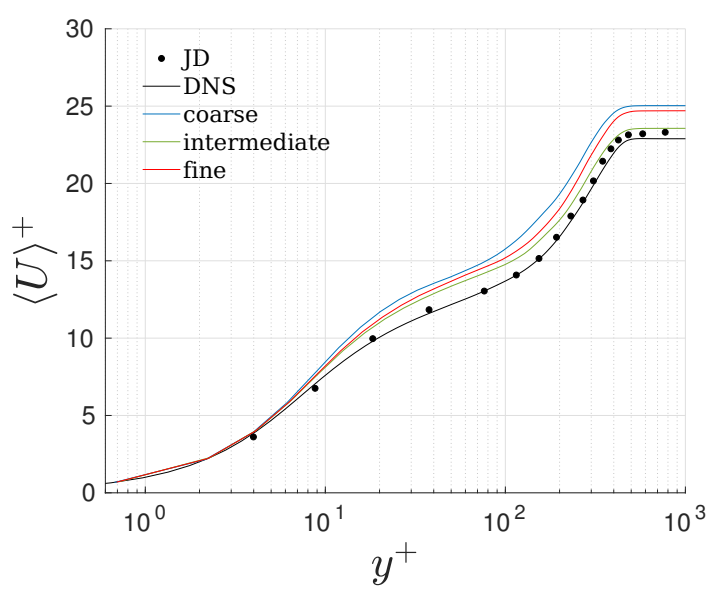

(b)

Figure 6-71: Inner-scaled mean streamwise velocity profiles versus the wall-normal coordinate taken at streamwise locations $x / h$ of approximately -3 (a) and 19 (f).

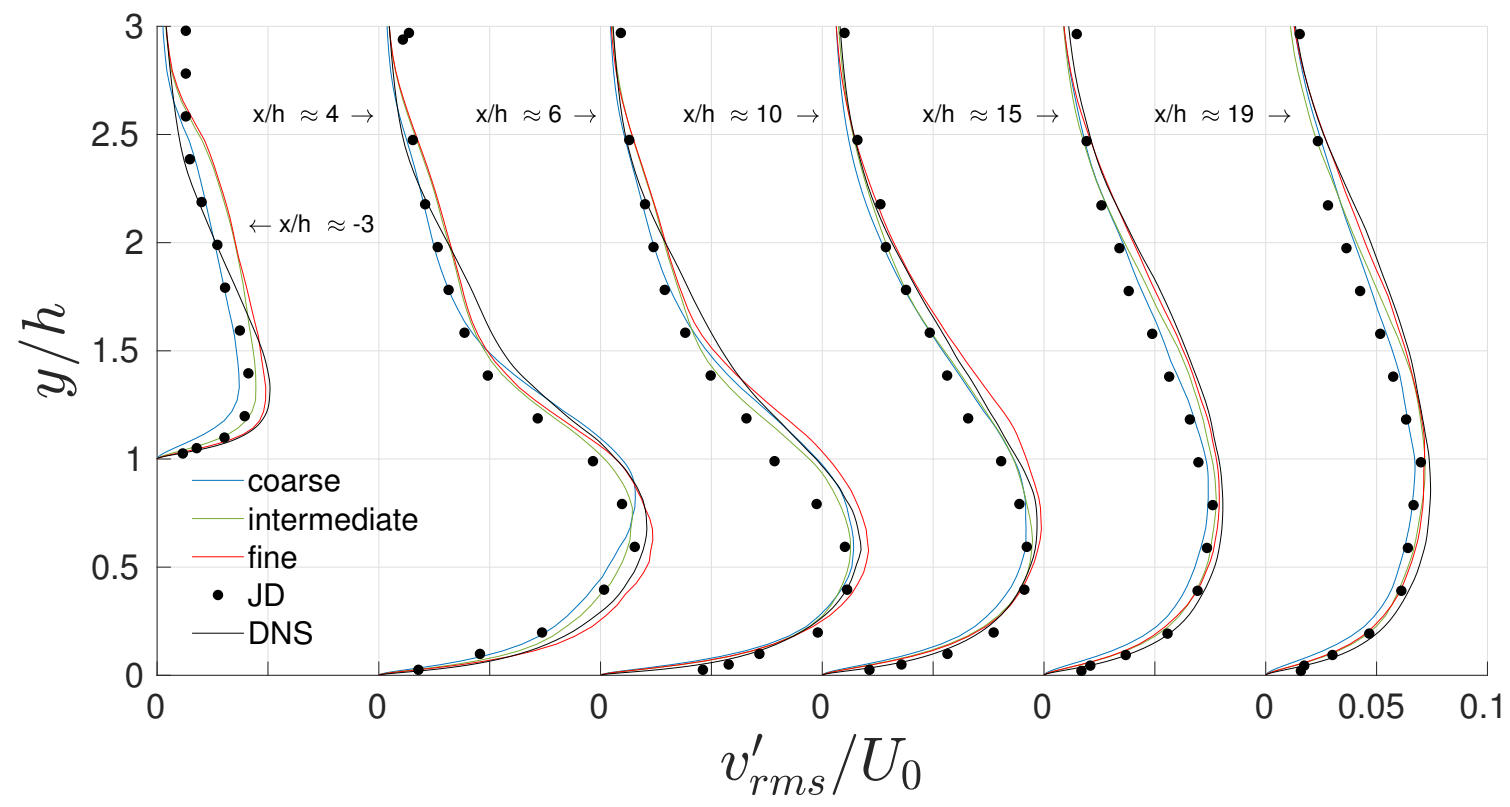

Figure 6-72: Outer-scaled $v_{r m s}^{\prime}$ profiles taken at six distinct locations in the streamwise direction in the flow over a backward-facing step. 


\subsubsection{Flow Reattachment Estimation}

In Table 6.5, a recap about the estimated reattachment locations is presented along with their respective relative error in absolute percentage form. The data are compared to both benchmark references. The relative error in percent is defined as:

$$
e^{r e l}(\Phi)=100\left|\frac{X_{r}-X_{r}^{\Phi}}{X_{r}^{\Phi}}\right|,
$$

where $X_{r}$ is the reattachment location and $\Phi$ is the reference benchmark data.

Table 6.5: Reattachment locations and their relative absolute error.

\begin{tabular}{c|cc|cc|c|c}
\multirow{2}{*}{ Parameter } & \multicolumn{2}{|c|}{ SGS-free } & \multicolumn{2}{|c|}{ WALE } & DNS $\dagger$ & JD $\dagger \dagger$ \\
& Intermediate & Fine & Intermediate & Fine & & \\
\hline$X_{r}$ & $6.48 h$ & $6.34 h$ & $5.99 h$ & $5.98 h$ & $6.28 h$ & $6 h$ \\
$e^{r e l}(\mathrm{DNS})$ & $3.18 \%$ & $0.95 \%$ & $4.62 \%$ & $4.78 \%$ & - & $4.46 \%$ \\
$e^{r e l}(\mathrm{JD})$ & $8.00 \%$ & $5.67 \%$ & $0.17 \%$ & $0.33 \%$ & $4.67 \%$ & - \\
\\
${ }^{\dagger}$ DNS data obtained from Le et al. [46]. ${ }^{\dagger \dagger}$ Experimental data obtained from Jovic and Driver [37].
\end{tabular}

\subsection{Concluding Remarks}

Three different grid resolution simulations are conducted in the flow over a BFS. The cells in each are refined in the streamwise and spanwise directions only; refer to Figure 6-4b. The wall-normal grid, motivated by the channel flow study in Chapter 5, is kept constant; refer to Figure 6-4a.

In subsection 6.4.1, a relatively coarse grid is used with a total of 704070 cells. Five different SGS models are used: the Smagorinsky, $k$-equation, dynamic $k$-equation, WALE and SGS-free models. Results indicate that the WALE, dynamic $k$-equation and SGS-free models perform much better than the Smagorinsky and $k$-equation models. For instance, when compared with the benchmark data, these results are illustrated in outer-scaled $\langle u\rangle$ and $u_{r m s}^{\prime}$ presented in Figures 6-6 and 6-7, respectively. The velocity profiles seem to deviate after the buffer zone from the expected behavior 
reported by the benchmark data. This is chiefly observed at the pre-expansion region in Figure 6-5. One reason for such behavior could be attributed to the unresolved inlet velocity profile using the precursor simulation. Moreover, the flow reattachment location computed from $C_{f}$ is determined to be $7.67 h, 8.98 h, 7.01 h, 6.55 h$ and $7.17 h$ for the Smagorinsky, $k$-equation, dynamic $k$-equation, WALE and SGS-free models, respectively. According to the DNS and JD findings, $X_{r}$ is $6.28 h$ and $6 h$, respectively. The $C_{f}$ profiles are illustrated in Figure 6-10b. This proves how bad the results the Smagorinsky, $k$-equation and SGS-free models return. Hence, before proceeding with further simulations on finer grids, the $k$-equation and Smagorinsky models are eliminated. As for the WALE model, it is interesting to note that, despite such a coarse-size grid, it manages to yield good agreement with both the DNS and JD data. This is evidenced in almost all velocity and Re-stress quantities and further advocated by $e^{r e l}<10 \%$ with respect to both benchmark data (see Table 6.5).

In subsection 6.4.2, an intermediate-size grid resolution consisting of 1893900 cells is used. The refinement is applied in both spanwise and streamwise directions (see Figure 6-69) in order to yield $\Delta x^{+} \leq 28$ and $\Delta z^{+} \leq 20$; at the pre-expansion region, the cell sizes are in the intervals $25 \leq \Delta x^{+} \leq 28$ and $12 \leq \Delta z^{+} \leq 20$, in accordance with previous channel flow analysis in Chapter 5. The SGS models used are the dynamic $k$-equation, WALE and SGS-free. The precursor's $\operatorname{Re}_{\theta}$ surprisingly converges into the same value as that of the coarser grid simulations which is $\operatorname{Re}_{\theta} \approx$ 630, although more than 2.5 times more cells are used (the target inlet $\operatorname{Re}_{\theta}=670$ ). The results of both WALE and SGS-free models display good agreement with the benchmark data, whereas the dynamic $k$-equation model shows more deviations from it - e.g. $\langle u\rangle^{+}$in Figure 6-12. This observation is also evident for $C_{p}$ and $C_{f}$ in Figure 6-17. At the pre-expansion region, the Re-stresses significantly under-predict the benchmark data in the case of the dynamic $k$-equation model. This could be explained by a low turbulence intensity occurring for such a model at that particular region. The flow reattachment location is approximately $5.99 h, 6.48 h$ and $6.84 h$ for 
the WALE, SGS-free and dynamic $k$-equation models, respectively. Therefore, the dynamic $k$-equation is neglected in further grid-refinement studies.

In subsection 6.4.3, a very fine grid in both streamwise and spanwise resolutions is used with a total of 7464006 cells. This results in $\Delta x^{+}<20$ and $\Delta z^{+} \lesssim 5$; in comparison to the coarse-size grid, on average, $\Delta x_{\text {fine }}^{+} \approx \Delta x_{\text {coarse }}^{+} / 2$ and $\Delta z_{\text {fine }}^{+} \approx$ $\Delta z_{\text {coarse }}^{+} / 6$; see Figure 6-69. Moreover, the spanwise cell sizes (based on the target inlet $\mathrm{Re}_{\tau}$ ) are about a third of that used in the DNS (see Table 6.4). The resulting inlet $\operatorname{Re}_{\theta}$ is approximately 687 , which is very close to the target 670 . All velocity and Re-stress quantities come into very good agreement with that of the benchmark data (Figures 6-20 to 6-23 and E-9 to E-12). Also, $X_{r}$ is estimated to be $5.98 h$ and $6.34 h$ for the WALE and SGS-free models, respectively.

In the case of the intermediate- and fine-size grid resolutions, both WALE SGS model and the SGS-free yield very good agreement with the DNS and JD benchmark data. Moreover, using the WALE SGS model in conjunction with the intermediatesize grid is most favorable; the additional computational and memory costs involved in using the fine grid seem to, arguably, outweigh the extra accuracy of the results. However, one interesting observation pertaining to the $X_{r}$ calculations is noted. With increasing grid resolution, the WALE model seems to converge more towards the JD experiment, while the SGS-free becomes closer to that of the DNS (see Table 6.5). The latter is expected, after all, a simulation without turbulence modeling on a well-refined grid should essentially converge towards a DNS (provided it resolves all integral-toKolmogorov length scales).

Finally, an exhaustive simulation is carried out using the finest grid in subsection 6.4.4. In terms of flow-through times, this results in an increase of more than $380 \%$ from the previous flow over a BFS simulations. The motivation behind such a study is to conduct a comprehensive simulation with extensive statistical data samples using the SGS-free and WALE SGS models. These data include the pressure, velocity, Re-stresses, $C_{p}$ and $C_{f}$, vorticity, high-order central statistical moments, TKE 
budget, one-dimensional PSD and ACC in the spanwise direction and probability of flow characteristics such as backflow and reattachment.

Quantities previously reported in the fine grid study (subsection 6.4.3) are dismissed, since they are more-or-less the same in this comprehensive investigation. The estimated $X_{r}$ computed from $C_{f}$ indicate it is approximately $6.143 h$ and $5.994 h$ for the SGS-free and WALE models, respectively. Compared to the same fine-size grid resolution in subsection 6.4.3, the SGS-free $X_{r}$ values have a relative error of just above $3 \%$. This possibly indicates that the SGS-free model requires additional sampling time than 5.6 flow-through times. However, when estimating $X_{r}$ using the probability of $P(u(x)<0)=0.5$, the observed results are approximately 6.216 and 6.085 for the SGS-free and WALE models, respectively; refer to Figure 6-68.

As for the TKE budget terms, the non-transport terms such as $\mathcal{P}_{k}, \mathcal{C}_{k}$ and $\varepsilon_{k}$ are in good agreement with the DNS data as reported in Figures 6-52 and 6-53. The most notable deviation is in the $\varepsilon_{k}$ profile, especially near the wall. This is expected, since the grid used in DNS is much more finer than LES in the viscous sublayer in order to fully resolve the Kolmogorov length scale. The ACC profiles in subsubsection 6.4.4.8 suggest the domain size in the spanwise direction is sufficient for this study, since $\rho\left(r_{z}>3\right) \approx 0$ for all velocity components. 


\section{Chapter 7}

\section{Conclusion and Future Work}

In this work, two separate and carefully conducted studies were performed on the statistically fully-developed turbulent channel flow and the turbulent flow over a backward-facing step. The studies were performed numerically with LES turbulence modeling using OpenFOAM. The aim of such studies was to address the effects of two important factors: grid resolution and subgrid scale models. Results were validated against available DNS and, whenever applicable, experimental data.

In the turbulent channel flow study, two target $\operatorname{Re}_{\tau}$ were considered: 180 and 300 . The first was used as a validation checkpoint before moving to higher Re-numbers, since the computational cost of the mesh is directly proportional to the target Renumber. A relatively fine grid resolution was used with both SGS-free and WALE models. Excellent agreement between the LES and DNS results were obtained.

In the turbulent channel flow with a target $\operatorname{Re}_{\tau}=300$, several different grid resolution and SGS models were investigated. Although this is still relatively low, yet this was selected on the basis of having a neighboring target $\operatorname{Re}_{\tau}$ to what is required at the inlet of the BFS simulations. Different wall-normal grid construction methods along with variants of equidistant cells in the remaining two homogeneous directions were considered. Furthermore, a SGS-free and two SGS models were used: WALE and

Smagorinsky models. The latter was tested with and without a van Driest damping 
function near the wall. All findings indicate that the SGS-free model performed the best for such a problem. Simultaneously, the M1 meshing strategy depicted the best compromise taking the computational cost into account. Moreover, the results exhibit more sensitivity towards the spanwise than the streamwise grid resolution. In wall units, a cell size between 25-28 and 12-20 in the streamwise and spanwise directions, respectively, was evidenced to have the best accuracy; although using $\Delta x^{+}<25$ and $\Delta z^{+}<12$ produces better results, nonetheless from an empirical standpoint, these were observed to yield a much higher computational overhead than accuracy gains. As for the wall-normal resolution, maintaining three cells in the viscous sublayer and about $\delta / 20$ cell sizes at the outer layer of the TBL with a geometric expansion in between was most preferred.

In the case of the turbulent flow over a BFS study, three distinct grid resolutions were explored. The target Re-number, based on the step-height, was $\mathrm{Re}_{h}=5100$. The grids were refined in the spanwise and streamwise directions, while the same wall-normal grid resolution was maintained. A SGS-free and four SGS models were used: $k$-equation, dynamic $k$-equation, WALE and Smagorinsky. Additionally, the BFS's inlet velocity TBL was sampled from a fully-developed turbulent channel flow simulation with matching flow characteristics.

Using the coarse-size BFS grid resolution, only the WALE SGS model managed to result in relatively good agreement with the benchmark data. The Smagorinsky and $k$-equation models, however, were significantly bad. The second study used an intermediate-size BFS grid resolution. The SGS models used were the dynamic $k$ equation, WALE and SGS-free models. Results of the latter two models showed very good agreement with the benchmark data. On the other hand, the dynamic $k$-equation model showed a poor outcome, especially at the pre-expansion region. Finally, an even finer BFS grid resolution resulting in about 7.5 million cells (i.e. roughly 4 times the number of cells in the intermediate grid) was used. Only the WALE and SGS-free models were utilized. The agreement with the benchmark data 
is very good in velocity, Re-stresses and $C_{f}$ and $C_{p}$ quantities.

Among all three different BFS grid resolutions, the intermediate grid seemed to yield the best compromise in between accuracy and computational/memory costs. Moreover, at the pre-expansion region, the cell sizes were about $\Delta x^{+} \approx 25$ and $\Delta z^{+} \approx 13$, which comply with the prior suggested values in the turbulent channel flow analysis (i.e. $\Delta x^{+} \approx 25-28$ and $\Delta z^{+} \approx 12-20$ ).

Additionally, a more detailed study was conducted for a relatively longer period of time using the finest grid and the SGS-free and the WALE SGS model. The results of the velocity and Re-stresses were almost identical to the ones reported in the 5.6 flowthrough time simulation using the same fine grid. Moreover, the TKE budget terms are computed and seem to be debatable. For instance, the non-transport terms show very good behavior while the transport terms, especially $\mathcal{T}^{(u u)}$, displayed noticeable deviations. Such behavior is attributed to the need of further refining the wall-normal grid for better outcomes with such targeted data. This hypothesis is further bolstered by the oscillatory behavior in the high-order statistical moments.

Additional sampled quantities accumulated are the mean pressure, one-dimensional ACC and PSD of the velocity in the spanwise direction, Re-stresses, vorticity, probability of reverse and reattachment of the flow. These data, along with case set-ups in OpenFOAM, are available online ${ }^{1}$ for open access and ease of research reproducibility.

Further future work may include different procedures. For instance, the numerical method could be replaced by a higher-order accurate method which could handle such simplistic geometry with relative ease. Another interesting variation is to experiment with unstructured and adaptive grid refinements that could better tackle the cells/nodes near the critical regions such as the TBL separation and recirculation. A more challenging option is to also consider different turbulence modeling procedures such as wall models via LES or even hybrid models that utilize RANS near the wall and LES far from it.

\footnotetext{
${ }^{1}$ https://bitbucket.org/inquisitor101/simulationdatasetsles/
} 
THIS PAGE IS INTENTIONALLY LEFT BLANK 


\section{Bibliography}

[1] DE Abbott and SJ Kline. Experimental investigation of subsonic turbulent flow over single and double backward facing steps. Journal of Basic Engineering, 84 (3):317-325, 1962.

[2] NA Adams and S Hickel. Implicit large-eddy simulation: Theory and application, 2009.

[3] Knut Akselvoll. Large eddy simulation of turbulent confined coannular jets and turbulent flow over a backward facing stepx. PhD thesis, Stanford University, 1995.

[4] J.D. Anderson. Computational Fluid Dynamics: The Basics with Applications. McGraw-Hill international editions. McGraw-Hill, 1995. ISBN 9780071132107.

[5] José Luis Aragón, Gerardo G Naumis, Ming Bai, Manuel Torres, and Philip K Maini. Turbulent luminance in impassioned van Gogh paintings. Journal of Mathematical Imaging and Vision, 30(3):275-283, 2008.

[6] Bassem F Armaly, F Durst, JCF Pereira, and B Schönung. Experimental and theoretical investigation of backward-facing step flow. Journal of fluid Mechanics, 127:473-496, 1983.

[7] Mustafa Barri and Helge I Andersson. Turbulent flow over a backward-facing step. part 1. effects of anti-cyclonic system rotation. Journal of Fluid Mechanics, 665:382-417, 2010.

[8] G Biswas, M Breuer, and F Durst. Backward-facing step flows for various expansion ratios at low and moderate Reynolds numbers. Journal of fluids engineering, 126(3):362-374, 2004.

[9] JP Boris, FF Grinstein, ES Oran, and RL Kolbe. New insights into large eddy simulation. Fluid dynamics research, 10(4-6):199-228, 1992.

[10] Jean Boussinesq. Essai sur la théorie des eaux courantes. Imprimerie nationale, 1877. 
[11] W Cabot. Near-wall models in large eddy simulations of flow behind a backwardfacing step. Annual Research Briefs, pages 199-210, 1996.

[12] Daniel C Chan and Rajat Mittal. Large-eddy simulation of a backward facing step flow using a least-squares spectral element method. 1996.

[13] JA Clark. A study of incompressible turbulent boundary layers in channel flow. Journal of Basic Engineering, 90(4):455-467, 1968.

[14] DE Coles and EA Hirst. Computation of turbulent boundary layers-1968. AFOSR-IFP-stanford conference. volume II-compiled data, 1969.

[15] Geneviève Comte-Bellot. Contribution a letude de la trubulence de conduite. These presente a la faculte des sciences de l'Universite de Grenoble, 1963.

[16] Augustus De Morgan. A budget of paradoxes. Longmans, Green, 1872.

[17] E. De Villiers. The Potential of Large Eddy Simulation for the Modeling of Wall Bounded Flows. PhD thesis, Imperial College of Science, Technology and Medicine, 2006.

[18] RB Dean. Reynolds number dependence of skin friction and other bulk flow variables in two-dimensional rectangular duct flow. Journal of Fluids Engineering, 100(2):215-223, 1978.

[19] James W Deardorff. A numerical study of three-dimensional turbulent channel flow at large Reynolds numbers. Journal of Fluid Mechanics, 41(2):453-480, 1970.

[20] Juan C Del Alamo, Javier Jiménez, Paulo Zandonade, and Robert D Moser. Scaling of the energy spectra of turbulent channels. Journal of Fluid Mechanics, 500:135-144, 2004.

[21] J Andrzej Domaradzki and Robert S Rogallo. Local energy transfer and nonlocal interactions in homogeneous, isotropic turbulence. Physics of Fluids A: Fluid Dynamics, 2(3):413-426, 1990.

[22] ER Van Driest. On turbulent flow near a wall. Journal of the Aeronautical Sciences, 23(11):1007-1011, 1956.

[23] F Ducros, F Nicoud, and T Poinsot. Wall-adapting local eddy-viscosity models for simulations in complex geometries, 1998.

[24] Helmut Eckelmann. The structure of the viscous sublayer and the adjacent wall region in a turbulent channel flow. Journal of Fluid Mechanics, 65(3):439-459, 1974. 
[25] Albert Einstein. Die grundlage der allgemeinen relativitätstheorie. Annalen der Physik, 354(7):769-822, 1916.

[26] Joel H Ferziger and Milovan Peric. Computational methods for fluid dynamics. Springer Science \& Business Media, 2012.

[27] Massimo Germano, Ugo Piomelli, Parviz Moin, and William H Cabot. A dynamic subgrid-scale eddy viscosity model. Physics of Fluids A: Fluid Dynamics, 3(7): 1760-1765, 1991.

[28] Fujihiro Hamba. A hybrid RANS/LES simulation of turbulent channel flow. Theoretical and computational fluid dynamics, 16(5):387-403, 2003.

[29] Kiyosi Horiuti. Large eddy simulation of turbulent channel flow by one-equation modeling. Journal of the Physical Society of Japan, 54(8):2855-2865, 1985.

[30] Sergio Hoyas and Javier Jiménez. Scaling of the velocity fluctuations in turbulent channels up to $\mathrm{Re}_{\tau}=2003$. Physics of fluids, 18(1):011702, 2006.

[31] AKMF Hussain and WC Reynolds. Measurements in fully developed turbulent channel flow. Journal of Fluids Engineering, 97(4):568-578, 1975.

[32] Raad I Issa. Solution of the implicitly discretised fluid flow equations by operatorsplitting. Journal of computational physics, 62(1):40-65, 1986.

[33] Kaoru Iwamoto, Y Suzuki, and N Kasagi. Database of fully developed channel flow. Department of Mechanical Engineering, The University of Tokyo, THTLAB Internal Report No. ILR-0201, 2002.

[34] Kaoru Iwamoto, Yuji Suzuki, and Nobuhide Kasagi. Reynolds number effect on wall turbulence: toward effective feedback control. International journal of heat and fluid flow, 23(5):678-689, 2002.

[35] H Jasak. Error Analysis and Estimation for the Finite Volume Method with Applications to Fluid Flows, 1996. PhD thesis, Ph. D. Thesis, University of London Imperial College, 1996.

[36] Arne V Johansson and P Henrik Alfredsson. On the structure of turbulent channel flow. Journal of Fluid Mechanics, 122:295-314, 1982.

[37] Srba Jovic and David M Driver. Backward-facing step measurements at low Reynolds number, $\operatorname{Re}_{h}=5000.1994$.

[38] Nobuhide Kasagi and Akio Matsunaga. Three-dimensional particle-tracking velocimetry measurement of turbulence statistics and energy budget in a backwardfacing step flow. International Journal of Heat and Fluid Flow, 16(6):477-485, 1995. 
[39] Steven Kay. Intuitive probability and random processes using MATLABß. Springer Science \& Business Media, 2006.

[40] J Kim, SJ Kline, and JP Johnston. Investigation of a reattaching turbulent shear layer: flow over a backward-facing step. Journal of Fluids Engineering, 102(3): 302-308, 1980.

[41] John Kim, Parviz Moin, and Robert Moser. Turbulence statistics in fully developed channel flow at low Reynolds number. Journal of fluid mechanics, 177: 133-166, 1987.

[42] Won-Wook Kim and Suresh Menon. A new dynamic one-equation subgrid-scale model for large eddy simulations. In 33rd Aerospace Sciences Meeting and Exhibit, page 356, 1995.

[43] Andrey Nikolaevich Kolmogorov. The local structure of turbulence in incompressible viscous fluid for very large Reynolds numbers. In Dokl. Akad. Nauk SSSR, volume 30, pages 299-303, 1941.

[44] J Kostas, J Soria, and M Chong. Particle image velocimetry measurements of a backward-facing step flow. Experiments in fluids, 33(6):838-853, 2002.

[45] John Laufer. Investigation of turbulent flow in a two-dimensional channel. 1951.

[46] Hung Le, Parviz Moin, and John Kim. Direct numerical simulation of turbulent flow over a backward-facing step. Journal of fluid mechanics, 330:349-374, 1997.

[47] Myoungkyu Lee and Robert D. Moser. Direct numerical simulation of turbulent channel flow up to $R e_{\tau} \approx 5200$. Journal of Fluid Mechanics, 774:395-415, 2015. doi: $10.1017 / \mathrm{jfm} .2015 .268$.

[48] Douglas K Lilly. A proposed modification of the germano subgrid-scale closure method. Physics of Fluids A: Fluid Dynamics, 4(3):633-635, 1992.

[49] Adrián Lozano-Durán and Javier Jiménez. Effect of the computational domain on direct simulations of turbulent channels up to $\mathrm{Re}_{\tau}=4200$. Physics of Fluids, 26(1):011702, 2014.

[50] Stephen L Lyons, Thomas J Hanratty, and John B McLaughlin. Large-scale computer simulation of fully developed turbulent channel flow with heat transfer. International journal for numerical methods in fluids, 13(8):999-1028, 1991.

[51] NN Mansour, John Kim, and Parviz Moin. Reynolds-stress and dissipation-rate budgets in a turbulent channel flow. Journal of Fluid Mechanics, 194:15-44, 1988. 
[52] PJ Mason and NS Callen. On the magnitude of the subgrid-scale eddy coefficient in large-eddy simulations of turbulent channel flow. Journal of Fluid Mechanics, 162:439-462, 1986.

[53] Johan Meyers and Pierre Sagaut. Is plane-channel flow a friendly case for the testing of large-eddy simulation subgrid-scale models? Physics of Fluids, 19(4): 048105, 2007.

[54] Parviz Moin and John Kim. Numerical investigation of turbulent channel flow. Journal of fluid mechanics, 118:341-377, 1982.

[55] Parviz Moin and Robert D Moser. Characteristic-eddy decomposition of turbulence in a channel. Journal of Fluid Mechanics, 200:471-509, 1989.

[56] Parviz Moin, WC Reynolds, and Joel H Ferziger. Large eddy simulation of incompressible turbulent channel flow. 1978.

[57] Todd K Moon and Wynn C Stirling. Mathematical methods and algorithms for signal processing, volume 1. Prentice hall New York, 2000.

[58] Robert D Moser, John Kim, and Nagi N Mansour. Direct numerical simulation of turbulent channel flow up to $\mathrm{Re}_{\tau}=590$. Physics of fluids, 11(4):943-945, 1999.

[59] Timofey Mukha. Inflow generation for scale-resolving simulations of turbulent boundary layers. PhD thesis, Uppsala University, 2016.

[60] Timofey Mukha and Mattias Liefvendahl. Large-eddy simulation of turbulent channel flow, 2015.

[61] Timofey Mukha and Mattias Liefvendahl. The generation of turbulent inflow boundary conditions using precursor channel flow simulations. Computers $\mathcal{E}$ Fluids, 156:21-33, 2017.

[62] Hassan M Nagib and Kapil A Chauhan. Variations of von kármán coefficient in canonical flows. Physics of Fluids, 20(10):101518, 2008.

[63] Franck Nicoud and Frédéric Ducros. Subgrid-scale stress modelling based on the square of the velocity gradient tensor. Flow, turbulence and Combustion, 62(3): 183-200, 1999.

[64] Balram Panjwani, IS Ertesvag, Andrea Gruber, and Kjell Erik Rian. Large eddy simulation of backward facing step flow. In 5th National Conference on Computational Mech, 2009. 
[65] Suhas V Patankar and D Brian Spalding. A calculation procedure for heat, mass and momentum transfer in three-dimensional parabolic flows. International journal of heat and mass transfer, 15(10):1787-1806, 1972.

[66] VC Patel and MR Head. Some observations on skin friction and velocity profiles in fully developed pipe and channel flows. Journal of Fluid Mechanics, 38(1): 181-201, 1969.

[67] Stephen B Pope. Turbulent flows, 2000.

[68] Saleh Rezaeiravesh and Mattias Liefvendahl. Grid construction strategies for wall-resolving large eddy simulation and estimates of the resulting number of grid points. In Technical Report 2017-005. Uppsala University, 2017.

[69] Jeffrey Rutledge and Charles A Sleicher. Direct simulation of turbulent flow and heat transfer in a channel. part I: smooth walls. International journal for numerical methods in fluids, 16(12):1051-1078, 1993.

[70] Yousef Saad. Iterative methods for sparse linear systems. SIAM, 2003.

[71] Pierre Sagaut. Large eddy simulation for incompressible flows: an introduction. Springer Science \& Business Media, 2006.

[72] Ulrich Schumann. Subgrid scale model for finite difference simulations of turbulent flows in plane channels and annuli. Journal of computational physics, 18(4): 376-404, 1975.

[73] Joseph Smagorinsky. General circulation experiments with the primitive equations: I. the basic experiment. Monthly weather review, 91(3):99-164, 1963.

[74] Steven Smith. Digital signal processing: a practical guide for engineers and scientists. Elsevier, 2013.

[75] Roland B Stull. An introduction to boundary layer meteorology, volume 13. Springer Science \& Business Media, 2012.

[76] Jonathan Swift. On poetry: A rhapsody (1733). Gulliver's Travels and Other Writings, Miriam Kosh Starkman (New York: Bantam, 1962), 633, 2012.

[77] Li Tan and Jean Jiang. Digital signal processing: fundamentals and applications. Academic Press, 2013.

[78] BA Toms. Large-eddy simulation of flow over a backward facing step: Assessment of inflow boundary conditions, eddy viscosity models, and wall functions. Journal of Applied Mechanical Engineering, 4:169, 2015. 
[79] Takahiro Tsukahara, Yohji Seki, Hiroshi Kawamura, and Daisuke Tochio. DNS of turbulent channel flow at very low Reynolds numbers. In TSFP Digital Library Online. Begel House Inc., 2005.

[80] Edward R Van Driest. On turbulent flow near a wall. J. Aeronaut. Sci, 23(11): 1007-1011, 1956.

[81] Henk Kaarle Versteeg and Weeratunge Malalasekera. An introduction to computational fluid dynamics: the finite volume method. Pearson Education, 2007.

[82] JC Vogel and JK Eaton. Combined heat transfer and fluid dynamic measurements downstream of a backward-facing step. Journal of heat transfer, 107(4): 922-929, 1985.

[83] John F Wendt. Computational fluid dynamics: an introduction. Springer Science \& Business Media, 2008.

[84] Akira Yoshizawa. Statistical theory for compressible turbulent shear flows, with the application to subgrid modeling. The Physics of fluids, 29(7):2152-2164, 1986.

[85] El-Sayed Mahmoud Zanoun. Answers to some open questions in wall-bounded laminar and turbulent shear flows. PhD thesis, Technische Fakultät der Universität Erlangen-Nürnberg., 2003. 
THIS PAGE IS INTENTIONALLY LEFT BLANK 


\section{Appendix A}

\section{Energy and Power Spectral Density}

\section{A.1 Energy/Power Spectra in One Dimension}

The process of computing the energy and power spectral densities will be considered in both time and space. Consider a general signal $f$, it can be either complex or real. It can be in space or time. This signal can be represented via a function $f(t)$ dependent on time $t$, or conversely, a function $f(r)$ dependent on the separation distance $r$. At first, the analysis shall be conducted in continuous space and then in its discrete counterpart. All in all, the explanation will be consistent with Pope [67, chap. 6].

\section{A.1.1 Continuous Space}

Definition A.1.1. Continuous Fourier transform pair. Consider a time-dependent continuous signal $f(t)$, its forward Fourier transform $F(\omega)$, denoted by $\mathscr{F}\{f(t)\}$, and the inverse Fourier transform, denoted by $\mathscr{F}^{-1}\{F(\omega)\}$, where $\omega=2 \pi f$ is the angular frequency and $f$ is the natural frequency.

$$
\left\{\begin{aligned}
F(\omega)=\mathscr{F}\{f(t)\} & \equiv \frac{1}{2 \pi} \int_{-\infty}^{\infty} f(t) e^{-i \omega t} d t \\
f(t)=\mathscr{F}^{-1}\{F(\omega)\} & \equiv \int_{-\infty}^{\infty} F(\omega) e^{i \omega t} d \omega
\end{aligned}\right.
$$


Similarly, for spatially dependent functions, consider $f(r)$ with wavenumber $\kappa=$ $2 \pi / r_{\max }$, where $r$ is the (uniformly equidistant) separation distance taken between any two points on the same line.

$$
\begin{cases}F(\kappa)=\mathscr{F}\{f(r)\} & \equiv \frac{1}{2 \pi} \int_{-\infty}^{\infty} f(x) e^{-i \kappa r} d r, \\ f(r)=\mathscr{F}^{-1}\{F(\kappa)\} & \equiv \int_{-\infty}^{\infty} F(\kappa) e^{i \kappa r} d \kappa\end{cases}
$$

The two equations in Equation A.1 and Equation A.2 form a Fourier transform pair each in continuous space for a given periodic signal. Next, an important theorem shall be introduced: Parseval's, also known as Plancherel's, theorem.

Theorem A.1.1 (Continuous Parseval's theorem).

$$
\int_{-\infty}^{\infty} f^{2}(t) d t=2 \pi \int_{-\infty}^{\infty}|F(\omega)|^{2} d \omega .
$$

Proof. Starting from the left hand side of Equation A.3:

$$
\begin{aligned}
\int_{-\infty}^{\infty} f^{2}(t) d t & =\int_{-\infty}^{\infty} f(t)\left[\int_{-\infty}^{\infty} F(\omega) e^{i \omega t} d \omega\right] d t \\
& =\int_{-\infty}^{\infty} F(\omega)\left[\int_{-\infty}^{\infty} f(t) e^{i \omega t} d t\right] d \omega \\
& =2 \pi \int_{-\infty}^{\infty} F(\omega) F(-\omega) d \omega \\
& =2 \pi \int_{-\infty}^{\infty}|F(\omega)|^{2} d \omega,
\end{aligned}
$$

where $F(-\omega)=F^{*}(\omega)$ denotes the complex conjugate of $F(\omega)$.

Note that the Fourier transform has many conventions when being defined, this is specially true for the normalization factor. Some prefer to split it in between the inverse and forward Fourier transforms (e.g. with a normalization factor of $1 / \sqrt{2 \pi}$ 
in each) while others might select to perform the normalization on either. In this description, the convention is adopted as that used by Pope [67, Appendix D].

The forward Fourier transform is used to convert a signal from time to frequency space, while the latter, the inverse Fourier transform, is used to go back from frequency to temporal space.

\section{A.1.2 Discrete Space}

The analysis here shall proceed similar to that of the continuous domain, however in the discrete space instead. First, a definition of the discrete Fourier transform pair shall take place.

Definition A.1.2. Discrete Fourier transform pair. Consider a discrete signal $f[n]$ composed of $N$ points, its forward discrete Fourier transform $\hat{F}(\omega)$, denoted by $\mathscr{F}\{f[n]\}$, and its inverse discrete Fourier transform, denoted by $\mathscr{F}^{-1}\{\hat{F}[k]\}$.

$$
\left\{\begin{array}{l}
\hat{F}[k]=\mathscr{F}\{f[n]\} \quad \equiv \frac{1}{N} \sum_{n=0}^{N-1} f[n] e^{-2 \pi i k n / N} \\
f[n]=\mathscr{F}^{-1}\{\hat{F}[k]\} \quad \equiv \sum_{k=0}^{N-1} \hat{F}[k] e^{2 \pi i k n / N}
\end{array}\right.
$$

Next, analogous to Theorem A.1.1, Parseval's theorem is introduced below in its discrete form.

Theorem A.1.2 (Discrete Parseval's theorem).

$$
\sum_{n=0}^{N-1}|f[n]|^{2}=N \sum_{k=0}^{N-1}|\hat{F}[k]|^{2} .
$$


Proof. Starting from the left hand side of Equation A.5:

$$
\begin{aligned}
\sum_{n=0}^{N-1}|f[n]|^{2} & =\sum_{n=0}^{N-1} f[n]\left[\sum_{k=0}^{N-1} \hat{F}[k] e^{2 \pi i k n / N}\right] \\
& =\sum_{k=0}^{N-1} \hat{F}[k]\left[\sum_{n=0}^{N-1} f[n] e^{2 \pi i k n / N}\right] \\
& =N \sum_{k=0}^{N-1} \hat{F}[k] \hat{F}^{*}[k] \\
& =N \sum_{k=0}^{N-1}|\hat{F}[k]|^{2},
\end{aligned}
$$

where the superscript in $\hat{F}^{*}$ denotes the complex conjugate of $\hat{F}$.

\section{A.1.3 Parseval's Relation to (homogeneous) Turbulence}

Moving to the relation of the prior exemplary signal, $f$, into turbulence spectra, the result is given by setting the original signal as the velocity fluctuation $f=u^{\prime}$ and its forward discrete Fourier transform as $\hat{F}=\hat{u}^{\prime}$. Since this procedure is restricted to a one-dimensional (homogeneous) spectra, it would be convenient to take for the sake of demonstration the velocity fluctuation value of only in the x-direction. Recall, the velocity fluctuation is defined as $u^{\prime}=u-U$, where $U=\langle u\rangle$ and the brackets $\langle$. denote the ensemble average taken in both space and time across a homogeneous flow direction. Therefore, after substitution, the following is inferred:

$$
\sum_{n=0}^{N-1}\left|u^{\prime}[n]\right|^{2}=N \sum_{k=0}^{N-1}\left|\hat{u}^{\prime}[k]\right|^{2} .
$$

Some care must be taken here. First, the mode corresponding to $n=0$ is the mean value (usually it is discarded and not accounted for). This can be easily illustrated when substituting in Equation A.6. Second, the frequencies (or similarly wavenumbers) are symmetric by means of the complex conjugate. This can also be 
easily demonstrated if the reader takes a simple small number of data points and apply Equation A.6. Also, whether or not the number of points, $N$, is even or odd is important during the process of obtaining the ESD. Generally, the ESD function can be expressed as:

$$
E[k]=2\left|\hat{u}^{\prime}[k]\right|^{2}
$$

The multiplier by two is because now, unlike in Equation A.6, the frequencies (or wavenumbers) do not reach the $(N-1)^{t h}$ value anymore, they, instead, only reach the mid-value (i.e. $k=n_{f}$ ). Thus, in order to account for the second-half of the frequencies (which are commonly referred to as 'negative-frequencies', e.g. see [74, chap. 12]), a multiplication by two is performed. Again, this is solely due to symmetry by complex conjugacy of the nature of the Fourier transform. For more information about spectral density and aliasing, refer to Stull [75, chap. 8].

Before proceeding, however, a crucial point must be addressed: the Nyquist frequency. In fact, this is the sole reason of 'folding' the signal at half-way through and in particular at $k=n_{f}$, where $n_{f}=N / 2$ denotes the Nyquist frequency. The reasoning behind the Nyquist theorem is that for a given digital ${ }^{1} \mathrm{~N}$-sample signal, it takes at least twice the number of samples (i.e. $2 N$ ) in order to fully capture it. For frequencies higher than $n_{f}$, aliasing takes place. For instance, if a wave signal is traveling with a period of $\tau$, then the number of samples used must be taken at least once every $\tau / 2$. In the case of a fluctuating velocity, that is why in actuality the maximum number of samples one can use without resorting to aliasing can be half of the original signal - i.e. $n_{f}=N / 2$. For a holistic overview of the pseudo-algorithm in computing the ESD, refer to Algorithm 2.

\footnotetext{
${ }^{1}$ In signal processing, an analog signal is any continuous signal, whereas a digital signal is a discrete signal; e.g. see Tan and Jiang [77, chap. 1].
} 


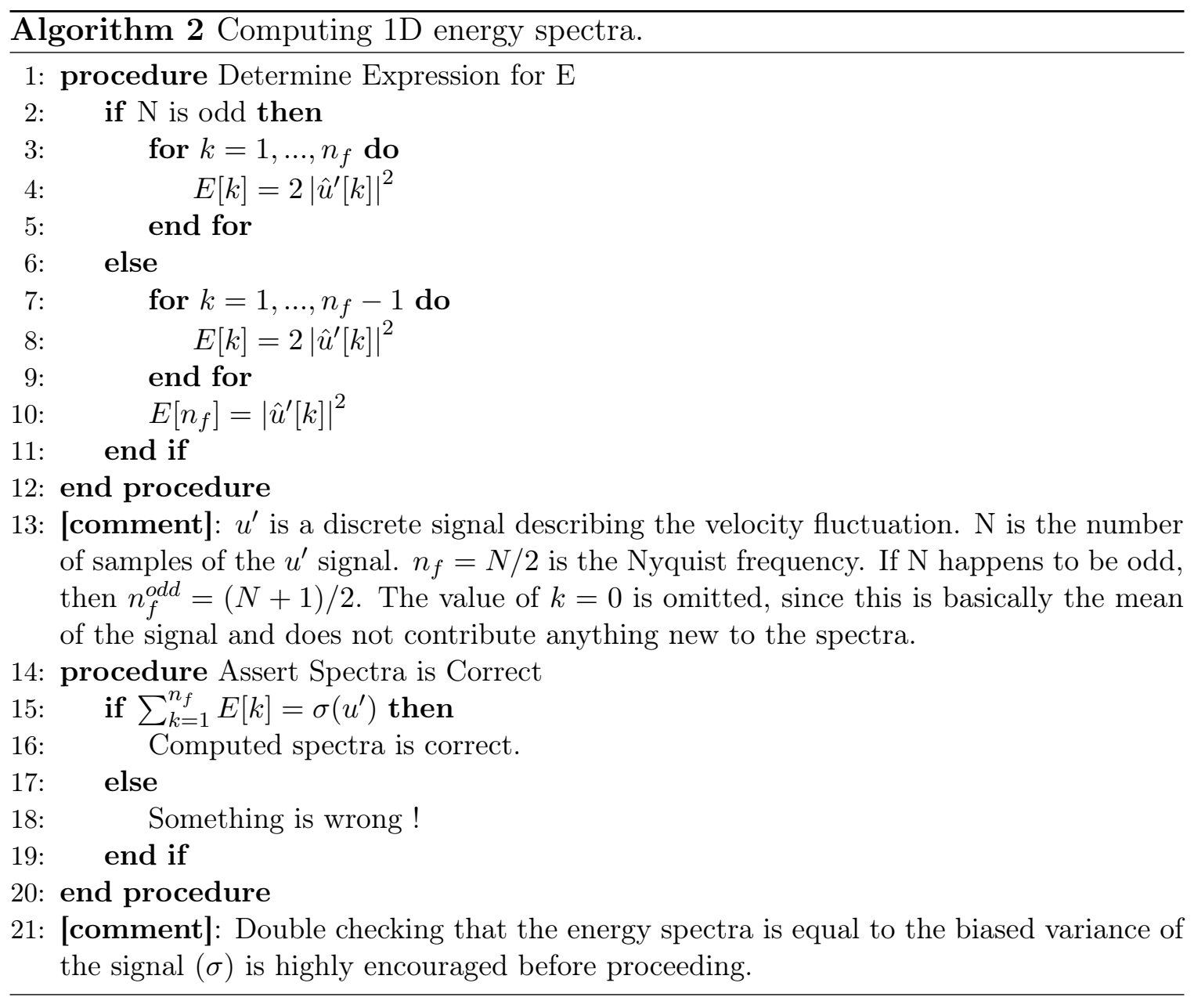

\section{A.2 Power Spectra in One Dimension}

Usually, in signal processing, there are two ways to perform spectral studies of a signal via either energy spectral density (ESD) or power spectral density (PSD) functions. If a signal has a finite amount of energy and consequently its average power is zero, then this signal is called an energy signal. If, however, a signal has infinite energy while having a finite average power, then it is called a power signal. More precisely, according to [57, p. 25], PSD is used for stochastic signals, whereas ESD is used for deterministic signals. Power is defined as the rate of energy. Hence, in this case, the PSD study is taken for a finite time (usually one period) over the energy of a signal. The following analysis will take place in continuous space (it is straightforward but 
tedious to include the discrete version as well).

Definition A.2.1. Truncated Fourier transform. Consider a signal $f(t)$, its forward Fourier transform taken on one period only is $F_{T}(\omega), \mathscr{F}\left\{f_{T}(t)\right\}$, where $\omega=2 \pi f$ is the angular frequency and $f$ is the natural frequency ${ }^{2}$.

$$
\begin{aligned}
F_{T}(\omega)=\mathscr{F}\left\{f_{T}(t)\right\} & \equiv \int_{-\infty}^{\infty} f_{T}(t) e^{-i \omega t} d t \\
& =\int_{-T / 2}^{T / 2} f(t) e^{-i \omega t} d t .
\end{aligned}
$$

Analogous to Theorem A.1.1, Parseval's theorem is introduced below in discrete form.

Theorem A.2.1 (Continuous PSD function). The continuous power spectral density function, denoted by $S_{p}$, applied to a truncated signal.

$$
S_{p}(\omega)=\lim _{T \rightarrow \infty} \frac{1}{T} \mathbf{E}\left[\left|F_{T}(\omega)\right|^{2}\right],
$$

where $\mathbf{E}$ is the expected value.

Proof. Given that the signal is a stationary random process, the expected value may be substituted by two instances of the signal in time, say for time $t$ and $s .{ }^{3}$ Starting from the right hand side of Equation A.9 and taking the expression of the expected

\footnotetext{
${ }^{2} \mathrm{~A}$ truncated Fourier transform is usually needed since the actual Fourier transform for many signals does not exist and so a portion taken for only one period is considered. Mathematically, this means:

$$
f_{T}(t)= \begin{cases}f(t) & \text { for } \quad-T / 2 \leq t \leq T / 2 \\ 0 & \text { for } \quad|t|>T / 2\end{cases}
$$

${ }^{3}$ Stationary random processes, especially wide-sense stationary (WSS), imply that, for such a signal, the mean and covariance do not change over time, e.g. see Kay [39, chap. 17].
} 
value first:

$$
\begin{aligned}
\mathbf{E}\left[\left|F_{T}(\omega)\right|^{2}\right] & =\mathbf{E}\left[F_{T}(\omega) \cdot F_{T}(-\omega)\right] \\
& =\mathbf{E}\left[\left(\int_{-T / 2}^{T / 2} f(t) e^{-i \omega t} d t\right)\left(\int_{-T / 2}^{T / 2} f(s) e^{i \omega s} d s\right)\right] \\
& =\int_{-T / 2}^{T / 2} \int_{-T / 2}^{T / 2} \mathbf{E}[f(t) f(s)] e^{i \omega(s-t)} d t d s .
\end{aligned}
$$

Introducing a lag parameter $\tau$, while performing a change of integration variables by letting:

$$
\left\{\begin{array}{l}
\tau=s-t \\
\zeta=s+t
\end{array}\right.
$$

An important note to take into consideration when changing the integration values of the integrals is that for a range of $-T / 2$ to $T / 2$, the domain shape is a square with side $T$. Using the new integration variables, the domain shape in now a $\pi / 4$ rotation of the original square - i.e. the shape of a diamond with larger sides. This can be easily shown when considering the intersection of the straight lines in Equation A.11 and the original $-T / 2$ to $T / 2$ square. Refer to Figure A-1.

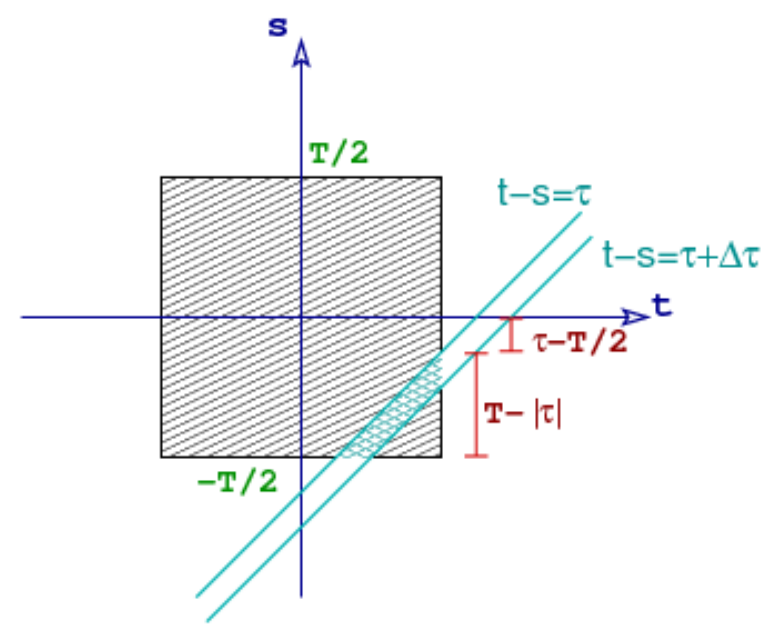

Figure A-1: Visual illustration of transformation of region of interest. 
Next, a relation between the old and new integration variables is constructed. This is based on an expression for the intersection region (between the old and new axis) and the formulation of a new integration parameters based on the difference of the the triangles situated at the bottom right corner with base lines of equation $t-s=\tau$ and $t-s=\tau+\Delta \tau$ each. Refer to Figure A-1, whereby the difference between areas of each of the larger and smaller triangles are given by:

$$
\frac{1}{2}(T-|\tau|)^{2}-\frac{1}{2}(T-|\tau|+\Delta \tau)^{2} \Longrightarrow(T-|\tau|) \Delta \tau+\frac{\Delta \tau^{2^{2}}}{2}
$$

where $\Delta \tau^{2} / 2 \approx 0$ is assumed to be of negligible effect and this in turn corresponds to the area of the region of interest (highlighted in shaded cyan in Figure A-1).

Substituting the new relation between the integration parameters into Equation A.10:

$$
\begin{aligned}
\mathbf{E}\left[\left|F_{T}(\omega)\right|^{2}\right] & =\int_{-T / 2}^{T / 2} \int_{-T / 2}^{T / 2} \mathbf{E}[f(t) f(s)] e^{-i \omega \tau} d t d s \\
& =\int_{-T / 2}^{T / 2} \mathbf{E}[f(t) f(s)] e^{-i \omega \tau}(T-|\tau|) d \tau .
\end{aligned}
$$

Finally, taking the PSD form of the above energy function (since without a rate, the power is considered energy), the outcome would be:

$$
\begin{aligned}
S_{p}(\omega) & =\lim _{T \rightarrow \infty} \frac{1}{T} \int_{-T / 2}^{T / 2} \mathbf{E}[f(t) f(s)] e^{-i \omega \tau}(T-|\tau|) d \tau \\
& =\lim _{T \rightarrow \infty} \int_{-T / 2}^{T / 2} \mathbf{E}[f(t) f(s)] e^{-i \omega \tau}(1-|\tau| / T) d \tau \\
& =\int_{-\infty}^{\infty} \mathbf{E}[f(t) f(s)] e^{-i \omega \tau} d \tau \\
& \equiv \lim _{T \rightarrow \infty} \frac{1}{T} \mathbf{E}\left[\left|F_{T}(\omega)\right|^{2}\right] .
\end{aligned}
$$




\section{A.2.1 Relation between PSD and ACF}

Conveniently, there exists a relation between the PSD function and the ACF. The following analysis will take place in continuous space as well.

Definition A.2.2. Autocorrelation function. Consider a statistically stationary homogeneous signal $f(t)$, its autocorrelation function is denoted by $R(\tau)$ and its autocorrelation coefficient is denoted by $\rho(\tau)$, where $\tau$ is referred to as a lag parameter.

$$
\begin{aligned}
R(\tau) & =\langle f(t) f(t+\tau)\rangle \\
\rho(\tau) & =\frac{\langle f(t) f(t+\tau)\rangle}{\sigma^{2}},
\end{aligned}
$$

where $\sigma$ is the standard deviation, $\sigma^{2}$ the variance and $|\rho| \leq 1$ while $\rho(0)=1$. Moreover, both autocorrelation function and coefficient are defined to be even functions.

The autocorrelation function is a measurement used to indicate how a given signal is correlated with a delayed copy of itself. This can be either time-dependent or spacedependent. In this analysis, however, temporal dependency is selected due to its more intuitive approach.

Definition A.2.3. Stationary ergodic hypothesis. Consider a stationary stochastic process $f(t)$, then an ergodic hypothesis allows the assumption that, due to stationarity, the time average of such a signal converges to the true mean when time approaches infinity. In such a case, the time average is equivalent to the ensemble average.

$$
\langle f\rangle \equiv \lim _{T \rightarrow \infty} \frac{1}{T} \int_{0}^{T} f(t) d t .
$$

Definition A.2.4. Homogeneous ergodic hypothesis. Consider a homogeneous stochastic process $f(x)$, then an ergodic hypothesis allows the assumption that, due to the invariance under spacial translation (i.e. homogeneity) the spatial average of such a 
signal converges to the true mean when the length approaches infinity. In such a case, the spatial average is equivalent to the ensemble average.

$$
\langle f\rangle \equiv \lim _{L \rightarrow \infty} \frac{1}{L} \int_{0}^{L} f(x) d x .
$$

Recall that for a given ergodic process, the expected value is similar to the ensemble average. Therefore, the autocorrelation function may be expressed as:

$$
R(\tau) \equiv \mathbf{E}[f(t) f(t+\tau)] .
$$

Theorem A.2.2 (Continuous Wiener-Khinchin theorem). Consider a continuous WSS signal $f(t)$ and its autocorrelation function $R(\tau)$, where $\tau$ is a lag parameter. Then, the PSD of the signal forms a Fourier transform pair with the ACF.

$$
\left\{\begin{aligned}
S_{p}(\omega)=\mathscr{F}\{R(\tau)\} & \equiv \int_{-\infty}^{\infty} R(\tau) e^{-i \omega \tau} d \tau \\
R(\tau)=\mathscr{F}^{-1}\left\{S_{p}(\omega)\right\} & \equiv \int_{-\infty}^{\infty} S_{p}(\omega) e^{i \omega \tau} d \omega
\end{aligned}\right.
$$

Proof. Stemming from the fact that the input signal $f(t)$ is a random stationary signal, WSS in particular, expanding on its PSD gives:

$$
\begin{aligned}
S_{p}(\omega) & =\lim _{T \rightarrow \infty} \frac{1}{T} \mathbf{E}\left[F_{T}(\omega) F_{T}(-\omega)\right] \\
& =\int_{-\infty}^{\infty} \mathbf{E}[f(t) f(s)] e^{-i \omega \tau} d \tau \\
& \equiv \int_{-\infty}^{\infty} R(\tau) e^{-i \omega \tau} d \tau \\
& =\mathscr{F}\{R(\tau)\},
\end{aligned}
$$

in which the steps involved are identical to the proof in Theorem A.2.1. 
THIS PAGE IS INTENTIONALLY LEFT BLANK 


\section{Appendix B}

\section{Turbulent Kinetic Energy Budget}

The TKE equation is defined as:

$$
\frac{D k}{D t}=\mathcal{P}_{k}+\varepsilon_{k}+\mathcal{T}_{k},
$$

where $k$ is the TKE, $\varepsilon_{k}$ is the dissipation of the TKE, $\mathcal{P}_{k}$ is the production of the TKE, $\mathcal{T}_{k}$ is the transport of the TKE and $D / D t$ is the material/substantial derivative defined as:

$$
\frac{D}{D t}= \begin{cases}\frac{\partial}{\partial t}+\langle\mathbf{u}\rangle \cdot \nabla & \text { (vector notation) } \\ \frac{\partial}{\partial t}+\left\langle u_{j}\right\rangle \frac{\partial}{\partial x_{j}} & \text { (Einstein notation) }\end{cases}
$$


In Einstein notation this may be expressed, expanded and rearranged as such:

$$
\begin{aligned}
\frac{1}{2} \frac{\partial}{\partial t}\left\langle u_{i}^{\prime} u_{i}^{\prime}\right\rangle & =\mathcal{C}_{k}+\mathcal{P}_{k}+\varepsilon_{k}+\mathcal{T}_{k} \\
=-\underbrace{\frac{1}{2}\left\langle u_{j}\right\rangle \frac{\partial}{\partial x_{j}}\left\langle u_{i}^{\prime} u_{i}^{\prime}\right\rangle}_{\mathcal{C}_{k}}-\underbrace{\left\langle u_{i}^{\prime} u_{j}^{\prime}\right\rangle \frac{\partial}{\partial x_{j}}\left\langle u_{i}\right\rangle}_{\mathcal{P}_{k}}-\underbrace{\nu\left\langle\frac{\partial u_{i}^{\prime}}{\partial x_{j}} \frac{\partial u_{i}^{\prime}}{\partial x_{j}}\right\rangle}_{\varepsilon_{k}} & \underbrace{\frac{1}{2} \frac{\partial}{\partial x_{j}}\left(\nu \frac{\partial}{\partial x_{j}}\left\langle u_{i}^{\prime} u_{i}^{\prime}\right\rangle-\frac{2}{\rho}\left\langle p^{\prime} u_{j}^{\prime}\right\rangle-\left\langle u_{i}^{\prime} u_{i}^{\prime} u_{j}^{\prime}\right\rangle\right)}_{\mathcal{T}_{k}} .
\end{aligned}
$$

Furthermore, the transport term $\left(\mathcal{T}_{k}\right)$ may be split into three separate terms corresponding to transport by: viscous diffusion $\left(\mathcal{T}^{(\nu)}\right)$, velocity-pressure gradient $\left(\mathcal{T}^{(u p)}\right)$ and turbulence (Re-stresses) $\left(\mathcal{T}^{(u u)}\right)$ accordingly:

$$
\mathcal{T}_{k}=\underbrace{\nu \frac{1}{2} \frac{\partial^{2}}{\partial x_{j}^{2}}\left\langle u_{i}^{\prime} u_{i}^{\prime}\right\rangle}_{\mathcal{T}^{(\nu)}}-\underbrace{\frac{\partial}{\partial x_{j}} \frac{\left\langle p^{\prime} u_{i}^{\prime}\right\rangle}{\rho} \delta_{i, j}}_{\mathcal{T}^{(u p)}}-\underbrace{\frac{1}{2} \frac{\partial}{\partial x_{j}}\left\langle u_{i}^{\prime} u_{i}^{\prime} u_{j}^{\prime}\right\rangle}_{\mathcal{T}^{(u u)}} .
$$

\section{B.1 Convection}

$$
\mathcal{C}_{k}=-\frac{1}{2}\left\langle u_{j}\right\rangle \frac{\partial}{\partial x_{j}}\left\langle u_{i}^{\prime} u_{i}^{\prime}\right\rangle
$$

In vector notation, this may be written as:

$$
\begin{aligned}
\mathcal{C}_{k} & =-\frac{1}{2}\langle\mathbf{u}\rangle \cdot \nabla\left\langle u_{1}^{\prime} u_{1}^{\prime}\right\rangle-\frac{1}{2}\langle\mathbf{u}\rangle \cdot \nabla\left\langle u_{2}^{\prime} u_{2}^{\prime}\right\rangle-\frac{1}{2}\langle\mathbf{u}\rangle \cdot \nabla\left\langle u_{3}^{\prime} u_{3}^{\prime}\right\rangle \\
& =-\langle\mathbf{u}\rangle \cdot \nabla k .
\end{aligned}
$$




\section{B.2 Production}

$$
\mathcal{P}_{k}=-\left\langle u_{i}^{\prime} u_{j}^{\prime}\right\rangle \frac{\partial}{\partial x_{j}}\left\langle u_{i}\right\rangle .
$$

In vector notation, this may be written as:

$$
\begin{aligned}
\mathcal{P}_{k} & =-\left\langle u_{1}^{\prime} \mathbf{u}^{\prime}\right\rangle \cdot \nabla\left\langle u_{1}\right\rangle-\left\langle u_{2}^{\prime} \mathbf{u}^{\prime}\right\rangle \cdot \nabla\left\langle u_{2}\right\rangle-\left\langle u_{3}^{\prime} \mathbf{u}^{\prime}\right\rangle \cdot \nabla\left\langle u_{3}\right\rangle \\
& =-\left\langle\mathbf{u}^{\prime} \otimes \mathbf{u}^{\prime}\right\rangle: \nabla\langle\mathbf{u}\rangle
\end{aligned}
$$

where the operators $\otimes$ and : denote the outer-product and double inner-product, respectively.

\section{B.3 Viscous Dissipation}

$$
\varepsilon_{k}=-\nu\left\langle\frac{\partial u_{i}^{\prime}}{\partial x_{j}} \frac{\partial u_{i}^{\prime}}{\partial x_{j}}\right\rangle .
$$

In vector notation, this may be written as:

$$
\begin{aligned}
\varepsilon_{k} & =-\nu\left\langle\nabla u_{1}^{\prime} \cdot \nabla u_{1}^{\prime}\right\rangle-\nu\left\langle\nabla u_{2}^{\prime} \cdot \nabla u_{2}^{\prime}\right\rangle-\nu\left\langle\nabla u_{3}^{\prime} \cdot \nabla u_{3}^{\prime}\right\rangle \\
& =-\nu\left\langle\operatorname{tr}\left(\left(\nabla \mathbf{u}^{\prime}\right):\left(\nabla \mathbf{u}^{\prime}\right)^{T}\right)\right\rangle
\end{aligned}
$$

where the operator tr is the trace of the second-order tensor and the superscript ${ }^{T}$ is the transpose.

\section{B.4 Transport by Viscous Diffusion}

$$
\mathcal{T}^{(\nu)}=\frac{\nu}{2} \frac{\partial^{2}}{\partial x_{j}^{2}}\left\langle u_{i}^{\prime} u_{i}^{\prime}\right\rangle .
$$


In vector notation, this may be written as:

$$
\begin{aligned}
\mathcal{T}^{(\nu)} & =\frac{\nu}{2} \nabla^{2}\left\langle u_{1}^{\prime} u_{1}^{\prime}\right\rangle+\frac{\nu}{2} \nabla^{2}\left\langle u_{2}^{\prime} u_{2}^{\prime}\right\rangle+\frac{\nu}{2} \nabla^{2}\left\langle u_{3}^{\prime} u_{3}^{\prime}\right\rangle \\
& =\nu \nabla^{2}\langle k\rangle
\end{aligned}
$$

where the operator $\nabla^{2}=\Delta$ is the Laplacian operator.

\section{B.5 Transport by Velocity-Pressure Gradient}

$$
\mathcal{T}^{(u p)}=-\frac{\partial}{\partial x_{j}} \frac{\left\langle p^{\prime} u_{j}^{\prime}\right\rangle}{\rho},
$$

where the operator $\delta_{i j}$ is the Kronecker delta. In vector notation, this may be written as:

$$
\begin{aligned}
\mathcal{T}^{(u p)} & =-\frac{1}{\rho} \frac{\partial}{\partial x_{1}}\left\langle p^{\prime} u_{1}^{\prime}\right\rangle-\frac{1}{\rho} \frac{\partial}{\partial x_{2}}\left\langle p^{\prime} u_{2}^{\prime}\right\rangle-\frac{1}{\rho} \frac{\partial}{\partial x_{3}}\left\langle p^{\prime} u_{3}^{\prime}\right\rangle \\
& =-\frac{1}{\rho} \nabla \cdot\left\langle p^{\prime} \mathbf{u}^{\prime}\right\rangle .
\end{aligned}
$$

\section{B.6 Transport by Turbulence}

$$
\mathcal{T}^{(u u)}=-\frac{1}{2} \frac{\partial}{\partial x_{j}}\left\langle u_{i}^{\prime} u_{i}^{\prime} u_{j}^{\prime}\right\rangle .
$$

In vector notation, this may be written as:

$$
\begin{aligned}
\mathcal{T}^{(u u)} & =-\frac{1}{2} \nabla \cdot\left\langle\mathbf{u}^{\prime} u_{1}^{\prime} u_{1}^{\prime}\right\rangle-\frac{1}{2} \nabla \cdot\left\langle\mathbf{u}^{\prime} u_{2}^{\prime} u_{2}^{\prime}\right\rangle-\frac{1}{2} \nabla \cdot\left\langle\mathbf{u}^{\prime} u_{3}^{\prime} u_{3}^{\prime}\right\rangle \\
& =-\nabla \cdot\left\langle\mathbf{u}^{\prime} k\right\rangle .
\end{aligned}
$$




\section{Appendix C}

\section{Implementation of Some SGS Models in OpenFOAM}

The following provides some comments concerning how the Smagorinsky SGS model and SGS kinetic energy equation model are implemented in OpenFOAM. The aim of this appendix is to serve as a guide to better shed a light on how the theory behind certain SGS expressions are translated numerically in OpenFOAM (version 3.0.x).

\section{C.1 Smagorinsky Model}

In chapter 2, the Smagorinsky model was expressed using the relation in Equation 2.14. This, however, is the classical version of the model. The model used in OpenFOAM uses a different definition for calculating the eddy-viscosity which depends on the SGS kinetic energy. This is an improvement of the classical eddy-viscosity model in which instead of using the rate-of-strain tensor, the SGS kinetic energy is computed from an equation based on local equilibrium. Thus, the resulting SGS viscosity expression is:

$$
\nu_{s g s}=C_{k} \Delta k_{s g s}^{1 / 2},
$$

where $C_{k}$ is a constant. 
The implementation process, as applied in OpenFOAM, is detailed in order to determine the equivalence of the Smagorinsky constant $C_{s}$ in the code (refer to Equation 2.14); all remaining constants and equations are discussed and presented according to OpenFOAM's source files. To start off, using some algebra on tensor properties, the SGS stress tensor may be broken down into an isotropic and a deviatoric part:

$$
\begin{aligned}
\tau_{i j}^{s g s} & =\left(\tau_{i j}^{s g s}-\frac{1}{3} \tau_{k k}^{s g s} \delta_{i j}\right)+\frac{1}{3} \tau_{k k}^{s g s} \delta_{i j} \\
& =2 \nu_{s g s} \bar{S}_{i j}+\frac{2}{3} k_{s g s} \delta_{i j},
\end{aligned}
$$

where the SGS kinetic energy is defined as $k_{s g s}=\tau_{k k}^{s g s} / 2$ and $\tau_{k k}^{s g s}=\overline{u_{k} u_{k}}-\bar{u}_{k} \bar{u}_{k}$.

The SGS kinetic energy is then computed via an extra equation that enforces local equilibrium between the SGS kinetic energy production $\mathcal{P}$ and the SGS kinetic energy dissipation $\varepsilon$ [73]:

$$
\underbrace{\left(2 \bar{S}_{i j} \bar{S}_{i j}\right)^{1 / 2} \tau_{i j}^{s g s}}_{\mathcal{P}}+\underbrace{\frac{C_{\varepsilon}}{\Delta} k_{s g s}^{3 / 2}}_{\varepsilon}=0,
$$

where $C_{\varepsilon}=1.048$ and $k_{s g s}$ is calculated as follows:

$$
\begin{aligned}
& \bar{S}_{i j}\left(\frac{2}{3} k_{s g s} \delta_{i j}-2 \nu_{s g s} \operatorname{dev}\left(\bar{S}_{i j}\right)\right)+\frac{C_{\varepsilon}}{\Delta} k_{s g s}^{3 / 2} \quad=0 \\
& \left(\frac{C_{\varepsilon}}{\Delta} k_{s g s}+\frac{2}{3} \bar{S}_{k k} k_{s g s}^{1 / 2}-2 C_{k} \Delta \operatorname{dev}\left(\bar{S}_{i j}\right) \bar{S}_{i j}\right) k_{s g s}^{1 / 2} \quad=0,
\end{aligned}
$$

where $C_{k}=0.094$ and the second line follows from substituting Equation C.1.

Finally, rewriting the above into a quadratic equation, the result is:

$$
a k_{s g s}+b k_{s g s}^{1 / 2}-c=0,
$$


where the quadratic equation's coefficients are:

$$
\left\{\begin{array}{l}
a=C_{\varepsilon} / \Delta \\
b=2 \operatorname{tr}\left(\bar{S}_{i j}\right) / 3 \\
c=2 C_{k} \Delta \operatorname{dev}\left(\bar{S}_{i j}\right) \bar{S}_{i j}
\end{array}\right.
$$

The solution to Equation C.4 lies in the form of a double-root being:

$$
k_{s g s}=\left[\frac{-b+\sqrt{b^{2}+4 a c}}{2 a}\right]^{2} .
$$

In relation to the older OpenFOAM versions (version 2.x and before), the SGS kinetic energy is computed differently. Instead of solving for an equation which dictates local equilibrium, the SGS kinetic energy is simply evaluated in the below expression:

$$
k_{s g s}=\frac{2 C_{k}}{C_{\varepsilon}} \Delta^{2}\left(\frac{\left(2 \bar{S}_{i j} \bar{S}_{i j}\right)^{1 / 2}}{\sqrt{2}}\right)^{2}
$$

Finally, taking the above expression for the SGS kinetic energy and replacing in Equation C.1, the outcome is:

$$
\nu_{s g s}=C_{k} \Delta^{2}\left(2 \bar{S}_{i j} \bar{S}_{i j}\right)^{1 / 2} \sqrt{\frac{C_{k}}{C_{\varepsilon}}}
$$

where by comparison with Equation 2.14:

$$
C_{s}^{2}=C_{k} \sqrt{\frac{C_{k}}{C_{\varepsilon}}} \Longrightarrow C_{s} \approx 0.16778594162
$$

where the constants used in OpenF0AM version 3.0.x are: $C_{\varepsilon}=1.048$ and $C_{k}=0.094$. 


\section{C.2 SGS Turbulent Kinetic Equation Equation Model}

Pertaining to the implementation of the SGS kinetic energy equation model in OpenFOAM, all the terms in Equation 2.16 are straightforwardly programmed except for the production term. For the sake of reproducibility, the difference will be highlighted. In the source files, the production term is programmed via the relation:

$$
\begin{aligned}
\mathcal{P} & =-\rho \tau_{i j}^{s g s} \bar{S}_{i j} \\
& =\left(-\frac{2}{3} \rho k_{s g s} \delta_{i j}+2 \rho \nu_{s g s} \operatorname{dev}\left(\bar{S}_{i j}\right)\right) \bar{S}_{i j} \\
& =-\frac{2}{3} \rho \frac{\partial \bar{u}_{k}}{\partial x_{k}} k_{s g s}+\rho G,
\end{aligned}
$$

where $\mathcal{P}$ denotes the SGS kinetic energy production term, $\delta_{i j}$ is the Kronecker delta and

$$
G=\rho \nu_{s g s} \frac{\partial \bar{u}_{i}}{\partial x_{j}}\left(2 \bar{S}_{i j}-\frac{1}{3} \operatorname{tr}\left(2 \bar{S}_{i j}\right) \delta_{i j}\right) .
$$




\section{Appendix D}

\section{High-order Statistics}

The high-order statistics defined below are all based on the central moment. The general expression for computing the $n$th central moment may be defined as:

$$
\begin{aligned}
\mu_{n} & \equiv\left\langle\phi^{\prime n}\right\rangle \\
& =\mathbf{E}\left[(\phi-\mathbf{E}[X])^{n}\right] \\
& =\left\langle(\phi-\langle X\rangle)^{n}\right\rangle \\
& =\int_{-\infty}^{\infty}(\phi-\langle\phi\rangle)^{n} f(\phi) d \phi,
\end{aligned}
$$

where $\mu_{n}$ denotes the $n$th central moment, $\mathbf{E}$ is the expected value, $\phi^{\prime}=\phi-\langle\phi\rangle$ is the fluctuation, $f(\phi)$ is the probability density function (PDF) describing $\phi$. By definition $\mu_{0}=1, \mu_{1}=0$ and $\mu_{2}=\sigma_{\phi}^{2}$ in which the latter is the variance and also happens to be the Re-stress component when the parameter of interest, $\phi$, is the velocity.

With regard to the velocity PDF, the skewness, $S$, and kurtosis, $K$, are used to characterize the asymmetry of the PDF and the tail profile, respectively. For more details pertaining to the probability behavior of the velocity, the reader is encouraged to read Pope [67, chap. 3]. 


\section{D.1 Third Moment}

The third central moment, commonly referred to as the skewness, is expressed as:

$$
\begin{aligned}
S_{\phi} & =\frac{\mu_{3}(\phi)}{\mu_{2}^{3 / 2}(\phi)} \\
& =\frac{\left\langle\phi^{3}\right\rangle-3\langle\phi\rangle\left\langle\phi^{2}\right\rangle+2\langle\phi\rangle^{3}}{\left(\left\langle\phi^{2}\right\rangle-\langle\phi\rangle^{2}\right)^{3 / 2}}
\end{aligned}
$$

\section{D.2 Fourth Moment}

The fourth central moment, commonly referred to as the kurtosis, is expressed as:

$$
\begin{aligned}
K_{\phi} & =\frac{\mu_{4}(\phi)}{\mu_{2}^{2}(\phi)} \\
& =\frac{\left\langle\phi^{4}\right\rangle-4\langle\phi\rangle\left\langle\phi^{3}\right\rangle+6\langle\phi\rangle^{2}\left\langle\phi^{2}\right\rangle-3\langle\phi\rangle^{4}}{\left(\left\langle\phi^{2}\right\rangle-\langle\phi\rangle^{2}\right)^{2}},
\end{aligned}
$$

\section{D.3 Fifth Moment}

The fourth central moment, commonly referred to as the hyperskewness, is expressed as:

$$
\begin{aligned}
\Pi & =\frac{\mu_{5}(\phi)}{\mu_{2}^{5 / 2}(\phi)} \\
& =\frac{\left\langle\phi^{5}\right\rangle-5\langle\phi\rangle\left\langle\phi^{4}\right\rangle+10\langle\phi\rangle^{2}\left\langle\phi^{3}\right\rangle-10\langle\phi\rangle^{3}\left\langle\phi^{2}\right\rangle+4\langle\phi\rangle^{5}}{\left(\left\langle\phi^{2}\right\rangle-\langle\phi\rangle^{2}\right)^{5 / 2}} .
\end{aligned}
$$


Appendix E

Additional Flow over a BFS Figures 


\section{E.1 Coarse Grid}

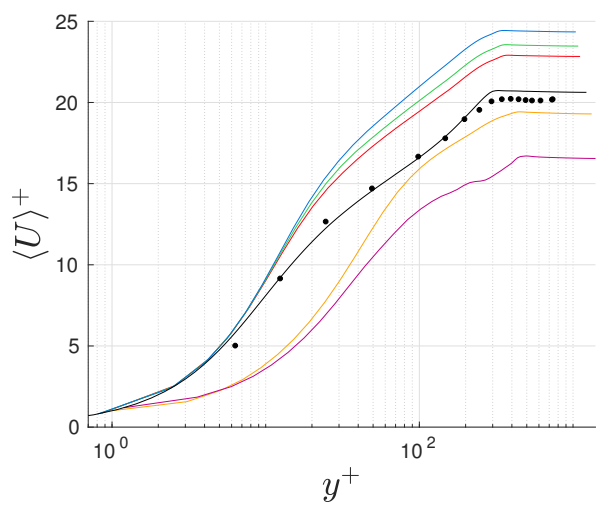

(a)

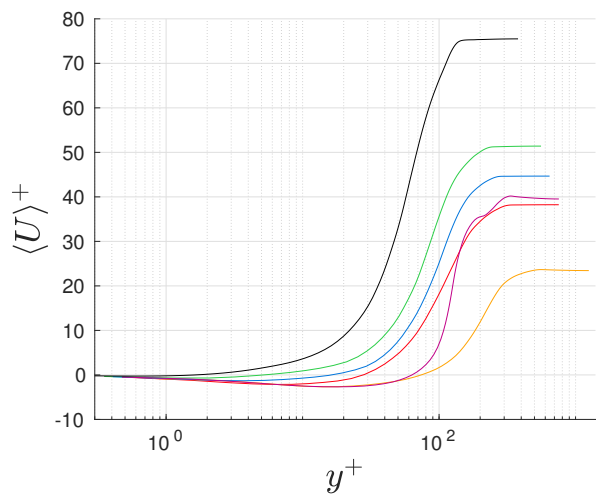

(c)

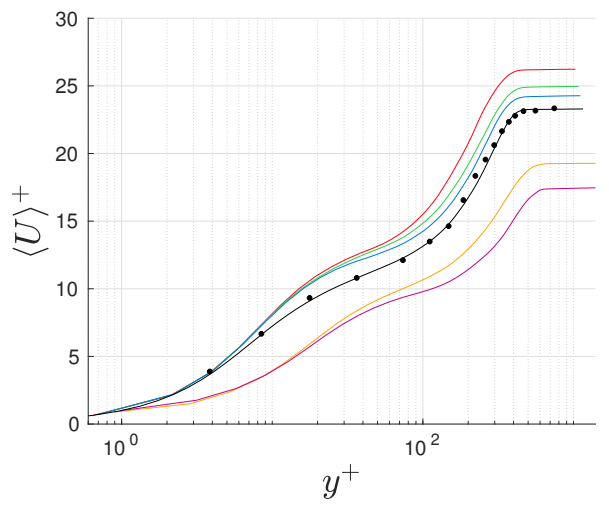

(e)

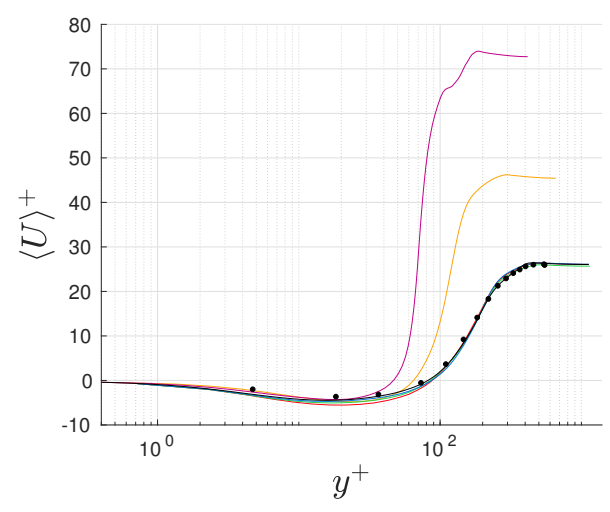

(b)

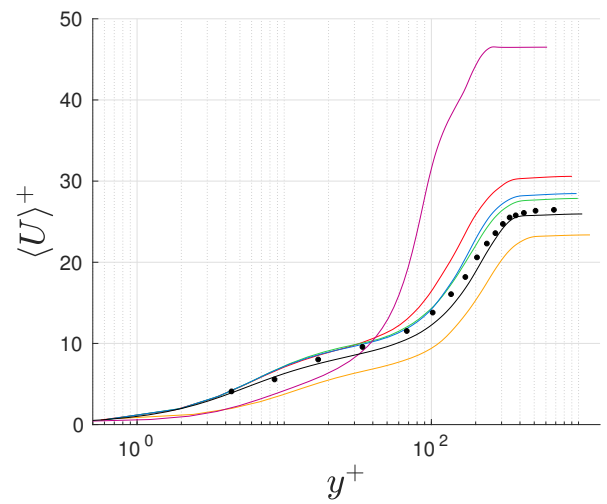

(d)

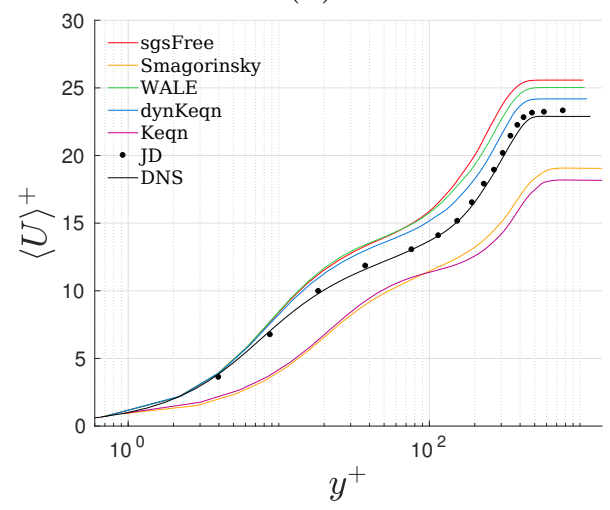

(f)

Figure E-1: Inner-scaled mean streamwise velocity profiles versus the wall-normal coordinate taken at dimensionless streamwise locations $x / h$ of approximately -3 (a), 4 (b), 6 (c), 10 (d), 15 (e) and 19 (f) using the coarse-size BFS grid resolution. 


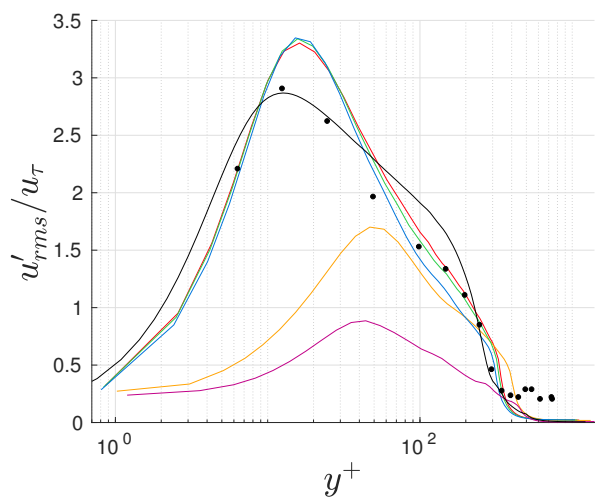

(a)

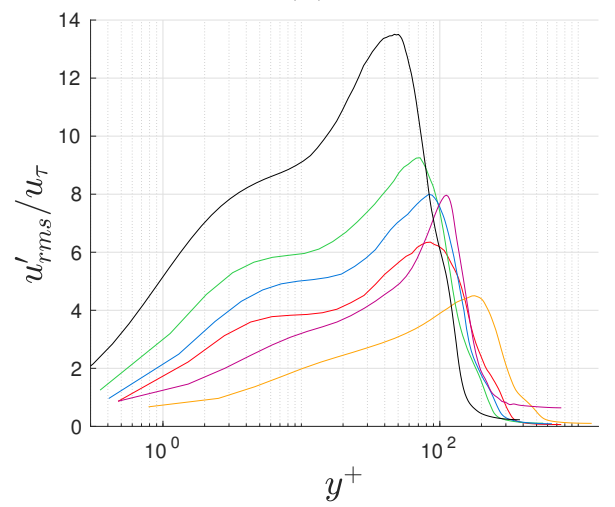

(c)

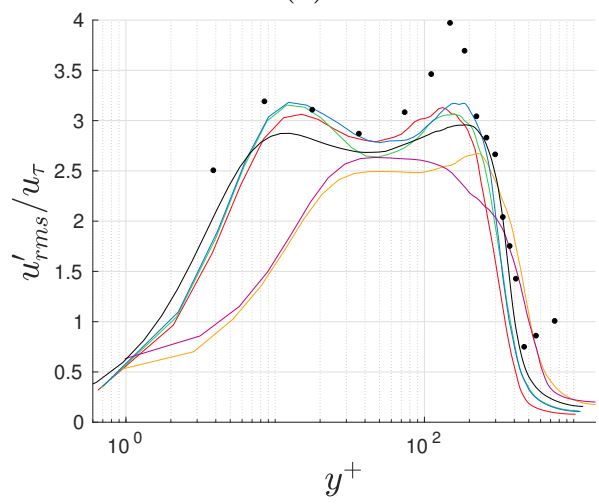

(e)

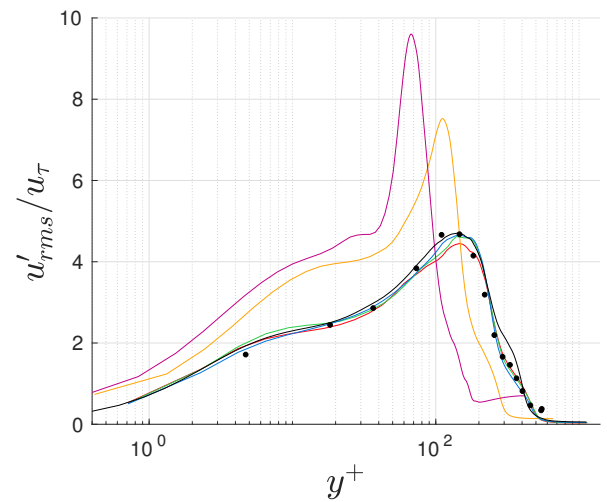

(b)

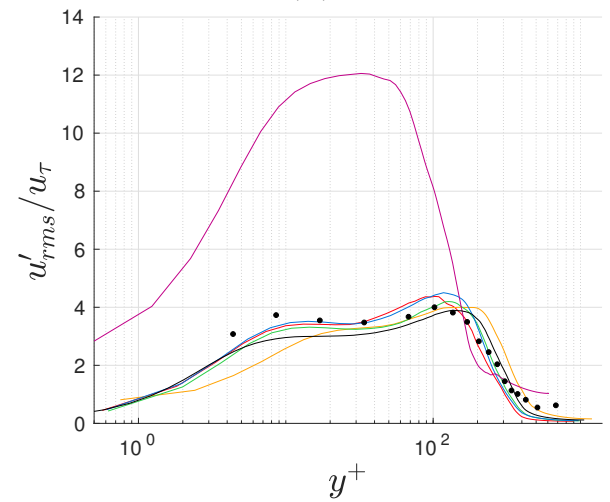

(d)

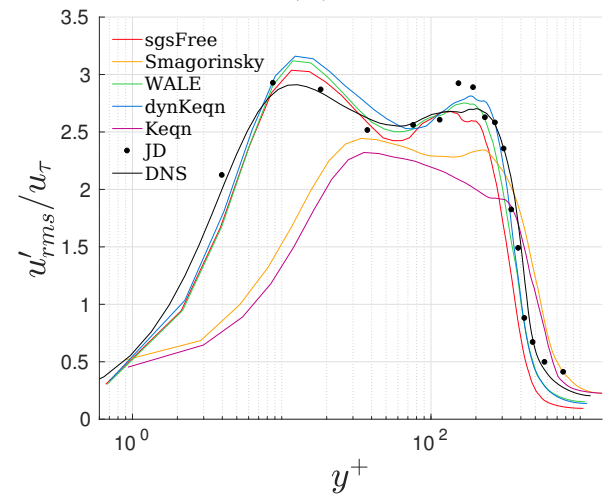

(f)

Figure E-2: Inner-scaled $u_{r m s}^{\prime}$ versus the wall-normal coordinate taken at dimensionless streamwise locations $x / h$ of approximately -3 (a), 4 (b), 6 (c), 10 (d), 15 (e) and 19 (f) using the coarse-size BFS grid resolution. 


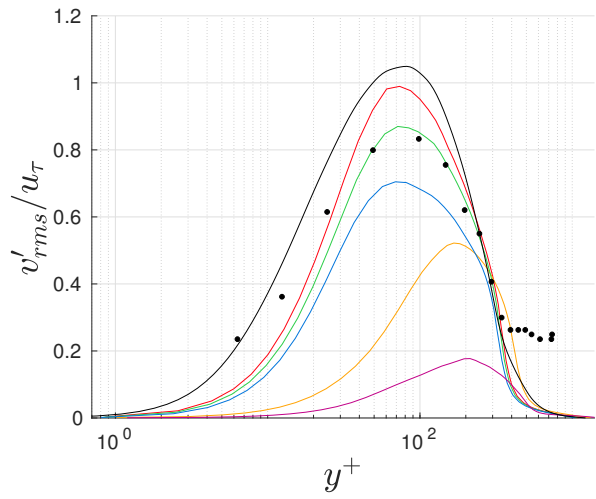

(a)

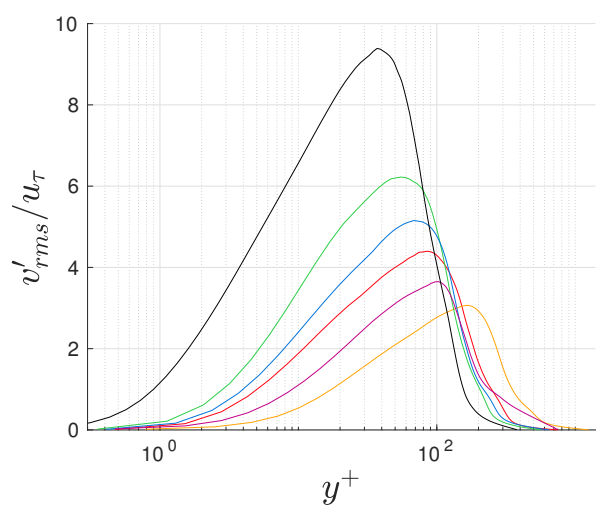

(c)

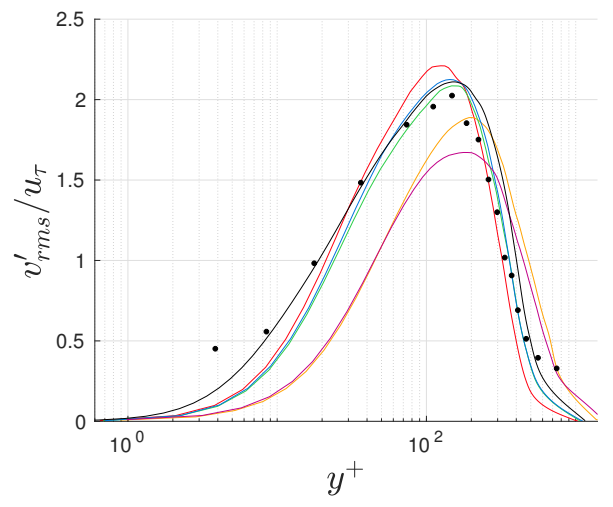

(e)

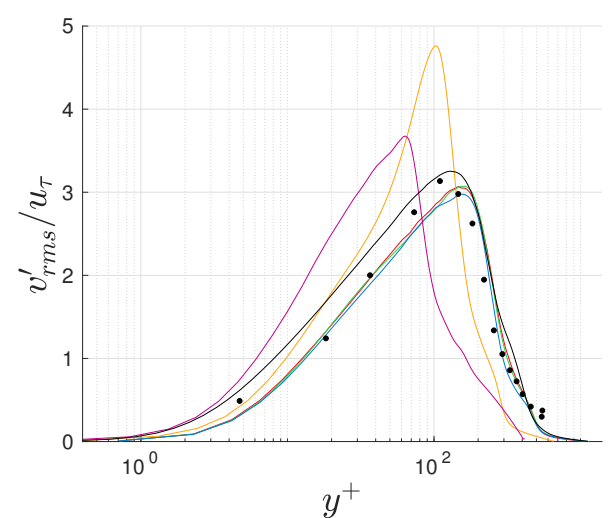

(b)

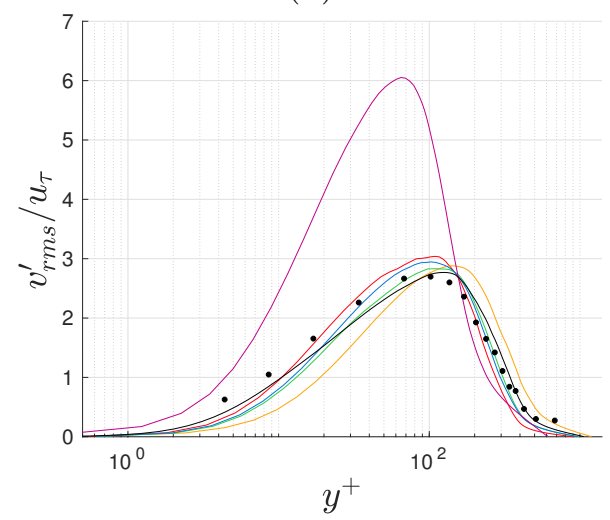

(d)

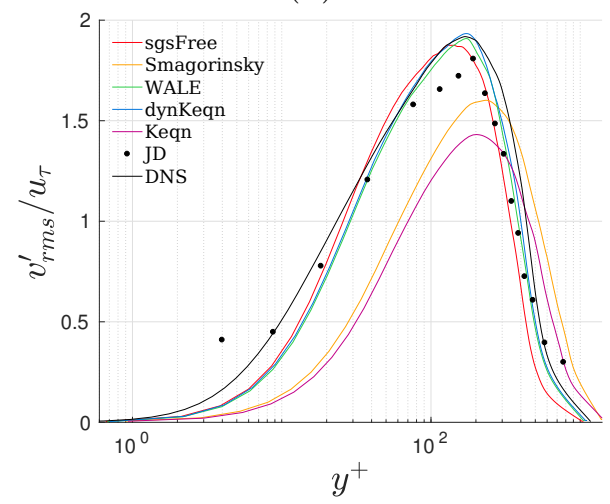

(f)

Figure E-3: Inner-scaled $v_{r m s}^{\prime}$ versus the wall-normal coordinate taken at dimensionless streamwise locations $x / h$ of approximately -3 (a), 4 (b), 6 (c), 10 (d), 15 (e) and 19 (f) using the coarse-size BFS grid resolution. 


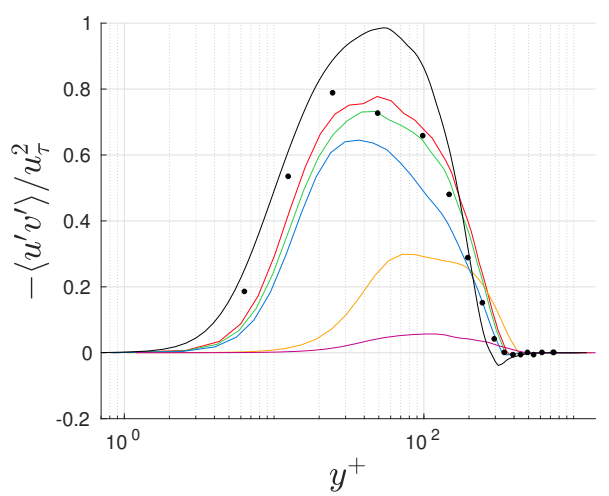

(a)

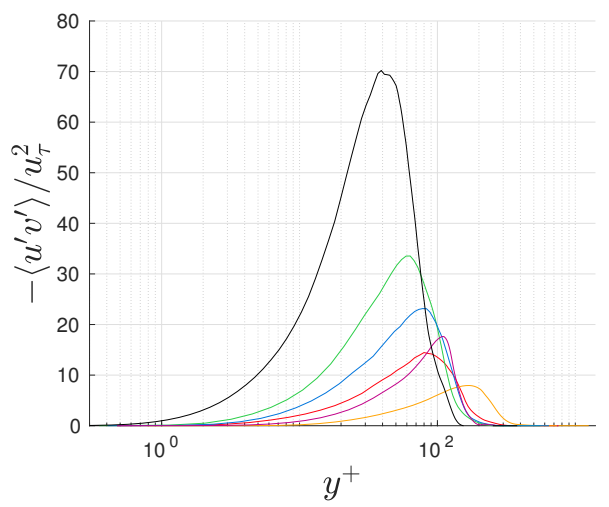

(c)

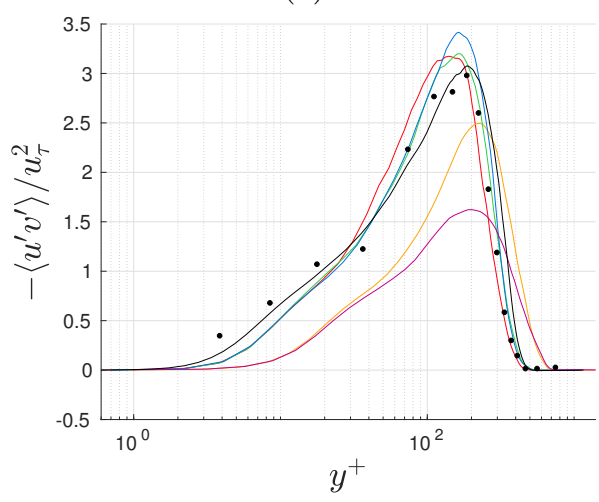

(e)

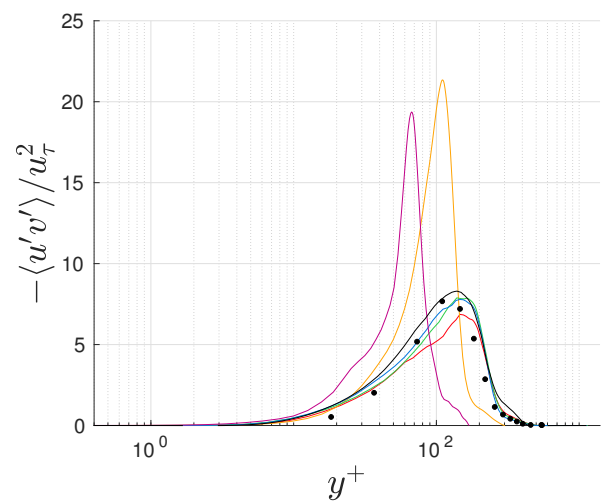

(b)

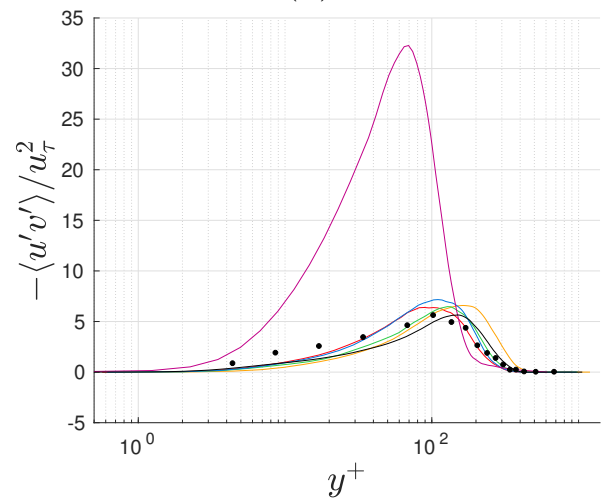

(d)

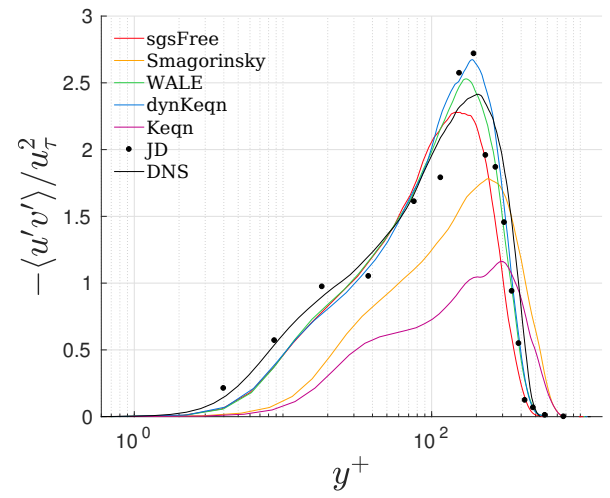

(f)

Figure E-4: Inner-scaled $-\left\langle u^{\prime} v^{\prime}\right\rangle$ versus the wall-normal coordinate taken at dimensionless streamwise locations $x / h$ of approximately -3 (a), 4 (b), 6 (c), 10 (d), 15 (e) and 19 (f) using the coarse-size BFS grid resolution. 


\section{E.2 Intermediate Grid}

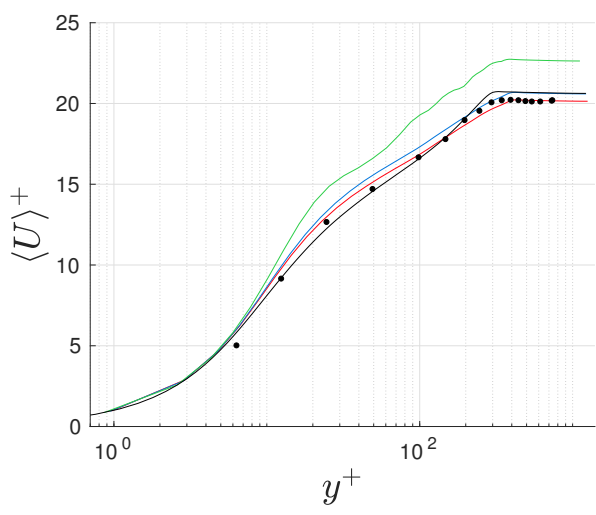

(a)

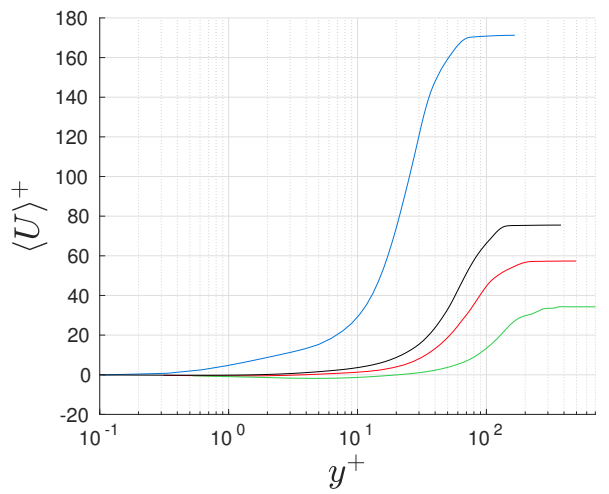

(c)

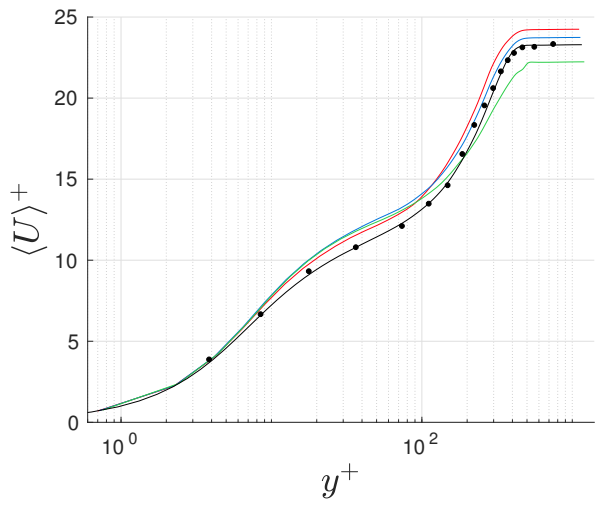

(e)

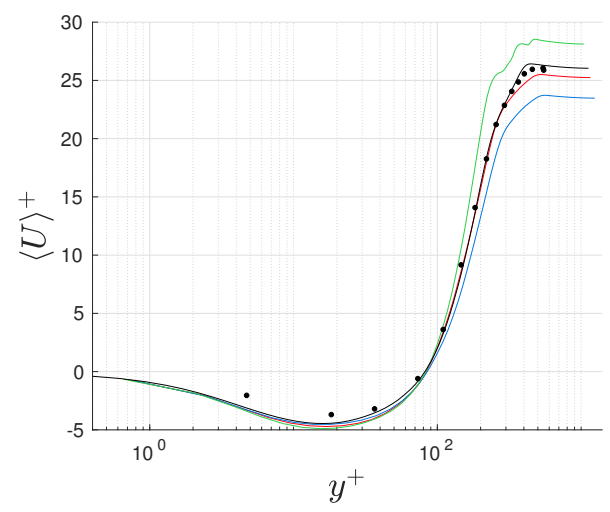

(b)

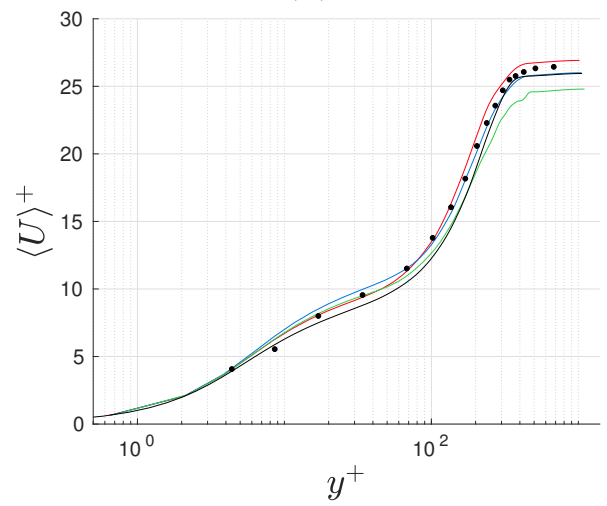

(d)

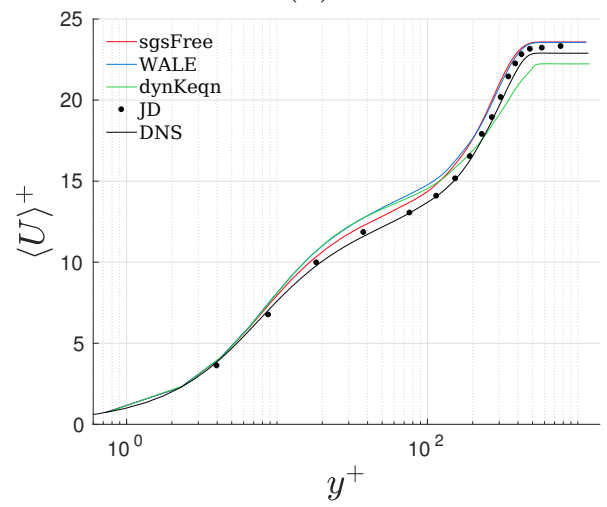

(f)

Figure E-5: Inner-scaled mean streamwise velocity profiles versus the wall-normal coordinate taken at dimensionless streamwise locations $x / h$ of approximately -3 (a), 4 (b), 6 (c), 10 (d), 15 (e) and 19 (f) using the intermediate-size BFS grid resolution. 


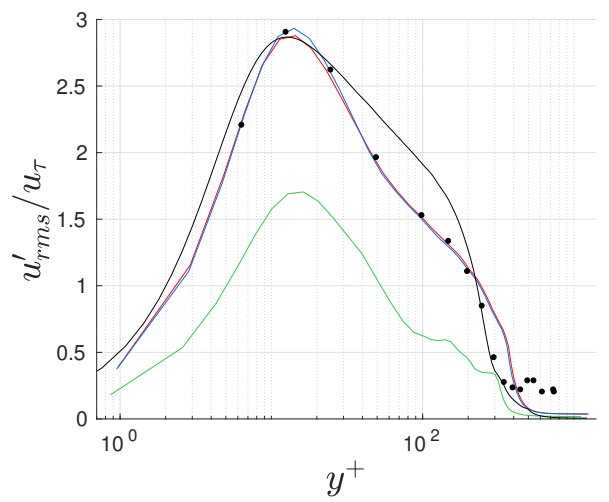

(a)

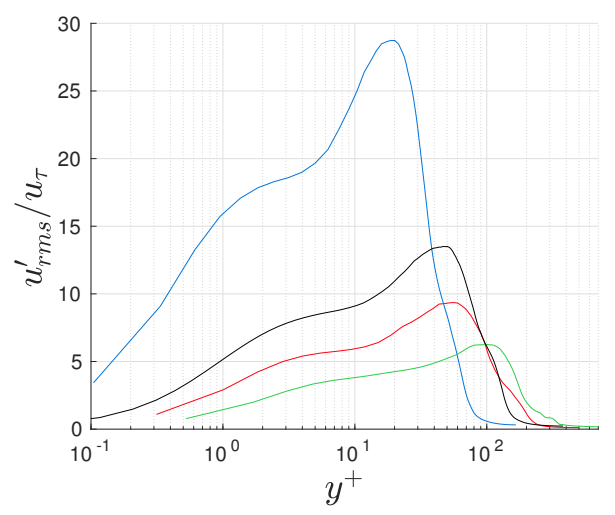

(c)

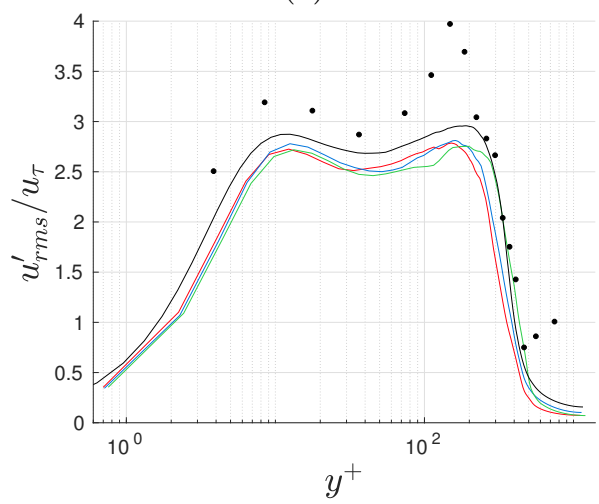

(e)

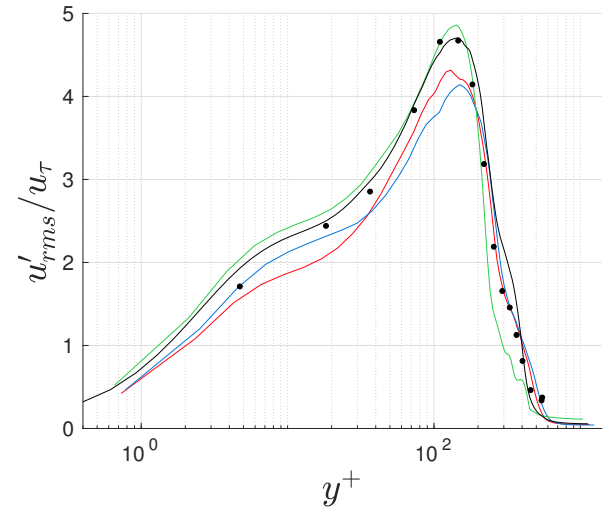

(b)

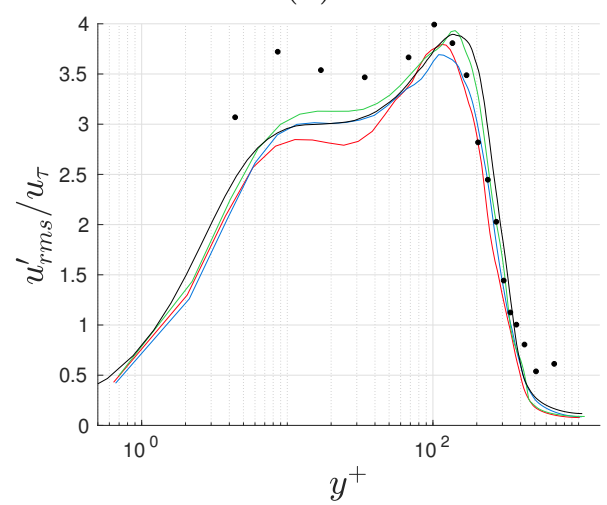

(d)

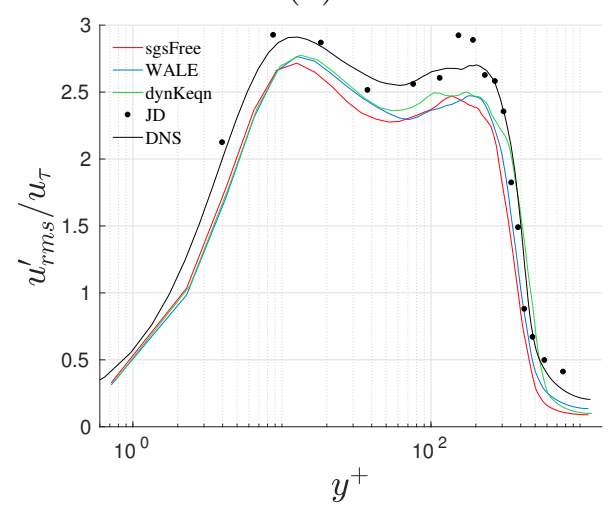

(f)

Figure E-6: Inner-scaled $u_{r m s}^{\prime}$ versus the wall-normal coordinate taken at dimensionless streamwise locations $x / h$ of approximately -3 (a), 4 (b), 6 (c), 10 (d), 15 (e) and 19 (f) using the intermediate-size BFS grid resolution. 


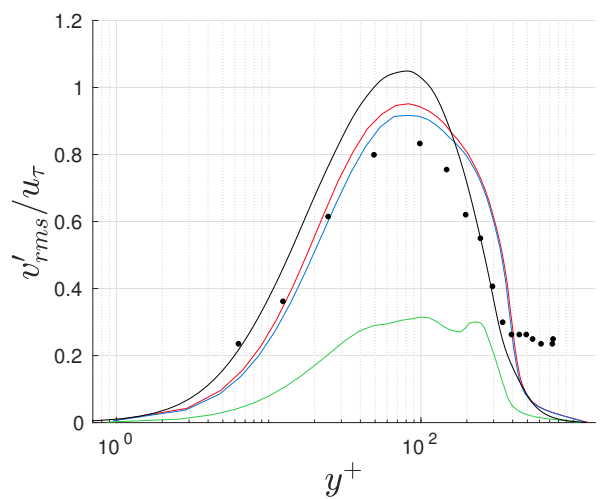

(a)

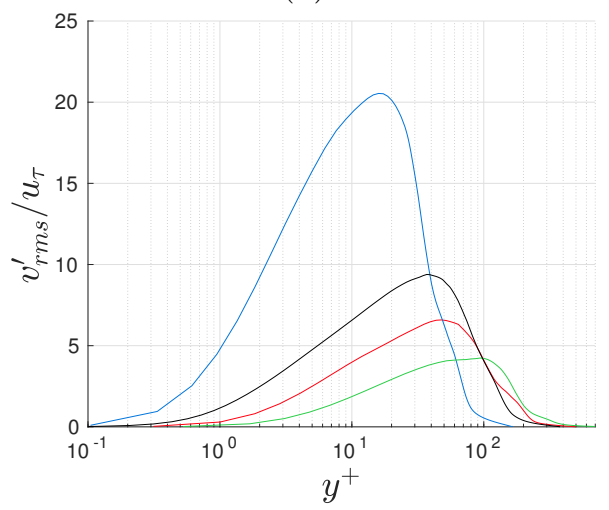

(c)

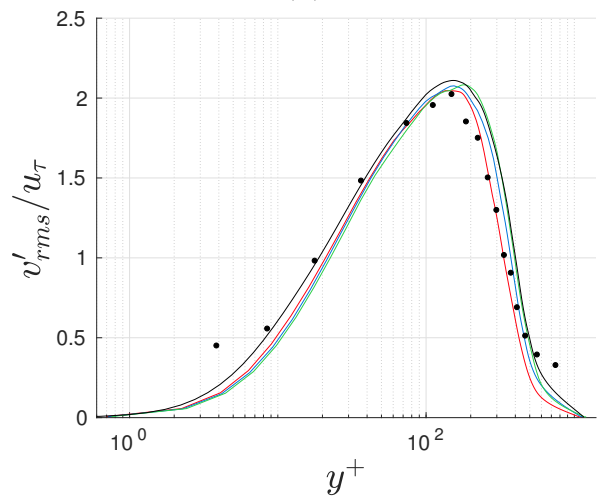

(e)

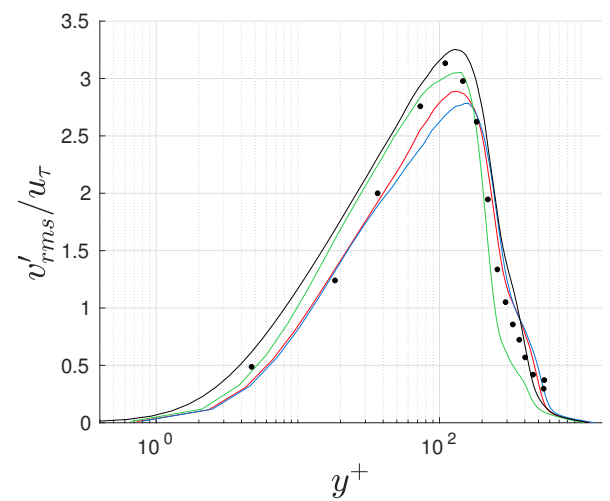

(b)

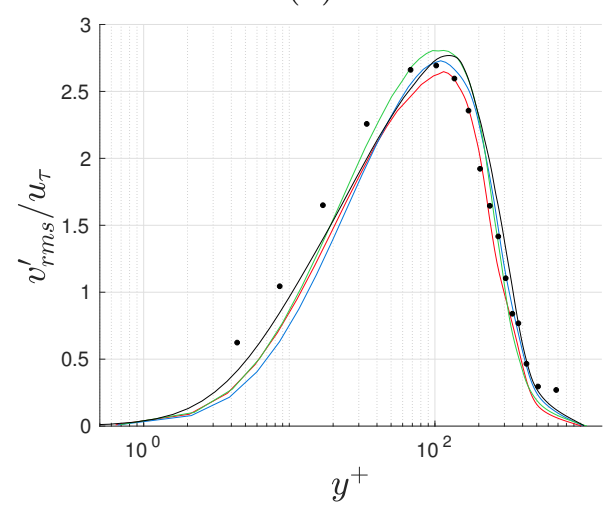

(d)

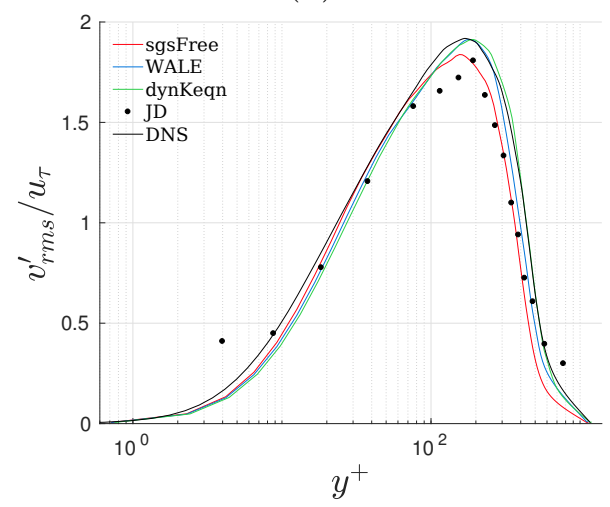

(f)

Figure E-7: Inner-scaled $v_{r m s}^{\prime}$ versus the wall-normal coordinate taken at dimensionless streamwise locations $x / h$ of approximately -3 (a), 4 (b), 6 (c), 10 (d), 15 (e) and 19 (f) using the intermediate-size BFS grid resolution. 


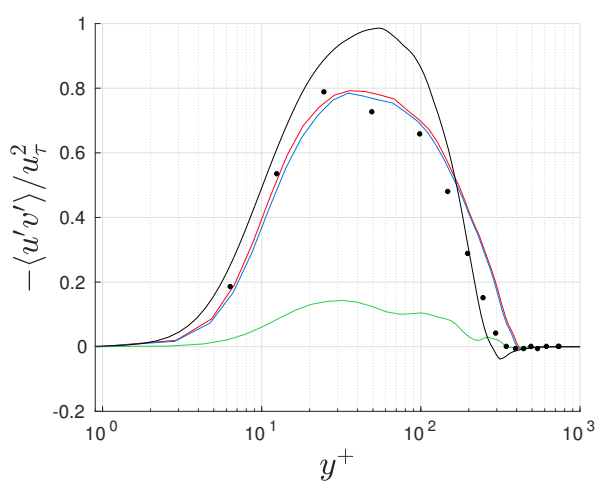

(a)

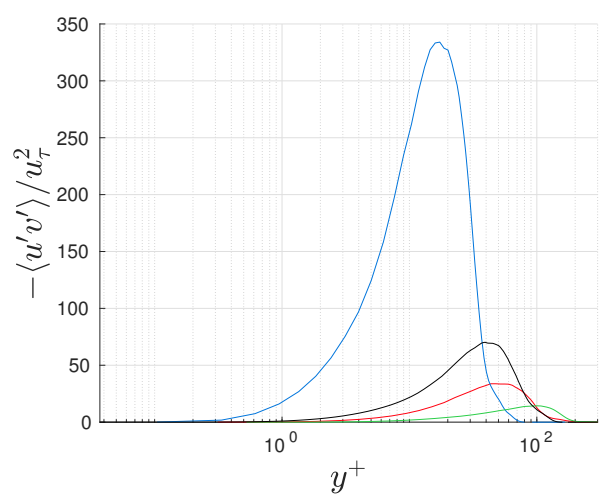

(c)

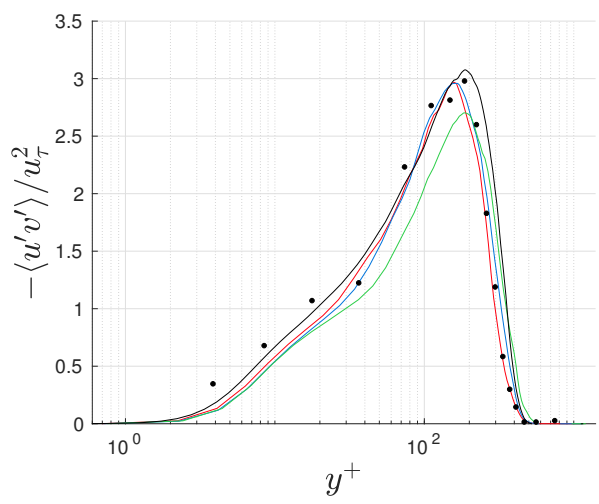

(e)

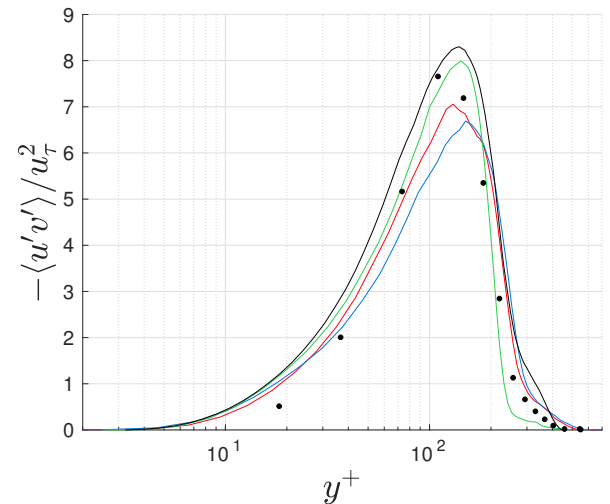

(b)

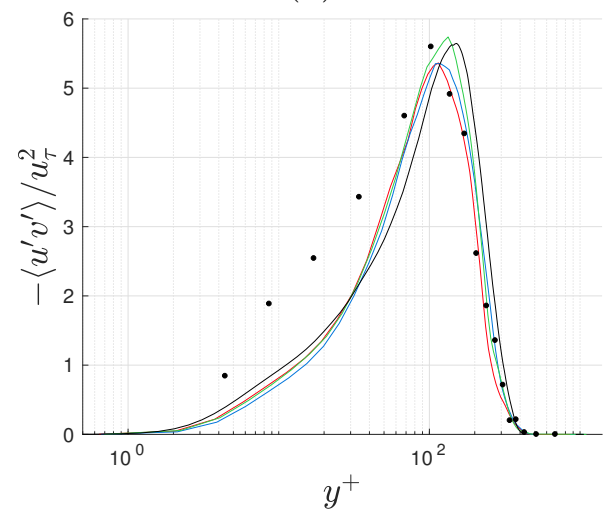

(d)

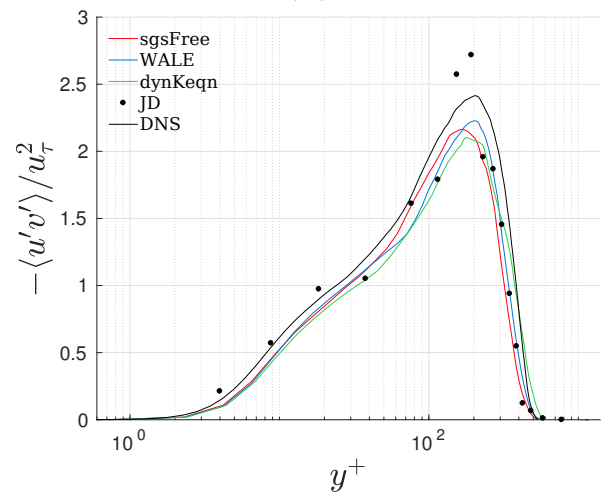

(f)

Figure E-8: Inner-scaled $-\left\langle u^{\prime} v^{\prime}\right\rangle$ versus the wall-normal coordinate taken at dimensionless streamwise locations $x / h$ of approximately -3 (a), 4 (b), 6 (c), 10 (d), 15 (e) and 19 (f) using the intermediate-size BFS grid resolution. 


\section{E.3 Fine Grid}

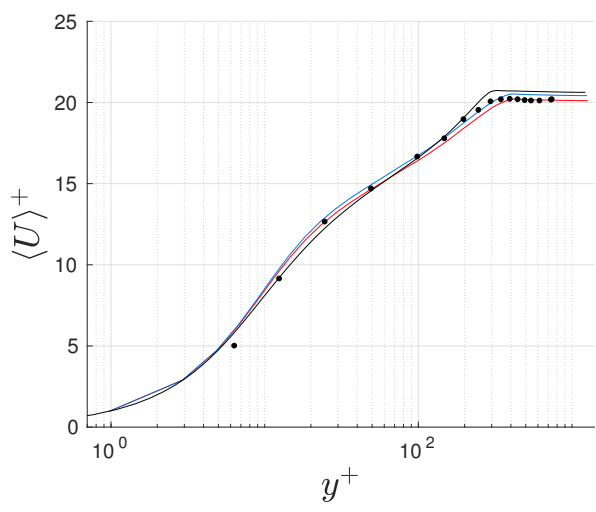

(a)

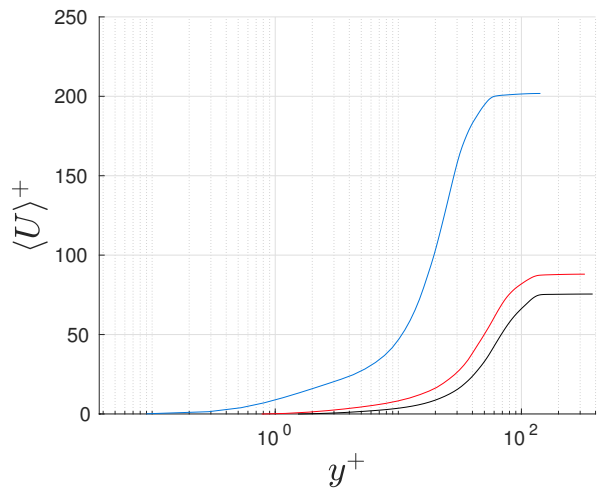

(c)

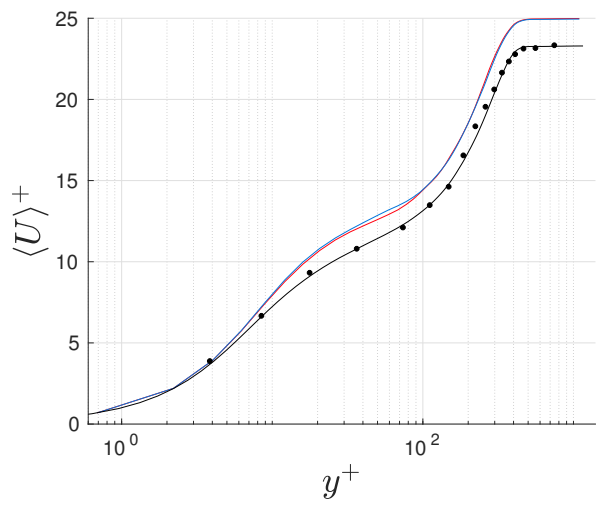

(e)

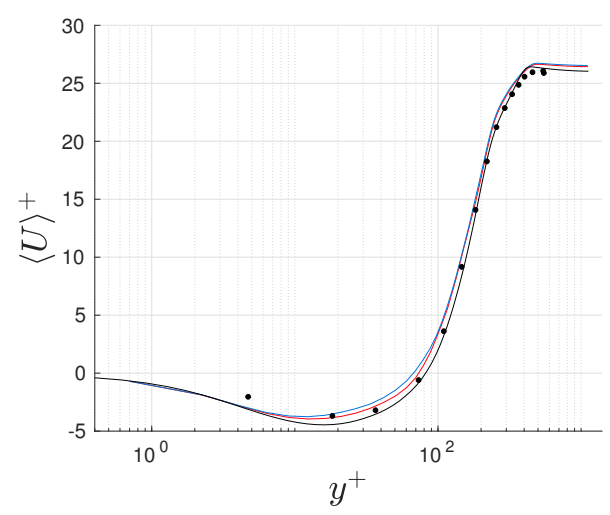

(b)

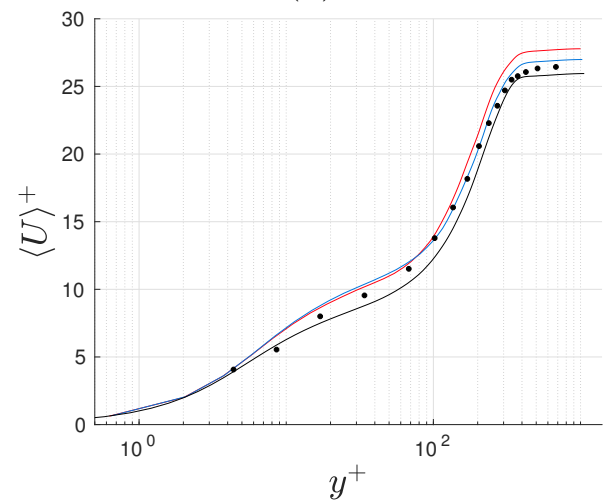

(d)

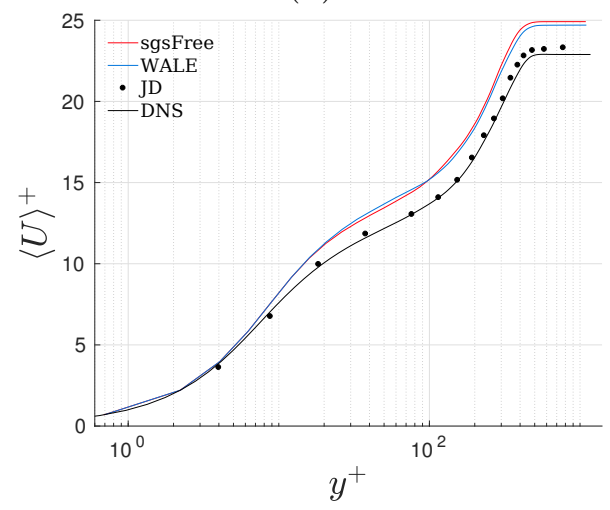

(f)

Figure E-9: Inner-scaled mean streamwise velocity profiles versus the wall-normal coordinate taken at dimensionless streamwise locations $x / h$ of approximately -3 (a), 4 (b), 6 (c), 10 (d), 15 (e) and 19 (f) using the fine-size BFS grid resolution. 


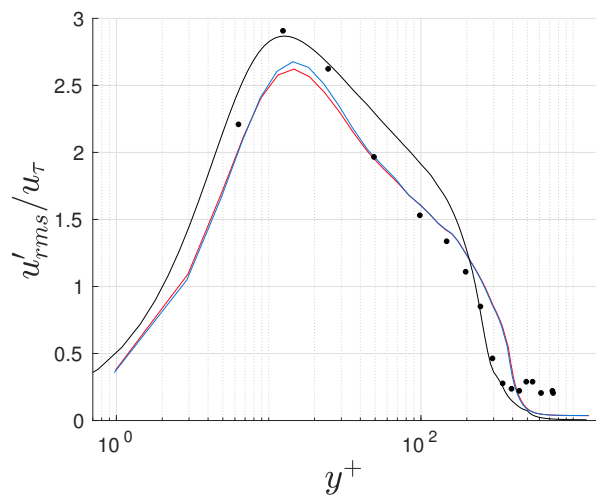

(a)

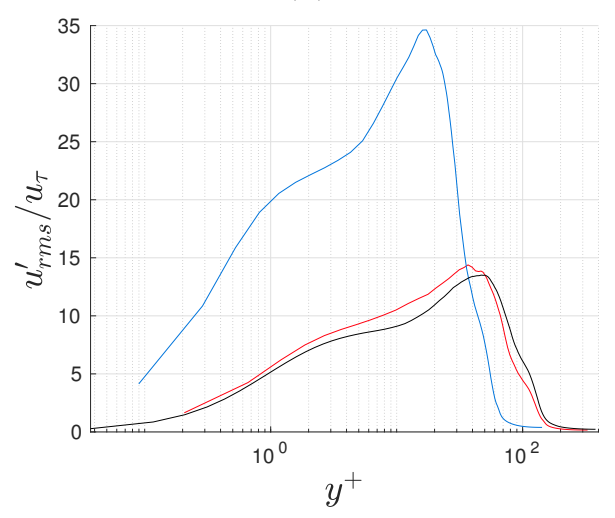

(c)

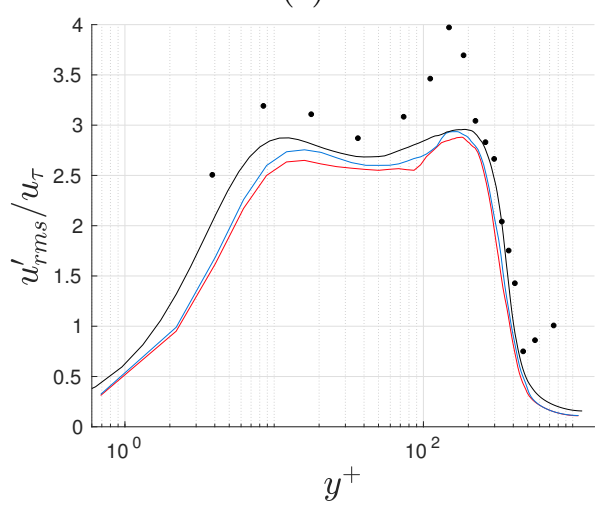

(e)

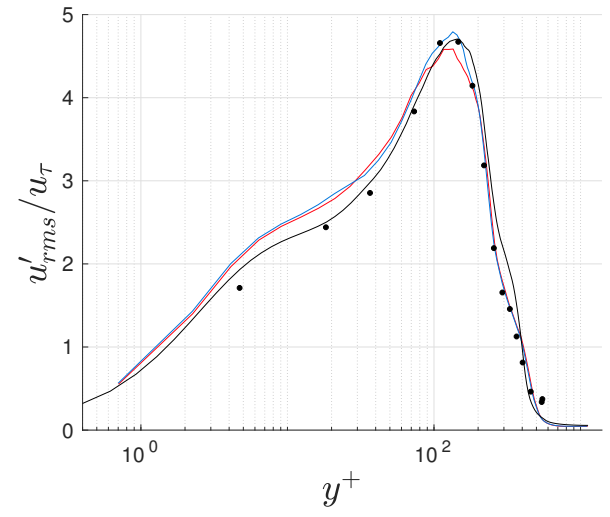

(b)

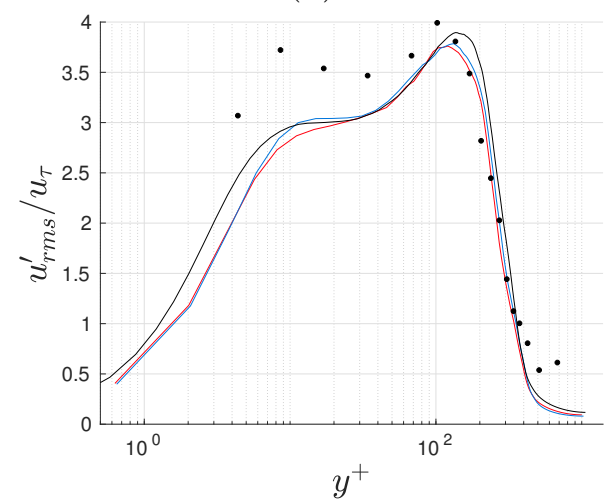

(d)

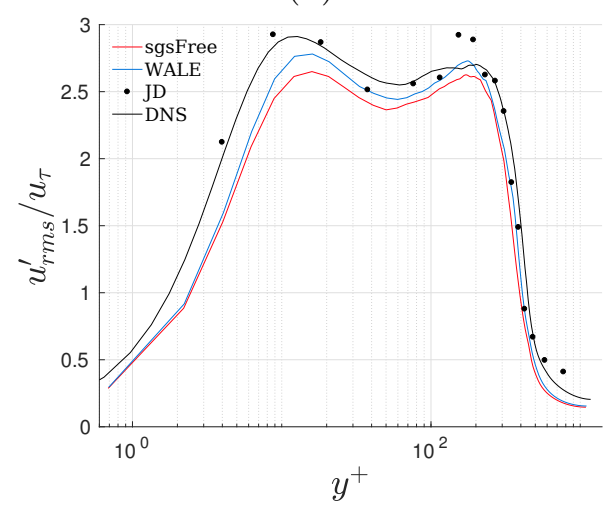

(f)

Figure E-10: Inner-scaled $u_{r m s}^{\prime}$ versus the wall-normal coordinate taken at dimensionless streamwise locations $x / h$ of approximately -3 (a), 4 (b), 6 (c), 10 (d), 15 (e) and 19 (f) using the fine-size BFS grid resolution. 


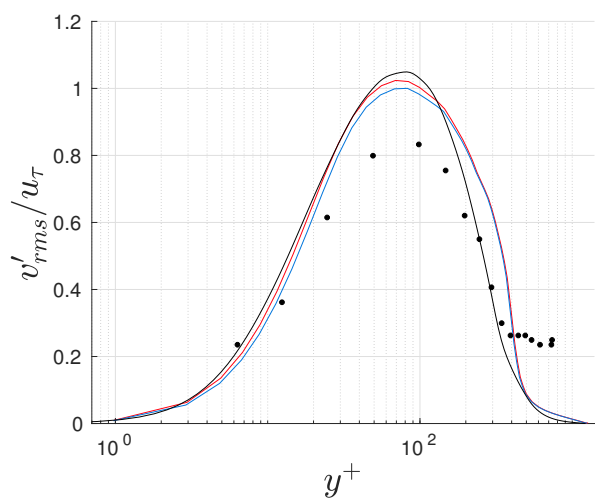

(a)

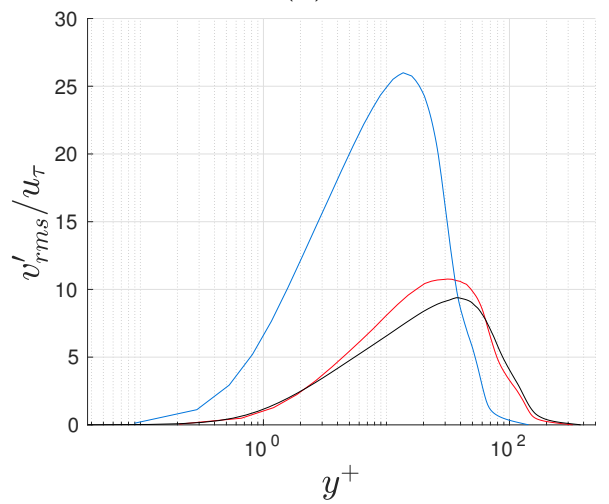

(c)

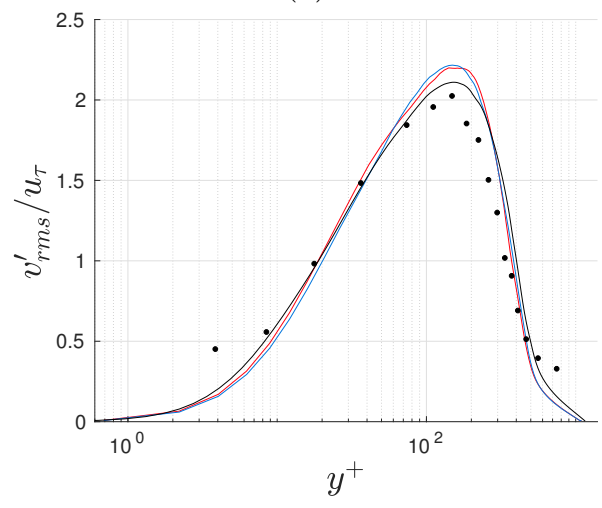

(e)

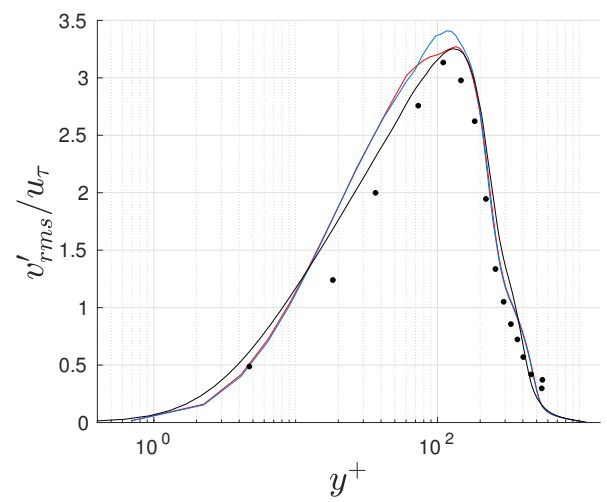

(b)

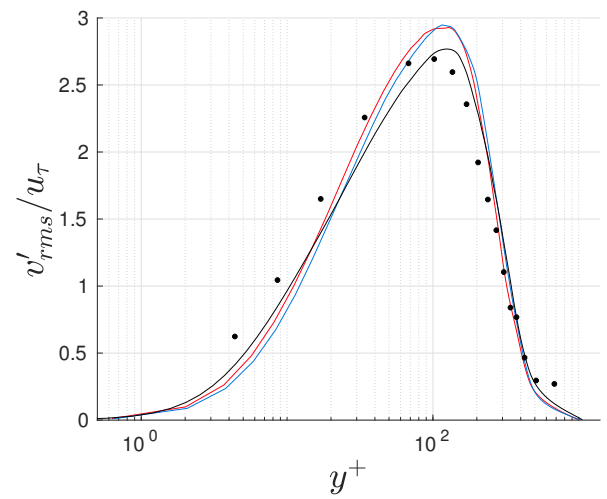

(d)

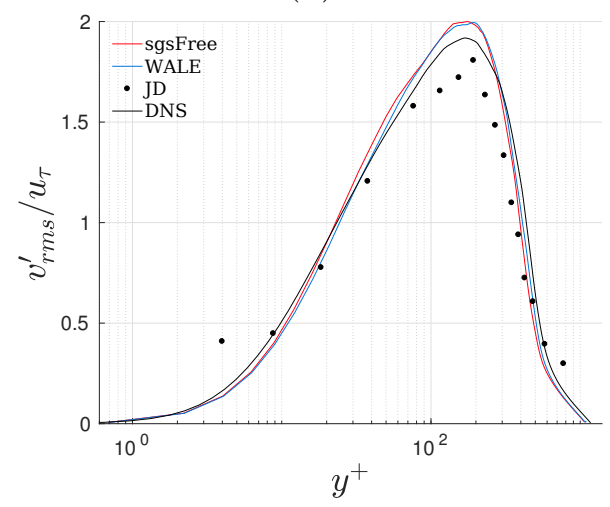

(f)

Figure E-11: Inner-scaled $v_{r m s}^{\prime}$ versus the wall-normal coordinate taken at dimensionless streamwise locations $x / h$ of approximately -3 (a), 4 (b), 6 (c), 10 (d), 15 (e) and 19 (f) using the fine-size BFS grid resolution. 


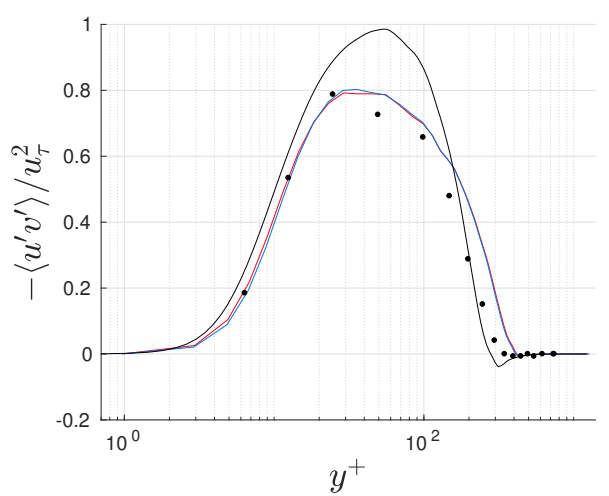

(a)

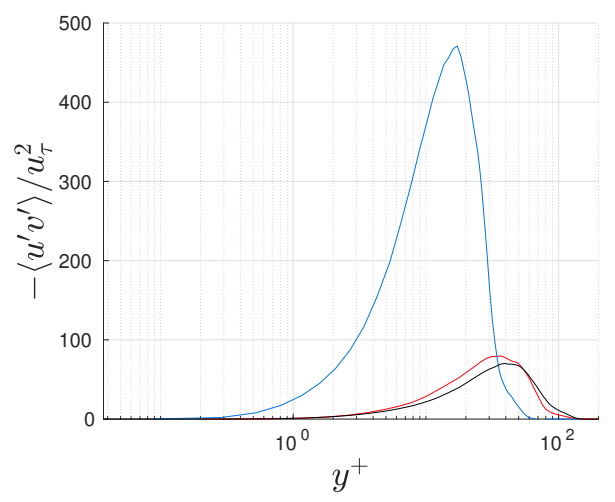

(c)

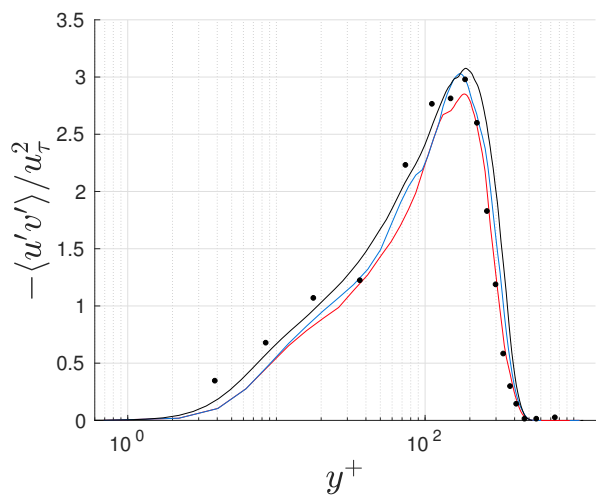

(e)

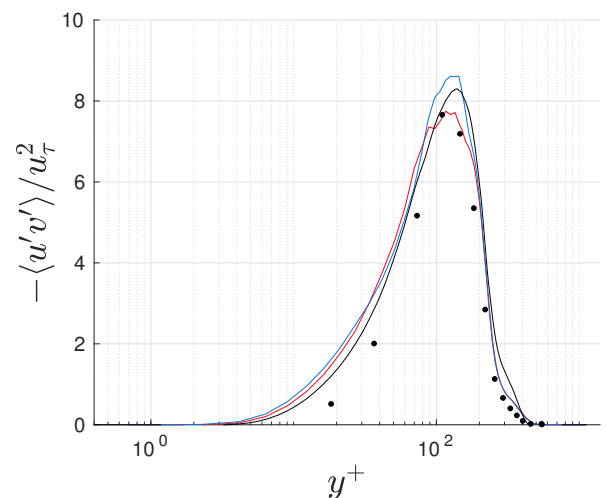

(b)

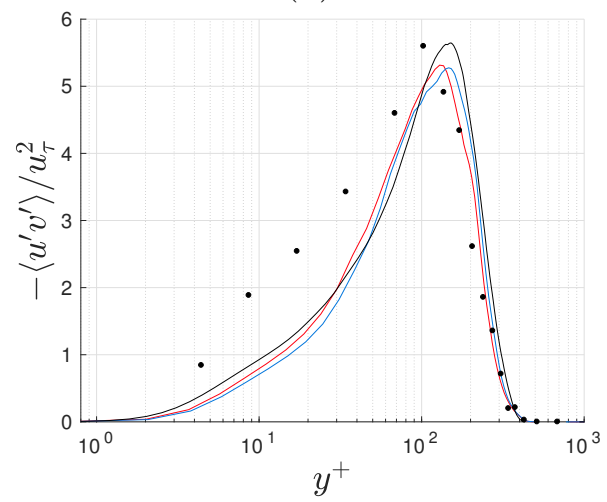

(d)

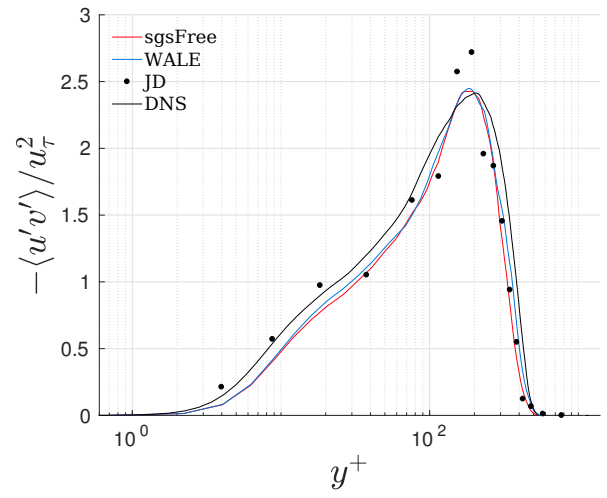

(f)

Figure E-12: Inner-scaled $-\left\langle u^{\prime} v^{\prime}\right\rangle$ versus the wall-normal coordinate taken at dimensionless streamwise locations $x / h$ of approximately -3 (a), 4 (b), 6 (c), 10 (d), 15 (e) and 19 (f) using the fine-size BFS grid resolution. 